Emanuela Chiapparini

Ehrliche Unehrlichkeit 
Emanuela Chiapparini

\section{Ehrliche Unehrlichkeit}

Eine qualitative Untersuchung der Tugend Ehrlichkeit bei Jugendlichen an der Zürcher Volksschule

Budrich UniPress Ltd.

Opladen, Berlin \& Toronto 2012 
Bibliografische Information der Deutschen Nationalbibliothek

Die Deutsche Nationalbibliothek verzeichnet diese Publikation in der Deutschen Nationalbibliografie; detaillierte bibliografische Daten sind im Internet über http://dnb.d-nb.de abrufbar.

Die vorliegende Arbeit wurde von der Philosophischen Fakultät der Universität Zürich im Herbstsemester 2011 auf Antrag von Prof. Jürgen Oelkers und Prof. Jan Skrobanek als Dissertation angenommen.

Gedruckt auf säurefreiem und alterungsbeständigem Papier.

Alle Rechte vorbehalten.

(C) 2012 Budrich UniPress, Opladen, Berlin \& Toronto

www.budrich-unipress.de

ISBN 978-3-86388-006-4

eISBN 978-3-86388-165-8

Das Werk einschließlich aller seiner Teile ist urheberrechtlich geschützt. Jede Verwertung außerhalb der engen Grenzen des Urheberrechtsgesetzes ist ohne Zustimmung des Verlages unzulässig und strafbar. Das gilt insbesondere für Vervielfältigungen, Übersetzungen, Mikroverfilmungen und die Einspeicherung und Verarbeitung in elektronischen Systemen.

Lektorat: Magdalena Kossatz, Berlin

Umschlaggestaltung: disegno visuelle kommunikation, Wuppertal - www.disenjo.de

Bildnachweis Umschlag: (C) shootingankauf - fotolia.com

Druck: paper\&tinta, Warschau

Printed in Europe 


\section{Inhaltsverzeichnis}

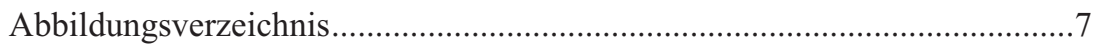

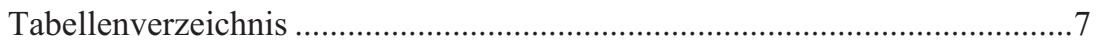

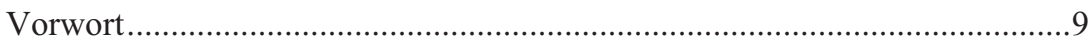

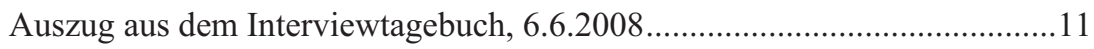

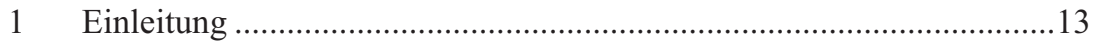

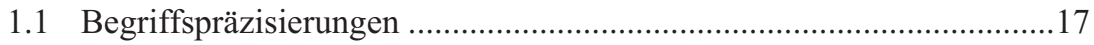

1.2 Aktualität der Tugenden in populären Erziehungsdebatten, in

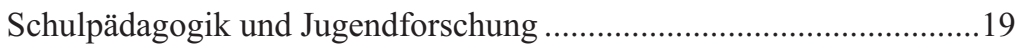

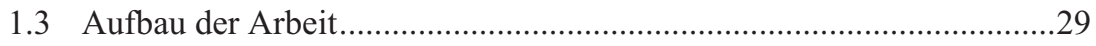

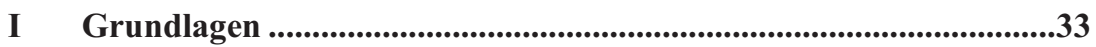

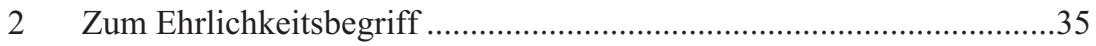

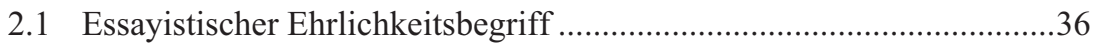

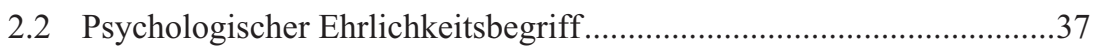

2.3 Erziehungsphilosophischer Ehrlichkeitsbegriff: Tugendkataloge ..........39

2.4 Ehrlichkeitsbegriff in der angelsächsischen Tugenddebatte..................49

2.5 Ehrlichkeitsbegriff als moralische Verhaltensweise.............................54

2.6 Ehrlichkeitsbegriff als loser theoretischer Rahmen für die Arbeit ........55

3 Kontextualisierung des Forschungsgegenstands .................................58

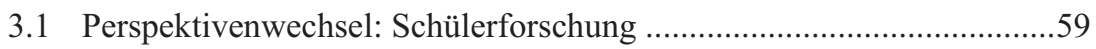

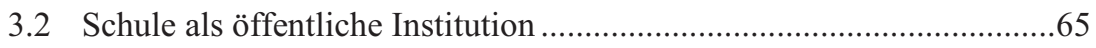

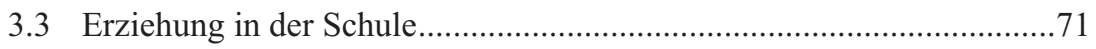

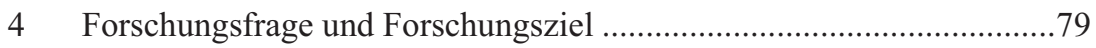

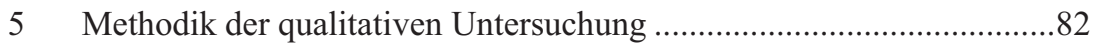

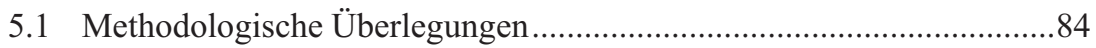

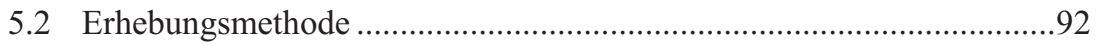

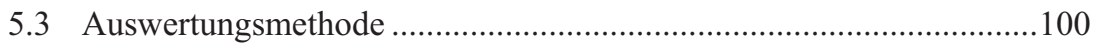

5.4 Forschungspraktisches Vorgehen ...................................................... 105

II Ergebnisse ...................................................................................121

6 Ehrlichkeitskategorien und Thematisierungsregeln.............................125

6.1 Ehrlichkeitskategorien und vielfältige Ehrlichkeitsregeln - Ein

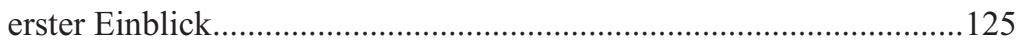

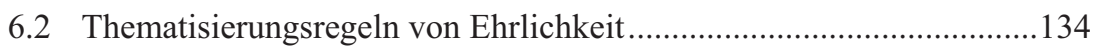


7 Von konventionellen Ehrlichkeitsregeln zu Ehrlichkeitsregeln der

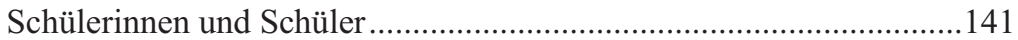

7.1 Zusammenhang von Ehrlichkeitsregeln und Schulregeln.....................142

7.2 Orientierungspunkt für Ehrlichkeitsregeln: Die Lehrperson ................146

7.3 Fachspezifische Varietät von Ehrlichkeitsregeln................................150

8 Komplexität der unkonventionellen Ehrlichkeitsregeln .....................151

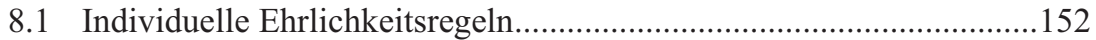

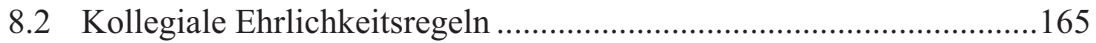

8.3 Ehrlichkeitsregeln zwischen Spaß und Ernst......................................182

8.4 Ehrlichkeitsregeln über den Schulkontext hinweg ............................186

9 Ehrlichkeitsregeln in realen Dilemmasituationen...............................190

9.1 Dilemma zwischen kollegialen und individuellen

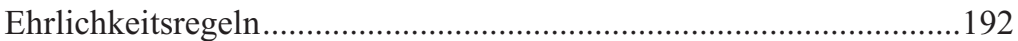

9.2 Dilemma zwischen individuellen und konventionellen

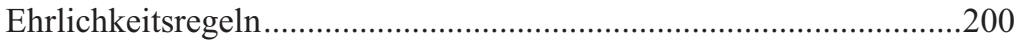

9.3 Dilemma zwischen kollegialen und konventionellen

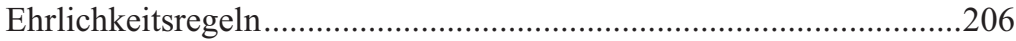

9.4 Dilemma zwischen individuellen, kollegialen und konventionellen

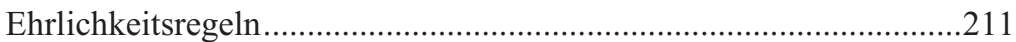

10 Zusammenfassung der Ergebnisse.................................................218

III Diskussion und Ausblick ..................................................................227

11 Diskussion der empirischen Ergebnisse ............................................229

11.1 Grenzen der Ehrlichkeitskategorien ,Wahrhaftigkeit',

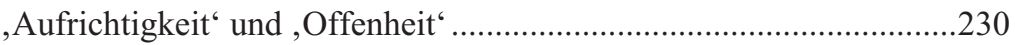

11.2 Grenzen der Thematisierung von Ehrlichkeit...................................232

11.3 Verhaltensorientierte Ehrlichkeitsregeln im Schulalltag .....................233

11.4 Verhaltensorientierte Ehrlichkeitsregeln in realen schulspezifischen Dilemmasituationen..............................................239

12 Zentrale Ergebnisse der Arbeit ..........................................................243

13 Ausblick: Schüler- und Jugendforschung ..........................................249

14 Ausblick: Erziehungsanforderungen und theoretisch fundierte

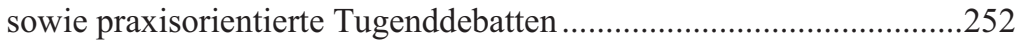

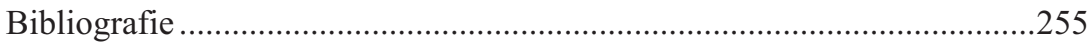

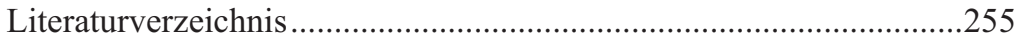

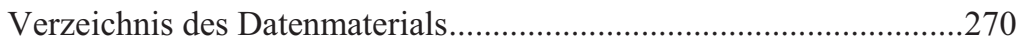

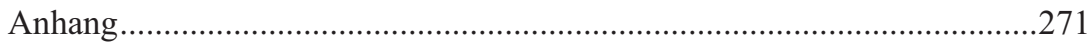




\section{Abbildungsverzeichnis}

Abb. 1: Sozialindex der Sekundarschulgemeinden des Kantons Zürich.....108

Abb. 2: Darstellungsweise der Ergebnisse..................................................124

Abb. 3: Darstellung von Ehrlichkeitsregeln in Dilemmasituationen und der damit zusammenhängenden Faktoren im Entscheidungsprozess

Abb. 4: Überblick der Ehrlichkeitsregeln in realen

Dilemmasituationen

\section{Tabellenverzeichnis}

Tab. 1: Aristotelische Tugenden

Tab. 2: Kindheitsforschung, Schulforschung, Schülerforschung und Jugendforschung im Vergleich

Tab. 3: Werte- und Tugenderziehung im Vergleich ....................................76

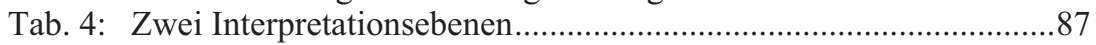

Tab. 5: Sinngehalte, empirische Erfassbarkeit, Interpretationsschritte und passende Fragestellungen

Tab. 6: Verhaltensweisen und Ehrlichkeitsregeln in Dilemmasituationen 



\section{Vorwort}

Die vorliegende Arbeit widmet sich der Frage, wie Schülerinnen und Schüler Ehrlichkeitspraxen an der Zürcher Volksschule deuten. Damit soll am Beispiel einer aktuellen Einzeltugend - Ehrlichkeit - in einem konkreten Kontext - Schule - die Tugenddebatte aus einer wissenschaftlichen und praxisorientierten Perspektive betrachtet werden. Die Perspektive der Adressatinnen und Adressaten von erzieherischen Bemühungen - Schülerinnen und Schüler - wird damit mittels eines sozialwissenschaftlichen Forschungszugangs erkundet und zugänglich gemacht.

Im Rahmen der Lizenziatsarbeit ${ }^{1}$ setzte ich mich bereits mit der theoretischen Begründung von Werteerziehung der gegenwärtigen Jugend auseinander. Ein Studienaufenthalt in Italien zu ethischen Themen weckte in mir das Interesse, mich mit dem Thema Tugenden und deren heutiger theoretischer und empirischer Relevanz zu beschäftigen. Im Zuge der alltäglichen Beobachtung stellte ich fest, dass in der Schulpraxis Fragen der Verhaltensweisen von Schülerinnen und Schülern zentral sind. Zudem stellen allzu oft Medienschaffende den Schulalltag anhand von Schlagwörtern wie ,Jugend ohne Tugend', ,Erziehungsdefizite' und ,ausgebranntes Lehrpersonal ${ }^{\text {* einseitig }}$ dar. Gleichzeitig befinden sich in der Schweiz genügend Kaderleute und Fachspezialisten, welche wöchentlich ihre drei wichtigsten Tugenden preisgeben, die im Eingangsinterview des Stellenmarkts derselben Zielgruppe (NZZexecutive) in der Zürcher Tageszeitung NZZ veröffentlicht werden.

Mit dem Dissertationsprojekt erhielt ich die Gelegenheit, mich mit den aufgeführten und angerissenen Themenkreisen vertieft zu beschäftigen.

Seit Beginn der Arbeit war es mir wichtig, die Jugendlichen selbst zu Wort kommen zu lassen. Motivation dazu gab mir ein langjähriges Engagement in der Jugendarbeit. Zudem fand ich eine Zusammenarbeit mit jungen Menschen spannend, welche mindestens acht Jahre die Volksschule besuchten und ein Erfahrungswissen im Schulkontext angesammelt haben, das bislang zu wenig zugänglich gemacht wird.

Zum Gelingen dieser Arbeit haben viele Personen beigetragen, denen ich an dieser Stelle meinen Dank aussprechen möchte.

Ein spezieller Dank geht an alle Schulleiter und Lehrpersonen, welche kollaborativ das Projekt unterstützten und mir den Zugang zu den erfragten Klassen ermöglichten. Insbesondere danke ich den Schülerinnen und Schü-

1 Chiapparini, Emanuela (2001): Werterziehung der gegenwärtigen Jugend? Eine Untersuchung zur theoretischen Begründung, ausgehend von der historischen und gegenwärtigen Autonomieforderung mit Schwerpunkt der Werterziehung gemäss dem Ansatz von R. S. Peters. Lizenziatsarbeit. Pädagogisches Institut der Universität Zürich. 
lern für ihre Teilnahmebereitschaft und Offenheit. Denn dank des Vertrauensvorschusses der Interviewten ist qualitative Forschung erst möglich.

Besonders danke ich Prof. Dr. Jürgen Oelkers für die Unterstützung, für die großzügigen Freiheiten in der Gestaltung der Dissertation und für seine ermutigenden und klärenden Hinweise.

Mein Dank gilt auch Prof. Dr. Jan Skrobanek, der sich als Zweitgutachter zur Verfügung gestellt hat. Sein Interesse für mein Forschungsthema und Diskussionen darüber waren motivierend und förderlich.

Ein Dank geht an alle, die kritische und konstruktive Kommentare in Forschungskolloquien, Tagungen und Forschungswerkstätten in der Schweiz, Deutschland und England zu verschiedenen Abschnitten dieser Arbeit gegeben haben.

Dem Schul- und Sportdepartement der Stadt Zürich, der Katholischen Kirche im Kanton Zürich und dem Jugendforschungsprojekt SoYouth an der Universität Zürich danke ich für die Publikationszuschüsse. Für das Lektorat richte ich Claudia Kühne ein besonderes Dankeschön aus.

Die Arbeit widme ich Chiara Lubich, welche in ihrem theologischen Gedankengut einen Zusammenhang zwischen Ethik und Alltag anstrebte und mich zu dieser Arbeit ermutigte. Sie verstarb 2008 - einige Monate nachdem ich die Forschungsarbeit aufnahm. 


\section{Auszug aus dem Interviewtagebuch, 6.6.2008}

Im Korridor des Schulhauses herrscht Stille. Es ist kurz vor Mittag. Die Unterrichtsstunden sind noch im Gang. Ein Schüler sitzt auf einer Bank neben dem Eingang zum Klassenzimmer, in dem ich mit einer Lehrerin verabredet bin. Er ist sichtlich verärgert. Ich spreche ihn an und erkundige mich, ob alles in Ordnung sei. Er erklärt, dass er in der Schulstunde frech gegenüber der Lehrerin gewesen ist. Diese habe ihn hinausgeschickt, was ihm aber egal sei, denn er habe bereits eine Lehrstelle. Die Pausenglocke läutet, und die Stille wird durch lautes Zurufen, Gelächter und Gespräche der aus den verschiedenen Schulzimmern herausströmenden Schülerinnen und Schüler schlagartig unterbrochen. Im Nebenzimmer höre ich das Dröhnen eines Staubsaugers, und ein Mädchen macht sich lustig über diejenigen, die drinnen noch etwas aufräumen müssen.

Drei großgewachsene Schüler kommen aus diesem Zimmer heraus und diskutieren eifrig und laut darüber, wie sie in der soeben geschriebenen Geometrieprüfung gespickt ${ }^{2}$ haben. Vorerst höre ich nicht richtig hin, denn ein Schüler der 3. Oberstufe (Typus B), den ich vor einem Monat interviewt habe, grüßt mich und wir unterhalten uns. (...) Als unser Gespräch langsam zu Ende geht, realisieren die besagten drei Burschen, dass ich als erwachsene Person präsent bin, und sie unterbrechen plötzlich ihr angeregtes Gespräch und schauen zu mir rüber. Eine peinliche Stille stellt sich ein. Ich kommentiere: „Ihr habt es ja ziemlich lustig!“. Sie stimmen dem zu, einer zeigt mir spontan eine Hand voll zerknüllter Spickzettel. Wir lachen alle, und sie erzählen stolz und ausführlich, wie sie beim Spicken vorgegangen sind. Leider habe der Lehrer einen Mitschüler auf frischer Tat ertappt. Nun ist dieser noch im Schulzimmer. Sie sind alle aus der dritten Oberstufe, Typus A, und zwar aus der Klasse des Schülers, den ich heute Morgen interviewt habe. Der Schüler mit den Spickzetteln in den Händen erklärt mir (ich verstehe ihn nicht richtig, da er gewisse Worte verschluckt), dass er mit Mitschülern während eines ganzen Semesters regelmäßig die Aufgaben der Geometrietests im Voraus aus dem Lehrerpult dieses Lehrers entwendet habe. Sie würden nicht klauen, denn sie legten die Aufgaben, nachdem sie diese abgeschrieben hatten, wieder an den gleichen Ort zurück. Sie ließen die Prüfungsaufgaben von einem Mitschüler lösen. Alle waren zuvor ziemlich schlecht in Geometrie. Mit dieser Technik erzielten sie nun nur noch Noten von 5.5. Dabei passten sie auf, nicht zu viele Noten 6 zu erhalten, weil dies sonst aufgefallen wäre.

2 Mit Spicken ist das schriftliche (ev. mit Notizen) Schummeln gemeint. 
Einige Mitschüler fanden ihr Verhalten, nicht richtig ' und verpetzten sie. Es gab ein Elterngespräch und die Zeugnisnote wurde beispielsweise bei dem Schüler, der mir die Sache erzählte, von einer 5 auf eine 4 gekürzt. Ich sage den Jungen, dass in dem Fall ihr Plan nicht aufgegangen sei. Sie sind anderer Meinung, denn sie hatten Spaß. Sie sind sich aber einig, in der Berufslehre diesen ,Sport' nicht weiter auszuüben. Alle Anwesenden haben eine Lehrstelle. Plötzlich bitten sie mich, nichts den Lehrpersonen zu verraten. Ich gebe mein Einverständnis. 


\section{$1 \quad$ Einleitung}

Der Tagebuchauszug, der dieser Einleitung vorangeht, gibt ein Ad-hocGespräch mit Schülern eines städtischen Oberschulhauses wieder. ${ }^{3}$ In den Äußerungen der drei Schüler kommen die expliziten und impliziten ,ehrlichen' Unehrlichkeitsregeln zum Ausdruck, nach denen sie sich verhalten. Diese lassen sich folgendermaßen zusammenfassen: Wer in die Schule geht, lernt, unehrlich zu sein, um gute Noten zu erzielen. Dies ist insofern zwiespältig, als so die unkonventionellen Ehrlichkeitsregeln des Schummelns mit den konventionellen Regeln des Nichtschummelns verbunden werden. ${ }^{4}$ Denn die drei Schüler sind sich einig, dass sie die konventionellen Regeln in der Berufsschule befolgen werden.

Die Verknüpfung von konventionellen und unkonventionellen Ehrlichkeitsregeln zeigt die Thematik der hier vorliegenden Untersuchung: Die schulrelevante Tugend Ehrlichkeit wird aus der Sicht der Jugendlichen untersucht. Dabei wird deren Begriff und Praxen in den Kontext der Zürcher Volksschule gestellt, in der neben Bildung ebenso sehr in Erziehung investiert wird. Der Titel Ehrliche Unehrlichkeit ist programmatisch zu verstehen. Die Zusammensetzung ist nur scheinbar unlogisch. Denn Ehrlichkeit als Verhaltensweise und Sekundärtugend zeichnet sich durch eine ambivalente Eigenschaft aus: Es ist nicht in jeder Situation angebracht, ehrlich zu sein so auch nicht im Schulkontext, wie ein weiteres Beispiel zeigt: Um die Klasse vor einer Kollektivbestrafung zu bewahren, meldet sich der 14-jährige Thomas $^{5}$ als Täter, obwohl er den Stuhl nicht beschädigt hat. Er muss zwar einen Nachmittag nachsitzen. Im Gegenzug erhält er die Aufmerksamkeit seiner Mitschülerinnen und -schüler, und sein Ansehen in der Klasse steigt. Die unehrliche Verhaltensweise gegenüber dem Lehrer ist gegenüber den Mitschülerinnen und -schülern ,ehrlich“ gemeint und damit zulässig.

Ehrlichkeit stellt also im Schulalltag eine hochaktuelle Tugend dar. Mit Tugend sind sowohl moralische Verhaltensweisen als auch innere Einstellungen und Charakterzüge erfasst. Der Begriff ,Tugend' in diesem allgemeinen Verständnis wird inflationär verwendet. Unter Tugenden summieren Philoso-

3 In qualitativen Forschungsverfahren ist es üblich, während der Erhebungsphase ein Interviewtagebuch zu führen, in dem Eindrücke, Beobachtungen und Reflexionen festgehalten werden (vgl. Kap. 5.2.2).

4 In dieser Arbeit wird die Unterscheidung in konventionelle versus unkonventionelle Ehrlichkeitsregeln vorgenommen (vgl. dazu Kap. 1.1 und Ergebnisse (Teil II)).

5 Alle Namen wurden anonymisiert. 
phen gemäss den jeweiligen theoretischen Ansätzen sowohl zentrale Tugenden (sog. Vernunftstugenden, Primärtugenden oder Kardinaltugenden) wie etwa Gerechtigkeit oder Tapferkeit als auch Einzeltugenden (sog. Charaktertugenden oder Sekundärtugenden) wie etwa Respekt, Ehrlichkeit und Fleiß. Allerdings besteht keine scharfe Grenze zwischen den Einzeltugenden, und ebenso fehlt es an klaren Auswahlkriterien, nach denen die Einzeltugenden zu übergeordneten Tugenden summiert werden.

Tugenden sind grundsätzlich Untersuchungsgegenstand in der Ethik unterschiedlicher Disziplinen wie Philosophische Ethik, Medizinische Ethik, Theologische Ethik oder Betriebswirtschaftliche Ethik, aber auch in der Praxis und in theoretischen Ansätzen der Erziehung wurden Tugenden im Verlauf der Geschichte diskutiert. Die Tatsache, dass sich unterschiedliche Disziplinen mit dem Thema Tugend beschäftigen, bringt zwangsläufig Überschneidungen und gegenseitige Durchdringungen mit sich. Es ist daher nicht einfach, einen Überblick über einzelne erziehungswissenschaftliche Traditionen zu gewinnen, die sich mit Tugenden und insbesondere mit Ehrlichkeit im Schulkontext auseinandersetzen. Im deutschsprachigen Raum gibt es keine übergreifende schulpädagogische Tugendforschung - übrigens ganz im Unterschied zur angelsächsischen Forschung, die seit etwa dreißig Jahren Tugenden unter character education und citizenship education diskutiert (vgl. Kap. 2.4).

Insofern kann es in dieser Arbeit nicht mein Anliegen sein, die Entstehung und den Verlauf der Tugendforschung chronologisch und auf Basis exklusiver Disziplinen bis ins Detail nachzuzeichnen. Gleichwohl scheint es sinnvoll zu sein, zuerst auf einer disziplinübergreifenden Ebene schlaglichtartig die Aktualität der Tugenden und damit der Ehrlichkeit aufzuzeigen und einen Einblick in den Forschungsstand zu geben (Kap. 1.2). Auf ähnliche Weise wird im Kapitel zum Ehrlichkeitsbegriff (Kap. 2) und zur Kontextualisierung des Forschungsgegenstands (Kap. 3) disziplinübergreifend verfahren. Vor dem Hintergrund der thematischen und wissenschaftlichen Kontexte, in denen meine Arbeit steht, begründe ich schließlich mein spezifisches Interesse an der Tugend Ehrlichkeit aus schulpädagogischer Perspektive und formuliere die sich daraus ergebenden Fragestellungen für meine Arbeit (Kap. 4).

Obwohl Tugenden gegenwärtig in den populären Erziehungsdebatten, Lehrerfachzeitschriften sowie Jugenduntersuchungen des deutschsprachigen Raums aktuell sind, ist der Tugendbegriff in der Alltagssprache unüblich, und als Erziehungsgegenstand sind Tugenden in der Schulpädagogik kaum zu finden. Gleichzeitig besteht in der Praxis und Theorie der Erziehung eine lange Tradition der Tugenden: Die Spannbreite reicht von den antiken Griechen über christliche und humanistische Tugendansätze bis zu heutigen erziehungsphilosophischen und bildungspolitischen Tugenddebatten, die besonders rege im angelsächsischen Raum geführt werden. 
Trotz der langen Tradition der Tugenden ist es keine Selbstverständlichkeit, dass Erziehungsphilosophen Ehrlichkeit zu den Tugenden zählen. So wird beispielsweise Ehrlichkeit von zwei griechischen Tugendklassikern nicht direkt erwähnt: Platon befasst sich nicht mit Ehrlichkeit und Aristoteles thematisiert sie unter dem Aspekt von ,Wahrhaftigkeit'. Im 18. Jahrhundert erfuhr die Tugend Ehrlichkeit im Zusammenhang mit den bürgerlichen Tugenden als gesellschaftlich anerkannte Tugend ihren Höhepunkt, was bis Mitte des 20. Jahrhunderts anhielt.

Über die historische Zeitspanne hinweg ist den unterschiedlichen Tugendansätzen gemeinsam, dass Erziehungsphilosophen diese in Form von Tugendkatalogen diskutierten. ${ }^{6}$ Die traditionellen Tugenden wurden dabei stark ideologisiert und reproduziert. Nach dem Zweiten Weltkrieg verloren theoretische Tugendansätze im Fachbereich der Erziehung an Bedeutung. Seit den 1960er Jahren werden sie vollständig durch angeblich neutralere Ansätze der Werteerziehung und sozialen Kompetenzen ersetzt. Spätestens seit der Pisa-Studie im Jahr 2000 dominieren Ansätze der sozialen Kompetenzen das wissenschaftliche Erziehungsfeld. Es stellt sich also die berechtigte Frage, weshalb ich mich dennoch mit dem scheinbar veralteten Tugendansatz im gegenwärtigen Schulkontext befasse. In einer sachlichen und kritischen Weise werde ich dies aus fünf zentralen Gründen tun:

- Der Tugendbegriff erlaubt Reibungen: Eine Person kann sozial kompetent und gleichzeitig unehrlich sein. So handelt Thomas im erwähnten Beispiel sozial kompetent, indem er einen Vorfall eingesteht. Gleichzeitig ist er unehrlich, weil er lügt, indem er vorgibt, die handelnde Person des Vorfalls zu sein. Nur mit dem neutralen Kompetenzbegriff oder Wertbegriff wäre es nicht möglich, diesen reibungsvollen Sachverhalt differenziert darzustellen.

- Die Tugend Ehrlichkeit ist schulrelevant: Lehrpersonen kontrollieren die Einzeltugend Ehrlichkeit im Schulalltag und sanktionieren festgestellte unehrliche Verhaltensweisen. Somit ist Ehrlichkeit von großer schulischer Relevanz. Jedoch steht die ambivalente Eigenschaft von Ehrlichkeit (die Frage nach der situativen Angemessenheit) dem Erziehungsauftrag der Volksschule entgegen, was zu individuellen, kollegialen und schulspezifischen Dilemmas im Schulalltag führen kann, wie das Beispiel von Thomas deutlich zeigt.

- Fehlende Sichtweise der Heranwachsenden: Erziehungsphilosophinnen und -philosophen formulieren theoretische Tugendansätze, ohne die Sichtweise der Heranwachsenden und damit die Adressatin sowie den Adressaten der Erziehungspraxis zu berücksichtigen - und das, obwohl vor rund dreißig Jahren ein Perspektivenwechsel in der Schulpädagogik

6 In der Philosophie werden Tugenden seit Mitte des 20. Jahrhunderts in der Tugendethik diskutiert (vgl. Kap. 1.2). 
stattfand, indem die Sichtweise der Schülerinnen und Schüler $(\mathrm{SuS})^{7}$ zum Forschungsgegenstand wurde und sich als ,Schülerforschung ' etablierte. ${ }^{8}$ Ein Erziehungsthema wie das der Tugenden wird also bislang ungenügend in der Erziehungswissenschaft aus der Sichtweise der SuS betrachtet.

- Fehlende praxisrelevante Tugend- und Ehrlichkeitsdefinitionen: Theoretische Tugendansätze berücksichtigen den Umgang mit Tugenden im Alltag nicht. Damit fehlen praxisrelevante Definitionen der Tugenden und folglich auch der Ehrlichkeit.

- Einstellungen zu einzelnen Sekundärtugenden, aber nicht deren Deutungen sind bislang empirisch zugänglich: Standardisierte Jugenduntersuchungen liefern das überraschende Ergebnis, dass Jugendliche Sekundärtugenden wie Ordnung und Fleiß regelmäßig höher bewerten als allgemein erwartet. Wie Jugendliche Sekundärtugenden deuten und wie sie sich im Alltag an diesen orientieren, bleibt hingegen ungeklärt, was jedoch mit einem qualitativen Forschungszugang untersucht werden kann.

Bezogen auf die vorliegende Untersuchung heißt das Folgendes: Das Forschungsprojekt untersucht unter dem Fokus einer schulrelevanten Tugend der Ehrlichkeit - die Sichtweise von Jugendlichen am Beispiel der Zürcher Volkschule qualitativ. Damit will die Untersuchung die Tugend Ehrlichkeit im Schulalltag und im Kontext der Erziehungsphilosophie wissenschaftlich ergründen und erstmals empirisch fundierte Aussagen zu Deutungen von Ehrlichkeitspraxen aus der Sicht der SuS machen.

Ausgehend vom Forschungsstand der Tugenden in der Schulpädagogik und Jugendforschung wird zunächst die leitende Forschungsfrage für das Forschungsprojekt entwickelt. Anschließend stellt sich in allgemeiner Weise die Frage nach der Definition des Ehrlichkeitsbegriffs und der dahinterstehenden theoretischen Ansätze, was in einem zweiten Schritt zu klären ist. In einem dritten Schritt werden die Schülerforschung, die Schule als öffentliche Institution, theoretische Erziehungsansätze im Bereich Schule sowie der Erziehungsauftrag der Zürcher Volksschule kritisch untersucht. Mit den ersten drei Arbeitsschritten (entsprechen den ersten drei Kapiteln) ist der Forschungskontext und das Vorwissen für die explorative Untersuchung festgelegt.

Im Zentrum der Arbeit steht der empirische Teil, in dem der Frage nach der Rekonstruktion von expliziten und impliziten Ehrlichkeitsregeln ausgehend von der Forschungsfrage nachgegangen wird: Wie deuten SuS Ehrlichkeitspraxen im Schulalltag? Zu vermuten ist, dass die Schule als Institution

7 Zwecks besserer Lesbarkeit werden im Folgenden ,Schülerinnen und Schüler ' mit ,SuS“ abgekürzt und sonst überwiegend weibliche und männliche Endungen verwendet.

8 Der Ausdruck ,Schülerforschung erfasst inhaltlich die Perspektive der Schülerinnen und Schüler. Zugunsten des Leseflusses ist der Term auf die männliche Form reduziert. 
Ehrlichkeit fördert und gleichzeitig Unehrlichkeit erzeugen kann, wie das Eingangsbeispiel der drei Schüler klar aufzeigt. Der Einblick in die Deutungen von Ehrlichkeitspraxen aus der Sicht der SuS und deren Ehrlichkeitsregeln (Ergebnis der Untersuchung) ermöglicht, öffentliche Tugendansätze, theoretische Tugendansätze und schulpädagogische Erziehungsanforderungen einer kritischen Überprüfung zu unterziehen, indem dazu empirisch begründete Hinweise geliefert werden. Weiter können wichtige Anhaltspunkte gewonnen werden, wie Lehrpersonen SuS angemessen in Ehrlichkeitsdilemmas begegnen und wo theoretische Ehrlichkeitsansätze sowie Ehrlichkeitsdefinitionen ansetzen können.

Das folgende Unterkapitel klärt vorerst zentrale Begriffe, welche nicht nur Grundlage für die Einordnung des Forschungsvorhabens, sondern auch die Basis für die empirische Analyse sind (Kap. 1.1). Anschließend wird die Aktualität der Tugenden aus der Perspektive der populären Erziehungsdebatte, der Schulpädagogik und der Jugendforschung skizziert (Kap. 1.2). Mit Schulpädagogik und Jugendforschung ist der Fokus auf jene zwei Einzelbereiche der Erziehungswissenschaft gerichtet, auf die sich mein Forschungsvorhaben gründet. Und schließlich gibt das dritte Kapitel eine Orientierung über den Aufbau der Arbeit (Kap. 1.3).

\subsection{Begriffspräzisierungen}

Ehrlichkeit wird als Verhaltensweise definiert, welche ausgehend von Erfahrungsberichten in Abgrenzung zu Täuschungen und möglichen taktischen und strategischen Spielen konzipiert werden kann. Im verhaltensorientierten Ansatz ist Ehrlichkeit als situationsabhängige und als kontextabhängige Tugend zu verstehen (vgl. Kap. 2).

Ehrlichkeit ist in dieser Arbeit gezielt als Verhaltensweise definiert, und nicht als Handlung. Mit Handlungen werden intentionale Tätigkeiten bezeichnet. Hingegen beziehen sich Verhaltensweisen auf intuitive und situationsabhängige Komponenten, welche in Umgangsformen vorkommen (vgl. Miebach 2010, S. 20ff.). Diese wiederum beruhen auf Gewohnheiten, Routinen oder Wiederholungen, die im Zuge einer Abstraktion zu Stereotypen formuliert werden können.

Ehrlichkeitspraxen: Im schulischen Kontext sind unterschiedliche Verhaltensweisen zu beobachten, die im Zusammenhang mit Ehrlichkeit und Unehrlichkeit stehen, wie beispielsweise das Schummeln während der Schultests oder das Eingestehen von unterlassenen Hausaufgaben. Eine Auflistung von ehrlichen und unehrlichen Verhaltensweisen kann lang ausfallen, denn die Zuordnung der einzelnen Aktivitäten ist von den jeweiligen Deutungen der involvierten Personen abhängig. Um einen möglichst breit angelegten 
Begriff zu verwenden, der die gedeuteten Tätigkeiten erfasst, die im Zusammenhang mit Ehrlichkeit stehen, bietet sich der Begriff der Ehrlichkeits- und Unehrlichkeitspraxen als passend an. Der Praxisbegriff ist in den kulturtheoretischen Ansätzen verankert (vgl. Überblick Moebius 2011; zu Praxistheorien Reckwitz 2003). Beispielsweise beruht Praxis in Anlehnung an Soeffners Ansatz auf sozialer und gedeuteter Wirklichkeit, die auf „Deutungsgewohnheiten und Reziprozitätsunterstellungen“ (Kurt 2011, S. 232) basiert, „die sich in der Praxis bewährt haben“" (ebd.). Folglich ist mit ,Ehrlichkeits- und Unehrlichkeitspraxen" der situative sowie der kontextabhängige Charakter von gedeuteten ehrlichen und unehrlichen Verhaltensweisen festgehalten, die sich an expliziten und impliziten Ehrlichkeitsregeln orientieren. Damit ist eine gedeutete Regel gemeint, welche eine ,implizite, informelle Logik der Praxis" (Reckwitz 2003, S. 282ff.) erfasst, die den sozialen Akteuren meistens nicht reflexiv zur Verfügung steht. Aus Gründen der Leserfreundlichkeit werde ich im Folgenden allein das Wort ,Ehrlichkeitsregeln' und ,Ehrlichkeitspraxen' verwenden und ,Unehrlichkeitsregeln“ sowie ,Unehrlichkeitspraxen' implizit mitdenken.

Subjektive Deutungen: Ehrlichkeit als eine Verhaltensweise wird nicht als solche beobachtet, sondern die Deutung von ehrlichen Verhaltensweisen der SuS wird auf Basis deren subjektiver Wahrnehmungen untersucht. Wie deuten SuS ihre Ehrlichkeitspraxen? Welche explizit oder implizit Ehrlichkeitsregeln, an die sie ihre Verhaltensweisen orientieren, lassen sich daraus gewinnen? Welche Unehrlichkeitsregeln werden dabei als zulässig gedeutet? Falls Wiederholungen der Deutungen von Ehrlichkeitsregeln im Fall selbst oder über den Fall hinweg auftreten, wird das fallimmanentes oder fallüberschreitendes ,Deutungsmuster' genannt. Der Begriff des Dilemmas wird für Reibungsfälle zwischen unterschiedlichen Ehrlichkeitsregeln verwendet. Dabei geht es nicht um das hypothetische Dilemma, wie es im Kohlbergschen entwicklungspsychologischen Ansatz thematisiert wird (vgl. Kohlberg et al. 1995; Latzko 2006, S. 23f.), sondern um die alltäglichen Dilemmas, von welchen die SuS berichten. Daher verwende ich den Ausdruck, reale Dilemmas', um eine sichtbare Abgrenzung zum verbreiteten Kohlbergschen Ansatz zu markieren.

Im Datenmaterial sind explizite und implizite Ehrlichkeitsregeln enthalten, an denen die SuS ihre Verhaltensweisen orientieren. Die Kategorie konventioneller und unkonventioneller Ehrlichkeitsregeln wird eingesetzt, um die vielfältigen Ehrlichkeitsregeln annähernd zu systematisieren. Konventionelle Regeln in der Schule können einen normativen Charakter haben. Jedoch wird im empirischen Teil ,Regel' nicht i. S. v. normativen Regeln wie Du sollst nicht lügen verwendet; der Fokus ist auf Normalität oder normalisierende Regeln gerichtet, etwa auf Wenn-dann-Konstruktionen.

Unter kollegialen Ehrlichkeitsregeln schließlich sind freundschaftliche Regeln zu verstehen, nach welchen SuS untereinander ihre Beziehungen 
pflegen. Hingegen beziehen sich kollektive Ehrlichkeitsregeln auf den ganzen Klassenverband. Sie setzen das Einhalten der Ehrlichkeitsregeln bei allen SuS einer Klasse voraus. Das Übertreten einer kollektiven Ehrlichkeitsregel kann entsprechend eine klasseninterne Sanktion zur Folge haben. Hingegen zieht das Nichteinhalten einer kollegialen Ehrlichkeitsregel nur einen möglichen Bruch mit den jeweiligen Kollegen nach sich.

Für die empirische Untersuchung gehe ich nicht in erster Linie von Normen und Moral aus, sondern arbeite sozialwissenschaftlich die Sichtweise der SuS heraus. Denn auf Anforderungen durch Normen reagiert man üblicherweise im öffentlichen Raum und im Gespräch zustimmend, allerdings können Verhaltensweisen und die an diesen orientierten Ehrlichkeitsregeln je nach Situation anders ausfallen (Tautologie). Im Ergebnisteil kann möglicherweise festgestellt werden, womit Normen und Moral zusammenhängen, worauf ich, falls nötig, in der Diskussion (Kap. 11) eingehen werde. Hier präsentiere ich vorerst die Begriffsverwendung von ,Normen “ und ,Moral', die dieser Arbeit zugrunde liegt:

Normen sind Verhaltensanforderungen und garantieren die Regelmäßigkeit von Verhaltensabläufen, die von allgemein vertretenen Werten geprägt sind.

Moral wird im üblichen Sinne verwendet als inhaltlicher Grundbestand von allgemeinen Wertvorstellungen, die sich unmittelbar auf singuläre Verhaltensweisen beziehen und damit dem gesellschaftlichen, historischen und kulturellen Wandel unterliegen. Hingegen bezieht sich Moralität auf die Grundlagen und Bedingungen sowie Prinzipien der Moral und situiert sich auf einer zeitlosen und abstrakten Ebene. ,Moral' und ,Sitte' gleichbedeutend verwendet ebenso wie ,Moralität‘ und ,Sittlichkeit‘ (vgl. Höffe 2008).

\subsection{Aktualität der Tugenden in populären Erziehungsdebatten, in Schulpädagogik und Jugendforschung}

Das Thema der Tugenden und damit auch der Ehrlichkeit ist in der heutigen Zeit zwar interdisziplinär relevant, es wird jedoch, was die begriffstheoretischen Voraussetzungen sowie die empirischen Befunde betrifft, einseitig und teilweise defizitär behandelt. Ehrlichkeit ist in der Schulpraxis hochaktuell, jedoch im Hinblick auf die wissenschaftliche Auseinandersetzung bislang vernachlässigt. Daher gehe ich zunächst auf die kontextuelle Verortung der Arbeit ein und untersuche an dieser Stelle ausgewählte Bereiche von Tugenddebatten, welche einerseits die Aktualität des Themas Tugend aufzeigen und andererseits in den Grundzügen den gegenwärtigen Forschungsstand für 
die Tugend Ehrlichkeit in der Schulpädagogik und Jugendforschung darstellen. Daraus entwickelt sich meine leitende Forschungsfrage.

Im Einzelnen zeigt das folgende Unterkapitel eingangs auf, wie heute Tugenden und damit auch Ehrlichkeit in den populären Erziehungsdebatten mittels Publikationen und Leserbriefen rege diskutiert werden (Kap. 1.2.1). Darüber hinaus wird die gegenwärtige geringe Bedeutung der Tugenden in der wissenschaftlichen Schulpädagogik beleuchtet (Kap. 1.2.2). Demgegenüber zeigen erstaunlicherweise Befunde aus neueren standardisierten Jugenduntersuchungen auf, dass Tugenden bei den Jugendlichen an Bedeutung gewonnen haben (Kap. 1.2.3).

\subsubsection{Florierende öffentliche Erziehungsdebatten zu Tugenden}

Am Beispiel der erfolgreichen Buchveröffentlichung Lob der Disziplin (Bueb 2006) und der heftigen Reaktion von Lesern auf den unkonventionellen Zeitungsartikel Wie Kinder verantwortungsvolles Lügen lernen (Mainpost Nr. 176, 2.8.2004, S. D1) lässt sich die Aktualität der Tugenden in öffentlichen Erziehungsdebatten des deutschsprachigen Raums klar aufzeigen.

Schulisch sind Tugenden durch Disziplin zu erwerben (Bueb 2006). Dies postuliert Bernhard Bueb, ehemaliger Schulleiter des Internats ,Schloss Salem‘. Mit strenger Führung durch die Lehrkräfte lassen sich gemäss Bueb viele Erziehungsschwierigkeiten in der Schule lösen, von denen die Medienschaffenden regelmäßig berichten. Solche und ähnliche Veröffentlichungen finden, trotz Einwänden gegen das wissenschaftlich nicht fundierte Vorgehen oder gegen totalitäre Bildungsideale (Brumlik et al. 2007), grosse Resonanz in den Medien und bei einer breiten Leserschaft. Bueb argumentiert moralisch, indem er voraussetzt, was gut und schlecht für die Heranwachsenden ist, ohne dabei Kontextwissen und die Sichtweisen der $\mathrm{SuS}^{9}$ zu berücksichtigen. Er beschreibt zwar punktuell Schul- und Unterrichtsbeispiele, jedoch unsystematisch und ohne den Qualitätskriterien der empirischen Forschung gerecht zu werden. Somit liefert er seine Ergebnisse der Kritik der Unwissenschaftlichkeit und Willkür aus.

So lassen sich auch die unverhältnismäßigen und feurigen Antworten auf den Artikel Wie Kinder verantwortungsvolles Lügen lernen in der Mainpost erklären. Journalist und Redaktionsmitglied Nils Graefe vertritt darin die unkonventionelle Meinung von Winfried Böhm zu Alltagslügen: Kleine Lügen machen das Leben erträglich. Daraus zieht Böhm den Schluss, es sei notwendig, dass Kinder verantwortungsbewusst lügen lernen, um sich in der Gesellschaft zurechtzufinden. Die unerwartet negative Reaktion der Leserschaft auf Böhms normabweichende Thematisierung der Untugend Lügen

9 In der Kinder- und Jugendforschung, die sich auf die Schule bezieht, ist ein Perspektivenwechsel von der Lehrperson zum SuS zu beobachten, auf den Kap. 3.1 eingeht. 
bzw. der Tugend Ehrlichkeit nötigte die Redaktion dazu, eine weitere Erklärung des Professors einzuholen und diese zu publizieren.

Zusammenfassend ist festzuhalten, dass in der populären Erziehungsdebatte, die in der Schweiz ähnlich wie in Deutschland verläuft, Erziehungsdefizite (vgl. bspw. Gerster und Nürnberger 2001) angeprangert sowie moralische Forderungen wie die Aneignung von Tugenden und hohe Erwartungen an die Heranwachsenden gestellt werden (vgl. Oelkers 2004). Pädagogische Programme werden mit Schlagworten wie ,Mehr Tugend für die Jugend“ versehen. Jedoch lässt sich die vielschichtige und komplexe Erziehungsrealität wie etwa in Familie und Schule nicht rhetorisch lösen. Darüber hinaus stellt sich die Frage, worin genau das offensichtlich grosse Interesse der Leserschaft besteht: in der Tugendthematik, im Bedürfnis nach klaren Handlungsanleitungen oder im unhinterfragten Festhalten an sozialen Erwartungen? Ohne darauf eine abschließende Antwort bereitzuhaben, scheint zumindest festzustehen, dass ein differenzierter Umgang mit Erziehungsfragen, zu denen wesentlich die Förderung von Tugenden zählt, nicht so sehr erwünscht zu sein scheint, wie es die breite Reaktion auf Böhms etwas provokative Meinung nahelegt.

Ein großes öffentliches Interesse an Tugenden kommt nicht nur dem Themenbereich der Erziehung zu, sondern auch der Psychologie und der Wirtschaft, worauf der folgende Exkurs einen kritischen Einblick gibt. Darin finden sich ähnliche Argumentationslinien bezüglich Tugenden und Ehrlichkeit wie in den soeben angesprochenen populären Erziehungsdebatten.

\subsubsection{Exkurs: Tugenden in der Positiven Psychologie und in der Betriebswirtschaft}

Die Aktualität des Tugendbegriffs zeigt sich derzeit ebenso in nichtpädagogischen Disziplinen wie der ,Positiven Psychologie“ oder Ökonomie: Ausgehend von Tugendkatalogen und der Nennung einzelner Schlüsseltugenden sollen alltägliche und berufliche Herausforderungen erfolgreich gelöst werden.

So konzentrieren sich Psychologen seit Mitte der 1990er Jahre immer mehr auf die positiven Eigenschaften von Menschen und weniger auf deren Fehler und Schwächen. Daraus entstand der neue akademische Zweig der Positiven Psychologie, in dem Tugenden benannt, als Stärken verstanden und als Basis für psychologische Therapien verwendet werden. Systematische Bemühungen zur Klassifikation der einzelnen Stärken und entsprechende Untersuchungen sind im umfassenden Handbuch Character Strengths and Virtues (Peterson und Seligman 2004) erfasst. Zu diesen zählen die Tugenden und Stärken Weisheit/Wissen, Mut, Liebe/Humanität, Gerechtigkeit, Mäßigung und Spiritualität/Transzendenz. 
In der Betriebswirtschaft wie auch in der ökonomischen Theoriebildung werden Modelle einzelner Tugenden propagiert. Laut einer Studie der Universität St. Gallen lässt sich zum Beispiel der Erfolg von Unternehmen zu einem Drittel durch tugendhaftes Verhalten der Mitarbeitenden erklären (Schönborn 2006). Zudem zeigen die Wirtschaftsprofessoren Ernst Frey (Universität Zürich) und Kurt Dopfer (Universität St. Gallen) die Bedeutsamkeit der Tugenden Fairness, Solidarität, Vertrauen und gegenseitigem/nichtgegenseitigem Altruismus in der ökonomischen Theoriebildung: Der homo oeconomicus sei ,lange nicht so rational und eigennützig wie im Standardmodell angenommen, denn er lässt sich auch von Kriterien wie Fairness, Solidarität, Vertrauen oder reziprokem und nichtreziprokem Altruismus leiten“ (Dopfer 2002, S. 103).

Ebenso werden in betriebswirtschaftlicher und medizinischer Ratgeberliteratur unterschiedliche Tugenden wie beispielsweise Vertrauen, Ehrlichkeit oder Respekt als Erfolgsgarantien für den beruflichen Erfolg aufgeführt.

Im Zusammenhang mit vielversprechenden Auswirkungen einzelner Tugenden stellt sich grundsätzlich die Frage, inwiefern Tugenden als solche tatsächlich als Therapie- und Erfolgsrezept verstanden werden sollen, da diese auch im falschen Moment, zu schwach oder zu spät eingesetzt werden können. Es fällt auf, dass die genannten Autoren den Tugendbegriff verkürzt, mit einem verzerrten Bezug zu alltäglichen Verhaltensweisen und eher als abstrakten Berufsethos thematisieren.

\subsubsection{Ehrlichkeit und Tugendansätze in der gegenwärtigen Schulpädagogik}

Eine quantitative Untersuchung der deutschen Bevölkerung belegt anhand einer Befragung von 2.000 Personen ab 14 Jahren, dass Ehrlichkeit als wichtigste Tugend der Deutschen gilt (Opaschowski 2006). So beschriebene Befunde können zu der Annahme verleiten, dass die Befragten das Wort ,Tugend' direkt verwenden würden, um Ehrlichkeit als praxisorientierte Verhaltensweise zu befürworten. Ehrlichkeit zählt in der Erziehungsphilosophie zu den Tugenden (vgl. Kap. 2), allerdings ist dies nicht im Alltagsdenken oder in der Schulpädagogik offensichtlich. Denn kaum jemand benutzt in den erwähnten Bereichen diesen Begriff.

In dieser Hinsicht zeigt das folgende Kapitel auf, wie Ehrlichkeit in der Schulpraxis erwünscht ist und wie sie in Lehrerzeitschriften thematisiert wird, obwohl in der wissenschaftlichen Schulpädagogik der Tugendansatz verabschiedet, gegenwärtig wenige theoretische Tugendansätze im Bereich Erziehung und Bildung situiert sind und damit bislang theoretische Fundierungen von Ehrlichkeit als Tugend im Schulkontext nicht bestehen. 
Ehrlichkeit in der Schulpraxis ohne Tugendbegriff und theoretische Tugendansätze

Wie im Eingangsbeispiel der drei Schüler aufgezeigt, ist Ehrlichkeit aus der Sicht des schulischen Erziehungsauftrags im Schulkontext wichtig. Dennoch gelten der Tugendbegriff und damit theoretische Tugendansätze spätestens seit den 1960er Jahren in der Schulpädagogik als „hoffnungslos veraltet“ und „moralinsauer“ (Dietenberger 2002, S. 49). Der Erziehungsauftrag der öffentlichen Schulen wird anhand von sozialisationstheoretischen Ansätzen und damit ausgehend von Begriffen wie ,Werte' (vgl. Uhl 1998; Reinders 2005b; Latzko 2006; Mokrosch und Regenbogen 2009; Schubarth et al. 2010), ,soziale Kompetenzen“ (vgl. Bieri Buschor und Forrer 2005; Rohlfs et al. 2008; Bormann und Haan 2008) und ,soziales Lernen' beschrieben. In pädagogischen Wörter- und Handbüchern findet sich der Tugendbegriff wenn überhaupt unter dem Stichwort, Werte' und im Zusammenhang mit anderen bürgerlichen Tugenden wie ,Fleiß', ,Anstand' sowie ,Gehorsam‘. Die Auseinandersetzung mit dem Begriff erfolgt dabei jedoch aus einem historischen Blickwinkel und zeigt geringen Gegenwartsbezug. Eine Ausnahme bildet Brumliks Beitrag im Handbuch der Nachbardisziplin der Sozialen Arbeit (Brumlik 2011), in dem er auf die wachsende Prominenz des Tugendbegriffs hinweist, die er allerdings als auf der philosophischen Ethik basierend ansieht. In einem knappen historischen Abriss (Brumlik 2011, S. 1695ff.) legt er die Bedeutung unterschiedlicher theoretischer Tugendansätze im Laufe der Zeit dar. Sie werden an dieser Stelle kurz skizziert, um an diesem Beispiel eine übliche und aktuelle Auseinandersetzung mit Tugenden aufzuzeigen, die sich auf einer philosophischen Ebene verortet.

Gemäss den Autoren der Antike ging es darum, sich vor allem über die Lehrbarkeit von Tugenden auszutauschen. Im Mittelalter hingegen befassten sich vornehmlich Theologen und Philosophen mit der Möglichkeit einer Rangordnung von Tugenden; also der Frage, welche Tugend zentral ist und welche nicht. Diese Beschäftigung mit Wertigkeiten von Tugenden ist im 18. Jahrhundert weitgehend durch funktionale Ansätze im Umgang mit Tugenden verdrängt worden, in denen die Bewältigung des Alltags im Vordergrund steht. Diese Zeitepoche ist gleichzeitig von einer Prinzipienethik geprägt (vgl. dazu Kants ,kategorische Imperative“ ([1797] 1966)), wobei Tugenden als Voraussetzung dafür verstanden werden, dass eine Person die regulative Eigenschaft der Prinzipien anzuwenden vermag. Tugenden vertreten deshalb eine Selbstbehauptung aus einer vernünftigen Selbstbestimmung.

Seit Mitte des 20. Jahrhunderts formulieren Philosophen theoretische Tugendansätze im Bereich der Tugendethik und grenzen diese scharf von der Prinzipienethik ab. Zudem gibt es gemäss Brumlik seit rund zwanzig Jahren innerhalb der Tugendethik Ansätze, die auf anthropologischen Universalien und gleichzeitig auf die entsprechend notwendigen Fähigkeiten (Tugenden 
einzusetzen) aufbauen. Brumlik bezieht damit die Tugendthematik in seinem aufgeführten Überblick auf die abstrakten Reflexionen zur philosophischen Ethik. Dem Einwand, dass Tugenden ,kulturrelativ ${ }^{`}$ seien und daher schwerlich Leitlinien für die Bildung liefern könnten, begegnet Brumlik, indem er gerade diese Eigenschaft der Tugendansätze positiv wendet: In einer Zeit der kulturellen Pluralität ist es zentral - anders als es moralische Prinzipienethiken vermögen -, auf gesellschaftliche und kulturelle Rahmenbedingungen einzugehen, was Tugendansätze zustande bringen (vgl. Brumlik 2011, S. 1700f.). Anders erklärt: Im Gegensatz zu den moralischen Prinzipien, die in einer abstrakten Form formuliert sind, wie etwa: „Handle so, dass die Maxime deines Willens jederzeit zugleich als Prinzip einer allgemeinen Gesetzgebung gelten könne“ (Kant ([1797] 1966), A 54), stellen Tugendansätze flexible Verhaltensregeln auf, wie etwa Anstand oder Respekt. Zudem sind Tugenden im Gegensatz zu Prinzipien direkt auf das Verhalten von Personen bezogen (Eykmann und Seichter 2007).

Neben diesen erziehungsphilosophischen Ansätzen machen regelmäßig Beiträge in Lehrerfachzeitschriften aus einer schulpädagogischen Sicht auf das Thema Tugenden, Einzeltugenden und Ehrlichkeit aufmerksam und geben praxisorientierte und didaktische Hinweise für Reflexionen; dies jedoch, ohne den Anspruch auf eine theoretische oder begriffliche Fundierung des Tugendansatzes zu erheben. Die Beiträge befassen sich z. B. mit den Themen Lügen (Berthold 2007), Höflichkeit (Groeben, 2000; Weidner 2004), Spicken (Grunder 1999), Nichtlehrbarkeit der Tugend (Heitger 2009), Respekt (Larson-Knight 2004), Disziplin (Krumm 2003), Achtsamkeit (Vorlauf 2010), Ehrlichkeit/Fairness/Glaubwürdigkeit (Silbernagel 1997), Sekundärtugenden (Seydel 2003) und Lügen/Stehlen/Weglaufen/Zündeln (Utz 1997), um nur einige zu nennen. Diese Vielzahl an Publikationen zu (Einzel-)Tugenden und Sekundärtugenden weist auf eine rege Nachfrage der Lehrerschaft hin, Tugenden in der Schulpädagogik zu thematisieren. Allerdings fehlen zu den thematisierten Tugenden, im Sinne von Verhaltensweisen, bislang Bezüge zu gegenwartsbezogenen theoretischen Tugendansätzen in der Schulpädagogik.

Wenige theoretische Tugendansätze in der gegenwärtigen Erziehungswissenschaft

Neben dem entgegengesetzten Interesse der praktischen und theoretischen Schulpädagogik gibt es einige wenige theoretische Ansätze in der Erziehungswissenschaft, die versuchen, jenen Mangel zu beheben, wie ich sie im Folgenden kurz vorstelle.

In Abgrenzung zur Werteerziehung, welche eher abstrakte Erziehungsziele anvisiert, strebt der Tugendansatz Erziehungsziele an, die im Alltag konkretisiert werden können (Ahlborn 1996). Es stehen also Verhaltensziele im Vordergrund, die die Gemüts- sowie Gesinnungsbildung fokussieren. 
Tugendhaftigkeit drückt damit einen gewünschten sozialen Charakter aus. Tugendhaft ist derjenige, ,der individuelle Interpretationen der Tugenden anstellt, seine sozialen Systeme stützt und auf Wandel drängt“ (ebd., S. 87f.).

Nicht von einer Verhaltensweise, sondern von einer Handlungsdisposition gehen Brumlik (2002) und Dietenberger (2002) im Rahmen eines entwicklungspsychologischen Ansatzes aus. Dabei verwendet nur Brumlik weiterhin den Tugendbegriff und plädiert für eine Aktualisierung der Kardinaltugenden (Gerechtigkeit, Tapferkeit, Mäßigung und Besonnenheit) sowie der theologalen Tugenden (Hoffnung, Glaube und Liebe) (Brumlik 2002, S. 149ff.).

Ebenso unternimmt Fellsches (1996) eine ,Modernisierung' der Tugenden. Er ersetzt beispielsweise den Begriff ,Tapferkeit' mit "Standhalten', womit der historische Bezug zum Krieger und Soldaten abgelegt und dennoch die Grundhaltung des Kämpferwillens unterstrichen wird. Im Grunde nimmt Fellsches damit eine ,sprachliche Modernisierung ' von Einzeltugenden der griechischen Antike vor. Den zweiten Teil seines Buches bildet ein Lexikon der Tugenden und Untugenden, das Begriffe von ,Achtung' und ,Anerkennung ${ }^{6}$ über ,Lüge' und ,Lust' hin zu ,Zuneigung ${ }^{6}$ und ,Zynismus ${ }^{6}$ umfasst, was an die historischen Tugendkataloge erinnert (vgl. Kap. 2.3).

Eykmann und Seichter präsentieren ausgehend von einer theoretischphilosophischen sowie historischen Perspektive Einzeltugenden und verstehen diese nicht im Kontext einer Tugendethik, sondern als Handlungsethik. Damit rückt nicht die Handlung oder eine Verhaltensweise an sich, sondern der Akteur in den Mittelpunkt (Eykmann und Seichter 2007).

Tugenden sind in den erwähnten erziehungstheoretischen Ansätzen als Verhaltensweisen oder Handlungsdispositionen ausgewiesen und zeichnen sich im Gegensatz zu philosophischen Ansätzen durch den Fokus auf das soziale Miteinander aus (sozialer Aspekt). Zudem stellen einige Autoren den Akteur und weniger die Handlung bzw. die Verhaltensweise ins Zentrum. In der vorliegenden Arbeit werden sowohl die Ansätze aufgegriffen, die auf die Verhaltensweise abzielen, als auch diejenigen, in denen die Akteure im Vordergrund stehen.

\subsubsection{Positive Bewertung der Tugenden in der Jugendforschung}

Eine Wiederaufwertung der Sekundärtugenden im Vergleich zur Untersuchung von 2002 zeigen Befunde der 15. Shell-Jugendstudie (Hurrelmann und Albert 2006) in Deutschland. Sekundärtugenden werden dabei als Respekt vor Gesetz und Ordnung, Streben nach Sicherheit und Fleiß sowie Ehrgeiz definiert. Die befragten 12- bis 25-Jährigen benoten die Sekundärtugenden auf einer Notenskala von 1 bis 7 wie folgt $(1=$ schlechteste Note, $7=$ beste Note) (ebd. 2006, S. 175ff.): 
- Respekt vor Gesetz und Ordnung: $\quad 5,6$

- Fleiß und Ehrgeiz: $\quad 5,6$

- Streben nach Sicherheit: $\quad 5,5$

Die Autoren sind über die hohe Wertschätzung Jugendlicher gegenüber Sekundärtugenden überrascht, weisen allerdings auf die Grenzen dieser Befunde hin: ${ }^{10}$ Die Zahlen sagen nichts darüber aus, wie Jugendliche die benannten Sekundärtugenden verstehen. Zudem können keine Zusammenhänge zwischen den Tugenden hergestellt werden.

,Tugendhafte' Heranwachsende finden sich ebenso in Jugendstudien der Schweiz: Die Längsschnittstudie COCON präsentiert in ihren ersten Ergebnissen einfühlsame, verantwortungsbewusste und anstrengungsbereite Kinder und Jugendliche (COCON 2006; Schultheis 2008). Die rund 1.200 befragten Jugendlichen gaben in der zweiten Erhebungswelle als wichtigste Tugend ${ }^{11}$ (86,6 \%) an: fair sein (COCON 2007, S. 2). Dabei sind auch geschlechtsspezifische Unterschiede auszumachen: Junge Frauen finden es wichtiger, fair zu sein, als junge Männer. Als weniger wichtig gaben die Heranwachsenden an: kritisch sein $(47,8 \%)$ und tun und lassen, was man will (45,4\%).

Darüber hinaus zeichnen die Ergebnisse der standardisierten Jugenduntersuchung Jugendbarometer 2010 (Jugendbarometer 2010) in der Deutschschweiz ein optimistisches Bild im Hinblick auf die Tugenden der 16- bis 25Jährigen. Hier findet sich an fünfter Stelle die Einstellung, sich selbst sein und sich nicht verstellen (75\%), was zur Tugend Ehrlichkeit, verstanden als Integrität, hinzugezählt werden kann.

Die drei Ergebnisse der ausgewählten neueren Jugendforschungen zeigen klar auf, dass einzelne Tugenden heute zu wichtigen Einstellungen der Heranwachsenden zählen, wobei der Begriff ,Tugend' als solcher nicht verwendet wird und fremd zu sein scheint. Weiter sind die Tugenden in den besagten Studien als Einzeltugenden zu verstehen und nicht als umfangreiche, mehrere Tugenden enthaltende Tugendansätze. Neben ,Ehrlichkeit‘ zählen außerdem die Einzeltugenden Respekt vor gesellschaftlichen Normen (Gesetz, Ordnung), Fleiß/Anstrengung, Empathie, Verantwortung, Fairness und Integrität dazu. Vor dem Hintergrund der Befunde aus den Jugenduntersuchungen

10 Eine ähnliche Schlussfolgerung ist aus den Ergebnissen der schulhausinternen Untersuchung (189 SuS) in einem Oberstufenschulhaus im Kanton Aargau zum Thema Schummeln zu ziehen (Stamm 1995). Darin zeigt Stamm auf, dass die meisten SuS unerlaubt fremde Hilfsmittel beim Lösen der Schultests verwenden: 71,9\% der Befragten geben an, in den letzten zwei Monaten bei Schultests ,gespickt“ zu haben. Die SuS erklären ihre Verhaltensmotivation als Mutprobe oder, wie es die Autorin bezeichnet, als „Kavaliersdelikt“ mit dem Ziel, bessere Noten zu erzielen. Damit zeigt die Erhebung Motive und eine Einschätzung der Häufigkeit von unehrlichen Verhaltensweisen auf. Jedoch bleibt die Bedeutung von Ehrlichkeit bei SuS ungeklärt.

11 Die Autoren sprechen von Werthaltungen, was gemäss Hillmann (2003) mit Tugenden gleichgesetzt werden kann. 
kristallisieren sich für das vorliegende Forschungsvorhaben folgende Fragen heraus:

- Was verstehen Jugendliche unter den aufgezählten Einzeltugenden? Würden sie die gleichen Einzeltugenden wie die Forschenden auflisten? Führen sie andere Tugenden auf, die ihnen wichtiger sind als die von den Forschenden erfragten?

- Die Vielzahl der Einzeltugenden wirft das Problem auf, wie Tugenden überhaupt als Gesamtes zu fassen sind. Denn über der Aufzählung von Einzeltugenden steht die begriffstheoretische Frage, welche und wie viele Tugenden unter welchen Bezeichnungen dazugehören (Begriffsumfang). Zudem stellt sich die Frage, ob alle Tugenden die gleiche Bedeutungsstärke aufweisen, welche weiteren Begriffsfacetten existieren und wo Überschneidungen vorliegen.

- Schließlich bleibt in den positiven Ergebnissen der Jugendforschung zu Tugenden offen, inwiefern die genannten Einzeltugenden Indikatoren für soziale Erwünschtheit, für persönliche Einstellungen, für Alltagspraxen oder für individuelle Erwartungen an sich selbst und an andere sind.

Meine Vermutung liegt darin, dass die Befragungen genau jene Einstellungen von Jugendlichen zu gesellschaftlichen und sozial erwünschten Tugenden untersuchen, welche die Befragten in der Alltagssprache nicht wörtlich als ,Tugenden' bezeichnen. Gleichwohl haben die Heranwachsenden ein Erfahrungswissen über die einzelnen Verhaltensweisen und die damit verbundenen sozialen Erwartungen. Damit lässt sich zwar der Widerspruch zwischen den populären Ansichten über ein Tugenddefizit bei Jugendlichen und den hier präsentierten positiven Ergebnissen entschärfen. Dennoch bleibt unklar, wie Jugendliche Einzeltugenden deuten.

Um die Perspektive der Jugendlichen, deren Deutungen von Praxen und die Bedeutung von Einzeltugenden zu untersuchen, bedarf es vorerst einer Ausklammerung von Sichtweisen der Erwachsenen. Insofern liegt ein qualitativer Zugang nahe, weil mit ihm subjektive Sichtweisen von Jugendlichen erhoben werden können. Denn quantitative Zugänge implizieren in den Fragestellungen bereits begriffliche und thematische theoretische Ansätze, die nicht zwangsläufig mit denen der Heranwachsenden übereinstimmen.

\subsubsection{Zusammenfassung}

Tugenden und die Einzeltugend Ehrlichkeit werden heute zwiespältig in populären Erziehungsdebatten, in der Schulpädagogik und in der Jugendforschung in Deutschland und in der Schweiz diskutiert:

- Vertreter gegenwärtiger populärer Erziehungsdebatten beklagen das Fehlen der Tugenden bei Jugendlichen, insbesondere in der Schule. 
Demgegenüber zeigen Befunde von standardisierten Jugenduntersuchungen die gestiegene Wichtigkeit von Einzeltugenden auf, zu denen auch Ehrlichkeit zählt.

- Ehrlichkeit wird im Schulalltag gefordert und in Lehrerfachzeitschriften diskutiert, in der wissenschaftlichen Schulpädagogik jedoch fehlen grundsätzlich theoretische Tugendansätze.

- Des weiteren sind Tugenden wie etwa ,Respekt` oder ,Disziplin“ attraktiv für schulpädagogische Untersuchungen, was auf die Tugend Ehrlichkeit nicht zutrifft, obwohl Ehrlichkeit als eine zentrale Verhaltensweise in der Schule kontrolliert und sanktioniert wird und zu deren Erziehungsauftrag zählt.

- In der Praxis und Theorie der Erziehung werden Tugenden seit den antiken Griechen behandelt. Dennoch sind Einzeltugenden bis heute ungenügend untersucht. Zudem sind die Rahmenbedingungen für die Förderung von Tugenden wie Schule, Peers oder Familie nicht differenziert berücksichtigt und Tugenden somit nicht praxisorientiert fundiert, was für eine wissenschaftliche Beschäftigung mit dem Schulalltag jedoch zentral wäre.

- Moralische Appelle und theoretische Abhandlungen zu Tugenden sind aus der Perspektive der Philosophen oder Erziehungsbeauftragten verfasst, ohne die Sichtweise der Adressatinnen sowie Adressaten zu berücksichtigen (womit hauptsächlich Heranwachsende gemeint sind).

- Befunde zu Einstellungen von Jugendlichen zu Tugenden sind in der Jugendforschung zugänglich, jedoch sind die Deutungen von Einzeltugenden und deren Praxen damit nicht erfasst.

- Es ist unumstritten, dass Tugenden und insbesondere Einzeltugenden in populären Erziehungsdebatten, in spektakulären Medienberichten, in fächerübergreifender Fachliteratur oder in breit angelegten Jugenduntersuchungen aktuell sind, auch wenn der Begriff ,Tugend' in der Alltagssprache und in schulpädagogischen Ansätzen kaum vorkommt resp. , out‘ ist.

Vor diesem Hintergrund steht fest, dass Erziehungsbeauftragte und an Erziehung Interessierte einen großen Bedarf haben, sich mit schulrelevanten Tugenden auseinanderzusetzen. Zugleich fehlen einerseits wissenschaftliche Tugendansätze in der Schulpädagogik und andererseits praxisorientierte erziehungsphilosophische Tugendansätze im Allgemeinen. Genau hier setzt mein Forschungsvorhaben an. Indem es die schulrelevante Einzeltugend ,Ehrlichkeit‘ fokussiert, kann am Beispiel einer Tugend aufgezeigt werden, wie SuS Tugendpraxen des Schulalltages deuten. Damit wird ausgehend von einem explorativen Forschungsansatz ein empirisch fundierter Beitrag zu den erwähnten Tugenddebatten im Bereich Erziehung und Schule gegeben. Zudem können populäre Erwartungen und moralische Appelle sowie Klagen bezüglich fehlender Tugenden bei SuS entschärft werden. 
Eine zentrale Eigenschaft der Tugend Ehrlichkeit besteht darin, dass es nicht in jeder Situation angemessen ist, sich ehrlich zu verhalten. Aufgrund der ambivalenten Bedeutung von Ehrlichkeit zählt sie zu den Sekundärtugenden und setzt die Fähigkeit der Akteure voraus, zu entscheiden, wann welches Verhalten gefragt ist. Das kann zu individuellen, kollegialen und schulischen Dilemmas führen, die SuS zu lösen haben. Der Einblick in die Deutungen der SuS dient dazu, dass Lehrpersonen dies besser verstehen können.

Kurz: Das Forschungsvorhaben untersucht die schulrelevante Einzeltugend Ehrlichkeit aus der Sicht von Jugendlichen, welche die Zürcher Volksschule besuchen. Die leitende Forschungsfrage lautet: Wie deuten Jugendliche Ehrlichkeitspraxen (und damit den Ehrlichkeitsbegriff) im Schulalltag? Damit sollen explizite und implizite Ehrlichkeitsregeln rekonstruiert und Ehrlichkeitspraxen der SuS nachgezeichnet werden.

\subsection{Aufbau der Arbeit}

Die Arbeit gliedert sich in drei thematische Teile, die jeweils mehrere Unterkapitel enthalten. Der erste Teil der Arbeit fasst die Grundlagen des Forschungsprojekts zusammen (Teil I), auf denen die empirischen Ergebnisse basieren, die im Hauptteil der Arbeit (Teil II) vorgestellt werden. Die Arbeit schließt mit der Diskussion der empirischen Ergebnisse und einen Ausblick (Teil III) ab.

Das einleitende Kapitel 1 zeigt zentrale Ausgangspunkte des Forschungsvorhabens auf. Teil I startet mit Kapitel 2, das sich fächerübergreifend mit dem Ehrlichkeitsbegriff und den damit verbundenen theoretischen Tugendansätzen befasst. Die Verwendung des Begriffs ,Ehrlichkeit' wird ausgehend von einer populären Betrachtungsweise, dann aber im Rahmen des bisherigen Forschungsstands der experimentellen Psychologie und Erziehungsphilosophie untersucht, und es werden zentrale Befunde und Problemlagen zum Begriff und theoretische Ansätze benannt. Die vielversprechenden angelsächsischen Tugenddebatten, in denen Tugenden und ebenso die Einzeltugend Ehrlichkeit im erziehungsphilosophischen und bildungspolitischen Kontext diskutiert werden, weisen allerdings theoretische und empirische Defizite auf, die umrissen werden. Dennoch wird aus diesen die schulrelevante dreifache Ehrlichkeitsdefinition (White 1996) gewählt und in Bezug zur Tugenddefinition als moralische Verhaltensweise (Reichenbach 2004, 2008) gesetzt. Der so definierte Ehrlichkeitsbegriff wird durch erste empirische Befunde zum Ehrlichkeitsverständnis (Chiapparini 2012) ergänzt und dient als loser theoretischer Rahmen für die vorliegende Arbeit. Damit ist das begriffstheoretische Vorwissen über den Forschungsgegenstand aufgezeigt: Deutungen von Jugendlichen bezüglich ihrer Ehrlichkeitspraxen im Schulall- 
tag. In Abgrenzung zum erziehungsphilosophischen Zugang basiert die vorliegende empirische Untersuchung auf einem sozialwissenschaftlichen $\mathrm{Zu}$ gang und auf der Sichtweise der Jugendlichen, die im Schulkontext untersucht wird.

In Kapitel 3 erfolgt die sozialwissenschaftliche Kontextualisierung des Forschungsgegenstands, indem die neuere Schülerforschung und die damit zusammenhängende Jugendforschung referiert wird sowie über die Funktion der Schule als Institution reflektiert und der öffentliche Erziehungsauftrag für die Zürcher Volksschulen eruiert wird. Dabei wird das für die Arbeit grundlegende sozialisationstheoretische Verständnis der SuS als produktiv verarbeitende Subjekte erklärt. Zudem sind zentrale Rahmenbedingungen für den Feldzugang in die Volksschule im Kanton Zürich festgehalten. Aus diesen Überlegungen zum Forschungsgegenstand heraus sind die Forschungsfragen ausführlich dargelegt und die Zielsetzungen der vorliegenden Untersuchung formuliert (Kap. 4).

Der Teil zu den Grundlagen der Untersuchung schließt mit dem Kapitel zur Methodik (Kap. 5), in dem der methodologische Ansatz des ,kontrollierten Fremdverstehens' vorgestellt wird, in dem die ausgewählten Erhebungsund Auswertungsmethoden situiert sind: das problemzentrierte Interview (Witzel 2000) und die dokumentarische Methode (Bohnsack 2003). Damit ist aufgezeigt, mit welchen Methoden die Forschungsfragen beantwortet werden. Zudem wird die Angemessenheit der zwei Methoden für das Erforschen von Deutungen der Ehrlichkeitspraxen bei Jugendlichen begründet. Besonderen Wert wird in diesem Kapitel auf die Skizzierung des praxisrelevanten Forschungsvorgehens gelegt, das die Umsetzung der reflektierten qualitativen Methoden in der Forschungspraxis widerspiegelt.

Teil II der Arbeit stellt den zentralen Teil des Forschungsprojekts dar, in dem die gewonnenen Ergebnisse der qualitativen Untersuchung dargestellt und diskutiert werden. Dabei bildet Kapitel 6 eine Hinführung zu den Hauptergebnissen, indem ein Überblick über den Ehrlichkeitsbegriff der SuS und dessen (begrenzte) Anwendung geleistet wird. Daraufhin werden in zwei weiteren Kapiteln die Ehrlichkeitsregeln der SuS präsentiert, die aus den subjektiven Deutungen von Ehrlichkeitspraxen rekonstruiert wurden. Diese sind einerseits in konventionelle Ehrlichkeitsregeln (Kap. 7) und andererseits in unkonventionelle Ehrlichkeitsregeln (Kap. 8) unterschieden. Das nächste Kapitel geht auf die realen Dilemmasituationen ein (Kap. 9). In Dilemmas werden Deutungen von Ehrlichkeitspraxen besonders wichtig und Entscheidungen für bestimmte Ehrlichkeitsregeln relevant. Anschließend werden die Forschungsergebnisse in Kapitel 10 zusammengefasst.

Teil III bildet den Abschluss der Arbeit. Zunächst werden die empirischen Befunde diskutiert (Kap. 11), womit die leitende Forschungsfrage nach den Deutungen von Ehrlichkeitspraxen von Jugendlichen an der Zürcher Volksschule beantwortet wird. Darüber hinaus werden Hinweise für Erklä- 
rungsansätze formuliert und die gewonnenen Befunde in den bestehenden Forschungsstand der Schulpädagogik und Jugendforschung eingeordnet. Anschließend sind in Kapitel 12 die zentralen Ergebnisse des ganzen Forschungsprojekts skizziert. Die nächsten zwei Kapitel befassen sich mit den Fragen, was die dargestellten Ergebnisse für die wissenschaftliche Erkenntnis zum Thema Tugend für die Schüler- und Jugendforschung (Kap. 13) und für die schulischen Erziehungsanforderungen sowie für eine theoretisch fundierte und praxisorientierte Tugenddebatte leisten (Kap. 14) und wo sie auf weitere Forschung verweisen. Pädagogische Forschung hat den Anspruch, nicht nur um ihrer selbst willen betrieben zu werden. Deshalb sind Folgerungen für die Praxis im abschließenden Kapitel III erwähnt, die sich aus den hier erarbeiteten Ergebnissen ableiten lassen. 

I Grundlagen 

Dieser erste Teil der Arbeit gibt einen klärenden Einblick in die Grundlagen der durchgeführten empirischen Untersuchungen und stellt das Vorwissen über den Forschungsgegenstand (Deutungen von Jugendlichen bezüglich ihrer Ehrlichkeitspraxen im Schulalltag), das Forschungsinteresse und den Forschungszugang vor. Zunächst befasst sich Kapitel 2 fächerübergreifend mit dem Ehrlichkeitsbegriff und den damit verbundenen theoretischen Tugendansätzen. Diese reflektiere ich kritisch, woraus ich Anhaltspunkte für einen losen theoretischen Rahmen für die vorliegende Arbeit definiere und die begriffstheoretische Ausgangslage des Forschungsgegensatands aufgezeige.

In Kapitel 3 erfolgt die sozialwissenschaftliche Kontextualisierung des Forschungsgegenstands, indem ich die neuere Schülerforschung und die damit zusammenhängende Jugendforschung diskutiere sowie die Funktionen der Schule als Institution thematisiere und den öffentliche Erziehungsauftrag für die Zürcher Volksschulen erkunde. Aus diesen Überlegungen zum Forschungsgegenstand heraus lege ich die Forschungsfragen ausführlich dar und formuliere die Zielsetzungen der vorliegenden Untersuchung (Kap. 4).

Der Teil zu den Grundlagen der Untersuchung schließt mit dem Kapitel zur Methodik (Kap. 5). Damit ist aufgezeigt, mit welchen Methoden die Forschungsfragen beantwortet werden. Zudem wird die Angemessenheit der zwei Methoden für das Erforschen von Deutungen der Ehrlichkeitspraxen bei Jugendlichen begründet.

\section{Zum Ehrlichkeitsbegriff}

Dieses Kapitel setzt sich differenziert mit dem Ehrlichkeitsbegriff und den damit zusammenhängenden theoretischen Ehrlichkeitsansätzen auseinander. Ehrlichkeit wird anhand essayistischer, psychologischer und erziehungsphilosophischer Verwendungen untersucht, womit zentrale Themenbereiche abgedeckt sind, in denen Ehrlichkeit heute diskutiert und erforscht wird. Ziel ist es, zentrale Befunde und Problemlagen des Ehrlichkeitsbegriffs aufzuzeigen, vor deren Hintergrund anschließend der Ehrlichkeitsbegriff für die vorliegende Arbeit formuliert wird.

Einleitend gehe ich auf populistische Abhandlungen von Ehrlichkeit ein, um die Unterdeterminiertheit des Begriffs aufzuzeigen (Kap. 2.1). In einem zweiten Schritt skizziere ich psychologische Ehrlichkeitsdefinitionen, die seit Anfang des 20. Jahrhunderts in der experimentellen Psychologie Forschungsthema sind. (Kap. 2.2). Anschließend zeige ich die erziehungsphilosophische Dimension des Ehrlichkeitsbegriffs auf, wobei hier ausführlich Ehrlichkeit im Bezug zum allgemeinen Begriff, Tugend' untersucht wird (Kap. 2.3). 
Damit soll zum einen auf ausgewählte traditionsreiche Ehrlichkeitsansätze hingewiesen werden. Zum anderen wird geklärt, worin der Bezug zwischen Tugenden und Einzeltugenden bestehen kann. Am Beispiel der angelsächsischen Tugenddebatte können besonders klar die Aktualität und die Grenzen von theoretischen Tugendansätzen in der gegenwärtigen Erziehungsphilosophie und Bildungspolitik aufgezeigt werden (Kap. 2.4). In Abgrenzung zum angelsächsischen Tugendbegriff basiert der Tugendbegriff dieser Arbeit auf Verhaltensweisen (Kap. 2.5) und wird nicht als innere oder ausschließliche Charaktereigenschaft verstanden. Abschließend definiere ich, auf welcher theoretischen Basis ich ,Ehrlichkeit' und ,Tugend' in dieser Arbeit verwende (Kap. 2.6), was als loser theoretischer Rahmen zu verstehen ist. Somit zeigt das zweite Kapitel das begriffstheoretische Vorwissen über den Forschungsgegenstand auf.

\subsection{Essayistischer Ehrlichkeitsbegriff}

Mit moralphilosophischen Themen wie Ehrlichkeit befassen sich Journalisten und Führungsberater in essayistischen Texten, so zum Beispiel der Journalist und Buchautor Ulrich Wickert in Büchern wie Gauner muss man Gauner nennen: Von der Sehnsucht nach verlässlichen Werten (Wickert 2007) oder Der Ehrliche ist der Dumme. Über den Verlust der Werte (Wickert 1994). Er empfiehlt Führungskräften Ehrlichkeit als das beste Mittel, um Respekt bei den Mitarbeitenden und unternehmerischen Erfolg zu erzielen. Nach Wickert setzt Ehrlichkeit die Fähigkeit voraus, sich selber einzuschätzen und Fehler einzugestehen. Jedoch seien aus Höflichkeit Aussagen möglich, die nicht ganz stimmen.

Demgegenüber bedeutet für den Journalisten Jürgen Schmieder (2010) Ehrlichkeit: Alles sagen, was man denkt. Ausgehend von diesem engeren Ehrlichkeitsverständnis hat der Autor während vierzig Tagen versucht, ohne zu lügen auszukommen. Nach dem Selbstexperiment stellt Schmieder fest, dass ein enges Ehrlichkeitsverständnis gesellschaftlich nicht erwünscht ist und damit Lügen in einer angepassten Hinsicht gesellschaftlich legitimiert wird. Deshalb empfiehlt er, die ,Lüge' ebenso wie die ehrliche Ansicht respektvoll zu formulieren.

Die Frage, ob ein moderates oder ein wörtliches Ehrlichkeitsverständnis gelten soll, greift der Journalist Nils Graefe in einem Artikel zur unkonventionellen Meinung bei Winfried Böhm auf (vgl. Kap. 1.2). Böhm postuliert nämlich ein verantwortliches Lügen. Die entrüsteten Antworten der Leserschaft verweisen darauf, dass die Tugend Ehrlichkeit als Nichtlügen einen rigiden und hohen Stellenwert einnimmt. Unklar bleibt, ob es sich hierbei 
mehr um eine allgemeine hohe soziale Erwartung handelt oder ob ein direkter Bezug zu den traditionellen Tugenden besteht (vgl. Kap. 2.3).

Die drei Beispiele aus Buchpublikationen und die Reaktion auf einen unkonventionellen Zeitungsartikel belegen eine allgemeine einseitige Wahrnehmung von Ehrlichkeit i. S. e. allgemeinen Vermeidens von Lügen. Darauf deuten diese kurzen Formeln hin: Alles sagen, was stimmt (inkl. Ausnahmen wegen Höflichkeit), Alles sagen, was man denkt und Nicht lügen. Zudem sind öffentliche Einstellungen zu Ehrlichkeit so stark von sozialen Erwartungen geprägt und bestimmt, dass differenzierte Thematisierungen wie verantwortliches Lügen oder Identifizierung von individuellen oder situationsabhängigen Absichten vorwiegend abgelehnt werden.

\subsection{Psychologischer Ehrlichkeitsbegriff}

In der Psychologie wird üblicherweise ein dualer Ehrlichkeitsbegriff ,ehrlich - unehrlich` verwendet, der nicht auf einem expliziten theoretischen Ansatz basiert.

Bereits Hugh Hartshorne (1926) untersuchte, wie ehrliche Verhaltensweisen gemessen werden können und führte die Befunde in einer umfangreichen Studie zum Charakter Studies in the Nature of Character (Hartshorne et al. 1929) weiter aus. Er ging von einem dualen Ehrlichkeitsbegriff aus und unterschied ehrliche und unehrliche Verhaltensweisen. Mittels Experimenten prüfte er die Unehrlichkeit von Schulkindern (vgl. Hartshorne 1926): Je ein Proband löste eine schriftliche Aufgabe ohne Aufsicht. Am nächsten Tag erhielten die Probanden die Korrekturen ihrer Aufgabe und Zeit, diese wieder ohne Aufsicht zu überprüfen. Damit erhielten sie die Gelegenheit, heimlich das Originalblatt zu verbessern, indem sie das Geschriebene löschen und die richtige Antwort hinsetzen konnten. Die Forscher überprüften im Nachhinein anhand einer angefertigten Kopie des Originalblattes vom ersten Tag, wie oft die Kinder unehrlich waren.

Solche nicht unumstrittenen Forschungsmethoden gehören heute zum ,letzten Jahrhundert". Allerdings basieren auch neuere psychologische Schuluntersuchungen auf dem dualen Ehrlichkeitsbegriff ,ehrlich - unehrlich“. So untersuchten beispielsweise Detlef H. Rost und Jörn R. Sparfeldt (2003) mittels Fragebögen ehrliche Verhaltensweisen von 524 Gymnasiasten aus 25 Klassen in Schultests. Ein zentrales Ergebnis weist darauf hin, dass höhere Selbstkompetenz zu ehrlicherem Verhalten bei Schultests führt (Rost und Sparfeldt 2003, S. 72).

Hingegen ist Ehrlichkeit in der Positiven Psychologie, etwa bei Peterson und Seligman (2004), explizit nicht erwähnt, obwohl Tugenden (Weis- 
heit/Wissen, Tapferkeit, Menschlichkeit, Gerechtigkeit, Mäßigung, Transzendenz) und zugeordnete 24 Charakterstärken thematisiert werden.

Die experimentelle Lügenforschung geht ebenfalls von einem dualen Ehrlichkeitsbegriff aus: prüfbar gelogen und wahrheitsgetreu (Krummenacher 2006). Zudem besteht der Versuch, Lügen anhand von inhaltlichen und non- und paraverbalen Merkmalen (z. B. Anzahl der Wortwiederholungen, Sprechlänge oder Stottern) differenziert zu identifizieren. Dabei werden kognitive, situative, motivationale, emotionale und soziale Kriterien innerhalb des Laborkontextes berücksichtigt. Trotz der differenzierten Vorgehensweise zeigt Krummenacher auf, dass teilweise widersprüchliche Befunde zu finden sind (ebd., S. 27ff.). Beispielsweise kann eine befragte Person, die unter Stress steht, ähnliche inhaltliche oder non- sowie paraverbale Merkmale im Sprechen aufweisen wie eine, die lügt (ebd., S. 29f.). Ebenso umstritten ist das Verfahren von Paul Ekman, der eine Checkliste von 28 Punkten zusammenstellt, um Lügen zu identifizieren. Dabei ist der Erfolg des Aufdeckens einer Lüge von der Art der Lüge, vom Lügner und vom „Lügenfänger“ abhängig (vgl. Ekman 2009, S. 240ff.).

Demgegenüber geht Martin Krummenacher in seiner Untersuchung von einem Lügenbegriff als einer von der Realität abweichende Aussage aus und zeigt zwei Weisen zu lügen auf: indem relevante Fakten weggelassen oder bestehende Fakten erweitert werden. Basierend auf der bisherigen Forschung zeigt Krummenacher, dass Lügen anhand von inhaltlichen Kriterien und der Mimik des Probanden am besten nachzuzeichnen seien (ebd., S. 32). Ein neues Kontrollkriterium sei ein erhöhter kognitiver Ressourcenverbrauch beim Lügen, was von neurowissenschaftlichen Studien untermauert wird (ebd., S. 152).

Krummenachers Forschungsergebnisse beziehen sich auf experimentelle Erkennungsprozesse von Lügen. Das Kontrollieren und Festmachen der Lüge an äußeren Faktoren und Merkmalen ist Ziel der experimentellen Lügenforschung. Darin ist Lügen allein verbal als Aussageinhalte definiert, die von der Realität abweichen oder die zurückgehalten bzw. verheimlicht werden. Zudem setzen sich die Probanden vorwiegend aus erwachsenen Personen zusammen und halten sich vorzugsweise im Untersuchungsort des Labors auf. Damit können die Untersuchungsvariablen besser kontrolliert und eine hohe Teilnehmerzahl erzielt werden. Allerdings stehen damit die Sichtweise der Probanden und alltägliche Situationen nicht im Fokus der Lügenforschung.

Die vorgestellten psychologischen Ansätze zu Ehrlichkeit sollen exemplarisch aufzeigen, dass der Ehrlichkeitsbegriff seit der klassischen Forschung Anfang des 20. Jahrhunderts anhand des Gegensatzpaares ehrlich - unehrlich untersucht wird. In der experimentellen Lügenforschung gilt dieser Ansatz sogar auf der verbalen Ebene: ,Lüge - Wahrheit sagen'. Unter Lügen wird dabei differenziert zwischen ,Falsch aussagen" und ,Aussagen vorenthalten“. 
Dennoch ist in diesem Forschungsfeld ein verkürzter und funktionaler Ehrlichkeitsbegriff vorzufinden. Obwohl die Positive Psychologie sich mit dem Ansatz der Tugenden auseinandersetzt, ist der Ehrlichkeitsbegriff in deren Systematik der Tugenden und Charakter nicht explizit aufgeführt. Hingegen wird ein dualer Ehrlichkeitsbegriff (Lüge - Wahrheit) im Zusammenhang mit entwicklungspsychologischen Ansätzen zur Werteerziehung in der Schule mit Blick auf die Einstellung der SuS untersucht (vgl. Kap. 3.3.2).

Vor dem Hintergrund eines dualen Ehrlichkeitsbegriffs in der Psychologie wird im folgenden Unterkapitel nach einer komplexeren Ehrlichkeitsdefinition in der Erziehungsphilosophie gesucht, welche die Theorie der Erziehung bis heute stark prägt und in der über Tugenden bereits in der griechischen Antike debattiert wurde.

\subsection{Erziehungsphilosophischer Ehrlichkeitsbegriff: Tugendkataloge}

Tugenden sind in der Praxis und Theorie der Erziehung seit der griechischen Antike ein Dauerthema (Hoyer 2005; Bonafaccia 2003; Uhl 1996). Die Bedeutung des Ehrlichkeitsbegriffs und die damit verbundenen theoretischen Ehrlichkeitsansätze haben sich im Laufe der historischen Erziehungsphilosophie vielfach gewandelt. Deshalb ist ein historischer Einblick in einzelne Ansätze angebracht, die für die Ehrlichkeitsthematik relevant sind.

Interessanterweise zeigt sich die wandelnde Bedeutung von Ehrlichkeit am Beispiel des Teilaspekts der Aufrichtigkeit in der angelsächsischen Literaturwissenschaft besonders klar, weshalb hier dieser eingangs zur erziehungsphilosophischen Untersuchung skizziert wird. In der angelsächsischen Literaturwissenschaft gilt das 18. Jahrhundert in Europa als ,Zeit der Aufrichtigkeit‘ (Trilling 1972; Geitner 1992). Aufrichtigkeit (sincerity) wird als Konstruktion und Erfindung dieser Zeitepoche betrachtet:

I propose the idea that at a certain point in its history the moral life of Europe added to itself a new element, the state of quality of the self which we call sincerity (Trilling 1972, S. 2).

Demgegenüber betonen andere Autoren in Bezug auf das 18. Jahrhundert nicht die Rolle der Aufrichtigkeit, sondern die Rolle der Verstellung (z. B. Davidson 2004). Oder sie gehen davon aus, dass Aufrichtigkeit als Ideal bereits im 16./17. Jahrhundert bedeutsam gewesen sein muss (vgl. Benthien 2006; Martin 1997).

Die literaturwissenschaftliche Skizzierung zeigt auf, dass es den Begriff ,Aufrichtigkeit' beispielsweise in England nicht schon immer gab, sondern dass er vom kulturhistorischen Wandel abhängig ist. Somit ist ,Aufrichtig- 
keit` eine kulturspezifische Konstruktion, deren historischer Ursprung schwer nachzuzeichnen ist. Ebenso schwierig ist es, den Ursprung des Ehrlichkeitsbegriffs aufzuzeigen, der inhaltliche und begriffstheoretische Überschneidungen mit ,Aufrichtigkeit" aufweist, worauf in diesem Kapitel hingewiesen wird.

Vor diesem Hintergrund erklärt sich zudem, weshalb theoretische Tugendansätze im Laufe der Geschichte ganz unterschiedliche Einzeltugenden berücksichtigen und diese unterschiedlich definieren. Meistens sind Reflexionen zu Tugenden in Form von Tugendkatalogen und eines Tugendkanons konzipiert, in denen nicht alle die Tugend Ehrlichkeit explizit oder mit bedeutungsähnlichen Begriffen aufführen. Daher wird im Folgenden aufgezeigt, dass der Ehrlichkeitsbegriff in den vielzitierten platonischen und aristotelischen Tugendlisten als solcher nicht vorkommt (Kap. 2.3.1). Anschließend wird der Ehrlichkeitsbegriff in Bezug auf die bürgerlichen Tugenden (Kap. 2.3.2), die Sekundärtugenden (Kap. 2.3.3) und die gegenwärtigen Tugendlisten (Kap. 2.3.4) untersucht und reflektiert. In diesen bis heute vielzitierten Listen ist der Ehrlichkeitsbegriff verortet. Den Abschluss bildet eine kritische Reflexion zu den Tugendkatalogen.

\subsubsection{Ehrlichkeitsbegriff in Tugendlisten der Klassiker der griechischen Antike}

Ein Blick in den theoretischen Tugendansatz von Platon und Aristoteles - auf den sich Erziehungsphilosophen und Erziehungswissenschaftler bis heute beziehen (vgl. Pieper 2004; Hoyer 2005; Brumlik 2002) - soll exemplarisch aufzeigen, dass Tugenden üblicherweise deskriptiv, funktional und normativ erklärt werden. Ehrlichkeit als solche oder als Wahrhaftigkeit ist nicht erwähnt.

Im Werk Politeia (Plato 2001) definiert Platon das Staatswesen als Bildungsanstalt im Großen und die menschliche Seele als Bildung im Kleinen. Dabei kommt die Erziehung nur wenigen auserwählten Knaben zugute (Höffe 1997). Vor dem Hintergrund dieser bildungspolitischen Reflexionen führt Platon die vier sogenannten Kardinaltugenden ${ }^{12}$ ein:

- Weisheit (sóphron): Vortrefflichkeit der Vernunft

- Gerechtigkeit (díkaios): Wissen, was für die Stände/Schichten (Handwerker/Bauer, Kriegerstand/Wächter, Philosophenherrscher) wesenseigene Tätigkeiten sind

12 Die Bezeichnung „Kardinaltugenden“ (von lat. cardo, „Türangel, Dreh- und Angelpunkt“) stammt nicht von Platon, sondern von dessen Rezeption im Mittelalter. Damit wird den vier platonischen Tugenden eine grosse Wichtigkeit und Abhängigkeit zu den anderen Tugenden zugesprochen, was in der Parallele der zentralen Bedeutung der Türangel für das Funktionieren der Türe veranschaulicht werden kann. 
- Tapferkeit (agathós): Einsicht, was wahrhaft zu fürchten sei und was nicht

- Besonnenheit (eusebés): Wissen von der Führungsfunktion der Vernunft gegenüber der Begierde und dem Willen

Platon geht von einer Analogie zwischen der menschlichen Seele und der Staatsform aus. Die Vortrefflichkeit der Seele und des Staates bestehe in der Tugend (areté), das Seinige - für die Seele sowie für den Stand - in der wesenseigenen Funktion zu tun. Ganz offensichtlich beschreibt Platon Tugenden funktional und normativ als Reproduktion der Gesellschaftsordnung. Die Einzeltugend Ehrlichkeit thematisiert Platon nicht. Ebenso erwähnt Aristoteles nur einen Teilaspekt der Einzeltugend Ehrlichkeit, die Wahrhaftigkeit. Allerdings tut er dies in einem spannenden theoretischen Tugendansatz, den er in seinem Werk Nikomachische Ethik ausführt (Aristoteles 2006). In den aristotelischen Reflexionen sind Tugenden nicht als einheitliche Regeln bestimmt, die a priori für jeden Einzelfall feststehen, wie dies im platonischen Ansatz der Fall ist. Aristoteles geht vielmehr von einem Verhaltensverständnis aus, das von den Umständen abhängig ist. Weiter unterscheidet er zwischen Vernunfttugenden (Herstellungswissen, Wissenschaft, Klugheit, Weisheit, intuitives Denken) und Charaktertugenden (instrumentelle bzw. technische Tugenden). Die Charaktertugenden werden von den Vernunfttugenden gelenkt. Daher kann der einzelne Mensch die Mitte zwischen zwei extremen Verhaltensweisen ausloten. Dabei geht es nicht um eine mathematische Mitte, sondern um das ,Beste', das im Bereich der Charaktereigenschaft erreicht werden kann. Diese Mitte ist subjektiv zwischen einem Extrem des ,Zuviel und des ,Zuwenig' bestimmbar.

Tabelle 1 gibt einen Überblick über die von Aristoteles beschriebenen Tugenden und deren jeweilige Über- und Untertreibungen.

Tabelle 1: Aristotelische Tugenden

\begin{tabular}{|l|l|l|}
\hline Extrem (,zu viel') & Mesotes (,Mitte') als Tugend & Extrem (,zu wenig') \\
\hline Tollkühnheit & Tapferkeit & Feigheit \\
\hline Übermäßigkeit & Mäßigkeit & Empfindungslosigkeit \\
\hline Verschwendung & Freigiebigkeit & Geiz (im Kleinen) \\
\hline $\begin{array}{l}\text { Geschmacklosig- } \\
\text { keit/Protzerei }\end{array}$ & Großzügigkeit & Kleinlichkeit (im Großen) \\
\hline Eitelkeit & Stolz & Kleinmütigkeit \\
\hline Ehrgeiz/Jähzorn & Sanftmut & Ehrgeizlosigkeit/Unerzürnbarkeit \\
\hline Angeberei & Wahrhaftigkeit & geheuchelte Bescheidenheit \\
\hline Poßenreißerei & Umgänglichkeit/Gewandtheit & Ungehobeltheit \\
\hline $\begin{array}{l}\text { Beliebtheits- } \\
\text { sucht/Schmeichelei }\end{array}$ & Freundlichkeit & mürrisches Wesen \\
\hline Schüchternheit & Scham & Schamlosigkeit \\
\hline Missgunst & berechtigte Entrüstung & Schadenfreude \\
\hline
\end{tabular}

Quelle: eigene Darstellung in Anlehnung an Aristoteles 2006 
Aristoteles gewinnt diese Verhaltensweisen aus dem Alltagswissen und erhebt mit ihnen keinen Anspruch auf Vollständigkeit. Vielmehr soll sein Tugendansatz eine Antwort auf ein gelungenes Leben geben.

Parallel zu Platon verleiht Aristoteles den Tugenden eine funktionale und normative Eigenschaft im Hinblick auf die Erhaltung einer Gesellschaftsordnung der Antike. Obwohl Ehrlichkeit als Tugend von beiden Autoren nicht erwähnt wird, verweist dies auf zwei wichtige Befunde für den Ehrlichkeitsbegriff. Zum einen haben die Tugenden bei Platon einen immer gültigen Aspekt, was anscheinend auf die Tugend Ehrlichkeit nicht zutrifft, weil diese nicht erwähnt wird. Dieser Befund verweist auf eine fließende und unklare Grenze zwischen den Tugenden im Allgemeinen und den Einzeltugenden und fordert eine Differenzierung des Begriffs, Tugend'.

Zum anderen verwendet Aristoteles in den Charaktertugenden den Ausdruck ,Wahrhaftigkeit' und die Gegenpole ,Angeberei' und ,geheuchelte Bescheidenheit ${ }^{\star}$ und fokussiert somit nur einen Teilbereich des Ehrlichkeitsbegriffs: die Wahrheit zu sagen. Gleichzeitig ist damit ein Indiz für die vielfältige Eigenschaft von Ehrlichkeit gegeben. Schließlich liefern beide Philosophen keine pädagogischen Ansätze, wie Tugenden zu erwerben sind. Somit schliessen beide philosophische Tugendansätze den Alltag und das damit zusammenhängende Erfahrungswissen aus.

Der platonische und aristotelische Tugendansatz ist im Verlauf der Geschichte der Philosophie unterschiedlich rezipiert worden. Eine besondere Aufnahme fand der aristotelische Tugendansatz im englischsprachigen Raum in den 1950er Jahren, welcher die Tugendethik (vgl. Nussbaum und PauerStuder 1999; Tugendhat 1984; MacIntyre 1981) in Abgrenzung zur kantianischen Prinzipienethik (vgl. Sommerfeld-Lethen 2005; Kant [1797] 1966) anstieß.

\subsubsection{Ehrlichkeit als bürgerliche Tugend}

$\mathrm{Zu}$ den bürgerlichen Tugenden, die sich größtenteils mit den preußischen Tugenden decken, zählen beispielsweise ,Ordnung', ,Disziplin', ,Pflichtbewusstsein und -erfüllung', ,Bescheidenheit', ,Unbestechlichkeit', ,bedingungsloser Gehorsam', ,Rechtschaffenheit" und ,Sparsamkeit" (Hillmann 2003, S. 206ff.; Bollnow 1958, S. 31ff.). In anderen Katalogen der bürgerlichen Tugenden wird Ehrlichkeit auch explizit aufgeführt (Münch 1984, S. 11; Junghänel et al. 1987, S. 145f.).

Der Zusammenhang zwischen Ehrlichkeit und bürgerlicher Tugend fordert eine knappe Erläuterung der bürgerlichen Tugenden, um deren bekannte missbräuchliche Verwendungen (s. weiter unten) aufzudecken und den Ehrlichkeitsbegriff in Abgrenzung dazu zu definieren. 
Ausgehend vom aufkommenden Pietismus im 17. Jahrhundert in Deutschland und dem anschließenden ,preußischen Beamtenideal' im 18. Jahrhundert war der gesellschaftliche Kontext gegeben, damit die preußischen Tugenden gefördert sowie bedeutsam wurden. Unter anderen Faktoren wird der Orientierung an diesen Idealen zugeschrieben, dass Deutschland eine wirtschaftliche, politische und militärische Großmacht wurde (Hillmann 2003, S. 206f.). Im 20. Jahrhundert sind die bürgerlichen Tugenden im Dienst der Macht des Dritten Reiches wiederum für politische Zwecke instrumentalisiert worden, wie beispielsweise im Postulat vom bedingungslosen Gehorsam bzw. ,Kadavergehorsam“ (ebd., S. 207). Zudem ist die ideologische Instrumentalisierung der Tugenden an zynischen Maximen festzumachen, beispielsweise wenn die Peiniger den Häftlingen im Konzentrationslager Dachau empfahlen:

Es gibt einen Weg zur Freiheit: Gehorsam, Fleiß, Ehrlichkeit, Ordnung, Sauberkeit, Nüchternheit, Wahrhaftigkeit, Opfersinn und Liebe zum Vaterland! (Münch 1984, S. 11)

Aus diesem geschichtlichen Abriss der bürgerlichen Tugenden wird verständlich, weshalb der Tugendbegriff zwischen den 1950er Jahren und heute weitgehend aus der pädagogischen Fachsprache und Alltagsprache verschwunden ist (vgl. Münch 1984, S. 17; Maier 1986, S. 40): Der eindimensional verwendete Tugendbegriff ist für ideologische Missbräuche anfällig. Der Tugendbegriff ist ab den 1960er Jahren durch den offenbar neutralen Wertebegriff ersetzt worden (vgl. Hillmann 2003; Hoyer 2005). Einen Rehabilitierungsversuch des Tugendbegriffs versuchte Bollnow (1958). Er wollte den terminologischen Verfallsprozess stoppen, indem er den Tugendbegriff als ,etwas Positives, eine wirkliche Kraft und Leistung“" (Bollnow 1958, S. 12) verstand und von „neuen Tugenden“ (ebd., S. 11) sprach. Bollnow expliziert in seiner Schrift Wesen und Wandel der Tugenden folgende Tugendliste, die sowohl ,alte Tugenden' mit neuen Bedeutungsinhalten sowie neue Tugendbegriffe enthält:

- bürgerliche Tugenden:

Ordnung, Sparsamkeit und Reinlichkeit

- Einzeltugend Fleiß

- drei ausdifferenzierte platonische Kardinaltugenden:

Tapferkeit (bürgerliche Tapferkeit; Mut und Tapferkeit, Kühnheit; Verschwiegenheit, Duldsamkeit und Standhaftigkeit)

Besonnenheit (Besinnung, Nähe zur Vernunft, Selbstbeherrschung)

Klugheit und Weisheit (Verstand und Vernunft)

- $\quad$ zwei Einzeltugenden:

Gelassenheit (innere Ruhe, gelassene Haltung, religiöses Grundgefühl)

Bescheidenheit (natürliche und neuartige Wertung, Tugend des Maßes, Demut, innere Sicherheit) 
- $\quad$ zwei Einzeltugenden, die missbräuchlich zu verwenden seien; beide Tugenden passen sich an Wertungen an, deren Verantwortbarkeit jeweils zu hinterfragen sei:

Wahrhaftigkeit (Wahrhaftigkeit, Ehrlichkeit, Aufrichtigkeit, Offenheit, Echtheit)

Treue (Gewohnheit, Ausdauer und Beständigkeit, Versprechen)

- vierte platonische Kardinaltugend sowie aristotelische und biblische Tugend:

Gerechtigkeit

Bei der Systematisierung der Tugenden stößt Bollnow ebenfalls an die Grenzen des Tugendbegriffs:

Je weiter die Betrachtung fortschreitet, desto schwerer wird es, die zu behandelnden Tugenden in eine systematische Reihenfolge zu bringen; denn mit jeder neu in Angriff genommenen treten neue Gesichtspunkte hinzu, die immer wieder den bisherigen Aufbau als ungenügend erweisen (Bollnow 1958, S. 135).

Otto Friedrich Bollnows Absicht ist es, den beständigen Wandel des Verständnisses von Tugenden aufzuzeigen, indem alte Tugenden verschwinden und neue Tugenden hinzukommen. $\mathrm{Zu}$ den neueren Tugenden zählt er beispielsweise Wahrhaftigkeit und Treue, weil es bei diesen „um das Verhalten des Menschen zu sich selbst geht" (ebd.) und damit um einen Bezug nach innen.

Wahrhaftigkeit als neuere Tugend zu bezeichnen überzeugt nicht, da diese bereits Aristoteles in der Nikomachischen Ethik erwähnt hat. Zudem ist die innere Charaktereigenschaft bei Tugenden wie Besonnenheit oder Gelassenheit viel offensichtlicher. Dennoch lohnt es sich, Bollnows Tugendliste genauer zu betrachten, da er einen differenzierten Fokus auf die Tugend Wahrhaftigkeit und deren bedeutungsähnliche Einzeltugenden richtet. So unterscheidet er:

- Wahrhaftigkeit:

Als Gesinnung wendet sich die Tugend Wahrhaftigkeit nach innen (Bollnow 1958, S. 139).

- Ehrlichkeit:

Ehrlichkeit ist ein Verhalten, das sich nach außen richtet und ,Ehre' zu bewahren hat, die nicht verletzt werden soll (ebd., S. 141).

- Aufrichtigkeit:

Aufrichtigkeit bezieht sich auf eine körperliche und eine symbolische Haltung, setzt einen sittlichen Willen und das Vertrauen in die eigenen Kräfte voraus (ebd., S. 143).

- Offenheit:

Der Mensch ist das, was er nicht verbirgt. ,Offenheit' setzt keinen Willen voraus, sondern ist von der Natur gegeben. Offenheit ist damit kein Verhalten, keine Gesinnung, sondern ein ,So-Sein“ (ebd., S. 144). 
- Echtheit:

Der Mensch ist so, wie er sich nach außen - meist unbewusst - gibt. Man kann sich auf ihn verlassen (ebd., S. 146f.).

Bollnow zeigt auf, wie ,Wahrhaftigkeit" die Verhaltensweisen ,Ehrlichkeit" und ,Offenheit' sowie ,Aufrichtigkeit' und ,Echtheit' berührt, jedoch nicht deckungsgleich damit ist. Von der begrifflichen Differenzierung verspricht sich Bollnow ein ,tieferes Verständnis' der einzelnen Tugenden.

Die begriffstheoretischen Ausführungen zu den Tugenden Wahrhaftigkeit, Ehrlichkeit, Offenheit, Aufrichtigkeit und Echtheit weisen wechselseitige Bezüge und Unterscheidungen auf, die Bollnow auf einer philosophischen Ebene abhandelt. Allerdings berücksichtigt und diskutiert er die genannten Bezüge und Unterscheidungen zwischen den Tugenden nicht im pädagogischen Kontext oder im Erfahrungsraum. Zudem ist in den erwähnten Tugendansätzen in Form von Tugendlisten der Zusammenhang zwischen Tugenden im Allgemeinen und Einzeltugenden durch die Subsumierung der Einzeltugenden unter den gesellschaftlich erwünschten Tugendbegriff hergestellt, aber dadurch noch nicht erklärt. Anders ist dies bei den Sekundärtugenden, wo die Beziehung der Tugenden und der Sekundärtugenden erklärt wird.

\subsubsection{Ehrlichkeit als Sekundärtugend}

Ehrlichkeit zählt nicht nur zu den bürgerlichen Tugenden, die Bollnow vergeblich zu rehabilitieren versuchte (vgl. Bollnow 1958), sondern auch zu den ,Sekundärtugenden'. Damit sind uneinheitlich bürgerliche Tugenden ab Ende der 1960er Jahre gemeint; der Begriff betont mißbrauchbare, aber dennoch unverzichtbare Eigenschaften der Tugenden (Fellsches 1996, S. 95; Spaemann 1988/1989, S. 82). Er ist von Unterscheidungskriterien der kulturellen Angemessenheit abhängig, nach denen Tugenden als Sekundärtugenden eingeordnet werden. Einige Autoren bezeichnen alle Tugenden als Sekundärtugenden, weil Tugenden grundsätzlich missbräuchlich verwendet werden können, wie beispielsweise während des Zweiten Weltkriegs in provozierender Weise offenbar wurde. Demgegenüber definiert Gamm Primärtugend als eine Tugend, die eine Person nicht zum Objekt und sie somit nicht von anderen Leuten abhängig macht. Vielmehr definiert er die Primärtugend als eine Tugend, die Personen als handelnde und aktive Personen und damit als ,Subjekte“ ansieht: „Als primäre Tugenden dürfen jene gelten, die den einzelnen ermächtigen, sich zum Subjekt seiner Geschichte zu formieren" (Gamm 1988, S. 99). Ein anderer Ansatz geht davon aus, dass Tugenden sich in Primärtugenden und Sekundärtugenden aufteilen. In diesem Sinne definiert Robert Spaemann Sekundärtugenden nach ihrer Zielfunktion: 
Sekundäre Tugenden nennen wir solche erworbenen Eigenschaften, die für sich betrachtet in ihrem Wert indifferent sind gegen ihre Funktion, die also ihren eigentlichen Wert erst erhalten durch das Ziel, dem sie dienen (Spaemann 1988/1989, S. 82).

Der Autor unterscheidet Sekundärtugenden von universellen Tugenden, die im Sinne von Platons Tugendansatz zu verorten sind. In Spaemanns Definition können Sekundärtugenden nur zum Tugendbegriff als solchem dazugezählt werden, wenn das Ziel der Tugend recht und gut ist.

Eine Begriffsunterteilung in Primärtugenden und Sekundärtugenden kann m. A. n. ebenfalls aus dem aristotelischen Ansatz abgeleitet werden: Aristoteles unterscheidet zwischen allgemeingültigen Vernunfttugenden und vielfältigen Charaktertugenden. Erstere gelten immer und führen zu positivem Verhalten, hingegen bestimmt der Situationskontext, ob eine Charaktertugend zu einem positiven Verhalten führt. So können Diebe die Charaktertugend ,Fleiß' auch bei einer unmoralischen Verhaltensweise wie beispielsweise Diebstahl aufweisen.

In den gegenwärtigen Tugendansätzen lehnt Fellsches die Unterscheidung zwischen Primärtugenden und Sekundärtugenden ab und Koch behält diese bei: Fellsches hebt die Unterscheidung zwischen primären und sekundären Tugenden für seinen theoretischen Tugendansatz auf, weil für ihn sämtliche Tugenden Primärtugenden sind und, zum Gelingen eines guten Lebens' passen. Zudem zählt er Ordnung, Fleiß und Disziplin nicht zu den Tugenden. Denn Tugenden beziehen sich nach Fellsches nicht auf Funktionalismen, wie dies Ordnung, Fleiß und Disziplin tun, sondern vielmehr auf Lebenshaltungen, welche auf, menschliche Begegnungen zielen' und auf Vertrauen, Gegenseitigkeit und Liebe basieren (Fellsches 1996, S. 97).

Friedrich Koch hingegen setzt Sekundärtugenden und bürgerliche Tugenden gleich und zeigt kritisch deren schädliche Eigenschaften auf. Dennoch empfiehlt er, Tugenden nicht abzuschaffen, sondern in einen neuen Zusammenhang zu setzen: „Wir müssen sie auf die primären Werte zuordnen, um ihre Bedeutung zu erkennen“ (ebd., S. 117). So werden heute - gemäss Koch - Abmachungen in einem Team oder abgesprochene Regeln nicht mehr als Tugend des Gehorsams bezeichnet. Damit weist er auf den immanenten ,Wandel der Tugenden“ hin: „Es geht nicht um eine Ablösung der überlieferten Sekundärtugenden durch neue Wertevorstellungen, sondern um die Zurechtrückung des Verhältnisses von primären und sekundären Werten“ (ebd., S. 118). Mit dem Wandel und der Unentbehrlichkeit der Tugenden vertreten Friedrich Koch und Otto Friedrich Bollnow den gleichen Standpunkte und die gleiche Forderung des Umgangs mit Tugenden.

Tugenden werden also in heterogenen Begriffssystemen verwendet. $\mathrm{Zu}$ den ordnenden Begriffssystemen gehören die bürgerlichen Tugenden sowie die Primär- und Sekundärtugenden. Die begriffstheoretischen Systematisierungsversuche ermöglichen es, eine funktionalistische Eigenschaft des Tu- 
gendbegriffs zu erfassen. Die vorgestellten Autoren konstituieren den Bezug zwischen Tugenden und Einzeltugenden folgendermassen: Erstens, indem Einzeltugenden unter den Tugendbegriff summiert werden. Zweitens, indem Primärtugenden und Einzeltugenden gleichgesetzt werden und drittens, indem bestimmte Einzeltugenden den Primärtugenden (quasi ,immergültige Tugenden') und andere Einzeltugenden den Sekundärtugenden zugeordnet werden (Wert der Einzeltugend liegt in dessen Ziel oder wenn die Person als Subjekt betrachtet wird). Damit wird zwar der Bezug zwischen Tugend und Einzeltugend angegangen, aber nicht vollständig geklärt. Denn weshalb eine bestimmte Anzahl Tugenden unter Primärtugenden oder Sekundärtugenden gezählt wird und nicht eine kleinere oder größere, bleibt unbestimmt.

Allerdings steht nach dem angeführten historischen Einblick zum Ehrlichkeitsbegriff und zu den dazugehörenden Ehrlichkeitsansätzen fest, dass in der vorliegenden Arbeit eine rigide Verwendung des Tugendbegriffs vermieden werden sollte, da diese zu einer missbräuchlichen Erziehung führen kann und um der situationsabhängigen Eigenschaft der Tugenden gerecht zu werden. Erziehungsansätze, die von einem Tugendbegriff ausgehen, haben m. A. n. folgende Orientierungspunkte zu beachten:

- persönliche Entscheidungsfreiheit der Heranwachsenden

- Reflexionsmomente der Heranwachsenden

- alternative Verhaltensweisen, die Heranwachsende annehmen können

Der Tugendbegriff soll daher in dieser Arbeit im Sinne einer offenen Verhaltensweise verwendet werden (vgl. Kap. 2.5).

\subsubsection{Gegenwärtige Tugendlisten und -kanons}

Tugendlisten sind noch immer aktuell. Ein Einblick in zwei Tugendkataloge soll eine ,modernisierte' Tugendvielfalt wie auch Systematisierungsschwierigkeiten aufzeigen.

Fellsches (1996) geht von einer Tugendliste aus, um einen modernisierten theoretischen Tugendansatz zu entwickeln. Dabei ersetzt er den Tugendbegriff durch den Haltungsbegriff (vgl. Kap. 2.3.3). Die Grund- und Haupthaltungen sind Liebe, Hoffnung und Vertrauen (Fellsches 1996, S. 127). Daneben zählen die vier umformulierten platonischen Kardinaltugenden zu den Haupthaltungen (ebd., S. 132ff.):

- Bewusstsein

- Klugheit

- Gerechtigkeit

- Standhalten 
Die Kardinaltugenden ergänzt Fellsches mit vier weiteren Haltungen bzw. Tugenden (ebd., S. 134ff.):

- Zusammenleben

- Bürgersinn

- Selbstkultur

- Treue zu sich selbst

- $\quad$ Lust und Freude

In Fellsches“ Listen der Einzeltugenden ist ,Ehrlichkeit“ nicht explizit erwähnt, aber mit der Einzeltugend Treue zu sich selbst ist ein Teilaspekt von Ehrlichkeit tangiert.

Eine weitaus umfangreichere Tugend- und Werteliste stammt von Hillmann (2003), in der er Werte und Tugenden in zahlreichen Oberkategorien ohne Anspruch auf Vollständigkeit systematisiert und mit empirischen Befunden untermauert. Die Oberkategorien heißen:

- $\quad$ humane und liberale Grundwerte (ebd., S. 188ff.)

- prosoziale Werte (ebd., S. 199ff.)

- Anstands- und Höflichkeitswerte (ebd., S. 203)

- bürgerliche Tugenden (ebd., S. 206)

- $\quad$ Arbeits- und Berufswerte (ebd., S. 212)

- materialistische Wertorientierungen (ebd., S. 220)

- $\quad$ hedonistische Wertorientierungen (ebd., S. 224)

- $\quad$ individualistische Wertorientierungen (ebd., S. 228)

- familienorientierte Wertvorstellungen (ebd., S. 236)

- $\quad$ konservative Wertvorstellungen (ebd., S. 224)

- $\quad$ ethnisch-nationale Wertorientierungen (ebd., S. 246)

- religiöse Wertorientierungen (ebd., S. 255)

- $\quad$ asketische Tugenden und Moralvorstellungen (ebd., S. 263)

- idealistisch-nichtmaterialistische Wertorientierungen (ebd., S. 267)

- ökologische Wertorientierungen (ebd., S. 272)

- $\quad$ politische Wertorientierungen (ebd., S. 276)

- $\quad$ negative Werte und Unwerte (ebd., S. 279)

Die Tugend Ehrlichkeit ist einmal unter den prosozialen Werten erwähnt: „Ehrlichkeit: Aufrichtigkeit, Wahrhaftigkeit, Vertrauen, Treue, Zuverlässigkeit, Offenheit, Natürlichkeit“ (Hillmann 2003, S. 199). Weiter ist Ehrlichkeit nicht explizit unter den bürgerlichen Tugenden zu finden, außer wenn inhaltliche Affinitäten $\mathrm{zu}$,Unbestechlichkeit' und ,Rechtschaffenheit' (ebd., S. 208) angenommen werden. Zu den anderen Oberkategorien wird Ehrlichkeit nicht dazugezählt, obwohl Affinitäten möglich wären, wie beispielsweise zu den Arbeitertugenden oder zu den religiösen Tugenden. 
Die beiden angeführten Tugendlisten aus der Gegenwart haben bezüglich der Definition von Ehrlichkeit ähnliche Grenzen, die nun zusammengefasst und reflektiert werden.

\subsubsection{Kritische Reflexionen zu Tugendkatalogen}

Anhand von Tugendkatalogen sollen Einzeltugenden möglichst vollständig systematisiert werden, um eine Übersicht zu gewinnen und Erkenntnisse für die Tugenddefinitionen zu liefern. Das hochgesetzte Ziel lässt sich schwer erfüllen, wie die unübersichtlichen und uneinheitlichen Tugendlisten belegen. Historische, sprachliche und kulturelle Faktoren erschweren ein solches Vorhaben. Der Bezug zwischen Tugenden und Einzeltugenden kann durch Unterscheidungen von Primär- und Sekundärtugenden (gleichbleibende oder ambivalente Eigenschaften der Einzeltugend) nur im Ansatz geklärt werden. Daher kann die Gruppierung von Einzeltugenden willkürlich erscheinen oder bestimmte Einzeltugenden mehreren ,Oberkategorien' zugeordnet werden. Zudem kann innerhalb von Tugendlisten nicht bei allen Tugenden eine scharfe Begriffsdefinition vorgenommen werden, sodass Überschneidungen vorkommen. Die allgemeine Schwierigkeit, Einzeltugenden wie beispielsweise Ehrlichkeit als eine Kategorie in Tugendkatalogen zu formulieren, zeigt die Grenzen der Tugendkataloge auf. Graham Haydon wies bereits 1999 auf die theoretische Vielschichtigkeit des Tugendbegriffs hin (Haydon 1999), die zu klären wäre - was bisher eine Forschungslücke bleibt. Zudem sind Tugenden in den Tugendkatalogen moralisch und funktional verwendet, um gesellschaftliche Auffassungen und Ordnungen zu erhalten, ohne die individuelle Mitbestimmung der Betroffenen zu berücksichtigen. Damit wurden Tugenden ideologisiert und repliziert. Indem alltägliche Kontexte durchgehend aus den Abhandlungen zu Tugenden ausgeschlossen waren, begünstigte dies den beschriebenen begriffstheoretischen Prozess.

\subsection{Ehrlichkeitsbegriff in der angelsächsischen Tugenddebatte}

Tugenden sind im angelsächsischen Raum seit den 1990er Jahren in neuer Form wiederentdeckt worden und werden auf die wissenschaftliche Schulpädagogik bezogen: Es fand ein Zusammenschluss von Erziehungsphilosophie und Bildungspolitik statt. Anhand der regen und aktuellen Tugenddebatten in Großbritannien, die als solche im Vergleich zu anderen europäischen Ländern einmalig sind, zeigt dieses Unterkapitel, welche Ehrlichkeitsdefinitionen 
und welche damit zusammenhängenden theoretischen Ansätze im angelsächsischen Kontext vorgeschlagen werden.

Der englische und traditionsreiche Terminus virtues gewinnt seit rund zwanzig Jahren an Aktualität und wird bis heute im Zusammenhang mit der öffentlichen Persönlichkeitsbildung (character education) und der politischen Bildung (citizenship education) in bildungspolitischen und erziehungsphilosophischen Debatten aktiv diskutiert. Citizenship education lautet die Bezeichnung des seit 2002 obligatorischen Schulfaches, in dem character education mit einem moralischen Gehalt neben bildungspolitischen Inhalten unterrichtet wird (Davies et al. 2005; Arthur 2005). Ziel der character education ist es, demokratische Tugenden zu fördern. Zudem sind Einzeltugenden seit 1999 im nationalen Lehrplan Englands erwähnt: „Education should also reaffirm our commitment to the virtues of truth, justice, honesty, trust and a sense of duty" (National Curriculum (NC) 2008 und 1999, S. 10).

Der Fokus der angelsächsischen Tugendthematik richtet sich auf die Persönlichkeitsbildung in der Schule (vgl. Arthur et al. 2009; Arthur 2003; McLaughlin und Halstead 1999), die demokratischen und pädagogischen Tugenden der Lehrpersonen und der Lehrerbildung (Kunzman 2006), die theoretischen Wurzeln der Tugenden im aristotelischen Ansatz (Carr 2007) sowie die kommunitaristischen und liberalistischen Ansätze der moralischen Erziehung (Haydon 2001).

Mit dem britischen Tugendbegriff sind ganz allgemein sowohl gute Eigenschaften des Charakters und der Persönlichkeit als auch Umgangsformen, Fertigkeiten und Fähigkeiten einer Person gemeint. Zivilcourage, Fairness, Anstand, Respekt, Ehrlichkeit, Hoffnung oder Vertrauen zählen zu den Einzeltugenden. Auf diese Tugenden kann gemäss den Autoren in einer demokratisch funktionierenden Gesellschaft kaum verzichtet werden.

Vertreter dieser Debatten grenzen sich von den amerikanischen Tugenddebatten $a b$ und kritisieren an diesen eine unscharfe und uneinheitliche begriffstheoretische und theoretische Fundierung des Tugendansatzes (vgl. McLaughlin und Halstead 1999; Horton und Mendus 1994). Zu den prominenten Exponenten der amerikanischen Tugenddebatte zählen Alasdair MacIntyre (1981) und Thomas Lickona (1991). Diese gehen von einer moralischen Krise der gegenwärtigen Gesellschaft aus, worauf eine Rückbesinnung auf und Förderung der antiken Tugenden eine Antwort wäre.

Tugenden sind gemäss beiden Autoren Charaktereinstellungen und als verinnerlicht zu verstehen. Lickona beispielsweise grenzt einen so verstandenen Tugendbegriff von Werteeinstellungen ab. Auf diese Weise definiert er Tugend wie folgt:

Character consists of operative values, values in action. We progress in our character as a value becomes a virtue, a reliable inner disposition to respond to situations in a morally good way (Lickona 1991, S. 51). 
Lickona geht hier von einem abstrakten Werteverständnis aus, das schwer zu erreichen ist, und fordert, dass Werte in Handlungen und in Aktionen festgesetzt werden. Gleichzeitig versteht er Tugenden als verinnerlichte Charaktereigenschaften. Jedoch führt Lickona nicht weiter aus, wie genau der Prozess von einem Wert zu einer Tugend verlaufen soll. Die Notwendigkeit und Legitimation von Tugenden macht er daran fest, dass er den Lesern diverse unmoralische Vorkommnisse aufzählt, was eine emotionale Zustimmung der Leserschaft hervorruft. Da Tugenden scheinbar kaum noch vorhanden sind, sollen diese gefördert werden, und zwar vorwiegend in der Schule. Lickona geht von einem allgemeinen Tugendbegriff aus und erklärt keine Einzeltugenden. Eine ähnliche Argumentationslinie ist in den populären deutschsprachigen Debatten wiederzufinden (vgl. Kap. 1.2).

Im Unterschied zum amerikanischen Tugenddiskurs, der unter dem Begriff character education steht, wird der angelsächsische Diskurs primär unter dem Begriff citizenship education geführt. Beiden Diskursen gemeinsam ist das Verständnis der Tugenden als Charaktereigenschaften, die zu bilden sind. So definiert der Erziehungsphilosoph James Arthur Charakter wie folgt:

First, there is such a thing as character, an interlocked set of personal values and virtues which normally guide conduct. Character is about who we are and who we become and includes, amongst other things, the virtues of responsibility, honesty, self-reliance, reliability, generosity, self-discipline, and a sense of identity and purpose (Arthur et al. 2009, S. 13).

Arthur et al. zeigt hier einen umfassenden Ansatz zum Verständnis von Charakter, der sowohl Werte als auch Tugenden enthält. Der Charakter bestimmt die Identität und Zukunftsausrichtung der Person und enthält unter anderem viele Tugenden, wovon Arthur sieben aufzählt. Er stellt zudem fest, dass der Tugendbegriff aus dem Sprechen über Charakter beseitigt wurde, was zu einer fehlenden Klarheit über moralische Themen in der Schule, beispielsweise bei der Selbstverantwortung, führte. Arthur schlägt vor, zwischen Charakter und Persönlichkeit zu unterscheiden:

Lastly, at a conceptual level it is important to distinguish between the qualities of character that define virtue from other qualities of the self and/or person which we are more inclined to associate with such notions as personality (Arthur et al. 2009, S. 13).

Die Tugenden bestimmen den Charakter, und andere Qualitäten sind der Persönlichkeit eigen. Vor dem Hintergrund der angelsächsischen und amerikanischen Tugenddebatten kann m. A. n. die Unterscheidung zwischen Charakter und Persönlichkeit noch schärfer formuliert werden bzw. zwischen Tugenden und anderen Qualitäten, was ich wie folgt formulieren will: Die Schule als öffentliche Instanz fördert soziale im Sinne von demokratischen Tugenden (Kunzman 2006; White 1996), welche als Verhaltensweisen und nicht als verinnerlichte Haltungen zu verstehen sind. Letztere ist Sache der 
individuellen Persönlichkeitsbildung. Allerdings ist nicht so klar, wo die Grenze zwischen Sozialem und Verinnerlichtem liegt, was in dieser Studie am Beispiel des Schülers Thomas gezeigt wird: Dieser verhält sich sozial gegenüber den Mitschülern und verinnerlicht gegenüber der Lehrperson.

Zurück zu den angelsächsischen Autoren, welche den amerikanischen Kollegen mangelnde theoretische Fundierung vorwerfen. Jene bieten selbst, trotz der regen Tugenddebatten, ebenso wenige einheitliche theoretische Tugendansätze. Noch schwieriger ist es, theoretische Ehrlichkeitsansätze zu finden.

Eine Ausnahme ist Patricia White, die sich mit Einzeltugenden, speziell im schulischen Kontext, befasst. Insbesondere in ihrer Veröffentlichung Civic Virtues and Public Schooling. Educating Citizens for a Democratic Society (1996) geht sie auf Einzeltugenden ein, die den Tugendbegriff im demokratischen Kontext neu verstehen lassen sollen. Dazu zählt sie sieben schulrelevante Tugenden auf und verwendet wie im deutschsprachigen Diskurs einen Tugendkatalog, der folgende Einzeltugenden beinhaltet ${ }^{13}$ :

- hope

Whites Hoffnungsbegriff folgt keinen frommen Eigenschaften, sondern es geht um soziale Vertrautheit, darum, dass die anderen Bürgerinnen und Bürger wichtig sind und dass sie gebraucht werden.

- courage

Beim Mutbegriff geht es um die Zuversicht und das Vertrauen in die eigenen Fähigkeiten und Kompetenzen.

- democratic self-respect and self-esteem

Selbstachtung und Selbstschätzung sind sowohl im individuellen als auch im sozialen Kontext zu betrachten.

- friendship

Freundschaft zeigt einen Ausgleich zwischen guten Wünschen und guten Verhaltensweisen.

- $\quad$ trust

Vertrauen hat einen individuellen und einen sozialen Aspekt und ist wichtig, um die grundlegende Verbindung zwischen Menschen und die Möglichkeit von sozialen Institutionen zu verstehen.

- honesty

Bei der Tugend Ehrlichkeit geht es um die Fähigkeit zu wissen, wann die Wahrheit, wann Halbwahrheit angebracht ist und wann geschwiegen werden soll (verschwiegene Wahrheit).

13 Die Übersetzung der englischen Tugenden ins Deutsche stellt ein schwieriges Unterfangen dar. Deshalb werden die englischen Originalbegriffe verwendet und umschrieben. Interessanterweise gelten Aspekte der Ehrlichkeit wie sincerity, frankness, plainess, ingenousness im 18. Jahrhundert als leicht verwechselbare Wörter, wie aus dem Wörterbuch von Trusler (1776) zu englischen Synonymen hervorgeht. Eine Parallele zu heutigen englischen Wortverwendungen und zu den zusätzlichen Deutschübersetzungsbemühungen ist offensichtlich. 
- decency

Anstand als ,negative Tugend' weist eine offene Definition auf, sodass nicht eindeutig gegeben ist, wann etwas richtig ist oder nicht. Daher kann auch nicht darauf insistiert werden.

Whites Definition von Einzeltugenden richtet sich auf die demokratische Gesellschaft. Sie geht davon aus, dass Heranwachsende die meisten Einzeltugenden in der Familie ansatzweise erwerben und dass sich diese in der Schule weiterentwickeln. Die Institution Schule hat damit einen erzieherischen Auftrag und Einfluss. Dabei soll der religiöse, ethische und kulturelle Hintergrund von SuS beachtet werden, was bezüglich der notwendigen Unterscheidung zwischen demokratischen und verinnerlichten Tugenden eine nicht einzulösende Erwartung ist. Vor dem Hintergrund des siebenteiligen Tugendkatalogs definiert White ,Ehrlichkeit' graduell (wie oben als die Wahrheit, Halbwahrheit und verschwiegene Wahrheit) und in Abgrenzung zur dualen Ehrlichkeitsdefinition (ehrlich - unehrlich) dreifach:

- veracity

- uprightness

- candor

Ehrlichkeit kann als veracity (White 1996, S. 67f.), Wahrhaftigkeit, verstanden werden, wenn Wahres und nichts Falsches ausgesagt wird. Dabei ist entscheidend $\mathrm{zu}$ wissen, wann der richtige Moment ist, um das ,Wahre“ preiszugeben. Diesen Aspekt erwähnt White, ohne weiter darauf einzugehen. Wenn Ehrlichkeit weniger auf das Reden, mehr auf Besitzangelegenheiten bezogen wird, bezeichnet White dies als uprightness (ebd., S. 66f.), Aufrichtigkeit. In der zweiten Differenzierung des Ehrlichkeitsbegriffs geht es darum, dass das Reden oder die Überzeugung mit den Verhaltensweisen übereinstimmt. White erklärt dies am Beispiel des Schummelns. Wenn beispielsweise SuS nicht aus eigener Leistung einen Schultest schreiben, sei dies nicht aufrichtig, und wenn Gegenstände im Schulzimmer im Verborgenen beschädigt werden, sei dies nicht ehrlich. Als pädagogisches Argument müsste nach White den Lernenden aufgezeigt werden, dass Schummeln gegenüber anderen Mitschülerinnen und Mitschülern unfair sei und dass Gegenstände im Schulzimmer, die durch Fehlverhalten beschädigt werden, der Allgemeinheit gehören. Schließlich bezeichnet White Ehrlichkeit auch als candor (ebd., S. 66f.), Offenheit; sie ist dann der Fall, wenn beispielsweise unter Freunden ohne Vorbehalte alles gesagt werden kann.

$\mathrm{Ob}$ es genau drei Aspekte von Ehrlichkeit oder mehr bzw. ob es sieben schulrelevante und demokratische Tugenden gibt oder mehr, lässt White parallel zu anderen Autoren von Tugendkatalogen ungeklärt. Weiter ist interessant, dass das Beschädigen von Gegenständen im Schulzimmer als Unehrlichkeit gilt. Meines Erachtens zählt das Verheimlichen von solchen Vorkommnissen als unehrlich, und Sachbeschädigungen ordne ich unter unacht- 
sames Verhalten ein, was zu Whites aufgeführter Tugend der, sozialen Selbstachtung' gut passt.

Whites dreifacher Ehrlichkeitsbegriff dient als Ausgangspunkt für die Bestimmung des Ehrlichkeitsbegriffs in Kapitel 2.6. Allerdings fehlt in ihrem Ansatz ein expliziter und erklärender Bezug zum Tugendansatz. Deshalb führt das nächste Unterkapitel einen Tugendansatz an, der sowohl für den dreifachen Ehrlichkeitsbegriff als auch für die empirische Untersuchung passend und erklärend ist.

\subsection{Ehrlichkeitsbegriff als moralische Verhaltensweise}

Whites Ehrlichkeitsbegriff (vgl. Kap. 2.4) basiert auf einem undefinierten Tugendbegriff, der mittels Reichenbachs Tugendbegriff (vgl. Reichenbach 2004 und 2008) im Folgenden geklärt werden soll. Reichenbach bestimmt Tugend als moralische Verhaltensweise und grenzt sich damit klar von Ansätzen der sozialen Kompetenz sowie von Tugendbegriffen ab, die als verinnerlichte Haltung oder allein als Charaktereigenschaft verstanden werden, wie dies beispielsweise in amerikanischen sowie teilweise in angelsächsischen Tugenddebatten der Fall ist (vgl. Kap. 2.4). Somit wird hier Whites Ehrlichkeitsbegriff in Bezug zum Tugendbegriff als moralische Verhaltensweise gesetzt.

\subsubsection{Tugenden als Kompetenz verbunden mit Moral}

Spätestens seit der internationalen PISA-Studie im Jahr 2000 dominiert im deutschsprachigen Raum der Kompetenzansatz das Feld der Erziehung und Bildung (vgl. Bieri Buschor und Forrer 2005; Rekus 2007), womit Ansätze der Werteerziehung weitgehend verdrängt wurden. In Abgrenzung zu den sozialen und überfachlichen Kompetenzen bietet Roland Reichenbach (2004 und 2008) einen aktuellen und komplexen Tugendansatz, der Tugenden als moralische Verhaltensweisen definiert.

Reichenbach bestimmt Tugend als „Kompetenz verbunden mit Moral“ (Reichenbach 2004, S. 7f.). Damit weist er auf einen zusätzlichen Aspekt des Tugendansatzes gegenüber dem Ansatz der sozialen Kompetenzen hin. Denn der Ansatz sozialer Kompetenzen geht gemäss Reichenbach von einem reibungslosen Begriff aus. So kann eine Verhaltensweise als sozial kompetent eingestuft werden, ohne sicher zu sein, ob diese der jeweiligen Situation angemessen ist. An einem Beispiel aus der eigenen Feldforschung soll dies veranschaulicht werden: Die Lehrerin fragt die Schülerin Sibylle, ob sie die Schokolade vom Lehrerpult mit den anderen gegessen hätte. Diese antwortet: 
„Nein, ich habe die Schokolade nicht gegessen“, obwohl sie sie gegessen hat. Das wollte sie aber der Lehrerin gegenüber nicht zugeben. In diesem Einzelfall lügt Sibylle. Somit kann man nicht sagen, Sibylle sei ehrlich. Sie kann aber in dieser Situation sozial kompetent sein und im Unterricht integer und aufrichtig agieren (vgl. Datenmaterial der ersten Erhebungsphase).

Reichenbachs Tugendansatz ist ein dreifacher (Reichenbach 2007, S. 195ff.): Erstens haben Tugenden mit dem Willen einer Person zu tun, womit der Bezug zur handelnden Person bestimmt ist. So kann die Schülerin Monika aus Eigeninitiative als Einzige in der Klasse ehrlich dem Lehrer gegenüber sein und ihm bestätigen, dass die Hausaufgaben tatsächlich bis zu diesem Tag zu erledigen gewesen waren. ${ }^{14}$

Zweitens sind Tugenden, an denen der Mensch sein Verhalten ausrichten soll, situativ gebunden. Der Schüler Daniel verwendet in Schultests prinzipiell keine unerlaubten Hilfsmittel. Wenn aber eine Lehrperson nicht gut kontrolliert oder er sich in einer Notsituation befindet, hat auch er sich schon durch Zuflüstern oder eine versteckte Notiz weiterhelfen lassen. ${ }^{15}$

Drittens haben Tugenden insofern mit dem Charakter zu tun, als nicht nur die positiven Eigenschaften, sondern auch die negativen - sogenannte Laster oder Untugenden - benannt werden können.

In diesem Sinne definiert Reichenbach Tugend als moralische Verhaltensweise auf dreifache Weise, womit ein Tugendbegriff für die empirische Untersuchung angemessen bestimmt ist. Zudem kann Whites Ehrlichkeitsbegriff, den sie nicht explizit auf einen theoretischen Tugendansatz bezieht, in einem solchen situiert werden. Somit sind der Ehrlichkeitsbegriff und der damit zusammenhängende Tugendbegriff geklärt.

\subsection{Ehrlichkeitsbegriff als loser theoretischer Rahmen für die Arbeit}

Die Befunde des zweiten Kapitels werden an dieser Stelle zusammengefasst und reflektiert. Daraus folgt die Bestimmung des Ehrlichkeitsbegriffs für die vorliegende Arbeit, der als loser theoretischer Rahmen zu verstehen ist.

Parallel zur fehlenden Tugenddefinition in populären Erziehungsdebatten (vgl. Kap. 1.1) gilt dasselbe für den Ehrlichkeitsbegriff: Dieser wird in essayistischen Ehrlichkeitsdebatten eher einseitig verwendet und mit sozialen Erwartungen verknüpft. Psychologische Untersuchungen untersuchen einen kontextabhängigen Ehrlichkeitsbegriff, gehen aber von einem dualen Ehrlichkeitsbegriff (,ehrlich - unehrlich') aus. Insbesondere in der Lügenfor-

14 Das Beispiel ist im Interview mit Lilian erwähnt, vgl. dazu Chiapparini 2011, S. 2ff.

15 Vgl. das Datenmaterial der zweiten Erhebungsphase. 
schung ist Unehrlichkeit relevant, allerdings auf der kommunikativen Ebene des verbalen Täuschens oder des Vorenthaltens von Informationen. Daher vermögen psychologische Ansätze keine weiteren begriffstheoretischen Anhaltspunkte zu bieten. Für die Untersuchung zu Deutungen von Ehrlichkeitspraxen ist jedoch ein eindimensionaler Begriff nicht hinreichend: Damit können die komplexen, dynamischen und vielfältigen Strukturen nicht aufgezeigt werden, die zu vermuten sind. Die Wortvielfalt aus der Alltagssprache weist bereits darauf hin, dass sie teilweise auch in den oben vorgestellten Tugendkatalogen wiederzufinden ist.

Ausgehend von Whites Ehrlichkeitsverständnis (White 1996) und den Ergebnissen meiner ersten Untersuchung (Chiapparini 2012) können folgende Begriffe unter Ehrlichkeit summiert werden:

- Wahrhaftigkeit (veracity)

- Aufrichtigkeit, Rechtschaffenheit (uprightness)

- Offenheit, Nettigkeit, Wohlwollen (candor)

- Echtheit

- Direktheit

- Transparenz

- Geradlinigkeit, unabhängig von gesellschaftlichen Konventionen und Schamgefühl der anderen (frankness)

- Integrität

- Loyalität

Diese unvollständige Liste zeigt auf, dass sich der Ehrlichkeitsbegriff als ein summierender Begriff auszeichnet. Das ist eine gemeinsame Eigenschaft der Einzeltugend Ehrlichkeit und des Tugendbegriffs, wobei dies beim Tugendbegriff auf einer allgemeineren und weiter zu fassenden Ebene zutrifft (unter Tugend steht eine größere Auswahl von Einzeltugenden zur Verfügung, die subsumiert werden können, hingegen können einer Einzeltugend wenige einzelne Einzeltugenden zugeordnet werden). Ferner steht der Ehrlichkeitsbegriff in einem engen begriffstheoretischen Bezug zum Tugendbegriff, was in den vorherigen Unterkapiteln belegt werden konnte. Die summierende Eigenschaft des Tugendbegriffs erklärt, weshalb der Tugendbegriff mit Recht als Sammelbegriff oder, inflationärer Begriff‘ verwendet wird, ähnlich wie der Bildungsbegriff als ,multidisziplinäre Substrat-Kategorie“ (Tenorth 2007, S. 975) oder als ,Container-Wort‘ (ebd.).

Eine beliebte Systematisierungsweise der Tugenden existiert seit der griechischen Antike in Form von Tugendkatalogen, welche je nach historischem Zeitabschnitt, Kultur und Sprache zu unterschiedlich komplexen und wenig übersichtlichen Listen führten. Den Tugendlisten gemeinsam ist, dass Tugenden allzu oft funktionale und moralische Verwendung fanden und damit ideologisiert und reproduziert wurden, ohne den Alltag und das darauf gründende Erfahrungswissen zu berücksichtigen. Zudem ist der Zusammen- 
hang zwischen Tugenden und Einzeltugenden weitgehend unreflektiert und unerforscht. Des weiteren konnte Münchs Beobachtung bestätigen, dass Ehrlichkeit zu den alltäglichen Tugenden zählt, welchen sich philosophische Untersuchungen zu wenig widmen (Münch 1984, S. 26). Solche theoretischen Arbeiten können auch in diesem Forschungsprojekt nicht geleistet werden; doch sind Zusammenhänge einzelner Tugenden anhand der empirischen Daten festzustellen und können daher vorerst dargelegt werden.

Vor dem Hintergrund eines ungenügend theoretisch definierten Tugendbegriffs fokussiert diese Arbeit auf eine Einzeltugend und nicht auf den Überbegriff Tugend. Damit kann am Beispiel einer Einzeltugend dennoch die Tugendthematik aufgezeigt werden, sofern Ehrlichkeit als moralische Verhaltensweise (Reichenbach 2004, 2008) zu verstehen ist (vgl. Kap. 2.5). Der verwendete Tugendbegriff ist daher nicht als innere Haltung oder lediglich als Charaktereigenschaft zu fassen. Vor diesem Hintergrund dient für den losen theoretischen Rahmen des Forschungsvorhabens zum einen Whites dreifache Ehrlichkeitsdefinition (White 2006): Wahrhaftigkeit, Aufrichtigkeit und Offenheit. Zum anderen bildet Reichenbachs Tugendbegriff die Basis für den gewählten Ehrlichkeitsbegriff. Schließlich konnte aufgrund des theoretischen Ansatzes zu Sekundärtugenden (Spaemann 1988/1989) die ambivalente Eigenschaft von Ehrlichkeit verortet werden.

In dieser Hinsicht kann Ehrlichkeit verstanden werden als aufrichtig zu anderen sein, das sagen, was man denkt, und entsprechend dazu sich verhalten. Mit dieser verhaltensorientierten Definition ist ein Spielraum eröffnet, in dem die Angemessenheit oder der Grad an Offenheit von der subjektiven Wahrnehmung abhängig ist. Der Bezug zur individuellen und operationellen Ebene ist gegeben. Oder negativ formuliert: Üblicherweise sind mit Ehrlichkeit keine strategischen Spiele, Taktiken oder Täuschungen verbunden.

Zum Abschluss des Kapitels über den Ehrlichkeitsbegriff sind die Argumente aufgeführt, die zur Auswahl der Einzeltugend Ehrlichkeit für die empirische Untersuchung führten. Dazu zählen unterschiedliche Gründe:

- Ehrlichkeit ist eine spannende Tugend bezüglich ihrer ambivalenten Eigenschaft: Ehrlichkeit ist nicht in jeder Situation angemessen, weshalb sie als Sekundärtugend bezeichnet wird.

- Ehrlichkeit ist eine schulrelevante Tugend und fordert aufgrund der ambivalenten Eigenschaft Mitschülerinnen und -schüler, Lehrpersonen und Eltern heraus. Dabei sind individuelle, kollegiale und schulische Dilemmas und Konflikte vorprogrammiert.

- Ehrlichkeit wird im Schulalltag kontrolliert und sanktioniert, scheinbar aber ohne Erfolg, weil im Alltagswissen Unehrlichkeit je nach Berufsfeld ohne Konsequenzen praktiziert werden kann.

- Ehrlichkeit ist im Vergleich zur Tugend ,Respekt` oder, Toleranz ‘ im Schulkontext scheinbar als Untersuchungsthema weniger attraktiv, ob- 
wohl sie im Alltagswissen als wünschenswerte Tugend bezüglich Heranwachsender deklariert und kontrolliert wird.

- Zur Stellungnahme, wie SuS die Tugend Ehrlichkeit in der Schule deuten, fehlen weitgehend empirische Daten.

- Weiter beziehen sich verschiedene Dokumente der Zürcher Volksschule implizit auf die Tugend ,Ehrlichkeit' (vgl. Kap. 3.3.3).

- Ehrlichkeit ist eine Einzeltugend, deren Bezug zum Tugendbegriff insbesondere im Alltag nicht offensichtlich ist. Womit auf einer theoretischen Abhandlung die Tugend Ehrlichkeit und im Gespräch mit den Jugendlichen Ehrlichkeit verwendet werden kann. Deshalb wird im empirischen Teil dieser Arbeit nur der Ehrlichkeitsbegriff benutzt. Hingegen wird hier im theoretischen Teil der Arbeit (Teil I) und ebenso im abschließenden Teil der Arbeit (Teil III) der Bezug zum Tugendbegriff hergestellt.

\section{Kontextualisierung des Forschungsgegenstands}

Aus dem vorangegangenen Kapitel geht hervor, dass keine stringenten theoretischen Ansätze zu ehrlichen Verhaltensweisen vorliegen, die von einem differenzierten Ehrlichkeitsbegriff ausgehen und zudem den Alltag berücksichtigen. Um den Forschungsgegenstand Deutungen von Ehrlichkeitspraxen aus der Sicht von jugendlichen SuS zu untersuchen, wurde daher ein explorativer Forschungszugang gewählt (vgl. Kap. 5). Der Forschungsgegenstand berührt zugleich die Schülerforschung, die Institution Schule und den öffentlichen Erziehungsauftrag, was als Forschungskontext zu berücksichtigen ist.

Dieses Kapitel der Arbeit skizziert die theoretischen Implikationen des Forschungskontexts und die damit verbundenen Reflexionen zum eigenen empirischen Forschungszugang. Damit wird das Vorwissen zum Untersuchungsgegenstand angegeben und Folgerungen für die Forschungsanlage fundiert. Zuerst wird die Untersuchungsperspektive der Schülerforschung erläutert und Bezug auf den dahinterstehenden Jugendbegriff genommen (Kap. 3.1). Anschließend werden die Chancen und Grenzen der Institution Schule bezüglich des Forschungsgegenstands umrissen (Kap. 3.2). Und schließlich wird das Thema des öffentlichen Erziehungsauftrags der öffentlichen Schulen diskutiert (Kap. 3.3). 


\subsection{Perspektivenwechsel: Schülerforschung}

Schule kann aus unterschiedlichen Forschungsperspektiven betrachtet werden, wie etwa derjenigen der Schulforschung oder aus der Sicht der Bildungsforschung. Ausgehend von der Kindheits- und Jugendforschung (vgl. Bucher 2001) entwickelte sich vor rund dreißig Jahren die Schülerforschung (vgl. Schweitzer und Thiersch 1983), da die Schulforschung sich nunmehr für die Adressatinnen und Adressaten interessierte, denen die Bemühungen von Unterricht gilt. Inzwischen hat sich die Schülerforschung etabliert (vgl. Brake und Bremer (2010); Schubarth und Speck 2008; Breidenstein und Jergus 2005).

Tabelle 2: Kindheitsforschung, Schulforschung, Schülerforschung und Jugendforschung im Vergleich

\begin{tabular}{|l|l|l|l|l|}
\hline & $\begin{array}{l}\text { Kindheitsfor- } \\
\text { schung }\end{array}$ & $\begin{array}{l}\text { Schulfor- } \\
\text { schung }\end{array}$ & $\begin{array}{l}\text { Schülerfor- } \\
\text { schung }\end{array}$ & $\begin{array}{l}\text { Jugendfor- } \\
\text { schung }\end{array}$ \\
\hline $\begin{array}{l}\text { Forschungs- } \\
\text { perspektive }\end{array}$ & vom Kind aus & $\begin{array}{l}\text { meistens von } \\
\text { den Erwachse- } \\
\text { nen aus }\end{array}$ & von den SuS aus & $\begin{array}{l}\text { von den Jugend- } \\
\text { lichen in der } \\
\text { Schule aus }\end{array}$ \\
\hline $\begin{array}{l}\text { Forschungs- } \\
\text { zugang }\end{array}$ & $\begin{array}{l}\text { Beschreibung und } \\
\text { Analyse von } \\
\text { Schule }\end{array}$ & $\begin{array}{l}\text { Debatte über } \\
\text { gute Schule }\end{array}$ & $\begin{array}{l}\text { Rekonstruktion } \\
\text { von Schule aus } \\
\text { Sicht der SuS }\end{array}$ & $\begin{array}{l}\text { jugendkulturelle } \\
\text { Orientierungen } \\
\text { von Heranwach- } \\
\text { senden }\end{array}$ \\
\hline $\begin{array}{l}\text { Pädagogi- } \\
\text { scher Aspekt }\end{array}$ & - & zentral & $\begin{array}{l}\text { wird vorerst } \\
\text { ausgeklammert }\end{array}$ & - \\
\hline $\begin{array}{l}\text { Grenzen des } \\
\text { pädagogi- } \\
\text { schen Han- } \\
\text { delns }\end{array}$ & werden markiert & - & werden markiert & werden markiert \\
\hline
\end{tabular}

Quelle: eigene Darstellung in Anlehnung an Breidenstein und Jergus 2005

Vor diesem Hintergrund schlagen Breidenstein und Jergus eine dreifache Unterteilung für die Forschungsperspektiven auf Schule vor: Kindheitsforschung, Schulforschung und Schülerforschung (Breidenstein und Jergus 2005). Diese Systematisierung ergänze ich um die Jugendforschung (vgl. Tab. 2), denn die Verknüpfung von Schüler- und Jugendforschung hat in den letzten Jahren an Bedeutung gewonnen (vgl. bspw. Bennewitz und Meier 2010; Schubarth und Speck 2008; Pfaff 2008). Anlass dazu gaben zwei Missstände in der Schulforschung:

Zum einen wurden die sozialen und jugendkulturellen Bedürfnisse der SuS unzureichend berücksichtigt, obwohl schulischer Misserfolg den Aufbau einer stabilen Identität von Heranwachsenden gefährden kann. Der Zusammenhang zwischen einer erfolgreichen Schulkarriere und der Entwicklung der Schülerpersönlichkeit ist insbesondere in den Forschungen zu Schülerbi- 
ografie, Schulkarriere (Helsper 2008) und Schülerpersönlichkeit (vgl. Dalbert und Stöber 2008) nachgewiesen worden.

Zum anderen hat sich die Meinung durchgesetzt, dass Heranwachsende nicht nur in ihrer ,Schülerrolle' und den damit verknüpften Entwicklungsaufgaben zu betrachten sind, sondern ebenso im ,Jugend-Sein', ein Aspekt, der über die ,Schülerrolle' hinaus ihre alltägliche Lebensführung betrifft (Schubarth und Speck 2008, S. 967).

In diesem Spektrum an Forschungsbereichen situiert sich die vorliegende Arbeit in der Schülerforschung, um dezidiert Ehrlichkeitspraxen aus Sicht der Heranwachsenden zu untersuchen. Denn die Sichtweisen der Lehrpersonen und Erwachsenen zu Erziehungsthemen sind allgemein bekannt (vgl. Ammann 2004; Mienert 2008; vgl. Kap. 3.3) und divergieren allzu oft mit den Perspektiven der Heranwachsenden (bspw. bzgl. der Beteiligungsmöglichkeit (Schubarth und Speck 2008, S. 972), bzgl. der Gewaltwahrnehmung (Ribeaud und Eisner 2009) und bzgl. ehrlichen Verhaltens (vgl. Bsp. in Kap. 3.3.2). Zudem ist die Sichtweise der Adressatinnen sowie Adressaten von Erziehungsbemühungen (vgl. zur schulischen Gewalt Drilling 2008) nur teilweise und zu Tugendanforderungen ungenügend erkundet.

Gleichzeitig liegen punktuelle Ergebnisse des ersten empirischen Schulforschungsprojekts in England vor (Arthur et al. 2009), in dem demokratische Werte und Tugenden als Einstellungen aus Sicht der Kinder und Jugendlichen untersucht wurden. Das Projekt umfasst die Ausbildungsstufen des Kindergartens bis zum Studium und folgt dem Erziehungsansatz der character education. Charakter ist in diesem Projekt als allgemeine Antwort auf die Fragen zu verstehen: „Wer sind wir?“ und „Wer wollen wir werden?“. So zeigt eine Studie aus dem Gesamtprojekt mittels Gruppendiskussion auf, dass 14- bis 16-jährige angelsächsische Jugendliche eher das Wort ,Charakter oder ,Werte' verwenden als ,Tugend'. Im schulischen Rahmen werten die befragten Jugendlichen die Charaktereigenschaft des Fleißes (work hard), der zu Leistungserfolg führt, höher als die Anerkennung durch die Lehrperson oder den Klassenzusammenhalt (ebd., S. 54).

Ebenso erweist sich bei Jugendlichen desselben Alters in Deutschland und in der Schweiz die Einstellung zu Fleiß als überraschend positiv (vgl. Kap 1.2). Die Forscher der 16. Shell-Jugendstudie folgern aus ihren Befunden, dass Jugendliche gesellschaftliche Moralordnung einfordern (Albert 2010, S. 29, 222ff.).

Allerdings liefern die angeführten quantitativ angelegten Studien nur Ergebnisse über Einstellungen zu Tugenden. Dadurch ist wie bereits erwähnt einerseits ein gesellschaftliches Tugendverständnis bereits vorausgesetzt, ohne das Eigenverständnis der Jugendlichen zu berücksichtigen. Andererseits geben die Befunde nur Auskunft über Einstellungen, die nicht notwendigerweise mit den Praxen übereinstimmen müssen. In diesem Sinne bekräftigen die Ergebnisse der Jugenduntersuchung zu Ziviltugenden in Deutschland 
(Nunner-Winkler et al. 2006), dass die meisten der rund 200 befragten 16Jährigen aus verschiedenen Schultypen sich ,zumindest in ihren verbalen Äußerungen an demokratischen Basisprinzipien orientieren“ (ebd., S. 192). Solche Ergebnisse beleuchten das Meinungsbild der Heranwachsenden und das, was sie als sozial erwünschte Werte wahrnehmen, was bedeutsam für Debatten zu demokratischen Werten sein kann. Jedoch führen solche Ergebnisse zu widersprüchlichen und polarisierenden Stellungnahmen: tugendhafte versus tugendlose Heranwachsende (vgl. Kap. 1.2). In solchen Debatten fehlen weitgehend Befunde dazu, wie Heranwachsende ihre Praxen deuten und an welchen gedeuteten Regeln sie sich orientieren.

Heranwachsende gehen in der Rolle der SuS unterschiedlichen Deutungen von schulischen Verhaltensanforderungen nach (vgl. Kap. 3.1) und gleichzeitig lassen sie als Heranwachsende jugendspezifische Eigenschaften in ihre Verhaltensweisen einfließen, die zu berücksichtigen sind (vgl. Kap. 3.2). An dieser Stelle zeigen die Schülerforschung und die Jugendforschung Überschneidungen mit dem verhaltensanleitenden und pädagogischen Bereich: In beiden Forschungsrichtungen wird vorerst der pädagogische Aspekt ausgeklammert. Zudem werden die Grenzen des pädagogischen Handelns bzw. der Erziehung markiert.

\subsubsection{Schülerinnen und Schüler}

Wie Heranwachsende ihre ,Rolle` als SuS wahrnehmen, zeigen Georg Breidenstein und Kerstin Jergus in einer ethnografischen Studie mit SuS der 7. und 8. Klasse auf (Breidenstein und Jergus 2005): Sie stellen fest, dass die Heranwachsenden in der Schule an ihrer Schülerrolle festhalten. Daraus folgern die Autoren, dass SuS sich gegenüber der Schule in einer Art ,Jobmentalität ${ }^{`}$ verhalten und nicht aus eigenem Antrieb lernen. Hinter dem Ansatz der ,Schülerrolle" stehen interaktionistische Reflexionen, welche das lebensweltliche Aushandeln und die interpretativen Deutungen der schulischen Akteure in den Mittelpunkt stellen (vgl. Helsper und Böhme 2010, S. 622; Tillmann 2010, S. 182ff.). Der interaktionistische Ansatz bietet nun einen theoretischen Rahmen, um eine aktive Bewältigung von Schule und der damit verbundenen Probleme aus Sicht der SuS zu erkunden.

Bewältigungsprobleme gibt es, weil die Lernenden in ihrer Schülerrolle mit unterschiedlichen Herausforderungen konfrontiert sind (vgl. Scherr 2009, S. 140f.):

- In der Schule werden Anforderungen gestellt, die über die familiären und jugendspezifischen Kommunikations- und Interaktionsformen hinausgehen.

- Eine sachlich zentrierte Beziehung zur Lehrperson soll aufgebaut werden. 
- Die SuS als soziale Akteure verhalten sich gemäss einer Schülerrolle und halten auch emotionale Distanz zueinander aus.

- SuS stehen in Selektions- und Leistungskonkurrenz und in erheblichem Zeitumfang unter Erwachsenenaufsicht.

Diese schulspezifischen Umstände können eine „belastende soziale Zumutung" (Scherr 2009, S. 140) für SuS sein und haben Einfluss auf ihre Verhaltensweisen und deren Deutungen. So zeigt eine Untersuchung mit 250 Gymnasiastinnen und Gymnasiasten (Eder 1987 in: Ulich 2008, S. 212), über welche unterschiedlichen Taktiken SuS verfügen, um mit den oben skizzierten schulspezifischen Herausforderungen umzugehen. Auf die Frage „Welchen Rat würden Gymnasiastinnen und Gymnasiasten der 8. Klasse den jüngeren Geschwistern geben, um besser durch die Schule zu kommen?" antworten diese wie folgt (in Anlehnung an Eder 1987, S. 106, zitiert in Ulich 2008, S. 212; gesamthaft mehr als 100\%, weil Mehrfachantworten möglich waren):
$94 \% \quad$ Mitarbeit und Lernen
$73 \%$ situationelle Anpassung (z. B. nicht negativ auffallen)
$38 \%$ personelle Anpassung (Wohlverhalten gegenüber Lehrpersonen)
$36 \%$ Ingratiation (z.B. ,Schleimen“ oder, Einschleimen')
$22 \%$ demonstratives Engagement
$20 \%$ Integration
$10 \%$ Identifizierung
$9 \% \quad$ Widerstand
$8 \%$ Distanz, Rückzug

In der Vielfalt der Bewältigungsmöglichkeiten dominiert also eine angepasste Haltung: Nur $9 \%$ der älteren SuS empfehlen Widerstand, worin Unterrichtsstörung und unterschiedliche Formen von Gewalt an Schulen impliziert werden kann. In diesem Sinne wird Lügen als ,externalisiertes Problemverhalten “ neben zerstörerischem Verhalten oder Stehlen kategorisiert (vgl. bspw. Eisner und Ribeaud 2007, S. 8). Die oben genannten Ergebnisse stammen vom leistungsorientierten Schultyp Gymnasium. Für ein umfassendes Verständnis von schulischer Erziehung wären weitere Ergebnisse aus leistungsschwächeren Schultypen und neuere Studien aufschlussreich. Gerade biografische Verläufe und die damit zusammenhängenden Anpassungsthematiken von Jugendlichen hinsichtlich Milieus, Lebensformen und Schulformen sind Forschungsdesiderata, welche sich die Forschung zu Schülerbiografie und Schulkarriere stellt (Helsper 2008, S. 939).

Zusammenfassend ist festzuhalten, dass SuS in der Schulforschung als ,lernende Subjekte“ oder als ,Leistungssubjekte“ und als ,soziale Akteure“ beschrieben sind. Damit sollte ihnen vom schultheoretischen Standpunkt nicht nur im Bereich der schulischen Bildung, sondern auch der schulischen Erziehung eine aktive und produktive Rolle zugesprochen werden. 


\subsubsection{Jugendphase}

Jugendliche sind demnach Belastungen ausgesetzt, die sie in der Schülerrolle zu bewältigen haben, wie aus den vorangegangenen Ausführungen hervorgeht. Hinzu kommen Belastungen, die mit entwicklungspsychologischen Veränderungen der Jugendphase zusammenhängen (vgl. Fend 2005, S. 367):

Die Jugendphase ist geprägt von der Entwicklung einer psychischen und sozialen Identität, eines stabilen Selbstbildes und der Ich-Identität, dem Aufbau von Beziehungen zu Gleichaltrigen, dem Erwerb der eigenen Geschlechterrolle und der Entwicklung eines Normen- sowie Wertesystems (vgl. z.B. Hurrelmann 2010; Ecarius 2011; Hurrelmann und Bründel 2003). Vor diesem Hintergrund sind SuS - neben der Schülerrolle - unter der Perspektive $J u$ gendliche zu betrachten, die sich außerhalb der Schule in weiteren Lebensbereichen aufhalten wie beispielsweise Familien, Freizeitbeschäftigungen oder Peer Groups (vgl. Wehr 2008; Esslinger-Hinz 2008; Krumm 2003; Wippermann 2007).

Das Verständnis der Jugendphase hat sich in den vergangenen Jahren verändert, wie in der gegenwärtigen Jugendforschung kritisch beleuchtet wird (vgl. Baacke 2004; Dudek 2010; Ferchhoff 2011; Scherr 2009; Muri 1999): Die Abgrenzung zwischen Jugend- und Erwachsenenalter hat an Prägnanz verloren. So wollen beispielsweise Erwachsene ebenso jugendlich wirken. Ehemals zuverlässige Kriterien für den Übergang wie Familiengründung oder berufliche Integration treten mittlerweile unabhängig voneinander auf. Deshalb ist der Übergang vom Jugend- zum Erwachsenenalter nicht mehr deutlich zu identifizieren.

Neuere Sozialisationsforschung und Jugendforschung (vgl. bspw. Mansel und Hurrelmann 2003) betonen die produktiv verarbeitende Realitätsbewältigung von Jugendlichen, indem sie sich im Spannungsverhältnis zwischen Fremdbestimmung und Selbstbestimmung bewegen und sich zwischen der Spannbreite beider Pole zu entscheiden und zu positionieren haben. Sie bilden ihre Identität in dieser Tätigkeit der Sozialisation, die als

produktive Auseinandersetzung des Menschen mit den sozialen und materiellen Lebensbedingungen und den sich in diesem Rahmen bietenden Entwicklungsmöglichkeiten auf der einen Seite und der eigenen Person in Form von Körper und Psyche, Bedürfnissen, Wünschen und Ansprüchen auf der anderen Seite (Mansel und Hurrelmann 2003, S. 75f)

betrachtet wird. Die Entscheidungsprozesse und die Deutungen von Praxen zwischen dem sozialen und materiellen Lebensrahmen einerseits und den eigenen Bedürfnissen und Wünschen andererseits führen dazu, dass den Jugendlichen als produktiv verarbeitende Subjekte eine aktive Rolle zugeschrieben wird:

Individuen sind demnach als Informationen verarbeitende und handelnde Subjekte sowohl an ihrer eigenen Entwicklung und dem jeweils realisierten Resultat als auch an der Be- 
schaffenheit und den Veränderungen der Umwelt beteiligt. Die Möglichkeit der aktiven Einflussnahme auf den eigenen Werdegang und die sozialen Prozesse sind ein konstitutives Element dieser Konzeptionen (Mansel und Hurrelmann 2003, S. 76).

Im Rahmen des so beschriebenen sozialisationstheoretischen Ansatzes wählen Jugendliche in einer produktiven Weise das möglichst Beste aus, um mit dem Spannungsfeld zwischen Umwelt und Selbst umzugehen.

Zudem sind Jugendliche als altersspezifische Gruppe als ein Produkt der bürgerlichen Schicht seit Anfang des 20. Jahrhunderts zu verstehen (vgl. von Trotha 1982; Roth 1983; Scherr 2009). An Jugendliche werden seitdem funktionale Erwartungen gestellt, die mit der Reproduktion und Stabilität der gesellschaftlichen Ordnung einhergehen. Dazu zählt primär ein angepasster Schul- und Ausbildungsbesuch, worauf sich die Persönlichkeitsentwicklung auszurichten hat.

Vor dem Hintergrund des gesellschaftlichen Bedeutungswandels von $J u$ gend kann nicht allgemein von dieser Phase gesprochen werden, sondern immer in einem gesellschaftshistorischen Kontext. Anders ausgedrückt: Die Jugendphase ist nichts ,naturhaft Vorgegebenes', sondern etwas historischgesellschaftlich Relatives und Konstruiertes.

Ausgehend von diesen Einsichten seien abschließend einzelne Befunde aus der Jugendforschung zusammengefasst, die für den Untersuchungsgegenstand relevant sind:

- Jugendliche gehen länger zur Schule als früher. Die Ausbildungszeit hat sich ausgedehnt.

- Jugendliche werden über entwicklungspsychologische und biologische Indikatoren definiert. So gelten beispielsweise Heranwachsende ab dem 14. Lebensjahr als urteils- und handlungsfähig (Palentien und Hurrelmann 1998).

- Weitere Anzeichen der gegenwärtigen Jugendphase sind die Geschlechtsreife, die ökonomische und die kulturelle Selbstständigkeit.

- Jugendliche verfügen über jugendspezifische Verhaltensweisen, welche sich je nach gesellschaftlichem Rahmen und dessen Repressivitätsgrad unterschiedlich entfalten.

- Das Interesse an Jugendlichen ist in den Medien, in der Wirtschaft, in der Politik, in Bildungs- und Erziehungswissenschaften sowie in der Soziologie gestiegen. Gleichzeitig ist die Überwachung der Jugendzeit entsprechend strikter.

- Jugendliche sind in neuerer Jugend- und Sozialisationsforschung als produktiv verarbeitende Subjekte zu betrachten, welche in der Spannung zwischen Fremd- und Selbstbestimmung alltägliche Realitäten zu bewältigen haben. 


\subsubsection{Zusammenfassung}

Die Schülerforschung untersucht Schule und Unterricht aus der Perspektive der SuS. Sowohl in der Schülerforschung als auch in der Sozialisationsforschung und Jugendforschung werden Heranwachsende als aktiv bezeichnet und als produktiv verarbeitende Subjekte betrachtet, welche unterschiedliche Bewältigungsprobleme in ihrem Alltag anzugehen haben, von dem der schulische Alltag einen großen zeitlichen Raum einnimmt. Die jüngere deutsche und angelsächsische Schülerforschung zu Tugenden und Werten liefert positive Befunde zu den Einstellungen der SuS. Jedoch fehlen weitgehend Ergebnisse zu Deutungen von Praxen, die Tugenden und Werte implizieren. Die vorliegende Studie beleuchtet diesen Aspekt genauer und untersucht, wie SuS Ehrlichkeitspraxen deuten, welche Ehrlichkeitsregeln sich rekonstruieren lassen und wie sich $\mathrm{SuS}$ in realen Dilemmasituationen orientieren (vgl. Kap. 9). Neben den schulischen Anforderungen sind SuS als Jugendliche von einem entwicklungspsychologischen, sich wandelnden gesellschaftlichen Kontext und von der Spannung zwischen Selbstbestimmung und Fremdbestimmung geprägt, was ebenso Einfluss auf den Forschungsgegenstand dieser Arbeit hat.

\subsection{Schule als öffentliche Institution}

Schule zählt neben der Familie und den Peer Groups zu einem wichtigen Sozialisationsbereich für die alltägliche Lebensführung eines Jugendlichen. 2010 gingen 30.100 SuS in die öffentliche Sekundarschule im Kanton Zürich (vgl. Bildungsstatistik des Kantons Zürichs 2011). Wöchentlich verbringen sie rund 33 Stunden in der Schule und weitere Zeit mit Hausaufgaben. Im Laufe ihrer Schulzeit erleben sie rund 15.000 Stunden in der Schule (vgl. Rutter und von Hentig 1980, S. 15), d. h. im Vergleich mit anderen Bereichen halten sie sich dort am meisten auf. Damit steigt auch die Bedeutung der Schule als öffentliche Institution und als Kontext für die Entwicklung der einzelnen SuS (vgl. dazu Kap. 3.1.1).

Vor diesem Hintergrund wird im Folgenden die gegenwärtige Schule als öffentliche Institution bezüglich deren Funktionen und der Bildungsungleichheit (Kap. 3.2) untersucht. Der Fokus wird auf den deutschsprachigen Raum unter besonderer Berücksichtigung der Zürcher Volksschule gesetzt. Damit soll an zwei aktuellen und zentralen schulpädagogischen Themen in den Grundzügen aufgezeigt werden, welches die schulischen Rahmenbedingungen sind, in denen sich die befragten SuS zurechtzufinden haben. 


\subsubsection{Gesellschaftliche Funktionen der öffentlichen Schule}

Der Schule als gesellschaftliche Institution kommen in der einschlägigen Bildungsliteratur vier zentrale Funktionen zu (in Anlehnung an Fend 2009, S. 49ff.):

- kulturelle Reproduktion:

Eine Funktion der Schule ist die Beherrschung grundlegender Symbolsysteme wie Sprache und Schrift bis zur Internalisierung grundlegender Wertorientierungen, z. B. der Vernunftfähigkeit und moralischen Verantwortlichkeit des Individuums. Was in der Gesellschaft als ,normal gelten soll, wird in der Schule repräsentiert. Somit werden die Heranwachsenden in die gesellschaftlich erwünschten Verhaltensweisen eingeführt, indem Schule Normen vermittelt und Persönlichkeit gebildet.

- Qualifikationsfunktion:

Alle Heranwachsenden sollen Wissen und Qualifikationen erwerben, um eine erfolgreiche Integration ins Berufsleben zu gewähren.

- Allokationsfunktion:

Jugendliche erzielen ungleichwertige Schulabschlüsse, was sich in der Berufswahl widerspiegelt. Die Zuführung ,,von Personen mit bestimmten Qualifikationen zu Aufgaben mit bestimmten Anforderungen" (ebd., S. 50) bezeichnet Fend als ,Allokation'. Er differenziert damit zwischen ,Selektionsfunktion' und ,Allokationsfunktion'. Denn es geht ihm nicht um den Ausschluss, sondern um eine legitimierbare Verteilung der zukünftigen Berufe.

- Integrations- und Legitimationsfunktion:

Dem Schulsystem kommt eine gesellschaftliche Integrationsfunktion zu, zugleich werden in ihm Werte, Normen und Weltansichten reproduziert und legitimiert.

Den vier gesellschaftlichen Funktionen der Schule entsprechen gemäss Fend vier individuelle Funktionen, die für die SuS in der sozialen Orientierung, im Qualifikationserwerb, in der Lebensplanung und in der Identitätsbildung bestehen (ebd., S. 53).

Aufgrund der gesellschaftlichen und individuellen Funktionen des Schulsystems gilt Schule als unverzichtbar, um die gesellschaftliche Ordnung aufrechtzuerhalten. Die Schule wird ebenso als ,Abbild der Gesellschaft' (repraesentatio mundi) bezeichnet. Sie ist der Ort,

an dem das Kapitel des gesellschaftlichen Wissens in die kleine Münze des individuellen Wissens umgewandelt wird. Darauf bezieht sich das pädagogische Handeln als Darstellung der Welt, wenngleich auch nur in ausgewählten Ausschnitten (Strobel-Eisele und Prange 2008, S. 219). 
Der öffentliche Schulauftrag besteht zusammenfassend in der Wissensvermittlung, Sozialisation und Erziehung für die SuS in Rahmen eines Schonraumes. Allerdings gibt es Normen, Zwänge, Werte und Regeln, die nicht zwingend mit dem Bildungs- und Erziehungsauftrag zu tun haben. So zeigen bildungssoziologische und sozialhistorische Studien (vgl. bspw. Scherr 2009, S. 140ff.), dass die schulische Zeitstruktur an die Zeitordnung der Industriearbeit bzw. an die Berechnung der Arbeitsstunden der Lehrpersonen (vgl. Döbrich 2009) adaptiert ist oder dass schulische Autoritätsbeziehungen und Geschlechterbeziehungen auf gesellschaftlich vorherrschenden Verhältnissen des Generationsverständnisses und der Geschlechterrollen beruhen. Wirft man einen Blick auf den gegenwärtigen Fächerkanon in der Schweiz, zeigt sich, dass die Fächerstruktur eine zwar ordnende, aber konservative Funktion hat, sodass die Fächer nicht gleichbehandelt werden (Oelkers 2009a, S. 310). Dabei stellt sich nicht die Frage, ob die vorhandenen Ressourcen innerhalb der Fächer genutzt werden, sondern wie diese genutzt werden: Die Ressourcennutzung hängt ab vom Interesse am Fach, wie es sich im Laufe der eigenen Schulkarriere entwickelt (ebd., vgl. S. 310ff.), woraus sich wiederum fachbezogene Zwänge seitens der SuS entwickeln können.

Somit ist die implizite erzieherische Funktion der Schule kritisch zu beleuchten: Erziehung ist nicht nur in der Vermittlung von Normen und in der Persönlichkeitsbildung gekoppelt mit Wissensvermittlung relevant, sondern auch in der nationalstaatlichen Erziehung, insofern als hier Landes- und Verkehrssprachen sowie ein Selbstverständnis als Staatsbürger erlernt wird. Zinnecker spricht in dem Zusammenhang vom ,heimlichen Lehrplan“ (Zinnecker 1975): In der Schule werden in verborgener oder indirekter Weise Kompetenzen erworben, beispielsweise den

Leistungsanforderungen $\mathrm{zu}$ entsprechen, sich nach Kriterien der Leistung bewerten zu lassen, sich in Konkurrenz und Wettbewerb einzuüben und Rangordnungen zu akzeptieren. (Strobel-Eisele und Prange 2008, S. 224).

An dieser Stelle ist m. A. n. eine schulische Aufklärungsarbeit angebracht, innerhalb derer Heranwachsende in der Schule und somit in Abwesenheit der Eltern angehalten werden, soziale Rollen und die damit verknüpften sozialen Erwartungen zu thematisieren, um sich damit auseinanderzusetzen und darin zu üben. Denn gerade diese impliziten Anforderungen können zu widersprüchlichen Verhaltensweisen der SuS führen. Im Kanton Zürich ist es in der Volksschule üblich, wöchentlich Klassenräte zu halten, was auf die Klassen der befragten SuS ebenfalls zutrifft. Eine Schülerin berichtet, dass sie in ihrer Schule die Klasse im Stufenrat mit einer Mitschülerin vertritt (klassenübergreifende Versammlungen mit Vertreter der jeweiligen Klassen). Mit solchen Initiativen soll die Partizipation der SuS gefördert werden. Allerdings sind die erwähnten schulischen Partizipationsformen punktuelle Momente im Regelunterricht, vom fachlichen Unterricht ausgeklammert und deren Wirksamkeit ist bislang nicht wissenschaftlich überprüft. 
Zudem stellt sich die Frage, ob im Klassenrat beispielsweise folgendes Problem angesprochen wird und Lösungsstrategien im Schulalltag möglich sind (Chiapparini 2011, S. 48ff.): Der Schüler Clemens weigert sich, Französisch zu ,pauken', weil er es für seine Berufswahl als nicht nützlich einschätzt. Als Konsequenz müsste er zu seinen schlechten Französischnoten stehen, was er gegenüber der Mutter auch tut. Gleichwohl versucht er während des Französischtests, mit unlauteren Hilfsmitteln bessere Noten zu erzielen. Damit folgt er wiederum den schulischen Leistungsanforderungen, jedoch nach individuellen Regeln.

Hier sind Anzeichen gegeben für einerseits ein widersprüchliches Schulsystem, das gute Leistungen und gleichzeitig ehrliche Verhaltensweise fordert. Andererseits führt der Anspruch der Leistungsorientierung und erwartetes ehrliches Verhalten zu widersprüchlichen Deutungen ihrer Ehrlichkeitspraxen von Seiten der SuS. Um beim Beispiel von Clemens zu bleiben, deklariert dieser die Sinnlosigkeit eines Schulfaches, steht aber nicht zu den Folgen seiner resignierten Haltung, welche sich in schlechten Schulleistungen ausdrückt, die er durch das Beiziehen von unlauteren Hilfsmittel verbessern möchte, was er wiederum als legitim deutet (vgl. ebd.).

\subsubsection{Schule und Bildungsungleichheit}

Neben den dargestellten Grenzen der schulischen Erziehungsfunktion besteht ein weiteres institutionelles Problem von öffentlichen Schulen darin, dass Bildungsgleichheit erstrebt wird, aber schwer zu kontrollieren und zu gewähren ist: Die gewachsene Bedeutung des sozialen und kulturellen Kontextes der Schule als öffentliche Institution ist klar an der Bildungsexpansion der letzten zehn Jahre zu beobachten. So hat sich die Schulzeit der Heranwachsenden ausgedehnt. Überdies werden im Vergleich zu früher vermehrt höhere Bildungsabschlüsse erworben, und schließlich ist eine gesteigerte Bildungsbeteiligung von Frauen zu vermerken (vgl. bspw. Strobel-Eisele und Prange 2008 S. 142).

Trotz dieser Bildungserfolge untermauern Untersuchungen (vgl. Bessoth 2000; Krüger und Deppe 2010) das Problem der Bildungsungleichheit, das auf folgendem Prinzip basiert: Je höher die soziale Position der Eltern (Einkommen, Bildungsniveau), desto grösser ist die Wahrscheinlichkeit, dass die Kinder einen höheren Bildungsabschluss erwerben. Ursachen, weshalb Bildungschancen ungerecht verteilt sind, lassen sich wie folgt aufschlüsseln (vgl. Scherr 2009):

- „Schulen bewerten Fähigkeiten, die nicht in der Schule, sondern vor und außerhalb der Schule erworben werden“ (ebd., S. 143), dazu gehören sprachliche Fertigkeiten sowie Allgemeinbildung (vgl. bspw. Moser et al. 2011). 
- Gymnasien richten sich nach der Herkunft des Bildungsbürgertums und der oberen Mittelschicht und nach deren Sprachgebrauch. So zeigt Basil Bernstein (1980) auf, dass Jugendliche, die nicht dieser Herkunft entsprechen, vor der Alternative stehen, sich von der eigenen Herkunft zu distanzieren oder Schule als eine ihnen fremde Institution zu erleben.

- Aus älteren Untersuchungen ist eine geringere Bildungsaspiration Jugendlicher niedriger Bildungsschichten festzustellen. Neuere Studien hingegen zeigen auf, dass Jugendliche niedriger Schichten die Bedeutung von Bildung vermehrt anerkennen.

- $\quad$ SuS aus unteren Schichten erfahren kaum Unterstützung bei schulischen Lernprozessen.

- In einem selektiven Schulsystem ist eine regelmäßig verteilte Anzahl an SuS über die Leistungsstufen impliziert, d. h. von der Organisationstruktur ist nicht vorgesehen, dass alle $\mathrm{SuS}$ in die Sekundarstufe A gehen könnten.

Anhand der Ursachen von Bildungsungleichheit lässt sich beispielsweise erklären, weshalb SuS nicht nur aufgrund der schulischen Leistungsstufen unterschiedlichen Schultypen zugeteilt werden, sondern auch aufgrund ihrer soziokulturellen und schichtspezifischen Hintergründe (Scherr 2009, S. 144; Moser 2011, S. 163ff.).

Soziale Herkunft scheint indes keinen Einfluss auf das Ehrlichkeitsverständnis von 14- bis 15-jährigen $\mathrm{SuS}$ zu haben. So zeigen erste Untersuchungsergebnisse (Chiapparini 2012) ein vielfältiges Ehrlichkeitsverständnis über alle drei Schulleistungsstufen gemäss des dreiteiligen Schulmodells ${ }^{16}$, das während der Untersuchung sowohl in der Stadt Zürich als auf dem Land einheitlich vorzufinden war, und über die kontrastierenden sozialen Belastungen der Schulgemeinden hinweg auf. Jedoch liegen Indizien vor, dass Lehrpersonen mit Verhaltensweisen der SuS je nach Leistungsstufe der Klasse unterschiedlich umgehen.

Darauf weist das Beispiel des Schummelns während der Schultests hin ${ }^{17}$ : In einem städtischen Oberstufenschulhaus dürfen die SuS einer Klasse der Sekundarstufe C mit einem ,Spick‘ die Prüfung lösen, weil dies die Lehrper-

16 Die Sekundarstufe im Kanton Zürich ist aufgrund des Volksschulgesetzes vom 7.2.2005 (Zürcher Kantonsrat 2005) im Umbau. Ab Schuljahr 2011/12 ist in allen Oberstufenschulen der Stadt Zürich nicht mehr die bis anhin Dreiteilige Sekundarschule (DS) mit drei nach Leistungen differenzierten Abteilungen (Abteilung A, B, C) sondern die Gegliederte Sekundarschule (GS) mit zwei nach Anforderungen differenzierten Stammklassen (Stammklasse G und E) eingeführt, solche mit grundlegenden und solche mit erweiterten Anforderungen. Zudem wird in den Fächern Mathematik sowie Deutsch oder Französisch in drei unterschiedlichen Leistungsniveaus unterrichtet: grundlegendes, mittleres und erweitertes Niveau (vgl. dazu Volksschulamt 2004). Anders haben die Gemeinden im Kanton Zürich grundsätzlich die Wahl zwischen beiden Schulmodellen.

17 Vgl. Verzeichnis des Datenmaterials in der Bibliografie: Datenmaterial aus der ersten Erhebungsphase (März bis Juni 2008). 
son bereits als Lernerfolg verbucht. In der Sekundarstufe B kontrolliert der Lehrer während Schultests, dass keine unerlaubten Hilfsmittel beigezogen werden; dennoch werden diese rege verwendet. Wenn er jemanden ertappt und Konsequenzen ausspricht, sind diese schwer einzufordern. In der Sekundarstufe A wiederum prüft die Lehrerin sehr streng, ob die Schultests selbstständig geschrieben werden, was die SuS wahrnehmen und veranlasst, von Spickversuchen eher abzulassen. Dennoch wird gespickt, was teilweise aufgedeckt wird und Konsequenzen nach sich zieht, die auch ausgeführt werden.

Solche Hinweise auf einen heterogenen und leistungsstufenabhängigen Umgang der Lehrpersonen mit Verhaltensweisen der SuS veranlassten mich, die vielfältigen Ehrlichkeitsregeln auf einer Leistungsstufe zu untersuchen, und zwar auf die Sekundarstufe A (vgl. Kap. 5.4.2). Damit sollen die Eigenart der an die Leistungsstufen angepassten Erziehungsmuster und die damit zusammenhängenden stark variierenden Sanktionsmuster der Lehrpersonen minimiert werden und, auf Basis derselben Leistungsstufe, vergleichbare Perspektiven der Heranwachsenden vertieft untersucht werden. Dennoch wird der soziokulturelle Rahmen angesichts der sozialen Belastung der Schulgemeinden und des Stadt-Land-Verhältnisses kontrastierend berücksichtigt. Denn Forschung zur Bildungsungleichheit weist einen Zusammenhang auf zwischen soziokultureller Herkunft und Bildungsfunktion (vgl. dazu die Längsschnittstudie von Moser et al. 2011), wobei ich obendrein einen Zusammenhang zwischen soziokultureller Herkunft und Erziehungsfunktion vermute. Auf eine fundierte Untersuchung dazu wird in dieser Studie aus Platzgründen verzichtet.

Zusammenfassend kann an dieser Stelle festgehalten werden, dass die öffentliche Schule bestimmte Rahmenbedingungen stellt, in denen SuS sich zurechtzufinden haben. Das konnte an zwei aktuellen und zentralen schulpädagogischen Themen, der gesellschaftlichen Schulfunktionen und der Bildungsungleichheit, aufgezeigt werden. Es zeichnete sich insbesondere in der schulischen Erziehungsfunktion der Widerspruch ab zwischen der Forderung des Schulsystems nach guten Leistungen und gleichzeitig der Forderung nach erwarteten Verhaltensweisen, wie beispielsweise Ehrlichkeit. Eine weitere Beobachtung ist in der Bildungsungleichheitsthematik zu finden, welche sich vornehmlich auf ungleiche Beurteilung von Schulnoten bezieht und damit die Bildungsfunktion kritisch reflektiert. Parallel dazu ist zu vermuten, dass eine herkunftsabhängige Erziehungsfunktion in der schulischen Ungleichheitsthematik vorhanden ist, was in einer späteren Untersuchung zu prüfen ist.

Aus der Ungleichheitsthematik präsentierten sich methodisch relevante Anhaltspunkte für das vorliegende Forschungsvorhaben: Zum einen wird aufgrund der leistungsabhängigen Bildungsungleichheit diese mit dem fokussierten Untersuchungsgegenstand einer Leistungsstufe minimiert. Zum anderen ist die soziale Belastung bei der Auswahl der Schulhäuser und das StadtLand-Verhältnis berücksichtigt, um der verbreiteten Meinung entgegenzu- 
wirken, dass auf dem Land die Verhaltensweisen der SuS im Vergleich zur Stadt angepasster seien.

\subsection{Erziehung in der Schule}

Wenn Erziehung in der Schule thematisiert wird, ist es keine Selbstverständlichkeit (wie in der Einleitung angedeutet), sich die Frage zu stellen, auf welchen theoretischen Ansätzen solche Überlegungen basieren können. Dieses Unterkapitel geht genau dieser Frage nach, um in einem ersten Schritt relevante Grenzen von theoretischen Erziehungsansätzen und Forschungsdesiderata nachzuzeichnen (Kap. 3.3.1). In der Schulpädagogik sind zudem Werteerziehungsansätze weit verbreitet. Diese werden in einem weiteren Arbeitsschritt auf deren Grenzen untersucht und bestimmte Parallelen zu Tugenderziehung aufgezeigt (Kap. 3.3.2). Gegenüber der theoretischen Beschäftigung mit Erziehung untersteht jede öffentliche Schule einem Erziehungsauftrag. In diesem sind die Erziehungserwartungen an die Schule formuliert, die an dieser Stelle ausgehend vom Erziehungsauftrag der Zürcher Volksschule im Hinblick auf die Tugend Ehrlichkeit untersucht werden (Kap. 3.3.3). Ausgehend von einer Zusammenfassung zum Thema Erziehung in der Schule werden Folgerungen für das Forschungsvorhaben skizziert (Kap. 3.3.4).

\subsubsection{Grenzen von theoretischen Erziehungsansätzen}

Nach einer Analyse von historisch-theoretischen Erziehungsansätzen stellt Jürgen Oelkers fest, dass diese im Laufe der Geschichte nicht als solche zu bezeichnen sind, weil sie sich grundsätzlich unter folgenden drei Merkmalen entwickeln (vgl. Oelkers 2001, S. 34f.):

- Reaktionen auf Moral

- Argumentationen, die auf Defizite hinweisen und an denen pädagogische Strategien anknüpfen

- Beschreibungen der Erziehungspraxis und Erziehungspersonen, welche Paradoxien, Dilemmas, Nöte, Zerbrechlichkeiten und Stile beinhalten.

Somit kann nicht von geschlossenen theoretischen Erziehungsansätzen gesprochen werden, sondern es muss vielmehr zwischen Beschreibungen von Erziehungspraxen sowie Reflexionen einerseits und theoretischen Ansätzen andererseits unterschieden werden.

Daneben registriert Michael Winkler für die gegenwärtige Erziehungswissenschaft eine ,Unsicherheit“ (Winkler 2010, S. 60f.) hinsichtlich Erzie- 
hung, weil diese eine Auseinandersetzung mit öffentlichen Erziehungserwartungen, eine Analyse der Semantik der Erziehung und einen Bezug zu sozialen Wirklichkeitskonstruktionen zu berücksichtigen habe. Winkler zeigt auf, dass gegenwärtige theoretische Erziehungsansätze diesen Anforderungen nicht entsprechen.

Hinzu kommt, dass theoretische Erziehungsansätze den sozialen und kulturellen Wandel zu berücksichtigen haben, welcher die Erziehungspraxis direkt betrifft. So sind im heutigen Lebenskontext, der von technologischen Errungenschaften geprägt ist, keine ,generationalen Erziehungsverhältnisse mehr gegeben (vgl. bspw. Thole 2009, S. 311f.): Erziehung kann zumindest nicht mehr durchgehend im klassischen Sinne verstanden werden, nach dem die älteren Menschen (Erzieherinnen und Erzieher) den Heranwachsenden (zu Erziehende) gesellschaftliche Regeln, Normen und Verständigungsformen vermitteln. So sind es gerade die jüngeren Menschen, die die älteren Menschen in die technologischen Errungenschaften einführen und damit ,zur Etablierung einer neuen Lernkultur beitragen“" (Thole 2009, S. 311).

Tholes Erziehungsverständnis geht von Erziehung aus als „ein bedingter und bedingender Faktor der gesellschaftlich-historischen Entwicklungs-, Auseinandersetzungs- und Entscheidungsprozesse" (ebd.). Daher kann Erziehung nur abhängig von gesellschaftlichen, sozialen und kulturellen Bedingungen und nicht als allgemeingültige Aktivität definiert werden. Darüber hinaus ist Erziehung eine praktische Tätigkeit ,spontaner Natur', die ,auf situative und persönliche Erfahrung" reagiert (Oelkers 2001, S. 16). Somit ist Erziehung nicht nur auf einer theoretischen und abstrakten Ebene zu reflektieren, sondern auch im konkreten Erziehungsalltag: „Der Begriff Erziehung trägt zur Bezeichnung von pädagogischen Handlungsprozessen nur dann noch zur Verständigung bei, wenn das jeweils mit ihm Gemeinte situationsbezogen präzise belegt wird" (Thole 2009, S. 311).

Zudem fordert Thole (Thole 2009, S. 310f.; vgl. dazu ebenso Oelkers 2009b; Oelkers 2004), dass die Erziehung zu Tugenden getestet wird: Reflexionen zu Erziehung und theoretische Erziehungsansätze scheinen, als ob sie nicht scheitern können, weil sie von bestehenden Erziehungswahrheiten ausgehen. Diese Erziehungswahrheiten sind mit Erziehungshypothesen gleichzusetzen, die jedoch nicht in der Praxis getestet werden, wie es dringend nötig wäre.

Die Erziehungspraxis ist eine hochkomplexe Angelegenheit, die vor allem in der Familie, in der Schule und in geleiteten Freizeitangeboten stattfindet. Nicht weniger bedeutend für Jugendliche sind das Zusammensein mit Peers, Medien oder informelle Freizeitangebote, was neueren Jugendstudien zu entnehmen ist (vgl. Trautwein und Kropf 2004; Jugendbarometer 2010). In diesen Bereichen sind die Heranwachsenden nicht direkt mit Erziehung, sondern mit Regeln, Werten und Normen konfrontiert, die nicht zwangsläufig 
mit denen der familiären und schulischen Erziehungsinstanzen übereinstimmen müssen.

Ebenso richtet sich das Verhalten der Jugendlichen in Erziehungskontexten nicht immer nach erlernten Einstellungen, sondern nach situationsabhängigen und emotionalen Faktoren wie beispielsweise Zugehörigkeit oder Kollegialität in Peer Groups (vgl. Wehr 2008; Riegel und Geisen 2010; Wetzstein et al. 2005).

Den vielfältigen und vielschichtigen Erziehungs- und Alltagssituationen können eindimensionale Erziehungsansätze nicht gerecht werden. Zu diesen zählen beispielsweise solche, die neuere neurowissenschaftliche Errungenschaften einbeziehen und sich als Verfechter einer ,gehirngerechten Pädagogik“ (Braun und Meier 2004, S. 518) bezeichnen. Nach ihnen soll ein linearer Zusammenhang zwischen neurowissenschaftlichen Befunden, wie beispielsweise Anzahl der Synapsen im Gehirn, und der Leistungsfähigkeit des Gehirns bestehen. Allerdings kann das neurowissenschaftliche Material dafür keine Legitimation anbieten: Neurobiologische Abläufe oder altersspezifische Fähigkeiten „können das Wissen und die Möglichkeit von Erziehung und Bildung absichern, keinesfalls jedoch ersetzen“" (Thole 2009, S. 313). Thole führt hierzu das Beispiel eines dreijährigen Kindes an, das nach einem neurologischen Test als fähig bezeichnet wurde, mathematische Aufgaben zu lösen. Folglich soll das Kind in diesem Bereich gefördert werden. Damit sei jedoch nicht geklärt, ob die Förderung tatsächlich sinnvoll ist.

An diesem Beispiel wird deutlich, dass biologisch gegebene Möglichkeiten von Lernen (mathematische Fähigkeit) als Notwendigkeiten des Lernens (Fördern der mathematischen Fähigkeit) ausgegeben werden. Allerdings kann die Gleichsetzung von Möglichkeit und Notwendigkeit nur für eine naturalistische Perspektive der Pädagogik von Bedeutung sein. „Dieses Ziel jedoch muss einem anderen untergeordnet sein: Die Pädagogik muss dem Kind als Person gerecht werden" (Giesinger 2006, S. 14). Somit kann die Pädagogik gehirngerecht ausgerichtet sein, wobei sie primär dem Heranwachsenden als Person gerecht zu werden hat.

Außerdem stoßen neurowissenschaftliche Ansätze an Grenzen, wenn es darum geht, den sozialen Kontext zu erfassen (Thole 2009, S. 313): Soziale Zugehörigkeiten im Bildungs- und Erziehungsprozess können nicht von neurowissenschaftlichen Ansätzen erfasst werden; diesen Zugehörigkeiten kommt jedoch für Heranwachsende nach der empirischen Jugendforschung eine zentrale Bedeutung zu (vgl. Kap. 3.1).

Zusammenfassend ist festzuhalten, dass gegenwärtig nicht auf theoretische Erziehungsansätze zurückgegriffen werden kann, die sowohl die öffentlichen Erziehungserwartungen, die Semantik der Erziehung als auch die vielfältigen Erziehungsrealitäten $\mathrm{zu}$ berücksichtigen vermögen. Theoretische Erziehungsansätze können nicht angemessen die Erziehungspraxis erfassen, weil die theoretischen Ansätze selten an der Praxis ausgerichtet und überprüft 
werden. In Erziehungsorten wie Familie oder Schule, aber auch in anderen Kontexten orientieren sich Heranwachsende nicht allein an erlernten Verhaltensregeln, sondern auch an anderen wie etwa gruppenspezifischen Faktoren. Zudem führen einseitige theoretische Erziehungsansätze, etwa solche der Neuropädagogik, dazu, eine Erziehung zu fördern, die sich durch einseitige naturwissenschaftliche Befunde legitimiert, ohne dem Heranwachsenden als Person gerecht zu werden oder ohne den sozialen Kontext zu berücksichtigen. In einem komplexen Alltags- und Erziehungsumfeld ist zudem keine passive Haltung der Heranwachsenden, sondern produktives Verhalten gefragt (vgl. Kap. 3.1.2). Die Erarbeitung von theoretischen Erziehungsansätzen, die den aufgeführten Anforderungen nachkommen, bleibt bislang ein Forschungsdesiderat.

\subsubsection{Weitere Grenzen am Beispiel der Werteerziehung}

Seit den 1960er Jahren sind theoretische Ansätze zur Werteerziehung aufgekommen. Diese sind heute in der Schulpädagogik relevant (vgl. Dubs 2009; Scherb 2004; Giesecke 2005; Wurz 2007; Latzko 2006; Mokrosch und Regenbogen 2009; Abendschön 2007). Mit Werteerziehung ist üblicherweise die ,Vermittlung ${ }^{6}$ ausgewiesener moralischer Werte an Kinder und Jugendliche gemeint. In Abgrenzung zum Tugendansatz versprechen sich Vertreter von Werteerziehungsansätzen eine neutral geführte Auseinandersetzung mit Moral und Erziehung.

Doch weist nur schon der Wertebegriff auf abstrakte und schwer erreichbare Erziehungsziele hin. Wenn beispielsweise Heranwachsende ,Respekt ${ }^{6}$ zu erwerben haben, steht nicht klar fest, wie der Aneignungsprozess zu diesem Wert aussehen soll. Zudem stellt sich die Frage, ob allen Menschen mit Respekt zu begegnen ist, ob Ausnahmen bestehen, bspw. ob etwas mit Nachdruck einzufordern manchmal angemessener ist, um ein gesetztes Ziel zu erreichen. Dubs (2009) geht von einem entwicklungspsychologischen Werteerziehungsansatz aus und schlägt eine Kombination von verschiedenen theoretischen Ansätzen der Werteerziehung vor, um damit die Wirksamkeit der Werteerziehungsansätze zu erhöhen (Dubs 2009, S. 394). Zugleich weist er, ausgehend von der Perspektive der Lehrperson, auf die begrenzte schulische Einflussmöglichkeit der Werteerziehung im Unterricht hin. Hierzu leistet er keinen Lösungsvorschlag. Jedoch bietet er Lehrpersonen didaktisches Material an, um den eigenen Unterricht kognitiv zu optimieren (vgl. ebd., S. 398ff.).

Darüber hinaus bleibt im Zusammenhang mit Werteerziehung die Bedeutung und Effizienz des Vermittlungsbegriffs unklar. Ist mit ,Vermitteln ${ }^{6}$ ,Übertragen' gemeint? Beispielsweise erzählte mir eine Klassenlehrerin aus der ersten Erhebungsphase (vgl. Anhang E), sie mache bereits in der ersten 
Stunde die Schülerinnen und Schüler $(\mathrm{SuS})$ ihrer gegenwärtigen Klasse darauf aufmerksam, dass es wichtig sei, ehrlich zu sein. Zur Untermauerung und als Erinnerungsstütze hängt ein Wortspiel zur Ehrlichkeit über der Wandtafel. Tagtäglich sehen die SuS das Plakat. Allerdings erwähnt keiner der vier interviewten SuS dieses Plakat. Auch nicht, als ich nach der Thematisierung von Ehrlichkeit im Schulunterricht nachfrage. Dieses Beispiel untermauert, wie unterschiedlich Tugendförderung von der Lehrperson und von den SuS wahrgenommen wird.

Weiter gibt es zahlreiche abstrakte und programmatische Veröffentlichungen zu Ansätzen der Werteerziehung. Empirische Untersuchungen dagegen fehlen weitgehend. So reflektiert Gundula Regenbogen in ihrem Beitrag zur Wahrhaftigkeit ausgehend von der Antike über gegenwärtige entwicklungspsychologische Ansätze (Regenbogen 2009), doch weder bezieht sie sich auf empirischen Daten noch implementiert sie ihre Gedankengänge in die alltägliche Unterrichtssituation.

Auf dieses Praxismanko und auf die fehlenden empirischen Untersuchungen zum theoretischen Werteansatz hat bereits Siegfried Uhl (1998) mehrfach aufmerksam gemacht: Eine

Schwierigkeit der Wirksamkeit von Werterziehungsprogrammen ist, dass man sich oft auf die Wirksamkeitsversprechen ihrer Befürworter und allenfalls auf einige Erfahrungsberichte und Einzelfallbeschreibungen aus der Praxis zu stützen hat (Uhl 1998, S. 158).

Hingegen existieren einzelne empirische Untersuchungen zu Einstellungen gegenüber Werten in der Jugendforschung (vgl. Jugendbarometer 2010; Hurrelmann und Albert Mathias 2006; COCON 2006) und in der Schulforschung (vgl. Latzko 2006). Jedoch trifft hier das gleiche Argument zu wie bei der Tugendforschung (vgl. Kap. 1.2, Kap. 2.4), nämlich dass nur Einstellungen untersucht werden, und nicht Aneignungsprozesse von Werten, nicht der Umgang mit Werten in der Praxis und nicht die Deutung sowie das Selbstverständnis von Werten aus der Alltagsperspektive von Jugendlichen.

Aufgrund des empirischen Forschungsdesiderats sowohl der Werteerziehung als auch der Tugenderziehung bestehen weitere Parallelen zwischen beiden Erziehungsansätzen. $\mathrm{Zu}$ diesen hat Timo Hoyer eine Gegenüberstellung vorgenommen, die ich tabellarisch dargestellt habe, um Grenzen und Chancen beider theoretischen Ansätze zu veranschaulichen (vgl. Tab. 3).

Gemäss Hoyer ist die Werteerziehung kein äquivalenter Ersatz für die Tugenderziehung, denn der Werteerziehung geht ein meist unterschätzter Erziehungs- und Sozialisationsprozess voraus (Hoyer 2005, S. 17). Ebenso unterstreicht Wolf, dass Verhaltensweisen bereits vor dem reflexiven Alter erworben werden:

Das liberale Ideal einer Erziehung zur eigenen Vernunft und Autonomie, welche inhaltliche Werte der späteren Entscheidung des erwachsenen Individuums überlässt, ist in dieser Hinsicht illusionär, weil sich Charakterhaltungen, die Reaktionen ebenso wie Ziele bestimmen, unvermeidlich schon im präreflexiven Alter verfestigen (Wolf 1999, S. 34). 
Tabelle 3: Werte- und Tugenderziehung im Vergleich

\begin{tabular}{|l|l|}
\hline Wertepädagogische Ansätze & Tugendpädagogische Ansätze \\
\hline Kritik & \\
\hline $\begin{array}{l}\text { Werteindoktrination } \\
\text { Fehndwertedogmatismus }\end{array}$ \\
\hline Erziehungsmethodische Seite \\
\hline Vermittlung, Klärung, Kommunikation & praktische Übung Gewöhnung \\
\hline Im Allgemeinen & $\begin{array}{l}\text { positiv bewertete Verhaltenseigen- } \\
\text { schaft }\end{array}$ \\
\hline $\begin{array}{l}\text { Wertebegriff verstanden als Bewusstseinsinhalte, } \\
\text { Sinnkriterien, Beurteilungsgepflogenheiten }\end{array}$ & $\begin{array}{l}\text { Tugenden werden gebildet, bevor das } \\
\text { Kind handlungsfähig und selbstver- } \\
\text { antwortlich ist }\end{array}$ \\
\hline $\begin{array}{l}\text { Werte werden im Reflexions- und Aufklärungsprozess } \\
\text { in der argumentativen Auseinandersetzung gebildet }\end{array}$ \\
\hline $\begin{array}{l}\text { Zeitpunkt des Beginns der Erziehung } \\
\text { Heranwachsende in fortgeschrittenem, meist schulfä- } \\
\text { higem Alter, denn Reflexions-, Begründungs- und } \\
\text { rationale Entscheidungsfähigkeit werden vorausgesetzt }\end{array}$ & seit der Kindheit \\
\hline
\end{tabular}

Quelle: eigene Darstellung in Anlehnung an Hoyer 2005

Hier zeigt sich eine weitere Schwierigkeit von theoretischen Erziehungsansätzen: Erziehung soll moralisches Handeln fördern, und zugleich soll dies der Lernende aus Selbsteinsicht und Selbstkompetenz (Roth 1971, S. 540) erwerben (vgl. zudem Drieschner 2007). Solche und andere Paradoxa finden sich in den unterschiedlichen Versuchen, über Erziehung zu sprechen (Oelkers 2004, S. 122) oder in Versuchen, Erziehung theoretisch zu fassen (Oelkers 2001).

An dieser Stelle werden am Beispiel der Werteerziehung mit Bezugspunkten zur Tugenderziehung bereits erwähnte Grenzen von theoretischen Erziehungsansätzen differenziert, die ich abschließend ohne Anspruch auf Vollständigkeit wie folgt zusammenfasse:

- Theoretische Erziehungsansätze sind allzu oft mit rhetorischen Erwartungen, Absichten und kommunikativen Absicherungen vermengt, was jedoch für die Differenzierung zwischen Erziehungsidealen und Erziehungsrealität zu unterscheiden wäre.

- In der Erziehungspraxis begegnet man oft unbeabsichtigten oder nichtkalkulierbaren Erziehungseffekten, was ebenso in theoretischen Erziehungsansätzen zu berücksichtigen wäre.

- Theoretische Erziehungsansätze sollten flexibler und komplexen Erziehungsphänomenen gerecht werden - denen gegenwärtige theoretische Ansätze nicht nachkommen, weil sie Dauergeltung beanspruchen und sich allzu oft auf lineare Erziehungsprozesse beschränken.

- In theoretischen Erziehungsansätzen werden Fragen, ob sich Erziehung lohne und welche Belastung damit verbunden sei, nicht gestellt, obwohl 
Auseinandersetzungen mit solchen Fragen Erschöpfungserscheinungen vorbeugen könnten.

\subsubsection{Tugend Ehrlichkeit und der Erziehungsauftrag der Zürcher Volksschule}

Ergebnisse der Längsschnittstudie zum Übergang von der Schule in den Beruf im Kanton Zürich (Neuenschwander et al. 2010) weisen darauf hin, es sei

nicht in erster Linie schulisches Wissen erforderlich, sondern eine Kombination von möglichst früh entwickelten klaren beruflichen Fähigkeitseinschätzungen und Interessen, positiven Attributen mit hoher Signalwirkung [...] hoher Flexibilität und Anpassungsfähigkeit, sowie einem wohlwollenden, ermutigenden und Autonomie förderlichen familiären Umfeld entscheidend (ebd., S. 97f.).

In diesem Zusammenhang zählen zu den positiven Attributen „soziale Kompetenzen im Sinne eines tugendhaften Verhaltens wie Pünktlichkeit, Sorgfalt, Anstand usw." (ebd., S. 98).

Tugenden sind somit für die erfolgreiche Suche nach einer Berufslehre im Anschluss an die obligatorische Schulzeit vorteilhaft. Dieser Befund unterstreicht die Bedeutsamkeit des Erziehungsauftrags der Zürcher Volksschule und der damit indirekt verbundenen Förderung von Tugenden. Ganz allgemein ist der Erziehungsauftrag im Volksschulgesetz des Kantons Zürich verfasst: „Die Volksschule erzieht zu einem Verhalten, das sich an christlichen, humanistischen und demokratischen Wertvorstellungen orientiert" (Zürcher Kantonsrat 2005, § 2).

Somit sollen in der Zürcher Volksschule - neben dem Erwerb von fachlichem Wissen - Wertvorstellungen (ebd., § 54) gefördert werden, die Tugenden wie Ehrlichkeit implizieren. In der Persönlichkeitsbildung und Förderung der Gemeinschaftsfähigkeit, die im Leitbild der Zürcher Volksschule im Zentrum steht, ist der Ehrlichkeitsbegriff notwendigerweise mitgedacht:

Der Erziehungs- und der Bildungsauftrag werden im Unterricht nicht getrennt, sondern beide Ziele werden bewusst gemeinsam angestrebt. Erzieherische Ziele sind dabei die Entfaltung einer lebensbejahenden und selbstständigen Persönlichkeit und die Gemeinschaftsfähigkeit des Individuums, das nicht nur Verantwortung für sich, sondern auch für seine Mitmenschen und für die Natur trägt und entsprechend handelt (Bildungsdirektion des Kantons Zürichs 2010, S. 18).

In Schulordnungen und insbesondere im Kontaktheft der SuS sind zwar Verhaltensregeln wie Respekt, Toleranz, Rücksichtnahme, Selbstständigkeit erwähnt, aber nicht die Ehrlichkeit. Im Schulzeugnis wird das Sozialverhalten unter zwei Punkten bewertet: ,Akzeptiert die Regeln des schulischen Zusammenlebens ${ }^{6}$ und ,Begegnet den Lehrpersonen und den Mitschülerinnen 
und Mitschülern respektvoll‘. In beiden Punkten sind ehrliche Verhaltensweisen impliziert. Diese werden also vorausgesetzt.

Im schulischen Alltag ist zu beobachten, dass Erziehungsziele allerdings nicht nur kantonal gesetzt sind, sondern schulhausintern anhand von Leitbildern und persönlichen Präferenzen der Schulleitung sowie von den einzelnen Lehrpersonen gefasst werden. Die Umsetzungsvielfalt des Erziehungsauftrags ist groß und nicht linear. Beispielsweise wird das unredliche Schreiben von Schultests unterschiedlich scharf kontrolliert bzw. gebilligt (vgl. Bsp. in Kap. 3.2).

\subsubsection{Zusammenfassung und Folgerungen}

In diesem Unterkapitel konnte aufgezeigt werden, dass bislang keine theoretischen Erziehungsansätze bestehen, welche die Erziehungspraxis in ihrer Alltäglichkeit zu verfassen vermögen, die von komplexen, paradoxen, unbeabsichtigten und nichtkalkulierbaren Erziehungsfaktoren bestimmt und damit vom situationsbedingten und sozialen Erziehungskontext stark geprägt ist. Daher wird die vorliegende Studie nicht an einen theoretischen Erziehungsansatz anknüpfen können.

Die zentrale Grenze der theoretischen Erziehungsansätze und damit ebenso der Ansätze von Werterziehung und Tugenderziehung besteht in der kaum ergründeten Erziehungspraxis. Dieses Manko ist Anlass für den empirischen Zugang dieser Arbeit.

Wenn im Folgenden die Schule als Erziehungsort thematisiert wird, ist immer zwischen öffentlichem Erziehungsauftrag und dessen realen Erziehungsmöglichkeiten im Unterricht und in der Schule abzuwägen. Weiter wird Erziehung als integrativer Bestandteil des Schulbetriebs betrachtet, wie dies der Erziehungsauftrag etwa der Zürcher Volksschule vorsieht. In diesem ist die Tugend Ehrlichkeit implizit als erwünschte, kontrollierte und sanktionierte Verhaltensweise enthalten, ebenso wie in den schulischen Dokumenten wie Lehrplan, Schulordnung, Kontaktheft oder Zeugnis.

Neben der Familie als primärer Erziehungsinstanz (vgl. Bergmann 2005; Bönsch 2002; Ahrbeck 2004; Thole 2008) kommt der Schule als sekundärer Erziehungsinstanz ein Erziehungsauftrag zu, jedoch mit eingeschränkten Rahmenbedingungen. Deshalb ist der Auftrag als ein solcher zu untersuchen. Zugleich handelt es sich bei der Schule um einen passenden Untersuchungsort. Denn gerade in der Schule und besonders im Unterricht findet sich ein System an Regeln, denen gegenüber man sich konform verhalten, an denen man sich reiben oder die man überschreiten kann. 


\section{Forschungsfrage und Forschungsziel}

Vor dem Hintergrund der vorgestellten Erkenntnisse aus dem gegenwärtigen Forschungsstand und der daraus resultierenden Kontextualisierung des Forschungsgegenstands (vgl. Kap. 1, 2, 3) werden in diesem Kapitel die Forschungsfrage und das Ziel der Untersuchung präsentiert.

\subsection{Forschungsfrage}

Die vorliegende Studie geht von der Schülerforschung und von SuS als produktiv verarbeitenden Subjekten aus (Kap. 3.1 und 3.2). Damit ist die erzieherische Rolle der Erwachsenen nicht negiert. Allerdings wird diese vorerst ausgeblendet, um die Sichtweise der SuS schärfer zu fokussieren. Denn die Sichtweise der SuS zu ihren Ehrlichkeitspraxen und damit die Deutung des Erziehungsalltags ist von den Erwachsenen kaum berücksichtigt, obwohl die Tugend Ehrlichkeit im Begriffsrepertoire, in Tugendkatalogen seit der griechischen Antike, in theoretischen Erziehungsansätzen sowie in populären Erziehungserwartungen weit verbreitet ist, wie in den vorangegangenen Kapiteln aufgezeigt wurde.

Daher sollen in dieser Studie zur Tugend Ehrlichkeit zum einen empirische Daten zum Thema gewonnen und zum anderen die Sichtweise der SuS zugänglich gemacht werden. Am Beispiel der Ehrlichkeitspraxen, die von den SuS gedeutet werden, kann grundsätzlich zum einen der Umgang mit der Tugend Ehrlichkeit aufgezeigt werden. Zum anderen wird eruiert, wie SuS den schulischen Erziehungsalltag wahrnehmen und wie sie Erziehungsprozesse sowie -methoden verarbeiten.

Ehrlichkeit ist eine Tugend, die als zentral und wünschenswert für den Schulalltag gilt (vgl. Kap. 3.3.3). Da zu den Tugenden im Allgemeinen sowie zur Tugend Ehrlichkeit im Spezifischen nur ungenügende empirische Forschungsbefunde zur Verfügung stehen (vgl. Kap. 1 und Kap. 2), fokussiert die vorliegende Untersuchung auf die schulrelevante Einzeltugend Ehrlichkeit und erkundet die subjektiven Deutungen von Ehrlichkeitspraxen aus Sicht der SuS (Forschungsgegenstand). Daraus können Ehrlichkeitsregeln rekonstruiert werden, an denen SuS ihre Verhaltensweisen orientieren.

Das primäre Forschungsinteresse besteht nicht darin, aufzuzeigen, wie ehrlich oder unehrlich die SuS sind. Es geht auch nicht darum, ihre Einstellungen zu ehrlichen Verhaltensweisen zu messen. Mein Forschungsinteresse zielt vielmehr darauf ab, subjektive Deutungen von expliziten und impliziten Ehrlichkeitsregeln nachzuzeichnen, an denen sich die Verhaltensweisen der $\mathrm{SuS}$ im schulischen Erziehungsalltag orientieren. Ziel ist dabei, die Sicht der 
Heranwachsenden zu beleuchten, um deren Bedeutung von Ehrlichkeit auf eine vielschichtige Weise aufzuzeigen. Anders ausgedrückt: Es geht darum, wiederzugeben, wie SuS die Tugend Ehrlichkeit im schulischen Erziehungskontext deuten und damit umgehen. Die empirische Hauptfragestellung lautet entsprechend:

- Wie deuten 14- bis 15-Jährige unterschiedliche Ehrlichkeitspraxen im Schulalltag?

Aus der Hauptfragestellung lassen sich folgende vier Unterfragenkomplexe ableiten:

- Welche Ehrlichkeitskategorien finden sich in den Berichten der Schülerinnen und Schüler?

- Wie thematisieren 14- bis 15-Jährige Ehrlichkeitspraxen?

- An welchen Ehrlichkeitsregeln orientieren sie ihre Verhaltensweisen in unterschiedlichen Situationen des Schulalltags?

- An welchen konventionellen und unkonventionellen Ehrlichkeitsregeln orientieren Schülerinnen und Schüler ihre Verhaltensweisen in realen Dilemmasituationen?

Dem Forschungsinteresse wird vor dem Hintergrund des sensitizing concept (Blumer 1954) nachgegangen. Aus dem Alltagswissen kristallisiert sich methodologisch das sensitizing concept im Rahmen der Grounded Theory:

A definitive concept refers precisely to what is common to a class of objects, by the aid of a clear definition in terms of attributes or fixed bench marks. A sensitizing concept lacks such specification of attributes or bench marks and consequently it does not enable the user to move directly to the instance and its relevant content. Instead, it gives the user a general sense of reference and guidance in approaching empirical instances. Whereas definitive concepts provide prescriptions of what to see, sensitizing concepts merely suggest directions along which to look (Blumer 1954, S. 7).

In diesem Sinne ist dieses Forschungsprojekt von der Annahme geleitet, dass das Thema Ehrlichkeit unter Berücksichtigung des Alltags, in diesem Fall des Schulalltags, in vielfältiger, eigenwilliger, nichtstatischer, situations- und kontextabhängiger Weise von den SuS gedeutet wird. Vor diesem Hintergrund gehe ich davon aus, dass eine situative und kontextabhängige Varietät von gedeuteten Ehrlichkeitsregeln zu finden ist. Weiter nehme ich an, dass der räumliche Kontext, die anwesenden Personen, die Zeit und die produktiv verarbeitenden Akteure eine eminent wichtige Rolle spielen. Gleichzeitig vermute ich, dass ihre Ehrlichkeitsregeln von sozialen Erwartungen geprägt sind. 


\subsection{Forschungsziel}

Das Ziel der vorliegenden Untersuchung ist, die Deutung der Tugend Ehrlichkeit aus der Sicht der SuS und ihre gedeuteten Praxen empirisch zu erkunden. Dabei sollen detaillierte Aussagen zu Deutungen von ehrlichen Verhaltensweisen und zu Ehrlichkeitsregeln im Schulalltag und in realen Dilemmasituationen des Schulalltags aus der Sicht von 14- bis 15-jährigen SuS gemacht werden. Zudem ist ein Einblick in den schulischen Erziehungsalltag aus ihrer Perspektive gegeben. Allgemeiner gefasst zielt die Studie darauf, zu erkunden, wie SuS ihre aktuelle Schulsituation erleben und bewältigen, und das unter besonderer Berücksichtigung der schulischen Erziehung.

Den Abschluss dieses Kapitels bildet die Präzisierung der Forschungsziele, die das so angelegte Projekt verfolgt:

- Ein moralisches Thema, die Tugenderziehung, soll am spezifischen Fall der Tugend Ehrlichkeit und im Schulkontext empirisch analysiert, differenziert und diskutiert werden.

- Erziehungsphilosophische Reflexionen zur Einzeltugend Ehrlichkeit sind in der Forschungsliteratur vorhanden, aber eher marginal. Noch weniger lassen sich empirische Untersuchungen solcher theoretischer Ansätze insbesondere des Schulalltags - finden. Dazu soll das vorliegende Forschungsprojekt einen Beitrag leisten.

- Üblicherweise wird Tugenderziehung seit der griechischen Antike aus der Perspektive der Erwachsenen betrachtet. Stellungnahmen der betroffenen Heranwachsenden fehlen weitgehend, obwohl die Erziehungsbemühungen gerade ihnen gelten und obwohl mit der Schülerforschung ein Perspektivenwechsel bereits vor rund dreißig Jahren stattgefunden hat. Entsprechend wird die Perspektive der SuS im Projekt vorgestellt und fokussiert.

- In der Literaturrecherche fällt zudem auf, dass zu Erziehungsfragen des Vorschulalters oder in der Primarschule viel publiziert wird. Hingegen findet sich wenig zur Erziehung im Oberstufenalter, obwohl anscheinend Tugenden für den Einstieg ins Berufsleben sehr zentral sind (vgl. Kap. 3.3.3). Daher fokussiert vorliegende Studie SuS aus der letzten Oberstufenklasse (14- und 15-Jährige), womit zentrale Aussagen über eine wichtige, aber bezüglich Tugend und Erziehung wenig erforschte Zielgruppe getroffen werden können.

- Subjektive Deutungen der SuS werden rekonstruktiv gewonnen: anhand von Ehrlichkeitsregeln, an denen SuS ihre Verhaltensweisen orientieren. Die Tiefenstruktur der Interviewaussagen und somit die Deutungen der SuS werden nachgezeichnet und Hinweise für Erklärungsansätze formuliert (vgl. Kap. 11). Eine Rückkoppelung zur Schüler- und Jugendfor- 
schung, zu öffentlichen Tugend- und Erziehungsdebatten wird abschließend vorgenommen (vgl. Teil III).

Viele Interviewaussagen der SuS werden sich für Lehrpersonen vertraut anhören; andere Aussagen und Denklogiken der Heranwachsenden hingegen können Erziehungsbeauftragte zum Nachdenken anstoßen. Jedoch liegt der eigentliche Erkenntnisgewinn in der Analyse der Hintergründe, Deutungen und Zusammenhänge der Aussagen und der Sichtweisen der SuS. Damit können die empirischen Befunde Anhaltspunkte sowohl für Erziehungspraxen als auch für zukünftige Erziehungsansätze oder jugendspezifische Theorieansätze bezüglich Verhaltensweisen von Jugendlichen liefern.

Kurz gefasst: Das vorliegende Forschungsprojekt soll zugleich einen Beitrag zur Versachlichung der oft emotional geführten Tugenddebatte leisten, das kritische Problembewusstsein für die Einzeltugend Ehrlichkeit schärfen, die oftmals in Erziehungsfragen vernachlässigte Perspektive der SuS berücksichtigen und für die komplexen, situationsabhängigen und teilweise widersprüchlichen Prozesse der gedeuteten Ehrlichkeitspraxen sensibilisieren, woran angepasste Tugenderziehung oder Tugendförderung anknüpfen kann.

\section{Methodik der qualitativen Untersuchung}

In einem dreiteiligen Forschungsprozess erarbeitete ich einen komplexen Ehrlichkeitsbegriff, Ehrlichkeitsregeln und Ehrlichkeitsmuster von 14- bis 15-jährigen Schülerinnen und Schülern (SuS). Dazu habe ich insgesamt 31 problemzentrierte Interviews (Witzel 2000) sowie sechs Gruppendiskussionen (Bohnsack 2006) mit Abschlussschülerinnen und -schülern an der Zürcher Volksschule in drei Erhebungsphasen (von März 2008 bis Dezember 2009) durchgeführt. Das Datenmaterial der ersten zwei Untersuchungsphasen wertete ich mit der qualitativen Inhaltsanalyse (Mayring 2008) aus. Die Interviewgespräche der dritten Untersuchungsphase analysierte ich in Anlehnung an die dokumentarische Methode (Bohnsack 2003, 2008; Przyborski und Wohlrab-Sahr 2009, S. 271ff.).

Die Ergebnisse der vorliegenden Arbeit beziehen sich auf die Einzelinterviews der letzten Forschungsphase, die in Zusammenhang mit den Zwischenergebnissen des dreiteiligen Forschungsprozesses stehen (Eine Kontextualisierung im Forschungsprozess skizziere ich in Kap. 5.4). Die Ausführungen zur Methodik sind insofern von großer Bedeutung, als anschließend die Ergebnisse vorgestellt werden, die vom ausgewählten methodologischen und methodischen Zugang geprägt sind. 
So ist im ersten Unterkapitel zum Thema,Methodologie' zu überprüfen, ob sich qualitative Interviews für die aufgeworfenen Fragestellungen eignen (Kap. 5.1). Hierbei ist zu klären, ob die Fragestellung und somit der anvisierte Forschungsgegenstand mit der Methode korrespondiert (vgl. dazu Przyborski und Wohlrab-Sahr 2009; Kleemann et al. 2009). Die anschließenden Unterkapitel klären die methodischen Ansprüche, welche an die Erhebungsmethode (Kap. 5.2) und die Auswertungsmethode (Kap. 5.3) gestellt werden.

Vor dem Hintergrund der methodologischen und methodischen Vorüberlegungen beschreibe ich das konkrete Forschungsvorgehen (Kap. 5.4), indem ich das Erhebungs- und Auswertungsverfahren dokumentiere. Die transparente Beschreibung des forschungspraktischen Vorgehens zählt neben der methodologischen Reflexion sowie der Konturierung der angewandten Methode zu den zentralen Qualitätsstandards der qualitativen Sozialforschung. In den Methodenbüchern und Debatten über Gütekriterien qualitativer Sozialforschung werden auch andere Aspekte und Akzentuierungen aufgeführt, die ich hier überblickshalber aufführe:

- Beschreibung angewandter Methoden und methodologische Reflexionen (Böhme 2008, S. 127f.)

- Definieren des Samplings, der Validität, der Reliabilität und der Objektivität (Silverman 2011, S. 268ff.)

- in der Regel von folgenden Aspekte geleitet: Intersubjektivität und Angemessenheit der Datenerhebung und Datenauswertung, sozialwissenschaftliche Situierung, Angaben zum Forschungskontext oder ethischer Bezugsrahmen, die Leitung durch theoretische Prämissen und Ansätzen, die Einbettung in die Forschungstradition, die Subjektsicht des Akteurs, die Entwicklung einer Interpretation der Forschenden, üblich theoriebildende Ergebnisse (Schweizerische Akademie der Geistes- und Sozialwissenschaften 2010, S. 19ff.)

- Qualität der Erhebungssituation, denn davon hängt die Qualität der Datenerhebung und -auswertung ab; methodologische Verortung des Forschungsgegenstands; Theorie- und Forschungstraditionen als Verortung methodologisch bestimmter Forschungsgegenstände; Klärung des Vorwissens und eines (zusätzlichen) Interesses am informativen Gehalt von Texten (Helfferich 2009, S. 99ff.)

- Anwendung der klassischen Gütekriterien auf den qualitativen Forschungszugang (Validität als Common-Sense-Konstruktionen und auf Standard der Verständigung aufbauend; Reliabilität als Reproduktionsgesetzlichkeit sowie systematisches Explizieren alltäglicher Standards der Kommunikation; Objektivität als Formalisierung von Erhebungsund Auswertungsschritten, was die Intersubjektivität erhöht), metatheoretische Fundierung von Methoden, methodologisch begründete Generalisierbarkeit von Ergebnissen, praxeologische Ausrichtung, Nutzung für 
Transdisziplinarität, Verbindung der Grundlagen- und Anwendungsforschung (Przyborski und Wohlrab-Sahr 2009, S. 25ff.)

- innerhalb der Kriterien für Wissenschaftlichkeit findet sich bspw. die Gütequalität als methodologische, Gegenstandsangemessenheit' (Breuer und Reichertz 2001, Abs. 17)

- fundierte Auswertung, inhaltliche und zeitliche Machbarkeit des Forschungsvorhabens, Sampling und dessen mögliche Generalisierbarkeit, auf Erklärung und Theorie ausgerichtete Forschung (nicht nur Beschreibung), schriftliche Qualität (Oswald 2010, S. 183ff.).

- Transparenz des Forschungsprozesses, Begründung des gewählten theoretischen Bezugsrahmens und methodisch kontrolliertes Fremdverstehen (Bennewitz 2010, S. 49).

- Nachvollziehbarkeit und Angemessenheit des Forschungsprozesses und der Ergebnisse, Reliabilität durch das Verfahren methodisch kontrollierten Fremdverstehens, theoretische Klarheit der Grundbegriffe, metatheoretische und erkenntnistheoretische Grundlagen des Verfahrens (Asbrand 2009; S. 135f.)

Es ist darauf hinzuweisen, dass auch bei akribischer Erfüllung der genannten Qualitätskriterien die Qualität der qualitativen Forschung nicht garantiert ist. Denn je nach Fachgebiet oder Forschungsmethode gibt es zusätzliche Qualitätskriterien (Schweizerische Akademie der Geistes- und Sozialwissenschaften 2010). Deshalb habe ich das erste Unterkapitel zur Methodik als einen methodologischen Abriss des gewählten methodischen Forschungszugangs geschrieben, unter besonderer Berücksichtigung des Fachbereichs der qualitativen Schulpädagogik.

\subsection{Methodologische Überlegungen}

Sprechen über Ehrlichkeit ist mit hohen sozialen Erwartungen verbunden: Grundsätzlich will niemand als unehrlich gelten - so auch nicht SuS. Zudem zeichnen sich Ehrlichkeitsmuster durch Situationsabhängigkeit, Vielfältigkeit und ungenügende theoretische Basis (vgl. Kap. 2) aus. Schließlich besteht ein zirkulärer Schluss darin, eine ehrliche Aussage verbal verifizieren zu wollen. Denn dass ich etwas ehrlich meine, kann auf jeden Fall nicht bewiesen werden, indem ich behaupte: „Ich meine es ehrlich.“ Daher lohnt es sich, Ehrlichkeitsmuster und Ehrlichkeitsregeln nicht allein auf der argumentativen Ebene, sondern vor allem auf der erzählenden und beschreibenden Kommunikationsebene zu untersuchen (vgl. Kap. 5.2).

Um den soeben aufgeführten Eigenschaften des Untersuchungsgegenstands gerecht zu werden, bietet insbesondere die qualitative Sozialforschung 
das passende Instrumentarium. Neuere Handbücher zur Erziehungswissenschaft belegen beispielsweise, dass qualitative Forschungszugänge meistens in der Mikroperspektive angesiedelt sind und pädagogisch relevante Einzelfälle fokussieren, was eine differenzierte Beleuchtung des komplexen Untersuchungsgegenstands ermöglicht (vgl. Prengel et al. 2010; S. 27).

Wegen der dünnen theoretischen Fundierungen und der komplexen Verläufe von ehrlichen Verhaltensweisen sind die Voraussetzungen nicht gegeben, um den Forschungsgegenstand mit quantitativen Forschungsmethoden als direkte Erfragung einzelner bereits definierter Kategorien zu erkunden. Zudem wären eigenwillige Ehrlichkeitsmuster von verzerrten Einstellungen durch soziale Erwartungen und das Streben nach Angepasstheit schwer zu differenzieren.

\subsection{1 ,Methodisch kontrolliertes Fremdverstehen' in der Datenerhebung und Datenauswertung}

Innerhalb der qualitativen Sozialforschung sind vorerst die ethnografischen Forschungszugänge auszuschließen, denn insbesondere unehrliches Verhalten zeichnet sich dadurch aus, dass es von außen nicht gesehen werden sollte und daher schwer zu beobachten ist.

Hinweise für die Angemessenheit eines kommunikativen Zugangs gab bereits der Ausschnitt aus dem Interviewtagebuch im Vorwort, in dem die SuS sich spontan mit mir über ihr akutes unehrliches Verhalten in der letzten Prüfungsstunde unterhalten hatten. Die verbale Kommunikation und die daran anknüpfende Methodik des qualitativen Interviews bietet ein passendes Erhebungsverfahren, um subjektive Ehrlichkeitsmuster im Schulalltag rekonstruktiv zu erfassen, wie in den nachfolgenden Ausführungen detaillierter aufgezeigt wird.

Als methodologischer Hintergrund dient das ,methodisch kontrollierte Fremdverstehen' (Schütze et al. 1973), worauf mit unterschiedlichen Akzentuierungen die qualitative Forschungsmethode basiert. Die methodologischen Prinzipien beziehen sich sowohl auf die Erhebungsphase als auch auf die Analysephase dieser Arbeit. Die zentralen Merkmale dieses Ansatzes werden im Folgenden hervorgehoben (vgl. bspw. Przyborski und Wohlrab-Sahr 2009, S. 28ff.) und innerhalb der der Erhebungs- und Auswertungsphase differenziert (vgl. Kruse 2009, S. 93):

- Die Forschungsperspektive ist an der Alltagspraxis des Erforschten angesetzt: „,Der Forschungsprozess nimmt also seinen Ausgang bei der Alltagspraxis und beim Alltagswissen der Erforschten“" (vgl. bspw. Przyborski und Wohlrab-Sahr 2009, S. 28).

- Das Gütekriterium der Offenheit ist zu berücksichtigen, das sich in der Datenerhebung dadurch auszeichnet, dass die Interviewperson das eigene 
theoretische und alltagspraktische Vorwissen reflektiert (vgl. Kap. 3 und Hinweise auf das Erhebungsjournal in Kap. 5.4), eine offene Haltung gegenüber dem Forschungsfeld einnimmt und offene Fragestellungen formuliert, damit der Befragte eigene Themen auswählen und damit thematische Präferenzen markieren kann. In der Datenanalyse geht es darum, den Text ,entfremdet ${ }^{\star} \mathrm{zu}$ interpretieren (Przyborski und WohlrabSahr 2009, S. 30 und Kap. 5.3).

- Die Verständigung im Alltag ist voraussetzungsvoll, was anhand des ,Krisenexperimentes' von Garfinkel aufgezeigt werden kann (vgl. dazu Garfinkel 1967, S. 35-75). Deshalb besteht eine zentrale Forderung darin, neben dem Wortlaut die Bedeutung des Gesagten zu berücksichtigen. Für das Verstehen sind damit der zeitliche Kontext, die sozialen, kulturellen und situativen Umstände sowie die biografische Situation des Sprechers prägend (vgl. weiterführende Reflexion dazu Kap. 5.3).

- Die Tatsache, dass sprachliche Äußerungen in einem Gesamtzusammenhang zu verstehen sind und damit nur auf Bedeutungsgehalte hinweisen, bezeichnen Garfinkel und Sack als ,Indexikalität" (Garfinkel [1961] 1981, S. 210ff.). Auf der kommunikativen Ebene ist eine ,unausweichliche Vagheit‘ (ebd., S. 204) der Alltagssprache zu identifizieren. In den Datenanalysen bieten unterschiedliche Methoden eine Differenzierung zwischen expliziten und impliziten Sinngehalten der Texte an (vgl. Kap. 5.1.2 und Kap. 5.4.2).

- Die spezifische Bedeutung des Gesagten wird im Alltag oftmals intuitiv bewältigt. Wenn jedoch die Kommunikationspartner biografisch und kulturell distanziert sind, kann die Kommunikation mehr oder weniger anhand von Fremdverstehen (Schütz [1932] 1960, S. 106ff.) erkundet werden. Schütz erklärt das Fremdverstehen am Beispiel des Holzfällens. Der Vorgang kann beobachtet werden: Das Holz wird mit einer Axt zerkleinert. Oder der fremde Leib, das Holzstück und dessen Veränderung kann analysiert werden, ohne nach dem Handeln und dem Motiv zu fragen. Schließlich kann der Holzfäller als ,handelnder Mensch ‘ betrachtet werden. In der letztgenannten Auslegungsmöglichkeit findet Fremdverstehen statt, was Schütz wie folgt erklärt: „Der Blick des Beobachters ist nicht auf die Anzeichen selbst gerichtet, sondern auf das, wofür sie Anzeichen sind, also auf den Erlebnisprozeß des Beobachteten selbst. (Echtes Fremdverstehen.)“ (Schütz [1932] 1960, S. 121; Hervorh. i. O.)

- Ebenso ist die Erklärung des Selbstverständlichen oder Intuitiven eine interpretative Herausforderung für die Forschungspraxis. Ein unmittelbares Verstehen kann sowohl für die interviewte Person als auch für die Forschenden problematisch sein (vgl. dazu bspw. Lilians Ehrlichkeitserklärung als Ehrlichkeit ist Ehrlichkeit in Kap. 6.1).

- Die methodische Kontrolle des Fremdverstehens wird gewährleistet, wenn die Darstellungsform von Untersuchten und Forschern systema- 
tisch differenziert wird. In der Erhebungsphase ist diese Unterscheidung dadurch gesichert, dass den Untersuchten die Möglichkeit gegeben wird, ihre Darstellung selbst zu gestalten. In der Analyse wird von der Kontextualisierung der Erforschten ausgegangen. Mit anderen Worten werden die Äußerungen im Kontext der Erzählung des Untersuchten interpretiert (vgl. Kap. 5.3).

\subsubsection{Zwei Interpretationsebenen}

Die Unterscheidung von zwei Interpretationsebenen ist für die hier angelegte Forschung sehr zentral. Denn Alltagswissen als Konstruktion kann auf der ersten Interpretationsebene oft nicht unmittelbar abgefragt werden:

Im Gegenteil: Viele sozialwissenschaftlich interessante Phänomene lassen sich nicht unmittelbar abfragen. Dies hat unter anderem damit zu tun, dass menschliches Handeln ernsthaft blockiert wäre, wenn wir uns alles, was wir tun, zurechtlegen und bewusst machen müssten (Przyborski und Wohlrab-Sahr 2009, S. 32; Hervorh. i. O.).

$\mathrm{Zu}$ den nicht unmittelbar abzufragenden Phänomenen zählt ehrliches Verhalten, das methodologisch vor dem Hintergrund des ,methodisch kontrollierten Fremdverstehens' ${ }^{\text {e }}$ erkundet werden kann. Mit der konkreten methodischen Umsetzung befasst sich Kapitel 5.2. An dieser Stelle soll die Differenzierung der zwei Interpretationsebenen vertieft werden, welche für die Erhebung und Auswertung des Forschungsvorhabens von zentraler Bedeutung sein werden.

Tabelle 4 soll einen kurzen Überblick über die unterschiedlichen Terminologien der zwei Interpretationsebenen geben, die aufgrund der methodologischen und methodischen Hintergründe mit unterschiedlichen Nomenklaturen verwendet werden.

Tabelle 4: Zwei Interpretationsebenen

\begin{tabular}{|l|l|l|}
\hline & 1. Interpretationsebene & 2. Interpretationsebene \\
\hline Alfred Schütz 1971 & Sinnstruktur 1. Grades & Sinnstruktur 2. Grades \\
\hline $\begin{array}{l}\text { Biografieforschung } \\
\text { Objektive Herme- } \\
\text { neutik }\end{array}$ & gemeinte, manifeste Sinnstruktur & objektive, latente Sinnstruktur \\
\hline Wissenssoziologie & explizite Wissensbestände & \\
\hline $\begin{array}{l}\text { Dokumentarische } \\
\text { Methode }\end{array}$ & $\begin{array}{l}\text { immanenter, wörtlicher Sinngehalt } \\
\text { (Was-Frage) }\end{array}$ & $\begin{array}{l}\text { implizite Wissensbestände } \\
\text { dokumentarischer Sinngehalt } \\
\text { (Wie-Frage) }\end{array}$ \\
\hline Narrationsanalyse & Erzählen & Argumentieren \\
\hline Gesprächsanalyse & wörtlicher Sinngehalt & kontextualisierter Sinngehalt \\
\hline
\end{tabular}

Quelle: eigene Darstellung in Anlehnung an Nohl 2008; Przyborski und Wohlrab-Sahr 2009; Kleemann et al. 2009; Flick 2008 
In der ersten Analyseeinstellung bzw. Beobachtungshaltung richtet sich der Blick auf das, was der Befragte meint oder sagen will. Diese Analyse hat einen deskriptiven Charakter. Wenn diese Konstruktion der ersten Analyseeinstellung in begriffstheoretische Explikationen überführt wird, die für die wissenschaftliche Erkenntnis notwendig und vom zeitlichen Handlungsdruck losgelöst sind, dann zählen diese Konstruktionen zur zweiten Analyseeinstellung. In der phänomenologisch begründeten Soziologie nach Alfred Schütz wird die zweite Analyseeinstellung als Konstruktion zweiten Grades benannt: „Es sind Konstruktionen jener Konstruktionen, die im Sozialfeld von Handelnden gebildet werden“" (Schütz 1971-1972, S. 7).

Somit zeichnet sich die zweite Analyseeinstellung dadurch aus, dass zunächst die formale Struktur von Interaktionen beschrieben und die Herstellungsregeln dieser Interaktionen aufgezeigt werden. In einem Beispiel von Garfinkel kann nachgewiesen werden, dass die Frage „Wie geht es dir?“ gleichzeitig eine Frage nach dem Wohlbefinden als auch eine Höflichkeitsgeste innerhalb einer sozialen Regel erfasst. Der wörtliche und der inhaltliche Sinn verorten sich in der ersten Analyseeinstellung. Darüber hinaus ist nach dem Sinn des inhaltlich bestimmten Verhaltens zu fragen. Die sinnstrukturierte Auseinandersetzung mit Aussagen und die Herausarbeitung sozialer Regeln charakterisiert die zweite Analyseeinstellung. Die Sinnstruktur, die dem Handeln zugrunde liegt, fokussiert die Prozessstrukturen der Herstellung der Interaktion.

In der Analyse zweiten Grades gilt es die Strukturgesetzlichkeit zu beleuchten, die Handeln und andere soziale Gegenstände hervorbringen. Die Strukturgesetzlichkeit bezeichnet Bourdieu als modus operandi (Bourdieu 1982, S. 728). Mannheim hingegen ordnet der Sinnstruktur zweiten Grades die Wie-Frage zu, welche die Herstellungsfunktion betont. Demgegenüber ist die Was-Frage zu differenzieren, die sich auf den kommunikativen Sinn einer Aussage bezieht (Mannheim und Kettler 1980, S. 201ff.).

Anhand der zwei Sinnstrukturen ist der Erkenntniszusammenhang zu rekonstruieren. Deshalb geht es nicht darum, reales Wissen zu produzieren: Eine reale Begebenheit spiegelt sich nicht im Interviewtext wider, sondern wird darin schon immer vielfach gedeutet. Hingegen wird soziale Wirklichkeit im Interview interaktiv und koproduktiv hergestellt (vgl. Kruse 2009, S. 109).

Abschließend möchte ich anhand einer Kette der sozialen Wirklichkeit die verschiedenen Deutungsstufen wie folgt veranschaulichen (in Anlehnung an Schütz 1971-1972, S. 3ff.):

Erfahrung machen $\rightarrow$ gemachte Erfahrung (Deutung $=2$. Analyseschritt $) \rightarrow$ Erinnerung an Erfahrung $\rightarrow$ Erzählung in einer Situation (Text $=$ Konstruktion=1. Analyseschritt) $\rightarrow$ Auswertungsverfahren, welche die zwei Analyseschritte unterscheiden 


\subsubsection{Interpretative bzw. rekonstruktive Sozialforschung}

Forschungsmethoden, welche die zwei Analyseverfahren differenzieren, sind in der interpretativen oder rekonstruktiven Sozialforschung zu situieren. Dazu gehört auch die dokumentarische Methode, mittels derer die Daten der vorliegenden Studie analysiert wurden (vgl. Kap. 5.3).

Eine weitere zentrale Unterscheidung der Alltagsrealität innerhalb von Kommunikationssituationen ist zwischen Sprechhandlung als spezielle Handlung im Interviewgespräch (es wird über etwas gesprochen) und der Handlungs- und Deutungspraxis selbst vorzunehmen. Ein Interview bildet, wie bereits oben erwähnt, nicht die Alltagspraxis ab. Dennoch hat die biografische Forschung den Anspruch, die Praxis möglichst nahe anhand narrativer Erzählungen zu rekonstruieren. Denn aufgrund der Zugzwänge des Erzählens sind Stegreiferzählungen besonders nahe an der Erfahrung des Erzählers (vgl. Schütze [1977] 1978). Allerdings soll es hier nicht darum gehen, welche methodischen Zugänge näher an der Praxis sind. Vielmehr verweist dieser Hinweis darauf, dass die Deutungsebene sowohl des Interviewten als auch des Forschenden sich auf den erzählten Text bezieht und voneinander unterschieden werden kann (vgl. Kap. 5.4.3).

Ein historischer Rückblick zeigt, dass das interpretative Paradigma bzw. die Alltagswende der Sozialwissenschaften sich in der qualitativen Schulforschung erst gegen Ende der 1970er Jahre etabliert hat, obwohl der Einsatz empirischer Verfahren bereits Anfang der 1960er Jahre begann. So fordert Heinrich Roth in seiner Antrittsvorlesung im Jahr 1962, dass die Erziehungswirklichkeit nicht nur historisch oder philosophisch erforscht wird, sondern auch direkt mit erfahrungswissenschaftlichen Methoden:

Es geht mit anderen Worten darum, die Methoden zu entdecken und zu schaffen, die es erlauben, die intuitive Hermeneutik der Erziehungswirklichkeit, die immer den problemerzeugenden Ausgangspunkt bilden wird, auf erfahrungswissenschaftliche Grundlagen zu stellen und dadurch deutlicher abzusichern (Roth 1963, S. 113).

Die Perspektive des Alltags als methodischen Ansatz beschreibt Thiersch für die Sozialpädagogik wie folgt:

Alltag ist z.Z. eine der Leitformeln progressiver sozial-pädagogischer Praxis und Diskussion. Alltag wird thematisiert, [...] das Tagesgeschäft wird analysiert; Handlungsforschung will am Alltag der Betroffenen partizipieren und in ihm aufklärend und aktivierend agieren. Alltagsorientierung [...] ist Indiz einer Sozialpädagogik, die Lebenswirklichkeit so, wie sie gegeben ist, ernst nimmt und sich von da aus orientiert (Thiersch 1978, S. 6).

Viel älter als der Ausgangspunkt der Alltagswende ist die allgemeine methodische Forderung, die Erziehungswirklichkeit zu verstehen. Sie gründet in der Tradition der geisteswissenschaftlichen Pädagogik (vgl. Bennewitz 2010, S. 43; Terhart 1997a, S. 31). In dieser entwickelten sich theoretische Ansätze des Verstehens in Form der philosophischen Hermeneutik. So befasste sich 
Friedrich Schleiermacher mit dem Verständnis des Autors eines Textes, wobei er eigenes Vorverständnis und Textverständnis unterschied (Böhme 2008, S. 128f.). Wilhelm Dilthey ging einen Schritt weiter und betonte den Erfahrungs- und Erlebnishintergrund des Interpreten als bedeutsam (ebd.). Es ging ihm um die Frage, was der Autor und der Interpret unter dem Gesagten verstehen. Hans-Georg Gadamer schließlich hat die Fixierung von Sinn über ein Textauslegen ausgeschlossen und verneint eine Gleichberechtigung von Interpret und Autor. Hingegen führt er die Geschichtlichkeit ins Verstehen ein: Die zentrale Frage im Verstehensverlauf besteht im Herausfinden, was der Interpret unter dem Gesagten aus seiner heutigen Situation heraus zu verstehen hat.

Zwei Grenzen der vorgestellten philosophischen Hermeneutik zeigen gerade Vertreter des ,interpretativen Paradigmas' auf: Erstens werde die aktuelle Erziehungswirklichkeit nicht berücksichtigt. Zweitens seien die gesellschaftlichen Konstitutionsbedingungen nicht reflektiert. Beide Aspekte werden hingegen in der interpretativen Forschung berücksichtigt.

Ebenfalls Ende der 1970er Jahre entfacht sich in der Schulforschung eine Debatte über Grenzen und Möglichkeiten qualitativer Methoden in Abgrenzung zu quantitativen Verfahren. Schon damals erklärt Radtke (1979) die „,chronische Bedeutungslosigkeit der Ergebnisse empirischer Schulforschung für die Gestaltung der Schulpraxis“" (Radtke 1979, S. 31). Er verweist darauf, dass durch die Generalisierbarkeit der Ergebnisse gleichzeitig das Subjekt ausgeschaltet werde.

Abschließend stellt Böhme in ihrer methodologischen Analyse qualitativer Methoden der Schulforschung eine Konzentration auf das Datenmaterial in Textform fest (Böhme 2008, S. 139). Dazu präsentiert sie einige gegenwärtig etablierte qualitative Methodenzugänge in der deutschsprachigen Schulforschung, wobei sie nicht zwischen Verfahren der Erhebung und der Auswertung unterscheidet: Objektive Hermeneutik, narrationsstrukturelles Verfahren, dokumentarische Methode und Ethnografie. Allerdings schlagen nicht immer alle Vertreter einer Erhebungsmethode zugleich eine passende Auswertungsmethode vor, wobei Passungsversuche angestrebt werden, was ich am Beispiel meines Forschungsprojekts zeigen möchte.

Für die vorliegende Studie habe ich die Daten mit problemzentrierten Interviews (vgl. Witzel 2000 und Kap. 5.2.1) erhoben, womit argumentative, beschreibende und erzählende Gesprächssequenzen die Basis bilden. Dabei richte ich in den Fragestellungen den Fokus auf Erzählungen aus dem Schulalltag, womit narrative Passagen generiert werden, die für die gewählte Auswertungsmethode vorausgesetzt werden. Andreas Witzel bietet als Begründer der problemzentrierten Methode in seinen mittlerweile klassischen Texten keine ausführlichen Hinweise für die Auswertungsmethode. In seiner Veröffentlichung von 2000 verweist er auf die Auswertungsmethode in Anlehnung an die Grounded Theory (vgl. Witzel 2000, Abs. 19ff.). Da jedoch Ehrlich- 
keitsorientierungen aus Alltagsrealitäten und nicht nur Ehrlichkeitskategorien aus den gewonnenen Daten rekonstruiert werden, eignet sich dazu die dokumentarische Methode besonders gut.

Zusammenfassend ermöglichen qualitative Interviewverfahren im Kontext der interpretativen Sozialforschung, Erziehungswirklichkeiten aus einer Tiefeninterpretation nachzuzeichnen und neben einer kommunikativen Ebene implizite Sinngehalte aus dem Datenmaterial zu gewinnen.

Eine scharfe Kritik an den qualitativen Interviewverfahren besagt, dass die Interviewten immer schon von dem beeinflusst seien, was der Forschende hören möchte (vgl. Atkinson und Silverman 1997). Diese Kritik ist sehr allgemein formuliert und vor dem Hintergrund zu entschärfen, dass im Interviewprozess offene Fragestellungen ausgesprochen werden und dem Interviewten die Möglichkeit gegeben wird, eigene Gewichtungen von Forschungsthemen vorzunehmen (vgl. bspw. narratives Interview bei Schütze 1987 oder problemzentriertes Interview bei Witzel 1982).

Eine weitere Grenze der Interviewführung bezieht sich auf die Sprachproblematik. Diese Kritik besagt, dass die Sichtweisen von Zielgruppen, die weniger ausdrucksstark sind, nicht gleichwertig zu erfassen sind (vgl. dazu frühe Soziolinguisten wie bspw. Bernstein 1980). Dieser Einwand kann durch eine adäquate Form der Interviewführung und mit verständlichen und nicht abschreckenden Fragen abgeschwächt werden. Zudem zeigt sich, dass Befragte mit einer knapperen Ausdrucksweise prägnanter und eindrücklicher Alltagserfahrungen versprachlichen (vgl. Kruse 2009, S. 103).

Soziale Wirklichkeiten und somit das Erfahrungswissen des Befragten sollen im Interviewgespräch erschlossen werden. In dieser Zielsetzung zeigt sich eine andere Grenze der Interviewmethode, die insbesondere von Harold Garfinkel vorgebracht wurde (vgl. ebd., S. 14ff.): Das Interviewgespräch ist kein natürliches Gespräch im Verhaltenskontext, sondern als künstliches Setting zu verstehen. Die Interviewsituation ist prägend für die Gesprächsführung, in der über den praktischen sozialen Sinn gesprochen wird. Dennoch bietet das Interviewgespräch einen ,sozialpraktischen Raum', in dem die Hervorbringung von Erfahrungswissen erklärlich gemacht werden kann. Entscheidend ist bei der Auswertung der Daten, dass die Aussagen nicht als rein sprachlich konstruierter Sinn gedeutet werden, sondern dass eine Differenzierung der Sinnebenen vorgenommen wird (vgl. Kap. 5.1.2).

Die Vielfalt der qualitativen Interviewmethoden zählt in der Forschungspraxis zu einer weiteren Herausforderung, weil damit keine scharfe Konturierung der einzelnen Methoden vollzogen werden kann. Daraus ergibt sich die Schwierigkeit, vergleichbare Kriterien $\mathrm{zu}$ finden, unter denen die Interviewmethoden systematisiert und differenziert werden können (Helfferich 2009, S. 35ff.). So bezieht sich beispielsweise die Bezeichnung ,problemzentriertes Interview“ (Witzel 2000) auf den Untersuchungsgegenstand. Dasselbe Interview kann als Leitfadeninterview (Schmidt 2010) eingeordnet 
werden, womit auf das Hilfsinstrumentarium des Leitfadens während des Interviewgesprächs hingewiesen wird. Und ebenso sinnvoll kann das problemzentrierte Interview als teilstrukturiertes Interview bezeichnet werden, womit auf die Strukturiertheit des Interviewverfahrens hingewiesen wird.

Kurz: Die begriffliche Unschärfe der Interviewmethoden fordert dazu auf, die verwendeten Erhebungsmethoden genau zu erklären (vgl. Kap. 5.2).

\subsection{Erhebungsmethode}

Qualitative Einzelinterviews sind Kommunikationen zwischen einem Interviewer und einem Interviewten, in denen die entscheidenden Daten ,in einer hochkomplexen und die Subjektivität der Beteiligten einbeziehenden Situation erzeugt" (Helfferich 2009, S. 9) werden. In demselben Sinne bezeichnen Mey und Mruck das Interview als ,soziales Arrangement' (Mey und Mruck 2007, S. 273f.). Der resultierende Text, ein Produkt des geführten Interviews, ist eine ,Ko-Konstruktion“ (Mecheril 2003, S. 32ff.). Die personelle und situationsabhängige Beeinflussung ist dabei offensichtlich und deshalb umso wichtiger zu reflektieren und zu kontrollieren (vgl. Helfferich 2009, S. 12).

Als gemeinsame Nenner qualitativer Interviewverfahren sind die Kommunikation und die Offenheit zu nennen (Helfferich 2009, S. 25). Hingegen reicht die Spannbreite von Interviewformen - wie bereits angedeutet - vom ununterbrochenen Erzählen bis zum Dialog zwischen Interviewten und Interviewern anhand von Nachfragen und Rückfragen (Helfferich 2009, S. 10). In der Vielfalt der Interviewformen (vgl. Übersicht in ebd., S. 36f. und Systematisierung ebd., S. 45; Mey und Mruck 2007, S. 256f.) drängt sich die Forderung auf, die ausgewählte Interviewform auf eine dem „Forschungsgegenstand angepasste Weise zu gestalten" (Helfferich 2009, S. 12).

Aus den genannten Gründen hängen die Qualität der Daten und die Auswertungsmöglichkeiten von der Auswahl der Interviewform und anschließend von der Qualität der Erhebungssituation ab (vgl. Kap. 5.4.3). Deshalb widmen sich die folgenden Ausführungen zunächst der getroffenen Auswahl der Erhebungsmethode.

\subsubsection{Problemzentriertes Interview mit Erzählsequenzen}

Das problemzentrierte Interview ist eine bewährte Interviewmethode in gegenwärtigen Forschungsprojekten, wobei ein Rückgriff auf die klassischen Texte (Witzel 1982, 1985) und methodologischen Hintergründe selten vorgenommen wird. Anders sollen hier die methodischen und methodologischen Voraussetzungen aufgezeigt werden. 
Das problemzentrierte Interview nach Andreas Witzel lässt sich auf die Sichtweise der Individuen ein, um ,subjektive Wahrnehmungen und Verarbeitungsweisen gesellschaftlicher Realitäten“" (Witzel 2000, Abs. 1) unvoreingenommen zu erfassen. Die subjektive Perspektive vom Untersuchungsgegenstand her gedacht, ein allgemeines Merkmal qualitativer Methoden, passt schon deshalb zum Forschungsinteresse der vorliegenden Arbeit, weil die subjektive Sichtweise der 14- und 15-Jährigen bezüglich ehrlicher Verhaltensweisen erfasst werden soll. Als ,gesellschaftliche Realitäten' sind die ehrlichen Verhaltensweisen im schulischen Umfeld zu verstehen, die unter dem Thema ,Tugendanforderung' oder ,Erziehungsauftrag ${ }^{6}$ im öffentlichen Kontext diskutiert werden.

Gleichermaßen ist die Unvoreingenommenheit im Forschungsinteresse zentral, um die Sichtweisen der Erziehenden möglichst ausgeschlossen zu halten. Dazu schlägt Witzel vor, den Wissenshintergrund des Forschers offen und systematisch darzustellen (Witzel 1985, S. 235), was ich in den ersten zwei Kapiteln dieser Arbeit umgesetzt habe (vgl. Kap. 2 und 3).

Das problemzentrierte Interview situiert Witzel eher in den theoriegenerierenden Verfahren der Grounded Theory, wobei er ein theoretisches „Vorwissen als Tabula rasa" (Witzel 2000, Abs. 3) ablehnt. Somit geht Witzel nicht von der Position des ,soziologischen Naturalismus' aus, der besagt, dass Forschende durch prinzipielle Offenheit dem Forschungsgegenstand gegenüberstehen. Indes schlägt Witzel vor, dass theoretisches Vorwissen anhand von sensitizing concepts (Blumer 1954 und vgl. Kap. 4) offenzulegen ist. Zudem grenzt er sein Verfahren bewusst gegen standardisierte Verfahren ab, da diese lediglich die im Vorfeld festgelegten Hypothesen überprüfen und nicht zur Theoriegewinnung beitragen. Vor diesem Hintergrund skizziert Witzel drei Prinzipien des pro $\neg$ blemzentrierten Interviews (Witzel 1982, S. $37 \mathrm{ff}$.$) :$

- Problemzentrierung

- Gegenstandsorientierung

- Prozessorientierung

Das Prinzip der Problemzentrierung zielt auf die Perspektive des Interviewten:

Sie [die Problemzentrierung] bietet dagegen die Möglichkeit, komplexe Vermittlungsprozesse von Handlungs- und Bewertungsmustern aufzudecken und in der Betonung der Sichtweise der Betroffenen deren Relevanzkriterien zu erfassen. Andererseits vermeiden sie auch die Beliebigkeit der Interviewfragen und damit zusammenhängende einseitige Materialorientiertheit der Alternativverfahren (Witzel 1982, S. 70).

Die Sichtweise der Schülerin und des Schülers (SuS) ist ein grundlegendes Kennzeichen des Untersuchungsgegenstands dieser Arbeit. Allerdings können, wie schon weiter oben erörtert, ehrliche Verhaltensweisen schwer direkt mit Heranwachsenden diskutiert werden, ohne von sozialen Erwünschtheiten 
beeinflusst zu sein. Zudem ist anzunehmen, dass die Forscherin zwar als anonyme Erwachsene und nicht als Lehrperson in positiver Weise wahrgenommen wird; allerdings nimmt sie den Status der Erwachsenen und nicht der Klassenkameradin wahr. Aus diesen Gründen ist teils Gehemmtheit, teils Offenheit seitens der Befragten zu erwarten. Diese Bedingungen habe ich reflektiert (vgl. Kap. 5.2.5) und in der Strukturierung des Leitfadens (vgl. Kap. 5.2.3) und in der Ausgestaltung des Interviewablaufs gezielt berücksichtigt (Kap. 5.4.3): Narrative Passagen habe ich anhand von Fragen gefördert, die auf Erzählungen und Beschreibungen von Schul- und Unterrichtssituationen zielten. Zudem habe ich die direkten Fragen zum Thema Ehrlichkeit erst in einem zweiten Schritt gestellt.

Als zu untersuchendes ,Problem' ist in dieser Arbeit, wie bereits erwähnt, Ehrlichkeitspraxen von SuS in der Schule sowie im Unterricht und die daraus resultierenden gedeuteten Ehrlichkeitsregeln als eine gesellschaftliche Tugend zu verstehen. Das Thema der Erziehung, welches ebenfalls ein zu untersuchendes Problem ist, verstehe ich als Hintergrundthema zur Ehrlichkeit.

Als zweites Prinzip nennt Witzel die Gegenstandsorientierung. Die Methode soll sich an den Anforderungen des Gegenstands orientieren und sich daraus entwickeln (vgl. Witzel 1985, S. 232ff.). Damit verweist Witzel auf den flexiblen Umgang mit narrativen Elementen und der Dialogform im Interview (ebd., S. 232). Insbesondere in Interviews mit Jugendlichen ist der flexible Umgang mit dem zuvor erarbeiteten Leitfaden zentral: Die Erzählbereitschaft kann andernfalls durch das Festhalten an vorgefertigten Fragen blockiert werden (mehr dazu in Kap. 5.2.3).

Das letztgenannte Kriterium, die Prozessorientierung, bezieht sich auf Erkenntnisgewinnung, welche direkt am gewonnen Material ansetzt und damit Affinitäten zum Ansatz der Grounded Theory (vgl. Glaser 1978) aufweist:

Es geht um die flexible Analyse des wissenschaftlichen Problemfeldes, eine schrittweise Gewinnung und Prüfung von Daten, wobei Zusammenhang und Beschaffenheit der einzelnen Elemente sich erst langsam und im ständigen reflexiven Bezug auf die dabei verwandten Methoden herausschälen. Entsprechend müssen Methoden entwickelt bzw. verschiedene Methodenelemente integriert werden, die diese gewährleisten. [...] So betrifft das Kriterium der Prozessorientierung neben der Gesamtgestaltung des Forschungsablaufs und der Entwicklung des kommunikativen Austauschs im Interview auch den Aspekt der Entwicklung des Verstehensprozesses im Interview bis hin zur kontrollierten Absicherung und Erweiterung der Interpretation im wissenschaftlichen Kontext. Des weiteren bezieht sich das Kriterium der Prozessorientierung auf den Untersuchungsgegenstand selbst (Witzel 1982, S. 71).

Das Kriterium der Prozessorientierung betrifft verschiedene Ebenen, die ich in Bezug auf die vorliegende Arbeit reflektiere und skizziere:

a) den Forschungsprozess 
b) den Interviewprozess

c) den Verstehensprozess im Interview selbst und im Theoriebildungsprozess

a) Hinweise auf den Forschungsprozess in der vorliegenden Arbeit habe ich weiter oben bereits gegeben, und ich werde im Kapitel 5.4 weiter darauf eingehen.

b) Wie bereits oben nehme ich die Möglichkeit in Anspruch, die Methode des problemzentrierten Interviews auf gezielte narrativen Passagen auszuweiten, um mich dem Untersuchungsgegenstand anzunähern, der von äußeren sozialen Erwartungen und Regeln geprägt ist.

c) Mein Vorgehen lehnt sich an das verstehende Vorgehen in der Interviewführung sowie in der Theoriebildung an, wozu die einzusetzenden Methoden dem Forschungsgegenstand angepasst werden. Jedoch beanspruche ich mit der Untersuchung nicht, einen theoretischen Ehrlichkeitsansatz präsentieren zu können. Vielmehr liefere ich wichtiges Material für zukünftige Erklärungsmodelle.

\subsubsection{Dokumentation des problemzentrierten Interviews}

Die Dokumentation des problemzentrierten Interviews ist insofern zentral, als an dieser das Auswertungsverfahren anknüpft und somit durch die Dokumentation vielfältige und theoriebildende Elemente geliefert werden. Die Erhebungsmethode des problemzentrierten Interviews bietet vier konkrete Erhebungsinstrumentarien an (Witzel 1985, S. 235ff.):

a) Kurzfragebogen

b) Leitfaden

c) Aufnahme des Interviewgesprächs

d) Postskriptum

a) Den Kurzfragebogen habe ich zu Datenblatt umbenannt und umfunktioniert, in dem ich neben sozialdemografischen Daten einige Angaben zu Freizeit, Schule sowie Teilnahmebereitschaft der SuS erfragte (s. Anhang A). Die im Datenblatt gewonnenen Informationen geben für die soziokulturelle, ökonomische und geografische Kontrastierung wichtige Hinweise, die bei der Auswahl der Teilnehmenden und für die Kontextualisierung in der Auswertung nützlich sind.

b) Auf den Leitfaden gehe ich später ein (Kap. 5.2.3).

c) Im Zusammenhang mit der Aufnahme der Gespräche, die ich digital festhielt, unterschrieben sowohl Interviewte als auch Interviewerin eine Vereinbarung (vgl. bspw. Arbeitsbündnis in Mey und Mruck 2007, S. 258f.). Darin 
sind die Interviewbedingungen in Form von Rechten und Pflichten beider Parteien schriftlich, transparent und verbindlich festgehalten - beispielsweise freiwillige Teilnahme, Sicherung der Anonymität oder die Möglichkeit, innerhalb einer bestimmten Frist Textpassagen zu widerrufen. Eine Kopie der Interviewvereinbarung konnten die Teilnehmenden mitnehmen.

d) Während der ganzen Erhebungsphase führte ich ein Interviewtagebuch, in dem ich Beobachtungen außerhalb der Interviewsituation, eigene Reflexionen und Befindlichkeiten festhielt. Im Postskriptum hingegen thematisierte ich dieselben Aspekte, die sich aber allein auf die Interviewsituation bezogen. Besonders nach dem Ausschalten des Aufnahmegerätes kommt es vor, dass die Interviewten aufgrund einer gelasseneren Stimmung interessante Informationen und Anmerkungen zum Thema ergänzen (vgl. bspw. Mey und Mruck 2007, S. 267f.). Diese hielt ich im Postskriptum fest. Zudem schrieb ich das Postskriptum unmittelbar nach dem Interview, wenn möglich vor Ort, damit ich flüchtige Gedanken sofort festhalten konnte.

Zusammenfassend dokumentiere ich jedes Einzelinterview mit folgenden Materialien, die ich ausgehend von Witzels Vorschlag für meinen Untersuchungsgegenstand erweitert und angepasst habe:

- Datenblatt

- Interviewtagebuch

- Interviewvereinbarung

- Leitfaden

- Aufnahme bzw. vollständige Transkription des Interviewgesprächs

- Postskriptum

\subsubsection{Leitfaden}

Das problemzentrierte Interview wird dem Leitfadeninterview zugeordnet, weil Witzel den Leitfaden als konkretes Interviewinstrument sieht:

Der Leitfaden hat nicht die Aufgabe, ein Skelett für einen strukturierten Fragebogen abzugeben, sondern soll das Hintergrundwissen des Forschers thematisch organisieren, um zu einer kontrollierten und vergleichbaren Herangehensweise an den Forschungsgegenstand zu kommen (Witzel 1985, S. 236; Hervorh. i. O.).

Die zentrale Funktion des Leitfadens zielt somit auf die Systematisierung und Aufdeckung des Vorwissens ab. Dem stehen Grenzen des Leitfadens gegenüber: Informationen, die sich im Gesprächsablauf präsentieren und den Befragten blockieren können. Christel Hopf bezeichnet dieses Phänomen als ,Leitfadenbürokratie" (Hopf 1978 S. 101ff.) und weist auf zwei Informationsblockaden hin (ebd., S. 102): Themen können als ,irrelevant' ignoriert werden, die nicht im Leitfaden aufgeführt sind, die jedoch der Befragte einbringt. 
Zudem können Nachfragen auf Reaktionen der Befragten zugunsten einer ,zügigen ' Gesprächsführung ausgelassen werden. Eine Haltung des Abfragens nehmen oft Interviewanfänger ein, wenn beispielsweise der Interviewte wortkarg ist oder der Interviewer wenig mit der Interviewsituation oder mit dem Thema vertraut ist. Geübten Interviewern empfiehlt Witzel einen flexiblen Einsatz des Leitfadens (vgl. ebenso Kruse 2009, S. 64ff. oder Helfferich 2009, S. 180). Zudem erklärt er, wieso das Wort ,Leitfaden“ ein unzutreffender Begriff ist: weil nämlich ,,der Gesprächsfaden des Interviewten im Mittelpunkt des Interesses steht, der Leitfaden diesen lediglich als eine Art Hintergrundfolie begleitet"“ (Witzel 1985, S. 236; Hervorh. i. O.).

In diesem Sinne habe ich den Leitfaden (s. Anhang B) erstens als Checkliste verwendet, mit der ich auf zentrale Themen zurückgreifen konnte, um eine spätere Vergleichbarkeit der Daten zu ermöglichen. Zweitens hatte ich die Grundzüge des Leitfadens vor dem Interviewgesprächs internalisiert, was mir ermöglichte, auf Themen und den Gesprächsverlauf der Interviewten offen einzugehen. Und drittens gab mir der Leitfaden Anhaltspunkte, um bei Wortkargheit des Gegenübers das Gespräch weiterzuführen. Schließlich systematisierte und reflektierte ich mein Vorwissen anhand des Leitfadens. An dieser Stelle zeigen sich das ungelöste Problem und das Spannungsverhältnis zwischen Strukturierung und Offenheit des Leitfadens.

Für den von mir verwendeten Leitfaden wählte ich sechs Themenkreise aus, bei denen SuS offen Alltagswissen zu ehrlichem Verhalten mitteilen konnten und die genügend Raum boten für subjektive thematische Relevanzsetzungen sowie für eigene Themen. Die ersten fünf erfragten Themenkreise umfassen: Verhaltensweisen im Unterricht, Schreiben von Schultests, Umgang mit Elternunterschriften, Klassenzusammenhalt und Gerüchte. Diese Themen habe ich exemplarisch für den Leitfaden ausgewählt, weil es hier möglich ist, zur Beantwortung der Fragen nach Ehrlichkeit aus dem Alltagswissen zu schöpfen. Es gibt weitere Themenkreise, die die SuS selber ansprachen, wie beispielsweise das Hausaufgabenlösen. Der letzte Themenkreis des Interviewleitfadens umfasst die direkte Frage nach dem Ehrlichkeitsverständnis.

Der Leitfaden ist gemäss Witzels Vorgaben wie folgt aufzubauen (Witzel 1985, Abs. 14ff.):

- Einstiegsfrage

- verschiedene Themenbereiche, die anhand von Sondierungsfragen und Ad-hoc-Fragen vertieft werden

Die Einstiegsfrage zielt auf ein Thema, auf das der Befragte etwas zu erzählen weiß. Damit soll dieser sich im Berichten aufwärmen und die Rolle des ,Experten“ wahrnehmen. Als Einstiegsfrage stellte ich die retrospektive Frage, was die Schülerin oder der Schüler in der letzten Stunde gemacht habe. 
Die darauffolgenden Themenbereiche sind als allgemeine und offene Sondierungsfragen, quasi als Einleitungsfragen ins Thema und erzählgenerierende Kommunikationsstrategie zu verstehen. Hingegen zählen die spezifischen Sondierungsfragen zu den verständnisgenerierenden Kommunikationsstrategie, die wie die allgemeinen Sondierungsfragen offen formuliert sind. Dazu gehören nachdoppelnde Verständnisfragen, Zurückspielen oder Konfrontationen mit Äußerungen. Im verwendeten Leitfaden kamen die allgemeine Sondierungsfrage und die zwei ersten Formen der spezifischen Sondierung zum Einsatz. Schließlich setzte ich auch die Ad-hoc-Frage ein, die während der Interviewsituation zu formulieren ist. Wenn etwa der Interviewte ein bestimmtes Thema ausklammert, ist eine Ad-hoc-Frage angebracht. Im Erhebungsprozess setzte ich beispielsweise die Ad-hoc-Frage ein, wenn bei der Frage zum Ablauf von Prüfungssituationen kein Hinweis auf das Spicken gegeben wurde. Dann erkundigte ich mich zunächst nach auffälligen Situationen in Schultests. Und in einem letzten Schritt fragte ich nach dem Einsatz unerlaubter Hilfsmittel.

Vor dem Ende des Interviews wiederholte ich die zentralen Aussagen der SuS, damit sie auf mögliche fehlende Themen eingehen konnten. Einige Teilnehmende gaben daraufhin ein Schlussstatement.

\subsubsection{Interviewablauf}

Den idealen Leitfaden sowie den idealen Ablauf eines Interviews gibt es nicht, auch wenn diese in vielen Methodenbüchern ausführlich beschrieben werden (vgl. bspw. Witzel 1985; Mey und Mruck 2007; Reinders 2005a, S. $63 \mathrm{ff}$. und 176ff.; Helfferich 2009, S. 167ff.). Die aufgeführte Literatur gibt jedoch wichtige Anhaltspunkte für die Interviewplanung und -durchführung, die ich reflektiert und an denen ich mich während des Forschungsprozesses orientiert habe. Aus pragmatischen Gründen habe ich mein Vorgehen stichwortartig in einem Interviewdossier zusammengefasst, das ich vor jedem Interview vergegenwärtigte.

\subsubsection{Rolle der Interviewenden}

Da man „die soziale Welt nicht erforschen kann, ohne selbst Teil davon zu sein" (Przyborski und Wohlrab-Sahr 2009, S. 58), worauf bereits die Ethnologen Hammersley und Athkinson 1983 hinwiesen (zit. in ebd.), ist es besonders wichtig, die Rolle der Interviewenden zu reflektieren. Anhand von zwei extremen Grundhaltungen der Forschenden lässt sich das Spannungsfeld in Interviewgesprächen umschreiben. Zum einen können die nötigen Kompetenzen, um ein Interview erfolgreich zu führen, als etwas intuitiv Erworbenes 
verstanden werden oder als etwas, das sich in der Interviewsituation ergibt. Dazu bemerkt Helfferich:

Es reicht nicht, auf Alltagskompetenz zu vertrauen oder darauf, dass sich Kompetenzprobleme ,mit der Narration sozusagen von selbst erledigen“ (Hopf 1995, S. 181) - wie schlecht geführte Interviews und Interviewfehler zeigen (Helfferich 2009, S. 11).

Zum anderen werden widersprüchliche Erwartungen an die Interviewenden gestellt: dass sie im Interview zwischen Offenheit und strukturierenden Vorgaben sowie zwischen Vertrautheit und Fremdheit zu vermitteln fähig sein sollen (vgl. ebd., S. 11). Dabei werden Interviewende schnell überfordert ,in dem Sinne, dass von ihnen allein die Lösung von strukturellen Spannungsverhältnissen verlangt wird, die allgemein konstitutiv für qualitative Verfahren sind" (ebd., S. 11; Hervorh. E. C.).

Es kann mit Helfferich festgehalten werden (vgl. ebd., S. 11f.), dass unterschiedliche Interviewformen unterschiedliche Kompetenzen fordern. Für narrative Interviews seien besonders die Fähigkeit des Zuhörens, Signale des aktiven Zuhörens und eine angemessene Intervieweröffnung gefragt, damit das Erzählen einsetzen kann. Hingegen erfordern es Leitfadeninterviews, unter Zeitdruck spontan die passende Frageformulierung zu finden, wobei gelernt werden muss, mit der eigenen selektiven Aufmerksamkeit und den eigenen Erwartungen umzugehen (vgl. Witzel 1982, S. 91).

Meine Interviewführung orientierte ich am zentralen Ziel einer Haltung, die Helfferich wie folgt definiert: Dem Interviewten soll

eine grundlegende Haltung der Offenheit für Fremdes, der Zurückstellung der eigenen Deutungen und der Selbstreflexion sowie schlicht und einfach die Fähigkeit zum Zuhören (eine Fähigkeit, die uns im Alltag weitgehend verloren gegangen ist) zu vermitteln (Helfferich 2009, S. 12).

Vor allem das Zuhören und das Ertragen von Denkpausen lernte ich während der Interviews bewusst einzusetzen. Bei Letzteren nutzte ich die Eselsbrücke, dass ich bei Sprechpausen der SuS innerlich bis zehn zählte. Das war i. d. R. zielführend (bis auf einen Fall, in dem Clemens erklärte, es störe ihn, dass ich so lange Pause mache, und fragte, ob ich schneller die nächste Frage stellen könne).

Besondere Aufmerksamkeit richtete ich auf die Frageformulierungen, indem ich offene Fragewörter einsetzte. Die Nachfragen formulierte ich zulasten meines eigenen Verständnisses, um die Expertenrolle der SuS zu wahren (vgl. Reinders 2005a, S. 64). Im Weiteren sind anhand des Leitfadens die Fragestellungen eingehend reflektiert, wobei in der Interviewpraxis mit vielen Eindrücken und Einflüssen umgegangen werden muss und zudem eine natürliche Interviewsituation aufrechterhalten werden soll. Daher versuchte ich bewusst, vor Ort Suggestivfragen oder geschlossene Fragen zu vermeiden. Ausnahme waren Situationen, in denen ich solche bewusst und begründet einsetzte: als es um spezifische Sondierungsfragen ging. Die so verwen- 
deten Suggestivfragen und geschlossenen Fragen werden nach Helfferich nicht als Interviewfehler bezeichnet (Helfferich 2009, S. 12f.). Auf diese Weise habe ich auffallende Frageformulierungen in der Analyse der Transkriptionstexte markiert, um diese in der Interpretation zu berücksichtigen.

Das problemzentrierte Interview, das ebenso als Leitfadeninterview oder teilstrukturierte Interviewmethode bezeichnet wird, zeigte dieses Unterkapitel im Hinblick auf den Forschungsgegenstands auf. Für die vorliegende Forschungsarbeit ist über die originäre Erhebungsmethode ein besonderer Fokus auf Erzählsequenzen gesetzt, um das Alltagswissen in seinen Facetten möglichst komplex zu erfassen. Weiter sind die Dokumentationsformen auf den Forschungsgegenstand erweitert worden. Schließlich sind die methodischen Reflexionen zum Leitfaden und zur Rolle der Interviewenden offen gelegt, um die intersubjektive Nachvollziehbarkeit des Erhebungsinstruments zu bewahren. Gleichzeitig konnte in diesem Unterkapitel die Angemessenheit der gewählten und modellierten Erhebungsmethode zum Forschungsgegenstand sichergestellt werden.

\subsection{Auswertungsmethode}

Die dokumentarische Methode geht ähnlich wie das Erhebungsverfahren der problemzentrierten Interviews von der Abgrenzung zu einer naturwissenschaftlichen Methodologie aus. Die Ursprünge gehen auf Karl Mannheim zurück, der bereits in den 1920er Jahren die ,dokumentarische Methode" als eine Methode der ,Weltanschauungsinterpretation' ausarbeitete und als sozialwissenschaftlich relevante Hermeneutik deklarierte (Bohnsack 2003, S. 550). Nach über dreißigjähriger Vergessenheit griffen die Ethnomethodologen, insbesondere Harold Garfinkel (1967), diesen Ansatz in ihren methodologischen Begründungen auf. Leider führt Garfinkel die Kommunikation auf der metakommunikativen Ebene nicht weiter aus, womit der Erklärungszusammenhang des Gesagten aufzuklären bleibt (vgl. Bohnsack 2008, S. 59; Przyborski und Wohlrab-Sahr 2009, S. 28f.). Die Frage „Wie geht es seiner Freundin?“ - um ein Beispiel von Garfinkel (Garfinkel 1967, S. 42f.) zu nennen - ist nicht wortwörtlich aufzufassen. Vielmehr ist sie in den Kontext von Höflichkeitsregeln zu stellen. Gerade diese zum Tragen kommenden Regeln der Kommunikation sollen aufgedeckt werden, was Ralf Bohnsack in den 1980er Jahren vornahm, als er die Anwendung der dokumentarischen Methoden fokussierte. Er wendete die dokumentarische Methode auf Gruppendiskussionen in Jugendforschungsprojekten an und entwickelte diese weiter, was zu einer breiten Anwendung bis heute führte. Inzwischen zählen zu den klassischen Erhebungsinstrumenten die Gruppendiskussion, die teil- 
nehmende Beobachtung und die Interviewführung (vgl. dazu Przyborski und Wohlrab-Sahr 2009, S. 273ff. oder Nohl 2008).

\subsubsection{Kommunikatives Wissen (Was-Frage) und atheoretisches Wissen (Wie-Frage)}

Die dokumentarische Methode verortet sich theoretisch zwischen einer subjektivistischen und einer objektivistischen Herangehensweise. Erstere wird üblicherweise der qualitativen Forschung und Letztere der quantitativen Forschung zugeschrieben. Dazwischen steht die dokumentarische Methode entsprechend in der Funktion einer „Vermittlung zwischen Konstruktivismus und Objektivismus“ (Bohnsack 2003, S. 563). Dazu schlägt Bohnsack eine ,methodische Fremdheitshaltung' (Bohnsack 2007, S. 12) in der Tradition von Karl Mannheim vor: Weder ein deduktiver noch eine induktiver Erkenntniszugang, sondern ein abduktiver Erkenntniszugang wird gewählt. Die Aufgabe des Beobachters besteht dann darin, ,ein den Erforschten bekanntes, von ihnen aber selbst nicht expliziertes handlungsleitendes (Regel-) Wissen (abduktiv) zur Explikation zu bringen" (ebd.). In dieser Fremdheitshaltung geht es nicht darum, dass der Forschende sein theoretisches Relevanzsystem zurückstellt, sondern dass er es zurücknimmt. Auf diese Weise geht der Forschende theoretisch sensibilisiert vor (vgl. dazu sensitizing concept in Kap. 4).

Im Zusammenhang mit Fremdverstehen in der Tradition von Alfred Schütz unterscheidet Karl Mannheim mit einer anderen Nomenklatur zwischen immanenten Sinngehalten und dokumentarischen Sinngehalten. Immanenter Sinngehalt bezieht sich auf kommunikativ-generalisierendes Wissen, dem eine öffentliche und gesellschaftliche Bedeutung zukommt und das daher als ,Common-Sense-Konstruktion“ und als ,Rollenerwartung' bezeichnet wird (Bohnsack 2003, S. 562). Kurz: Es geht um ein kommunikatives Wissen, das deskriptiv zu erfassen ist. Damit kann die Frage nach dem Was beantwortet werden.

Demgegenüber zielt der dokumentarische Sinngehalt auf ein , atheoretisches Wissen' (Mannheim 1964, S. 100ff.). In anderen Theorietraditionen spricht man vom ,impliziten Wissen“ (Polanyi 1985). Damit ist ein Wissen gemeint, über das in der Handlungspraxis verfügt wird, das aber im Alltag nicht erklärt werden muss. Das atheoretische Wissen ist somit erkenntnislogisch auf einen milieuspezifischen oder individuell-spezifischen Eigensinn zurückzuführen. Der dokumentarische Sinn ist als ,handlungsleitendes Wissen' oder ,inkorporiertes Erfahrungswissen' zu verstehen (Begrifflichkeiten der Habitustheorien aufgeführt unter Przyborski und Wohlrab-Sahr 2009, S. 275). Er beruht auf kollektivem Wissen und greift folglich auf einen kollektiven Handlungsraum zurück, den Mannheim als ,konjunktive Erfahrungs- 
räume' (Mannheim und Kettler 1980, S. 100) bezeichnet. Es geht um ein unmittelbares Verstehen (und nicht um ein Interpretieren) aufgrund wechselseitig gesellschaftlich bedingter Kriterien. Der kollektive Sinn liegt zwar im Akteur, ist aber nicht als subjektiver Sinn, sondern als Herstellungssinn zu verstehen. Vor diesem Hintergrund hat der Forschende keinen Wissensvorsprung gegenüber dem Akteur, sondern einen Zugang zum impliziten Erfahrungswissen, dessen sich der Akteur nicht bewusst ist. Bohnsack drückt das treffend aus, dass die Interpretierende nicht davon ausgehen, dass die Forschenden

mehr wissen als die Akteure oder Akteurinnen, sondern davon, dass letztere selbst nicht wissen, was sie da eigentlich alles wissen, somit also über ein implizites Wissen verfügen, welches ihnen reflexiv nicht so ohne weiteres zugänglich ist (Bohnsack 2007, S. 11; Hervorh. i. O.)

Kurz: Mit dem dokumentarischen Sinn kann die Frage nach dem Wie und Wozu beantwortet werden (Bohnsack 2003, S. 560f.).

In Anlehnung an Mannheim ist zu unterscheiden, „was kulturelle oder gesellschaftliche Phänomene oder Tatsachen sind" (Bohnsack 2003, S. 556, Hervorh. i. O) und ,wie diese hergestellt werden“ (ebd., Hervorh. i. O.). Einen Überblick zu den verschiedenen Sinngehalten, der empirischen Erfassbarkeit, den Interpretationsschritten und den passenden Fragestellungen gibt Tabelle 5 .

Tabelle 5: Sinngehalte, empirische Erfassbarkeit, Interpretationsschritte und passende Fragestellungen

\begin{tabular}{|l|l|l|l|}
\hline Sinngehalt & & Empirische Erfassbarkeit & Interpretationsschritte \\
\hline $\begin{array}{l}\text { immanenter } \\
\text { Sinngehalt }\end{array}$ & $\begin{array}{l}\text { Intentionale } \\
\text { Ausdrucksform }\end{array}$ & nicht erfassbar & keine \\
\cline { 2 - 4 } & objektiver Sinn & thematisch zu identifizieren & $\begin{array}{l}\text { formulierende Interpretation, } \\
\text { Frage nach dem Was }\end{array}$ \\
\hline \multicolumn{2}{|l|}{ dokumentarischer Sinngehalt } & $\begin{array}{l}\text { anhand des Herstellungs- } \\
\text { prozesses zu rekonstruieren }\end{array}$ & $\begin{array}{l}\text { reflexive Interpretation, } \\
\text { Frage nach dem Wie und } \\
\text { Wozu }\end{array}$ \\
\hline
\end{tabular}

Quelle: erweiterte Darstellung zu Nohl 2008, S. 9

Diese methodologischen Überlegungen finden in der Forschungspraxis in zwei Interpretationsschritten ihren Ausdruck: der formulierenden Interpretation und der reflektierenden Interpretation, die Bohnsack wie folgt voneinander unterscheidet: „In diesem Sinne geht es darum, das, was (wörtlich) gesagt wird, also das, was thematisch behandelt wird, von dem zu unterscheiden, wie ein Thema, d. h. in welchem Rahmen es behandelt wird" (Bohnsack 2003, S. 263; Hervorh. i. O.).

Innerhalb der zwei Interpretationsschritte nimmt der Forscher vorerst eine Perspektive des erforschten Akteurs ein. Von dieser Position fasst er das 
zusammen, was der Akteur verbal formuliert. Damit ist das, „was von den Akteuren im Forschungsfeld bereits selbst interpretiert, also begrifflich expliziert wurde, noch einmal zusammenfassend ,formuliert"“(ebd.). AnschlieBend wechselt der Forscher die Perspektive und nimmt die Position des Beobachters ein, indem er sequenziell den Text analysiert. Anders als die objektive Hermeneutik ist ,die empirisch gegebene Relation von empirisch beobachteter Äußerung und empirisch beobachteter Reaktion bzw. Nachfolgeäußerung oder -handlung der Ausgangspunkt der Interpretation“ (ebd., S. 265).

Die dokumentarische Methode analysiert eine Sinneinheit im Text nach der anderen, was als Sequenzanalyse auf der Bedeutungsebene zu bezeichnen ist. Gleichzeitig setzt eine fallübergreifende Suche nach empirischen Äußerungen oder Reaktionen ein, die von der Funktion her äquivalent oder homo$\log$ sind. Diesen Analysevorgang nennt Bohnsack ,empirisch komparative Sequenzanalyse“ (Nohl 2008, S. 11f.).

Nach diesem Abriss der methodologischen Grundüberlegungen zur dokumentarischen Methode wird ausgeführt, wie diese Methode umgesetzt wird.

Methodische Umsetzung: Formulierende und reflektierende Interpretationsschritte sowie Darstellung der Ergebnisse

$\mathrm{Zu}$ den vorgeschlagenen Interpretationsstufen der dokumentarischen Methode zählen (Bohnsack et al. 2007; Przyborski und Wohlrab-Sahr 2009):

a) formulierende Interpretation

b) reflexive Interpretation

c) Typenbildung

Diese werden für den Forschungsgegenstand der vorliegenden Arbeit wie folgt angewendet:

a) Die formulierende Interpretation geht von einem thematischen Verlauf aus, nach dem Interviewausschnitte zum Transkribieren ausgewählt werden. Kriterien für die Auswahl sind erstens Relevanz zum Forschungsthema, zweitens ,Fokussierungsmetaphern', womit Themen gemeint sind, die die befragte Person besonders ausführt, die sie engagiert und metaphorisch äußert, und drittens Themen, die in unterschiedlichen Fällen ähnlich behandelt werden, womit eine empirisch-komparative Analyse möglich wird (vgl. Nohl 2008, S. 45ff.). Demgegenüber transkribierte ich alle 16 Interviews auf Schweizerdeutsch, um die Möglichkeit zu haben, auf die Interviewfälle immer wieder zurückzugreifen und auch andere, vielleicht anfänglich unbedeutende Textstellen in der Originalsprache zu berücksichtigen. Um einen thematischen Überblick über alle Fälle zu gewinnen, legte ich dennoch einen thematischen Verlauf aller Interviews fest, gemäss den drei oben genannten Auswahlkriterien der dokumentarischen Methode. Mit diesem Verlauf waren Interviewausschnitte zu identifizieren, die explizit mit ehrlichen oder unehrli- 
chen Verhaltensweisen zu tun haben, die ähnlich bei verschiedenen SuS vorkamen und die eine engagierte Erzähl- und Beschreibungsstruktur aufweisen. Diese Interviewabschnitte habe ich gemäss der formulierenden Feininterpretation nach Bohnsack analysiert, womit eine Antwort auf die WasFrage zum kommunikativen Gehalt gegeben wurde.

b) Auf der reflexiven Interpretationsstufe habe ich zunächst, wie der vorgeschlagene erste Schritt besagt, die Textabschnitte nach formalen Textsorten unterteilt: Erzählung, Beschreibung und Argumentation. Diesen Interpretationsschritt bezeichnet Nohl als ,formale Interpretation mit Textsortentrennung' (Nohl 2008, S. 45). Daran knüpft die semantische Interpretation an. Die Autoren schlagen vor, anhand der Wie-Frage das Herstellungswissen des Gesagten zu erfassen. Demnach stellte ich folgende Frage an den Text: Wie bzw. auf welche Art und Weise wird Ehrlichkeit thematisiert? Insbesondere für den reflexiven Interpretationsschritt sind formale und semantische Aspekte zentral und miteinander verschränkt. Denn die formale Ebene gibt Auskunft über die Relevanz der Bedeutungsebene. Deshalb unterzog ich die Textstellen zunächst der formalen Analyse und dann der semantische Analyse. Fokussiert habe ich in Anlehnung an die dokumentarische Methode die Abschnitte mit alltäglichen Erzählungen, weil diese wegen der Dynamik und der Zugzwänge des Erzählens (vgl. Schütze 1978 und zusammengefasst in Nohl 2008, S. 29) besonders nahe an den Erfahrungen des Erzählers liegen. Nicht zu übersehen ist, dass die Erzählungen eng mit Beschreibungen und Argumentationen verknüpft sind, was die oberen Autoren auch vermerken.

Aufgrund der erzählgenerierenden Fragen im Leitfaden finden sich in den erhobenen 16 Interviews zahlreiche alltägliche Erzählungen, die ich gemäss der beschriebenen Interpretationsschritten empirisch-komparativ analysiert habe. An dieser Stelle ist der Qualitätsstandard der Passung der Erhebungs- und Auswertungsmethode klar ersichtlich und erfüllt. Denn nur anhand problemzentrierter Interviews mit Erzählsequenzen ist eine Textanalyse in Anlehnung an die dokumentarische Methode möglich.

c) Es macht wenig Sinn, aus Ehrlichkeitsorientierungen Typen zu bilden: Ehrlichkeitsorientierungen sind situationsbedingt und sehr variabel - und könnten daher nur auf unbefriedigende Weise in Typen gedrängt werden. Daher habe ich eine andere Möglichkeit gesucht, ausgehend von den Funden im Datenmaterial die Ergebnisse darzustellen. Es kristallisierten sich einerseits fallübergreifende Regelmäßigkeiten im Umgang mit Ehrlichkeit heraus, welche ich als ,Ehrlichkeitsregeln' benannt habe, die bei wiederholtem Vorkommen bei derselben Person als fallimmanent und bei mehreren Personen als fallübergreifend bezeichnet wird. Andererseits präsentierten sich Erzählungen von Situationen, in denen die SuS von Dilemmas berichteten, die sich aus unterschiedlichen Regeln ergeben. Die Ehrlichkeitsregeln in Dilemmasituationen untersuchte ich fallimmanent. Daraus ergab sich auch die Struktur, 
um die Analyseergebnisse vorzustellen: Konventionelle und unkonventionelle Ehrlichkeitsregeln (vgl. Kap. 7, 8) und Ehrlichkeitsregeln in realen Dilemmasituationen (vgl. Kap. 9).

\subsubsection{Zusammenfassung der Interpretationsschritte}

Zusammenfassend halte ich stichwortartig die Interpretationsschritte fest, an denen ich mich in der Textanalyse aller 16 Interviews orientierte:

a) formulierende Interpretation:

- vollständige Transkription der 16 Interviews

- thematischer Verlauf über alle 16 Interviews

- formulierende Feininterpretation gezielter Interviewausschnitte (Frage nach dem Was)

b) reflexive Interpretation:

- formale Interpretation mit Textsortentrennung

- semantische Interpretation mit komparativer Sequenzanalyse (Frage nach dem Wie und nach dem Wozu)

c) Darstellung der Ergebnisse:

- fallübergreifende Ehrlichkeitsregeln und fallimmanente Ehrlichkeitsregeln in realen Dilemmasituationen

\subsection{Forschungspraktisches Vorgehen}

Dieses Unterkapitel richtet sich darauf, wie ich die gewählten Forschungsmethoden im forschungspraktischen Vorgehen reflektiert habe. Damit situiere ich die gewonnenen Daten in einem forschungspraktischen Rahmen. Zuerst bette ich die Forschungsergebnisse, die in dieser Arbeit vorgestellt werden, in einen größeren Forschungsprozess ein (Kap. 5.4.1). Weiter zeige ich auf, nach welchen Kriterien ich die SuS ausgewählt habe. Zudem stelle ich anhand von zusammenfassenden Informationen alle $16 \mathrm{SuS}$ vor, um deren kontrastierende soziokulturelle Hintergründe aufzuzeigen (Kap. 5.4.2). Die Umsetzung der Erhebungs- und Auswertungsmethode bezogen auf meinen Forschungsgegenstand habe ich bereits in Kapitel 5.2 und 5.3 erwähnt. An dieser Stelle halte ich für das Erhebungs- und Auswertungsverfahren (vgl. Kap. 5.4.3, Kap. 5.4.4) das gewonnene Erfahrungswissen fest. Denn die Forschungspraxis bildet einen ebenso relevanten Rahmen der gewonnenen Daten wie die methodologischen sowie methodischen Reflexionen und wird deshalb hier berücksichtigt. Zudem erfülle ich mit der offenen Darstellung des For- 
schungsvorgehens einen weiteren Qualitätsstandard des qualitativen Forschungszugangs im Feld.

\subsubsection{Drei Feldphasen}

Mit dem Forschungsprojekt sind drei Erhebungsphasen verbunden, die sich aus der ,zirkulären Forschungsstrategie“ theoretisch erklären lassen. „Das heißt, dass in den qualitativ angelegten Projekten eine bestimmte Aufeinanderfolge von Forschungsschritten idealerweise mehrmals durchlaufen wird“ (Bennewitz 2010, S. 48). Damit kann das Vorverständnis des Forschungsgegenstands sukzessive erweitert und präzisiert werden, womit der Forschungsprozess als ,entwicklungsoffen' bezeichnet wird (ebd., S. 49).

Entsprechend unterscheiden sich die drei Feldphasen meines Untersuchungsprojekts in der Variation und Präzisierung des Samplings, in der Erweiterung und Schärfung des eingesetzten Forschungsinstrumentariums und in der Hinzunahme von weiteren Erhebungs- sowie Auswertungsmethoden, die sich aus den Forschungserkenntnissen ergeben. Angewendet auf die einzelnen Forschungsphasen heißt das:

Die Ergebnisse der ersten Feldforschung (März-Juni 2008) umfassen drei leistungsstufenübergreifende 3. Oberstufenklassen (Schultyp A, B, C) eines städtischen Schulhauses in Zürich. Mit acht SuS sowie mit drei Klassenlehrpersonen führte ich Einzelinterviews durch (Witzel 2000). In der zweiten Erhebungsphase (Juni-Juli 2009) untersuchte ich zwei sozial kontrastierende Schulhäuser in der Stadt und in der Agglomeration von Zürich. Dabei befragte ich sieben SuS in Einzelinterviews, 16 weitere SuS in zwei Gruppendiskussionen (Bohnsack et al. 2010) und zwei Klassenlehrpersonen in Einzelinterviews. Die Daten der ersten zwei Erhebungsphasen wertete ich nach der qualitativen Inhaltsanalyse in Anlehnung an Mayring aus (Mayring 2008), unterstützt vom Softwareprogramm MAXQDA (2010). Zu den zentralen Ergebnissen zählen sieben Ehrlichkeitsverständnisse (Chiapparini 2012).

Das Forschungsvorgehen der dritten Erhebungsphase (erste Hälfte des Schuljahrs 2009/2010) zielt darauf ab, über die Ehrlichkeitsverständnisse hinaus Ehrlichkeitsmuster zu erarbeiten. Damit können über die Einstellungen hinweg Aussagen über verhaltensorientierte Ehrlichkeitsregeln gewonnen werden, woraus wichtige Hinweise für die Erziehungspraxis sowie für die Debatten zu Tugenden und Erziehung gewonnen werden können.

Die vorangegangenen Unterkapitel (Kap. 5.1, 5.2, 5.3) bezogen sich insbesondere auf die Methodologie und Methode des dritten Forschungsprozesses. Was das Forschungsvorgehen im Feld betrifft, fasse ich anhand einiger Merkmale zusammen: Angesichts der sozialen Belastung und des StadtLand-Verhältnisses habe ich vier kontrastierende 3. Oberstufenklassen des Leistungstypus' A untersucht (vgl. Kap. 5.4.1). Pro Klasse befragte ich je- 
weils zwei Schülerinnen und zwei Schüler in Einzelinterviews, vier weitere Schülerinnen und vier Schüler in Gruppendiskussionen und die Klassenlehrpersonen sowie eine Lehrperson eines Nebenfaches in Einzelinterviews (vgl. Kap. 5.4.2 und Kap. 5.2). In den zwei ersten Forschungsprozessen konnte ich ein vielfältiges Ehrlichkeitsverständnis der SuS mittels problemzentrierter Interviews und Gruppendiskussionen ermitteln, weil die Aussagen anhand des kommunikativen Sinnes in Anlehnung an die Inhaltsanalyse ausgewertet wurden. Die vorliegende Studie zielt darüber hinaus darauf ab, das Erfahrungswissen bezüglich Ehrlichkeitspraxen von SuS zu erheben. Deshalb habe ich das Leitfadeninterview mit erzählgenerierenden Fragen ergänzt (vgl. Kap. 5.2) und die Daten in Anlehnung an die dokumentarische Methode ausgewertet (vgl. Kap. 5.3).

Die Interviews mit den Lehrpersonen und die Gruppendiskussionen sind aus zwei Gründen bis jetzt nicht ausgewertet: Nach einer ersten Sichtung der Interviewaussagen der Lehrpersonen stellte sich heraus, dass diese stark von einem erzieherischen Wunschdenken und von Rechtfertigungen ihres eigenen Verhaltens geprägt sind. Zudem haben die meisten Lehrpersonen eine begrenzte Einsicht in das, was in den einzelnen SuS, in der Klasse und im Klassenverband vor sich geht. Somit würde eine Analyse der Interviews mit Lehrpersonen auf unbefriedigende Weise über ehrliche Verhaltensweisen der SuS Auskunft geben. Dies ist eine Erkenntnis, die ich erst im Laufe des Forschungsprozesses gewonnen habe.

Zweitens sind die Gruppendiskussionen noch nicht ausgewertet, weil ich mich mit den Einzelinterviews vorerst dem individuellen und danach dem kollektiven Ehrlichkeitssinn in den Gruppendiskussionen widmen wollte. Allerdings entdeckte ich bereits im Auswertungsprozess der Einzelinterviews kollegiale und kollektive Ehrlichkeitsmuster. Da diese bereits substanzielle Ergebnisse für diese Forschungsarbeit lieferten und einen großen Analyseumfang erreichten, entschied ich mich, die Gruppendiskussionen zu einem späteren Zeitpunkt auszuwerten.

\subsubsection{Sampling}

An dieser Stelle beleuchte ich die Auswahlkriterien, mit denen ich die 16 SuS ausgesucht habe, und stelle zudem das Sampling anhand vergleichender Kurzportraits. Die Auswahl und Transparenz der Untersuchungseinheiten spielt im Zusammenhang mit der Frage nach der Verallgemeinerung der Befunde qualitativer Studien wie auch nach den Gütekriterien qualitativer Forschung eine zentrale Rolle (vgl. Przyborski und Wohlrab-Sahr 2009, S. 173). 
Der Forschungsgegenstand zielt auf Ehrlichkeitsregeln und Ehrlichkeitsmuster von $\mathrm{SuS}$, die einem Klassenverband und einem Schulkreis mit spezifischen sozialen Rahmenbedingungen angehören. Daher soll das Sampling die Klassendynamik sowie den sozialen Hintergrund berücksichtigen.

Der soziale Hintergrund kann anhand des statistischen Sozialindexes im Kanton Zürich bei der Auswahl von kontrastierenden Schulgemeinden in der Stadt und auf dem Land ermittelt werden. Der Sozialindex wird aus der Arbeitslosenquote, der Ausländerquote, dem Anteil an Eigentumshäusern und dem Sesshaftigkeitsquotienten errechnet und ist ausschlaggebend für die Höhe der öffentlichen Finanzierung der einzelnen Schulgemeinden. Die höchste soziale Belastung ist in den Agglomerationen der Stadt Zürich und in der Stadt Zürich selbst zu finden. Hingegen sind ländliche Schulgemeinden gering sozial belastet (vgl. Abb. 1).

Aufgrund dieses institutionellen Zugangs beruht das Sampling der befragten SuS auf einem gezielten Sampling bzw. auf einem „Sampling nach bestimmten, vorab festgelegten Kriterien“" (Przyborski und Wohlrab-Sahr 2009, S. 178). Auf diese Weise können Rahmenbedingungen wie soziale Belastung der Schulklassen und Stadt-Land-Verhältnisse kontrastierend berücksichtigt werden.

Abbildung 1: Sozialindex der Sekundarschulgemeinden des Kantons Zürich: $100=$ niedrige soziale Belastung, $120=$ hohe soziale Belastung

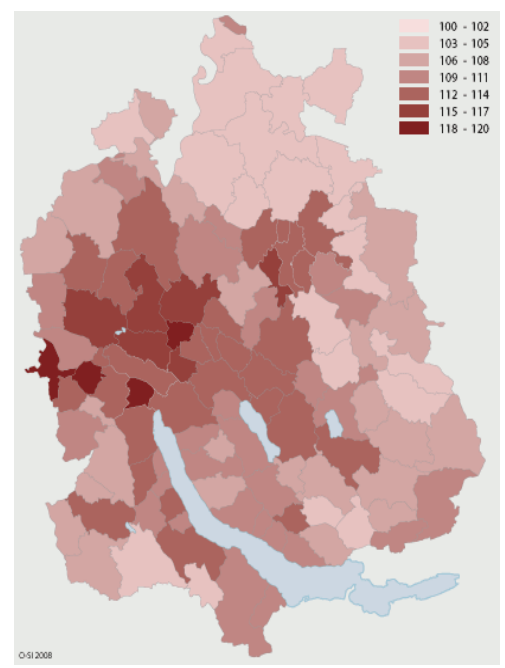

Quelle: Bista 30.10.2009 
Um verschiedene Perspektiven innerhalb der Klassen zu repräsentieren und um den Geschlechterausgleich zu sichern, sind vier Schüler pro Klasse ausgewählt worden. Daraus ergab sich, dass vier SuS aus einer Oberstufenklasse jeweils aufgrund der kontrastierenden Achse der sozialen Belastung und des Stadt-Land-Verhältnisses untersucht wurden: eine Klasse aus einer belasteten Agglomerationsgemeinde und eine aus einer kaum belasteten Schulgemeinde sowie eine Klasse aus einer stark belasteten und eine Klasse aus einer gering belasteten städtischen Schulgemeinde.

Die SuS habe ich bewusst aus dem letzten Schuljahr der obligatorischen Schulzeit ausgesucht, weil neue Studien auf die Wichtigkeit der Tugenden für die Berufsausbildung hinweisen (vgl. bspw. Neuenschwander et al. 2010), womit es umso relevanter ist aufzuzeigen, inwiefern Abschlussschülerinnen und -schüler über Tugendauffassungen verfügen, wie sie Ehrlichkeitspraxen deuten und nach welchen Ehrlichkeitsregeln sie sich orientieren. Das Durchschnittsalter der Befragten war 14 bis 15 Jahre, womit sie gemäss der Entwicklungspsychologie urteils- und handlungsfähig sind (Palentien und Hurrelmann 1998). Zudem ist gezielt die dritte Oberstufe ausgewählt worden, um eine noch wenig erforschte Altersgruppe bezüglich schulischer Erziehungsfragen $\mathrm{zu}$ untersuchen ${ }^{19}$ und somit das wenig zugänglich gemachte Erfahrungswissen zu erkunden, das auf mindestens acht Schuljahren basiert.

Wie bereits weiter oben aufgezeigt, beeinflusst die Leistungsstufe den erzieherischen Umgang mit Ehrlichkeit. Deshalb fokussiere ich absichtlich nur die Leistungsstufe A, um gezielt die Bedeutung von Ehrlichkeit innerhalb einer Leistungsstufe kontrastierend zur sozialen Belastung und zum StadtLand-Vergleich einzubeziehen. Befunde zu anderen Leistungsstufen können interessante Erkenntnisse liefern, dem in weiterführenden Studien nachzugehen ist.

In Schulgemeinden, in denen mehrere Oberstufenschulhäuser und mehrere Schulklassen vorhanden sind, wählte ich die Schulhäuser nach dem $\mathrm{Zu}$ fallsprinzip aus.

Das so angelegte Auswahlvorgehen unterscheidet sich vom Verfahren, das Witzel für das problemzentrierte Interview vorschlägt, der von einem theoretical sampling nach Glaser und Strauß ausgeht (Witzel 1982, S. 37, 78ff.). In deren Vorgehen wird das Sampling im Forschungsprozess fortwährend und aufgrund theoretischer Überlegungen festgelegt und nicht im Vorherein, wie ich es vornahm. Neben den bereits weiter oben erwähnten Gründen ist das gezielte Sampling insofern für meinen Forschungsgegenstand passender, als die populistischen Erziehungsdebatten entweder den soziokulturellen Hintergrund kaum berücksichtigen oder nur in einer pauschalen Art und Weise. Dazu zählt die verbreitete Meinung, dass ländliche und sozial

19 In der Literaturrecherche fällt auf, dass zu Erziehungsfragen des Vorschulalters oder in der Primarschule viel publiziert wird (vgl. zu demokratischen Werten und Normen bspw. Abendschön 2007). 
wenig belastete Schulgemeinden ebenso wenige schulische Erziehungsprobleme vorweisen. Um diese zusätzliche Differenzierung in die Ergebnisse einzubeziehen, habe ich das gezielte Sampling gewählt.

Bilanzierend steht das vorliegende Sampling für vier kontrastierende 3. Oberstufenklassen im Kanton Zürich, ausgehend von den Extremen, die die soziale Belastung als auch das Stadt-Land-Verhältnis betreffen.

\section{Informationen zu den Schülerinnen und Schülern}

Um die interviewten SuS in ihren Schul-, Freizeit- und Familienkontexten zu situieren, sind einige soziale, kulturelle und ökonomische Aspekte im Datenblatt erfragt worden. Die SuS der Klasse D (Lara, Valentina, Adrian und Clemens) kommen aus einem sozial belasteten Stadtschulhaus. Sie weisen unterschiedliche kulturelle Herkünfte auf: Lara und Adrian sprechen zu Hause Schweizerdeutsch. Valentina und Clemens sprechen zusätzlich noch andere Sprachen (Portugiesisch und Brasilianisch). In der Freizeit sind alle außer Valentina in einem oder mehreren Freizeitvereinen. Taschengeld erhalten sie zwischen 50 und 100 Franken im Monat. Drei planen, eine Lehre im Sommer zu absolvieren, wobei einer diese mit der Berufsmittelschule kombiniert; eine weiß noch nicht, was sie will. Sie geben an, dass sie eher gute Schulleistungen (in Deutsch und Mathematik) erbringen.

Aus der kontrastierenden Klasse E (Regula, Lara, Fabian und Marco) hat es zwei Lernende, die nur Schweizerdeutsch zu Hause sprechen, eine Französisch und einer Deutsch. Eine Schülerin und ein Schüler sind in Freizeitvereinen. Sie erhalten weniger Taschengeld im Monat als die SuS der Klasse D. Nur einer plant eine Lehre, und die anderen orientieren sich nach weiterführenden Schulen. Die Schulleistungen sind ebenso gut wie in der Klasse D.

Ilona, Claire, Christoph und Emil kommen aus einer Klasse in einer sozial stark belasteten Schulgemeinde in der Agglomeration. Die kulturelle Herkunft ist vielfältig. Keiner der SuS spricht Schweizerdeutsch zu Hause. Nur einer ist in einem Fußballverein. Zum Taschengeld geben zwei an, dass sie um die 30 Franken erhalten. Eine verdient nebenbei und erreicht eine verfügbare Geldsumme bis zu 400 Franken im Monat. Und einer erhält, was er braucht. Drei planen eine Lehre zu beginnen, davon einer mit Berufsmatura. Claire möchte eine weiterführende Schule absolvieren. Die Schulleistungen sind niedrig eingestuft und reichen von ,ungenügend ' bis zu ,befriedigend".

Die SuS der Klasse G (Sandra, Nora, Stefan und David) sprechen alle Schweizerdeutsch zu Hause und kommen daher aus einem homogenen kulturellen Hintergrund, was eine klare Kontrastierung zur vorherigen Klasse F sicherstellt. Ebenso ist die Klasse G in einer sozial kaum belasteten Schulgemeinde situiert. Alle besuchen Freizeitvereine, davon ist Nora sogar in drei Vereinen aktiv. Taschengeld erhalten sie zwischen 32 und 80 Franken. Alle werden eine Lehre beginnen, einer mit Berufsmatura. Im Vergleich zu den 
anderen Klassen haben diese vier SuS bereits einen Lehrvertrag. Die Schulnoten in Deutsch und Mathematik reichen von der Bewertung ,genügend' bis ,sehr gut .

Die ausgeübten Berufe der Eltern sowie die Anzahl der Geschwister zeigen vor allem, dass die SuS der sozial weniger belasteten Schulklassen (E und G) qualifiziertere Berufe ausüben und weniger Geschwister haben, womit der geplante kontrastierende Aspekt unterstrichen wird.

Zusammenfassend kann festgehalten werden, dass kontrastierende Klassen bezüglich sozialer Belastung und Stadt-Land-Verhältnisse mit dem Sampling tatsächlich erfasst sind. Darüber hinaus ist eine Kontrastierung der kulturellen Herkunft, der Teilnahme an Freizeitvereinen zu erkennen und an zugesicherten Lehrstellen festzustellen. Damit sind weitere Kontrastierungen erfasst, die parallel zur Gegenüberstellung von Stadt-Land-Verhältnissen und zur sozialen Belastung verlaufen.

\subsubsection{Erhebungsverfahren}

Die Beschreibung des Erhebungsverfahrens und des anschließenden Auswertungsverfahrens (vgl. Kap. 5.4.4) zählt einerseits zu den Qualitätsmerkmalen der qualitativen Sozialforschung. Andererseits wird hier besonderes Erfahrungswissen aus der Feldforschung geliefert. Denn eine Sache ist es, die theoretischen Ansätze zu reflektieren, und eine andere Sache, im Feld die reflektierten Vorgänge in einer alltagsangemessenen Weise umzusetzen, ohne sophistische Formulierungen $\mathrm{zu}$ verwenden, und dennoch reflektiert und nicht willkürlich zu forschen. Anschließende Reflexionen basieren auf dem ,Fahrplan' für die Rekrutierung und Erhebung, der Dokumentation von Rekrutierungsgesprächen und auf Interviewtagebüchern.

\section{Rekrutierung}

Allgemein bekannt ist, dass städtische, aber bereits auch ländliche Volksschulen im Kanton Zürich überforscht sind und restriktiv gegenüber Forschungsanfragen reagieren. Dies konnte ich bei den Kontaktaufnahmen mit den insgesamt acht Schulleitungen bestätigen.

Den ersten Feldzugang eröffnete mir ein Studienkollege. Es handelte sich um ein Oberstufenschulhaus in einer sozial belasteten städtischen Schulgemeinde. Während der Zehnuhrpause sprach ich im Lehrerzimmer die ersten drei Klassenlehrpersonen der 3. Oberstufe, die je eine Klasse aus dem Schultyp A, B und C unterrichteten, persönlich an, ob sie mit ihrer Klasse an einer Untersuchung zu Umgangsformen im Unterricht teilnehmen wollen. (Um unnötige Voreingenommenheit und soziale Erwartung bei den Befragten zu vermeiden, verwendete ich zunächst den allgemeinen Titel Umgangsformen 
im Unterricht für die Vorstellung meines Projekts.) Ich erhielt sofort von allen drei Lehrpersonen eine Zusage. Sie schätzten die unkonventionelle Anfrage sehr und fand das Forschungsthema interessant.

Auf dem großen Tisch im Lehrerzimmer lag ein unberührter Berg Fragebögen einer universitären Untersuchung. Dazu erfuhr ich, dass briefliche Zustellungen von Fragebögen zwei der drei angefragten Lehrpersonen aus Prinzip nicht beantworteten. Die meisten Lehrpersonen, die ich während der Feldforschung kennenlernte, klagten unaufgefordert über die vielen Anfragen von Forschenden und berichteten, dass eingehende Briefe, die ,nach Untersuchung aussehen“, ungeöffnet im Papierkorb landen können. Aber auch telefonische Anfragen sind nicht sehr willkommen. Auch dies erfuhr ich in der Praxis selbst: Ein Schulleiter wies mich vorerst ab, weil er im Durchschnitt wöchentlich zwei telefonische Anfragen von Forschenden erhalte.

Die Nennung der Auswahlkriterien für das jeweilige Schulhaus, die Wichtigkeit von Erziehungsthemen in der Schule, der geringe Zeitaufwand für die Lehrpersonen, die Einsicht in die anonymisierten Ergebnisse, die Empathie für die schulpolitische Unzufriedenheit der Lehrpersonen und ein gewisser Grad an Hartnäckigkeit in meinen Nachfragen führte dazu, dass mit einer Ausnahme alle angefragten Schulleiter innerhalb einer gewissen Zeitspanne positiv auf meine Anfragen antworteten. Anstelle des einen abgesagten Schulhauses suchte ich ein neues Oberstufenschulhaus mit den gleichen Auswahlkriterien aus.

Die Konzentrierung vieler Forschender auf das Untersuchungsfeld der Schule, wozu auch ich mich zähle, erklärt die anfänglichen Vorbehalte der Schulleiter und Lehrpersonen gegenüber meiner Untersuchung. Letztlich waren jedoch alle Lehrpersonen, die sich an der Studie beteiligten, sehr kooperativ und konstruktiv.

Anders als im oben beschriebenen ersten Feldzugang kontaktierte ich im zweiten und dritten Feldzugang die Schulleiter der ausgewählten Schulhäuser (vgl. Kap. 5.4.2) zuerst per E-Mail, dann telefonisch und falls nötig mit abklärenden Schulbesuchen. Damit holte ich in einem aufwändigen hierarchischen Verfahren die Informationen und die Einwilligung der Schulleiter für die Untersuchung ein. Einige Klassenlehrpersonen sind mir zugewiesen worden, andere rekrutierte ich mit Zustimmung des Schulleiters selbst. Die Klassenlehrpersonen bestätigten schriftlich ihre freiwillige Einwilligung in die Untersuchung.

Die SuS rekrutierte ich ausschließlich selbst, um nicht die Auswahl der SuS den Auswahlkriterien der Lehrperson zu überlassen, was zu verzerrenden Ergebnissen führen würde. Vielmehr setzte ich auf die freiwillige Teilnahme und das Teilnahmeinteresse der SuS. Mit dem Einverständnis der Lehrpersonen stellte ich mich und mein Vorhaben kurz am Rande einer regulären Unterrichtsstunde vor. Um die Freiheit der SuS zu wahren, war während dieser Zeit - mit einer Ausnahme - die Lehrperson abwesend. Die kon- 
krete Anfrage zur Teilnahme an die SuS sowie deren Einwilligung holte ich zusammen mit einem Datenblatt schriftlich ein (s. Anhang A). Üblicherweise dauerte die Rekrutierung rund zehn Minuten. Mit dieser ersten Kontaktaufnahme sahen die SuS mich und gewannen einen ersten Eindruck, mit wem sie das Interview führen werden.

Weiter hielten die SuS schriftlich ihre freiwillige Teilnahme an der Erhebung in einem Interviewvertrag fest, in dem auch ihre Rechte und Pflichten vermerkt sind. Die Eltern der Befragten informierte ich über die Erhebung schriftlich (passive Einwilligung). Dabei war keine schriftliche Einwilligung der Eltern einzuholen, weil die Jugendlichen bereits das urteilsfähige Alter erreicht haben und die Befragung innerhalb des Schulkontextes stattfindet.

Ich stellte fest, dass für Gruppendiskussionen eine größere Teilnahmebereitschaft seitens der SuS bestand als für Einzelinterviews. Für Letztere musste ich in einem sozial belasteten Schulhaus sogar in zwei weiteren Schulstunden an verschiedenen Tagen vorbeigehen, um die SuS für eine Teilnahme am Interview nachzufragen. Die Gehemmtheit der SuS erkläre ich mit diffusen Gründen, etwa dass sie sich nicht genau vorstellen konnten, was in einem Interview zu leisten ist. Hingegen war in demselben Schulhaus die Bereitschaft für die Gruppendiskussion so groß, dass ich sofort genügend Interessierte für zwei statt einer Gruppendiskussion gehabt hätte.

Falls sich mehr SuS meldeten, als das Forschungsdesign vorsieht, loste ich die Teilnehmenden aus, indem der Geschlechterausgleich berücksichtigt wurde. Das kam in zwei von vier Klassen vor.

\section{Forschungsethik und Datenschutz}

Meiner Forschungsarbeit liegt eine Forschungsethik (vgl. bspw. Helfferich 2009, S. 190ff.) zugrunde, welche sich auf das Datenschutzgesetz des Kantons Zürich (Gesetz über die Information und den Datenschutz IDG vom 12. Februar 2007, 2011) bezieht. Dessen praktische Einhaltung ist erstens durch die Zusicherung des Datengeheimnisses und der Anonymisierung während der schriftlichen Rekrutierung den Eltern, Teilnehmenden und Klassenlehrern gewährt und während der Einzelbefragung über die Interviewvereinbarung wiederholt worden.

Zweitens ist Anonymität durch Vergabe von Decknamen an die Interviewten oder von diesen genannten Personen und Orten gewährt. Zudem habe ich keine spezifischen Merkmalkombinationen gewählt, die Rückschlüsse auf einzelne Schülerinnen oder Schüler und Klassen zulassen. Ferner ist das Trenn- und Löschungsgebot befolgt, indem die Kontaktdaten und die Transkripte an unterschiedlichen Orten aufbewahrt und Erstere nach Beendigung der Transkriptionsphase zerstört wurden. (Nur eine Liste mit Personen, die an den Forschungsergebnissen interessiert sind, habe ich aufbewahrt, um ihnen später die Ergebnisse übermitteln zu können.) Schließlich 
arbeitet das Verstreichen der Zeit zugunsten der Anonymisierung: Spuren, die eine Rückführung auf einzelne Personen ermöglichen könnten, werden mit der Zeit und durch den Wechsel in Berufs- oder weiterführende Schulen verwischt.

\section{Interviewdurchführung}

Die Interviews dauerten zwischen 30 und 60 Minuten und konnten mit Ausnahme der Klasse F parallel zum Unterricht gehalten werden. Die SuS der Klasse F nahmen außerhalb der Unterrichtszeit an den Interviews teil. Alle Interviews habe ich auf expliziten Wunsch der einzelnen Teilnehmenden in einem abgeschlossenen Gruppenraum oder in der abgeschlossenen Bibliothek der Schule und nicht an einem außerschulischen Ort wie etwa einem Café durchgeführt.

Der Ablauf der Interviews folgte den Anhaltspunkten des Interviewdossiers (vgl. Anhang C). Alle Interviews habe ich auf Schweizerdeutsch geführt, weil dies die Teilnehmenden wünschten. Dies ermöglichte den Befragten, sich freier und gelassener in ihrer Alltagssprache auszudrücken.

In der Interviewdurchführung ist eine grosse Flexibilität der Forschenden gefragt (vgl. Kap. 5.2.5). Auch hat die Qualität der Interviewsituation, wie bereits weiter oben erwähnt, Einfluss auf die Qualität der gewonnenen Daten und anschließend auf die Datenauswertung (vgl. Helfferich 2009; S. 9). Deshalb ist es zentral, die realen Interviewsituationen annähernd zu beschreiben, in denen ich als Forscherin und Interviewerin arbeitete. ${ }^{20}$ Heinz Reinders zählt beispielsweise folgende Aspekte der Interviewdurchführung auf, an denen ich mich in meinen anschließenden Reflexionen orientiere:

- Ausfälle von Interviews

- Art des Interviewortes

- Anwesenheit von anderen Personen

- hartnäckige Mütter

- geringer Informationsfluss

- $\quad$ soziale Erwünschtheit

- technische Probleme (vgl. Reinders 2005a, S. 64).

Von den 16 geführten Interviews ist keines ausgefallen wegen Nichterscheinens des Interviewers, der Interviewten oder wegen grösserer technischer Probleme. Hingegen kam es dreimal vor, dass das Aufnahmegerät Warnsignale von sich gab; die jeweiligen kleineren Probleme konnten innerhalb weniger Minuten geklärt werden und führten zu einer kurzen Unterbrechung der Interviews. Die Interviewgespräche konnten mit einer Ausnahme ungestört von Drittpersonen durchgeführt werden. Die Ausnahme betrifft ein Inter-

20 Leider sind solche Ausführungen in den wenigsten Forschungsberichten zu finden. 
viewgespräch, während dem eine Lehrperson ins Zimmer kam. Nach einem kurzen informativen Hinweis meinerseits verließ diese schnell wieder den Raum, und der Schüler fragte mich rhetorisch: „Wo simmer schtaa gblibe?“ und fuhr nahtlos mit seinen Ausführungen weiter. Somit konnte das Interviewgespräch auch in diesem Fall ungestört weitergeführt werden.

Den Interviewort durften die SuS frei wählen, wobei sich alle für die Gruppenräume innerhalb des Schulareals entschieden. Die räumlichen Voraussetzungen können unterstützend darin wirken, dass die SuS in ihrer Schülerrolle sprechen. Dass die Schulräume geschlossen und anonym waren, begünstigte einen geschützten äußeren Rahmen und somit ein offenes Gesprächsklima. Zudem brachte das bekannte Umfeld den Vorteil des Vertrauten. Und schließlich konnte aufgrund des schriftlichen Rekrutierungsverfahrens im Klassenverband, bei Abwesenheit der Lehrperson (vgl. Kap. 5.4.3) und anhand der Interviewvereinbarung den SuS vermittelt werden, dass ich den Institutionszugang gewählt habe, um den Zugang zu den SuS zu gewinnen, und dass es sich um ein unabhängiges Forschungsprojekt handelt.

Mit hartnäckigen Eltern hatte ich es nicht zu tun. Jedoch erkundigte sich die Mutter einer Interviewten aufgrund des Informationsblatts an die Eltern, weshalb ich gerade ihre Tochter ausgewählt hätte. Ich wies sie auf die freiwillige Teilnahme hin. Anschließend erzählte sie mir von ihrer Unzufriedenheit mit der Schulleitung.

Auf die Minderung der sozialen Erwünschtheit und auf den geringen Informationsfluss bin ich bereits an verschiedenen Stellen eingegangen (vgl. Kap. 5.2), sodass ich dies hier nicht wiederhole.

Bei der Durchführung der Interviews achtete ich darauf, dass ich nur ein Interview pro Tag führe (vgl. dazu bspw. Mey und Mruck 2007, S. 267), damit ich genügend Zeit für die Vor- und Nacharbeit und genügend Ressourcen hatte, um den Ansprüchen in der Interviewsituation gerecht zu werden (vgl. Kap. 5.2.5).

\section{Datenbearbeitung}

Alle geführten Interviews und Diskussionen sind mittels digitaler Aufnahme festgehalten (vgl. Übersicht in Anhang E). Die 16 Interviews mit den SuS habe ich vollständig auf Schweizerdeutsch situations- und inhaltsgetreu mittels des Transkriptionsprogramms $f 4$ (f4 2004) transkribiert (vgl. Dresing und Pehl 2011; Dittmar 2009). Alle Transkriptionstexte sind im Beiheft Datenmaterial einsehbar (vgl. Verzeichnis des Datenmaterials). Die systematische und sorgfältige Transkription ist zwar aufwändig, aber eine wichtige und notwendige Voraussetzung für die anschließende Datenanalyse (Przyborski und Wohlrab-Sahr 2009, S. 162). Denn anhand der Transkription können die Textstellen ausgelegt werden, und die daraus erarbeiteten Interpretationen 
müssen wiederum eindeutig auf die entsprechenden Textstellen zurückgeführt werden können.

Allerdings stellte sich die Frage, wie genau das vorliegende Audiomaterial transkribiert werden soll. In der Literatur der Sozialforschung finden sich nur vage Ratschläge wie: ,[...] nur so viel und so genau zu transkribieren, wie von der Fragestellung tatsächlich notwendig erscheint" (vgl. Flick 2009, S. 380). Der persönliche Spielraum ist demnach groß.

Die Genauigkeit der Transkriptionsregeln (Bohnsack 2008) passte ich den Anforderungen der Auswertungsmethode an, in der beispielsweise parasprachliche Phänomene wie Sprech- oder Lachpausen für die inhaltliche Analyse von Bedeutung sind (s. Transkriptionsregeln in Anhang C). Nonverbale Zeichen wie zum Beispiel Gesten vermerkte ich so weit als möglich. Ganz klar sind damit Verzerrungen der realen Gesprächssituation unvermeidlich. Da es in der folgenden Untersuchung weniger um linguistische Aspekte des Quellenmaterials geht, werden Füllwörter wie ,äh` oder ,hmm` sowie Wörter, welche das aktive Zuhören der Interviewerin unterstreichen, wie ,mhm' oder , ja' ausgeblendet, soweit sie keinen inhaltlichen Bezug aufweisen. Hingegen werden Versprecher wiedergegeben.

Um die Anonymität zu gewährleisten, erhielt jedes Interview einen Code; Personen und Orte wurden seit Beginn der Transkription mit Decknamen versehen.

Die Transkription von Audio- in Textform behält das Schweizerdeutsch (Originalsprache) bei, damit die Authentizität des Gesprächs und der Äußerungen so weit wie möglich gewahrt werden konnte. Zur Schreibweise der Mundart finden sich viele individuelle Arten, den Dialekt zu schreiben. Der Leitsatz „Schreibe wie du sprichst, wie du es hörst und empfindest“ (Dieth und Schmid-Cadalbert [1938] 1986, S. 22), den die Autoren erstmals bereits 1938 in der Ausgabe der Schwyzertütschen Dialäktschrift festhielten, ist bis heute in der verschriftlichten Dialektologie sowie im alltäglichen Gebrauch gültig (vgl. bspw. Schobinger 2010; Gallmann 2010). Die Entfremdung des standardsprachlichen Schriftbildes wird ebenfalls in der vorliegenden Arbeit in Kauf genommen, denn jeder Buchstabe soll gesprochen werden. Mit Ausnahme der langen Laute, die doppelt geschrieben werden, werde ich anders als die Autoren keine weiteren Vokalqualitäten unterscheiden, wie beispielsweise offene und geschlossene Vokale. Die differenzierte Vokalschreibweise kann insofern weggelassen werden, als durch die Umschreibung mehr inhaltliche und weniger linguistische Ziele verfolgt werden. Weiter wird, anders als die Autoren vorschlagen, für die leichtere Lesbarkeit der Texte die Groß- und Kleinschreibweise berücksichtigt.

Grundsätzlich habe ich in der Transkription die Sprachverwendung der Befragten möglichst getreu wiedergegeben, sodass Abweichungen zur standardisierten Zürcherdeutschen Grammatik und der Zürcherdeutschen Verschriftung zugunsten der real gesprochenen Texte hingenommen werden. 
Während der Transkription hielt ich meine theoretischen, methodischen und inhaltlichen Reflexionen in Form eines Journals fest.

\subsubsection{Auswertungsverfahren}

Für jedes Interview erstellte ich ein Auswertungsdossier mit einem Titelblatt (Codename, Eckdaten zur Erhebung und zur Transkription und zentrale inhaltliche Aussagen), der Transkription, dem thematischen Verlauf des Interviews, der formalen Auswertung und der reflexiven Auswertung. Während des Auswertungsprozesses hielt ich meine Reflexionen in einem Auswertungsjournal fest.

Für die formulierende und reflexive Auswertung stellte ich ausgehend von den Überlegungen in Kapitel 5.3.2 einige praktische Richtlinien zusammen, die im Folgenden zu erwähnen sind (in Anlehnung an Przyborski und Wohlrab-Sahr 2009, S. 277ff.):

\section{Formulierende Interpretationsphase}

In der formulierenden Interpretationsphase fragte ich mich, was der Text sagt. Ich las die Transkripte und teilte den Inhalt nach Ober- und Unterthemen ein. Damit erzielte ich eine thematische Struktur des Textes, sodass deren thematische Gliederung nachvollziehbar erschlossen wird. Diese muss nicht notwendigerweise mit der Gliederung des Leitfadens übereinstimmen. In diesem Interpretationsschritt blieben einzelne Begriffe oder Wendungen auch unverständlich, weil der implizite Bezugsrahmen noch nicht erschlossen war.

Als ,analytische Trennung' ist die Unterscheidung der beiden Sinnebenen (kommunikative und dokumentarische Sinnebene) zu verstehen, sodass der erste Interpretationsschritt nicht mehr Gegenstand des nächsten Interpretationsschritts (reflexive Interpretation) ist. Dann werden die Textstellen für die reflektierende Interpretation ausgewählt, nach den bereits erwähnten Kriterien.

\section{Reflexive Interpretationsphase}

In der reflektierenden Interpretation versuchte ich, anhand folgender Fragen (in Anlehnung an Przyborski und Wohlrab-Sahr 2009, S. 284ff.) den dokumentarischen Sinngehalt zu erschließen:

- Wie wird erzählt?

- Was zeigt sich über den Fall?

- Welche Bestrebungen und/oder welche Abgrenzungen sind in den Redezügen impliziert? 
- Welches Prinzip, welcher Sinngehalt kann die Grundlage der konkreten Äußerung sein?

- Welches Prinzip kann mir (thematisch) unterschiedliche Äußerungen verständlich machen, womit der zugrunde liegende Sinn gewonnen wird?

Die Sinnmuster bzw. Orientierungen werden in (einzelnen) Verhaltensweisen hervorgebracht. Dabei zeichnen sich drei Eckpunkte des Orientierungsgehalts bzw. -wissens ab:

- Wiederholungen, Agency:

Wo strebt die oder der Befragte hin?

- Erschließung des Orientierungsgehalts durch einen begrenzenden Horizont:

Wovon wendet oder grenzt sich die oder der Befragte ab?

- Einschätzung der Realisierungsmöglichkeiten, verschiedene Lesarten werden entwickelt:

Wo werden Durchführungsmöglichkeiten oder -probleme gesehen?

Wie, auf welche Weise erfolgt eine Reaktion?

Wie, in welchem Rahmen wird ein Thema behandelt?

Während der Auswertungsphase verfasste ich einen Textkorpus von über 200 Seiten, in dem die unterschiedlichen Interpretationsschritte und Reflexionen festgehalten sind. Davon ausgehend systematisierte ich die Forschungsergebnisse (vgl. Teil II).

Als Co-Leiterin und Co-Gründerin der Forschungswerkstatt QUALIzüri boten sich viele Gelegenheiten, Interpretationsausschnitte während des Auswertungsverfahrens in interdisziplinären Forschungswerkstätten auch auf internationaler Ebene zu diskutieren und damit intersubjektiv zu überprüfen. Die Rückmeldungen erlaubten, die Spannung zwischen Vertrautheit und Fremdheit zu den anonymisierten Texten aufrechtzuerhalten. Der gezielte Austausch war für den Interpretationsprozess selbst sehr fruchtbar. Zudem zählt die Nachvollziehbarkeit der Interpretationsergebnisse zu einer weiteren Qualitätssicherung der qualitativen Sozialforschung.

Abschließend ist zu vermerken, dass die Daten im Ergebnisteil jene Fälle umfassen, die ich zur Beantwortung der Forschungsfrage gefunden habe. Dabei ist mir bewusst, dass außerhalb des Materials weitere Ehrlichkeitsregeln oder Ehrlichkeitsmuster existieren können.

\subsubsection{Zusammenfassung}

Bilanzierend betten sich die gewonnenen Forschungsbefunde in ein komplexes empirisches Forschungsvorgehen ein, das in einer dreiteiligen Feldphase situiert ist. Weiter liegt dem Forschungsvorgehen ein gezieltes Sampling 
zugrunde, das vier Schülerinnen und vier Schüler aus je vier kontrastierenden Oberstufenklassen im Kanton Zürichs zählt, womit die soziale Belastung, das Stadt-Land-Verhältnis und der Geschlechterausgleich sichergestellt ist. Schließlich dokumentierte und reflektierte ich in diesem Unterkapitel die Umsetzung des Erhebungs- und Auswertungsverfahrens angemessen und biete damit einen Einblick in die Feldforschung. 

II Ergebnisse 

Nachdem ich in Teil I der Arbeit die thematischen, theoretischen und methodischen Grundlagen erläutert habe, auf denen die qualitative Untersuchung basiert, präsentiere ich in diesem Teil die Ergebnisse der Analyse, die ich aus einem umfangreichen Datenmaterial mehrdimensional analysiert habe (vgl. Verzeichnis des Datenmaterials in der Bibliografie). Forschungsgegenstand sind subjektive Deutungen von Ehrlichkeitspraxen aus der Sicht von Abschlussschülerinnen und -schülern. Damit wird die Tugend Ehrlichkeit anhand von Deutungen praktizierter Verhaltensweisen explorativ erkundet, aus denen sich Ehrlichkeitsregeln rekonstruieren lassen. Mit der gewählten offenen Vorgehensweise sind unvermeidlich einige Doppelungen von Ehrlichkeitsregeln verbunden. Gleichzeitig kann damit die Vielfältigkeit, die Verzahnung und Verstrickung von diesen in deren Situationsabhängigkeit aufgezeigt werden.

Geteilte Ehrlichkeitsregeln können als Muster bezeichnet werden und weisen auf gemeinsame Erlebniszusammenhänge hin, die gemäss Mannheim als existentielle Hintergründe (Mannheim 1980, S. 211f.) bzw. konjunktiver Erfahrungsraum der Schülerinnen und Schüler (SuS) zu betrachten sind (vgl. Kap. 5.1, 5.2, 5.3). Aus den expliziten Aussagen der SuS gehen bereits folgende existentiellen Hintergründe mit unterschiedlichen Gewichtungen hervor:

- Leistungs- und Schulstufen

- $\quad$ soziale Belastung der Schulhäuser

- Unterrichtssituation sowie Art der Beziehungsnetze mit Lehrpersonen, Elternhaus, Mitschülerinnen/-schülern und anderen Gleichaltrigen

Die unterschiedliche Gewichtung dieser Aspekte, welche die SuS teils explizit nennen, fließt vorwiegend implizit in die subjektiven Ehrlichkeitsregeln ein. Eine Aufschlüsselung der Zusammenhänge kann mittels Gruppendiskussionen gezielt gewonnen werden, was jedoch in der Präsentation der vorliegenden Ergebnisse nicht verfolgt werden kann.

In der Darstellung der Ergebnisse räume ich den SuS über Originalzitate $^{21}$ viel Raum ein (vgl. Bohnsack et al. 1995, S. 15f.; Pfaff 2006, S. 193). Dies aus zwei Gründen: Erstens soll an die in den Aussagen vorgetragenen Stellungnahmen zu einzelnen Verhaltensweisen sowie Kritik an schulischen Erwartungen für sich sprechen. Damit kann auch denjenigen Lesern ein $\mathrm{Zu}-$ gang eröffnet werden, die weniger ein Interesse an theoretischen Reflexionen haben und mehr Auskunft über den praktischen Umgang mit Ehrlichkeit und die Deutungsregeln der Heranwachsenden erhalten wollen. Zweitens soll mit einer eher ausführlichen Wiedergabe der Originaltexte und die darauf zu

21 Die Originalzitate basieren auf dem Beiheft ,Transkriptionen der 16 Interviews mit Schülerinnen und Schüler an der Züricher Volksschule“ (Chiapparini 2011). Um den Lesefluss zu begünstigen sind die Transkriptionsstellen im Ergebnisteil mit dem Namen des interviewten SuS im Fliesstext versehen, aber nicht mit genauem Quellennachweis. 
beziehenden Interpretation dem Anspruch des gewählten rekonstruktiven Vorgehens nachgekommen werden: Der Blick auf die Deutungen der jugendlichen Akteure soll nicht durch die vorgenommene Konstruktion zweiten Grades (Schütz 1971-1972, S. 7; Bohnsack et al. 1995, S. 15) verstellt, sondern auf diese rückbezogen werden.

Der Einbezug von Zitaten und die damit vertiefte Auslegung der Texte variiert gering je nach Funktion der Kapitel. So kommt dem Einleitungskapitel eine hinführende Funktion zu, sodass die einzelnen Quellentexte einen Einblick ins Forschungsfeld geben sollen (vgl. Kap. 6). Hingegen zeigen die Kapitel zu den konventionellen (Kap. 7) und unkonventionellen Ehrlichkeitsregeln (Kap. 8) einen fallübergreifenden Zusammenhang der Ehrlichkeitsregeln, sodass eine vertiefte Interpretation nötig ist. Schließlich ist bei der fallimmanenten Untersuchung von Ehrlichkeitsregeln in Dilemmasituationen die Tiefeninterpretation entscheidend und damit die Berücksichtigung der ausgelegten Textstellen zentral, um die unterschiedlichen beeinflussenden Faktoren und die expliziten und impliziten Ehrlichkeitsregeln zu differenzieren und zu beleuchten (vgl. Kap. 9).

Abbildung 2: Darstellungsweise der Ergebnisse

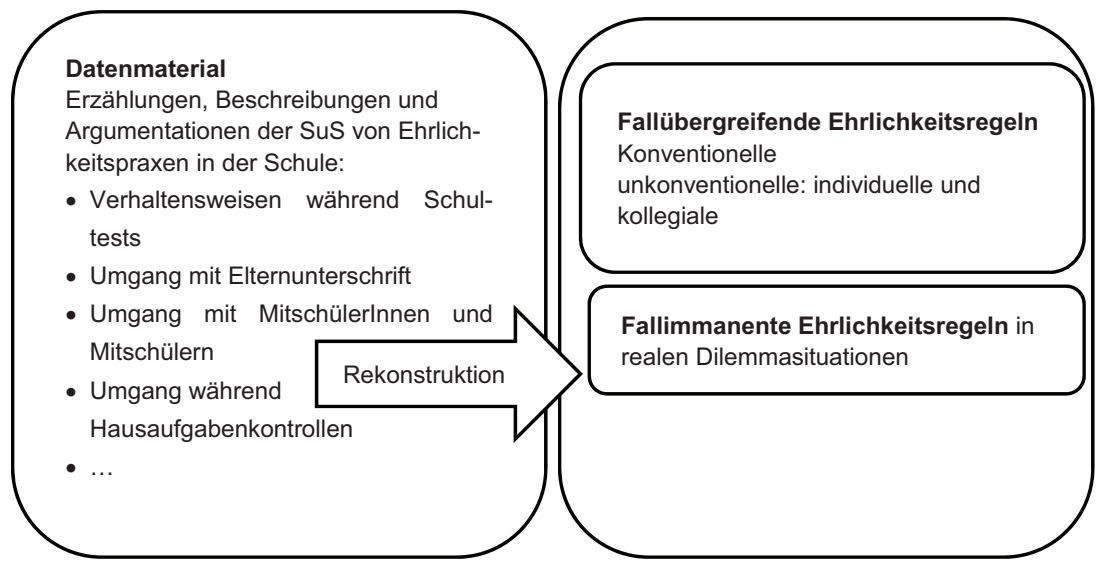

Quelle: eigene Darstellung

Die Ergebnisse stelle ich thematisch dar (vgl. Abb. 2). Einleitend gebe ich einen knappen Überblick zu sechs Ehrlichkeitskategorien und zu Thematisierungsregeln von Ehrlichkeit der SuS (vgl. Kap. 6). Vor diesem Hintergrund rekonstruiere ich aus ihren berichteten Ehrlichkeitspraxen fallübergreifende subjektive Ehrlichkeitsregeln von 14- bis 15-Jährigen. Die gewonnenen Ehrlichkeitsregeln lassen sich in den breitgefassten Begriffskategorien der konventionellen Ehrlichkeitsregeln (vgl. Kap. 7) und der unkonventionellen Ehrlichkeitsregeln (vgl. Kap. 8) einteilen. Konventionelle Ehrlichkeitsregeln 
sind in dieser Arbeit solche, die schulischer Usus sind und von der Lehrperson kontrolliert werden, etwa Nicht spicken oder Nicht täuschen.

Einige Interviewpassagen zeigen klar, wie die SuS in realen Dilemmasituationen in Interessenkonflikte geraten können. Anhand dieser Beispiele untersuche ich fallimmanent, welche Ehrlichkeitsregeln sich durchsetzen und somit, welche subjektiven Ehrlichkeitsregeln in realen Entscheidungsprozessen vorzufinden sind (Kap. 9).

Die präsentierten Ergebnisse aus den einzelnen Kapiteln (vgl. Kap. 6, 7, $8,9)$ werden abschließend zusammengefasst (vgl. Kap. 10). Damit strebe ich an, wichtige empirische Hinweise für den theoretischen Erklärungsbedarf bezüglich schulischer Ehrlichkeitspraxen zu liefern (vgl. Teil III), an denen SuS ihre Verhaltensweisen orientieren.

\section{Ehrlichkeitskategorien und Thematisierungsregeln}

Als Hinführung zum Hauptteil der Ergebnisse sollen die zwei einleitenden Unterkapitel einerseits Einblick in die Vielfalt der Ehrlichkeitskategorien leisten, die ich anhand von Ehrlichkeitsregeln aus dem empirischen Material generiert habe (vgl. Kap. 6.1). Damit ist vorerst aufgezeigt, über welche Ehrlichkeitsregeln SuS verfügen und wie Ehrlichkeitskategorien wegen der Situationsabhängigkeit schwer stringent $\mathrm{zu}$ fassen sind. Dem schließt sich eine Analyse der Thematisierungsregeln von Ehrlichkeit an, womit Einsicht in die Artikulationsweisen der SuS zum Thema Ehrlichkeit gegeben wird (vgl. Kap. 6.2). Mit der Einleitung soll der Leserschaft ein roter Faden gegeben werden, der sich durch die darauffolgenden Kapiteln zieht, in denen fallübergreifende und fallimmanente Analysen von Ehrlichkeitsregeln vorgestellt werden.

\subsection{Ehrlichkeitskategorien und vielfältige Ehrlichkeitsregeln - Ein erster Einblick}

SuS berichten über unterschiedliche ehrliche Verhaltensweisen aus dem schulischen Alltag. Besonderes Augenmerk richte ich in diesem Unterkapitel auf die am häufigsten explizit geäußerten Ehrlichkeitsregeln, die ich annähernd an die Ehrlichkeitskategorien von Whites Ehrlichkeitsdefinition (vgl. Kap. 2.4) zusammengefasst habe. Damit soll ein erster Überblick über die facettenreichen Eigenschaften von Ehrlichkeitsregeln gegeben werden, die 
nur annähernd nach erziehungsphilosophischen Ehrlichkeitskategorien einzuteilen sind:

- Ehrlichkeit als selbstsprechendes Selbstverständnis

- Ehrlichkeit als Wahrhaftigkeit

- Ehrlichkeit als Aufrichtigkeit gegenüber anderen SuS

- Ehrlichkeit als Aufrichtigkeit gegenüber Lehrpersonen

- Ehrlichkeit als Aufrichtigkeit in Schultests

- Ehrlichkeit als Offenheit

Diese Ehrlichkeitskategorien werden anhand einer ersten Textanalyse präzisiert. Die Textauszüge beziehen sich einerseits auf Situationsbeispiele, welche die SuS in Eigeninitiative eingebracht haben. Andererseits sind sie den teilweise ausführlichen Antworten auf die direkte Interviewleitfrage nach dem Ehrlichkeitsverständnis entnommen.

\subsubsection{Ehrlichkeit als selbstsprechendes Selbstverständnis}

Was mit Ehrlichkeit gemeint ist, ist schwierig in Worte zu fassen. Zudem braucht es nicht erklärt zu werden. Das selbstsprechende Selbstverständnis drückt Lilian so aus: „(3) Ich cha das nöd erklääre. Eerlichkäit isch Eerlichkäit (2)“. ${ }^{22}$ Die Aussage impliziert ein informelles Selbstverständnis von Ehrlichkeit, worüber alle informiert sein sollen: Ehrlichkeit ist selbsterklärend oder setzt einen kollektiven Erfahrungsraum voraus, weshalb sich eine Erklärung erübrigt. In derselben Weise argumentiert Ilona, dass es selbstverständlich sei, ehrlich zu sein. Darüber hinaus weist Lilian insbesondere auf die verbalisierende Schwierigkeit hin, Ehrlichkeit in Worte zu fassen. Dem schließt sich Clemens an:

(2)@1@ Eerlichkäit erklääre? Ui, das isch ja seer schwirig. (1) Und vor allem de Schüeler z erklääre? Isch noo schwiriger! (3) Eerlichkäit erklääre, (1) phuu (4) Eerlichkäit erklääre, was würd ich da säge. (3) Ja, da mues ich emaal überlegge. (I: Mhm) (6).

Der in anderen Interviewabschnitten schlagfertige Clemens hat sichtlich Mühe, Ehrlichkeit zu erklären. Wobei unklar bleibt, ob es tatsächlich die verbale Schwierigkeit ist, die ihn hindert, eine Antwort zu finden, oder ob ein vielfältiges ehrliches Verhalten oder die davon abweichenden Verhaltensweisen ihm diese Aufgabe so schwierig erscheinen lassen. Er wiederholt viermal das Verb erklääre und zwei Mal das Wort schwirig. Dadurch gewinnt er neben den Denkpausen Reflexionszeit, was er verbal nochmals unterstreicht. Anschließend an diese Gesprächspassage definiert Clemens Ehrlichkeit im Sinne von Aufrichtigkeit als Antwort auf Anfrage hin (vgl. Kap. 8.1.4). Damit präsentiert sich ein Aspekt von ehrlichen Verhaltensweisen. Es dokumentiert

22 Die hier verwendeten Transkriptionsregeln sind in Anhang C einzusehen. 
sich sowohl eine reale Erklärungsschwierigkeit sowie eine punktuelle Ehrlichkeitsregel. Zudem gibt dies ein Indiz darauf, dass Clemens Ehrlichkeit anhand von Beispielen erklären kann, aber dies in einer direkten und nur reflexiven Abfrage schwererfällt. Dabei müssen nicht nur kognitive Kompetenzen, sondern auch emotionale Faktoren und kulturelle Sensibilitäten des Themas eine Rolle spielen.

Die Ehrlichkeitskategorie des selbstsprechenden Selbstverständnisses von Ehrlichkeit ergänzt Whites dreiteilige Ehrlichkeitskategorien. Sie führt zur Ehrlichkeitsregel: Ehrlichkeit ist nicht zu definieren oder mit Worten zu fassen, sondern vorerst eine Praxis. Diese Regel ist zentral, weil nicht nur einzelne SuS der untersuchten Schule Ehrlichkeit als Verhaltensweise einfach voraussetzen; ebenso nehmen populäre Tugenddebatten selbsterklärende Einzeltugenden an, die scheinbar keiner differenzierten Erörterung bedürfen (vgl. Kap.1.2, Kap. 2.1).

\subsubsection{Ehrlichkeit als Wahrhaftigkeit}

Der Ehrlichkeitskategorie der Wahrhaftigkeit ordnet sich die Ehrlichkeitsregel Nicht lügen bzw. Die Wahrheit sagen zu, die als sozial erwünschte Verhaltensweise und allen SuS bekannt ist. Unterschiede bestehen, in welchen Unterrichts- und Schulbereichen diese Ehrlichkeitsregel vorzufinden ist und wie die SuS sie darin werten. So benutzt David auf die Frage, was Ehrlichkeit sei, beide Formulierungen zugleich: ,(1) Wämmer halt nöd lüügt und wämmer d Waret säit (5)“. Das Neutrum der handelnden Person mer kennzeichnet die kollektive Form der Regel. Sie ist allgemein gesetzt, sodass diese nicht zu hinterfragen ist. Offenbar soll kein Raum zur Interpretation verbleiben. Zudem unterstreicht das Partikel halt die Normalität der Ehrlichkeitsregel; anscheinend ist keine subjektive Stellungnahme zu berücksichtigen, sondern es besteht eine unhinterfragte Konvention. Kurz: Diese konventionelle Ehrlichkeitsregel impliziert ein Selbstverständnis, das auf eine eindeutige Wahrheit zeigt.

Nora verwendet darüber hinaus die Definition des Nichtlügens auf der Beziehungsebene:

(3) Ja, (2) ja, wänn mer nöd so umelüügt, also mir händ äini ghaa id de- Die isch früüener i de Parallelklass gsii, die isch nach de zwäite Sek is Gymi (1) und die isch für mich es guet s Biischpiil@als uneerlichi Person@, will si isch immer zu alne Lüüt so, uu nett gsii und so. (1) Und sobald irgendöpper wäggloffe isch vo ire, hät si grad mit de reschtlichi Persoon über die aafange läschtere und so.

Lügen (umelüügen) setzt Nora hier mit lästern gleich. Lästern heißt, dass jemand über eine abwesende Person schlecht redet und in deren Anwesenheit wieder nett ist. Das so verstandene Lügen bezieht sich nicht nur auf die ver- 
bale Aktivität, es berücksichtigt auch den kollegialen und sozialen Kontext der anwesenden Personen.

Lara sind die sozialen Beziehungen grundsätzlich wichtig, wie an verschiedenen Stellen im Interview zu erkennen ist. So thematisiert sie beispielsweise gleich zweimal die freie Sitzwahl, obwohl nur zwei Lehrpersonen freie Sitzwahl unter besonderen Umständen erlauben und diese strikt aufgehoben wird, sobald Mitschülerinnen und -schüler sich zu laut verhalten.

Ilona beurteilt die Ehrlichkeitsregel Nicht lügen unter Kolleginnen in der Schule als sehr wichtig, womit sie ebenfalls einen sozialen Aspekt aufwirft. Beispielsweise flippt sie selber aus, wenn eine Kollegin unehrlich bzw. nicht direkt ist: „Ich wird au wüetig, wenn Kolleegin mich aalügt oder e so, dänn (2) flipp ich@mäischtens@ völlig uus. Ich find das s schlimmschte (2).“ Ilona erwartet, dass ihre Mitschülerinnen nicht nur das angenehme, sondern auch das unangenehme Wissen weitergeben. Wenn dies nicht der Fall ist, trifft sie das persönlich, und sie reagiert stark emotional.

Die Ehrlichkeitsregel des Nichtlügens ist den SuS bekannt sowie erlernt und findet je nach dem sozialen und personalen Kontext unterschiedliche Anwendungen. Darauf gehe ich in Kapitel 7 genauer ein. Zudem zeigt die Ehrlichkeitsregel des Nichtlügens im Zusammenhang mit Freundschaften Affinitäten zur besonderen Form der Ehrlichkeitskategorie Offenheit als Direktheit (Kap. 6.1.5). In diesem Sinne setzt Ilona die Ehrlichkeitsregeln des Nichtlügens und der Direktheit gleich bzw. die der Lüge und der fehlende Direktheit.

\subsubsection{Ehrlichkeit als Aufrichtigkeit gegenüber anderen SuS}

Verpetzen im Sinne von Verraten beurteilen die meisten SuS als eine negative Verhaltensweise. In ihrer Wahrnehmung ist der Verrat eines anderen etwas, das üblicherweise nicht in der eigenen Klasse, dafür aber in anderen Klassen vorkommt. Adrian erklärt das Verpetzen in der folgenden Textpassage in diesem Sinne:

(2) Ja, wänn jetzt irgendöpper öppis aagschtellt hät und nachheer (1) säit s irgend öpper andersch de Leerer, wer das gsii isch und- (2) das chunt mängisch voor. (1) Jetzt i öisere Klass machet mir s äigentli nöd soo. Aber mängi, irgendöpper vonere andere Klass, säget s dänn mängisch. (3) Und s isch amigs nöd so luschtig. (2) Ich find s au irgenwie (2) es muess nöd sii. (5)

Adrian beurteilt das Verpetzen hier eindeutig negativ (nöd so luschtig, muess nöd sii). In einer späteren Textstelle formuliert er das Nichtverpetzen positiv und als Widerspiegelung eines guten Klassenzusammenhalts.

Clemens schildert ebenfalls, dass seine Klasse gut zusammenhält, indem keiner den anderen verrät. Clemens ist ein Tracker (vgl. auch Kap. 8.2.2): Er setzt Schriftzüge an öffentlich und zugängliche Wände. Über seine unerlaub- 
te Tätigkeit sind seine Mitschülerinnen und Mitschüler informiert. Einige sahen ihn sogar, als er die WC-Wände im Schulhaus beschriftete. Jedoch haben sie ihn nie verraten - auch nicht, als die Lehrpersonen direkt danach fragten.

Clemens ist der Ansicht, dass es nie einen Grund gibt, jemanden zu ,verpetzen':

(1) Aber s git äigentli nie Moment, wo (1) me sött verpetze. Also ich ha daas no nie erläbt, wo me würkli sött: „Dee und dee isch s gsii.“ (1) Nähe. (2) Wänn, würd ich s nur immer uf miini Schuld nee. Aber nie verpetze.

Der Konjunktiv und das Verb sollen verweisen in diesem Textabschnitt auf Clemens' Erwartungshaltung. Das Nichtverpetzen konstruiert Clemens als idealisierte Verhaltensweise: Er ist sogar bereit, eine Schuld auf sich selbst zu nehmen, um nur niemanden zu verraten. Diese Bereitschaft bezeichnet er im vorangegangenen Abschnitt als heldenhaft, wobei er selbst noch nie Anlass hatte, sich danach zu verhalten - wohl aber ein Mitschüler, Otto, dessen Courage Clemens entsprechend lobt und bewundert (vgl. Kap. 8.2). Clemens erklärt, die gesamte Klasse habe auf diese Heldentat positiv reagiert.

Zusammenfassend zeigt sich: Die Verhaltensweise des Nicht verratens fungiert als eine feste klasseninterne und unausgesprochene Ehrlichkeitsregel, die zur Ehrlichkeitskategorie der Aufrichtigkeit gegenüber anderen SuS gezählt werden kann. Allerdings weisen einige wenige SuS auf Grenzen dieser Ehrlichkeitsregel hin, worauf ich in Kapitel 8.2.1 ausführlich eingehe.

\subsubsection{Ehrlichkeit als Aufrichtigkeit gegenüber Lehrpersonen}

Die Ehrlichkeitsregel des Nicht verratens betrifft die grundsätzliche und allgemeine Verhaltensweise der Aufrichtigkeit der SuS gegenüber anderen SuS, aber nicht gegenüber den Lehrpersonen. Ehrlichkeitsregeln, welche unter der Ehrlichkeitskategorie Aufrichtigkeit gegenüber der Lehrperson erfasst werden, zielen hingegen auf die Aufrichtigkeit gegenüber Lehrpersonen. Es handelt sich um spezifische Situationen meistens einer einzelnen SuS und setzt die Bedingung des Nachfragens der Lehrperson voraus. Das typische Schulbeispiel dazu ist, wenn jemand die Hausaufgaben nicht gelöst hat und bei der Nachfrage der Lehrperson dies nicht kaschiert, sondern dazu steht. Darauf gehen die folgenden Ausführungen ein.

Regula und Adrian halten fest, dass bei Nachfragen der Lehrperson ehrliche Antworten zu geben sind. Dabei unterscheiden sie genau zwischen wichtigen Tätigkeiten und Ausnahmen. Zu den wichtigen Tätigkeiten zählt, wenn Briefe zu Hause abzugeben sind oder wenn Material für einen Vortrag mitzunehmen ist. $\mathrm{Zu}$ den Ausnahmen zählen die vergessenen Hausaufgaben.

Keine Aussage der SuS bezieht sich auf eine genuine Form der Aufrichtigkeit; vielmehr wird die Art und Weise der Aufrichtigkeit differenziert. 
Exemplarisch hierfür ist eine Textstelle aus dem Interview mit Lara. Lara zeigt eine spezifische Ehrlichkeitsregel bezogen auf die Ehrlichkeitskategorie der Aufrichtigkeit gegenüber Lehrpersonen, wie sie in ähnlicher Weise bei Claire und Clemens zu finden ist: Aufrichtig sollte man insbesondere bei wichtigen sowie nachweisbaren Tätigkeiten und auf äußere Anfrage hin sein. In diesem Zusammenhang sind Hausaufgabenkontrollen oft erwähnte Schulsituationen, in denen eine differenzierte Ehrlichkeitsregel bezüglich Aufrichtigkeit erkennbar ist. So berichtet Lara:

Scho klar versuecht mer zeerscht, das d Leerer nöd gseend, aber wänn s dänn gsee händ, nöd irgendwelches Züüg z versueche: „Ja, ich has aber daa.“ Und es isch, ja, dänn no irgendwelchi Uusrede erfinde (3).

Lara setzt mit großer Selbstverständlichkeit voraus, dass ungemachte Hausaufgaben kaschiert werden; sie fordert aber die Aufrichtigkeit des ertappten Schülers, sobald die Lehrperson die Unterlassung bemerkt. Dabei beurteilt Lara es negativ, wenn sich die Betroffenen mit Ausreden zu rehabilitieren versuchen. In der angeführten Passage bezieht sich Aufrichtigkeit gezielt auf die Hausaufgabenkontrolle und darauf, dass die Lehrperson bereits jemanden ertappt hat - in genau einer solchen Situation sollte man ehrlich sein. Diese Aufrichtigkeit setzt Zivilcourage voraus, was Lara mit dem Hinweis auf die Ausrede andeutet. Zugleich kann die so verstandene Aufrichtigkeit durch das Risiko motiviert sein, mögliche Sanktionen zu meiden.

Für andere schulrelevante Situationen - etwa den Umgang mit Unterschriften der Eltern oder dem Schummeln in Schultests - fordert Lara verschiedene Facetten der Aufrichtigkeit, auf die ich in Kapitel 7.1 eingehe.

In der Interviewanalyse findet sich also keine Kategorie für Aufrichtigkeit als Verhaltensweise in einer genuinen Art. Vielmehr untermauern zahlreiche Aussagen der SuS die Kategorie der Aufrichtigkeit gegenüber Lehrpersonen, wobei auch hier situationsabhängige Ausnahmen genannt werden.

\subsubsection{Ehrlichkeit als Aufrichtigkeit in Schultests}

Die Ehrlichkeitsregel des Nichtspickens (schriftliches Schummeln) ist nur an zwei Gesprächsstellen explizit genannt. Nora gesteht sich ein, dass Spicken Selbstbetrug ist: „, isch ja sälber Betruug“; das neutrale Subjekt sowie das Partikel ja signalisieren die gefestigte und konventionelle Ehrlichkeitsregel des Nichtspickens, die allgemein klar zu sein scheint. Sandra ist ebenfalls der Meinung, dass Spicken unehrlich gegenüber der Lehrperson sei, allerdings fügt sie Bedingungen an, die das Spicken legitimieren: ,(2) Ja@1@. Ich find s äigentli scho echli (1), ja (1) me hindergaat d Leerer scho echli, aber ich find jetzt, wänn d Leereri halt nöd luegt, isch si halt sälber gschuld.“ Die fehlende Kontrolle des Lehrers nennt Sandra also als legitimen Grund, um zu 
spicken. Eine weitere Bedingung ist der Druck, eine gute Leistung zu erzielen.

(1) Äigentli macht mer s scho für sich d Prüefig, zum Luege wie guet, das ich äigentli bin. Ich mäin die Noote chunt nachheer trotzdem is Zügnis und me mues sich mit dene (1) sich bewerbe und so. (2) Also versuecht mer doch lieber e gueti Noote z haa @1@ (2).

Sandra sieht einerseits ein, dass Schultests der eigenen Lernkontrolle dienen. Gleichzeitig stellt sie die Noten aus den Schultests in einen größeren und pragmatischen Zusammenhang: Die Noten der Schultests fließen ins Zeugnis, mit dem sich die SuS auf der Suche nach einer Lehrstelle erfolgreich zu bewerben haben. Die Lehrstellensuche ist ein legitimer Grund, weshalb die Schultests nicht nur als Lernkontrolle betrachtet werden können, sondern den Zweck implizieren, gute Noten zu schreiben, um in der Zukunft erfolgreich zu sein. Die Spannungssituation zwischen fehlende Leistungserfüllung und Notwendigkeit von guten Noten (Leistungsanforderung), um mit einem guten Zeugnis eine Lehrstelle zu suchen, legitimiert den Einsatz von unlauteren Hilfsmitteln.

Ebenso legitimiert Valentina die Ehrlichkeitsregel des Nichtspickens mit einem ästhetisch-sozialen sowie mit einem verallgemeinernden Argument (vgl. Kap. 7.1). Nach der Beschreibung, wie ihre Klasse vor allem in der ersten Oberstufe unlautere Hilfsmittel während der Schultests auf unterschiedliche Weise verwendete, erklärt sie: „(2) Es isch au so Kollege und echli mitenander hälfe, find ich schöön@1@, obwoohls ja echli Betrug isch, ja das isch@1@. Äigentli hät s scho jede maal gmacht, äigentli (5)“.

Die konventionelle Ehrlichkeitsregel, unlautere Hilfsmittel während der Schultests nicht zu verwenden, ist offensichtlich sehr präsent. Valentina etikettiert dieses Verhalten mit dem kriminologischen Wort ,Betrug'. Demgegenüber positioniert sie ihre grosse Bedeutung der kollegialen Beziehungen, indem sie diese als schöön bezeichnet. Das Adjektiv ,schön“ kann auch als , gut' gelesen werden und weist auf ein ästhetisch-soziales Argument hin. Dass ein Betrug vorliegt, wird eingesehen, obwohl die Termini echli, ja, äigentli auf Unsicherheit hinweisen und damit die moralische Grenze zwischen ehrlichen und unehrlichen Verhaltensweisen verwischt. Der Betrug wird schließlich dadurch legitimiert, dass viele ihn begehen. Mit diesem verallgemeinernden Argument schiebt Valentina die Verantwortung für die unehrliche Verhaltensweise ab.

\subsubsection{Ehrlichkeit als Offenheit}

Auf die Frage, was Valentina unter Ehrlichkeit versteht, antwortet sie mit zwei Ehrlichkeitsregeln:

Eerlichkäit? (4) Also, ich find äifach bi Eerlichkäit git s zwäi Sache, wo- also zwäi Aarte das zsäge, äifach e Aart, wo verletzt, wo so echli zuu diräkt isch und echli unhöflich und 
vor allem vor allne Lüüt oder so. (1) Und e Art, wo (3) halt ebe echli scho d Waarheit aber echli netter gsäit und so.

Sowohl die unhöfliche als auch die nettere Art, die Wahrheit zu sagen, betont die kommunikative Art und Weise einer gemeinsamen Ehrlichkeitsregel: Direkt die Wahrheit sagen. Was Valentina unter dem Wahrheitsbegriff versteht, erklärt sie nicht, sie setzt diesen voraus.

Die Ehrlichkeitskategorie zeichnet sich hier in einer besonderen Form der Direktheit aus, von der Claire, Valentina, Lara, Sandra, Ilona und Lilian ausführlich berichten und die in den Erzählungen und Beschreibungen einheitlich dargestellt wird. Darauf gehe ich in Kapitel 8.1.3 und Kapitel 9.1.2 ausführlicher ein.

Die Ehrlichkeitsregel des Direktheitseins spielt im Zusammenhang des Lästerns ebenfalls eine zentrale Rolle. Mit Lästern ist die üble Nachrede gemeint, wobei die adressierte Person nicht präsent ist. Dies war der Fall in Laras Klasse. Die unerwünschte Schülerin aber wechselte bald darauf ins Gymnasium. Als sie nach dem Klassenwechsel einen Besuch bei der ehemaligen Klasse abstattet, sind Laras Mitschüler ehrlich bzw. direkt - sie teilen ihren Ärger direkt mit: „Ja, chasch grad wider gaa. Mir@wänd jetzt nöd mit dir rede@." Lara selbst bejaht diese Umgangsform, obwohl sie sich in jener Aussprache nicht direkt beteiligte. An diesem Beispiel ist klar erkennbar, dass die ,Wahrheit' darin besteht, das auszudrücken, was man denkt.

Darüber hinaus setzt sich Ilona für die Ehrlichkeitsregel des Direktheitseins ein, die meistens unabhängig vom Sympathiegrad des Gegenübers einzuhalten ist. Im Gegensatz zu Laras Ansicht, nur SuS gegenüber ehrlich zu sein, hat Ilona den zusätzlichen Anspruch, ebenfalls gegenüber Erwachsenen direkt zu sein; zudem fordert sie eine höfliche und von Respekt geprägte Gesprächskultur:

Wänn mich öppis schtöört, dänn säg ich s dere Person grad is Gsicht. (1) Und mäischtens isch s mir egaal, wer die Person isch. Au wänn s en Erwachsene isch- Ich han Reschpäkt vor erwachseni Persoone und alles, aber (2) wänn mich öppis schtöört vo dere Persoon und (2) irgendwie, dänn gaan ich und säg das au höfflich.

Auslöser des direkten Mitteilens ist der Störfaktor, was Ilona als Wenn-dannRegel verbalisiert: Wenn sie etwas stört, dann spricht sie das direkt an. Die Regel des Direktheitseins wird auch assoziiert mit Offenheit bzw. der Bereitschaft, alles zu sagen. Die Metapher is Gsicht ist aussagestark. Es impliziert eine couragierte und unmittelbare Verhaltensweise. In diesem Sinne erzählt Ilona von einer Begebenheit, wie sie und alle Mädchen im Geheimen die Haarverlängerung einer Mitschülerin negativ begutachten. Ilona schildert ihr Vorgehen in einem solchen Fall wie folgt:

Aber ich ha si uf em Wäg gsee und han ire gsäit: „Hey, wiso häsch daas gmacht?“ und so. „Ja, ich find s schöön“, und ich soo: „Aha, iisi. (1) Aber ich has hübscher gfunde oni“, und fertig, also ebe (1). 
Das Gespräch ist auf einer sachlichen Ebene geführt. Ilona stellt eine Frage, die rhetorisch oder empathisch zu interpretieren ist. Sie reagiert verständnisvoll und deponiert kurz ihre Meinung. Die Reaktion der Kollegin erwähnt Ilona nicht, als ob diese nicht wichtig sei. Wichtiger scheint, dass Ilona ihre Meinung mitgeteilt hat, auch wenn diese sehr kurz ausgefallen ist.

Unter allen $16 \mathrm{SuS}$ ist Ilona die Einzige, die explizit erklärt, wie sie ihre Meinung gegenüber Erwachsenen und Lehrpersonen direkt anbringt. Beispielsweise bezieht sie Stellung, nachdem die Lehrerin sie ungerecht im Zeichnungsunterricht zurechtgewiesen hat: „Ich chan nöd immer Gschuld sii. Si gseent immer nur mich und die andere gseent si nie“. Auf diesen Hinweis folgt ein längeres Gespräch mit der Lehrerin, und Ilona teilt ihre Meinung respektvoll mit, was die Lehrerin annimmt oder annehmen muss. $\mathrm{Zu}$ einer Antwort darauf fehlen weiterführende Informationen.

Ilonas bevorzugte Verhaltensweise bei zwischenmenschlichen Schwierigkeiten mit einer Person ist also, sie direkt darauf anzusprechen. Dabei ist mit persönlichen Verletzungen zu rechnen. Diese nimmt Ilona in Kauf, weil sie der Ansicht ist, unangenehme Bemerkungen seien angenehmer von der betroffenen Person direkt zu erfahren, als später über Dritte zu vernehmen. Ilonas Verständnis setzt ein starkes Selbstbewusstsein voraus, das nicht bei allen SuS gleich ausgeprägt ist.

Aus den Schilderungen lässt sich ableiten: Hinter den Kategorien der Ehrlichkeit als Direktheit verbirgt sich der Wunsch nach Vertrauen und Offenheit. Die Ehrlichkeitskategorien der Direktheit als spezielle Form der Offenheit sind bei zahlreichen SuS zu finden, wobei auch hier verschiedene Nuancen in der Anwendung festzustellen sind, auf die ich später genauer eingehe.

\subsubsection{Zusammenfassung}

Einleitend ist auf die Grenze von Ehrlichkeitskategorien hingewiesen, zu denen in der Erziehungsphilosophie ,Wahrhaftigkeit', ,Aufrichtigkeit' und ,Offenheit‘ zählen. Es konnte gezeigt werden, dass die erwähnten Ehrlichkeitskategorien nur ungenügend die Vielfalt von Ehrlichkeitspraxen zu berücksichtigen vermögen:

- Ehrlichkeit als selbstsprechendes Selbstverständnis:

Ehrlichkeit ist nicht zu definieren oder in Worte zu fassen, sondern vorerst eine Praxis

- Ehrlichkeit als Wahrhaftigkeit:

Nicht lügen als erlernte und bekannte Ehrlichkeitsregel

- Ehrlichkeit als Aufrichtigkeit gegenüber anderen SuS:

Nicht verraten als klasseninterne und unausgesprochene Ehrlichkeitsregel 
- Ehrlichkeit als Aufrichtigkeit gegenüber Lehrpersonen:

Aufrichtig sein, insbesondere bei wichtigen sowie nachweisbaren Angelegenheiten und auf Anfrage von außen

- Ehrlichkeit als Aufrichtigkeit in Schultests:

Nicht spicken

- Ehrlichkeit als Offenheit:

Alles direkt sagen

Die meisten SuS formulieren zunächst explizit und auf Anfrage eine allgemein gehaltene Definition von Ehrlichkeit. Im Verlauf des Berichtens jedoch beziehen sie sich nahezu ausschließlich auf schulische Alltagsbeispiele, womit die SuS die erziehungsphilosophischen Ehrlichkeitskategorien teilweise erwähnen, mehrheitlich ergänzen und präzisieren. Damit sind konventionelle Ehrlichkeitsregeln, die in Berührung mit dem Schulalltag kommen, Abweichungen, Spezifizierungen und verschiedenen Sichtweisen der SuS ausgesetzt und entwickeln sich dadurch zu situations- und kontextabhängigen Ehrlichkeitsregeln. Deshalb ist es notwendig, gezielt thematisch und kontrastierend Ehrlichkeitsregeln aus dem Datenmaterial herauszukristallisieren (vgl. dazu Kap. 7, 8).

Darüber verweist die Präzisierung der Ehrlichkeitskategorien auf das Spannungsfeld zwischen konventionellen und unkonventionellen Ehrlichkeitsregeln. Unterricht- und Schulsituationen können für einzelne SuS zu einem Dilemma werden. Denn die aufgeführten Aussagen der SuS zeigen auf, dass unterschiedliche explizite und implizite Ehrlichkeitsregeln gleichzeitig Geltung beanspruchen und damit Entscheidungsprozesse in Gang gesetzt werden (vgl. Kap. 9).

Doch zunächst ist aufzuschlüsseln, wie SuS über die Tugend Ehrlichkeit und über Ehrlichkeitspraxen sprechen (vgl. Kap. 6.2).

\subsection{Thematisierungsregeln von Ehrlichkeit}

Ausgehend vom eigenen Erfahrungswissen nahm ich in der Felduntersuchung an, dass SuS nicht über Tugenden sprechen, was sich bestätigte. Jedoch überraschte mich die Tatsache, dass ebenfalls das Substantiv ,Ehrlichkeit' sowie das Adjektiv, ehrlich' keine grosse Aufnahme in ihre Alltagssprache findet, worauf die anschließenden Ausführungen eingehen (Kap. 6.2.1).

Es lassen sich aus den Daten vier Arten generieren, wie sich SuS zu Ehrlichkeit äußern. Die erste Art bezieht sich vornehmlich auf die letzte Leitfadenfrage, in der die SuS eingeladen werden, zu erklären, was Ehrlichkeit sei; die anderen drei Arten sind aus den Berichten über den Schulalltag extrahiert: 
- $\quad$ stereotype und explizite Ehrlichkeitsdefinition (Kap. 6.2.2)

- $\quad$ altersstufenabhängige explizite Ehrlichkeitsregeln (Kap. 6.2.3)

- $\quad$ graduelle und kontextsensible Ehrlichkeitsregeln (Kap. 6.2.4)

- formale Struktur der expliziten Regeln (Kap. 6.2.5)

Diese vier formalen Sprechweisen über Ehrlichkeit werde ich im Folgenden anhand von Textbeispielen vorstellen. Damit ist der formalsprachliche Rahmen skizziert, in dem die Rekonstruktion von expliziten und impliziten Ehrlichkeitsregeln (vgl. Kap. 7, 8, 9) zu situieren ist.

\subsubsection{Marginalisierter Ehrlichkeitsbegriff in der Alltagssprache der Schülerinnen und Schüler}

SuS verwenden in den Interviewgesprächen den Begriff ,ehrlich' oder ,Ehrlichkeit' nicht in Eigeninitiative, obwohl in den Interviewgesprächen Themen des Schulalltags fokussiert werden, die mit Ehrlichkeit zu tun haben, beispielsweise Verhaltensweisen in Schultest oder der Umgang mit Elternunterschriften.

Das Attribut ,ehrlich“ benutzen Stephan, Clemens, Valentina und David allerdings in einer adverbialen Form, wie beispielsweise eerlich gsäit. Mit ,ehrlich“ ist damit nicht der wörtliche, sondern der rhetorische Sinn gemeint. Sie betonen damit den Inhalt und Realitätsbezug ihrer Aussagen. Zum Beispiel verwendet Clemens den Ausdruck, eerlich gsäit', um zu bestärken, wie sehr es ihn in den kurzen Unterrichtspausen stört, wenn die anderen ihn nicht in Ruhe eine Aufgabe fertig lösen lassen: „(1) Was mich schtöört, isch zum Biischpil- daas schtöört mich äigentli grausam, daas find ich (1) eerlich gsäit absoluut (1) birrewäich vo däne Chinde (1)“. Der Ausdruck, eerlich gsäit“ erlaubt Clemens zudem, den anschließenden vulgären Ausdruck absoluut birrewäich (im Sinne von dumm) im konventionell geführten Interviewgespräch einzuleiten. Damit kommt in diesem Kontext dem Ausdruck ,eerlich gsäit‘ eine Einleitungsfunktion zu.

Den Begriff ,Ehrlichkeit‘ führe ich im Zusammenhang mit der letzten Interviewleitfragestellung direkt ins Interviewgespräch ein, um die direkte Stellungnahme zum Ehrlichkeitsverständnis und zu ehrlichen Verhaltensweisen der SuS einzuholen (vgl. Anhang B). Interessanterweise greifen Regula, Emil, Nora, Stefan und David dabei nicht den Begriff ,Ehrlichkeit' oder ,ehrlich' in ihren Ausführungen auf. Zudem beobachtete ich, dass die anderen SuS das Attribut, ehrlich' häufiger verwenden als das Nomen ,Ehrlichkeit'.

Aus den genannten Feststellungen, dass die SuS den Ehrlichkeitsbegriff kaum verwenden, dokumentiert sich der geringe begriffliche Vertrautheitsgrad. Zugleich wissen die SuS sehr wohl über ehrliche Verhaltensweisen in der Schule zu berichten oder mit alternativen Begriffen eigene Ehrlichkeits- 
regeln kundzutun. Dass SuS Ehrlichkeitspraxen nicht unbedingt mit ,Ehrlichkeit' oder ,ehrlich' kennzeichnen, fordert auf, verstärkt zu verstehen, wie sie denn über Alltagspraxen sprechen, die mit Ehrlichkeit zu tun haben. Welche Denk- und Sprachmuster sind vorzufinden?

Beispielsweise zählt der Begriff ,Offenheit' oder ,offen' zu einem von den SuS oft verwendeten Begriffen, um in einem spezifischen Sinn über Ehrlichkeit zu sprechen. So benennt Sandra in Eigeninitiative ,Offenheit' als ein positives Verhalten der Mitschüler. Damit ist sie die einzige Schülerin, welche im Interviewgespräch Verhaltensweisen mit Offenheit in Bezug setzt.

\subsubsection{Stereotype und explizite Ehrlichkeitsdefinition}

In der Antwort auf die direkte Frage nach dem Ehrlichkeitsverständnis definieren die SuS Ehrlichkeit oft explizit mit der positiven und negativen Formulierung: Nicht lügen und Die Wahrheit sagen. Beide Formulierungen zugleich verwendet David: „(1) Wämmer halt nöd lüügt und wämmer d Waret säit.“

David spricht in einer distanzierten Weise über Ehrlichkeit, die im neutralen Subjekt (mer) gekennzeichnet ist. Der persönliche Bezug fehlt, was ihm ermöglicht, eine stereotype Aussage zu formulieren. In diesem Sinne kann zwischen Lüge und Wahrheit gewählt werden, ohne eine Alternativmöglichkeit zu berücksichtigen. Zwischen den beiden Extrempositionen finden sich jedoch keine Differenzierungen bzw. Abstufungen.

Neben den SuS, die eine solche stereotype und allgemeine Ehrlichkeitsdefinition verwenden, gibt es SuS, die sich keiner abstrakten Sprache bedienen. Diese erklären ihr Ehrlichkeitsverständnis anhand von Alltagsbeispielen. Eine dritte Gruppe kombiniert abstrakte Ansichten und Unterrichts- und Schulbeispiele.

Insbesondere in der letztgenannten Gruppe zeigen sich Ausnahmen von der polarisierenden und stereotypen Ehrlichkeitsdefinition. Ausgehend von stereotypen Ehrlichkeitsdefinitionen wird deren Eindeutigkeit mit Partikeln wie ,vielleicht' oder ,eher' relativiert. In den geschilderten Fällen geht es meistens um sozial unerwünschte Verhaltensweisen, die in die individuellen Ehrlichkeitsverständnisse zu integrieren sind. Dies zeigt beispielhaft die Aussage von Adrian auf: Bei unüberprüfbaren oder unwichtigen Verhaltensweisen ,dörf me schoo, un- (1) mängisch vilicht (2) nöd eerlich sii (2).“ Das Wiederansetzen des Satzes und der Einschub mängisch vilicht relativiert die rigide Ehrlichkeitsauffassung, pauschal, d. h. unter allen Umständen und in jedem Fall, ehrlich sein zu müssen.

Hiermit zeigt sich, dass SuS in Form von Polarisierungen und Stereotypen von Ehrlichkeit sprechen, wenn es darum geht, ein Ehrlichkeitsverständnis zu formulieren. Sobald sie Alltagsbeispiele beiziehen, relativieren sie die 
polarisierenden und stereotypen Ehrlichkeitsdefinitionen. Dieser Befund ist insofern interessant, da er zusätzlich aufzeigt, wie ausgehend von der Semantik und Form der Fragestellung die Antworten von Jugendlichen vorausgesagt werden können. Zielen die Fragen auf eine abstrakte Ebene ohne die Möglichkeit, eigene Alltagsbeispiele einzubringen, so besteht die Gefahr, dass die Antworten ebenfalls auf einer abstrakten Ebene erfolgen. Wenn hingegen die Fragen inhaltlich und formal offen gestellt und die Antworten nicht vorgegeben sind, steht es den SuS dennoch frei, stereotype Ehrlichkeitsverständnisse zu nennen. Zudem erhalten sie damit den Freiraum, anhand von eigenen Ehrlichkeitspraxen in der Schule zu berichten, aus denen explizite und implizite Ehrlichkeitsregeln gewonnen werden können. Zusammenfassend unterstreicht dieser Befund, dass Ehrlichkeitsdefinitionen zwar allgemeingültig sind, aber nur einen Teilbereich der Ehrlichkeitspraxis abdecken. Hingegen ermöglicht ein offener und explorativer Forschungszugang, explizite und implizite situations- und kontextabhängige Ehrlichkeitsregeln zu entschlüsseln.

\subsubsection{Altersstufenabhängige explizite Ehrlichkeitsregeln}

Wie bisher gezeigt, verstehen viele SuS unter Ehrlichkeit Nicht verpetzen und Nicht spicken (vgl. Kap. 6.1). Gerade bei diesen beiden Ehrlichkeitsregeln grenzen sich Marco, Valentina, Nora und Ilona explizit von Ehrlichkeitsregeln der Kindheitsphase ab: In der 3. Oberstufe wird nicht verpetzt. Das war in der Primarschule so (Valentina) und früher ebenso (Ilona). Marco geht sogar bis zur Kindergartenzeit zurück und äußert sich in Abgrenzung zu dieser Altersphase wie folgt:

Ich persönlich find das [Verpetzen] zimlich (1) Schäisse, wänn mer so öppis macht. (2) Und mir sind äigentli au us dem Alter use und das find ich äigentli guet. (1) Will im Chindergarte macht mer das no und ich hasse so Sache@1@.

Aus den negativ abgrenzenden Aussagen gegenüber jüngeren Klassenstufen spricht eine Souveränität der gegenwärtigen Ehrlichkeitsregeln. Gleichzeitig greift Marco das Wort verpetzen nicht auf, sondern verweist auf dieses mit neutralen Relativpronomen öppis und das. Dieser formalsprachliche Hinweis unterstreicht zum einen die negative und distanzierte Haltung gegenüber der Tätigkeit des Verpetzens. Zum anderen kann diese Haltung von einem Schamgefühl geprägt sein, wodurch ein solches Wort nicht in den Mund genommen werden will. Am Anfang der Schulzeit, in diesem Fall des Kindergartens, wurde noch gepetzt, aber jetzt sind die SuS in einer anderen Altersphase. Anscheinend soll die Bezugnahme auf die früheren gegenteiligen Regeln die gegenwärtigen stärken. In diesem Sinne erklärt Nora, weshalb sie heute während der Schultests nicht mehr spickt: „Früener han ich s au 
gmacht- no i de Primaar. Aber jetzt mach ich s nüme will- will ich wett au öppis leere."

In der Primarschule verwendete Nora also unlautere Hilfsmittel während der Schularbeiten. Die Tätigkeit des Spickens bezeichnet sie mit dem sachlichen Relativpronomen $s$, was eine distanzierte Haltung zum Gegenstand signalisiert. Interessant ist, dass Nora den Istzustand mach ich s nüme fliessend feststellt, jedoch bei der Begründung den Sprechfluss absetzt und das Konjunktivpronomen weil verdoppelt verwendet. Hier dokumentiert sich, dass es Nora einfacher fällt, über einen Sachverhalt zu berichten, als Reflexionen dazu anzustellen.

Das ausdruckstarke Worte Schäisse und das moralische Bewertungsadjektiv guet sind emotional geladene Ausdrücke, in welchen die Brisanz der Abgrenzung gegenüber Jüngeren dokumentiert ist. Zudem ist hier ein Paar von einem dichotomen Denkmuster ,Schäisse" als etwas Negatives und Schlechtes gegenüber dem Guten impliziert.

Darüber hinaus ist Clemens der Ansicht, dass das Thema Ehrlichkeit als konventionelle Verhaltensweise nur in der ersten Oberstufe im Fach Mensch und Umwelt thematisiert werden kann, aber nicht mehr in der dritten Oberstufe:

(2) I de dritte Klass eerlich gsäit nöd, aber i de eerschte Klass chönnd me soo halt Verhaltenssache scho präsentiere.

Der Zeitbezug kann auf zwei Arten gelesen werden: Entweder ist man in der dritten Oberstufe so weit, dass die konventionellen und unkonventionellen Regeln klar sind, wann Ehrlichkeit und wann Unehrlichkeit angebracht ist. Dadurch kann die Thematisierung von Ehrlichkeit in der dritten Oberstufe keine nachhaltigen Folgen haben. Oder die Förderung konventionelle Ehrlichkeitsregeln ist wirkungslos. Ausgehend vom Interviewkontext passt die erste Lesart, denn Clemens inszeniert sich und ebenso den Klassenverband als souveräne Menschen, die cleverer sind als die Lehrpersonen. Er und seine Mitschüler sind an die Unterrichtssituationen äußerlich angepasst und zugunsten der Kollegialität des Klassenverbands je nach dem gezielt ehrlich oder unehrlich (vgl. Kap. 8.2).

Die ausgewählten Interviewpassagen zeigen einen expliziten Entwicklungsprozess und eine Art und Weise, über Ehrlichkeit und Ehrlichkeitspraxen zu sprechen, dass SuS sich von früheren Ehrlichkeitsregeln abgrenzen und ihre neuen Ehrlichkeitsregeln als altersgerechten, souveränen und positiven Entwicklungsschritt präsentieren.

\subsubsection{Graduelle und kontextsensible Ehrlichkeitsregeln}

Insbesondere Sandra und Lilian sprechen implizit in graduellen Kategorien von Ehrlichkeit. Dazu führt Lilian in einer Selbstbeobachtung aus: 
Ich, also ich bin dänn au mängisch echli zu eerlich, (1) was dänn au chan verletze und so, aber (1) ich find es isch d Warhäit gsii.

Lilian fügt eine Messbarkeit und Veränderbarkeit von ehrlichem Verhalten ein: Man kann mehr oder weniger ehrlich oder auch $z u$ ehrlich sein. In ihrem Fall ist sie zuweilen zu ehrlich, womit sie andere verletzen kann. Obwohl die Angesprochenen die zu starke Ehrlichkeit von Lilian negativ beurteilen, steht Lilian zu ihren wahren Aussagen. Damit liefert Lilian ein pragmatisches und messbares Argument für graduelle Ehrlichkeitsregeln in Deutungen von Ehrlichkeitspraxen im Gegensatz zum dichotomen Ehrlichkeitsverständnis ehrlich versus unehrlich.

Sandra ist der Auffassung, es sei ehrlich, wenn SuS nur auf Anfrage der Lehrperson Auskunft über Schulangelegenheiten geben (vgl. auch Kap. 6.1.4). Gleichzeitig findet sie es aber noch ehrlicher, wenn SuS direkt ohne Anfrage der Lehrperson Auskunft geben:

Aber (1) ich find jetzt wämer vo sich uus, sälber zu dere Person gaat und öppis verzelt, das isch au eerlicher.

Die beiden von Sandra geschilderten Verhaltensweisen sind also beide ehrlich, unterscheiden sich aber in der Intensität: Ehrliche Verhaltensweisen in Selbstinitiative sind ehrlicher als ehrliche Verhaltensweisen auf Anfrage hin. Sandra verwendet die Vergleichsfolie, um Ehrlichkeit als Regel in Bezug der Kategorie der Aufrichtigkeit zu erklären. Unhinterfragt bleibt, ob eine Auskunft wahrheitsgetreu sein soll. Somit gibt es einerseits ein Übermaß an Ehrlichkeit und andererseits ein mehr oder weniger ehrliches Verhalten, wobei das ,weniger ehrliche Verhalten' noch nicht zur Unehrlichkeit zählt. Hieran dokumentiert sich, wie differenziert SuS über ehrliche Verhaltensweisen berichten.

Zudem vertreten viele SuS unterschiedliche Ehrlichkeitsregeln, die aus den Ehrlichkeitspraxen generiert und gedeutet werden. Daher ist es nicht in jeder Unterrichtssituation angebracht, ehrlich $\mathrm{zu}$ sein (vgl. insbesondere Kap. 8, 9). Das situationsgebundene Merkmal von Ehrlichkeitsregeln wird insbesondere in erzählten Unterrichts- und Schulbeispielen der SuS sichtbar. Zwar sprechen sie in den berichteten Praxen nicht explizit von Ehrlichkeit; jedoch werden die Merkmale von Ehrlichkeitsregeln explizit und implizit aus dem Material ersichtlich: Die scheinbar statischen Ehrlichkeitskategorien und Ehrlichkeitsdefinitionen, die einzelne SuS in ihren anfänglichen Ausführungen aufgreifen, relativieren sie mit der subjektiven Deutung von Ehrlichkeitspraxen im Unterricht und in der Schule. 


\subsubsection{Formale Struktur der expliziten Ehrlichkeitsregeln}

Wenn SuS über Ehrlichkeit reden, dann tun sie dies auffallend häufig anhand von Wenn-dann-Sprachkonstruktionen. Diese sind formal insbesondere mit dem Konjunktiv wenn oder mit der Konstruktion wenn - dann gekennzeichnet. So formuliert z. B. Christoph eine explizite Ehrlichkeitsregel wie folgt: „Eerlichkäit isch, wänn me zu siinere Mäinig schtaat.“ $\mathrm{Zu}$ dieser abstrakten Regel führt er einige Unterrichtsbeispiele an. Beispielsweise beurteilt er es als negativ, wenn Mitschülerinnen und -schüler, wie unlängst geschehen, diese Regel nicht einhalten (vgl. Chiapparini 2011, S. 185). Die Mitschüler gaben in diesem Fall nicht zu, dass sie im Schulzimmer bei Abwesenheit der Lehrperson geraucht haben.

Die formale Struktur Ehrlichkeit ist/Ehrlich ist/Das ist, wenn ... kommt in den Schilderungen besonders oft vor. Regula verwendet die komplexere Struktur von Ehrlichkeitsregeln ,wenn - dann', beispielsweise für den Fall, dass bei guten Noten die Elternunterschrift vergessen wurde einzuholen und daher von Mitschülerinnen und -schülern oder von ihr selber gesetzt wird:

(3) Und es isch au nöd nume bi de schlächte Noote ebe, ich mäine, wänn- (2) wänn mer ebe e gueti Noote hät und d Prüefig vergisst, dänn (1) macht mer s trotzdem no churz, will suscht bechunt mer halt en Iitraag (3).

Aus dieser Textpassage geht Regulas implizite Verhaltensregel hervor: Wenn ein Schüler eine gute Note schreibt, aber vergisst, die Prüfung von den Eltern unterschreiben zu lassen, dann setzt sie ihre Unterschrift selber - ist unehrlich aber zulässig, um keinen Eintrag ${ }^{23}$ zu kassieren. Einträge gelten bei Regula als wirksames Sanktionierungsmittel. Die Struktur der expliziten Ehrlichkeitsregel hier ist komplex: Wenn ..., aber ..., dann ..., um ... Implizit enthält diese Passage die Ehrlichkeitsregel: Unehrlichkeit durch Unterschriften fälschen ist legitim, wenn vergessen ging, die Unterschrift der Eltern einzuholen, und wenn ihnen nichts verheimlicht werden sollte.

Die SuS formulieren explizite Wenn-dann-Ehrlichkeitsregeln sowohl in Form von abstrakten Gedanken als auch in Form von abstrahierten Beispielen. Darüber hinaus lassen sich implizite Ehrlichkeitsregeln aus beschriebenen oder erzählten Beispielen rekonstruieren. In den Textpassagen dokumentiert sich ein Regeldenken der SuS, das teils von ihren Definitionsbemühungen geprägt ist, aber vermutlich auch von einer schulischen Sozialisation in einer solchen Regeldenkweise gefördert werden. Allerdings liegen bislang nur Befunde von ,schulischen Logiken“ (Jünger 2010) und Spannungen zwi-

23 Im Zürcher Schulsystem wird das Verhalten der SuS während dem Unterricht zeugniswirksam beurteilt. Daher sind Lehrpersonen beauftragt, negative Verhaltensweisen der SuS in deren Kontaktheft zu notieren (= Eintrag). Bei 12 Einträgen wird ein Abzug im Schulzeugnis vorgenommen. 
schen Schulordnung und Gleichaltrigenkultur (Boer und Deckert-Peaceman 2009) vor, die sich auf das Vorschulalter und die Primarschule beziehen.

\subsubsection{Zusammenfassung}

Das Unterkapitel zu den Thematisierungsregeln hat gezeigt, dass in den Aussagen der SuS eine Abgrenzung zur jüngeren Altersstufe, eine Kontextsensibilität und eine Gradualität des Ehrlichkeitsbegriffs zu entnehmen ist. Bei Letzterem thematisieren SuS Ehrlichkeit oft explizit oder implizit in formalen Regeln: Dazu verwenden sie häufig die einfache Sprachfigur Wenn ... und die komplexere Wenn ... dann...

Darüber gilt Ehrlichkeit bei den Jugendlichen nicht als eingespielter Begriff aus der Alltagssprache. Auf Anfrage der Interviewerin verwenden die SuS den Ehrlichkeitsbegriff - mit einzelnen Ausnahmen eines Einstiegspassus - nicht statisch und nicht aufgrund einer stringenten Definition, wie dies aus der Erziehungsphilosophie und aus populären Debatten zu Tugenden bekannt ist. Es scheint gar, als handle es sich hierbei ausschließlich um einen Begriff der Erziehenden und damit der Erwachsenenwelt, der im Sprachgebrauch der SuS als solcher kaum vorkommt. Daher ist die nachfolgende Analyse der Ehrlichkeitsregeln von SuS nicht auf das Wort ,Ehrlichkeit' oder ,ehrlich“ ausgerichtet. Vielmehr suche ich nach expliziten und impliziten Ehrlichkeitsregeln, an denen die SuS ihre Verhaltensweise in der Schule orientieren.

\section{Von konventionellen Ehrlichkeitsregeln zu Ehrlichkeitsregeln der Schülerinnen und Schüler}

Von der Schule wird das Einhalten schulspezifischer Ehrlichkeitsregeln, die den SuS bekannt sein sollen und das schulische Zusammenleben fördern, wie beispielsweise Nicht schummeln, stillschweigend erwartet (vgl. Kap. 3.3). Diese schulspezifischen und vorausgesetzten Ehrlichkeitsregeln ordne ich unter der Kategorie der konventionellen Ehrlichkeitsregeln ein. Natürlich stellt der schulische Kontext gewisse Ehrlichkeitsregeln auf, die als solche in anderen Lebensbereichen anders vorzufinden sind.

Innerhalb der konventionellen Ehrlichkeitsregeln differenzieren sich je nach Situation und Kontext folgende thematische Gruppen an Ehrlichkeitsregeln: Ehrlichkeitsregeln als Schulregeln (Kap. 7.1), die Lehrperson als Orien- 
tierungspunkt für Ehrlichkeitsregeln (Kap. 7.2), fachspezifische Variation von Ehrlichkeitsregeln (Kap. 7.3).

Die von den SuS wahrgenommenen konventionellen Ehrlichkeitsregeln zeige ich als Nächstes auf und verweise zugleich auf deren Grenzen.

\subsection{Zusammenhang von Ehrlichkeitsregeln und Schulregeln}

Aus der eigenen Schulerfahrung sind gewisse schulspezifische Ehrlichkeitsregeln wohl bekannt, die auch heute noch ihre Geltung beibehalten haben und von denen die SuS berichten. Insbesondere zählen dazu: Nicht schummeln, Ungelöste Hausaufgaben nicht verheimlichen und Elternunterschriften nicht selber setzen. Es geht darum, selbstständig Schultests zu schreiben, ungelöste Hausaufgaben einzugestehen oder noch besser, Hausaufgaben selbstständig zu lösen, und sich derer zu erinnern, Schultests und andere Blätter den Eltern einsehen zu lassen und mit deren Originalunterschrift abzugeben. Bei dieser Auflistung fällt auf, dass es sich vorerst nur um Ehrlichkeitsregeln handelt, die kontrolliert werden können.

\subsubsection{Nicht schummeln als bekannte aber überschrittene Schulregel}

Die Schule gibt klare Regeln vor, was zu einer ehrlichen und was zu einer unehrlichen Verhaltensweise gehört. Darüber sind die SuS informiert. In ihren Ausführungen benennen sie selten diese Regeln direkt, häufiger implizit. Beispielsweise gehen alle $\mathrm{SuS}$ von der Untersagung von unlauteren Hilfsmitteln während Schultests aus. Allerdings bezeichnen allein Valentina und Nora die Verwendung von unlauteren Hilfsmitteln als Betrug, und dies in einer ihnen eigenen Weise.

Valentina streicht den kollegialen Aspekt heraus:

Es isch au so Kolleege und echli mitenander hälfe, find ich schöön@1@, obwool s ja echli Betrug isch, ja das isch @1@, äigentli hät s scho jede maal gmacht, äigentli (5).

Die Schulregel, dass Schultests nicht zusammen gelöst werden sollen, anerkennt Valentina; sie bezeichnet einen Verstoß gegen die Verhaltensweise mit dem Ausdruck ,Betrug ${ }^{6}$ - ein Terminus aus dem kriminellen Bereich. Dennoch erklärt Valentina ihr Verhalten aus einer Perspektive des Klassenzusammenhangs als kollegial und aus einer ästhetischen Perspektive als schön. Die Spannung zwischen der Schulregel und der kollegialen Regel ist offensichtlich. Sie wird durch das punktuelle Lachen, durch die Verkleinerungsformen echli und die Wiederholungen äigentli unterstrichen. ,Äigentli‘ ist ein interessantes Adverb, welches auf eine Doppelwahrheit hinweist: auf das, 
was sein sollte, und das, was ,äigentli‘ ist. Anders ausgedrückt: Die Schulregel gegen unlautere Hilfsmittel während Schultests gilt, und bei Regelbruch liegt ein Betrug vor, was ebenfalls vorkommt und daher ebenfalls gilt.

Allerdings gewichtet Valentina den kollegialen Aspekt beim Regelbruch höher als die Regel selbst. Zudem legitimiert sie ihre Ehrlichkeitsregel, indem sie das Argument der allgemeinen Praxis beizieht: „... alle haben es bereits einmal gemacht." Somit ist festzuhalten, dass die Schulregel Valentina dazu nutzt, um ihre kollegiale Regel zu favorisieren.

Besagte Schulregel thematisiert auch Nora, nachdem sie ihre Vermutung preisgibt, dass die Lehrpersonen die unlauteren Hilfsmittel während der Schultests entdecken und sehen. Allerdings intervenierten diese nicht, weil die SuS sich damit selbst betrügen:

$\mathrm{S}$ isch äigentli- $\mathrm{s}$ isch ja sälber Betruug. Und ich machs au nöd- früener han ich $\mathrm{s}$ au gmacht- no i de Primaar. Aber jetzt mach ich s nüme will- will ich wett au öppis leere und (1) ich gsee au nöd wie guet, dass ich bi, wänn ich alles abschriibe (4).

Während Nora in der Primarklasse zuweilen schummelte, haben sich inzwischen ihre Ehrlichkeitsregeln gegenüber Schummeln geändert. Gegenwärtig hält sie sich an diese Schulregel - jedoch nicht blindlings, sondern aus genau zwei Gründen: aus der Motivation, etwas zu lernen, und als Leistungskontrolle.

Sowohl Valentina als auch Nora kennen also sehr wohl die Schulregel Nicht schummeln während der Schultests und bezeichnen diese Tätigkeit als ,Betrug'. Sie orientieren daran ihre individuellen Regeln. Dabei differenziert bzw. relativiert Valentina die Regeln, indem sie der kollegialen Regel der gegenseitigen Hilfe den Vorrang lässt. Nora hingegen folgt ausschließlich der Schulregel, und zwar mit der Begründung, selbst lernen zu wollen.

Die Schulregel des Nichtschummelns ist ein Nichtdürfen, was Stefan als solches bezeichnet: „Dörfet mer nöd.“ Die Regel ist durch die Negation, das neutrale und kollektive Subjekt mer und durch das Modalverb dürfen klar als distanzierte und statische Regel gekennzeichnet. Einige Zeilen später erzählt derselbe Schüler in einer Selbstverständlichkeit, wie jede und jeder in seiner Klasse schummelt, beispielsweise indem das Lösungsblatt unter dem Pullover versteckt oder die Lösung auf die Hand geschrieben wird.

Die Schulregel und die praktizierenden Regeln scheinen sich auf zwei Bedeutungsebenen zu situieren, sodass sie für Stefan keinen Widerspruch bilden. Die Unterscheidung widergespiegelt sich in der Textsorte, in der die Regeln formuliert sind: Bei der Schulregel handelt es sich um eine Argumentation, und die praktizierte Regel ist in Form von Beschreibungen und einer Erzählung eingebunden. 


\subsubsection{Hausaufgaben als Gefährdung von ehrlichem Verhalten}

Ein großes Thema der SuS sind die Hausaufgaben - ein Themenfeld, das ich im Leitfaden nicht berücksichtigt habe, dessen Vergessen mit ehrlichem Folgeverhalten zu tun hat und das die Befragten von selbst in verschiedenen Zusammenhängen erwähnen. Insbesondere gilt das Lösen von Hausaufgaben als unbeliebte Schulregel. Aus der Sicht der Schule wird das Verheimlichen von ungelösten oder vergessenen Hausaufgaben als Unehrlichkeit und Regelbruch verstanden, das Konsequenzen, wie beispielsweise einen Eintrag ins Kontaktheft, nach sich zieht.

Fabian klagt darüber, dass diese Schulregel von den Lehrpersonen eingesetzt wird, ohne an die vielen Hausaufgaben der SuS zu denken:

Ich mäine mer chan nöd verlange, dass mer das und das und dänn no das und das. Also d Leerer söllet emaal im Schtundeplan ineluege, wänn s zum Biischpiil gseend- Mir händ s Klassenbuch, wo alles ufglischtet ist, aber wänn mer s gseet, guet morn hämmer zwei Tescht und übermorn au wider eine und dänn chan mer nöd verlange, dass mer hundertprozent d Ufzgi debii hät, will, ja, mer hät äifach anders und me will au no Freiziit haa. (1) Okey, das isch äigentli seer sälte. Würkli en schöne- en freie Tag, aber (2), jaa also mer chan nöd immer, dass mer hundertprozent d Ufzgi hät.

Die Schulregel des Hausaufgabenlösens wird in dieser Textpassage als Erwartung der Lehrpersonen definiert, die sich nicht mit den Bedürfnissen und der Zeiteinteilung von Fabian deckt. Wenn zu viele Tests anstehen, kommt Fabian der Schulregel des Hausaufgabenlösens nicht nach, was ihm legitim erscheint. Denn die Schule auferlegt, Hausaufgaben zu lösen, ohne die SuS nach Zeitressourcen und nach deren Bedeutung von Hausaufgaben zu fragen. Die zu vielen Hausaufgaben als strukturelle Eigenschaft des Unterrichts beeinflussen und gefährden das ehrliche Verhalten. Ein wichtiges Bedürfnis von Fabian ist auch die Freizeit, die kaum vorhanden sei. Hausaufgabenlösen ist für Fabian negativ besetzt, weil es die Freizeit einschränkt oder verhindert. Die Hausaufgaben sind nicht mit Lernmotivation verknüpft, sondern mit Gefährdung der Freizeit. Daraus gewinnt Fabian das Selbstverständnis, dass Hausaufgaben nicht immer gelöst werden können.

Somit dient die Schulregel des Hausaufgabenlösens dazu, individuelle oder kollektive Regeln kontrastierend zu dieser zu bilden. Die Schulregel: Hausaufgaben müssen gelöst werden fordert die SuS auf, alternative Regeln zu bilden, die aus der Sicht der Lehrpersonen unaufrichtig, aber aus der Sicht der SuS meistens durch kollegiale oder individuelle Regeln legitimiert sind. 


\subsubsection{Elternunterschrift nicht selber setzen: Ausnahmen und Resistenz}

Wenn die Lehrperson vergessene oder ungelöste Hausaufgaben entdeckt, wozu auch das Vergessen von Elternunterschriften gehört, oder wenn eine Schülerin oder ein Schüler sich nicht an die im Unterricht erwarteten Umgangsformen hält, besteht eine schulische Ehrlichkeitsregel darin, diesen Missstand in das Kontaktheft einzutragen, das jeder SuS mit sich führt. Das wird als Iitraag von den SuS bezeichnet und muss von den Eltern eingesehen werden, was mit deren Unterschrift bestätigt wird, die wiederum vergessen werden kann.

Elternunterschriften setzten die SuS weniger, weil sie keine schlechte Prüfungsnote zu Hause zeigen wollen, sondern vielmehr weil sie diese vergessen haben einzuholen, was Regula bestätigt und so ausdrückt: „Mir passiert daas mee, (1) als dass ich si fälsch will ich e schlächti Noote ha.“"

Hingegen zählt Adrian zu den SuS, die Unterschriften nicht selber setzen und die sich somit an die schulische Ehrlichkeitsregel hält: Unterschriften werden nicht selber gesetzt. Das ist auch durch den moralisch negativ geprägten Begriff fälsche unterstrichen, den Adrian in Eigeninitiative verwendet. Ausgehend von einem aufgedeckten Vorfall in der 6. Klassen zieht er folgenden Schluss:

... und (2) deet isch s n- isch s zwar no ggange, (1) aber jetzt i dem Alter macht mer sich scho zimli schtraafbar. (1) Deet isch es i de Primaarschuel gsii und deet isch s dänn- (2) irgendwänn hät s äifach s Elteregschprööch und soo gee. (3) Aber jetzt git s nüme so vill, will me wäiss, wänn mer entdeckt wird, dass- (2) Konsequänze sind zimli groos (2), mit de Underschriftefälsche und so (6).

Adrian nennt nicht direkt die schulische Ehrlichkeitsregel: Elternunterschriften dürfen nicht selber gesetzt werden. Stattdessen appelliert er an die Folgen wie Strafbarkeit und Konsequänze in Abgrenzung zu früher. Konkret gibt er keinen Vorfall an, in dem diese Konsequenzen ausgesprochen wurden. So ist für Adrian die schulische und von ihm unausgesprochene Ehrlichkeitsregel bedeutungsvoll und als internalisiert präsentiert.

Zusammenfassend werden Elternunterschriften zweckgebunden, um direkte und größere Konsequenzen (Einträge) zu meiden, selber gesetzt. Gleichzeitig gibt es SuS, die keine Elternunterschrift selber setzen.

\subsubsection{Zusammenfassung}

Schulische Ehrlichkeitsregeln wie Nicht schummeln, Hausaufgaben lösen oder Elternunterschrift nicht selber setzen stehen in einem Zusammenhang mit allgemeinen Schulregeln, die den SuS bekannt sind. Die meisten SuS verfügen über unkonventionelle Ehrlichkeitsregeln. Dazu konnte ein erstes 
Motiv gewonnen werden: größere Sanktionen durch die Lehrperson zu vermeiden. Hier zeigt sich zudem, dass Ehrlichkeitsregeln nicht als eine Auflistung von Regeln anzutreffen sind, sondern aus dem Schulalltag zu generieren sind.

\subsection{Orientierungspunkt für Ehrlichkeitsregeln: Die Lehrperson}

Über Lehrpersonen haben die SuS unaufgefordert viel zu berichten. Die Gespräche beispielsweise in den Gruppendiskussionen sind vom Thema Lehrpersonen stark geprägt, sodass das Thema Ehrlichkeit zuweilen in den Hintergrund rückt. Allerdings geht aus dem Datenmaterial der Einzelinterviews hervor, wie bedeutsam die Rolle der Lehrperson bezüglich der ehrlichen und unehrlichen Verhaltensweisen ist. Daher zeige ich als Erstes auf, wie die SuS die Lehrerrolle wahrnehmen. Zweitens ist herauszuarbeiten, wie sie die Kontrollfunktion der Lehrperson deuten, und schließlich, wie relevant die Sympathie oder Distanz zur Lehrperson ist.

\subsubsection{Punktuelle Widerstände und grundsätzliche Anpassung an personalisierte Ehrlichkeitsregeln}

Die meisten SuS nehmen die Lehrperson als personalisierte Regel für Verhaltensweisen wahr, was auch für die Ehrlichkeitsregeln zutrifft. Mit deren Regeln stimmen die der SuS nicht immer überein. So kann beispielsweise Regula kaum verstehen, weshalb sich die Lehrer aufregen, wenn die Hausaufgaben nicht gelöst und deshalb abgeschrieben werden:

Ich dänk bi de- bi de Ufzig oder so, wänn mer- wämmer si ebe ämaal vergisst und öpper irgendwie, käi Aanig öpper vom andere irgendöppis chan nee, dänn find ich s egaal. (2) Ich wäis nöd, vilicht schtöört das d Leerer würkli, aber (2) also ich find s äigentli nöd so schlimm (1).

Regula ordnet das Vergessen der Hausaufgaben oder das Abschreiben von den anderen nicht negativ ein und hat deshalb kein Problem damit. Hingegen nimmt sie wahr, dass solche Taten der SuS für die Lehrpersonen ein Problem sein können und dies sie deshalb schtöört. Hier ist sehr schön erkennbar, wie eine abstrakte Regel auf die persönliche Einschätzung einer Lehrperson runtergebrochen wird. Nehmen wir das Beispiel des Abschreibens von Hausaufgaben, was allgemein untersagt ist. Auf diesen Fall angewendet, wird diese Ehrlichkeitsregel personalisiert als Regel des Lehrers, der sich daran stören 
kann, wenn diese nicht eingehalten wird. Damit ist die Ehrlichkeitsregel vom Lernauftrag abgekoppelt und legitimiert.

Die Rolle der Lehrperson als personalisierte Ehrlichkeitsregel wird von den Lehrern selbst unterstrichen. Sandra erzählt von einer Prüfungsstunde, in der die Lehrerin fälschlicherweise meinte, Sandras Kollegin beim Spicken erwischt zu haben:

Irgendöppis isch gsii. (1) Si hät irgendwie s Fäischter zue taa oder so. Dänn hät d Leereri dänkt: „Du häsch gschpickt“ und so. Dänn hät si irgendwie en Äiner überchoo. Debii hät si gar nöd gschpickt. Und all händ gsäit: ,Ja si, si hät nöd gschpickt. Sie hät nur s Fäischter wele zue tue.“ Dänn hät si gsäit: „Ja, ich laa mich nöd so hindergaa“"und @so@ (2) Ja.

Sandra nimmt wahr, dass sich die Lehrperson hintergangen fühlt. Sie erzählt die Begebenheit, in dem sie die Lehrerin als handelnde Person schildert und die Mitschülerin als Opfer. Ob tatsächlich ein Nichteinhalten der Ehrlichkeitsregel vorliegt oder nicht ist mit der Lehrperson verknüpft und wird in diesem Fall sekundär behandelt. Zentral ist die benachteiligte Schülerin und die Lehrerin, die sich hintergangen fühlt und für die aus der Perspektive von Sandra ungerechte Behandlung. In diesem Sinne schiebt auch Fabian den Lehrpersonen die Verantwortung für das Nichtlösen von Hausaufgaben und das Beiziehen von unlauteren Hilfsmitteln zu (vgl. Kap. 7.1.2).

Nicht nur die Verantwortung wird den Lehrpersonen zugespielt, sondern auch die Richterfunktion, die mit dem Verteilen von Einträgen zusammenhängt. So berichtet Marco darüber, wann Einträge tatsächlich vorgenommen werden und wann nicht:

(1) Es chunt drufaa bi welne Leerer. (2) Aber mängmaal git s käine und mängmaal git $s$ äine, wänn- Es chunt mängmaal au uf d Luune vom Leerer drufaa (5).

Der Lehrperson kommt gemäss Marco eine Entscheidungsfunktion zu, die von der einzelnen Lehrperson oder von deren Gemütszustand (Luune) abhängt. In dieser Passage dokumentieren sich die Grenze einer transparenten Ehrlichkeitsregel und deren Ausnahmefälle, die die Lehrperson scheinbar willkürlich festsetzt.

An dieser Stelle könnten einige Überlegungen zur Rolle der SuS interessieren. Aus dem Interviewmaterial geht klar hervor, dass die meisten SuS sich den schulischen Ehrlichkeitsregeln und den Regeln der Lehrpersonen grundsätzlich fügen oder dass sie heimlich Notlösungen finden. Nur einer der Klassen gelang es, sich durch ihr eigenwilliges Verhalten und die Unterstützung der Eltern gegen eine Lehrerin durchzusetzen. Anlass war, dass die SuS nach ihrer Auffassung nichts lernten, was alle vier Interviewten derselben Klasse von sich aus thematisierten. Eine positive Haltung nimmt Clemens für seine Schule insofern vor, als er im Abschlussvotum des Interviews die Offenheit seiner Schule honoriert:

[...] ich wett emaal säge die Schuel (1) isch relativ offe für alles. Also solang me chan, irgendwie rede oder zum Leerer gaa, ich würd gern, daas und daas mache. Dänn wird daas 
au so gaa. Daas isch seer guet, a dere Schuel- find ich jetzt. (2) S git au da weniger Schlegereie. Ich würd einmal säge, mir sind daa, mee multikulti als im X oder soo (3).

Clemens' Schulhaus zeichnet sich durch kulturelle Vielfalt aus und beschäftigt offene und zugängliche Lehrkräfte. Weil das positive Statement von Clemens eine allgemeine Äußerung ist, wird nicht klar, ob Ausnahmen bewusst nicht erwähnt sind. Jedenfalls begrüßen die SuS, wenn ihre Sichtweisen von Lehrpersonen oder Erwachsenen berücksichtigt werden. Diesen Eindruck bestätigen nicht wenige SuS während des Interviews. Beispielsweise freut sich Christoph, der aus einer streng geführten Klasse kommt, seine Sichtweise kundzutun:

Also ebe ich find s guet, dass me au äinisch über d Mäinig vo de Schüeler tuet öppis mache, schriibe so. (1) Ja. (1) Ich find s äifach guet (3).

Aus der Perspektive von Christoph besteht ganz allgemein kein großes Interesse daran nach der Sichtweise der SuS zu fragen. Andere Sichtweisen, die er aber nicht näher ausführt, sind scheinbar bekannt und setzen sich durch. Der Terminus äinisch weist auf den Ausnahmefall hin und bestätigt damit den Regelfall: Andere Meinungen als die der SuS sind relevant.

Ilona stellt die Sichtweise der SuS denen der Lehrpersonen gegenüber:

(1) Ebe ich find au interessant, dass mal öpper- Das s us de Sicht vo der Chinder und vo de Schüeler- ää@vo de Chinder@und nöd vo de Leerer (1) gsee wett. Ja (4).

Aus der Textpassage geht erneut hervor, dass die Sichtweise der SuS nicht so sehr gefragt ist wie die der Lehrpersonen. Zu berücksichtigen ist hierbei: Die $\mathrm{SuS}$ waren vor dem Interview informiert, dass Umgangsformen das Gesprächsthema sein werden. Verbunden mit der obigen allgemeinen Aussage kann somit Folgendes festgehalten werden: Den SuS ist bewusst, dass sie üblicherweise nicht nach ihrer Sichtweise zu Umgangsformen gefragt werden.

Dies ist insofern eine interessante Feststellung, weil die ersten Analysen darauf hinweisen, dass die schulische Ehrlichkeitsregeln oder die Regeln der Lehrperson zwar respektiert, doch mit individuellen und kollegialen Regeln ergänzt, modelliert oder ersetzt werden. Hier dokumentiert sich die erzieherische Partizipation und Eigentätigkeit der SuS, was ein Potenzial umreißt, das bislang Erziehungsbeauftragte noch zu wenig berücksichtigen und nutzen.

\subsubsection{Erwartung an Kontrollinstanz und Angst}

Die Lehrperson übt eine Kontrollfunktion aus, indem sie die Hausaufgaben kontrolliert, bei Schultests das selbstständige Arbeiten oder das Einholen von Elternunterschriften für erledigte Schultests oder für Absenzen überprüft. Dabei setzen nicht alle Lehrpersonen die gleichen Maßstäbe an. Nur in einer der vier Klassen zieht der Klassenlehrer die Unterschrift der Eltern nicht 
mehr ein mit der Begründung, dass die SuS inzwischen genug groß seien. Grundsätzlich aber kontrollieren die meisten Lehrpersonen, ob die SuS ihren Pflichten nachkommen und führen durchweg ein Kontaktheft. Allerdings sehen nicht alle SuS ein, weshalb ein Eintragsheft $\mathrm{zu}$ führen sei. Fabian drückt seinen Unmut so aus:

Die Iiträg daa, wo mer hät, die sind au lächerlich. Was gits für Konsequänze, also en Iitraag sorry, so e komischi Underschrift. Mee git s da nüm. Und (1) wänn mer zwölf hätt gits waarschinli en Zügnisiitraag oder so. Das bringt doch gar nüüt. Me söll da lieber öppis anders mache, wie zum Biischpiil (1) so e Schtrichlilischte.

Fabian sieht keinen Nutzen in der Führung des Kontaktheftes mit den Einträgen. Bei zwölf Einträgen gibt es einen Vermerk ins Schulzeugnis, was - so Fabian - für die Lehrstellensuche relevant sei, aber keine Verhaltensänderung der SuS hervorbringe. Das Verb bringt ... nü̈̈t kann zweifach interpretiert werden: als ,keine Verhaltensänderung ' oder ,kein persönlicher Gewinn`. Hingegen kann beides beim sanfteren Prinzip der Strichliste berücksichtigt werden, wie es ein Lehrer aus der Grundstufe praktizierte. Dieses Prinzip ist flexibler, weil jede Woche ein Strich abgezogen wird und weil bei einer gewissen Anzahl Striche ein so langweiliger Text abzuschreiben wäre, dass Fabian alles unternimmt, um nicht so weit zu kommen.

Auf die Spannung zwischen Freiheit und Kontrolle aus der Sicht der SuS werde ich in Kapitel 8 weiter eingehen. Hier ist jedoch festzuhalten, dass gewisse SuS die Kontrolle wünschen, die ihnen als Anerkennung oder Leitplanke für Verhaltensweisen dient. Dieselben SuS drücken ihren Ärger aus, wenn Lehrpersonen nicht gründlich kontrollieren oder wenn sie vergessen zu kontrollieren. So hat Sandra kein Verständnis für die Lehrerin, wenn diese während der Schultests nicht genügend kontrolliert, ob jemand schummelt: „... wänn d Leereri halt nöd luegt, isch si halt sälber gschuld.“ Das Kontrollieren gehört zur Aufgabe der Lehrperson, der die SuS gegenüberstehen. In Sandras Aussagen haben internalisierte Ehrlichkeitsregeln scheinbar keine Wichtigkeit. Die Ehrlichkeitsregel richtet sie hier nach der Kontrollschärfe der Lehrperson. Daraus lässt sich die Ehrlichkeitsregel generieren: Wenn die Lehrperson nicht kontrolliert, darf geschummelt werden. Die Kontrollintensität der Lehrpersonen überprüfen die SuS und wissen dann genau, bei welchen Lehrpersonen in Schultests geschummelt werden kann und bei welchen nicht. Sandra bringt diese Schülerkompetenz wie folgt auf den Punkt:

Mir wüsset natürli au gnau, bi welne Leerer, dass mer chönd schpicke und bi welne nöd. (1) Bi de äinte Lüüt, würdet mir eener schpicke, mit de andere würdet mir s öis nie im Läbe getraue, so echli umezluege (1).

Gewisse Lehrpersonen lassen Raum, um schummeln zu können, und bei anderen wagt sich keiner umzuschauen. Wagen hat immer mit der Angst zu tun, die es zu überwinden gilt. Wenn jemand etwas nicht wagt, kann das darauf hinweisen, dass Angst das Verhalten bestimmt. Damit ist der Hinweis 
gegeben, dass neben der Kontrolle auch die Angst eine zentrale Rolle spielt, nach denen SuS Ehrlichkeitsregeln anwenden.

\subsubsection{Mit Distanz, keine Nähe und damit legitimierte Unehrlichkeit}

Die Kontrollfunktion der Lehrperson steht im Spannungsverhältnis zur Sympathie sowie Nähe der Lehrperson zu den SuS. Oder ist es gar so, dass nur ohne Kontrolle Sympathie sowie Nähe gewonnen werden kann und umgekehrt? Dazu passte Clemens' Forderung, die sich so als individuelle Ehrlichkeitsregel formulieren lässt: Nur gegenüber denjenigen Menschen braucht man ehrlich zu sein, die einem wichtig sind.

[...] (2) ich würd, wänn ich MU²4-Leerer wär, würd ich säge, dass me eerlich sii sött gegenüber sinere Fründin, gegenüber sim Leermäischter, gegenüber jede Mueter oder was au immer- S mues nöd emaal sii gegenüber Leerer oder soo, aber würd gegen- gegenüber die Lüüt, wo dir am Herze liget söttsch eerlich sii (1).

Gemäss Clemens zählen Lehrpersonen nicht unbedingt zu den Menschen, die einem am Herze liegen und zu denen man deshalb ehrlich sein muss. ,Herz ist eine starke Metapher, die in den Zusammenhang von Wärme und liebgewonnenen Leuten gesetzt wird, wozu nicht unbedingt die Lehrpersonen zu zählen haben. Es stellen sich dennoch die Fragen:

- Inwiefern kann oder soll eine Lehrperson mit Kontrollfunktion zu dieser Kategorie von Leuten gehören?

- Inwiefern kann oder soll eine Lehrperson zu einer Vertrauensperson werden, worauf Ehrlichkeit basieren kann?

Die Wechselbeziehung zwischen Kontrolle, Ehrlichkeit und Vertrauen soll hier vorerst vermerkt sein. Darauf wird noch später im Zusammenhang von Kollektivität eingegangen (vgl. Kap. 8.2).

\subsection{Fachspezifische Varietät von Ehrlichkeitsregeln}

Grundsätzlich sind sich alle SuS einig: Ehrliche Verhaltensweisen sind neben der Abhängigkeit zur Lehrperson ebenso von einzelnen Schulfächern abhängig und stehen im engen Zusammenhang zu diesen, insbesondere beim Schreiben von Schultests. Kriterien, an denen SuS ihre Ehrlichkeitsregeln orientieren, können wie folgt zusammengefasst werden:

- Stellenwert des spezifischen Schulfachs im Fächerkanon

24 MU ist die Abkürzung für das Schulfach „Mensch und Umwelt“. 
- Gelegenheiten für unkonventionelle Ehrlichkeitsregeln

- inhaltliche und didaktische Beschaffenheit des Schultests (bspw. Beweiskonstruktion oder Wörterabfrage) und räumlicher Ausstattungen, in denen Schultests geschrieben werden

Tatsächlich zählen die Noten bestimmter Unterrichtsfächer mehr für das Zeugnis als andere. So ergibt es sich, dass SuS bei Schultests im Kochunterricht oder im Fach Mensch und Umwelt risikoreicher handeln als in den Hauptfächern wie Mathematik oder Deutsch.

Je nach Fach und Themen ergeben sich unterschiedliche Alternativen zum selbstständigen Schreiben von Schultests: Bei Sprachtests, in denen der Wortschatz und somit einzelne Wörter abgefragt werden, eignet es sich, die schwierigen Wörter auf die Handfläche, zwischen die Finger oder bei langärmligen Kleidungsstücken auf den Arm zu notieren. Hingegen ist es in Geometrie schwer zu schummeln, weil Konstruktionen abgefragt werden. Zudem führt Regulas Klasse ein Theorieheft in Geometrie, das nicht so leicht zu verstecken sei wie ein Theorieblatt in Biologie. In der Mathematik eignet sich der Taschenrechner, um einen kleinen Zettel mit Lösungen zu verstecken.

Schließlich bieten sich nicht in jedem Schulzimmer die gleichen räumlichen Bedingungen: In kleinen und verwinkelten Schulzimmern lässt es sich einfacher schummeln. Trennwände oder der Abstand zwischen den Tischen erschweren die Gelegenheiten zum Schummeln. Schließlich eignen sich gestuft gestuhlte Zimmer (bspw. Biologie- oder Physikräume) sehr gut zum Schummeln. Zudem ergeben sich viele Schummelgelegenheiten, wenn eine Prüfung allein in einem separaten Zimmer nachgeholt werden soll.

Die vorgenommene Analyse weist bereits auf Modifikationen der konventioneller Ehrlichkeitsregeln in der Schulpraxis. Im nächsten Kapitel sind die Modifikationen der konventionellen Ehrlichkeitsregeln als ,unkonventionelle Ehrlichkeitsregeln' bezeichnet und werden aus den Ehrlichkeitspraxen rekonstruiert sowie genauer überprüft.

\section{Komplexität der unkonventionellen Ehrlichkeitsregeln}

SuS vermitteln zwischen konventionellen schulischen Ehrlichkeitsregeln und unkonventionellen Ehrlichkeitsregeln. Im Spannungsfeld zwischen der Kontrolle der Lehrpersonen und individueller sowie kollegialer Freiheit entwickeln die SuS unterschiedliche Regeln, die sie sowohl explizit als auch implizit beschreiben. Inhaltlich könnten gewisse Ehrlichkeitsregeln der SuS als punktuelle Notlösungen festgehalten werden. Hingegen weist der analytische 
Blick auf die positive Funktion der Notlösungen hin, die darin bestehen, nichtkonventionelle Regeln zu legitimieren. Zudem können auf diese Weise Indizien gewonnen werden, wie SuS mit Ehrlichkeitsregeln in Abhängigkeit von Vorder- und Hinterbühne (Goffman [1959] 1983, S. 99ff.; Verwendung für Unterrichtsanalysen Zinnecker 1978, S. 103f.; Wagner-Willi 2010, S. 50) im schulischen Alltag umgehen.

Interessanterweise stellen sich bei den unkonventionellen Regeln fallübergreifende Zusammenhänge dar. Unter bestimmten Umständen gelten individuelle Regeln, welche die SuS selber als zulässige Unehrlichkeitsregeln festlegen, wie beispielsweise Unlautere Hilfsmittel in Tests heranziehen (Kap. 8.1). Besonderes Interesse besteht für kollegiale Ehrlichkeitsregeln, die im Spannungsfeld zwischen der Kollegialität innerhalb des Klassenverbands und der individuellen Freiheit situiert sind (Kap. 8.2). Gerade in diesem Kontext werden schulspezifische Regeln oft außer Kraft gesetzt.

Spezielle Ehrlichkeitsregeln bestehen in der Wechselwirkung zwischen Spaß und Ernst (Kap. 8.3) sowie in den Schulkontext überschreitenden Regeln (Kap. 8.4).

Zusammenfassend ist der Fokus in diesem Kapitel auf folgende Aspekte von unkonventionellen Ehrlichkeitsregeln gerichtet:

- individuelle Ehrlichkeitsregeln

- kollegiale Ehrlichkeitsregeln

- Ehrlichkeitsregeln zwischen Spaß und Ernst

- Ehrlichkeitsregeln über den Schulkontext hinaus

\subsection{Individuelle Ehrlichkeitsregeln}

Unter gewissen Umständen sind während Schultests Unlautere Hilfsmittel beizuziehen (Kap. 8.1.1), Informationen vorzuenthalten (Kap. 8.1.2), Elternunterschriften selber zu setzen (Kap. 8.1.3), ist Aufrichtigkeit nur bei Anfrage nötig (Kap. 8.1.4) und Direktheit mit oder ohne Respekt gegenüber Lehrpersonen wichtig (Kap. 8.1.5). Die ersten drei Regeln beziehen sich auf die drei schulspezifischen und konventionellen Ehrlichkeitsregeln, die die SuS explizit genannt haben (vgl. Kap 7).

\subsubsection{Unlautere Hilfsmittel beiziehen}

Eine alternative Ehrlichkeitsregel geben sich SuS im Rahmen der Schultests, die selbstständig zu lösen sind. Sie wissen genau, in welchem Fach, bei wel- 
chen Lehrpersonen und bei welchen Prüfungsformen geschummelt werden kann und wo nicht. Nora beschreibt dies in einer distanzierten Form:

Me kännt scho echli d Leerer und so, uf was s lueget und uf was überhaupt nöd lueget (1).

Aus dieser kurzen Textpassage geht hervor, wie genau die SuS das Verhalten der Lehrpersonen durchschauen. Nora setzt ihr Verhalten in ein Spannungsfeld zwischen zwei extreme Positionen, das durch den Partikel überhaupt verstärkt wird. Die Lehrpersonen achten einerseits auf gewisse Zwischenfälle und haben andererseits blinde Flecken, welche die SuS kennen und ausnützen. Sandra beschreibt dies so:

Ja, zum Biischpiil, ob mir wüsset, ja d Leereri oder de Leerer wird schtinksauer, wänn mir emaal schpicket und me chunt en Iitraag über und vilicht werdet d Eltere kontaktiert. Bim andere, (1) ja de merkt gar nöd, dass me schpickt oder wämer schpickt, nimmt- überseet er s oder (1) nimmt s äifach nöd so eernscht (3).

Sandras Regel Schummeln ist unter bestimmten Umständen möglich und deshalb legal beruht auf Erfahrungswerten und ist auf die Aufmerksamkeit, Reaktion und Konsequenz der Lehrperson ausgerichtet. Sie schildert einen Lehrertyp, der emotional (schtinksauer) und mit klaren Konsequenzen (Iitraag und kontaktiert Eltere) reagiert. Der andere Lehrertyp kontrolliert nicht während Schultests oder ist unachtsam, oder aber er nimmt das Schummeln nicht so ernst.

Zudem bestimmen die einzelnen Lehrpersonen den Ablauf von Schultests und treffen zum Teil räumliche Vorkehrungen. So schieben sie etwa Tische auseinander oder stellen Trennwände auf. Dabei zeichnet sich aus den Beschreibungen und Erzählungen der SuS eine Ritualisierung ab. Sie beschreiben in klaren Ablaufschritten das Hineinkommen ins Schulzimmer, die Nervosität der Mitschülerinnen und -schüler, die räumliche Vorkehrungen, den Beginn der Unterrichtsstunde und die Phase des Abgebens der Schularbeit. Ausgehend von diesen Möglichkeiten berichten die SuS von Regeln, die unter bestimmten Umständen das Schummeln untersagen, und solchen, die alternative Regeln vorschlagen:

- Wenn man schummelt, hat man ein schlechtes Gefühl und Angst, erwischt zu werden, daher konzentriert sich beispielsweise Ilona auf das Schreiben ihrer Schularbeit.

- Wenn für die Schularbeit gelernt wurde, kann diese selbstständig gelöst werden (Claire, Christoph und Nora wollen so eigene Leistungen überprüfen, Emil will in der anstehenden Lehre keine Wissenslücke haben, Lilian spickt nicht und hilft auch niemandem, weil es ihre eigene Schularbeit ist).

- Scharfe Kontrolle, kluge Sitzordnung und harte Konsequenzen sind Faktoren, die das Schummeln während der Schultests als riskant einstufen lassen (Christoph, Emil, Adrian, Lilian, Valentina David, Fabian, 
Sandra) - doch die meisten Lehrpersonen sind ,umstimmbar' (Valentina, David, Fabian, Nora).

- Schummeln ist erlaubt, wenn es nur punktuell vorkommt, bei einem akuten Blackout aushilft, als Ausdruck der Hilfsbereitschaft steht und wenn es alle tun können (Lara, Regula, Valentina, Fabian) - oder wenn es mit einem ,Aha-Erlebnis‘ verknüpft ist und somit ,auf die Sprünge hilft' (Lara).

Interessanterweise erwähnt nur Clemens explizit, wie nützlich das Spicken sei, um gute Noten zu erzielen, ohne gelernt zu haben. Diese Befunde, die aus Erzähl- und Beschreibungssequenzen stammen, zeigen auf, dass die SuS prinzipiell lernen, jedoch äußere Faktoren, die konkrete räumliche, fachspezifische und personale Testsituation sowie die eigene Lerneinstellung Schummeln unterstützt oder hemmt. Zudem betonen Adrian, Clemens und Nora in Abgrenzung zum aktuellen Klassenverband, dass früher mehr geschummelt wurde und wie verbreitet diese Praxis in den Parallelklassen sei.

Zusammenfassend geht aus den bisherigen Ausführungen hervor: Die SuS sind lernorientiert, und das Schummeln hat für sie nicht erste Priorität. Entscheidend ist die Kontrolle der Lehrpersonen, um unlautere Hilfsmitteln in Schultests aufzudecken. Allerdings zählt Kontrolle der Lehrpersonen als eine von vielen Anhaltspunkten, an denen sich die SuS orientieren. Hinzu kommen noch andere: Interesse am Lernen, Art des Lernstoffs, Kontrolle des eigenen Lernerfolgs, gegenseitige Hilfsbereitschaft oder Überbrückung eines akuten Blackouts während der Schultests. Ein weiterer Aspekt ist, dass Hilfsmittel punktuell eingesetzt werden und Lehrpersonen umgestimmt werden können. Dieser Spielraum der Verhaltensweisen und die damit zusammenhängenden Ehrlichkeitsregeln der SuS sind nach der Kontrolle der Lehrperson und der daraus folgenden Angst der SuS ausgerichtet.

\subsubsection{Informationen vorenthalten}

Aus den Aussagen der SuS lassen sich drei Umstände identifizieren, unter denen Informationen vorenthalten werden dürfen:

- $\quad$ wenn die Tatbestände nicht überprüfbar sind oder nicht überprüft werden

- wenn grosse Spannungen herrschen

- wenn es die Privatsphäre zu schützen gilt

Nicht alle SuS setzen das Vorenthalten von Informationen mit unehrlichen Aussagen oder Verhaltensweisen gleich und kennzeichnen diese als solche. Allerdings ist unabhängig davon, ob die SuS einen direkten oder indirekten Zusammenhang zwischen ihren Verhaltensweisen und ihren Ehrlichkeitsregeln herstellen, das Vorenthalten von Informationen als legitime Verhaltensweise zu sehen, wenn eine der oberen drei Regeln eintritt. Anhand von aus- 
gewählten Textausschnitten untermaure ich diese Regeln, damit deren konkrete Anwendung nachvollziehbar ist.

Informationen dürfen vorenthalten werden, wenn die Tatbestände nicht überprüfbar sind oder nicht überprüft werden

Wie bereits erwähnt nehmen SuS schnell wahr, ob Lehrpersonen Hausaufgaben kontrollieren oder der Ursache von Vorfällen auf den Grund gehen. Die Überprüfbarkeit der Tatbestände spielt dabei eine entscheidende Rolle. Dies erklärt Adrian, als er über die ehrliche Antwort bei Anfragen von Lehrpersonen reflektiert:

(3) Ob mer das wüürkli gmacht hät oder nööd und (3) vorher dörf me schoo, un- (1) mängisch vilicht (2) nöd eerlich sii. (2) Wenn mer s nöd umbedingt chan naawiise (2), dänn gaat $\mathrm{s}$ au. (2) Aber sobald mer s äigentli chan naawiise, ob das würkli passiert isch oder nööd, sött me äigentli eerlich sii. (1) Deet isch äigentli Gränze (10).

Adrian schildert die Grenzziehung zwischen erwarteter Ehrlichkeit und der legitimen Möglichkeit, Informationen vorzuenthalten: Solange die Lehrperson einen Vorfall nicht überprüft oder dieser nicht überprüfbar ist, darf man unehrlich sein. Wenn hingegen die Lehrperson nachfragt, sollte man ehrlich sein. Das Modalverb ,sollen' hat hier eine entscheidende Funktion, denn nicht in allen Fällen der Nachfrage sind die SuS tatsächlich ehrlich. Beispielsweise spielt die Kollegialität hierbei eine wichtige Rolle (vgl. Kap. 8.2) oder die Zivilcourage (vgl. Kap. 8.4.1).

Darüber hinaus weist diese Interviewsequenz bereits auf die Regel der Aufrichtigkeit bei Anfrage hin (s. Kap. 8.1.4), die aber nicht bei allen Befragten gleich ausgelegt wird, wie das Interviewmaterial zeigen wird. Festzuhalten ist hier, wie die einzelnen Regeln miteinander verwoben sind.

Der Fokus soll nochmals darauf gerichtet werden, dass SuS tendenziell bis zur Anfrage oder bis zur Kontrolle der Lehrperson ein unehrliches Verhalten als legitim einstufen. Hingegen ändert sich inhaltlich die einheitliche individuelle Ehrlichkeitsregel der SuS in dem Moment schlagartig, wenn eine direkte Anfrage des Lehrers gestellt wird. Die vielfältigen Möglichkeiten von Regeln sind in Kap. 8.1.4 aufgeführt.

Informationen dürfen vorenthalten werden, wenn grosse Spannungen herrschen

Wenn eine angespannte Stimmung unter den Fragenden und den potentiellen Tätern wahrzunehmen ist, darf über das Vorenthalten von Informationen hinaus gar falsch ausgesagt werden. Dies ist eine Regel, die viele vertreten, aber zu der keiner der Befragten ein Erfahrungsbeispiel nannte. Die Erzählung von Marco bringt eine angespannte Situation mit einem Lehrer auf den Punkt, in der er zumindest erwogen hat, eine falsche Information zu geben: 
[...] ich bin emaal uf em Pausepalatz gsii und dänn isch irgend so en Leerer, dee isch Pauseuufsicht und dee isch äigentli e zimlichi Schpaasbrämse. (2) Deet tuet würkli irgendwie- Mir händ d tschuutet und nachheer mit de Chline und mir händ $\mathrm{s}$ äigentli luschtig ghaa. (1) Und dänn chunt dee: „Wänd ir mit dene tschuute?“ und nachheer dörfet mer nüme und dänn han ich churz wiiter gschpilt. (2) Und nachheer han ich- Hät er gsäit: „Näi, si wänd nüme zäme schpiile“ irgendwie. (1) Und dänn bin ich wäggange und dänn han ich gsäit: „Si sind so e Schpaasbrämse.“ Und dänn hät er gsäit: „Wie häisisch du?" Und dänn han ich mir zeerscht überläit, ob ich imm sött veraarsche und irgendwie säge, ich säig de Pascal.@1@ (2) Ja (I: Und dänn?) Näi, dänn han ich scho s Richtige gsäit, aber (1) deete find ich müsst mer äigentli nöd grad. Müesst mer äigentli scho rächt eerlich sii, ussert es schiist dich würkli aa. Und deet hät s mich würkli aagschisse. @1@ (I: Und warum häsch trotzdem de richtig Name gsäit?) (2) Will ich nöd no mee Schwiirigkäite han wele. @1@ (3) Ja, und (1) es isch ee echli en ecklige Leerer. (2) Und ja (3).

Marco spielt Fußball mit jüngeren Schülern, was offenbar nicht erlaubt ist, denn ein vorbeikommender Lehrer verbietet es, indem er Marco erklärt, dass die jüngeren Schüler nicht mit ihm spielen wollen. Nach der wiederholenden Aussage des Lehrers, dass die Kinder nicht mit ihm spielen wollen, stellt Marco das Fußballspiel ein. Seine Wut drückt er in der Bemerkung aus: „Si sind so e Schpaasbrämse." Worauf die negative Spannung zwischen beiden den Höhepunkt erreicht hat. Der Lehrer erkundigt sich nach seinem Namen, wobei Marco überlegt, ob er wirklich seinen Namen nennen soll, was er schließlich tut, um sich weitere Konsequenzen zu ersparen.

Die Erzählung schildert ein Spannungsverhältnis, in welchem sich die Option der unehrlichen Aussage als ein Lösungsansatz für Marcos Unterlegenheit zeigt. Marco stellt sich mit der Bemerkung Schpaasbrämse und mit der Option der Unehrlichkeit der Machtasymmetrie entgegen. Denn aus seiner Sicht ist die Intervention der Lehrperson ungerechtfertigt. Seine Gefühlslage drückte er mit der unhöflichen Aussage aus: Schiisst mich aa. Nach Marco dürfen bei ungleichen Machtverhältnissen Informationen vorenthalten werden. Wenn jedoch der SuS nicht auf der überlegenen Seite steht, ist mit Strafen zu rechnen. Angst und negative Folgen veranlassen, dass die alternativen Ehrlichkeitsregel (Informationen zurückhalten) nicht in Kraft tritt.

Informationen dürfen vorenthalten werden, wenn es die Privatsphäre zu schützen gilt

Über persönliche Angelegenheiten ist man nicht gezwungen, Auskunft zu geben. Jedoch können diese beispielsweise im Klassenrat angesprochen werden. Eine Möglichkeit ist es, die Informationen zurückzuhalten und bei direkter Nachfrage eine Unwahrheit zu sagen. Als Lara über die Funktion des Klassenrates informiert, bezieht sie sich kurz auf dieses Thema:

Also, mir händ so en Klasseraat und es isch äigentli innerhalb vo de Klass, das d Lüüt so eerlich sind zunenand. Das isch äigentli klar. Also- (1) ussert es isch irgendöppis, wo me findet, das gaat die andere äifach wükli nüt aa und dänn lügt mer halt irgendöppis, aber dänn gaat s ja scho wider. (1) Aber susch, also ebe im Klasserat chan mer äigentli alles 
aneschriibe, wo äim nöd würd passe oder so und dänn (1) wird das au i de Klass dänn thematisiert werde (4).

Innerhalb der Klasse sind gemäss Lara die SuS ehrlich. Jedoch kann es im Klassenrat vorkommen, dass zu persönliche Dinge (gaat die andere äifach würkli nüt $a a) \mathrm{zu}$ besprechen sind. In diesem Fall ist eine Lüge quasi als Schutz möglich. Interessanterweise hat eine solche Lüge keine Folgen, denn gemäss Lara wird das Gespräch einfach weitergeführt. Hier kristallisiert sich die Spannung zwischen Offenheit i. S. v. Alles sagen und Wahrung der Privatsphäre i. S. v. Gewisse Angelegenheiten sind nicht zu sagen heraus.

Im Klassenrat herrschen gleiche Machtverhältnisse, weil sich die Lehrperson im Hintergrund hält. Weil keine Machtasymmetrie besteht, fühlen sich die SuS freier und können persönliche Informationen zurückgehalten werden.

Dieser Bereich der Offenheit ist mit Leitfadeninterview schwer zu erschließen, weil ein sensibler Themenbereich angeschnitten wird, in dem offensichtlich die Rolle der Interviewerin als Außenstehende und nicht Eingeweihte an Grenzen stößt.

\subsubsection{Elternunterschriften selber setzen}

In fast allen Schulfächern ist es üblich, die Noten der Schultests den Eltern zu Hause zu zeigen und dies mit deren Unterschrift beglaubigen zu lassen. Für Emil, Ilona, Claire, Valentina, Emil, Adrian, Clemens, Lara, Christoph und David kommt es nicht in Frage, wegen schlechter Noten oder um einen Eintrag zu umgehen die Elternunterschrift selber zu setzen. Dafür geben sie verschiedene Gründe an:

- Verständnis der Eltern für schlechte Noten und keine Angst vor den Eltern (Ilona, Claire, Valentina, Emil und seine Klasse)

- Selbstverständlichkeit, dass Eltern über Schulleistungen informiert sein müssen (David)

- Strafbarkeit (Adrian)

- Angst vor dem Lehrer und den Konsequenzen (Nora, Lara)

- zur eigenen Note stehen (Christoph)

- Gleichgültigkeit gegenüber der negativen Reaktion der Mutter (Clemens)

Daraus lassen sich zusammenfassend Ehrlichkeitsregeln der SuS formulieren, wann Elternunterschriften nicht selber gesetzt werden sollen. Ehrlichkeit wird von sich und den Mitschülern erwartet,

- wenn seitens der Eltern trotz misslungener Schulleistungen Verständnis erwartet werden kann

- wenn Angst vorhanden ist, weil eine Schulregel gebrochen wurde 
- wenn die Courage vorhanden ist, zu den eigenen Schulleistungen und versäumnissen zu stehen

Somit orientieren sich neun von $16 \mathrm{SuS}$ nicht unreflektiert an der schulischen Ehrlichkeitsregel Elternunterschriften nicht selber setzen, vielmehr ist diese Regel in ihren Verhaltensorientierungen mit eigenen Erklärungsansätzen und damit persönlichen Ergänzungen bereichert.

Im Zusammenhang mit Elternunterschriften formulieren Nora, Sandra, Lilian, Fabian, Stefan, Regula und Marco unkonventionelle Regeln, die in zwei Regeln zusammengefasst werden können: Elternunterschriften dürfen selber gesetzt werden,

a) wenn bei schlechten Noten ein fehlendes Verständnis der Eltern wahrge nommen wird (Marco, Fabian) oder

b) wenn ein Eintrag umgangen werden soll (Sandra, Lilian, Stefan, Regula).

Ausschlaggebend für beide alternativen Regeln ist die Strafe, die vermieden werden soll: zeugniswirksame Einträge, emotionale Reaktionen der Eltern, Freizeitentzug und Lernzwang. Dazu beschreibt Marco genauer, was ihn an der Reaktion der Eltern ,nervt":

Ja ebe, dänn zäigsch si: „Ou bisch nöd e so guet gsii.“ Und dänn muesch äifach (1) anschtatt- Dörfsch mit dem Kolleeg go tschuute, musch schtundelang mit dine Eltere oder zwäi Schtund lang oder jede Tag irgendwie Franzwoggiwörter abschribe oder so. Und das närvt uuhuere und dänn (3). Ja.

Marco spielt in seiner Freizeit lieber mit seinen Freunden Fußball, anstatt Französischwörter zu pauken. Es nervt ihn dann sehr, dass er bei schlechten Noten Letzteres zu tun hat. Offen bleibt, ob ihn eher die Tätigkeit an sich nervt oder ob er sich gegen die Konsequenz der Eltern stellt.

Fabian berichtet von der negativen Reaktion der Mutter, wenn sie schlechte Schultests zu unterschreiben hat:

(1) Ich säge, mini Mueter würd au nöd immer grad säge (1): „Fabe, das häsch super gmacht, nächscht Maal au“ und so. (2) Ja, und vor allem, mäischtens tüemer das vorheer aaluege und so. Und wänn ich genau das nöd chan, wo $n$ ich het sölle aaluege, dänn regt si sich mäischtens extrem uuf. Nöd extrem, aber es regt äifach uf (1) und so. Und ich säg mäischtens, ja: „Das bringt mir gar nüd, wänn du mich aaschräisch“ und so. Denn eener „Lern!“ und so. Dänn- (2) Dänn chunt immer das, de Enthusiasmus oder @wie das häisst@. Ich chan das nie richtig uusspräche. Vo dem Dings und dänn will si grad (1) morn lerne und übermorn und nomee lerne und so und immer mee lerne und dänn (1) legt sich das wieder und dänn nach de Prüefig will si grad no me und so (2).

Die negative Reaktion der Mutter hängt mit dem gemeinsamen Lernen und dem Lernstoff zusammen, den der Sohn hätte anschauen sollen. Fabians Mutter scheint sich stark mit Fabian zu identifizieren. Durch das gemeinsame Lernen sichert sie eine gute Testvorbereitung, weshalb sie auch entsprechende Resultate sehen möchte. Fabian begründet nicht, ob er den abgesproche- 
nen Lernstoff tatsächlich nicht anschaut oder ob er ein Blackout in der Schule hat. Aus Fabians Beschreibung geht auch eine Routine hervor: ,„[...] dänn (1) legt sich das wieder und dänn nach de Prüefig will si grad no mee“, mit der er sich offenbar abgefunden hat.

Die beiden Regeln - Unterschriften nicht setzen und Unterschriften unter bestimmten Umständen setzen - sprechen für ein ungleiches Abhängigkeitsverhältnis zwischen den Eltern und den Heranwachsenden. Darauf antworten Marco und Fabian punktuell mit unkonventionellen Regeln, wobei die meisten SuS sich anpassen. In diesem Sinne hält Lara fest:

(1) Scho klar git s Lüüt, wo säget: „Oo näi, das wett ich lieber au nöd Häi bringe!“ oder so, aber (1) die tüend dänn nöd extra daa go Underschrifte fälsche. Die tüend s dänn doch underschriibe laa. (4) So ja (4).

Damit beschreibt Lara einen allgemeinen Reibungsfall bezüglich ehrlicher Verhaltensweisen, den sie bei ihren Mitschülerinnen und -schülern wahrnimmt. Schlechte Noten führen zu negativen Emotionen seitens der Eltern und des SuS. Demgegenüber betrachtet Lara das Fälschen von Unterschriften abschätzig und bekräftigt, dass die SuS dennoch die Elternunterschrift einholen. In diese Entscheidung kann die Abhängigkeit von den Eltern mitgedacht werden.

\subsubsection{Aufrichtigkeit bei Anfragen}

Die Regel, dass bei einer Anfrage der Lehrperson oder eines Mitschülers eine ehrliche Antwort erwartet wird, vertreten die meisten SuS, wobei es situative Unterschiede gibt. So erklärt Adrian:

(3) Aber mängisch isch mer au nöd eerlich, (1) wänn jetzt irgendwie de Leerer fröögt, ob me d Uufgabe gmacht hät, (2) dänn isch me halt nöd eerlich und säit: „Ja, ich has gmacht!““

(2) obwool mer s äigentli nöd gmacht hät (1) und dänn laat me sich irgendwie- e Uusreed: „Ich has di Häi vergässe“" oder irgendöppis ,aber ich has gmacht.“ Und ich find, das isch au soo- (1) daa i soo Situatione isch s vilicht au nööd soo schlimm, (1) will mängisch muemmer s äifach umgaa. (1) Aber suscht (1) so ganz (1) schpezielli Fraage, wänn ich au nöd $\mathrm{z}$ vill mit dere Persoon $\mathrm{z}$ tue hät $<$ ha $>$, (1) muemer äigentli scho eerlich sii. (3) Will, wänn de Leerer würkli öppis wichtig s fröögt, (1) dänn isch mer äigentli scho eerlich oder au underenand, (3) find ich s äigentli scho zimli wichtig, dass me dänn eerlich isch, aber mängisch- (1) mueme halt echli uneerlich sii@1@(4).

Adrians Ehrlichkeitsregel ist stark situativ und an die Wichtigkeit eines Gegenstands gebunden. Hausaufgaben fallen unter die unwichtigen Dinge, und daher dürfen hierfür Ausreden verwendet werden. Hingegen erwartet Adrian bei wichtigen Anfragen der Lehrperson, dass man ehrlich sein solle - wobei er nicht weiter darauf eingeht, was er damit genau meint. Adrian bezieht seine abstrakten Erklärungen sowohl auf Lehrpersonen als auch auf Mitschülerinnen und -schüler. Insbesondere bei Mitschülerinnen und -schülern, die 
man nicht so gut kennt, sei die Ehrlichkeit umso wichtiger, wenn diese einen etwas fragen. Dies gilt jedoch nur für Mitschüler der eigenen Klasse, welche in die kollegialen Regeln oder Späße noch nicht eingeweiht sind. Dem ist anders bei SuS aus anderen Klassen (vgl. Kap. 8.3, 8.4). Schließlich ist interessant, wie Adrian Unehrlichkeit als etwas Zulässiges und mit einem Muss in Verbindung bringt. Als ob Unehrlichkeit ein Zwang sei, dem man unterliegt.

Emil bringt ein weiteres Argument ein, wenn es darum geht, bei Anfragen ehrlich zu sein:

(1) Ja, ebe wänn mer en Säich macht. (1) Dänn sött mer s äigentli grad säge, (1) wer s gsii isch oder ob mer s sälber gsii isch, (2) will suscht (1) hät sicher au Konsequänze für die anderi zäme (6).

Dieser Textabschnitt impliziert die Anfrage einer Lehrperson an die SuS, auf die unmittelbar eine aufrichtige Antwort zu geben ist. Emils Erklärungen sind mit dem Modalverb ,sollen' formuliert, woraus geschlossen werden kann, dass die Regel, auf Anfrage ehrlich zu antworten, nicht immer eingehalten wird und dass daher die Lehrpersonen Kollektivbestrafungen tatsächlich aussprechen. Dieselbe Erwartung teilt Nora: Bei Anfragen der Lehrperson soll der SuS diese nicht anlügen und auch nichts erfinden.

Kontrastierend zur bisherigen Ehrlichkeitsregel Aufrichtigkeit bei Anfrage steht Sandras Erwartung an SuS, aus Eigeninitiative - ohne eine Anfrage die Wahrheit zu sagen:

Aber (1) ich find jetzt wämer vo sich uus, sälber zu dere Person gaat und öppis verzelt, das isch au eerlicher. Es isch scho, käi Lüüg, wämer nöd gaat öppis go verzele, aber es isch äifach eerlicher, wämer öppis vo sich us verzelt (7).

Sandra führt eine Gradierung der Ehrlichkeit ein: Eine Aussage kann mehr oder weniger ehrlich sein, je nachdem, ob eine Nachfrage oder eine Eigenmotivation vorhanden ist. Im gleichen Sinne wie die anderen Mitschüler erklärt auch Sandra das Vorenthalten von Informationen nicht als Lüge. Schließlich zeigt sie an einem Beispiel die Sinnlosigkeit dessen auf, dass Lehrpersonen immer nachzufragen haben:

(4) Ja, ebe zum Biischpiil, (2) wänn (2) wänn öpper gmobbt wird oder so, (1) das nöd d Leereri mues frööge, wird bi öi öpper gmobbt, sondern dass en andere Mitschüeler sälber zu de Leereri gaat: „Ja, dee und die wird gmobbt“ und so. Das d Leerer nöd immer- Ich mäine süscht müsst d Leereri jedi Wuche naafrööge: „Ja, wird bi öi öpper gmobbt“ (6).

Dies ist ein fiktives Beispiel, das aber den Wunsch nach Eigeninitiative und Partizipation der Schülerin sichtbar macht. Zudem zeigt es die Abhängigkeit der Lehrperson von der aktiven Teilnahme der SuS.

Die Regel der Ehrlichkeit auf Anfrage hin gilt in den meisten Fällen als Verhaltensorientierung. Ausnahmen werden im Konjunktiv oder anhand von fiktiven Beispielen vorgestellt. 


\subsubsection{Direktheit mit oder ohne Respekt gegenüber Lehrpersonen}

Aus den bisher dargestellten unkonventionellen Regeln geht hervor, welche Auswege in heiklen Schulsituationen gesucht werden, d. h. in solchen Situationen, in denen das eigene Verhalten nicht mit den konventionellen Ehrlichkeitsregeln in der Schule übereinstimmt - besonders in der direkten Konfrontation mit der Lehrperson. Auswege werden nur bei einzelnen SuS nicht in Betracht gezogen.

Adrian zählt zu diesen wenigen Ausnahmen. Beispielsweise berichtet er von einer Hausaufgabenkontrolle, bei der er einen Eintrag ins Kontaktheft erhält, weil er Unterrichtsmaterial vergessen hat. Mitschülerinnen, die das Material ebenfalls vergessen haben, besorgen es kurz vor der Schulstunde bei der Parallelklasse. Indem Adrian zu seiner Unterlassung steht und diese nicht vertuscht, akzeptiert er auch die Konsequenz (Eintrag ins Kontaktheft). Er respektiert so in einer deutlichen Selbstverständlichkeit die konventionelle Ehrlichkeitsregel $\mathrm{Zu}$ vergessenen Hausaufgaben stehen und Konsequenzen akzeptieren. Das heißt noch nicht, dass er die konventionellen Ehrlichkeitsregeln im Ganzen verträte. Sicher lässt sich jedoch sagen, dass hier nicht ein Fall der Resignation gegenüber Autoritätspersonen vorliegt.

Fabian kann seinerseits Eigeninitiative ergreifen, indem er sich gegen konventionelle Regeln in der Schule stellt. Beispielsweise setzt er zuweilen selbst eine Elternunterschrift, um damit der Mutter eine schlechte Note zu verheimlichen, wie weiter oben bereits aufgezeigt wurde (vgl. Kap. 8.1.3). Demgegenüber erklärt Ilona, dass sie allen, unabhängig ob Peers oder Erwachsenen, direkt und mit Respekt ihre Meinung mitteilt:

Wänn mich öppis schtöört, dänn säg ich s dere Person grad is Gsicht. (1) Und mäischtens isch s mir egaal, wer die Person isch. Au wänn s en Erwachsene isch- Ich han Reschpäkt vor erwachseni Persoone und alles, aber (2) wänn mich öppis schtöört vo dere Persoon und (2) irgendwie, dänn gaan ich und säg das au höfflich.

Die Haltung von Ilona ist, Personen, an denen sie etwas stört, höflich zu begegnen. Ihr Lösungsansatz besteht darin, Angelegenheiten direkt mit der betroffenen Person zu besprechen. Ilona meint, sich darin gesellschaftskonform zu verhalten, sofern sie sich höflich gibt. Auf Anfrage erzählt sie das Beispiel, wie sie von der Zeichnungslehrerin Frau Z ungerecht behandelt wird und wie sie auf diese Situation reagiert:

(5) Ich han (2) mit de Frau Z @1@ han ich scho emaal öppis ghaa. (1) Mir händ s äigentli (1) am Aafang nöd so guet ghaa. (1) Ich bin au scho maal frächer gsii und so. Und dänn bin ich- Han ich mich irgendwie beruigt und so. Hämmer s guet ghaa und mir händ immer so zäme glacht und s isch äigentli no rächt luschtig i de Schtund gsii, (1) aber ebe zum Biischpiil paar Maal, wo si mich gsee hät, öppis mache und de ander hät si nie gsee. (1) Isch halt immer wider passiert. Und ich bin immer wider gschuld gsii, bis ich uusgflippt bin. (1) Ja ich han gsäit: ja „Ich chan nöd immer gschuld sii. Si gseent immer nur mich und die andere gseent si nie." (1) Und (2) halt ebe, nachheer hämmer megalang gredet und so und 
dänn han ich ire äifach d Mäinig gsäit, (3) Reschpäktvoll, aber d Mäinig gsäit, also ich has si nöd wele beschuldige wäge irgendöppisem. Aber ich han ir au wele mal zäige, us miinere Sicht, wie daas isch. (2) Und dänn hät si es paar Maal gsäit: „Näi, ich wett käin Kommentar. Ich wett dich nöd ghööre.“Han ich mich@duuregsetzt@ han wiiter gredet, bis si zuegloost hät. (2) Ja, will paar Maal au, au, (1) obwool si Leerer sind, obwool si Erwachse sind, (1) ich find äifach mängmaal sött mer au d Mäinig vo de Chinde (1) loose, also. (2) Das wär s (1).

In dieser Textpassage zeigt sich sehr schön ein nichtstatisches, organisches Beziehungsverhältnis zwischen Schülerin und Lehrerin aus der Sicht von Ilona. Deshalb analysiere ich diese Passage anhand der verschiedenen Thematiken ausführlicher.

Zunächst charakterisiert Ilona das Beziehungsverhältnis wertend: Sie greift in ihrer Schulbiografie etwas zurück und zeigt eine Entwicklung des Beziehungsverhältnisses von nicht gut zu gut. Das erste negative Stadium erklärt sie am eigenen frechen Verhalten. Den Wechsel zum zweiten Stadium bezieht sie wieder auf sich: wie sie sich beruhigt habe, woraus ein gutes Verhältnis folgte. Die Wertung gutes Verhältnis leitet Ilona aus der Tatsache ab, dass beide, Lehrerin und Schülerin, oft miteinander lachen.

Dennoch beschäftigt sich Ilona während der Zeichnungsstunde mit etwas (nicht näher Beschriebenem) Anderem, das nicht regelkonform ist. (Die Zeichnungsstunde bezeichnen verschiedene SuS als Entspannungsstunde; daher nehmen sie es hier mit den Verhaltensregeln nicht so genau.) Die Zeichnungslehrerin bemerkt Ilonas Fehlverhalten und weist sie zurecht. Ein wesentlicher Grund für das Eskalieren der Situation ist Ilonas Ungerechtigkeitsgefühl; die Folge ist, dass sie ,ausflippt ${ }^{\star}$. Der Lösungsansatz für diese Situation ist das Gespräch, das Ilona als sehr lang (megalang) empfindet und positiv bewertet. Sie ist sich anscheinend bewusst, dass es nicht üblich ist, der Lehrperson die eigene Meinung zu sagen. Dies wird unterstrichen, indem sie präzisiert, dass sie die Lehrperson nicht beschuldigt habe. Zunächst setzt sich Ilona gegen die Weigerung der Lehrperson durch, ihre Sichtweise kundzutun. Dass ihr Verhalten - sich gegenüber einer Lehrperson durchzusetzen unkonventionell ist, signalisiert sie mit einem kurzen Lachen. Ilona gelingt es, ihre Meinung respektvoll auszudrücken, wie sie betont.

Durch ihre Beharrlichkeit erreicht Ilona also, dass die Lehrperson ihr zuhört. Es scheint, als sei sie darüber überrascht, denn sie schließt diese Sprechsequenz mit einem Postulat ab: Lehrpersonen und Erwachsene und somit Autoritätspersonen hätten zwischendurch auch auf die Meinung der Kinder zu hören. Allerdings berichtet Ilona nicht, wie die Lehrerin oder die Klasse den Disput wahrnehmen.

In anderen Beispielen erzählt Ilona, wie sie sich auch an anderer Stelle durchgesetzt hat. Ein Beispiel stammt aus dem privaten Lebensbereich; jedoch bat sie mich einige Tage nach dem Interviewgespräch, diese Aufzeichnungen zu streichen. Eine ähnliche Hartnäckigkeit in der Beziehung zu Lehr- 
personen findet sich bei Nora, die ihre Meinung bekundet, als ein Lehrer einen Schüler regelmäßig ungerecht behandelt:

@1@ (2) Ja, zum Biischpiil s letscht Jaar hämmer immer so en Religionsleerer ghaa, woo(1) immer de äint hät er immer voll gmobbt. Und hät er amigs so d Hand, soo begrüesst, so zäge und d Hand so fescht zuedruckt bis im Träne choo sind und so. Und dänn han ich dem Leerer äifach äinmaal gsäit, ja, das göngi nööd. Und dänn hani gschtritte mit em Leerer und han im würkli d Mäinig gsäit und das gaat nöd und- und nachheer hät er mich au nüme chöne uusschtaa, aber wenigschtens- nachheer isch er nüme so gemäin gsii. Daas isch äifachtsoo@1@. Isch jetzt echli es Ego-Biischpiil@1@. Aber ja (4).

Der Lehrer behandelt einen Mitschüler ständig ungerecht. Für Nora ist das Motiv einzugreifen, gegenüber einem Lehrer ihre direkte Meinung zu äußern, also die Ungerechtigkeit gegenüber einem anderen. Im Unterschied zu Ilona erwähnt Nora nicht, ob sie sich respektvoll verhält. Die Intensität des negativen Umgangs spiegelt sich im ausdrucksstarken Wort gemobbt wider, das üblicherweise zwischen SuS, aber nicht im Verhältnis zu den Lehrpersonen verwendet wird.

Der Lehrer mobbt den Schüler, indem er diesem die Hand sehr fest zudrückt, bis Letzterer Tränen in den Augen hat. Zunächst sagt Nora dem Lehrer, dass ein solcher Umgang nicht gehe. Eine Steigerung des Gespräches findet statt, und sie stellt fest, dass sie fast streiten. Zudem bekräftigt Ilona mit dem Partikel würkli und der Wiederholung, dass sie ihre Meinung kundtat, dass sie wirklich so gehandelt habe. Das impliziert, dass es nicht wirklich oder dass es nicht üblich ist, so zu reagieren.

Resultat des Streitgesprächs ist ein schlechtes Verhältnis zwischen Nora und dem Lehrer. Nora kann positiv aus dem Ganzen nur verzeichnen, dass der Lehrer nicht mehr so gemein zu dem Mitschüler ist. Ebenso signalisiert Nora mit dem Lachen und mit der Feststellung Daas isch äifacht soo ihr Erstaunen darüber, wie eine so unkonventionelle Situation entstehen kann. Sie schließt die Erzählung, indem sie das Beispiel als Ego-Beispiel bezeichnet.

Diese Metapher Ego verweist auf ihr Verhalten als Einzelgängerin, was einen weiteren Hinweis auf unkonventionelles Verhalten gibt. Scheinbar besteht die Annahme, dass konventionelles Verhalten von vielen geteilt wird und spezifisches Verhalten, wie Nora es beschrieben hat, allein angepackt werden kann. Darüber hinaus ist festzustellen, dass Nora dennoch einen Gewinn aus dem Streit ziehen konnte: Sie hat ihr Ziel erreicht, dass der Mitschüler vom Lehrer besser behandelt wird.

Die Beispiele von Ilona und Nora zeigen sehr schön den couragierten Umgang mit Autoritätspersonen auf. Zudem weisen sie darauf hin, wie Ehrlichkeit ebenfalls darin besteht, die eigene Meinung auszudrücken. In beiden Beispielen spielt die starke Sensibilität für Gerechtigkeit eine wichtige Rolle. Zugleich zeigen die Beispiele sehr klar das latente Machtverhältnis zwischen 
Lehrperson und Lernenden, das hier ausnahmsweise zugunsten der Schülerin entschieden wird.

Die Beispiele von Ilona und Nora illustrieren wie Ungerechtigkeitsgefühle veranlassen, das asymmetrische Machtverhältnis zwischen SuS und Lehrperson zu brechen. Ilona lässt die Zeichnungslehrerin nicht ausreden und Nora sagt dem Lehrer ihre Meinung schonungslos. Im Moment, in dem Verhaltensweisen gefragt sind, übernahmen die Schülerinnen die Machtposition. Beiden Schülerinnen ist es wichtig, ihre Sichtweisen den Lehrpersonen kundzutun. Das erreichen beide. Bei Ilona bleibt unklar, ob sich das Verhalten der Lehrerin geändert hat. Nach Noras Stellungnahme hingegen behandelt der Lehrer den gemobbten Schüler besser. Das asymmetrische Machtverhältnis hat sich wieder eingestellt, insofern Nora feststellt, dass der Lehrer sie nicht mehr mag.

\subsubsection{Zusammenfassung}

Unkonventionelle individuelle Ehrlichkeitsregeln gehören zum Schulalltag der SuS. Sie werden von außen als Regeln der Unehrlichkeit wahrgenommen. Aus der Sicht der SuS sind unkonventionelle Ehrlichkeitsregeln in den fünf thematisierten Schulbereichen von klaren Bedingungen abhängig und nicht rigide.

- Spicken ist unter gewissen Umständen möglich und daher legal. Zudem hilft es bei punktuellen Überbrückungen von Blackouts. Die SuS prüfen aktiv die Intensität der Kontrolle und wägen ab, ob es zu riskant ist oder ob die Lehrpersonen umstimmbar sind. Gleichzeitig ist ein Interesse am Lernen und ein schlechtes Gefühl oder Angst beim Spicken festzustellen. SuS verstehen Spicken als gegenseitige Hilfsbereitschaft.

- Informationen dürfen vorenthalten werden, wenn die Tatbestände nicht überprüfbar sind oder nicht überprüft werden. Eine weitere Bedingung besteht darin, ob grosse Spannungen herrschen oder ob die Privatsphäre zu schützen ist, denn dann sei es legitim, die Informationen zurückzubehalten.

- Elternunterschriften dürfen selber gesetzt werden, wenn die Noten schlecht sind und das Verständnis der Eltern fehlt. Zudem können die Elternunterschriften selber gesetzt werden, wenn damit ein unnötiger Eintrag umgangen werden kann, weil das Einholen der Elternunterschrift vergessen ging und ein Eintrag ins Kontaktheft vermieden werden soll.

- Direktheit wird von wenigen SuS als eine besondere Form der Ehrlichkeit vorgestellt, die mit der ausformulierten subjektiven Wahrheit zu tun hat. Insbesondere im Verhältnis zwischen Lehrperson und SuS sind dafür eine ausgeprägte couragierte Haltung und Sensibilität für Ungerechtigkeit Voraussetzung, welche das Machtgefälle und den sozialen Kodex 
der Höflichkeit zu umgehen vermögen, womit offenbar ungerechte Zwischenfälle direkt mit der Lehrperson geklärt werden können.

- Erst bei der Anfrage von Lehrpersonen erwarten SuS von sich, dass sie eine aufrichtige Aussage machen. Zuvor ist dies nicht nötig.

Diese unkonventionellen Ehrlichkeitsregeln sind als Orientierungen zu verstehen, die Ausnahmen zulassen.

\subsection{Kollegiale Ehrlichkeitsregeln}

Um die Klasse vor einer Kollektivbestrafung (Eintrag und Elterngespräche) zu bewahren, meldet sich der 14-jährige Otto als Täter, obwohl er die Eier während der Kochschule nicht auf die vorbeifahrenden Autos geworfen hat. Er muss ein ernstes Elterngespräch über sich ergehen lassen und wird von den Eltern drangekommen, aber im Gegenzug erhält er Aufmerksamkeiten der Mitschülerinnen und -schüler, und sein Ansehen in der Klasse steigt. ${ }^{25}$

Das ist ein eher extremes Beispiel aus den zahlreichen Erzählungen und Beschreibungen der SuS zum Klassenzusammenhalt in Bezug auf Ehrlichkeitsregeln. Die Kollegialität in Ottos Klasse ist so stark ausgeprägt, dass sich Otto aus Loyalität bereiterklärt, sowohl für den anscheinend schüchternen Täter als auch für den ganzen Klassenverband einzustehen. Sein Mitschüler Clemens bezeichnet die Verhaltensweise von Otto als heldenhaft. Die meisten interviewten SuS weisen ähnliche kollegiale Ehrlichkeitsregeln auf: Die grosse Bedeutung der Kollegialität innerhalb des Klassenverbands reflektieren ihre Berichte zur grundsätzlichen Ablehnung des Verpetzens (Verratens) und zur Hilfsbereitschaft im Klassenverband.

Unabhängig von der Motivation für eine solche Verhaltensweise (bspw. das gewachsene Ansehen innerhalb der Klasse) geht aus dem Beispiel Ottos klar die hohe Bedeutung der Kollegialität hervor, welche die Grenze zur Ehrlichkeit überschreitet. Denn Otto handelt zugunsten der Kollegialität und zuungunsten der Lehrperson, der gegenüber er unehrlich handelt. Somit verschieben sich die Werte des moralischen Maßstabs. Indem Otto sich meldet, ist er kollegial gegenüber der Klasse und unehrlich gegenüber dem Lehrer, was positiv von der Klasse und von Otto gewertet wird.

Welcher Bezug besteht nun zwischen Kollegialität und Ehrlichkeit? Die SuS nehmen mit Ausnahme von Emil keinen verbalen und direkten Bezug auf Ehrlichkeit. Allerdings sind indirekte Bezüge festzustellen: Wenn jemand einen Mitschüler nicht verpetzt, ist diese Person gegenüber dem Mitschüler kollegial, aber beispielsweise gegenüber der Lehrperson oder einem geschä-

25 Vgl. Interview mit Clemens (Chiapparini 2011, S. 28ff.). 
digten Mitschüler unehrlich. In den meisten Situationen ist Hilfsbereitschaft zugunsten eines Mitschülers und zuungunsten der Lehrperson oder eines anderen Mitschülers positiv konnotiert. Ehrlichkeit gegenüber Lehrpersonen ist somit zwar eine wichtige Tugend, wenn aber zwischen Kollegialität und Ehrlichkeit zu entscheiden ist, hat Kollegialität das größere Gewicht.

Anders ausgedrückt: Aus der Sicht von SuS, die beispielsweise in einer Schularbeit unerlaubte Hilfsmittel beiziehen, um einen Mitschüler zu unterstützen, sind solche Verhaltensweisen zulässig und ehrlich. Hingegen sind diese aus Sicht der Lehrperson oder der Schulleitung unehrliche Verhaltensweisen, die zu ahnden und zu unterbinden sind. Somit können Ehrlichkeitsregeln angeführt werden, die von der Perspektive des Betrachters abhängen.

Der indirekte Bezug der Kollegialität auf Ehrlichkeitsregeln veranlasst dazu, vorerst fallübergreifend die Beispiele von Kollegialität vorzustellen, in denen eine grundsätzliche Ablehnung des ,Verpetzens' (Kap. 8.2.1) und eine Hilfsbereitschaft im Klassenverband (Kap. 8.2.2) ausgedrückt werden. Auch hier teilen nicht alle SuS in jeder Situation die gleichen Ehrlichkeitsregeln, worauf ich im Folgenden eingehe. Abschließend fasse ich die Ehrlichkeitsregeln bezüglich Klassenverband in Bezug auf Kollegialität (Kap. 8.2.3) zusammen.

\subsection{1 ,Verpetzen' als besondere Form der kollegialen Unehrlichkeit}

Alle SuS thematisieren, meist in Eigeninitiative, das Problem Vertädeln und Verpetzen. Damit ist die Aktivität bezeichnet, dass SuS andere Peers, die gegen eine Schulregel gehandelt haben, einer Lehrperson verraten.

Wenn verpetzt wird, dann vornehmlich von SuS aus anderen Klassen (a), in leistungsschwächeren Klassen (b) und in der Primarschule (c). Das sind alles Personen, die gegenüber dem eigenen Klassenverband eine bedeutungsärmere Stellung einnehmen, was in den Textpassagen zu allen drei Personengruppen ersichtlich wird:

(a) Adrian berichtet von einem Vorfall, in dem jemand beleidigende Bilder eines Schülers aus der Parallelklasse in den öffentlichen Schulcomputer stellt (Bulling) und dieser sich darüber beschwert. Eine lange Suche nach dem Täter beginnt. Schließlich verrät ein Schüler aus der Parallelklasse, dass es jemand aus Adrians Klasse sei. Einzelgespräche und Gruppengespräche mit Lehrpersonen starten, und irgendwann findet man den Täter. Wichtig für Adrian ist, dass die Leute nicht nachtragend sind und dass der Vorfall schnell vergessen wird: ,(3) Aber es isch dänn mäischtens i paar Taag au vergässe. @1@ Das find ich äigentli au wichtig, dass es nööd z lang aahebt (6)“. Adrian geht in dieser Passage nicht auf die Tragweite des Vorfalls ein, lediglich darauf, dass dieser möglichst bald zu vergessen sei. Unterstrichen wird dies von der abgrenzenden Konjunktion, aber'. Adrian zieht also nicht den 
Schluss, dass nicht verpetzt werden soll oder dass aus der negativen Verhaltensweise Anhaltspunkte für künftige Verhaltensweisen zu gewinnen sind. $\mathrm{Ihm}$ ist offenbar wichtiger, dass der historische Vorfall vergessen wird. Eine Lesart der Textpassage kann darin bestehen, dass der Vorfall dadurch entdramatisiert werden soll, dass er möglichst bald vergessen wird.

(b) Lilian, seit kurzem in der Sekundarstufe A, stellt fest, dass in ihrer früheren Klasse B oft verpetzt wurde, in der neuen Klasse hingegen nicht. Diese Veränderung erklärt sie sich so: „Ja, (1) will ali echli mittänket und so. (1) Händ echli die bessere Schtrategie@1@ sozsäge (6)““. Lilian ist der Ansicht, dass die SuS der Sekundarstufe A aus der Perspektive des Klassenverbands und in dessen Interesse denken, was impliziert, dass die SuS aus der leistungsschwächeren Stufe weniger die Sicht der Lehrperson reflektieren. Lilian spricht von Schtrategie, womit im Voraus geplante, teils erprobte und gezielte Verhaltensweisen bezeichnet werden. Aufgrund Lilians Aussage ist ,Verpetzen' leistungsstufenabhängig, wobei die leistungsstärkere Stufe eher nicht verpetzt und daher konventionellen Unehrlichkeitsregeln oder unkonventionelle und loyale Ehrlichkeitsregeln befolgt.

(c) Nicht wenige SuS nehmen Bezug auf Primarklassen, wenn sie das Verpetzen im Interviewgespräch erläutern. Den Ehrlichkeitsregeln dieser Befragten ist gemeinsam, dass sie sich klar von der Praxis und der Zeit in den niedrigen Schulstufen abgrenzen. So berichtet beispielsweise Nora in Erzählbruchstücken:

I de Primaarschuel isch s soo: „Ja das säg ich de Leereri, wänn jetzt nöd uufhörsch!“ (1) Jetzt so i de Oberschtufe isch s äigentli- (2) Näi s Petze isch nöd soo- Also au wänn jetzt irgendöpper so en Säich macht oder d Underschrift gfälscht hät, säit s äigentli niemert de Leereri. Zwar d Jungs säget amigs: „Oh, bisch wider am Abschriibe!“, wänn me d Uufzgi über de Mittag no schnäll abschriibt oder soo, (1) aber es würd nie de Leeri säge oder irgendöppert anderem (4).

Aus Noras Erklärungen geht klar eine Dichotomie zwischen Primarstufe und Sekundarstufe hervor: In der Primarstufe wird bei Belästigungen gepetzt, und in der Sekundarstufe wird beim Brechen von schulischen Verhaltensregeln (Säich mache) und bei unlauterem Verhalten (Unterschrift fälschen, Hausaufgaben abschreiben) nicht gepetzt. Noras klare Ehrlichkeitsregel wird durch den absoluten Terminus nie unterstrichen.

Sogar einen Bezug zum Kindergarten stellt Marco her, um das Verpetzen zu erklären. Dies tut er in einer zugespitzten und emotional abwertenden Form. Er will damit aufzeigen, dass seine Klasse aus dem Alter heraus sei, in dem verpetzt wird, und er verurteilt das Verpetzen mit einer persönlichen Einschätzung: „Ich persönlich find das zimlich (1) Schäisse“. Er verwendet anschließend den emotionalen Terminus hassen, um die extreme Abneigung gegen das Verpetzen zu unterstreichen, und begründet diese mit dem Freiheitsentzug: ,(1) Will mer sött doch de ander mache laa, was er möcht (2)“ 
(Chiapparini 2011, S. 128). Somit kann das Verpetzen eine persönliche Freiheitsgrenze beschneiden.

Marco selbst erzählt, wie ihn einmal zwei Kollegen verpetzten (vgl. ebd., S. 128ff.): Unerlaubterweise verlassen alle drei während der Zehnuhrpause den Pausenplatz, um in einem Lebensmittelladen Getränke und Snacks einzukaufen. Beim Rückgang werden die anderen zwei erwischt. Er aber nicht. Jedoch verpetzen die zwei Kollegen Marco bei der Befragung durch die Lehrperson. Es gibt für alle drei eine Schulverwarnung unterschrieben vom Schulleiter. Marcos Eltern sehen nicht ganz den Sinn der Schulregel Pausenplatz während der Pause nicht verlassen ein. Denn sie sind, so Marco, der Meinung, dass Marco jetzt genug alt dafür sei. Inzwischen hat Marco die unerlaubten Ausflüge während der Pause wieder aufgenommen - jetzt aber allein.

In diesem Fall sind die zwei Kollegen ehrlich gegenüber der Lehrperson - aber nicht kollegial mit Marco. Alle drei haben das Bedürfnis, etwas für die Pause einzukaufen; weil das nicht erlaubt ist, machen sie es heimlich. Darin, dass drei Schüler ihre Initiative und ihre Bedürfnisse zugunsten der konventionellen Ehrlichkeitsregel verdecken, präsentieren sie sich in einer unterlegenen Rolle. Die Themen Mündigkeit, Sanktion und Freiheit werden hier im Zusammenhang mit der Ehrlichkeitsregel und der Tugendfrage berührt.

Fazit: In Abgrenzung zu anderen Klassen, leistungsschwächeren Klassen und Primarklassen lehnen die meisten SuS der dritten Oberstufe das Verpetzen explizit und grundsätzlich ab. De facto aber wird auch hier zuweilen noch gepetzt.

a) Verpetzen: „Das fänd ich jetzt nöd so cool!“

Die Schüler haben keine Schwierigkeit zu erklären, was mit vertädele und verpetzen gemeint ist, und tun dies in unterschiedlicher, doch mehrheitlich ablehnender Weise. So bezieht Lara ihre Definition allgemein auf das Vergessen von Hausaufgaben und speziell auf ihr Vergessen von diesen im Aufgabenheft:

(2) Ja, verpetze, (1) das isch äifach, wänn mer irgendöppis @vergässe hät und s dänn so Lüüt git@, wo (2) findet, sie müend jetzt de Leerer säge (1), dass mer das nöd hät oder so. Oder au wänn au so (2) Ufgabe im Heft und dänn gseet mer, dass es irgendwie de Naachbar nöd hät, wänn mer dänn <Husten>, (1) säit: ,Ja, si, er hät s nöd.“ (1) Das isch bi öis no nie passiert, aber das fänd ich jetzt nöd so cool.

Wie Lara verneinen die SuS einstimmig, dass in ihrer Klasse irgendjemand verpetzt wird. Das Verraten von Mitschülerinnen und Mitschülern ist negativ konnotiert, wie es der absolute Terminus no nie unterstreicht. Lara schließt ihr Statement mit einer personifizierten Beurteilung des Verpetzens, wenn sie sie in dem relativierten Modus nöd so cool zusammenfasst. 
Ebenso unterstreicht Regula, dass in ihrer Klasse Mitschüler nicht verpetzt werden, wenn auch die meisten von ihnen über die Anwendung von unlauteren Mitteln während Schultests informiert sind.

Wänn mer e Prüefig naahole mues, mues mer immer in es anders Zimmer gaa, will es isch ja luut im andere. (2) Und dänn chan mer ja mäischtens- Daa isch mer aläi im Zimmer und dänn (1) chan mer äigentli en Schpick mitnee. Also en richtige. Da chan mer (1) würkli zum Biischpiil es Grammatikblatt oder so mitnee (2). Und au ganz (1) schön öffne uf em Tisch oder so. Will d Leererin chunt äigentli nöd ie go luege. (3) Und ich würd drunter verschtaa, wänn jetzt öpper das wäiss, und dass nachher de Leererin gaat go säge. (1) Aber das passiert nöd. (1) Ich mäine, mer wäis under de Klass, wänn jetzt öpper es Grammatikblatt mitnimmt. Aber niemert hät s (1) irgendwie gsäit oder so.

Dieses Beispiel dokumentiert, dass Sandras Klasse über die Anwendung von unlauteren Hilfsmitteln informiert ist, die in Nachholtests, die in einem separaten Zimmer stattfinden, sehr gut eingesetzt werden können. Ausschlaggebend für das Verhalten der SuS ist nicht die Ehrlichkeit speziell gegenüber der Lehrperson, welche eben nicht kontrolliert, sondern die kollegialen Regeln innerhalb des Klassenverbandes. Kurz: Ehrliches Verhalten ist hier durch kollegiale Regeln ersetzt.

In gleicher Weise verurteilt Stefan das Verpetzen eines Mitschülers besonders scharf als absurd, wenn dieser unlautere Hilfsmittel während der Schultests einsetzt:

(5) Also ich glaub es würd niemert uf d Idee cho, go säge, ja: „Dee hät im Fall en Schpick ghaa" (4).

Für Stefan ist es selbstverständlich, dass niemand in seiner Klasse irgendjemanden im Rahmen von Schultests bei der Lehrperson verraten würde. Hier dokumentieren sich ebenfalls der starke Klassenzusammenhalt und die Selbstverständlichkeit des Nichtspickens.

Darüber hinaus vertritt Clemens eine explizite und absolute Haltung des Nichtpetzens, die sich nicht nur auf die genannten Unterrichtspraktiken Vergessen von Hausaufgaben oder die Anwendung unerlaubter Hilfsmittel während der Schultests - beschränkt, sondern immer gilt: Es gebe keinen Grund, jemanden zu verpetzen. Davon habe er selbst auch schon profitiert:

(3) Ich bin ja anonym, da chan ich s ja @säge@. (1) Mir händ i de Schuel (1), also bi de WCs und soo. Si kännet sicher Träckers, die wo so de Name uufschriibet und so. Das han ich au gmacht. Also mit emene ganz dicke fette Schtift- i de WC Saal hinder de Tüüre, überall, rächt oft heregschribe. Und dänn händ si [die Lehrpersonen] s halt gsee, ich mäine, daas isch ja nöd $\mathrm{z}$ übersee. Und dänn sind $\mathrm{s}$ halt immer $\mathrm{zu}$ öis choo und so und händ gfröögt, so- und ali [SuS] händ s gwüsst, dass ich s bin gsii, aber niemert het öppis gsäit. (1) Si händ nur so Aaschpilige gmacht, so: „Hey, C, kännscht du dee, wo das gmacht het“ oder soo. Aber gsäit würkli, wer das gsii isch, händ s äigentli nie. (1) Und de Leerer het s au nie gmerkt.

Clemens ist sich bewusst, dass seine Tätigkeit als Tracker gegen die Schulregel verstößt. Daher vergewissert er sich nochmals, bevor er zu erzählen be- 
ginnt, dass das Interviewgespräch anonym ist. Ohne zu begründen, warum er ein Tracker ist, erklärt er das Tracken als Beschreibung der WC-Wände mit einem dicken Stift. Die Lehrpersonen versuchen, den Autor der Schriftzüge ausfindig zu machen, aber ohne Erfolg. Einzelne aus Clemens Klasse spielen öffentlich auf ihn an, indem sie ihn vor dem Lehrer befragen, ob er denjenigen kenne, der die Wände beschrieben hat. Jedoch wird er nicht verraten, trotz der wiederholten Nachfrage der Lehrpersonen und des Mitwissens der SuS.

Mit der Selbstsicherheit und Selbstwahrnehmung von Clemens sprechen nicht alle SuS über das Nichtverpetzen. Nur in einer der befragten Klassen (D) wird öfters innerhalb der Klasse verpetzt. Claire berichtet beispielsweise von einer Schulstunde, in der der Lehrer abwesend und die Klasse so laut ist, dass eine andere Lehrerin vorbeikommt, um sich zu erkundigen, weshalb die Klasse so laut sei. Die lauteste Person wird verpetzt. Hingegen wird in jener Klasse nach Aussage von Christoph dichtgehalten, wenn jemand in Schultests schummelt.

Aus den Interviewdaten präsentiert sich somit ein eindeutig negatives Bild vom Verpetzen innerhalb des Klassenverbands, insbesondere im Bereich des Vergessens von Hausaufgaben oder der Anwendung unlauterer Hilfsmittel während der Schultests.

\section{b) Konfliktsituationen beim Verpetzen}

Das Bestehen der Regel, dass innerhalb der Schulklasse nicht verpetzt wird, bedeutet nicht, dass keine klasseninternen Konflikte bestehen oder in bestimmten Situationen einzelne SuS die kollegiale Regel nicht einhalten. $\mathrm{Zu}$ solchen Situationen zählen besonders die, die mit Gerechtigkeitsfragen zu tun haben, mit schwerer Manipulation wie Erpressung, mit ernsten Zwischenfällen wie Unfällen sowie mit Iischläimi, also dem Einschmeicheln/Anbiedern.

Konflikte durch gefährdete Gerechtigkeit

Clemens sieht einen Konflikt in Situationen, in denen eine Kollektivbestrafung für die ganze Klasse ausgesprochen wird. Als erprobte und gelungene Alternative schlägt er vor, die Schuld auf sich zu nehmen. Wie dies Otto, ein Mitschüler von Clemens, gemacht hat (vgl. zu Beginn von Kap. 8.2).

Nicht alle SuS vertreten eine bedingungslose kollegiale Haltung wie Clemens. Ebenso sind nicht alle bereit, negative Konsequenzen für das Verschulden von anderen auf sich zu nehmen. Von heldenhaften Verhaltensweisen, die mit Beispielen untermauert sind, berichten punktuell nur Clemens (Otto und die Kollektivstrafe, vgl. Kap. 8.2) und Nora (Einsatz für einen ungerecht behandelten Mitschüler, vgl. Kap. 8.1.5). 
Über die Kollektivbestrafung hinweg, sieht Clemens auch bei Mitwissen um Gewaltpläne von Mitschülern keinen Grund, diese zu verpetzen. Dazu nennt er ein hypothetisches Beispiel. Er erklärt, dass er bei Mitwissen um den Plan eines Mitschülers, jemanden blutig zuschlagen, diesen nur mit direkten und kollegialen Empfehlungen zurückhalten würde. Ein Verpetzen bei der Lehrperson sei kein Lösungsweg. Auf diese Weise vertritt Clemens seine absolute Ablehnung gegenüber dem Verpetzen konsequent durch das ganze Interviewgespräch.

Unklar bleibt, weshalb Clemens die absolute Haltung so sehr betont. Denn die Lehrpersonen sind nicht direkt seine Feindbilder, wie aus dem Umgang mit einem Lehrer hervorgeht, mit dem er sich während des Interviews spontan unterhält (vgl. Kap. 5.4.3).

Im Gegensatz zu Clemens' Ehrlichkeitsregel nimmt Lilian eine positive Haltung gegenüber dem Verpetzen ein, sofern es darum geht, kollektive Bestrafungen zu umgehen. Ihre Ehrlichkeitsregel teilt Emil. Er erzählt, wie in einer Singstunde jemand Papierchügeli umherwirft. Weil der Lehrer nicht herausfindet, wer es ist, spricht er eine Kollektivstrafe aus, die zusätzliche Hausaufgaben umfasst. Die Klasse verrät nichts. Allerdings gesteht am Ende der Schulstunde der betreffende Schüler seine Tat und der Lehrer nimmt die Kollektivstrafe zurück, was Emil als sehr positiv einschätzt.

Regula berichtet von einem ähnlichen Vorfall: In der Kochstunde werfen einige SuS Spaghetti an die Wand. Die Klassenlehrerin kündigt der Klasse eine Kollektivstrafe (Telefonanruf bei den Eltern) an, falls die Täter sich nicht freiwillig melden. Die Klasse weiß zwar, wer die Täter sind, aber niemand klärt die Lehrerin darüber auf. Erst gegen Unterrichtsende gestehen die Täter der Lehrerin ihr Vergehen. Regula findet es fair, dass die Betroffenen ihre Tat eingestehen und somit nicht die ganze Klasse die Strafe antreten muss.

Nicht wenige SuS berichten, wie einzelne Schüler nicht nur bei stillen und konzentrierten Arbeiten stören, sondern auch Ursache für kollektive Konsequenzen sind. So erzählt Lara von einer Schulstunde in Mensch und Umwelt. Sie hat ein spannendes Thema: den Schwangerschaftsverlauf. Es gibt freie Sitzwahl, was Lara besonders mag. Allerdings sind einige SuS zu laut, und die Lehrerin bestraft alle, indem die SuS sich wieder an die gewohnten Arbeitsplätze zurückzusetzen haben: „Das (Zurücksitzen) isch echli blööd gsii.“ Regula nimmt hier eine passive Rolle ein, denn sie wehrt sich nicht gegenüber der kollektiven Strafe, die sie nicht verursacht hat. Das kann daran liegen, dass sie nicht couragiert genug ist, der Lehrerin diese ungerechte Behandlung aufzuzeigen. Oder sie fügt sich den Kollektivregeln, welche diejenigen diktieren, die laut waren.

Die kollegiale Regel des Nichtverpetzens wird auch gebrochen, wenn einzelne SuS zu ihrem Recht kommen wollen. So erzählt beispielsweise Lara von Roger: 
Oder zum Biischpill au wänn s so- wänn mer müend es Heft abgee, das hät s äinmaal gee. Mir händ müese es Heft abggee und dänn hät s mindeschtens d Hälfti vo de Klass, hät s nöd fertig ghaa und äine dee hät s aber fertig ghaa und dee hät gfunde- Also d Leereri hät s dänn vergässe, iizzie, und dänn hät er gfunde, er gaat das ire jetzt goge säge, dass si das sött iizie, dänn er hegi bis am Elfii deet draa gschaft und jetzt wett er s au abgee. (1) Jetzt will er au irgendwie zu sim Rächt choo, dass s er gmacht hät. (2) Das händ dänn au echlii blööd gfunde. Also ich cha s au verschtaa, das würd mir au echli närve, wänn ich so lang draa gschaffet hät und dänn (1) ali wo s nöd händ äifach so devoo chömet (1).

Die Mehrheit der Klasse erfüllt eine Aufgabe nicht. Allerdings erledigt Roger diese noch bis spät in die Nacht und unter Anstrengung, um sie termingerecht abzugeben. Da die Lehrerin am nächsten Tag vergisst, die Aufgabe einzusammeln, fühlt sich Roger ungerecht behandelt, weil er sich so außergewöhnlich eingesetzt hat und dafür nicht belohnt wird, sondern fast bestraft wird, weil die anderen ohne Einsatz gut wegkommen. Daher geht er zur Lehrerin und gibt seine Arbeit ab, was die anderen in der Klasse nervt. Denn mit dieser Geste erinnert er die Lehrerin daran, den Arbeitsauftrag einzuziehen. Jedoch kann Lara Rogers Verhaltensweise gut nachvollziehen.

Obwohl es eine Erzählung aus der Perspektive einer zweiten Person ist, zeigt dieses Beispiels sehr schön auf, wie das Verpetzen auch indirekt geschehen kann. Roger will zu seinem Recht kommen und nimmt dafür in Kauf, dass über die Hälfte der Klasse in ein schlechtes Licht gerückt wird, weil sie die Hausaufgaben nicht gelöst hat. Roger verpetzt indirekt, indem er der Lehrerin seine erledigten Hausaufgaben zeigt und die Lehrerin damit daran erinnert, dass sie diese kontrollieren sollte.

Valentina erzählt auf Nachfrage der Interviewerin folgendes Beispiel für ein Verpetzen aus Verletzung des Gerechtigkeitsgefühls:

(10)@1@Ja zum Biischpiil im Turne, wänn e Person gwüssi Sache nöd macht, sondern zum Biischpiil bim Volleyball- deet sitzt immer irgend öpper use. Es isch immer am Rotiere. Dänn wänn die Person, wo use sitzt, also überspringt äifach und so säit: „Hey ich bin immer no dusse, mir isch grad schlächt“, und dänn verpetzt die Person@1@ja. (2) Aber wänn s so zum Biischpiil gaat (1) zum Schuel-Turne schwänze. Das machet mir Mäitli äigentli scho zimlich vill @1@(2). Dänn immer so: ,Ja sii, ich han mega Ruggewee“ oder „Ich han irgend öppis am Fuess" oder „Mir isch schlächt", „Ich han Chopfwee, Mens“, irgend so öppis. Und dänn säit niemert irgend öppis. (2) Ussert paar maal säget Buebe: „Aa ja, häsch du das, grad?“ Dänn grinst er echli tumm @und ja@, aber süsch nöd. (5) Äifach nur wämmer echli fuul isch und das echli unfäär fint, dass die Person es nümme mache muess, will si e tummi Uusreed gfunde hät (5).

Die Schülerinnen sind sehr ideenreich mit Ausreden, um nicht am Turnunterricht teilzunehmen. Dazu zählen Übelkeit, Kopf-, Rücken-, Fuß-, Bauchschmerzen und monatliche Frauenbeschwerden. Ausschlaggebend für das Verpetzen sind die eigene Faulheit und die selbst wahrgenommene Ungerechtigkeit, dass einige dank der Ausreden nicht turnen müssen. Für das Verpetzen ist damit die Gerechtigkeit bezogen auf die eigene Person ausschlaggebend. 
Valentina berichtet in einer neutralen Erzählperspektive von den Praktiken im Turnen, womit die Regelhaftigkeit dieser Vorgänge unterstrichen wird.

Konflikte durch schwere Manipulation

Obwohl Fabian das Verpetzen als sinn- und nutzlos bezeichnet, gesteht er dieser Regel eine Ausnahme zu: Bei schwerer Manipulation ist ein Verpetzen der Täter sinnvoll.

Verpetze git s bi öis fascht gar nöd. (2) Verpetze häisst, de andere verrate, so gsäit, aber (1) die mäischte sind s schweers Vergee. Aber wänn s würkli en Iifluss hät uf irgendöppis, also, wänn zum Biischpiil de äint de anderi erprässt oder so, dänn würd ich s au säge, will es hät schweeri Ifluss uf de ander oder so. (1) Ich han grad us Tüütschland Nachrichte göört, dass sich en Schüeler, sich umbracht hät, will er vom andere erprässt worden isch mit irgendöppisem (2). Bis s soo wiit chunt, okey.

Fabian berichtet in einer hypothetischen Beschreibung, dass Verpetzen unter bestimmten Bedingungen erlaubt ist: wenn eine Verhaltensweise zu schwerer Manipulation einer Zweitperson führt, konkret wenn ein Schüler wegen Erpressung sich das Leben zu nehmen droht.

Diese Reflexion ist durch eine Medienmeldung aus Deutschland angeregt, wo ein Schüler sich tatsächlich wegen Erpressung das Leben nahm. Anhand dieses Beispiels erklärt Fabian die Grenze der Regel des Nichtverpetzens: Verpetzen ist erlaubt, um schwere Manipulation zu vermeiden.

Dennoch bleibt Fabians Extrembeispiel eine Hypothese. Es bleibt offen, ob er auf dieses Beispiel zurückgreift, weil es weniger verfänglich oder belastend ist oder weil er einem ähnlichen Fall offenbar in seiner bisherigen Schulzeit nie begegnet ist. Der Konjunktiv in der Beschreibung unterstreicht die hypothetische Sichtweise.

Konflikte durch ernste Zwischenfälle

Sandra differenziert ähnlich wie Fabian zwischen ernsten und anderen Situationen. In Ersteren sei das Verpetzen gerechtfertigt:

Also ich find, wänn en Umfall passiert oder so, zum Biischpiil, wänn (1) irgendöpper verletzt wird oder so, find ich schoo, dass mer s müsst verpetze. Ich mäin s isch äigentli eernscht.

Die kollegiale Regel Nicht verpetzen darf laut Sandra gebrochen werden, wenn ein Unfall passiert. Sandra argumentiert hier mit der Ernsthaftigkeit der Situation. Wie Fabian reflektiert sie in der Möglichkeitsform und berichtet nicht von einem realen Vorfall. Inwiefern diese Ehrlichkeitsregel auch Anwendung in der Schulpraxis findet, bleibt unbeantwortet. 
Allerdings erzählen die SuS auch von erlebten Beispielen, in denen sie oder Mitschüler jemanden beim Lehrer verpetzt haben: Christoph selbst muss niemanden verpetzen, weil er angespannte Situationen mit Mitschülerinnen und -schülern meidet. Hingegen weiß er von einem längeren Konflikt zwischen zwei Mitschülern zu berichten, in den schließlich der Lehrer einbezogen wurde:

De C hät en andere Schüeler vo öisere Klass- Si sind sich immer id Haar graate. (1) Und händ sich halt Schimpfwörter- (1) Und dänn hät dänn- Dänn isch s äinisch z wiit worde und dänn hät de $\mathrm{C}$ am andere e Fuscht is Aug gee. Das sind s au- Daas isch dänn au so öppis- Wämmer jetzt zum Biischpiil er isch miin Kolleeg oder wänn dänn so drüü, vierVilicht jetzt nöd äifach deet schtönd, säget so: „Tubel“ oder so. Aber nur äine jetzt zum Biischpiil, de Bueb, wo jetzt aktiv jetzt gsii isch und mit im jetzt Schimpfwörter gsäit hät und so. Dänn isch s für mich au blööd. (2) Wäis nöd. Äinersiits hät er ja vilicht rächt. Es chan ja emaal öppis vorgfalle gsii, wo de ander natürli en Säich gmacht hät. Aber anderersiits isch au nöd rächt, wämmer immer uf de gliich loos gaat. Das isch vilicht e Wuche oder zwäi gange. Das wäis ich nüme gnau. (3) Süscht, (1) wäis ich jetzt nüüt. (3) (I: Wie hät sich das glööst, jetzt bi dim Kolleeg?) (3) Ja, si sind- (1) Wie isch das jetzt fertig gsii? (1) Si sind sich dänn us em Weeg gange (1) und mir händ dänn au glueget, dass si sich, äifach nöd znööch chömet. Mir sind dänn au dezwüsche, dänn händ si öis usenandgnoo. (1) Ich glaub, dänn isch irgendöppis vorgfalle, dass $<_{\mathrm{S}}>$ dänn de $\mathrm{C}$ am Herr $\mathrm{H}$ gsäit hät und dee hät dänn es Machtwort gschproche und nachheer isch $<_{\mathrm{s}}>$ glaub au verfloge, dänn äifach (5).

In dieser Erzählung fällt die mediatorische Rolle auf, die Christoph und andere Mitschüler einnehmen, um die streitenden Parteien ( $\mathrm{C}$ und einen Mitschüler) zu beschwichtigen. Als letzte Lösung entscheidet sich $C$, den Lehrer einzubeziehen, und verpetzt damit quasi den Mitschüler. Gepetzt haben hier nicht die Mitschüler, sondern der betroffene C, der erst zu diesem Ausweg greift, nachdem zwei schwere Vorfälle eingetroffen sind, von denen Christoph sich nur an ein blaues Auge erinnern kann. Konsequenz ist das Machtwort des Lehrers, was eine ausdruckstarke Metapher ist. Der Lehrer nimmt aus der Sicht von Christoph keine vermittelnde Rolle, sondern eine hegemoniale Rolle ein, welche schließlich die spannungsreiche Beziehung zwischen $\mathrm{C}$ und dem Mitschüler zu lösen scheint.

In diesem Beispiel zeigt sich schön, wie mit dem Verpetzten die Hilfesuche bei der Lehrperson angestrebt wird, die dann handeln kann. Den SuS gelingt es offenbar nicht, den Konflikt selber zu lösen; daher greifen sie zur Lösung des Verpetzens.

Konflikte durch unsympathische Schülerinnen und Schüler

Kleinere Zänkereien - chiflet oder Beschimpfungen - kommen in der eigenen Klasse und auch in anderen Klassen vor. In solchen Fällen wird dann verpetzt, wenn der Grad an Antipathie eine bestimmte Grenze erreicht hat. Bei- 
spielsweise erzählt Ilona von ihrem Banknachbar, der sie so sehr stört, dass sie sein Verhalten schließlich dem Lehrer verpetzt:

(3) Ich han so z säge, ich bin nüün Jaar mit em Bueb id Schuel. (1) Gaan immer noo. Sit de Dritte bis jetzt. (1) Immer i de gliich Klass (1) und halt ebe, mir händ Zikkereie, will eer isch ebe luut und schpilt i de Schtund, er konzentriert sich nöd. Und daa länkt er mich au ab. Und zum Biischpiil grad geschter bim Choche, (2) bin ich duregloffe und er hät mich uf de Chopf glanget. Nachheer bin ich uusgflipt.@1@(1)@1@ Nachheer bin ich uusgflipt und nachheer han ich s- Nachheer han ich s halt a de Leereri gsäit: „Wänn jetzt nöd uufhöörsch, gaan ich zum Heer B. Will daas schtresst." Und de Herr B hät s warschiindli au gmerkt, dass er und min Banknachbaar vill immer gredet händ. Und hät min Banknachbaar umgsetzt und jetzt isch en andere näbet mir (2).

In Ilonas Erzählung geht es vorerst nicht um ein unerlaubtes Verhalten, sondern um eine nichtkonforme Verhaltensweise eines Mitschülers. Sie bezeichnet dies als Zikkereie, was üblicherweise gleichgeschlechtlich und insbesondere unter Schülerinnen verwendet wird. In vier Verhaltensschritten erreicht sie, dass der Banknachbar den Platz wechselt: Zuerst flippt sie aus, nachdem er ihr de Chopf glanget hat (Ausgangslage). Sie teilt es der anwesenden Kochlehrerin mit (1. Schritt), warnt den Mitschüler (2. Schritt), dass sie zum Lehrer B gehe (3. Schritt), und tut dies auch, womit sie Erfolg hat (4. Schritt).

Interessanterweise berichten alle vier SuS des Schulhauses F, dass üblicherweise nichtkonformes Verhalten untereinander dem Lehrer gepetzt wird, eine Praxis, die in den Berichten der anderen drei Klassen kaum vorkommt.

So berichtet Adrian von zwei Schülern, die sich gegenseitig stressen, wobei einer den Lehrer einbezieht. Nach längerer gegenseitiger Schuldzuweisung endet das Ganze in einem Witz, und der Lehrer empfiehlt ihnen, aufzuhören. Hier präsentiert sich das Verpetzen in Form eines Spieles, das von einem emotionalen Auslöser ausgeht. Diese Ursache bestätigt auch Emil: Verpetzt werde selten, und wenn, dann weil ein Schüler den anderen einfach nervt.

Folglich sind es emotionale und personenspezifische Gründe, die Schüler veranlassen, die kollegiale Regel des Nichtverpetzens zu brechen.

Lara berichtet von einem Beispiel für die Regel Wenn jemand besonders wütend auf jemand anderen ist, dann wird verpetzt. Sie erzählt von einer Hausaufgabenkontrolle, in der jeder SuS die Lösungen vorzulesen hat. Damit folgt die Lehrerin dem Prinzip: Wer vorlesen kann, hat die Hausaufgaben auch gelöst.

@ Also, das chunt amigs scho vor, wänn Lüüt so grad unglaublich sauer sind ufenand@, will irgendöppis passiert isch vorheer oder so. (1) Und dänn findet mer halt: „Ja, si, er hät das aber nöd gmacht" oder so. Also wänn dänn d Persoon, dänn d Löösige list und si [die Lehrerin] es findet, si hät s gmacht und so. (1) Dänn git s scho, das Lüüt dänn äifach säget: „Ja, du häsch es gar nöd gmacht.“ Dänn ghöört s dänn de Leerer und so. Aber äigentli nume wänn mer würkli sauer isch, passiert das. 
Die SuS lesen die Hausaufgaben vor, und die Lehrerin merkt nicht, dass einer die Hausaufgaben nicht gelöst hat, denn er liest ja die Lösungen vor. Das deckt ein Mitschüler auf, der besonders sauer auf den anderen ist.

Lara erzählt in einer neutralen und allgemeinen Form, sodass nur aus der direkten Rede hervorgeht, dass es sich beim Vorlesenden um einen Schüler, und nicht um eine Schülerin handelt. Ferner markiert sie die Intensität, mit der die Person sauer sein muss, indem sie diese Aussage am Anfang und am Ende der Erzählung wiederholt und dem Attribut die Verstärkungswörter unglaublich bzw. würkli voranstellt. Weiter verwendet Lara das passive Verb geschehen, womit den sich so verhaltenden Personen die Verantwortung für solche Begebenheiten abgesprochen wird. Schließlich kommt das Verpetzen aus Antipathie nur selten vor, wie Lara zu Beginn der Erzählung versichert.

Vor diesem negativen Hintergrund des Verpetzens scheint Lara ihre Regel des Nichtverpetzens zu bestätigen. Anders ausgedrückt: Lara erklärt, dass selten jemand verpetzt wird, und dann nur, wenn die Person besonders wütend auf eine andere Person ist. Weil das selten vorkommt, dominiert im Klassenverband offenbar die Regel des Nichtverpetzens. Demgegenüber vertritt Lilian offen die Regel des Nichtverpetzens unabhängig von der Sympathie der Mitschüler. Dabei bezieht sie sich auf das Thema Hausaufgaben.

(2) Wänn irgendöppert- (3) ich wäis au nöd. (11) Wänn irgendöpper d Uufzgi nöd gmacht hät und de Leerer bemerkt s nöd, dänn git s halt sönigi, wo säget: „Jaa, die hät d Uufzgi nöd gmacht.“ Und daas git s bi öis nöd, wänn öpper nöd d Uufzgi gmacht hät, dänn chan mer s irgenwie so halbe abschriibe, (1) äifach, dass me öppis uf em Blatt hät und so. (2) Me wird nöd verpetzt irgendwie dass- (2) dass mer dänn am Leerer säit, au wenn- wenn ich jetzt öpper hasse us minere Klass und die hät d Uufzgi nöd gmacht hät, dänn chan mir das äigentli egaal sii. Dänn muess ich si nöd no goge verpetze, dass si mich no mee hasst @1@ (9).

Lilian beschreibt in allgemeiner Form, dass die vergessenen Hausaufgaben zumindest teilweise abgeschrieben werden können, damit dem Lehrer nicht ein leeres Hausaufgabenblatt auffällt. Die allgemeine Form kann auf die Routine dieser Vorgehensweise hindeuten. Allerdings bezieht Lara den Schlusssatz auf die eigene Person: „[...] dass si mich no mee hasst.“ Das kann ein Hinweis darauf sein, dass sie das bereits erlebt hat. Leider beschreibt sie dies nicht weiter, sondern stellt eine längere Sprechpause ein.

Aus Lilians Berichten zu schliessen, petzt sie nicht aus einem Grund: Ihr ist der gehasste Mitschüler nicht gleichgültig; aber sie kalkuliert, dass sie noch mehr gehasst würde, wenn sie sein Unterlassen verraten würde, und nur aufgrund dieser Kalkulation unterlässt sie das Verpetzen und vermeidet damit, dass dieser sie noch mehr hasst. Dem Interviewkontext kann entnommen werden, dass Lilian von der ganzen Klasse ausgeschlossen wird, was sie selber an einer anderen Stelle bestätigt. Daher stehen Lilians Aussagen in einer besonders spannungsreichen Beziehung zu ihren Mitschülerinnen. 
Konflikte durch Einschmeicheln

Ein Grenzfall des Verpetzens sind Iischläimer, welche im Klassenverband die kollektive Meinung vertreten und bei Präsenz der Lehrpersonen deren Partei ergreifen. Sie verpetzen die Klasse durch einen Wechsel der Partei und sind insofern unehrlich, als sie je nach hierarchiespezifischem Umfeld ihre Meinung ändern. Dies steht in Zusammenhang damit, dass diese SuS im Klassenverband oder gegenüber einzelnen SuS nicht zu ihrer Meinung stehen können. In diesem Sinne erklärt Stefan anhand störender Verhaltensweisen von Mitschülerinnen und -schüler, was Iischläimer sind:

(3) Jaa, die wo, (1) sich entweder (1) zeerscht über- (1) zum Biischpiil über öppis fluechet (1) und wänn s zur Diskussion chunt, (1) dänn säget: „Also näi. Ich han also- ich han äigentli gar nüd gmacht", und so. (2) Also äifach so, Iischläimer (2). Das find ich dänn äifach denäbet.

Stefan zeigt den Parteienwechsel der Iischläimer auf, welche zunächst eine Situation kritisieren (darüber fluchen), aber einen Rückzieher machen, wenn die missliche Situation zur öffentlichen Aussprache (Diskussion) kommt. Ein solches Verhalten beurteilt Stefan negativ (denäbet). Distanzierung von solchen Mitschülern signalisiert Stefan, indem er eingangs ein anonymes Subjekt für die Iischläimer verwendet: die.

In diesem Abschnitt setzt Stefan voraus, dass die Bestimmung der Ischläimer in engem Bezug zur Präsenz oder Absenz der Lehrperson steht; später expliziert er das an einem Beispiel: Die ganze Klasse schreibt in der Klassenstunde der Turnlehrerin einen Brief, in dem sie ihre Unzufriedenheit mit der Turnlehrerin festhält, weil diese ständig zu spät in die Turnstunden komme. Bei der Übergabe des Briefes an die Lehrerin zieht sich zuerst eine Schülerin zurück und anschließend auch zwei Kolleginnen. Die erste Schülerin erklärt, dass sie inhaltlich nicht daran beteiligt sei. Durch diese Parteinahme ist das Anliegen der ganzen Klasse geschwächt, und Stefan schließt daraus auf den fehlenden Erfolg des Anliegens.

Daraus resultiert die Regel: Ein Anliegen des Klassenverbands gegenüber einer Lehrperson kann nur durch die kollegiale Haltung aller SuS Erfolg haben. Und: Wenn eine Schülerin sich aus der kollegialen Haltung des Klassenverbands zurückzieht, verrät sie die ganze Klasse.

Die hier implizierte Erwartung des Klassenzusammenhalts setzt Emil explizit in Bezug zu Vertrauen und Ehrlichkeit, als er auf die Frage nach seinem Ehrlichkeitsverständnis antwortet: ,(4) Ja, dass mer em andere vertaue chan. (2) Dass er nöd lüügt also. (1) Und (3) ja immer zämehebet so zsäge (4).“

Emils Antwort ist eine Aufzählung von kontextlosen Aspekten eines Ehrlichkeitsverständnisses: jemandem vertrauen können, nicht lügen, zusammenhalten. In diesem Textausschnitt schwingt ein Selbstverständnis von Ehrlichkeit mit, das zu Beginn der Aussage mit Ja, dass signalisiert wird und mit den Eigenschaften Vertrauen oder Zusammenhalt attribuiert wird, die 
vorausgesetzt sind. Dieselben Eigenschaften werden bei den anderen kollegialen Regeln des Nichtverpetzens angeführt (vgl. Kap. 8.2.1).

Somit haben diese Darlegungen aufgezeigt, was SuS unter Verpetzen als eine besondere Form der kollegialen Unehrlichkeit verstehen und wo Konfliktsituationen zwischen kollegialen und individuellen Regeln bestehen. In diesem Spannungsfeld ist auch die Hilfsbereitschaft innerhalb des Klassenverbands zu situieren, wie dies im Folgenden thematisiert wird.

\subsubsection{Unlautere Hilfsbereitschaft im Klassenverband}

Die SuS berichten aus verschiedenen Schulbereichen über kollegiale Hilfsbereitschaft, deren Relevanz grundsätzlich als sehr hoch betrachtet wird. Hilfsbereitschaft thematisieren sie in folgenden Kontexten: im Klassenlager, auf dem Pausenplatz, beim Lernen, beim Erklären von Hausaufgaben und während des Schreibens von Schultests.

Marco erklärt, dass ein guter Klassenzusammenhalt an der Hilfsbereitschaft der Mitschüler zu erkennen ist: ,(2) Me hilft sich. Mer laat sich gägesiitig, irgendöppis vilicht emaal abschriibe oder ebe öppe- Me hilft sich mit emene Schpick oder so.“

Die Hilfsbereitschaft unter den SuS zeigt sich bei unlauteren Verhaltensweisen: Man lässt abschreiben (Hausaufgaben oder Lösungen während der Schultests - das bestimmt Marco nicht klar) oder man hilft sich mit einem Spick aus. Die Regelmäßigkeit dieser Hilfe drückt sich durch die wiederholte Nennung der Hilfe und des neutralen Subjekts me aus. Zudem benennt Marco die Gegenseitigkeit als Eigenschaft der Hilfsbereitschaft. Die Vorsicht oder Unsicherheit bei der Einordnung dessen, was eine unlautere Verhaltensweise sei, wird mit den Begriffen vilicht, emaal oder öppe signalisiert. Offen bleibt, ob alle SuS von der Hilfsbereitschaft profitieren oder nur einzelne.

Hilfsbereitschaft im Klassenverband hat für Marco eine so grosse Relevanz, dass er in Kauf nimmt, die Grenze der Unehrlichkeit gegenüber der Lehrperson zu überschreiten und sich eher an den kollegialen Regeln (Hilfsbereitschaft und Vertrauen im Klassenverband) zu orientieren als an der sozial erwarteten Ehrlichkeit gegenüber der Lehrperson - die er kennt. Fabian hinterfragt, ob unlautere Hilfsbereitschaft tatsächlich dem Empfänger etwas nützt:

[...] mer hilft de andere. Ich han nüüt degäge, (1) wänn mer vor de Schtund äim abschriibe laat, mäin das isch zum Wool vo allne. Okey für im nöd grad, also vo dem, wo s abschriibt, will eer chan s dänn nöd, aber wänn er s Bedürfnis hät (6).

Gegenseitige Hilfsbereitschaft besteht auch für Fabian darin, Hausaufgaben abschreiben zu lassen, was er als ein Wool vo allne (Wohl für alle) versteht. Worauf das ,Wohl' sich bezieht, ist unklar. Auf jeden Fall bezieht es sich 
nicht auf den Empfänger der Hilfsbereitschaft, denn dieser wird, so Fabian, den Schulstoff nicht beherrschen. Das ist ein lernorientiertes Argument, das über das Bedürfnisargument des Nutzers dominiert. Anders ausgedrückt: Zuerst sind die individuellen Bedürfnisse zu erfüllen, und in diesem Zusammenhang gelten die individuellen Regeln. Erst in einem zweiten Schritt zählen dann die lern- oder schulorientierten Regeln, welche die Bildungsbedürfnisse abdecken.

In gleicher Weise legitimiert Lara die unlautere Hilfsbereitschaft während Schultests, die sie stark mit Herausforderungen und persönlichem Interesse verbindet und die nach ihrer Darstellung punktuell und sehr selten vorkommt:

(3) Ja, also Schpicke jetzt, (2) also es git- Also es git halt so Lüüt, die dänn immer so Zädeli oder so, aber das- Dänn tüen mir halt es Zädeli is Etui, aber ich glaub, die mäischte benutzes dänn nöd. Also so als Sicherheit oder so, aber. (1) Also es isch au no nie öpper verwütscht worde oder so. Ich glaub es isch nöd so hüfig und wänn dänn, isch s halt so, dass mer am Nachbaar s Blatt (1) irgendwie zäigt oder so. Es hät sogar äimaal (1) hämmer bi- (1) In Gschicht hämmer e Prüefig ghaa und öisi Leereri isch deet vorne gschtande und hät äifach so uf ires Blatt gluegt. (1) Und dänn händ hine d Lüüt sogar d Blätter tuuscht (1) und si hät s nöd gmerkt. (1)@Das isch dänn hält au no luschtig@, also. Und si hät s au nachheer nöd gmerkt- Also bim Korrigiere oder so. (2) Und au- (1) Hüt im Französisch hämmer s im Klassezimmer ghaa und dänn sitzt öpper- mues amigs vorne am Leererbank, äifach damit s (1) gnueg Abschtand hät zwüsche de Lüüt. (2) Und dänn chan mer halt, wie mit dene Lüüt vor äim ooni Ton rede oder so. (1) Oder am Schluss i de letschte Minuute, wänn ali scho am Abgee sind, chan mer vilicht no schnäll öppis aneschriibe, wänn s äim öpper säit, aber susch. (4) So richtig gschpickt, dass jetzt en Groose irgendwie maal öpper gmacht- gmacht hät, hät s äigentli nie gee (3).

Erst nach einer Bezugnahme auf die unlautere Selbsthilfe während Schultests widmet sich Lara dem Thema der Hilfsbereitschaft und veranschaulicht diese anhand von drei Beispielen: Während einer Geschichtsprüfung, in der die Lehrerin nicht kontrolliert, gelingt es zwei Schülern sogar, eine Prüfung auszutauschen. Während der Französischprüfung hilft derjenige Schüler, der aus Platzgründen am Lehrerpult sitzt und somit zur Klasse schaut, anderen, indem er ohne Ton Lösungen mitteilt. Wenn alle dabei sind, die Prüfung abzugeben, gelingt es einem manchmal noch, eine Lösung aufzuschreiben, die jemand anderer ihm zuflüstert.

Insbesondere im ersten Beispiel zeigt sich, dass mit Hilfsbereitschaft auch $\mathrm{Spa} ß$ verbunden ist: Die Situation, Prüfungsblätter austauschen zu können, ist geradezu luschtig, was Lara auch beim Erzählen zum Lachen bringt. In den Berichten der anderen SuS bestätigt sich, dass Hilfsbereitschaft mit einem Unterhaltungswert verbunden sein kann.

Nur für Emil und Lilian spielt die Hilfsbereitschaft während der Schultests keine Rolle. Sie lösen die Schultests selbstständig, und ihnen ist gleichgültig, ob andere die Schultests selbstständig schreiben. So erklärt Emil auf die Nachfrage, wie er zum Helfen während der Schultests steht: 
(3) Ja, mir isch s äigentli egaal. (2) Also ich mach mini Arbet äigentli (1) alläi, (1) ussert wänn s Gruppearbet isch. (1) Und (1) isch iiri Entschäidig, die wo s machet (2).

Emil entbindet sich der Verantwortung dafür, wie die anderen Schultests schreiben. Für sich hat er entschieden, Schultests selbstständig zu schreiben, wofür er keine Begründung gibt. Auf dieselbe Frage der Interviewerin antwortet Lilian:

(3) Oft@1@ (4) Ja, (1) seer oft. (1) Also ich machs jetzt- ich machs nöd. Ich find (2), das isch e Prüeffig äignetlich für mich. Also (2) nur mängisch, wänn ich gar nüüt me wäis, wänns sowisoo egal isch, dänn- (4) aber. (3) Ich hilf au niemertem. (2) Und darum bin ich au wider die Giizig und so@1@. Aber (2) ich find s blööd. (5)

Lilian weiß, dass in ihrer Klasse eine grosse unlautere Hilfsbereitschaft während Schultests besteht, die sie aber nicht in Anspruch nimmt, weil sie die Schultests für sich schreibt. Kleine Ausnahmen bestehen, wobei tragender ist, dass sie selber auch niemandem hilft, womit sie sich aus dem Klassenverband ausschließt. Dennoch findet sie eine solche Hilfsbereitschaft nicht wünschenswert, sondern blööd.

Die unkonventionelle Ehrlichkeitsregel der unlauteren Hilfsbereitschaft ist ein wichtiges Thema bei den SuS. Diese Regel ist eng verbunden mit Kollegialität, Lernorientierung und Unterhaltung. Nur Emil und Lilian teilen diese Regel explizit nicht.

\subsubsection{Zusammenfassung: Ehrlichkeitsregeln im Klassenverband}

Nicht verpetzen kann als eine unausgesprochene Ehrlichkeitsregel im Klassenverband verstanden werden. Dabei bezieht sich Ehrlichkeit auf Beziehungen zu den Mitschülerinnen und -schülern, die zu schützen sind und denen eine größere Wichtigkeit zukommt als die Ehrlichkeit gegenüber Lehrpersonen. Diese Ehrlichkeitsregel ist stark verbunden mit der Kollegialität und Loyalität gegenüber der Schulklasse, sodass auch bei Nachfrage der Lehrperson unlautere Vorgänge verborgen bleiben können.

Die SuS präsentieren die Ehrlichkeitsregel des Nichtverpetzens als eine absolute Regel, die offenbar keine Ausnahmen zulässt: Das Verraten von Mitschülerinnen und -schülern ist grundsätzlich nicht erwünscht und nöd so $\mathrm{cool}$. Verpetzt wird lediglich in der Primarschule, in anderen Klassen und in leistungsschwächeren Klassen. Innerhalb der eigenen Klasse soll ,Verpetzen“ nicht vorkommen (vgl. Parallele zum Spicken Kap. 7.1.1).

Erst in den Beschreibungen und Erzählungen aus dem Schulalltag werden Konfliktsituationen sichtbar, die auf Ausnahmen der kollegialen Ehrlichkeitsregel des Nicht verratens innerhalb des Klassenverbands hinweisen. In diesem Zusammenhang lassen sich modifizierte Ehrlichkeitsregeln zum Nichtverpetzen, zu kollegialen Regeln und somit zu Ehrlichkeitsregeln formulieren: 
- Unehrlichkeit ist legitim, wenn zugunsten eines eigenen heldenhaften Einsatzes ein Mitschüler zu decken ist, indem freiwillig die Täterschuld und die Strafe übernommen werden und damit eine ausgesprochene Kollektivbestrafung aufgehoben werden kann. In diesem Fall ist Unehrlichkeit gegenüber der Lehrperson zugunsten der kollektiven Regel innerhalb des Klassenverbands positiv gewertet.

- Üblicherweise wird bei Kollektivbestrafungen erwartet, dass der Täter sich meldet. Dies aufgrund der Gerechtigkeitsfrage und weniger aus Gründen der Ehrlichkeit.

- Bei Mitwissen um gewalttätige Pläne von Mitschülern mit anderen SuS müssen die Planenden nicht verraten werden. Vielmehr sollen mitwissende SuS die Planenden selbst beraten und überzeugend von ihren Vorhaben abhalten. Mitwisser nehmen hier also die Rolle von Mediatoren ein.

- Verpetzen ist legitim, um zum eigenen Recht zu kommen und um ungerechte Situationen aufzudecken.

- Verpetzen ist erlaubt, um schwere Manipulationen zu vermeiden, etwa eine Erpressung - oder um einen Unfall aufzuklären. Diese Regel ist nicht mit Beispielen untermauert, sondern wurde hypothetisch von einigen SuS formuliert.

- Verpetzen ist erlaubt, wenn Konflikte mit Mitschülern nicht selbstständig gelöst werden können.

- Verpetzen ist erlaubt, wenn unsympathische Mitschüler den Befragten stressen, sodass er unglaublich sauer wird. Diese Regel erfährt eine Ausnahme: Eine Schülerin (Lilian) findet ihre Mitschülerinnen unsympathisch. Sie sind ihr so gleichgültig, dass sich die Anstrengung nicht lohnt, sie zu verpetzen; zudem verpetzt sie nicht, weil sie nicht noch mehr gehasst werden will. In diesem Ausnahmefall präsentiert sich das Nichtverpetzen als eine Überlebensstrategie und weniger als eine Frage der Ehrlichkeit und Unehrlichkeit.

- Verpetzen kann man auch in der Rolle des Iischläimis, d. h. wenn SuS im Klassenverband die kollektive Meinung vertreten, aber in Anwesenheit der Lehrperson ihre Meinung zugunsten der Lehrperson ändern. Das sind Ausnahmen, die an Beispielen von Zweitpersonen genannt und negativ gewertet werden. Unklar bleibt das Motiv, ob diese SuS etwa aus Angst gegenüber dem Klassenverband oder gegenüber der Lehrperson ihre Ehrlichkeitsregeln ändern. Auf jeden Fall orientieren sich ,Ischläimis` weniger an einem Ehrlichkeitsverständnis, sondern mehr an Machtverhältnissen, seien diese kollegial oder durch die Hierarchieungleichheit gegenüber der Lehrperson bedingt.

- Kollegiale und unlautere Hilfsbereitschaft im Klassenverband ist beispielsweise bei Schultests oder beim Abschreiben von Hausaufgaben grundsätzlich sehr relevant. Der einzige Einwand besteht aus eigenen 
Prinzipien und damit in der Gleichgültigkeit gegenüber den anderen Mitschülern. Zudem stellen sich einzelne SuS offene Fragen nach der Wirkung und Nachhaltigkeit ihrer Verhaltensweise, d. h. ob mit unlauterer Hilfe dem Empfänger tatsächlich geholfen wird.

Aus der Auflistung gehen einzelne Tendenzen hervor, in denen sich eine Vielfältigkeit und Verflechtungen von Ehrlichkeitsregeln zeigen. Daher können keine eindeutigen Ehrlichkeitsregeln formuliert werden, sondern nur solche, die vom Schulkontext und vom Kontext des Klassenverbands abhängig sind.

\subsection{Ehrlichkeitsregeln zwischen Spaß und Ernst}

In der bisherigen Textanalyse konnte besonders im Zusammenhang mit Schummeln gezeigt werden, dass Ehrlichkeitsregeln mit Spaß und Ernst verbunden sind sowie von emotionalen Reizen stimuliert werden. Wenn beispielsweise eine Lehrperson die unlauteren Hilfsmittel nicht entdeckt oder die Hausaufgaben vergisst zu kontrollieren, ist dies Grund zur Freude. Zudem erweist sich mit dem Auflachen der Befragten während dem Berichten solcher Schulerlebnisse die erheiternde Stimmung als immer noch präsent. In solchen Beispielen wird die Ehrlichkeitsregel vom Unterhaltungswert mitgeprägt.

Allerdings kann der Spaß sich schnell ins Doofe wandeln. Claire beispielsweise freut sich über lustige Vorfälle im Unterricht, in denen sie beispielsweise ihre Lehrerinnen in der ersten Sekundarstufe reinlegen kann und ihnen gegenüber damit auf lustige Weise unehrlich ist:

Aber die sind würkli, also (2) tummi Leererinne@gsii@. (1) Die äint isch liecht zum Verarsche gsii und die ander isch- Si sind halt no serigi- Sie sind bäidi no seer jung- (2).

Andererseits beurteilt Claire Ausreden, die nicht überzeugend sind, als doof. Somit ist für Claire nicht jede unehrliche Unterrichtssituation grundsätzlich lustig. Wo genau die Grenze zwischen Ernst und Spaß liegt, ist aus ihren Ausführungen nicht hinreichend nachvollziehbar. Es muss mit dem Selbstverständnis und der Rolle der Lehrperson und mit der Überzeugungskraft des Schülers zusammenhängen.

Zentral ist die Fähigkeit der Differenzierung von lustigen und ernsten Unterrichtssituationen. Dabei verlieren konventionelle Ehrlichkeitsregeln in lustigen Situationen tendenziell an Bedeutung, und bei ernsten Begebenheiten können Ehrlichkeitsorientierungen auch von Angst bestimmt sein (bspw. Nicht vom Lehrer angeschrien werden). 
Die verschiedenen Realitäts- und Bedeutungsebenen sind in den anschließenden Erklärungen von Regula erkennbar: Für Eingeweihte ist das ,Fertigmachen' von Mitschülern bzw. fehlender integrer Umgang mit Mitschülerinnen und -schüler mit Spaß verbunden. Hingegen beurteilen andere solche Verhaltensweisen als zu ernst und als Mobbing. So berichtet Regula:

Es wird ja immer so vo Mobbing und so gredet und so. Aber ich glaub, das git s i öisere Klass nöd. (9) Es wird immer scho echli fertig gmacht oder so, aber das isch underenander und das- (2) Ich wäis nöd, es git Lüüt, die verschtönd (1) das- (2) die nämet das vilicht mängisch echli z eernscht oder so. (3) Oder vilicht chan mer das nur echli eernscht nee, will- (2) aber ich glaub, niemert mäint s würkli böös oder so. (4) (I: Chasch echli beschriibe, was es häisst, wänn säisch echli fertig mache, was wird dänn so gsäit?) (9) Ja, so echli beläidigt. (1) Äifach (2), ich wäis nöd. Ich ha jetzt käi Biischpiil. (10) Ja, wänn irgend öpper ebe öppis uselaat und dänn isch s nöd luschtig, dänn (2) dänn macht- dänn säit halt irgendöpper: „Ja, das hetsch jetzt nöd müese säge“" oder so. (3) Und das isch dänn scho echli so echli (2), dänn piinnlich dänn für die ander Persoon und das zum Biischpiil, aber (1) das isch ja nöd eernscht und die Persoone verschtönd sich nachheer i de Pause. Es isch nachheer nöd, dass si irgendwie Schtriit händ oder so. Es sich nur so en chline Witz gsii überenand. Ich mäine, das macht mer ja immer. (2) Oder so, wänn irgendöppertem öppis Päinliches passiert oder (3) umghäit oder so (3), dänn lacht mer halt oder so. (1) Aber ich mäine schlussändlich hilft mer dänn äim trotzdem no uufschtaa oder so. (9) Ich wäis nöd, ich han jetzt nöd so (1) Biischpiil mit so würkli fertigmache oder so (4).

Regula unterteilt Personen in den Klassenverband (underenander) und außenstehende Personen (Lü̈̈t). Dieses Erklärungsmodell ruft Schwierigkeiten hervor. Beispielsweise nehmen die Außenstehenden Personen gewisse Unterrichtssituationen $z u$ ernst, und gleichzeitig stimmt Regula diesen Personen indirekt zu, dass gewisse Situationen vielleicht echli ernst zu nehmen sind. Die Gradierung zu ernst und ein wenig ernst verweist auf Schattierungen des Ernstes: zu ernst, ernst, etwas ernst, ein wenig ernst. Regula schließt aber pauschal aus, dass solche Situationen des ,Fertigmachens' in ihrer Klasse böse gemeint sind. Die zwei Perspektiven (Schülersicht und Außensicht) und die Gradierung des Ernstes weisen auf verschiedene Realitätsauffassungen hin: Beispielsweise zeigt sich aus Schülersicht der Ernst, wenn der Schüler nicht lachen und erklären würde, dass er gemobbt wird. Für die Außensicht reicht bereits die Beobachtung, dass die Klasse immer mit dem gleichen Schüler Späße treibt. Eventuell verfügen Außenstehende, wie Lehrpersonen, über zusätzliche Informationen, weil sie Einzelgespräche mit ausgeschlossenen $\mathrm{SuS}$ und dessen Eltern durchgeführt haben.

Worin das ,Fertigmachen' besteht, erklärt Sandra auf Nachfrage, allerdings ohne ein konkretes Beispiel beizuziehen: Das ,Fertigmachen“ präsentiert sich offenbar als ein Tabuthema, sodass Beispiele nicht zu nennen sind. Dass es ein Tabuthema ist, unterstreicht Sandras Kommentar, die Beleidigungen könnten von Außenstehenden nicht nachvollzogen werden, weil die Beispiele den sozialen Erwartungen widersprächen. Von diesen Erwartungen scheint Sandra beeinflusst zu sein. Sie nennt ein allgemeines Beispiel, dem- 
zufolge ein Schüler etwas ,herausgelassen“ (gesagt) hat, was ,nicht lustig“ gewesen sei. Damit hält sie nur indirekt fest, dass Beleidigungen ausgesprochen werden, um jemanden ,fertigzumachen': ,Nicht lustig' und ,herauslassen' sollen auf beleidigende Worte hinweisen, die Sandra nicht direkt benennt. Allerdings beschreibt sie die Folgen einer solchen Situation: Ein Mitschüler ergreift Partei für den beleidigten Mitschüler und weist den Redner auf seine unpassende Aussage hin. Dadurch wird dem Redner die Situation peinlich. Die ganze Situation beurteilt Sandra als nicht ernst, denn alle verstehen sich in der Pause wieder.

Hier stellt sich die Frage, ob bei den SuS die Sensibilität für andere als soziale Kompetenz fehlt oder ob Situationsspiele die Regeln der Realität Offen sein und Ehrlich sein ausschalten und andere Regeln in Kraft setzen. Die Lesart des Spiels wird durch die Aussage unterstrichen, dass das Ganze ein Witz sei. Zudem sucht Sandra solche Situationen zu rechtfertigen, indem sie aufzeigt, dass andere spielerische Beleidigungen immer wieder aussprechen. Hier wird Verantwortung an die Allgemeinheit delegiert. Die Realitätsebene ist am Schluss des Abschnitts mit dem Begriff würkli direkt angesprochen. Es gibt scheinbar eine Form des ,Fertigmachens', die wirklich ist, und eine Form, die in der Wirklichkeit eines Spiels zu verstehen ist (Was immer Wirklichkeit heißen mag!).

Im selben Sinn unterscheidet Nora zwischen ernstem Mobbing und spaßhaftem Mobbing und sagt aus, dass es in ihrer Klasse allein die Variante des spaßhaften Mobbing gäbe:

Es wird äigentli i öisere Klass, wird au niemert so gmobbt. Und wänn d Jungs maal öpper mobbet, machet s eher so als Schpass. (1) So chlii, so ernscht nimmt s äigentli niemert.

Interessanterweise spricht Nora nur von Jungen, die mobben. Weiter zögert sie, Mobben mit Spaß gleichzusetzen, indem sie den Begriff eher verwendet. In ihrer Interpretation sind solche Vorfälle nicht ernst zu nehmen; jedenfalls werden sie in ihrer Klasse nicht ernst genommen. Unausgesprochen bleibt, dass Mobbing im spaßigen Sinne spielerischen Regeln folgt, die als kollegiale Ehrlichkeitsregeln zu verstehen sind.

Als lustig benennt Adrian auch die Zuweisung bewusst falscher Unterstellungen:

(4) Me säit äifach, dass irgendöpper schwuul isch. Und dänn (2) frögt me dee natüürli au und dee säit natüürli: „Näi“. Und nachheer säit mer: „,Ja, doch bisch s schoo““ Oder „Ali säget s!“ und so. Und irgenwänn läit sich das äigentli irgend- (1) Das isch äifach für es paar Taag- isch es luschtig oder. Gaat me immer zu dem: „Ja, bisch schwuul!“ und so. Aber irgendwänn isch es natürli nüme luschtig (2).

$\mathrm{Zu}$ falschen Unterstellungen zählt beispielsweise die Aussage, dass jemand schwul sei, was der Betroffene verneint und einen Wortwechsel auslöst. Anscheinend treten solche Unterstellungen regelmäßig auf, denn Adrian erzählt davon in einer verallgemeinernden Weise in direkter Rede. Mehr als ein Spiel 
drückt sich hier eine Ritualisierung aus, die ein Ende nimmt, wenn es nicht mehr lustig ist. Leider beschreibt Adrian nicht näher, wann dieser Endpunkt erreicht wird; er hält nur fest, dass der Wortwechsel sich legt. Das kann ein weiterer Hinweis darauf sein, dass gerade die unausgesprochenen kollegialen Regeln gelten wie Wenn es nicht mehr lustig ist, ist das Spiel fertig. In diesem Kontext haben falsche Unterstellungen eine spielmotivierende Wirkung und verweisen weniger auf einen ernsten Zusammenhang zwischen Wahr und Falsch. Die Bedeutung von Ehrlichkeit ist kein Thema. Allerdings könnte es sogar sein, dass die durchschaute Unehrlichkeit eine tragende Funktion für den Unterhaltungswert hat. Solange alle Beteiligte in die kollegialen Regeln eingeweiht sind, scheint diese Art von Kommunikationsspielen niemanden zu schädigen.

Parallel zu Adrian erklärt Emil, wie die SuS sich gegenseitig nur zum Spaß abemachet: „Näi, mir machet s äigentli nur zum Schpaas und de ander verschtaat das au zum Schpaas (2). Wänn s es mir machet, nimm ich s au so (2).“

Emil zeigt hier die Gültigkeit der kollegialen Regel Necken nur solange es allen Beteiligten Spaß macht innerhalb des Klassenverbands, die für den Ausübenden sowie für den Empfänger zutrifft. Er untermauert seine Sichtweise mit einer persönlichen Bezugnahme. Mit dem kollektiven vertritt er die ausübenden SuS, und mit dem nimm ich zählt er sich selbst zu den empfangenden SuS. Hier dokumentiert sich die Normalität und Alltäglichkeit der kollegialen Regel aus der ersten Person.

Das Lästern kann zugleich als lustig und gemein gedeutet werden. So beschreibt Valentina:

(2) Oder paarmal, wänn mer äifach so Gruppeaarbet hät und sch- mir föif amene Tisch und vomene andere und so, (1) wänn grad mit diine Kolleginne bisch, dänn @läschterisch@ halt echli und isch ebe (1) äigentli noluschtig@1@. Das isch zwar fiis, aber-@1@ ja(1).

Valentina lästert also gelegentlich mit ihrer Kollegin während Gruppenarbeiten, was luschtig (unterhaltsam), aber fiis (gemein) sei. Mit dieser Spannung geht sie beim Erzählen so vor, dass sie in diesem kurzen Abschnitt dreimal lacht. In Kapitel 6.1.2 ist aufgezeigt, dass Lästern von den SuS zwar als Lügen verstanden, aber dennoch praktiziert wird. Da Lästern neben dem Spaß mit Lüge und Unehrlichkeit zu tun hat, ist dessen Ausüben gemein. Dennoch hält Valentina sich am Unterhaltungswert (dem Lustigen) fest. Hier dokumentiert sich ein Reibungsfeld, aus dem auf keine klare Ehrlichkeitsregel geschlossen werden kann. Vielmehr ist situativ zu bestimmen, ob Lästern in einer bestimmten Unterrichtssituation eher als lustig oder als fiis auszulegen ist.

Vor dem Hintergrund, dass die Schule aus Sicht der meisten Befragten Spaß machen soll, schliesse ich dieses Unterkapitel mit einer entsprechenden Erklärung von Claire und füge eine Reflexion an: 
Dänn säit zum Biischpiil- Äini hät s letscht Maal uselaa, (1) also hät s letscht Maal gsäit ghaa (1) ,Pferdeschwanz' statt ,Rossschwanz'. Und über daas hämer äifach müese lache und wänn dänn öpper säit: ,Zzz. Was lachet ihr, chönd ir öi nöd konzentriere?“ Das nervt mich dänn, will ich find mer sött s nöd zu eernscht nee- $d$ Schuel, suscht macht si gar käi Schpass@1@(1).

Claire baut hier ein dualistisches Ansatz auf: Die Schule ist ernst und kann durch lustige Unterrichtssituationen wie Versprecher (Pferdeschwanz statt Rossschwanz) aufgeheitert werden, indem darüber gelacht wird. Beim einen ist die Konzentration gefragt und beim anderen wird die Konzentration gestört. Das eine macht keinen Spaß, das andere schon. Darüber hinaus sind in der Schule Schulregeln einzuhalten und im Klassenverband kollektive Regeln. Erstere stützen sich auf die Prinzipien der Ehrlichkeit, und die anderen erlauben Ausnahmen wie z. B. den Spaß.

In diesem Kontext sind somit Ehrlichkeitsregeln nur i. S. v. offiziellen Schulregeln eindeutig. Es besteht ein Spielraum, in welcher Unterrichtssituation kollektive Regeln und wann individuelle Regeln greifen. Daher können schulische, kollektive und individuelle Ehrlichkeitsregeln nur annähernd die Bedeutung der Ehrlichkeit von SuS und deren Orientierungen in den Verhaltensweisen veranschaulichen.

Eine weitere Annäherungsmöglichkeit ist die Analyse von Entscheidungsfällen einzelner SuS, was in Dilemmasituationen der Fall ist. Welche Ehrlichkeitsregeln in solchen Situationen gelten, ist in den einzelnen Schulsituationen genauer nachzuvollziehen (Kap.9). Doch zunächst werden noch Ehrlichkeitsregeln ergänzt, die über den Schulkontext hinausreichen. Diese sind ebenfalls zu berücksichtigen, weil die Schule nicht als isolierte Erziehungsinstanz zu verstehen ist.

\subsection{Ehrlichkeitsregeln über den Schulkontext hinweg}

Der Interviewleitfaden enthält keine Fragen, die direkt auf Lebensbereiche außerhalb der Schule abzielen. Dennoch beziehen sich die Interviewten punktuell auf die Bereiche Gesellschaft, Familie und Peers. Ich skizziere in diesem Unterkapitel entsprechende Passagen, um auf den fließenden Übergang zwischen den einzelnen Lebensbereichen hinzuweisen und einen Einblick in die Ähnlichkeiten der Ehrlichkeitsregeln im Schulkontext zu solchen in anderen Lebensbereichen aufzuzeigen. Das Unterkapitel stellt damit Ehrlichkeitsregeln in Lebensbereichen in den Vordergrund, welche einzelne SuS nur am Rande der Gespräche erwähnten. 
Gesellschaftlich geprägte Ehrlichkeitsregeln

Bereits in Kapitel 8.1.1 sind Ehrlichkeitsregeln genannt worden, die das Heranziehen unlauterer Hilfsmittel betreffen: Wenn potenziell alle spicken können, dann ist Spicken erlaubt. Diese Regel basiert auf einem Prinzipiendenken, dessen Legitimationsstrukur an den kategorischen Imperativ (Kant [1797] 1966) erinnert, der zusammenfassend Handlungen an Maximen orientiert, die ein allgemeines Gesetz ermöglichen, bei dem die handelnde Person sowie die anderen Personen nicht als Mittel gebraucht werden sollen. So begründete Ehrlichkeitsregeln müssten sich in andere Lebensbereiche adaptieren lassen. In einer weitführenden Studie wäre zu untersuchen, ob und inwiefern Jugendliche tatsächlich diese Ehrlichkeitsregel außerhalb der Schule adaptieren.

Zudem ist an verschiedenen Interviewstellen das Bewusstsein der SuS erkennbar, sich nach einer sozialen Erwartung auszurichten, die darin besteht, grundsätzlich ehrlich zu sein. Es ist offensichtlich, dass sich die soziale Erwartung nicht auf den Schulkontext reduzieren lässt. In der Analyse der Textstellen ist bereits verschiedentlich darauf hingewiesen worden. So drückt dies Stefan in Bezug auf alle Schultests direkt aus: „Ja, me sött im Allgemäine scho eerlich sii." Einige Zeilen später nennt er aber Ausnahmen.

Zusammenfassend lässt sich sagen: Die SuS äußern sich zu gesellschaftlichen Ehrlichkeitsregeln in einer allgemeinen Weise, welche im ersten Moment keine Ausnahmen zulässt und die von der sozialen Erwartung (ehrlich zu sein) geprägt ist. Damit ist eine weitere Aussage über Ehrlichkeitsregeln aufgezeigt.

Ehrlichkeitsregeln in der Familie

Die Familie und insbesondere die Eltern sind für die SuS Referenzpunkt ihrer Ehrlichkeitsregeln. Dies zeigte sich schon anschaulich im Umgang mit der Fälschung von Elternunterschriften (Kap. 8.1.3). Interessanterweise verbinden Marco und Clemens Ehrlichkeit in der Familie mit schlechten Gefühlen, die zu beseitigen sind, und mit einem schlechten Gewissen: Wenn zu Hause etwas beschädigt wird, so erklärt Marco, muss dies mitgeteilt werden. Anstoß gibt das Vermeiden eines negativen Gefühlszustands: um es so vo de Seel geredet und vo dir wäg zu haben und um nöd es schlächts Gwüsse zu haben. Mit dieser Wortwahl begründet Marco Aufrichtigkeit. Die Wortwahl mag erstaunen, weil er im Interview sehr aufgeklärt und selbstsicher auftritt. Die Begriffe ,Seele' und ,Gewissen' sind aus dem religiösen Sprachschatz bekannt und weisen auf Denkkonzepte hin, die Marco von zu Hause mitbekommen haben muss. Denn in der Schule ist ihre Anwendung nicht üblich. Marcos Ehrlichkeitsregel könnte demnach so formuliert werden: Man soll 
aufrichtig sein, um eigene emotionale negative Gefühlszustände zu vermeiden.

Hier dokumentiert sich, wie Ehrlichkeitsregeln in unterschiedlichen Kontexten andere inhaltliche und kulturelle Prägungen erhalten. Die SuS stehen vor der Herausforderung, je nach Situation die verschiedenen Ehrlichkeitsregeln angemessen einzusetzen.

Wie Marco verwendet auch Clemens emotionale Sprachkategorien, um über Ehrlichkeit im Familienbereich zu sprechen. Clemens ist überzeugt, dass es entscheidend ist, zu Personen ehrlich $\mathrm{zu}$ sein, die einem wichtig sind (Kap. 7.2), wozu nicht notwendigerweise Lehrpersonen gehören. Er untermauert seine Ehrlichkeitsregel mit einem fiktiven Beispiel:

(1) Will ich mäine- (3) S wär- s sich rächt fiis, wenn me, säget me maal, du häsch diinere Grossmueter no nie gsäit, dass du iire de Pulli verbrännt häsch und iiri Schtricksache ali kaputt gmacht häsch. Und sägemer emaal si stirbt dänn irgenwänn mal und du chasch s iire nie mee säge. Dänn häsch äifach e schweeri Lascht uf diim Herz, wo niemertem chasch säge. Chasch schoo jedem säge, aber das nimmt s nöd weg. Du muesch s ja ire säge und dere Person säge, wo s au betrifft. Und wänn si dir dänn verzäiit, isch okey, und wenn nöd, dänn häsch Päch ghaa. Aber oft isch s soo, dass si dir äigentli verzäiit. (1) Aber au wänn s nöd säge chasch, isch halt (3) schöni Schäisse für dich (7).

Clemens zählt fiktive Kinderstreiche auf, die ein Enkel seiner Großmutter nicht eingestanden hat, etwa ihren Pullover verbrennen oder ihre Stricksachen zerstören. Die Folge davon erklärt er mit der Metapher schweeri Lascht uf diim Herz, die allein durch das Eingeständnis bei der betroffenen Person aufgehoben werden könne. Clemens reflektiert detailliert und chronologisch die fiktiven Beispiele nach Verhaltensschritten und berücksichtigt dualistische Optionen wie beispielsweise: Wenn die betroffene Person verzeiht, ist es okey, andernfalls ist es Päch. Wenn das Eingestehen eines Streiches nicht gelingt, ist das für Clemens ein Problem: schöni Schäisse. Dieser und andere emotionale Ausdrücke weisen darauf hin, dass Clemens' Ehrlichkeitsregeln mehr auf emotionalen als rationalen Kategorien beruhen.

Nunmehr könnte überlegt werden, weshalb gerade Marco und Clemens sich auf Situationen in der Familie beziehen und ihre Berichte sich nicht auf den Schulkontext beschränken. Das ist indes eine psychologische Frage, die hier nicht beantwortet werden kann. Es fällt aber auf, dass Clemens und Marco die einzigen Befragten sind, welche einen Familienbezug herstellen, und dass sie als einzige emotionale Metaphern verwenden.

Hier dokumentiert sich, dass die Ehrlichkeitsregel je nach Kontext kommunikativ verschieden ausfällt und dass sie im Familienkontext zusätzliche emotionale Ebenen berührt, welche die SuS als solche im Schulkontext nicht benennen. 


\section{Ehrlichkeitsregeln unter Peers in der Freizeit}

Bereits die Analyse der kollegialen Ehrlichkeitsregeln (Kap. 8.2) im Klassenverband zeigt die Eigenheit dieser Regeln. Zudem beziehen sich insbesondere die individuellen Ehrlichkeitsregeln der Direktheit (Kap. 8.1.5) auf solche, die von sozialen Beziehungen gesteuert sind. Auch außerhalb des Schulkontexts bestimmen diese Regeln das Zusammensein von Peers.

Sensibel reagieren SuS auf falsche Aussagen unter Kollegen außerhalb der klasseninternen Spiele. Dasselbe trifft auf Freundeskreise zu. So berichtet Claire von unterschiedlichen Freizeitsituationen, in denen Kolleginnen falsche oder beleidigende Aussagen über Claires Person und ihr Aussehen anderen gegenüber kundtun, es ihr aber nicht direkt mitteilen. Die fehlende Direktheit beurteilt sie als negativ; sie bezieht sich zugleich auf die Ehrlichkeitsregel der Direktheit (Kap. 6.5).

Lilian kommuniziert klar, welchen hohen Stellenwert die Peers in ihrer Freizeit haben und dass ihre Ehrlichkeitsregeln zu ihnen ganz andere sind als in der Schule (vgl. Kap. 9.1.1): Was die Peers im Klassenverband über sie denken, ist ihr egal. Nicht egal ist ihr die Meinung der anderen über sie im Fußballclub. Aus der Gegenüberstellung der Peers in der Schule mit denen im Freizeitsport gehen unterschiedliche Ehrlichkeitsregeln hervor, wobei Lilian nur ihre Ehrlichkeitsregel im Schulkontext erwähnt (Direktheit und Gleichgültigkeit, was die anderen über sie denken). Ihr Wohlbefinden im Sport dagegen vergleicht sie mit dem Gefühl, zu Hause zu sein, ohne hierfür eine Ehrlichkeitsregel zu beschreiben. Aus dem Kontext kann geschlossen werden, dass gegenüber den Peers in der Freizeit eine moderierte Direktheit und keine Gleichgültigkeit herrscht. Der Ehrlichkeitsbegriff basiert hier auf einer intensiven Kollegialität und Freundschaft.

Abschließend zu diesem Kapitel über unkonventionelle Ehrlichkeitsregeln ist festzuhalten: Die Komplexität der fallübergreifenden Ehrlichkeitsregeln, insbesondere in der Einbettung des Schulalltags, verweist nicht nur auf die Vielfältigkeit von Ehrlichkeitsregeln, sondern auf Konfliktsituationen zwischen Ehrlichkeitsregeln (vgl. bspw. Kap. 8.2.2), die ich als ,reale Dilemmas “ bezeichne. Denn nicht immer ist für die SuS eindeutig, an welchen Ehrlichkeitsregeln sie sich orientieren wollen. Daher werden im Folgenden reale Dilemmasituationen genauer auf die Entscheidungsprozesse zwischen Ehrlichkeitsregeln untersucht. 


\section{Ehrlichkeitsregeln in realen Dilemmasituationen}

Die bisherigen Ergebnisse zeigen, dass SuS sich in unterschiedlichen Unterrichts- und Schulsituationen für bestimmte Ehrlichkeitsregeln entscheiden. Weiterführend geht dieses Kapitel anhand von realen Dilemmasituationen den Fragen nach, wie SuS sich für bestimmte Ehrlichkeitsregeln entscheiden und welche Faktoren dabei bedeutungsvoll sind. An Dilemmasituationen lassen sich besonders deutlich Entscheidungsprozesse nachzeichnen. In der Entwicklungspsychologie wird die Entwicklung der moralischen Urteilsbildung anhand hypothetischer Dilemmas und der Stellungnahme der Befragten untersucht (vgl. Kohlberg et al. 1995). Demgegenüber handelt es sich bei den realen Dilemmasituationen um schulalltägliche Situationen, in denen SuS bezüglich Ehrlichkeitsregeln Interessekonflikte zu bewältigen haben, von denen sie explizit oder implizit berichten. Damit soll der reale Bezug zu den Dilemmas und die Deutung der SuS bewahrt werden.

Im Interviewmaterial sind zahlreiche Dilemmasituationen zu finden, von denen ich acht ausgewählt habe, welche die Vielfalt und eine möglichst grosse Breite von Dilemmasituationen widerspiegeln sollen. Zur Veranschaulichung einer realen Dilemmasituation soll hier Marcos Dilemmasituation auf dem Pausenplatz betrachtet werden (vgl. Beispiel in Kap. 8.1.2). Folgende Rahmenbedingungen kennzeichnen jene Dilemmasituation:

Marco spielt Fußball mit jüngeren Schülern auf dem Pausenplatz (Kontext). Das ist jedoch eine verbotene Verhaltensweise, was den Lehrer, der Pausenaufsicht hat, veranlasst, verbal zu intervenieren. Er fordert Marco auf, das Spiel einzustellen (intervenierende Maßnahme I). Der neue Kontext besteht nun darin, dass Marco sich zugunsten des unterhaltsamen Spiels und seines Gerechtigkeitssinns (Ursache) nicht an die Aufforderung des Lehrers halten will und weiterspielt (Verhaltensweise I, Kontext II). Da interveniert der Lehrer zum zweiten Mal verbal (intervenierende Maßnahme II). Daraus resultiert Marcos Verhaltensweise (II): Er stellt das Spiel widerwillig ein und wendet sich vom Lehrer mit einem beleidigenden Ausdruck Spaßbremse ab (Kontext III). Das gibt dem Lehrer Anlass, barsch nach Marcos Namen zu fragen (intervenierende Maßnahme III).

Die Dilemmasituation zu diesem Zeitpunkt besteht darin, dass Marco sich grundsätzlich an einer individuellen und mit anderen Peers geteilten Ehrlichkeitsregel orientiert: Unter großen Spannungen dürfen Informationen vorenthalten oder verdeckt werden (vgl. Kap. 8.1.2). Zudem kennt er die konventionelle Ehrlichkeitsregel Nicht täuschen bzw. Nicht lügen. Vor diesem Hintergrund löst er die Dilemmasituation, in dem er seinen Namen preisgibt und auf die Anforderung des Lehrers eingeht (Verhaltensweise III). Mit dieser resultierenden Verhaltensweise orientiert er sich an der konventionellen Ehrlichkeitsregel des Nichtlügens. Auf die Nachfrage der Interviewe- 
rin, was der Anlass für seine Entscheidung war, erwähnt Marco die negativen Konsequenzen, die hätten folgen können. Die Ursache seiner Verhaltensweise ist mit der Vorsicht und der Wahl des geringeren Widerstands zu erklären. Marco berichtet von keinen Konsequenzen, woraus zu vermuten ist, dass sein Kalkül aufgegangen ist.

Die Faktoren, welche Marcos Dilemmasituation beeinflussen, sind auf verschiedenen Ebenen (I, II, III) situiert, die nach einer impliziten und expliziten Textanalyse (vgl. Kap. 5.3) anhand des Rasters (Ursachen, Kontext, intervenierende Maßnahme, Dilemma unterschiedlicher Ehrlichkeitsregeln und Verhaltensweise) auf eine differenzierte und systematische Weise präsentiert werden können (in Anlehnung an das Kodierparadigma nach Strauß 1991 [1987], S. 48f.; Przyborski und Wohlrab-Sahr 2009, S. 204ff.).

Das Analyseverfahren und die Darstellung der Dilemmasituationen sind zusammenfassend in zwei Arbeitsschritte und damit mehrdimensional erfasst: In einem ersten Interpretationsschritt geht es darum, den Text zu analysieren und explizite sowie implizite Ehrlichkeitsregeln in der Dilemmasituation herauszuarbeiten. In einem zweiten Interpretationsschritt sollen Zusammenhänge der gefundenen Ehrlichkeitsregeln mit dem situativen Kontext, den Ursachen, den intervenierenden Maßnahmen und den resultierenden Verhaltensweisen aufgezeigt werden (vgl. Abb. 3). Auf diese Weise kann gezielt der Entscheidungsprozess zwischen unterschiedlichen Ehrlichkeitsregeln samt der daraus resultierenden Verhaltensweisen der SuS fallimmanent nachgezeichnet und präsentiert werden.

Die vielfältige Selbstdarstellung der SuS während der Interviewgespräche zeigt sich bereits darin, dass nicht alle Befragten reale Dilemmasituationen als solche wahrnehmen und problematisieren. So berichtet beispielsweise Emil in einer Selbstverständlichkeit von seinem Lehrer, der die SuS schreiend zurechtweist, wenn sie festgesetzte Schulregeln nicht einhalten (vgl. Chiapparini 2011, S. 190).

Abbildung 3: Darstellung von Ehrlichkeitsregeln in Dilemmasituationen und der damit zusammenhängenden Faktoren im Entscheidungsprozess

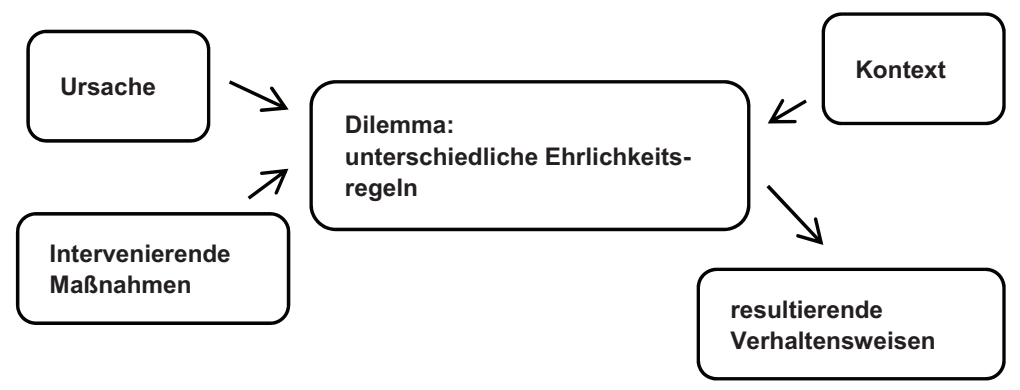

Quelle: eigene Darstellung in Anlehnung an Strauß 1991 [1987] 
Die Adressatinnen sowie Adressaten wagen dem Lehrer gegenüber nicht, eine Richtigstellung von missverständlichen Sachverhalten (z.B. nicht geschummelt, sondern Namen hingeschrieben) vorzubringen. Von einer solchen Situation berichtet Claire, eine Mitschülerin von Emil; als der Lehrer sie ungerechtfertigt anschreit und brüsk eine Testaufgabe durchstreicht, weil sie nach dem Schlusszeichen des Schultests noch ihren Namen auf das Blatt geschrieben und nicht den Stift abgelegt hat. Claire bekundet Verständnis für die unverhältnismäßige Reaktion des Lehrers (vgl. Chiapparini 2011, S. 156).

Daher fokussiert das folgende Kapitel Schulsituationen, in denen die SuS aus eigener Initiative von realen Dilemmasituationen berichten und diese explizit oder implizit problematisieren.

Die acht Dilemmasituationen habe ich nach der Analyse in vier thematischen Gruppen angeordnet. Da die Ehrlichkeitsregeln im Zentrum der Betrachtungsweise der Dilemmasituationen stehen, richtet sich die Anordnung der Dilemmas nach der Grobgliederung der Ehrlichkeitsregeln in konventionelle, unkonventionelle und individuelle Regeln: Dilemma zwischen kollegialen Ehrlichkeitsregeln (vgl. Kap. 9.1), Dilemma zwischen individuellen und konventionellen Ehrlichkeitsregeln (vgl. Kap. 9.2), Dilemma zwischen kollegialen und konventionellen Ehrlichkeitsregeln (vgl. Kap. 9.3) und Dilemma zwischen individuellen, kollegialen und konventionellen Ehrlichkeitsregeln (vgl. Kap. 9.4). Die Dilemmas zwischen individuellen oder zwischen konventionellen Ehrlichkeitsregeln gibt es auch, sie kristallisieren sich allerdings nicht so deutlich im Datenmaterial heraus. Ebenso sind die Dilemmas zwischen individuellen und kollegialen Ehrlichkeitsregeln in Kapitel 8.2 angesprochen. Allerdings sind die Berichte zu diesen Dilemmasituationen kurz gehalten und der Übergang zwischen individuellen und kollegialen Ehrlichkeitsregeln teilweise fließend problematisiert (vgl. Lilian Kap. 9.1.1), sodass ein Beispiel in der vorliegenden Darstellung unter Dilemmas zwischen kollegialen Ehrlichkeitsregeln situiert ist.

\subsection{Dilemma zwischen kollegialen und individuellen Ehrlichkeitsregeln}

Kollegiale Ehrlichkeitsregeln sind eine grobe Kategorie, unter der sowohl eine Regel zu verstehen ist, die etwa zwischen zwei Kollegen gilt (bilaterale kollegiale Ehrlichkeitsregel) als auch eine, die kollektiv für den ganzen Klassenverband gilt (kollektive Ehrlichkeitsregel). Zudem sind die Beziehungen mehr durch freundschaftliche oder interessegeleitete Motive charakterisiert. In der Vielfalt der Formen von Kollegialität, welche die kollegialen Ehrlichkeitsregeln unterschiedlich charakterisieren, zeichnen sich in den Dilemmasituationen folgende Formen aus: 
- gewachsene Gleichgültigkeit gegenüber kollegialen Ehrlichkeitsregeln (vgl. Kap. 9.1.1)

- $\quad$ zwei unterschiedliche Verständnisse von kollegialen Ehrlichkeitsregeln (vgl. Kap. 9.1.2)

\subsubsection{Gewachsene Gleichgültigkeit gegenüber kollegialen Ehrlichkeitsregeln: ... will s mer äigentli schäissegal isch, was die über mich dänket}

Lilian berichtet auf eindrückliche Weise, wie ihre Ehrlichkeitsregel darin besteht, das zu sagen, was sie denkt - ohne Angst vor der Reaktion der anderen zu haben (vgl. Kap. 6.5). Sie ist sich bewusst, dass sie mit dieser Verhaltensweise $z u$ ehrlich ist und das bei den anderen Mitschülerinnen und schülern nicht gut ankommt. An ihre expliziten Ehrlichkeitsregel schließt sie weiterführende Erklärungen an, aus denen sich eine Dilemmasituation nachzeichnen lässt:

Aber bi miir isch es halt soo, ich bin da i de Schuel, drüü Jaar und ich han s au nöd so extrem guet mit minere ganze Klass- Kamerade und so. (4) Und dänn isch es mir au echli egaal, was die vo mir dänket und so. Aber (2) ich schpile Fuessball und deet bin ich eener so echli di Häi, inere Mannschaft und so- (5) darum isch es mir da echli- ja es isch mir äigentli egaal, was die über mich dänket. (1) Am Aafang nöd und jetzte- letschte Jaar, au scho s letscht Jaar han ich aagfange- (2) chan ich äigentli- (2) chan ich mich uuffüere, wie ich wett (2) bi dene, will s mer äigentli (2) schäissegal isch, was die über mich dänket (11).

Die Textsorten der Interviewpassage ordnen sich als retrospektivische Argumentationen mit beschreibenden Textelementen und mit biografischen Bezügen dazwischen. Im Verlauf des Interviewgesprächs fällt auf, dass Lilian keine Beispiele nennt, um ihre selbstformulierte und individuelle Ehrlichkeitsregel des Zu-ehrlich-Seins zu erklären. Sie tut dies auch nicht auf Nachfragen der Interviewerin. Anders ist dies bei der funktionierenden kollegialen Ehrlichkeitsregel Verdecken der ungemachten Hausaufgaben von Mitschülerinnen und -schülern vor der Lehrperson (vgl. Kap. 9.3.2). In Lilians $\mathrm{Zu}-$ rückhaltung, Beispiele zu ihrer explizierten Ehrlichkeitsregel zu nennen, obwohl sie zu kollegialen Ehrlichkeitsregeln über Schulsituationen detailliert erzählt, dokumentiert sich eine Weigerung, Beispiele zu ihrer individuellen Ehrlichkeitsregel vorzubringen. Zwar ist Lilian bereit, ihre eigenwillige Ehrlichkeitsregel zu formulieren. Allerding geht sie auf die Nachfrage der Interviewerin nicht ein. Hingegen beschreibt sie argumentativ und teilweise beschreibend ihre Ehrlichkeitsregel im Schul- und Klassenkontext, zu dem sie sich nicht zugehörig fühlt.

$\mathrm{Zu}$ Beginn der Textpassage setzt Lilian vorerst den Ich-Bezug fest und fährt dann mit anschließenden Aussagen fort. Damit definiert sie bewusst die Sprechposition und die Perspektive der Aussagen, die von ihrer Wahrneh- 
mung ausgehen. Die Selbstpositionierung des Berichtens kann gelesen werden als Abgrenzung zu den anderen Mitschülerinnen und -schülern, auf welche die Ausführungen nicht zutreffen. Weiter erinnert Lilian an die schulischen Rahmenbedingungen: dass die Schulzeit drei Jahre dauert. Hier dokumentiert sich ein vorübergehender und limitierter Zustand: der Schulkontext. Daraufhin stellt sie in einer abgeschwächten und ironisch wirkenden Weise fest, dass sie es nöd so extrem guet mit ihrer ganzen Klasse habe. Damit verweist sie auf ihre Desintegration im Klassenverband. Allerdings identifiziert sie sich an dieser Stelle noch mit der Klasse, indem sie sich mit einem Possessivpronomen minere auf diese bezieht.

$\mathrm{Ab}$ hier ist eine emotionale Steigerung der Abgrenzung zur Klasse als Klassenverband und zu dem, was diese über Lilian denkt, feststellbar. Das ist zum einen formal anhand des Wandels der Ausdrücke echli egaal zu schäissegal unterstrichen. Zum anderen wird die emotionale Abweisung von der Klasse durch die Betonung der inhaltlichen Aussage der Gleichgültigkeit egal unterstrichen. Die emotionale Abweisung wird zudem durch die dreifache Wiederholung der fast identischen Aussage egal, was die über mich dänket hervorgehoben und verstärkt. Die anfängliche Bezeichnung der Mitschülerinnen und -schüler mit minere ganze Klass wandelt sich im Verlauf des Berichtens zum neutralen und abwertenden Pronomen die oder bi dene. $\mathrm{Zu}-$ dem dokumentiert sich hier im wertenden Wandel vom integrierenden zum distanzierenden Bezug von Lilian zum Klassenverband ein weiteres Merkmal dieses Bezuges. Dieser richtet sich zu Beginn des Abschnittes nur einmal auf die ganze eigene Klasse und dieser dann richtet sich zweimal auf die vielen neutralen Individuen im Klassenverband.

Diese Hinweise können auf Lilians ursprünglich ganzheitliche Integration in die Klasse verweisen und auf die gegenwärtige Desintegration und zerstückelte Distanzierung zu den einzelnen Mitschülerinnen und -schülern. Darüber hinaus zeichnet eine solche Lesart und die fehlenden Erklärungsbeispiele für ihre Ehrlichkeitsregel klar die emotionale Involviertheit von Lilian mit dem Inhalt ihres Berichtens ab. Als Kontrastierungsfolie zum negativ erlebten Klassenverband führt Lilian die Fußballmannschaft ein, in der sie sich di Häi fühlt. Die ausdrucksstarke Metapher des zu Hause vermittelt das vertraute und friedvolle Klima des Heims gegenüber dem von ihr erfahrenen divergierenden Klima im Klassenverband.

Lilians soziales Netzwerk bildet die Peer Group der Fußballmannschaft. Aus der Zugehörigkeit zu dieser Peer Group erklärt sie - signalisiert durch das Wort darum - ihre Gleichgültigkeit gegenüber der Meinung des Klassenverbandes zu ihrer Person und ihren Verhaltensweisen. Lilians Erklärung kann auf zwei Weisen gelesen werden. Entweder erklärt sie, weshalb sie die Gleichgültigkeit erträgt, in dem sie in der Fußballmannschaft ihren sozialen Bedürfnissen nachkommen kann. Oder die Erklärung richtet sich auf ihren Umgang des negativen Klimas im Klassenverband, in dem Sinne, dass dies 
nicht verändert, sondern nur ignoriert werden kann. Ihre Verhaltensweise der Gleichgültigkeit war nicht immer so: Am Aafang nöd. Der prozesshafte Wandel dokumentiert sich in den verzögerten Ausdrücken Jetzt ... letzte Jahre ... bereits im letzten Jahr (,,jetzte- letschte Jaar, au scho s letscht Jaar"). Somit fand der Positionswechsel von der Wichtigkeit dessen, was die Klasse über sie denkt, hin zur Gleichgültigkeit nicht plötzlich statt - er fand aber statt, doch er ist nicht genau nachzuzeichnen. Weil die Meinung der Klasse für Lilian gegenwärtig bedeutungslos ist, kann sie sich verhalten (uuffüere), wie sie will. Damit ist indirekt auf Lilians individuelle Ehrlichkeitsregel des Direkt-Sagens bzw. des Zu-ehrlich-Seins verwiesen. Es ist offensichtlich, dass diese eingesetzt werden kann, obwohl sie damit die Mitschülerinnen und schüler verletzt, was für sie scheinbar bedeutungslos ist. Hier dokumentiert sich, dass Lilian sich früher nicht aufführen konnte, wie sie wollte. Dies ist jetzt möglich, weil keine Loyalität zum Klassenverband mehr besteht und damit auch nicht $\mathrm{zu}$ den daraus folgenden kollegialen verpflichtenden Regeln.

In diesem kurzen Abschnitt betont Lilian ihre Gleichgültigkeit gegenüber dem Klassenverband dreimal mit dem Wort egaal. Die emotionale Steigerung ist mit dem Adverb untermauert, das zunehmend mit Zusatzworten verstärkt wird. Bei der ersten Erwähnung steht noch das abschätzende Wort echli. Beim zweiten Mal wird das Zusatzwort echli widerrufen, indem sich Lilian für äigentli egaal entscheidet. Abschließend verwendet Lilian das zusammengesetzte Wort schäissegal. Die Wiederholungen, die steigende aktive Bekräftigung von ,egal' und die dazwischenliegende Unklarheit, ob es ein bisschen oder gänzlich egal ist, kann auf eine Unsicherheit in Lilians Positionierung in der Klasse verweisen. Eine Ungereimtheit in der Positionierung dokumentiert sich weiter in der anfänglichen Identifikation mit der Klasse, dem Bedürfnis nach einem sozialen Netzwerk in der Fußballmannschaft und im Nichtberichten von einem realen Beispiel ihrer Ehrlichkeitsregel.

Das Dilemma zwischen den Ehrlichkeitsregeln ist in diesem Fallbeispiel nicht situationsbedingt, es bezieht sich auf eine Zeitspanne. Dennoch ist ein Dilemma zwischen Lilians individuellen Ehrlichkeitsregeln des DirektSagens bzw. des Zu-ehrlich-Seins und den kollegialen Ehrlichkeitsregeln zu erkennen, welche implizit darin bestehen, nicht immer alles zu sagen, was man denkt, weil dies im Regelfall das Gegenüber nicht gleichgültig lässt.

Zusammenwirkende Ehrlichkeitsregeln (Kap. 9.1.1, Lilian):

individuelle Ehrlichkeitsregel: Direkt Sagen bzw. Zu ehrlich Sein

versus

kollegiale Ehrlichkeitsregeln: Angemessene Meinungsäußerung berücksichtigen die Meinung der anderen Peers 
Bezogen auf die Veranschaulichung des Dilemmas mittels der zusammenwirkenden Faktoren (vgl. Faktorenstruktur in Abb. 3) kann Lilians Dilemma wie folgt beschrieben werden:

Kontext des Dilemmas ist das Faktum, dass Lilian nicht in die Klasse integriert ist. Es besteht der Wunsch nach Anerkennung und sozialem Netzwerk, wie sie der Fußballverband bietet, aber nicht die Schulklasse. Die Anerkennung unter Peers und das soziale Netzwerk kann als Ursache des Dilemmas bezeichnet werden. Der kollegialen Ehrlichkeitsregel, welche Lilian aus dem Fußballverband kennt, stellt sie im Klassenkontext ihre individuelle Ehrlichkeitsregel gegenüber, worin sich die Dilemmasituation abzeichnet. Darüber berichtet Lilian nicht direkt. Ebenso stehen keine Informationen zur Verfügung, welche die intervenierende Maßnahme erklären können. Anzunehmen ist, dass verschiedene Vorfälle Lilian zu ihrer Entscheidung führten. Der Auslöser, der eine Entscheidung von Lilian forderte, bildet in der Interpretation von Lilians Dilemma einen blinden Fleck. Aus dem Dilemma resultiert Lilians freie Verhaltensweise gegenüber kollegialen Regeln und Konventionen. Lilians Ehrlichkeitsregel scheint im Kontext dieses Abschnittes von den äußeren Umständen im Klassenverband aufgedrängt zu sein. Die Annahme der Ehrlichkeitsregel von Lilian als Reaktion auf von ihr negativ empfundene Verhaltensweisen der Mitschülerinnen und -schüler wird durch explizite Aussagen von Valentina untermauert: Lilian habe andere Mitschülerinnen und -schüler verpetzt. Daher habe eine Mädchengruppe Lilian gemobbt, womit sie jetzt eine Außenseiterin sei (vgl. Chiapparini 2011, S. 27). Ebenso weist die Klassenlehrperson auf die Desintegration von Lilian hin (vgl. Anhang, Datenmaterial aus der zweiten Erhebungsphase).

\subsubsection{Zwei unterschiedliche Verständnisse von kollegialen Ehrlichkeitsregeln: ... dänn han ich äifach abblockt}

Christoph zählt explizit drei Ehrlichkeitsregeln auf, die für ihn wichtig sind (Chiapparini 2011, S 184ff.): Ehrlich verhalte sich jemand, wenn dieser

- zur eigenen Meinung steht

- bei Streitigkeiten nicht falsch aussagt

- nicht über jemanden hinter dessen Rücken spricht

$\mathrm{Zu}$ jeder dieser Ehrlichkeitsregeln erwähnt er ein reales Beispiel aus dem Schulalltag, wobei es sich beim letzten Erläuterungsbeispiel um eine Dilemmasituation handelt, die ich an dieser Stelle genauer analysiere. Christoph nimmt vorerst stichwortartig Bezug auf seine Ehrlichkeitsregel Nicht hinter dem Rücken über jemanden sprechen auf:

Daas (1) und hinderem Rugge. (1) Das isch mir jetzt persönlich maal passiert. Es Mäitli hät mit mir gredt, mir sind Kolleege. (1) Und dänn han ich äifach erfaare, dass si hinder mim 
Rugge dänn über mich verzelt hät. (1) Dänn han ich äifach gsäit: ,Jetzt isch verbii“ oder. Dänn hät si wider emaal wele mit mir rede, dänn han ich äifach abblockt (3).

Christoph erzählt auf sehr knappe, strukturierte und chronologische Weise von einem für ihn negativen Erlebnis bezüglich des Bruchs mit dem kollegialen Vertrauen zu einer Mitschülerin. Der Interviewabschnitt strukturiert sich erstens in einer anfänglichen expliziten und stichwortartigen Bezugnahme auf seine als dritte genannte Ehrlichkeitsregel, zweitens mit einem persönlichen Bezug zum Beispiel, von dem er berichten wird, und schließlich in fünf Erzählungssätze, die parallel zueinander mit dänn beginnen.

Im ersten Textsegment dominiert der Ausdruck hinderem Rugge. Die Aussage ist ohne Verb positioniert, obwohl die Metapher für eine Tätigkeit steht, die nicht offen, sondern von der Wortdefinition her versteckt und vor der betroffenen Person geheimgehalten stattfindet. Eine versteckte und geheime Verhaltensweise verbindet Christoph hier indirekt mit Ehrlichkeitsregeln. Allerdings nennt er den Zusammenhang zwischen Ehrlichkeitsregeln und versteckten sowie geheimen Verhaltensweisen explizit in der oberen Auflistung von Ehrlichkeitsregeln. Somit ist der Ausgangspunkt der Erzählung die unehrliche Verhaltensweise, die sich darin charakterisiert, dass etwas im Verborgenen und in Unwissenheit einer betroffenen Person passiert.

Die Person, um die es in Christophs realem Beispiel geht, ist er selbst. Dies deklariert Christoph im anschließenden Satz, bevor er mit der Erzählung beginnt. In der ersten Erzählsequenz stellt Christoph fest, dass ein Mädchen mit ihm gesprochen hat. Christoph verwendet für die Gesprächsführung nicht das kollektive Subjekt wir bzw. ein wir haben gesprochen, sondern die distanziertere Formulierung der dritten Person Einzahl: Das Mädchen habe mit ihm gesprochen. Die Wahl der Erzählsubjekte verweist auf ein distanziertes Verhältnis von Christoph zu dem Mädchen. Zudem geht die Initiative für das Gespräch von dem Mädchen aus, indem sie für ein Gespräch zu Christoph geht. Auch verweist das Partizip gredt nicht zwingend auf ein gleich partizipiertes und teilgenommenes Gespräch. Es ist vorstellbar, dass eine Gesprächspartei unverhältnismäßig mehr gesprochen hat oder ein Abhängigkeitsverhältnis besteht, was zum einseitigen Ergreifen der Gesprächsinitiative passen würde. In dieser Lesart wäre das Mädchen von Christoph abhängig.

Über den Inhalt des Gesprächs äußert sich Christoph nicht. Das kann so gedeutet werden, dass der Inhalt irrelevant für das ist, was Christoph in diesem Beispiel mitteilen will. Es kann auch sein, dass Christoph den Inhalt nicht preisgeben will. Christoph deklariert anschließend mit dem kollektiven Subjekt mir, dass die Art der Beziehung zwischen dem Mädchen und ihm kollegial ist. Hier dokumentiert sich wiederum eine Unklarheit, ob die Beziehung gegenseitig oder einseitig aus der Perspektive von Christoph als kollegial zu verstehen ist.

Zudem fällt auf, dass Christoph das Mädchen nicht als Schülerin bezeichnet und es auch nicht beim Namen nennt. Das könnte mit der Wahrung 
der Anonymität der Schülerin zu tun haben. Zugleich ist festzuhalten, dass er in anderen Textpassagen andere SuS namentlich nennt. Diese Unklarheit in der Interpretation muss vorübergehend stehen gelassen werden, weil sachliche Informationen fehlen. Im schweizerdeutschen Schulkontext werden in der Grundschule die SuS als ,Mädchen“ und ,Buben' bezeichnet, wobei damit keine pejorative Wertung verbunden ist. Etwas unüblicher ist es, dies in der Oberstufe zu tun. Doch auch andere interviewte SuS verwendeten für sich oder für andere diese kindlich scheinenden Ausdrücke. Da durch den Ausdruck ,Mädchen' noch nichts über das Beziehungsverhältnis zu diesem Mädchen geklärt ist, präzisiert Christoph, dass es sich um eine Kollegin handelt. Im Schweizerdeutschen werden mit dem Wort ,Kollege“ sowohl Mitschülerinnen und -schüler als auch gute Freunde bezeichnet. Hier lässt sich nicht identifizieren, um welche der beiden Arten von Kollegen es sich handelt.

Im dritten Erzählfragment berichtet Christoph, wie er passiv die Information erhalten hat, dass jenes Mädchen über ihn mit anderen gesprochen habe. Es ist unklar, ob es negativ oder nur über den Inhalt des Gesprächs gesprochen hat, ob es dies vor vielen und vor welchen Leuten getan hat. Zudem bleibt unklar, welchen Gesprächsinhalt es mitgeteilt hat. Trotz dieser offenen Sachverhalte für den Leser ist für Christoph der Entschluss gefällt: Die Kollegschaft mit dem Mädchen isch verbii. Das ist ein brüsker Entscheid, wenn bedacht wird, dass Christoph sich nicht darüber äußert, ob er die Richtigkeit und Gültigkeit der Information überprüft hat. Anscheinend reicht die Tatsache, dass das Mädchen über ihn und den besprochenen Sachverhalt anderen etwas mitgeteilt hat, um die Vertrauensbeziehung mit dem Mädchen abzubrechen. Darin steckt die Annahme, dass für Christoph der Gesprächsinhalt persönlich und nicht zum Weitererzählen war. Hingegen hat das Mädchen eine andere Wahrnehmung vom Sachverhalt, deshalb kommt sie ein anderes Mal wieder zu Christoph, um mit ihm zu reden. Auch hier betont Christoph implizit mit den Worten wele mit mir die Initiative und den Wunsch seitens des Mädchens für das gemeinsame Gespräch. Seine Reaktion beschreibt Christoph knapp mit abblockt. Damit kann sowohl das Abbrechen des Gesprächs als auch das Abbrechen der kollegialen Beziehung gemeint sein. Mit großer Plausibilität hat er Letztere bereits vorgenommen, als er die Informationen der Nachrede erhalten hat. Doch physisch bot sich erst jetzt die Möglichkeit, den Beziehungsabbruch anzukündigen.

Christophs Reaktion erscheint brüsk: ohne Erklärung und ohne das Bedürfnis, die kollegiale Beziehung aufrechtzuerhalten oder ihr eine zweite Chance zu geben. Die abrupte Art dokumentiert eine Reaktion von Christoph, die er nicht verbal, sondern durch sein Verhalten markiert. Es bleibt offen, ob sich Christoph tatsächlich ohne Kommentar von dem Mädchen abgewendet hat oder ob er etwas gesagt hat und sich dann abgewendet hat. Unabhängig von dieser Unklarheit kann geschlossen werden, dass eine persönliche und von Christoph unerwartete Verletzung seiner Vorstellung von Zweiergesprä- 
chen und von Beziehungsverhältnissen vorliegt, die sich hier als delikate Angelegenheiten dokumentieren.

Der Inhalt des Gesprächs scheint für Christoph und für seine Reaktion nicht relevant zu sein. Vielmehr ist der Vertrauensbruch bedeutend. Deshalb hat sich Christoph im Dilemma zwischen der kollegialen bilateralen Ehrlichkeitsregel Nicht hinter dem Rücken über jemanden sprechen (Offenheit gegenüber Kollegen, die in einen Sachverhalt eingeweiht und davon betroffen sind) und der anderen kollegialen Ehrlichkeitsregel Es ist ehrlich, wenn man über andere Kollegen spricht, die abwesend sind (Offenheit gegenüber allen Kollegen, ob anwesend oder nicht) in dieser Situation zu entscheiden. Zudem unterstreicht die chronologische und knappe, auf die Verhaltensebene fokussierte Erzählung mehr den Ablauf als den Inhalt des Vorgehens. Damit hebt Christoph den Verhaltensablauf im erzählten Beispiel als modellhaft für andere Dilemmasituationen hervor und generalisiert diesen auf einer abstrakten Ebene zu seinem Regelverhalten.

Zusammenwirkende Ehrlichkeitsregeln (Kap. 9.1.2, Christoph):

kollegiale, bilaterale Ehrlichkeitsregel: Nicht hinter dem Rücken über jemanden sprechen

(Offenheit gegenüber Kollegen, die in einen Sachverhalt eingeweiht und davon betroffen

sind)

versus

kollegiale, allgemeine Ehrlichkeitsregel: Es ist ehrlich, wenn man über andere Kollegen

spricht, die abwesend sind (Offenheit gegenüber allen Kollegen, ob anwesend oder nicht)

Ich fasse die einzelnen Faktoren zusammen, die aus Christophs Erzählung eine Dilemmasituation ergeben (vgl. Faktorenstruktur in Abb. 3). Der kontextuelle Hintergrund bildet ein Zweiergespräch zwischen Christoph und einer Schülerin. Die Ursache des Dilemmas liegt in der unterschiedlichen Gewichtung der Vertrautheit des geführten Gesprächs. Für Christoph handelt es sich um ein vertrautes Zweiergespräch, dessen Inhalt nicht Dritten weiterzuerzählen ist. Neben dem Vertrauen steht auch die Loyalität, das Zusammenhalten gegenüber Dritten im Vordergrund. Hingegen trifft diese Wahrung bei dem Mädchen nicht zu, das ausgehend von den Gesprächsinhalten über Christoph bei anderen spricht. Daraus positionieren sich zwei kollegiale Ehrlichkeitsregeln zur Offenheit in kollegialen Beziehungen und dazu, wie gegenüber Dritten mit den erhaltenen Informationen umgegangen wird: Nicht hinter dem Rücken über jemanden sprechen und Es ist ehrlich, wenn man über andere Kollegen spricht, die abwesend sind.

Auslöser für Christophs Reaktion ist die Information, dass das Mädchen über ihn in seiner Abwesenheit gesprochen hat. Diese intervenierende Maßnahme führt zu einem brüsken und unhinterfragbaren Beziehungsabbruch mit dem Mädchen - zuerst entscheidet Christoph sich persönlich für dieses Verhalten. Erst bei der nächsten Anfrage des Mädchens für ein neues Gespräch kündigt er öffentlich, aber indirekt diesen Entscheid an, indem er sich nicht auf das Gespräch einlässt. Christoph verzichtet auf die Kollegschaft mit dem 
Mädchen zugunsten seiner Ehrlichkeitsregel Nicht hinter dem Rücken über jemanden sprechen.

In der brüsken Reaktion von Christoph könnte sich eine Verletzung seiner Person dokumentieren, wozu Anhaltspunkte im Interviewtext darin zu finden sind, dass er sich im Klassenverband als souverän und gegenüber den Lehrpersonen als ein Musterschüler präsentiert. Durch die Offenheit der Mitschülerin könnten seine Stellung und sein Image in der Klasse getrübt worden sein.

\subsection{Dilemma zwischen individuellen und konventionellen Ehrlichkeitsregeln}

SuS kennen die Bandbreite an konventionellen Ehrlichkeitsregeln, die sowohl einen schulspezifischen als auch gesellschaftlichen Ursprung haben können. Gleichzeitig stellen konventionelle Ehrlichkeitsregeln im Schulalltag eine Herausforderung für die SuS dar, die sie unterschiedlich angehen. In diesem Zusammenhang ist eine oft erwähnte Dilemmasituation für SuS, wenn diese die Elternunterschrift für zurückerhaltene und schlecht ausgefallene Schultests einzuholen haben. Diesbezüglich berichten Clemens, Marco und Stephan von Dilemmasituationen und wie sie diese bewältigen. Im Unterschied zu Stephan und Marco lässt Clemens die unzufriedene Mutter verbal und mit Nachdruck ihre Erwartungen einfordern. Er wartet, bis die Mutter sich beruhigt, denn dann unterschreibt sie seine Prüfung (vgl. Kap. 8.1.3). Indes hat Marco für sich auszuhandeln, ob er die ungenügende Note eingestehen oder partiell kaschieren soll (vgl. Kap. 9.2.1). Bei Stephan stellt sich bei diesem Thema zusätzlich der drohende Freizeitentzug (vgl. Kap. 9.2.2).

\subsubsection{Eingestehen von ungenügenden Noten anhand partieller Kaschierung: ... ich han amigs en Trick, äifach...}

Ausgehend von der Interviewfrage „Wänn ir so Prüefige gschribe händ, müender s amigs de Eltere gee zum Underschriibe? (M: Mhm) Und wie isch das so, wänn du das so abgee muesch di Häime?", erklärt Marco anhand von zwei Verfahrensweisen, wie er bei schlechten Noten die Unterschrift der Eltern einholt:

Wänn s e Schäissnote isch, isch es scho nöd so aagneem, aber (3) ich han amigs en Trick, äifach am Morge, (1) grad drüü Minute bevor gaasch, gisch es churz und si underschriibet und tu säisch: „Ou, ich mues jetzt gaa“ und dänn gang@ich@ (2) Und ja, käi Aa- Ja, mängmaal lueg ich au (1), dass ich- Wänn ich e schlächti Noote han, (1) dass ich no e gueti 
Prüefig dazue han, dänn chan ich bäides abgee. (1) Und dänn isch s wie echli kompensiert. (2) Ja (7).

Marco beginnt seine Ausführungen mit einer verallgemeinernden Aussage (neutrales Subjekt es). Darin dokumentiert sich die Allgemeingültigkeit von dem, was folgen wird. Zudem ist in der verallgemeinernden Formulierung ein vager Hinweis auf die Häufigkeit von schlechten Noten in Schultests und die persönliche Distanzierung von diesen gegeben. Es handelt sich nicht um einen Einzelfall. Wenn schlechte Noten zu Hause zu zeigen sind, um die Elternunterschrift einzuholen, ist das keine ,angenehme' Aufgabe. Marcos negative Gefühlslage wird nicht allein durch den Ausdruck nöd so aagneem signalisiert, sondern ebenso im Wort Schäissnote. Dieses Wort zählt ausschließlich zur verbalen Alltagssprache und wird verwendet, wenn etwas misslungen ist. Hier dokumentiert sich Marcos Einsicht, dass Schultests misslingen können. Eine dazu ergänzende Lesart des Wortes ,Schäissnote“ ist in der hintergründigen Erwartung und im Prinzip zu finden, dass Schultests zu gelingen haben, was an den erfolgreichen Noten abzulesen sei. Da dem nicht immer so ist und durch die Regelmäßigkeit der unangenehmen Aufgaben, Elternunterschriften für ungenügende Noten einzuholen, dokumentiert sich, dass Marco eine bewährte Verfahrensweise gefunden hat: einen Trick. Damit ist eine Taktik bezeichnet, mittels der eine Person etwas vortäuscht und dadurch eine schwierige Aufgabe gelöst wird. Marcos Trick besteht darin, gezielt einen günstigen Zeitpunkt zu wählen, um die Unterschrift der Eltern einzuholen: der Morgen. Dieser eignet sich dafür besonders gut - kurz bevor er in die Schule gehen muss. Das Füllwort äifach kann als Hinweis der Alltäglichkeit von Marcos Verhaltensweise gelesen werden. Drü̈̈ Minute ist als kurze Zeiteinheit und nicht wortwörtlich zu interpretieren. Die Zeitknappheit wird zudem mit der Bemerkung unterstrichen, die als direkte Rede formuliert ist: Ou, ich mues jetzt gaa. Das Wort ou kann als Entschuldigung und als überraschende Feststellung gedeutet werden, dass er gehen muss. Es hat den Nachklang von etwas Theatralischem und Gestelltem. Das Subjekt si verweist, dass die Mutter unterschreibt. Vom Vater ist nicht die Rede. Die Zeitknappheit impliziert ebenso die Tugend der Pünktlichkeit, die an dieser Textstelle implizit von Marco starkgemacht wird. Anders formuliert: Zugunsten der Pünktlichkeit gerät die Forderung in den Hintergrund, zu einer misslungenen Note aufrichtig zu stehen.

Zusammenfassend sind folgende Faktoren in der geschilderten Dilemmasituation erkenntlich: Den Kontext bildet die unangenehme Aufgabe, bei misslungenen Schultests die Unterschrift der Eltern (in diesem Fall der Mutter) einzuholen. Das Dilemma steht zwischen zwei konventionellen Ehrlichkeitsregeln der Aufrichtigkeit, von denen er einer nachgeht und der anderen nicht. Die konventionelle Ehrlichkeitsregel verfolgt er: Elternunterschriften nicht fälschen. Jedoch will er die konventionelle Ehrlichkeitsregel $\mathrm{Zu}$ schlechten Schultests stehen umgehen. 
Zusammenwirkende Ehrlichkeitsregeln (Kap. 9.2.1, Marco):

konventionelle Ehrlichkeitsregel: Elternunterschrift nicht fälschen

versus

konventionelle Ehrlichkeitsregel: Zu den eigenen Schulleistungen/Noten stehen

daraus

individuelle Ehrlichkeitsregel: Bei schlechten Schultests kann die Elternunterschrift mit einer partiellen Täuschung eingeholt werden

Die Ursache dieses Konflikts findet sich in der fehlenden Courage, zu schlechten Noten zu stehen, oder in der Befürchtung von negativen Reaktionen der Mutter und den daraus resultierenden möglichen Sanktionen. Allerdings geht aus der genannten Textpassage nicht klar hervor, worin genau diese Ursachen bestehen. Daher lasse ich die Vermutungen hier so stehen.

Eine intervenierende Maßnahme besteht im Hausaufgabenauftrag, die Elternunterschrift ohne Widerstand einzuholen. Marco bleibt zum einen implizit dabei, die konventionelle Ehrlichkeitsregel der Aufrichtigkeit einzuhalten, in diesem Fall: Elternunterschrift nicht fälschen. Zum anderen möchte er die unangenehme Situation der Konfrontation der Eltern mit seiner ungenügenden Schulleistung meiden und weigert sich, die zweite konventionelle Ehrlichkeitsregel anzuwenden: Zu schlechten Schultests stehen. Daraus resultiert die Verhaltensweise, die von einer individuellen Ehrlichkeitsregel geprägt ist: Bei schlechten Schultests kann die Elternunterschrift mit einer partiellen Täuschung eingeholt werden. Die Verhaltensweise ist ein ,Trick': Vordergründig wird Aufrichtigkeit vorgespielt, indem scheinbar die Unterschrift offen eingeholt wird. Allerdings wird die Dauer dafür knapp gehalten. Marco expliziert dazu keine Gründe. Jedoch ist zu vermuten, dass damit ein spontanes Gespräch über die Prüfung oder eine gründliche Prüfung der erbrachten Schulleistung umgangen werden kann. Die Dauer für das Unterschreibenlassen wird von Marco kontrolliert, indem er gezielt einen Zeitpunkt auswählt (morgens kurz vor der Schule) und das Ende des Verhaltens anhand der Aussage vorgibt, dass er gehen müsse, die er mit dem als Überraschung wirkenden Wort ou initiiert. In diesem Abschnitt dokumentiert sich sehr klar die Hinterbühne, wie Marco sich weigert, die schlechte Note den Eltern zu zeigen und wie er eigene Wege (,Tricks') festlegt, um diese alltägliche Herausforderung anzugehen.

Den Übergang von der Beschreibung der ersten Vorgehensweise zur zweiten bildet der Satzabbruch $\mathrm{Ja}$, kai Aa-. Aus dem semantischen Kontext lässt Aa auf Aanig (Ahnung) schliessen. Zu dieser abgebrochenen Aussage ist eine zweifache Deutung angebracht. Zum einen ist Marco sich nicht ganz über seine Verfahrensweise im Klaren, weil er nie darüber nachgedacht hat. Zum anderen kann dieser Ausdruck eine Ablenkung vom Gesprächsthema sein und ein Signal, dass er darüber nicht sprechen will. Dennoch kommt ihm plötzlich ein Beispiel in den Sinn. Die Unmittelbarkeit des Einfalls ist mit dem Wortabbruch angezeigt. Seine zweite Vorgehensweise bezieht sich auf das gleichzeitige Abgeben eines guten und eines schlechten Schultests. Das 
Wort kompensiert deutet auf den Ausgleich der guten und schlechten Note hin. Die zwei moralischen Terme dokumentieren die soziale Erwünschtheiten, dass Schultests gut auszufallen haben.

Kontext und Ursache sind bei Marcos zweiter Vorgehensweise der ersten gleich. Hingegen fügt sich der ersten intervenierenden Maßnahme noch eine zweite an, die in der Gelegenheit besteht, eine gute Prüfung gleichzeitig beilegen zu können. Das ist ein situationsabhängiger Faktor. Falls dieser vorhanden ist, resultiert daraus die Verhaltensweise, dass Marco beide Schultests zur Unterschrift vorlegt.

In Marcos Beschreibung kommt weder das Thema noch das Schulfach des Schultests vor. Er fokussiert die Beschreibung seines Vorgehens und weniger den Kontext. Daher geht er nicht gezielt auf die Interviewfrage ein, die Darstellung einer Situation, in der misslungene Schultests den Eltern vorgelegt werden müssen, um deren Unterschrift einzuholen. In Marcos Beschreibung dokumentiert sich, wie er mit solchen Situationen umgeht und welche Bewältigungsweisen er erfolgreich anwendet. Marcos Eigenart im Sprechen über das Unterschrifteneinholen bei schlechten Noten verweist auf ein Schamgefühl, sich über solche Angelegenheiten mit einer Fremdperson zu unterhalten. Das wird mit der Unterbrechung der verbindenden Aussage zwischen beiden Verfahrensweisen von Marco unterstrichen. Scham weist auf Verhaltensweisen hin, die vorhanden sind, aber über die nicht gesprochen wird, weil sie als nicht wünschenswert erachtet werden. Dennoch sind $\mathrm{m}$. E. gerade diese Verhaltensweisen und deren Beleuchtung interessant, um zu verstehen, worin alltägliche schulische Herausforderungen bestehen und wie SuS diese bewältigen.

\subsubsection{Schulisches Engagement und Überzeugungskraft gegen Freizeitentzug:... wänn die nächscht Noote ungnüegend isch, dänn isch de PC echli länger weg}

Stefan erklärt offen, dass er noch nie die Elternunterschrift selber gesetzt hat, und situiert sich damit als Schüler, der die konventionellen Ehrlichkeitsregeln kennt und befolgt. Dennoch ist das Einholen der Elternunterschrift eine diffizile Aufgabe, denn seine Leistungsresultate haben reale Sanktionen, die von der Mutter ausgesprochen werden und die seine Freizeitaktivitäten einschränken. Vor diesem Hintergrund veranschaulicht Stefan an zusammenhängenden Vorfällen sein Bewältigungsverfahren:

I: Und wie machsch s du, wänn du schlächti Noote häsch?

S: (2) Ich zäigs- Also ich han nonie d Underschrift gfälscht. (2) (I: Was säget dänn dini Eltere?). (1) Chunt ganz druf aa i welem @Fach@ (3). Also ja, ich ha letschthin grad, en Drükommavier ghaa, (1) im Französich und süscht bin ich äigentli nienets ungnüegend. (2) Und däne, ja isch de letschti Voggitescht gsii. Isch en drüünün gsii. Und deet hät si [Mut- 
ter] mir drüü Tääg PC-Verboot ggee. (3) Und dänn, wo mer in zrugg überchoo händ, hät s ghäisse, nächschte Friitig- Dunschtig und Friitig hämmer au zwäi französisch Tescht (3) und dänn (3) hät s äifach ghäisse di Häi, ja wänn die nächscht Noote ungnüegend (1) isch, dänn isch de PC echli länger weg. (2) Und nachheer isch dee Tescht am Dunschtig- Hämmer müese (1) Tegscht schriibe (1) und am Friitig (1) Hörverstehen, Wäg iizäichne. (1) Und deet isch Qualität akuut schlächt gsii. Es hät immer es Rusche im Hindergrund ghaa und bim eerschte Maal han ich gnau verschtande, das ich links abbüüge sött und nachheer nüt mee. (2) Und dänn hät si no gsäit- Ja, (2) also es händ no vill gsäit, d Qualität isch seer schlächt gsii und (2) wänn s öppis würd uusmache im Zügnis, (1) schtatt wäis au nöd Vierenhalber schtatt en Föifer (1). Näi schtatt en Föifer en Vierehalber, zält si nöd. (3) Und im Tegscht hän ich zum Glück en Föifkommadrüü ghaa. (3) Und de Tüütschtescht, wo n i no naagholt han, (1) will- also letscht Wuche- (1) Vorletscht Wuchenend isch Doorffäscht gsii. (2) Und mer hät- Mir händ am Morge- (1) Hämmer Französisch (1) und ich han am Morge vom Nüüni bis am Zäni Schuel. Näi vom Achti bis am Zäni und dänn Mittag bis am halbi Drüü. (1) Und dänn han ich, Englisch, Tüütsch und es Buech müese läse. (1) Han ich gsäit: „Daas mach ich über de Mittag“, will ich han föifezwänzg Schtund gschafft am Dorffäscht. (2) Und dänn (2) hät s am Morge ebe en Tescht ggee im Französisch. (1) Und dänn händ au wider es paar proteschtiert: ,Ja, si, ich has nonig glässe. Es isch s Doorffäscht gsii“" und so, und dänn am Mittwoch nach em Klasseraat hämmer d Prüefig zrugg überchoo.(2) Und deet hät s dänn ghäisse, ja: (1), Wer wott cha s am Dunschtig am föifi naahole." Und dänn han ich si deet no nagholt (1) und dänn han ich deet no en Sächser ghaa. (3) Und dänn han ich äifach die drüü zäme anegläit und han gsäit ja- (2) Dee Franztescht hät si scho seer kritisch aagluegt. Und so: „Ja, daas und daas.“ Ich so: „Isch en Föifdrüü nöd gnueg guet.“ Und so. Dänn hät si gfunde: „Ja doch.“ Dänn hät si endlich Maal underschribe (1) und bim Drüüvier han ich äifach gsäit: „Ja, es isch e akut schlächtsschlächti Qualität gsii und si zält nöd im Zügnis. Und ooni die han ich en Viernüün im Schnitt.“ (2) Und ja, dänn hät si underschriibe (8).

Die Frage der Interviewerin ist zu direkt gestellt, sodass Stefan zunächst darauf hinweist, dass er misslungene Schultests den Eltern zeigt. Allerdings bricht er diese Aussage ab, um klarzustellen, dass er die Unterschrift der Eltern nicht fälscht. Darin zeigt sich zum einen, dass Stefan sich durch die Fragestellung angegriffen fühlt und sich daher rechtfertigt. Zum anderen dokumentiert sich neben dem Befolgen der konventionellen Ehrlichkeitsregeln Elternunterschriften werden nicht gefälscht und Erzielte Noten in Tests werden den Eltern offen gezeigt die individuelle Ehrlichkeitsregel $\mathrm{Zu}$ seinen erzielten Noten stehen. Auf die anschließende Fragestellung nach der Reaktion der Eltern auf misslungene Schultests antwortet Stefan präzisierend, dass dies fächerabhängig sei, und beginnt eine längere Erzählung, in der der Inhalt der Prüfung und der Kontext detailliert nachgezeichnet werden: Wenn Stefan ungenügende Schultests schreibt, folgt zu Hause eine Sanktion, wie beispielsweise, als unlängst ein dreitägiges Computerverbot verhängt wurde, weil er zwei ungenügende Noten in Französisch schrieb. Wer genau die Sanktion ausgesprochen hat, wird nicht erwähnt. Darin dokumentiert sich, dass diese Sanktion nicht personengebunden, sondern als allgemeine Konvention von Stefan aufgefasst ist. In der Erzählung berichtet Stefan sehr präzise von jeder Note bis auf eine Dezimalzahl nach dem Komma. Es fällt auch 
auf, dass gewisse Dinge unwiderrufbar festgesetzt sind, wie das Ansetzen einer Prüfung oder die Sanktionen für schlechte Noten. Das wird mit der sprachlich neutralen Form häts ghäisse bestärkt und dokumentiert sich so als feste Regel. Trotz der hohen Leistungserwartungen von zu Hause unternimmt Stefan außerschulische Aktivitäten wie die aktive und zeitintensive Vorbereitung und Durchführung des Dorffestes - in Kauf nehmend, dass er das Deutschbuch erst über den Mittag fertiglesen kann. Dahinter steht ein Kalkül für ein zielstrebiges Engagement für die Schule: Die Deutschprüfung findet am Nachmittag statt, und deshalb kann das Buch über Mittag fertiggelesen werden. Dass dieser Plan aufgegangen wäre, zeigt sich darin, dass Stefan in anderen Schultests ebenfalls gute Leistungen erbringt, wenn die Prüfungsumstände es zulassen. Stefans Kalkül wird jedoch von der Stundenverschiebung der Lehrerin durchbrochen, welche die Unterrichtsstunden Deutsch und Französisch an jenem Tag gewechselt hat, sodass die Deutschstunde ausnahmsweise am Morgen anstelle der Französischstunde stattfindet. Zudem überrascht sie die SuS mit einem Test zum gelesenen Buch. Damit hat Stefan in seinem Lernplan nicht gerechnet; er erzielt eine ungenügende Note. Jedoch schlägt die Lehrerin eine Nachholprüfung für Donnerstag vor. Stefan ergreift die Gelegenheit und berichtet, dass er in jener Prüfung die Höchstnote erzielt habe. In dieser Textpassage dokumentiert sich Stefans kalkuliertes Engagement, um gute Schulleistungen zu erzielen.

Die Schultests im Französischfach werden besonders genau von der Mutter überprüft: hät si scho seer kritisch aagluegt. Stefan erzählt, wie er bei einer Französischprüfung in den schriftlichen Aufgaben (auf dem Blatt) eine 5.4 erzielt und im Hörverständnis wegen der schlechten Qualität eine ungenügende Note (3.4) erlangt. (Allerdings wird die Hörverständnisnote nicht im Zeugnis zählen, falls sie den Notendurchschnitt zu stark drückt.) $\mathrm{Zu}$ den Resultaten des Deutschtests teilt Stefan die Reaktion der Mutter nicht mit. In Stefans Erzählung dokumentiert sich ein Dilemma, das im Zusammenhang steht mit den konventionellen Ehrlichkeitsregeln Die erzielten Schulnoten den Eltern zeigen und Elternunterschrift nicht fälschen, der impliziten individuellen Ehrlichkeitsregel $\mathrm{Zu}$ den Noten stehen, die erzielt werden und der individuellen Ehrlichkeitsregel Ungenügende Schulleistungen verweisen nicht notwendigerweise auf ungenügendes schulisches Engagement. Die letztgenannte Ehrlichkeitsregel entwickelt sich im Spannungsfeld zweier impliziter Faktoren, die in der genannten Textpassage dokumentiert und mittels der Textanalyse herausgearbeitet sind: Auf der einen Seite steht die scharfe Kontrolle der Mutter und deren Beurteilung der erzielten Noten, woraus bei ungenügenden Noten Sanktionen folgen. Auf der anderen Seite steht Stefans kalkuliertes Engagement für Schulleistungen. (So berechnet Stefan, dass das Fertiglesen des Deutschbuches während der Mittagspause noch ausreichen sollte. Zudem holt er den Deutschtest nach, der ungenügend ausgefallen ist, weil die Lehrerin die Überraschungsprüfung über die 
Deutschlektüre in der Französischstunde schreiben ließ). In diesen zwei Faktoren ist eine individuelle Ehrlichkeitsregel von Stefan impliziert: Ungenügende Schulleistungen verweisen nicht notwendigerweise auf ungenügendes schulisches Engagement. Diese Regel führt zur Dilemmasituation, in der sie zwei konventionellen und einer weiteren individuellen Regel gegenübersteht.

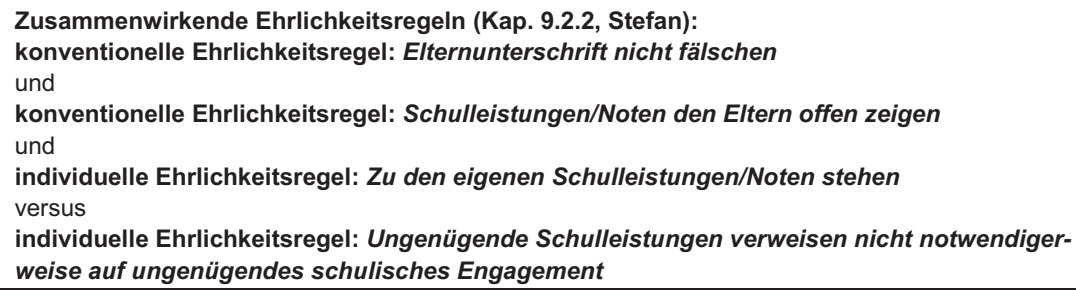

Stefans Entscheidungsprozess auf die wirkenden Faktoren zurückzuführen erweist sich als ein komplexes Unterfangen, das ich in einer vereinfachten und reduzierten Art vornehme. Denn das Faktum der Kontextabhängigkeit und die daraus resultierende Verhaltensweise ist Ziel der Analysearbeit und weniger die genaue Nachzeichnung der Prozesse: Der Kontext dieses Dilemmas bezieht sich auf das Einholen der Elternunterschrift für erzielte Noten in Schultests. Im konkret beschriebenen Fall handelt es sich zum einen um eine zweiteilige Französischprüfung mit der gelungenen schriftlichen Aufgabe und der misslungenen Aufgabe des Hörverständnisses und zum anderen um eine misslungene Deutschprüfung wegen Verschiebung der Unterrichtsstunde und deren gelungene Nachholprüfung. Die Ursachen bestehen in einem Dreifachen: Integrität (Stefan möchte transparent handeln), Anerkennung für sein schulisches Engagement und Loyalität gegenüber der Mutter (Aushandlungsmomente ermitteln). Als intervenierende Maßnahme steht die implizite Hausaufgabe, Prüfungsnoten von den Eltern unterschreiben zu lassen. Aus diesen Faktoren und den oben beschriebenen Dilemmas zwischen unterschiedlichen Ehrlichkeitsregeln resultiert Stefans Vorgehensweise, die darin besteht, die vier Schultests gleichzeitig vorzulegen und bei der Kontrolle und den Kommentaren der Mutter mit Überzeugungskraft die eigene Sichtweise seines schulischen Engagements vorzubringen.

\subsection{Dilemma zwischen kollegialen und konventionellen Ehrlichkeitsregeln}

Der Schulkontext, in dem Schultests stattfinden, bietet den Freiraum, sowohl individuelle als auch kollegiale Ehrlichkeitsregeln zu entwickeln, die mit konventionellen Ehrlichkeitsregeln konfligieren. Über das Spannungsfeld 
zwischen individuellen und konventionellen Ehrlichkeitsregeln gab Kapitel 8.1.1 bereits Auskunft. Eine komplexere Dilemmasituation bildet das $\mathrm{Zu}-$ sammentreffen von kollegialen und konventionellen Ehrlichkeitsregeln während Schultests, von dem Valentina erzählt (vgl. Kap. 9.3.1). Ein anderer Schulkontext, in dem kollegiale und konventionelle Ehrlichkeitsregeln regelmäßig zusammenstoßen, sind die Hausaufgabenkontrollen, wozu Lilian von einer Dilemmasituation berichtet und einen Leistungsstufenvergleich vornimmt (vgl. Kap. 9.3.2).

\subsubsection{Hilfsbereitschaft oder Betrug während Schultests: ... echli mitenander hälfe, find ich schöön, obwoohl s ja echli Betrug isch}

Als Valentina berichtet, wie eine Unterrichtsstunde verläuft, in der ein Schultest geschrieben wird, erklärt sie, wie sie gezielt Hilfe bei einer Mitschülerin einholt. Sie schaut, wer um sie herum sitzt, um zu verstehen,

wo die Person grad isch und ob si äim helfe chan. Zum Biispil Ufgab sächs verstaan ich grad nöd und dänn lueg ich die Person aa und dänn säit si, irgendwie so: <Direkte Rede ganz leise gesprochen> „Es isch so und so.“@1@ Und sehr liislig und ja (3).

Valentina kann bei einer ganz bestimmten Person Hilfe während der Schultests einholen, was mit dem bestimmten Artikel die unterstrichen wird. Die Routine dieser Tätigkeit dokumentiert sich in der abstrahierenden Beschreibung, welche eine direkte Rede impliziert. Nicht die ganze Klasse ist während der Schultests hilfsbereit, sondern nur eine Schülerin. Die duale Beziehung, die aus dem Geschilderten auf eine einseitige Hilfestellung verweist, ist ein Indiz für eine bilaterale kollegiale Relation, die eine freundschaftliche Ursache hat und weniger eine interne Klassenregel ist. Wenn Valentina die Lösung einer Testaufgabe nicht weiß und in der Nähe dieser Schülerin sitzt, kann sie diese Schülerin nach der Lösung fragen, die ihr in einer unauffälligen Weise zugeflüstert wird.

Valentina geht nicht direkt auf die Perspektive der Kollegin ein. Allerdings ist aus ihrer Verhaltensweise zu vermuten, dass die Kollegin wie selbstverständlich ihre Testarbeiten unterbricht und sich taktvoll und den Umständen angepasst Valentinas Fragestellung widmet. In dieser unausgesprochenen, aber nach den geschilderten Umständen anzunehmenden Verhaltensweise dokumentiert sich nochmals die freundschaftliche Beziehung, welche die beschriebene und riskante Verhaltensweise der Hilfeleistung voraussetzt. Die Hilfestellung ist nur möglich und erfolgreich, solange diese genannte Schülerin in der Nähe von Valentina sitzt und solange die Lehrperson nicht kontrolliert.

Auf die Möglichkeit, erwischt zu werden, geht Valentina anschließend ein, nachdem sie erklärt hat, dass die beschriebene Verhaltensweise während 
Schultests gut funktioniert, wenn die Lehrerkontrolle fehlt: „Also wänn s de Leerer nöd merkt und so. @Süsch isch es echli krass@1@ echli schlächt (3)“. Das Aufdecken der kollegialen Hilfsbereitschaft beurteilt Valentina in moralischen Kategorien als krass und schlächt und somit negativ. Das Auflachen verweist, wie aus der unteren Interviewstelle noch klarer hervorgeht, weniger auf eine unterhaltsame Tätigkeit, eher auf das Schamgefühl und auf das Wissen um die konventionelle Ehrlichkeitsregel Unlautere Hilfsmittel bei Schultests nicht beiziehen. Hierin dokumentiert sich, dass die kollegiale Ehrlichkeitsregel der Hilfestellung situationsabhängig ist. Zentrale Faktoren dazu nennt Valentina implizit: die Sitzordnung, die Hilfsbereitschaft von Mitschülerinnen sowie -schülern und die Kontrollschärfe der Lehrperson.

Auf die gezielte Nachfrage der Interviewerin nach dem Realitätsbezug und der Reaktion der Lehrperson auf ihre unkonventionelle Tätigkeit erzählt Valentina in derselben Interviewpassage:

Und dänn hät er äifach gsäit- dänn hät er äifach de Name grüeft, so: „Ja hey“@1@ (3) (I: Und dänn?) Dänn hämmer öis wider zruggdräit und es isch öis echli@2@. Also daas isch echli piinlich gsii@1@ Jetzt chunds mer grad @1@(3) (I: Und nachher, was isch nachher passiert?) Nachher hämmer äifach wiiter gschribe @1@ (4).

Valentina erzählt mit Lachpausen und zieht die direkte Rede bei. Für sie ist klar, dass die beschriebene bilaterale einseitige und kollegiale Hilfestellung während Schultests unehrlich ist. An anderer Stelle bezeichnet sie eine solche Verhaltensweise sogar als Betrug. Den geschilderten Vorfall beschreibt sie als ,peinlich', wobei der Lehrer außer einem nichtpersonalisierten Appell keine weiteren Konsequenzen ausspricht. Das peinliche Gefühl, worauf Valentina wörtlich und durch die lachende Sprechweise hindeutet, weist auf einen Zusammenhang mit dem Schamgefühl hin, was weiter oben bereits gedeutet ist. Zudem kann der Ausdruck jetzt chunds mer grad auf einen Verdrängungsmechanismus des unangenehmen und in der Erinnerung fast vergessenen Vorfalls deuten. Beide Schülerinnen kennen sehr wohl die konventionelle Ehrlichkeitsregel Selbstständiges Arbeiten während Schultests und richten sich nach dem Appell des Lehrers kommentarlos nach dieser Regel, indem sie selbstständig den Prüfungstest weiter lösen. In der Unmittelbarkeit dokumentiert sich die geteilte und nicht zu widersprechende konventionelle Ehrlichkeitsregel.

Dennoch zeigt sich vor dem Ertapptwerden eine Dilemmasituation zwischen der kollegialen Ehrlichkeitsregel Versteckte Hilfestellung während Schultests und der konventionellen schulischen Ehrlichkeitsregel Nicht schummeln. Diese haben die beiden Schülerinnen nach dem Ertapptwerden zu lösen. 
Zusammenwirkende Ehrlichkeitsregeln (Kap. 9.3.1, Valentina):

bilaterale, kollegiale Ehrlichkeitsregel: Zuflüstern von angefragten Lösungen während Schultests

versus

konventionelle Ehrlichkeitsregel: Nicht schummeln

Aus der Textanalyse ergeben sich zwei Dilemmasituationen: eine Dilemmasituation ohne Kontrolle der Lehrperson (a) und eine Dilemmasituation mit Kontrolle der Lehrperson (b), die ich anhand der unterschiedlichen Faktoren (vgl. Faktorenstruktur in Abb. 3) zusammenfasse:

Der Kontext der ersten Dilemmasituation (a) besteht in der Unterrichtssituation, in der Schultests geschrieben werden. Die Ursache der Dilemmasituation besteht in der gegenseitigen Loyalität und Freundschaft - nicht alle Mitschülerinnen und -schüler helfen Valentina. Als intervenierende Maßnahme kann eine Wissenslücke von Valentina betrachtet werden. Zudem ist mit dieser Maßnahme die Voraussetzung der Sitznähe von Valentinas Kollegin und die geringe Kontrolle der Lehrperson verbunden. Treffen diese Aspekte in der Situation zu, orientiert sich Valentina an der bilateralen kollegialen Ehrlichkeitsregel, welche sich in der Verhaltensweise der Hilfestellung der Kollegin ausdrückt: Zuflüstern von angefragten Lösungen.

Der Kontext der zweiten Dilemmasituation besteht in der ausübenden Hilfestellung der Kollegin während eines Schultests, was der resultierenden Verhaltensweise entspricht, die weiter oben genannt ist. Der Faktor der Ursache wechselt von der Loyalität zur Empathie gegenüber der Lehrperson und zur Einsicht in die konventionelle Ehrlichkeitsregel des selbstständigen Arbeitens während Schultests. Als intervenierende Maßnahme selbst ist der Appell des kontrollierenden Lehrers zu bezeichnen, welcher die beiden Schülerinnen bei ihrer Tätigkeit ertappt. Die resultierende Verhaltensweise zeigt sich im nahtlosen und kommentarlosen Entscheid von beiden, sich an der konventionellen Ehrlichkeitsregel zu orientieren. Diese Verhaltensweise ist von einem Schamgefühl geleitet.

Die Handlungsmacht des Lehrers dringt nur unterschwellig durch, weil er ohne direkte Ansprache und ohne Sanktionsdrohung interveniert. Valentina und ihre Kollegin prüfen die Grenzen der konventionellen Ehrlichkeitsregeln in einer spielerischen Weise, wobei beide sich grundsätzlich an diesen orientieren.

\subsubsection{Verwechslung des Abgabetermins eines Hausaufgabenauftrages: Es hät natürli niemert äis Wort gsäit}

Rund um Hausaufgabenkontrollen bieten sich Freiräume für unterschiedliche Verhaltensweisen, mit denen Ehrlichkeitsregeln verbunden sind. Im Themenkreis des Klassenzusammenhalts berichtet Lilian ausführlich und kon- 
trastierend verschiedene Episoden aus der Sekundarstufe B, die sie bis vor einem Jahr besucht hat, und stellt diese Episoden aus der jetzigen Klasse (Sekundarstufe A) gegenüber. Im Unterschied zur früheren Klasse beurteilt Lilian den Klassenzusammenhalt in der gegenwärtigen Klasse als groß. Diese Beurteilung ist auf den Kontext der Hausaufgabenkontrollen beschränkt, denn im Allgemeinen grenzt sich Lilian von der Klasse ab (vgl. Kap. 9.1.1). Den großen Klassenzusammenhalt im Rahmen von Hausaufgabenkontrollen untermauert Lilian anhand folgender Erzählung:

Zum Biischpiil hämmer emaal müesse- (1) also i de Englischschtund isch es gsii- (1) Händ (2) ali äigentli d Uufzgi gmacht, (2) ussert ganz wenig. Und dene hät d Leererin gsäit: ,,(1) Das isch- (2)“ - Die Siite 23 hämmer ali gmacht - und, ,die Siite 23 isch uf $<$ di $>$ näscht Wuche Uufzgi.“ Es hät natürli niemert äis Wort gsäit, vo (1): „Das hämmer scho uf hüt müesse mache“"(4).

Lilian erzählt eine Episode zur Hausaufgabekontrolle in der Sekundarstufe A, in der die Englischlehrerin fälschlicherweise einen Arbeitsauftrag für die nächste Stunde gibt, den sie bereits für die gegenwärtige Stunde gegeben hat. Die SuS klären die Verwechslung nicht auf und verschweigen diese bewusst, um die wenigen Mitschülerinnen und -schüler, die diese Aufgaben nicht ausgeführt haben, zu decken. Darin dokumentiert sich eine kollegiale Ehrlichkeitsregel: Das Vergessen von Hausaufgaben ist legitim, passiv zu verdecken, um Mitschülerinnen und -schüler vor möglichen Sanktionen zu schützen. In der Textanalyse fallen Dichotomien in Lilians Formulierungen auf: ali-ganz wenige und niemert- äis. Darin dokumentiert sich ein Ungleichgewicht, das nicht zur Auseinandersetzung führt, was hingegen in der Dilemmasituation mit Roger geschieht (vgl. dazu Kap. 9.4.1). Vielmehr sieht Lilian in diesem Ungleichgewicht und dem Zusammenhalt der Klasse ein Indiz für den starken Klassenzusammenhalt. Diesen wertet sie im Vergleich zu dem aus der Sekundarstufe B sehr positiv. Es könnte auch sein, dass Lilian die Hausaufgaben nicht gelöst hat und daher vom Schutz des Klassenverbandes profitiert. Allerdings ist dem Interviewkontext zu entnehmen, dass sie selbstständig Schultests löst, ohne anderen zu helfen, und dass sie deshalb als Streberin bezeichnet wird. Daher kann davon ausgegangen werden, dass Lilian auch bei den Hausaufgaben nicht auf die Unterstützung der Mitschülerinnen und -schüler zählt, sondern diese selber löst. Ausgehend von dieser Lesart möchte weder Lilian noch die Mehrheit der Klasse, welche die Hausaufgaben gelöst hat, für ihre gemachte Arbeit anerkannt und belohnt werden. Aus Lilians Sicht wird das Faktum, dass die ganze Klasse die Verwechslung des Hausaufgabenauftrags durch die Lehrerin verschweigt, als selbstverständlich aufgefasst, was mit dem natürli unterstrichen wird. Darin dokumentiert sich ebenso die Kollektivität dieser kollegialen Regel, die vorausgesetzt wird.

Neben der kollegialen Ehrlichkeitsregel ist in dieser Unterrichtssituation auch die konventionelle Ehrlichkeitsregel der ungelösten Hausaufgaben mitzudenken. 
Zusammenwirkende Ehrlichkeitsregeln (Kap. 9.3.2, Lilian):

kollegiale Ehrlichkeitsregel: Das Vergessen von Hausaufgaben ist legitim, passiv zu verdecken, um Mitschülerinnen und -schüler vor möglichen Sanktionen zu schützen

und

kollegiale Ehrlichkeitsregel: Sachverhalte (verursacht durch die Lehrperson) müssen nicht richtiggestellt werden, wenn kollegialer Vorteil daraus gezogen werden kann

versus

konventionelle Ehrlichkeitsregel: Ungelöste Hausaufgaben werden deklariert

und

konventionelle Ehrlichkeitsregel: Sachverhalte aktiv richtigstellen

Hinter der analysierten Episode steht eine Dilemmasituation, die aus Sicht von Lilian und der Klasse einen positiven Ausgang nimmt: Kontext ist die Hausaufgabekontrolle in der Englischstunde. Die Ursache des Dilemmas besteht im Klassenzusammenhalt, der so dominierend ist, dass ein möglicher individueller Gerechtigkeitssinn oder ein individuelles Bedürfnis nach Anerkennung für die erledigten Schulaufgaben wirkungslos sind. Intervenierende Maßnahme ist die Verwechslung des Lehrers bei der Erteilung einer Hausaufgabe, die bereits in der letzten Schulstunde ausgesprochen wurde und bis heute zu erledigen gewesen wäre. Die Dilemmasituation enthält zwei konventionelle Ehrlichkeitsregeln: Ungelöste Hausaufgaben werden deklariert und Sachverhalte aktiv richtigstellen und zwei kollegiale Ehrlichkeitsregeln: Das Vergessen von Hausaufgaben ist legitim, passiv zu verdecken, um Mitschülerinnen und-schüler vor möglichen Sanktionen zu schützen und Diskussionslos resultiert die Verhaltensweise, welche sich an der kollegialen Ehrlichkeitsregel orientiert, auf Kosten der Anerkennung der Arbeitsleistung der Mehrheit der Klasse. Ungelöst bleibt, ob die Ursache tatsächlich in der kollegialen Hilfsbereitschaft liegt, die Mitschülerinnen und -schüler zu decken, welche die Hausaufgaben nicht gelöst haben, oder ob mehr der Unterhaltungswert entscheidend ist, dass die Lehrperson etwas verwechselt, was ausgenützt wird.

\subsection{Dilemma zwischen individuellen, kollegialen und konventionellen Ehrlichkeitsregeln}

SuS berichten seltener von Schulsituationen, in denen explizit oder implizit gleichzeitig Entscheidungen zwischen individuellen, kollegialen und konventionellen Ehrlichkeitsregeln zu fällen sind. Dennoch kommt das vor. Lara und Valentina schildern eine solche Dilemmasituation, wobei bei Lara alle drei Ehrlichkeitsregeln explizit in der Textpassage angeschnitten werden. Hingegen resultiert im Fallbeispiel von Valentina die individuelle Ehrlichkeitsregel erst in der Textanalyse anhand von implizitem Erfahrungswissen. 


\subsubsection{Indirektes Verpetzen von Mitschülerinnen und -schülern durch die Richtigstellung eines Hausaufaufgabenauftrags: ...zu sim Rächt choo}

Im Themenkreis des Verpetzens, welches Lara grundsätzlich als eine negative Verhaltensweise beurteilt und was ihrer Ansicht nach in ihrer Klasse üblicherweise nicht vorkommt, situiert sich folgende Interviewpassage, in der Lara von einem Vorfall erzählt, den sie miterlebt hat, aber in dem Roger der Hauptakteur war:

Oder zum Biischpill au wänn s so- wänn mer müend es Heft abgee, das hät s äinmaal gee. Mir händ müese es Heft abggee und dänn hät s mindeschtens d Hälfti vo de Klass, hät s nöd fertig ghaa und äine dee hät s aber fertig ghaa und dee hät gfunde- Also d Leereri hät s dänn vergässe, iizzie und dänn hät er gfunde, er gaat das ire jetzt goge säge, dass si das sött iizie, dänn er hegi bis am Elfii deet draa gschaft und jetzt wett er s au abgee. (1) Jetzt will er au irgendwie zu sim Rächt choo, dass s er gmacht hät. (2) Das händ s dänn au echlii blööd gfunde. Also ich cha s au verschtaa, das würd mir au echli närve, wänn ich so lang draa gschaffet hät und dänn (1) ali wo s nöd händ, äifach so devoo chömet. (1) Aber das isch dänn au nöd so (1). Si hät s sowieso eerscht spööter iizoge oder so. Das isch au nöd so tragisch gsii. (4) (I: Also er isch s äifach goge säge gange und d Leereri hät s trotzdem nöd gmacht). (1) Näi d Leeri isch isch dänn irgendwie wäg gsii oder er isch s trotzdem nöd goge säge so. (1) Oder isch s goge säge oder er hät siis scho chöne abgee, aber (4) die andere händ wie nomaal e Schanse überchoo oder so (7).

Vor dem Hintergrund, dass die Lehrerin ein Heft mit Hausaufgaben in einer Stunde nicht wie üblich eingezogen hat, kommt es zu konfligierenden Stellungnahmen in der Klasse, von denen Lara berichtet. Die Mehrheit der Klasse hat die Arbeit im Heft nicht fertiggestellt, sodass sie nicht bereit ist, dieses abzugeben, obwohl dies zum Arbeitsauftrag zählt. Ein Schüler, Roger, der am Vorabend bis $23 \mathrm{Uhr}$ daran gearbeitet hatte und somit fleißig war, findet das ungerecht und will zu seinem Recht kommen.

Lara berichtet, dass die ganze Klasse das Verhalten des einzelnen Schülers negativ beurteilt, und zwar als blööd. Der Klassenmeinung schließt sich Lara an, und gleichzeitig zeigt sie empathische Impulse für Roger. Diese differenzierte Stellungnahme zeigt sich nicht nur in der inhaltlichen Aussage, sie dokumentiert sich auch im Perspektivenwechsel der Erzählposition: $\mathrm{Zu}$ Beginn der Erzählung spricht Lara mit einem kollektiven Subjekt und erwähnt Roger distanziert mit dem neutralen Relativpronomen dee. Erst als Lara ihr Verständnis für Rogers Verhalten erwähnt, nimmt sie einen Perspektivenwechsel vor und argumentiert in der Ich-Form. Sie zeigt Verständnis für seine Verhaltensweise, weil er viel ins Erledigen der Hausaufgabe investiert habe. Daher kann sie sein Bedürfnis nachvollziehen, für seinen Einsatz eine Anerkennung zu erhalten.

Lara teilt mit Roger den negativen Eindruck, dass seine nächtliche Investition sich nicht gelohnt hätte. Das signalisiert Laras Wortwahl zu sim Rächt choo: Roger ist nicht zu seinem Recht gekommen, womit die ungerechte 
Behandlung angedeutet wird. Als Gegenfolie dazu ist Laras Aussage zu lesen ali wo s nöd händ, äifach so devoo chömet. Darin dokumentiert sich ein Gerechtigkeitsgefühl, das sie mit Roger teilt und das verletzt ist. Anders ausgedrückt: Er befürchtet, benachteiligt zu sein, weil diejenigen SuS (Mehrheit der Klasse), die den Auftrag nicht erfüllt haben, vorteilhafter gehandelt haben. Denn die Lehrerin hat ihre Unterlassung der Hausaufgaben übersehen. Gleichzeitig deklariert Lara ihr Unverständnis bezüglich Rogers Verhaltensweise und relativiert seine Sichtweise, indem sie darauf hinweist, dass zu einem späteren Zeitpunkt sowieso alle Hefte eingezogen worden wären. Lara erzählt chronologisch und fließend von der Episode. Jedoch mag sie sich nicht mehr genau erinnern, wie die Episode ausgegangen ist. Das lässt sich anhand widersprüchlicher Verben dokumentieren (nöd goge säge, goge säge). Am Ende dieser Interviewpassage hält Lara fest, dass Roger sein Heft abgab und die andern Mitschülerinnen und -schüler einen zweiten Abgabetermin erhielten. Damit ist für Lara die Spannung zwischen den Verhaltensweisen von Roger und den restlichen SuS gelöst. Ob dies auch für Roger zutrifft, bleibt ungeklärt.

An dieser Schulepisode zeigt sich sehr klar eine Dilemmasituation, in der vier Ehrlichkeitsregeln in Berührung kommen:

Zusammenwirkende Ehrlichkeitsregeln (Kap. 9.4.1, Lara):

individuelle Ehrlichkeitsregel: Wenn ich etwas geleistet habe, dann deklariere ich das

versus

kollegiale Ehrlichkeitsregel: Sachverhalt nicht klären, um Mitschülerinnen und -schüler zu schützen, auf Kosten der eigenen erbrachten Schulleistungen

versus

konventionelle Ehrlichkeitsregel: Ungelöste Hausaufgaben werden deklariert

und

konventionelle Ehrlichkeitsregel Wenn Hausaufgaben nicht kontrolliert werden, wird das richtiggestellt

Als Kontext der Dilemmasituation steht die Hausaufgabenkontrolle. Die Ursache ist je nach Perspektive auf die Ehrlichkeitsregeln unterschiedlich. Innerhalb der konventionellen Ehrlichkeitsregel zählt die Loyalität zur Lehrperson. Hingegen gehört zur kollegialen Perspektive die Loyalität zum Klassenverband. Schließlich ist die Ursache für die individuelle Perspektive der Gerechtigkeitssinn. Zur intervenierenden Maßnahme zählt die Unterlassung der Lehrerin, das Hausaufgabenheft einzuziehen. Aus der Perspektive von Roger resultieren der Wunsch nach Gerechtigkeit und die Orientierung an der individuellen Ehrlichkeitsregel, sodass er seine Sichtweise den anderen Mitschülerinnen und -schülern verkündet, wodurch Lara über den Vorfall berichten kann. 


\subsubsection{Kollegiale Ehrlichkeitsregel einer kollegialen Regel: ... dänn gömmer zäme i $d$ Schuel und tüend soo, als wär s so lang gange}

Im Themenkreis des Klassenzusammenhalts erzählt Valentina von einer Dilemmasituation, in der konventionelle, kollegiale und individuelle Ehrlichkeitsregeln bedeutsam sind:

Mir sind alli zum Arzt gange. Alläige, also zum Schuelarzt. Und dänn (1) simmer ebehämmer öis uuftäilt. D Mäitli sind i d Stadt, nach em Ding bis zu de Zänipause. Und Buebe sind halt echli am Hänge gsii@1@. Mir händ nöd wele id Schuel gaa bis am Zäni und dänn (2) hämmer gsäit, ja dänn träffet mer öis am Zäni wider da i de Schuel und dänn gömmer zäme i d Schuel und tüend soo, als wär s so lang gange@1@. Und dänn simmer ebe deete gsii und sind echli z spaat cho und die sind äifach ali scho ineggange. Da hät s de Leerer natürlich komisch gfunde und es isch echli schlächt gsii@1@, aber ja (3) (I: Und dänn, was isch nachher passiert?) Ja, dänn hät er äifach gsäit: „Warum sind iir spööter cho?" und so und dänn hämmier gsäit: (1) „Jaa, irgendwie hämmer uf die und die gwartet und si isch no schnäll zum Schulepsycholog und dädädä“ und @ja@. Und dänn hät er s glaubt, aber es isch trotzdem echli unagneem gsii (5).

Valentinas Klasse geht außerhalb des Schulhauses und ohne Begleitung der Lehrperson zum Schularzt. (Diese Informationen gibt Valentina zu Beginn der Erzählung nicht vollständig, aber sie generieren sich aus der Erzählung.) Offenbar ist die Klasse früher fertig mit dem Arztbesuch als geplant. Statt direkt in die Schule zurückzugehen, entscheidet sich die Klasse - so Valentina im kollektiven Subjekt für diese sprechend -, dass sie bis $10 \mathrm{Uhr}$ etwas anderes unternimmt. Der Plan war, sich um $10 \mathrm{Uhr}$ beim Schulhaus zu treffen und vorzutäuschen, dass der Arztbesuch so lange gedauert hätte: ,tüend soo, als wär s so lang gange @1@."“Die kollegiale Regel der Täuschung beendet Valentina mit einem Lachen, womit die Unkonventionalität der Regel und der Spaß daran signalisiert wird. Die Klasse teilt sich auf: Die Bubengruppe hängt herum, und die Mädchengruppe geht in die Stadt. Die Mädchengruppe verspätet sich, sodass die Bubengruppe bereits ins Unterrichtszimmer hineingegangen ist, als die Mädchengruppe den Treffpunkt erreicht.

An dieser Verhaltensweise ist interessant, dass die Bubengruppe im Zeitalter des Handys keine Rücksprache mit der Mädchengruppe nimmt. Allerdings formuliert Valentina nicht solche Gedanken, sondern verwendet in ihrer Erzählung das Wort einfach, womit auf die Selbstverständlichkeit der kollektiven Verhaltensweise der Bubengruppe hingewiesen wird. Persönlich nimmt Valentina keine Stellung zu deren Verhaltensweise, sondern erzählt in einer Selbstverständlichkeit (natürli), dass der Lehrer die Ungleichzeitigkeit der Rückkehr der geschlechtsspezifischen Gruppen komisch und damit ungewöhnlich fand. Im Zusammenhang mit dieser wahrgenommenen negativen Reaktion der Lehrperson bezeichnet Valentina ihre Lage mit dem moralischen Wort echli schlächt (,echli` kann im diesem Kontext als Füllwort be- 
trachtet werden). Damit beurteilt Valentina nicht das vorzeitige Hineinkommen der Buben, sondern ihre Dilemmasituation. Valentina lacht nach dem Wort schlächt auf, was als Indiz für Unwohlgefühl gelesen werden kann. Das Fragment aber ja kann als Indiz dafür gedeutet werden, dass die Mädchengruppe nicht hinterfragt, wie es zu dieser ungünstigen Situation kam, sondern das angeht, was ansteht: die Beantwortung der Frage des Lehrers nach dem Grund ihrer Verspätung. Aus Valentinas Erzählung geht nicht hervor, wer und wie viele der Mädchen auf die Frage des Lehrers antworten, auf jeden Fall bringen sie zwei für den Lehrer - aus Valentinas Sicht - überzeugende Argumente: Aufeinander warten und Jemand ging kurz zum Schulpsychologen. Valentina lacht nach der Aufzählung der zwei Argumente zum dritten Mal auf, was wieder als Unwohlsein und als Peinlichkeit interpretiert werden kann. Diese Auslegung des negativen Schamgefühls wird dadurch untermauert, dass Valentina in der anfänglichen Erzählung mit einer Ausnahme nicht lacht (Die Ausnahme erfolgt, als sie erklärt, dass die Buben herumhingen.) Die Gemeinsamkeiten der drei genannten Stellen, in denen Valentina lacht, beziehen sich vielmehr auf das Unwohlsein und die Ungewohntheit bezüglich der vorangegangenen Aussagen. Die zwei überzeugenden Argumente überprüft der Lehrer nicht, denn er glaubt den Schülerinnen. Mit anderen Worten: Valentina nimmt wahr, dass den Lehrer ihre unwahren Ausreden tatsächlich überzeugen.

Valentinas Beispiel enthält zwei kollegiale Ehrlichkeitsregeln und eine konventionelle Ehrlichkeitsregel des Nichttäuschens bzw. des Nichtlügens. In der ersten kollegialen Ehrlichkeitsregel geht es darum, dass eine unehrliche Verhaltensweise gegenüber der Lehrperson legitimiert wird. Die kollegiale Ehrlichkeitsregel lautet in diesem Fall: Wenn der Klassenverband sich darüber einig ist, die freigewordene Schulzeit - wegen fehlender Aufsicht - als persönlichen Freiraum zu nutzen, soll die Lehrperson getäuscht werden. Für die zweite kollegiale Ehrlichkeitsregel ergreift die Mädchengruppe die Initiative. Zudem besteht diese in der Ablehnung der konventionellen Ehrlichkeitsregel Nicht täuschen. Auf die Fragen der Lehrperson nach dem Grund der Verspätung nennen die Mädchen Argumente, die im Klassenkontext real auftreten können: Mädchen warten üblicherweise aufeinander, und eine Schülerin der Klasse besucht tatsächlich den Schulpsychologen. Damit täuschen sie zum einen die Lehrperson. Zum anderen decken sie nicht nur die Verhaltensweise der Mädchen, sondern auch die Bubengruppe, was als eine doppelte kollegiale Regel bezeichnet werden kann: Situative und verbale Täuschung des Lehrers für eine vorausgegangene kollegiale Verhaltensweise, die sich an einer kollegialen Ehrlichkeitsregel orientiert, die besagt: Wenn der Klassenverband sich darüber einig ist, die freigewordene Schulzeit - wegen fehlender Aufsicht - als persönlichen Freiraum zu nutzen, soll die Lehrperson getäuscht werden. Anders formuliert: Die Mädchengruppe deckt durch die verbale Täuschung nicht nur sich selbst, sondern auch die Bubengruppe, 
welche die anfangs formulierte kollegiale Ehrlichkeitsregel nicht kollegial verwenden, die auf Täuschung von frei verfügbarer Schulzeit zurückzuführen ist. Die zweite kollegiale Ehrlichkeitsregel basiert auf einer starken Loyalität im Klassenverband als Ganzes.

Ohne Überprüfung der Tatbestände schenkt die Lehrperson den vorgebrachten Argumenten der Schülerinnen Glauben, und die Dilemmasituation hat ein positives Ende für die SuS genommen. Dennoch schließt Valentina die Erzählung mit Worten des Unwohlseins ab: aber es isch trotzdem echli unagneem gsii. Als Gegenfolie der erfolgreichen Wendung des Dilemmas durch die zweite kollegiale Ehrlichkeitsregel verbleibt ein unangenehmes Gefühl in Valentina zurück. Es ist unklar, worauf sich das neutrale Pronomen es bezieht: Es kann an den ganzen Vorfall anknüpfen, das heißt an die erste kollegiale Ehrlichkeitsregel. Eine zweite Lesart ist die Bezugnahme des Pronomens auf die Verhaltensweise der Burschen und somit auf das Nichteinhalten der ersten kollegialen Ehrlichkeitsregel. Eine weitere Lesart bezieht sich auf die unmittelbar erzählte Situation, in der die Mädchen erfolgreich und aktiv Stellung gegenüber der Frage des Lehrers (nach dem Grund der verspäteten Rückkehr der Mädchen) nehmen. In der letzten Lesart dokumentiert sich ein produktives verarbeitendes Moment des Dilemmas.

Den drei genannten Lesarten liegt das Indiz zugrunde, dass Valentina genau die konventionelle Ehrlichkeitsregel des Nichtlügens kennt und ihr entgegengesetztes Verhalten das Unwohlsein produziert. Interessanterweise drückt Valentina keine Wut gegenüber den Buben aus, welche die Dilemmasituation auslösten. Valentinas Unwohlsein kann anhand der Diskrepanz erklärt werden zwischen dem Wissen um die konventionelle Ehrlichkeitsregel des Nichtlügens und deren Bruch, indem sie und ihre Klasse sich für die kollegialen Regeln in scheinbarer Freiheit entschieden haben. Die Lesart der Diskrepanz wird durch Valentinas gezielte Lachmomente an drei Stellen erhärtet, an denen sich die konventionelle und kollegiale Ehrlichkeitsregel berühren.

Zusammenwirkende Ehrlichkeitsregeln (Kap. 9.4.2, Valentina):

zwei kollegiale Ehrlichkeitsregeln: Kollektiv unterrichtsfreie Zeit genießen und Loyalität: ...

dänn gömmer zäme i d Schuel und tüend soo, als wär s so lang gange.

versus

eine konventionelle Ehrlichkeitsregel: Nicht täuschen oder (Not-)Lügen anwenden

und

eine individuelle Ehrlichkeitsregel: (Not-)Lügen anwenden

Zusammenfassend ist die Dilemmasituation, von der Valentina erzählt, mit folgenden Faktoren zu beschreiben: Der Kontext (I) des Dilemmas ist der Arztbesuch der ganzen Klasse ohne Lehrperson. Ursache des Dilemmas ist die Loyalität und das Vertrauen der SuS untereinander. Als intervenierende Maßnahme zeichnen sich die Dauer des Arztbesuches, der schneller als geplant vorbeigeht, und die daraus gewonnene freie Schulzeit ab. Das Dilemma 
besteht zwischen der konventionellen Ehrlichkeitsregel Nicht täuschen und der kollegialen Ehrlichkeitsregel Wenn der Klassenverband sich darüber einig ist, die freigewordene Schulzeit - wegen fehlender Aufsicht - als persönlichen Freiraum zu nutzen, soll die Lehrperson getäuscht werden. Der Entscheid des Klassenverbandes fällt auf die kollegiale Ehrlichkeitsregel. Daraus resultiert die Verhaltensweise der SuS, dass während der verbleibenden Zeit die Mädchengruppe in die Stadt geht und die Bubengruppe ,herumhängt'.

Ein zweiter Kontext schließt sich diesem Verhalten an, weil die Mädchengruppe verspätet zum vereinbarten Treffpunkt beim Schulhaus kommt und die Bubengruppe bereits ins Unterrichtszimmer hineingeht. Die Ursache der Loyalität bleibt bestehen. Intervenierende Maßnahme ist die Fragestellung der Lehrperson nach dem Grund der Verspätung. Die zweite Ehrlichkeitsregel wird beigezogen: Situative und verbale Täuschung des Lehrers für eine vorausgegangene kollegiale Verhaltensweise, die sich an einer kollegialen Ehrlichkeitsregel orientiert, die besagt: Wenn der Klassenverband sich darüber einig ist, die freigewordene Schulzeit - wegen fehlender Aufsicht als persönlichen Freiraum zu nutzen, soll die Lehrperson getäuscht werden. Daraus resultiert die Verhaltensweise der Mädchengruppe, den Lehrer mit zwei Scheinargumenten zu überzeugen. Die Verhaltensweise der Bubengruppe wird von Valentina nicht beschrieben und kann als zurückhaltende Verhaltensweise interpretiert werden. Hingegen resultiert bei Valentina aus der gewählten kollektiven Ehrlichkeitsregel und der damit verbundenen kollektiven Verhaltensweise ein Unwohlgefühl: unagneem.

Angewendet auf die genannten Faktoren lässt sich hier ein dritter Kontext beschreiben, der aus dem erfolgreichen Täuschen des Lehrers besteht. Die Ursache kann als Empathie zur Lehrperson oder als Integrität beschrieben werden. Für eine Präzisierung fehlen dazu weitere Informationen. Als intervenierende Maßnahme ist Valentinas Unwohlgefühl zu betrachten. Aus diesen neuen Faktoren fügt sich den vorhandenen drei Ehrlichkeitsregeln eine implizite individuelle Ehrlichkeitsregel hinzu: Nicht täuschen. Diese hat einen ähnlichen Charakter wie die konventionelle Ehrlichkeitsregel, ist aber nicht von außen, sondern von den Umständen und der Gefühlslage initiiert. Valentina hält sich an die konventionelle Ehrlichkeitsregel. Daraus resultiert die Verhaltensweise des Ignorierens des unangenehmen Gefühls. 


\section{Zusammenfassung der Ergebnisse}

Über die empirischen Befunden, die in den vorangegangen Kapiteln thematisch und getrennt vorgestellt wurden, gibt dieses Kapitel einen zusammenhängenden Überblick.

Einleitend ist zum einen auf die Grenze von Ehrlichkeitskategorien hingewiesen, zu denen in der Erziehungsphilosophie ,Wahrhaftigkeit', Aufrichtigkeit‘ und ,Offenheit' zählen. Es konnte gezeigt werden, dass die erwähnten Ehrlichkeitskategorien nur ungenügend die Vielfalt von Ehrlichkeitspraxen $\mathrm{zu}$ berücksichtigen vermögen (Kap. 6.1). Interessanterweise ist ein Wandel im Berichten über Ehrlichkeitspraxen festzustellen, in dem die philosophischen Kategorien differenziert wurden:

- Ehrlichkeit als selbstsprechendes Selbstverständnis:

Ehrlichkeit ist nicht zu definieren oder in Worte zu fassen, sondern vorerst eine Praxis

- Ehrlichkeit als Wahrhaftigkeit:

Nicht lügen als erlernte und bekannte Ehrlichkeitsregel

- Ehrlichkeit als Aufrichtigkeit gegenüber anderen SuS:

Nicht verraten als klasseninterne und unausgesprochene Ehrlichkeitsregel

- Ehrlichkeit als Aufrichtigkeit gegenüber Lehrpersonen:

Aufrichtig sollte man insbesondere bei wichtigen sowie nachweisbaren Angelegenheiten und auf Anfrage von außen sein

- Ehrlichkeit als Aufrichtigkeit in Schultests:

Nicht spicken

- Ehrlichkeit als Offenheit:

Alles direkt sagen

Die meisten SuS formulieren zunächst explizit und auf Anfrage eine allgemein gehaltene Definition von Ehrlichkeit. Im Verlauf des Berichtens jedoch beziehen sie sich nahezu ausschließlich auf schulische Alltagsbeispiele, womit die SuS die erziehungsphilosophischen Ehrlichkeitskategorien teilweise erwähnen, mehrheitlich ergänzen und präzisieren. Die Präzisierung der Ehrlichkeitskategorien verweist auf das Spannungsfeld zwischen konventionellen und unkonventionellen Ehrlichkeitsregeln.

Darüber hinaus konnte aufgezeigt werden, dass Ehrlichkeit bei den Jugendlichen kein eingespielter Begriff aus der Alltagssprache ist (Kap. 6.2). Den Aussagen ist zudem eine Abgrenzung zur jüngeren Altersstufe, eine Gradualität des Ehrlichkeitsbegriffs und eine Kontextsensibilität zu entnehmen.

Ausgehend von diesen ersten Erkenntnissen sind nicht Ehrlichkeitskategorien, sondern explizite und implizite Ehrlichkeitsregeln aus den Interview- 
aussagen der SuS auf eine differenzierte Art und Weise rekonstruiert worden, um präziser die Deutungen von Ehrlichkeitspraxen zu beschreiben. Gerade weil Ehrlichkeit als Wort nicht in schulischen Dokumenten wie Zeugnis oder persönlichem Kontaktheft artikuliert ist und die meisten SuS sich nicht mehr daran erinnern können, ob Ehrlichkeit bislang überhaupt ein Thema im Unterricht war, ist es erstaunlich, wie genau sie die konventionellen und anscheinend unausgesprochenen Ehrlichkeitsregeln wie Nicht lügen, Nicht schummeln oder Elternunterschrift nicht selber setzen kennen (vgl. Kap. 7). Darin dokumentiert sich ein ausgeprägtes Erfahrungswissen.

Zentrale Befunde konnten aufgrund von fallübergreifenden Ehrlichkeitsregeln gewonnen werden, die aus Ehrlichkeitspraxen rekonstruiert und in zwei Gruppen eingeteilt wurden: konventionelle Ehrlichkeitsregeln (vgl. Kap. 7) und unkonventionelle Ehrlichkeitsregeln (vgl. Kap. 8).

Anhand der gewonnenen fallübergreifenden Ehrlichkeitsregeln konnte aufgezeigt werden, dass diese schulspezifische Rahmenbedingungen widerspiegeln. Beispielsweise zählt zu einer konventionelle Schulregel, dass Hausaufgaben gelöst werden. Wenn diese jedoch vergessen werden, haben sich $\mathrm{SuS}$ zu entscheiden, ob sie ehrlich sind und das Vergessen der Hausaufgaben eingestehen oder ob sie eine alternative Verhaltensweise wählen. Ebenso zählt die Kontrollfunktion und Sanktionsbereitschaft der Lehrperson als schulspezifische Eigenheit: Die SuS loten das Kontroll- und Sanktionsverhalten der Lehrpersonen so genau aus, dass sie recht gut wissen, wo deren Toleranzgrenze liegt.

Konventionelle Ehrlichkeitsregeln implizieren sozial erwartete Regeln, beispielsweise dass eine richtige Antwort auf Anfragen zu geben ist; zudem sind leistungsorientierte Erwartungen zu vermerken, die sich durch gute Noten bei Schultests oder das Fehlen von Einträgen ins Kontaktheft erfüllen. Wenn solche konventionellen Ehrlichkeitsregeln modifiziert werden, ergeben sich unkonventionelle Ehrlichkeitsregeln (vgl. Kap. 8).

Ein weiterer zentraler Befund besteht in der Vielfalt an gewonnenen unkonventionellen Ehrlichkeitsregeln, welche die heterogenen Deutungen von Ehrlichkeitspraxen bestätigen. Zudem konnte ein Zusammenhang festgestellt werden zwischen Unterrichtssituation, individuellen Ehrlichkeitsregeln und sozialen Beziehungen zu Mitschülerinnen und -schülern sowie zu Lehrpersonen.

Unkonventionelle Ehrlichkeitsregeln normalisieren und legitimieren die $\mathrm{SuS}$ in ihren Aussagen. $\mathrm{Zu}$ einem zen $\neg$ tralen Ergebnis zählt die grosse Bedeutung von kollegialen und kollektiven Ehrlichkeitsregeln, denen sich üblicherweise individuelle Ehrlichkeitsregeln unterordnen. Ausnahmen dazu bilden die Stellungnahmen von Lilian (Kap. 9.1.1) oder Roger (Kap. 9.4.1), die von Vorfällen berichteten, in denen eine individuelle eine kollektive Ehrlichkeitsregel dominierte. In den Ergebnissen wurden Ehrlichkeitsregeln berührt, welche außerschulische Kontexte betreffen. Zudem konnte aufge- 
zeigt werden, dass SuS Ehrlichkeitsregeln mit Spaß und Ernst in Verbindung bringen.

In Konsequenz dieser Arbeit sind Auflistungen von Ehrlichkeitsregeln oder Ehrlichkeitskategorien ohne Praxisbezug zu verabschieden: Mit wachsendem Abstraktionsgrad der Regel verblasst deren Kontext- und Situationsbezug, der jedoch für pädagogische Ansätze von großer Relevanz ist. Wenngleich fallübergreifende Ehrlichkeitsmuster, also geteilte Ehrlichkeitsregeln, aufgezeigt werden können, bestehen doch immer wieder situations- und kontextspezifische Ausnahmen, sodass eher Tendenzen als Muster zu erkennen sind.

Immerhin sind zwei Tendenzen von Ehrlichkeitsregeln festzumachen: Ehrlichkeitsregeln reagieren sowohl stark auf die Kontrolle der Lehrperson als auch auf kollegiale und kollektive Regeln.

In Kapitel 9 konnte aufgrund einer mehrdimensionalen Textanalyse fallimmanente Ehrlichkeitsregeln in realen Dilemmasituationen extrahiert werden. Aus den Fallbeispielen kristallisierten sich konventionelle, kollegiale und individuelle Ehrlichkeitsregeln heraus (vgl. Abb. 4), deren Gleichzeitigkeit und drei weitere Eigenschaften auffallen: Erstens entscheiden sich die SuS für bestimmte Ehrlichkeitsregeln, womit der Entscheidungsprozess zentral ist. Zweitens berücksichtigen nicht alle SuS alle potenziell möglichen Ehrlichkeitsregeln in einem Entscheidungsprozess. Drittens präsentieren sich komplexe Wechselwirkungen zwischen den einzelnen Ehrlichkeitsregeln. Neben den Ehrlichkeitsregeln sind zusammenhängende Faktoren wie ,Ursache', ,Kontext' und ,intervenierende Maßnahmen' differenziert und analysiert worden. Auf diese Weise konnten aus realen Dilemmasituationen subjektive Deutungen und Erfahrungswissen der SuS generiert werden.

Als grundsätzliches und deutliches Ergebnis stellt sich heraus: Dilemmasituationen forcieren unter Einbezug unterschiedlicher Faktoren einen differenzierten Entscheidungsprozess zwischen unterschiedlichen Ehrlichkeitsregeln. Daraus resultiert eine Verhaltensweise, die für die spezielle Situation vom Akteur als angemessen erachtet wird und belegt, dass SuS sich an gewissen Ehrlichkeitsregeln situativ und kontextabhängig orientieren.

Ebenso setzten SuS dieselbe Ehrlichkeitsregel in einem Kontext ein und in einem anderen nicht. Diese individuelle Logik belegt das Beispiel von Lilians kollegialer Ehrlichkeitsregel Die eigene Meinung direkt sagen besonders klar. Diese Regel verwendet Lilian nur im Klassenverband, was den von ihr empfundenen Ausschluss aus dem Klassenverband festigt. Hingegen in ihrer Fußballmannschaft richtet sie sich nach der moderierten Ehrlichkeitsregel Angemessene Meinungsäußerung unter Berücksichtigung der Meinung der anderen Peers.

Die Kategorie der kollegialen Ehrlichkeitsregeln differenziert sich darüber hinaus zwischen zwei Polen: der gänzlichen Offenheit gegenüber Dritten und der Offenheit gegenüber einer begrenzten Anzahl an Peers. Aller- 
dings bleibt gerade bei den referierten und angewendeten kollegialen Ehrlichkeitsregeln unklar, ob alle SuS mit diesen aktiv einverstanden sind. Hier schliessen sich offene Fragen zur Gruppendynamik, zum Ausgeschlossensein oder zu Mobbing an (vgl. Kap. 11.3, Abs. c). Geschlechtsspezifische Eigenheiten von Klassen kommen besonders in kollegialen Ehrlichkeitsregeln zum Tragen (vgl. 9.4.2). Jedoch kann aus den analysierten Dilemmasituationen keine evidente Aussage über geschlechtsspezifische Eigenheiten von Ehrlichkeitsregeln abgeleitet werden.

Gleichermassen zeichnet sich neben den konventionellen Ehrlichkeitsregeln - etwa Nicht lügen - eine individuelle Ehrlichkeitsregel Nicht lügen aus eigener Motivation ab, die ebenfalls das Nichtlügen beinhaltet, deren Motivation aber intrinsisch und nicht extrinsisch ist. Damit zusammenhängend können sich individuelle Ehrlichkeitsregeln in Dilemmasituationen selbst entwickeln, etwa aufgrund dessen, dass sich jemand schämt (vgl. Valentinas Aussage in Kap. 9.4.2). Diese Beobachtung ist mit weiterführenden und gezielten Studien zu untermauern, denn sie verweist auf die Annahme, dass individuelle Ehrlichkeitsregeln sich innerhalb von Schulpraxen situativ entwickeln, verstärken und erhärten.

Im Sprechen über einzelne Dilemmasituationen berühren die SuS persönliche Verhaltensweisen, die beispielsweise verletzende Erinnerungen oder Befangenheitsgefühle hervorrufen können. Dies wird etwa mit lachender Rede, mit einem Auflachen oder mit der Auslassung weiterer Aussagen zum Thema signalisiert. Darin dokumentiert sich ein Modus, über Verhaltensweisen zu sprechen und nachzudenken. Gleichzeitig ist es erstaunlich, wie aufgeschlossen und detailliert SuS eigene und fremde Verhaltensweisen in ihren Schulpraxen wahrnehmen, damit umgehen und davon zu berichten wissen.

Die SuS haben sich in den acht realen Dilemmasituationen für Verhaltensweisen entschieden, die sich an Ehrlichkeitsregeln orientieren, wie sie Tabelle 6 zusammenfasst. Diese Aufzählung der gewählten Ehrlichkeitsregeln, aber auch die Darstellung der zusammenhängenden Ehrlichkeitsregeln in den einzelnen realen Dilemmasituationen (vgl. Abb. 4) beschreiben die Vielfältigkeit, Differenziertheit, Situationsabhängigkeit, Kontextabhängigkeit und Widersprüchlichkeit von Ehrlichkeitsregeln und weniger deren Vollständigkeit.

Tabelle 6: Verhaltensweisen und Ehrlichkeitsregeln in Dilemmasituationen

\begin{tabular}{|l|l|}
\hline Verhaltensweise & Ehrlichkeitsregel \\
\hline Sich aufführen, wie man will & $\begin{array}{l}\text { Die eigene Meinung direkt sagen, ohne diese } \\
\text { an die Meinung der anderen Peers anzupassen }\end{array}$ \\
\hline Abbruch der kollegialen Zweierbeziehung & $\begin{array}{l}\text { Kollegiale Offenheit ist beschränkt auf die } \\
\text { eingeweihten und betroffenen Personen }\end{array}$ \\
\hline $\begin{array}{l}\text { Anwendung von Tricks, um Elternunterschrift } \\
\text { bei ungenügenden Noten einzuholen }\end{array}$ & $\begin{array}{l}\text { Partielle Täuschung ist erlaubt, um nicht die } \\
\text { eigenen Misserfolge im Schultest vor den Eltern } \\
\text { zu verantworten }\end{array}$ \\
\hline Schultests von der Mutter kontrollieren lassen & Für die geleisteten Schulleistungen einstehen \\
\hline
\end{tabular}




\begin{tabular}{|l|l|}
\hline & $\begin{array}{l}\text { und die Mutter überzeugen, dass ungenügende } \\
\text { Noten nicht notwendigerweise mit ungenügen- } \\
\text { dem schulischem Engagement zu tun haben } \\
\text { müssen }\end{array}$ \\
\hline $\begin{array}{l}\text { Gelegentliches Anfragen und Zuflüstern mit } \\
\text { derselben Kollegin }\end{array}$ & $\begin{array}{l}\text { Gelegentliches Zuflüstern von angefragten } \\
\text { Lösungen während Schultests ist legitim }\end{array}$ \\
\hline $\begin{array}{l}\text { Verwechslung des Abgabetermins einer } \\
\text { Hausaufgabe durch die Lehrerin wird nicht } \\
\text { gemeldet }\end{array}$ & $\begin{array}{l}\text { Sachverhalte, die durch die Lehrperson verur- } \\
\text { sacht werden, müssen nicht richtiggestellt } \\
\text { werden, wenn kollegialer Vorteil daraus zu } \\
\text { erkennen ist }\end{array}$ \\
\hline $\begin{array}{l}\text { Trotz unterlassener Hausaufgabenkontrolle } \\
\text { durch die Lehrerin eigenes Hausaufgabenheft } \\
\text { abgeben }\end{array}$ & $\begin{array}{l}\text { Eigene erfüllte Hausaufgaben sollen deklariert } \\
\text { werden, auch wenn dies Mitschülerinnen und - } \\
\text { schüler benachteiligt, die diese nicht erledigt } \\
\text { haben }\end{array}$ \\
\hline $\begin{array}{l}\text { Mit einer (Not-)Lüge dem Lehrer die unaufge- } \\
\text { deckte und unerlaubte Verhaltensweise des } \\
\text { Klassenverbands überzeugend verheimlichen }\end{array}$ & $\begin{array}{l}\text { Täuschung und (Not-)Lügen sind legitim, um } \\
\text { kollektive und unkonventionelle Verhaltenswei- } \\
\text { sen und Regeln zu verdecken }\end{array}$ \\
\hline
\end{tabular}

Quelle: eigene Daten

Ein weiteres Ergebnis ist, dass sich SuS in Entscheidungsprozessen an unterschiedlichen Faktoren orientieren, so etwa: die sich bietende Gelegenheit, Gefühlszustände, kollegiale und freundschaftliche Beziehungen, Präsenz der Lehrperson, aktive Kontrolle und Überprüfung von Tatbeständen und Arbeitsaufträgen durch die Lehrperson, Selbstdarstellung, individuelle Überzeugungskraft und individuelle Initiativen.

Abbildung 4: Überblick der Ehrlichkeitsregeln in realen Dilemmasituationen

\section{Dilemmasituationen zw. kollegialen Ehrlichkeitsregeln \\ kollegiale Ehrlichkeitsregel: \\ Angemessene Meinungsäusserung unter Berücksichtigung der Meinung der anderen Peers \\ versus}

individuelle Ehrlichkeitsregel (Punktuelle Ablehnung von kollegialen Ehrlichkeitsregeln in der Klasse):

Meinung direkt sagen bzw. zu ehrlich sein

(vgl. Kap. 9.1.1, Lilian)

\section{kollegiale, bilaterale Ehrlichkeitsregel:}

Nicht hinter dem Rücken über jemanden sprechen (Offenheit gegenüber Kollegen, die in einen

Sachverhalt eingeweiht und davon betroffen sind)

versus

kollegiale, allgemeine Ehrlichkeitsregel:

Es ist ehrlich, wenn man über andere Kollegen spricht, die abwesend sind (Offenheit gegenüber allen Kollegen, ob anwesend oder nicht)

(vgl. Kap. 9.1.2, Christoph) 


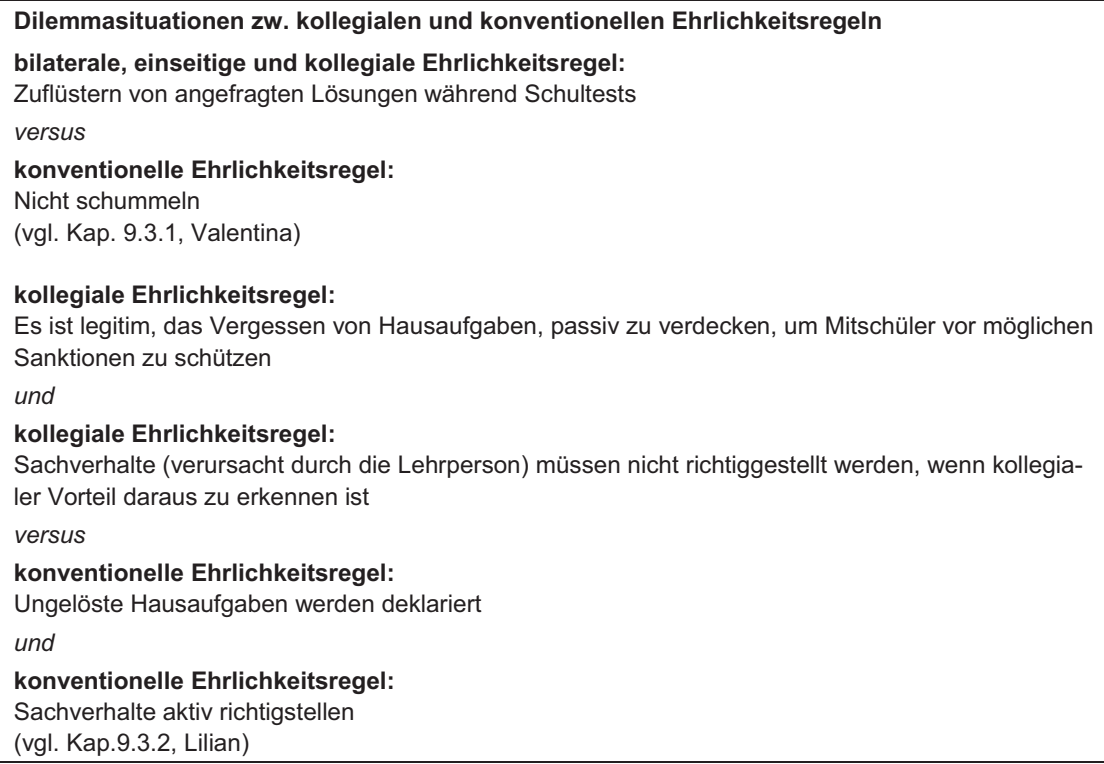

Dilemmasituationen zw. individuellen und konventionellen Ehrlichkeitsregeln

konventionelle Ehrlichkeitsregel:

Elternunterschrift nicht fälschen

versus

konventionelle Ehrlichkeitsregel:

Zu den eigenen Schulleistungen/Noten stehen

daraus

individuelle Ehrlichkeitsregel:

Bei schlechten Prüfungen können die Elternunterschriften mit einer partiellen Täuschung eingeholt werden

(vgl. Kap. 9.2.1, Marco)

konventionelle Ehrlichkeitsregel:

Elternunterschrift nicht fälschen

und

konventionelle Ehrlichkeitsregel:

Schulleistungen/Noten den Eltern offen zeigen

und

individuelle Ehrlichkeitsregel:

Zu den eigenen Schulleistungen/Noten stehen

versus

individuelle Ehrlichkeitsregel: Ungenügende Schulleistungen verweisen nicht notwendigerweise auf ungenügendes schulisches Engagement

(vgl. Kap. 9.2.2, Stefan) 


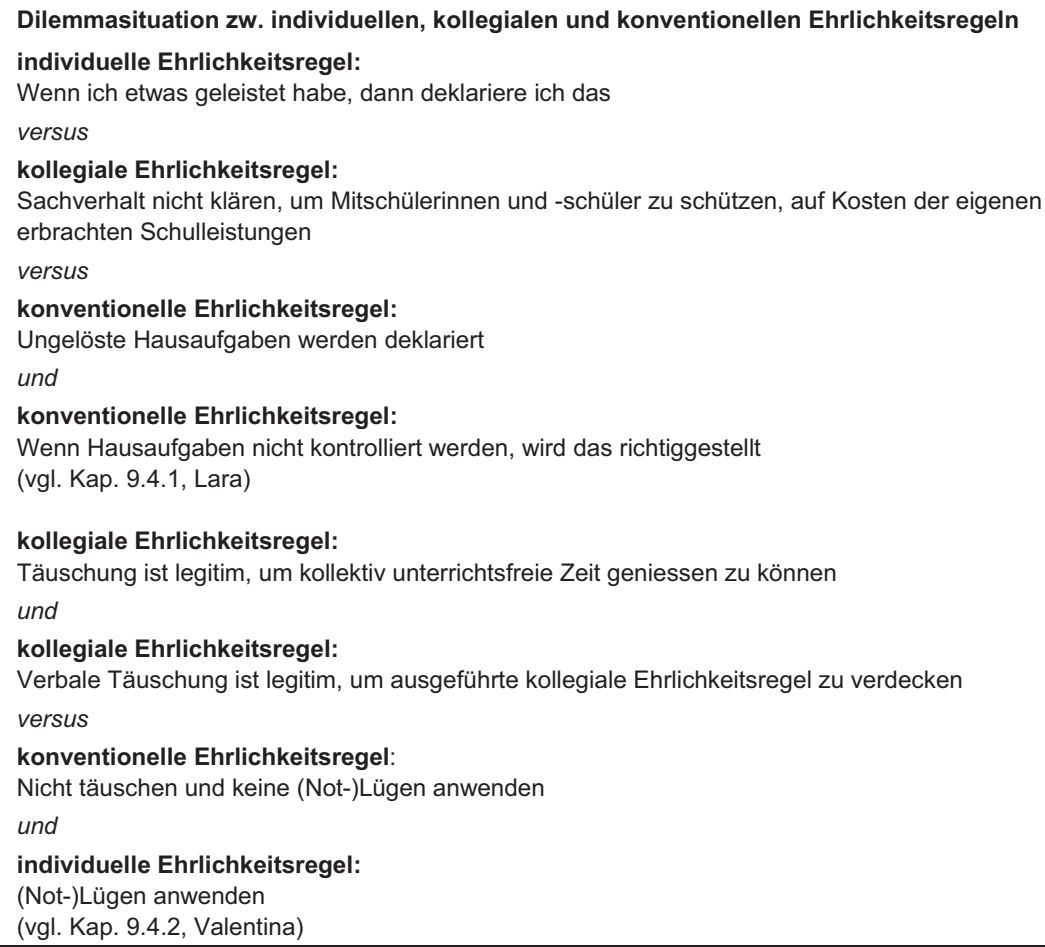

Quelle: eigene Darstellung und Daten

Der Fokus der Ehrlichkeitsregeln ist auf die Beziehungsebene gerichtet, also auf Mitschülerinnen und -schüler, Lehrpersonen, Eltern oder andere Peers. Zudem weisen die Verhaltensweisen, die sich an unterschiedlichen Ehrlichkeitsregeln ausrichten, auf Aktivitäten hin, die sich sowohl auf der Verhaltensebene als auch auf die argumentative Ebene beziehen (vgl. dazu Stephans Aussagen in Kap. 9.2.2).

Einzelne SuS verwenden Taktiken, also wiederholte Verhaltensweisen, um Zweitpersonen zu täuschen. In einzelnen Taktiken ist ein schulischer Oppositionscharakter zu vermerken, beispielsweise bei Marcos räumlichem Freiheitsbedürfnis, das er verdeckt auslebt (Kap. 9.2.4). Die Verhaltensweisen anderer SuS stellen Aktivitäten dar, die sich durch Transparenz auszeichnen (vgl. Fabian, Kap. 9.2.3). Den unterschiedlichen Verhaltensweisen gemeinsam ist, dass sie dazu dienen, alltägliche und schulische Herausforderungen zu bewältigen. 
Als Ursache für Dilemmasituationen, die aus unterschiedlichen Ehrlichkeitsregeln entstehen, sind bedeutsam: Loyalität, Gerechtigkeitssinn, Integrität, Anerkennungsbedürfnis, Empathie gegenüber Lehrpersonen oder Eltern, Schamgefühl, Abhängigkeitsverhältnisse oder Unsicherheitsgefühle. Diese Faktoren geben Indizien dafür, worin schulalltägliche Herausforderung bestehen, denen sich $\mathrm{SuS}$ zu stellen haben, und die Gegenstand für weiterführende Forschung sein können.

Schließlich resultiert aus der Analyse der Dilemmasituation, dass SuS angesichts konventioneller und wirksamer Ehrlichkeitsregeln im Rahmen der Schulordnung gewisse Spielräume ausloten, um mit schulalltäglichen Herausforderungen umzugehen, indem sie sich an unterschiedlichen konventionellen, kollegialen und individuellen Ehrlichkeitsregeln orientieren. 



\section{Diskussion und Ausblick}



Die Einzeltugend Ehrlichkeit wurde in der vorliegenden Arbeit aus dem Blickwinkel des schulischen Alltags und der Deutungen der Schülerinnen und Schüler (SuS) betrachtet. Der sozialwissenschaftliche und schülerzentrierte Zugang zum Thema Tugend leistet einen zentralen schulpädagogischen Beitrag zu gegenwärtigen deutschsprachigen Tugenddebatten, welche sich bislang auf erziehungsphilosophische, entwicklungspsychologische sowie populäre Fundamente stützten, sich vorwiegend auf die Sichtweise der Erziehungsbeauftragten beschränkten und in der Jugendforschung allein die Einstellungen der Heranwachsenden zu Sekundärtugenden betrachteten.

Dieser abschließende Teil der Arbeit diskutiert die empirischen Befunde, fasst die bisherigen thematischen und theoretischen Ergebnisse zusammen und formuliert weiterführende Reflexionen. Dies erfolgt in vier Schritten: Zunächst diskutiere ich die empirischen Befunde (Kap. 11). In einem zweiten Schritt zeichne ich die zentralen Ergebnisse der ganzen Arbeit nach (Kap. 12). Drittens zeige ich auf, was diese Befunde in der Jugend- und Schülerforschung leisten können und an welchen Stellen Anlass für weitere Forschung besteht (Kap. 13). In einem vierten Schritt lote ich die Bedeutung und Perspektive der Ergebnisse im Hinblick auf die schulischen Erziehungsanforderungen und auf empirisch fundierte öffentliche Tugenddebatten aus (Kap. 14). Damit sind einzelne Faktoren genannt, die in einer theoretisch fundierten und praxisorientierten Tugenddebatte im Bereich Schule und Erziehung zu berücksichtigen sind.

\section{Diskussion der empirischen Ergebnisse}

Die vorausgegangenen Kapitel stellten anhand von Ehrlichkeitsregeln dar, wie SuS Ehrlichkeitspraxen im Schulalltag deuten. Eine Vielfalt an Ehrlichkeitsregeln konnte rekonstruiert werden, an denen SuS ihre Verhaltensweisen orientieren. Damit zeichnen sich Ehrlichkeitsregeln als ein geeignetes Begriffsinstrumentarium ab, um Deutungen von Ehrlichkeitspraxen aus Sicht der SuS darzustellen. Hingegen erweisen sich philosophische Ehrlichkeitskategorien wie ,Wahrhaftigkeit', ,Aufrichtigkeit' oder ,Offenheit' als zu undifferenziert, um die Vielfältigkeit und die Nuancen der Deutungen der Ehrlichkeitspraxen zu erfassen.

Um die Befunde systematisch zu beschreiben, sind die gewonnenen Ehrlichkeitsregeln in die Sammelkategorien von konventionellen und unkonventionellen Ehrlichkeitsregeln unterteilt. Zudem beziehen sich wichtige Befunde auf die komplexen Entscheidungsprozesse von Ehrlichkeitspraxen, die anhand realer Dilemmasituationen erkundet wurden. 
Die ausführliche Darstellung der Ehrlichkeitsregeln (vgl. Kap. 6 bis 9) will den Beitrag für die Schulpädagogik leisten, empirische Daten zum noch ungenügend erforschten Thema der Tugenden aus Sicht der SuS (vgl. Kap. 1 bis 3) bereitzustellen.

Vor dem geschilderten Hintergrund wird in diesem Kapitel die zentrale Forschungsfrage beantwortet, wie SuS unterschiedliche Ehrlichkeitspraxen im Schulalltag deuten. Dies erfolgt, indem der Analyseverlauf verfolgt wird: Ehrlichkeitskategorien (Kap. 11.1), Thematisierungsregeln (Kap. 11.2), konventionelle sowie unkonventionelle Regeln im Schulalltag (Kap. 11.3) und in realen Dilemmasituationen (Kap. 11.4). Gleichzeitig erfolgt eine Einordnung der Ergebnisse in den bestehenden Forschungsstand der Schulpädagogik und Jugendforschung.

\subsection{Grenzen der Ehrlichkeitskategorien ,Wahrhaftigkeit', ,Aufrichtigkeit' und ,Offenheit'}

Weitgehend übereinstimmend äußern sich SuS zur Ehrlichkeitskategorie der Wahrhaftigkeit, die sie als schulspezifische Ehrlichkeitsregel mit Nicht lügen benennen. Diese Regel ist ihnen bekannt, und fast alle SuS erwähnen sie als Erstes, wenn sie zu ihrem Ehrlichkeitsverständnis befragt werden. Darüber hinaus zeigt sich die Tendenz, dass die meisten SuS die Ehrlichkeitsregel des Nichtlügens im Verlauf ihrer Aussagen präzisieren und relativieren, indem sie spontan ihren Alltag einbeziehen und weitere Ehrlichkeitskategorien entwickeln. So bezieht Nora das Nichtlügen auf soziale Beziehungen und formuliert eine weitere Ehrlichkeitsregel: Nicht lästern. Sie schreibt beiden Regeln die gleiche Relevanz zu. Darüber hinaus fokussiert Ilona den sozialen Aspekt des Nichtlügens auf den sozialen Kontext und limitiert dessen Geltungsgrad auf die Kollegen und Peers, womit die Lehrpersonen ausgeschlossen sind. Auf Verhaltensregeln in Peer Groups verweisen neuere außerschulische Jugendstudien (vgl. bspw. Wetzstein et al. 2005; Bütow 2006; Wehr 2008).

Parallel zur Ehrlichkeitskategorie der Wahrhaftigkeit beziehen sich viele SuS auf die Ehrlichkeitskategorie der Aufrichtigkeit, indem sie Ehrlichkeitsregeln erwähnen wie Nicht verraten, Nicht spicken oder Wahre Aussagen auf Anfragen bezüglich wichtiger und nachweisbarer Tätigkeiten. An diesen Ehrlichkeitsregeln werden die Präzisierung und der Bezug zum Alltag der Ehrlichkeitskategorie ,Aufrichtigkeit' besonders ersichtlich. So präzisiert die Ehrlichkeitsregel Nicht spicken die Aufrichtigkeit im Setting eines Schultests. Damit findet ein Perspektivenwechsel von einer allgemeinen Bedeutungsebene zu einer kontextabhängigen Bedeutungsebene statt. Zudem wird die Ehrlichkeitskategorie der Aufrichtigkeit ausdifferenziert, indem die SuS diese auf die Funktion der betroffenen Personen beziehen. In diesem Sinne be- 
zeichnen einige SuS ,Aufrichtigkeit“ als Aufrichtigkeit gegenüber anderen Mitschülerinnen und-schülern sowie gegenüber Anfragen von Lehrpersonen.

Auf Mitschülerinnen und -schüler wenden viele SuS die Ehrlichkeitsregel Nicht verraten gegenüber Lehrpersonen an. Damit messen die SuS der Aufrichtigkeit gegenüber SuS größere Wichtigkeit zu als der Aufrichtigkeit gegenüber Lehrpersonen. Das Zusammenspiel der Ehrlichkeitsregel des Nichtverratens mit der Ehrlichkeitsregel Aufrichtigkeit nur auf Anfragen leisten eröffnet den SuS einen Spielraum für unkonventionelle Ehrlichkeitsregeln. Dies unterstreicht Latzko mit Befunden aus einer Schülerbefragung zur Überschreitung von moralischen Regeln. Die 54 befragten SuS aus drei unterschiedlichen Klassenstufen eines deutschen Gymnasiums bestätigten, dass Lügen gegenüber der Lehrperson legitimer sei als gegenüber den Eltern (vgl. Latzko 2006).

Parallel zu den anderen zwei Ehrlichkeitskategorien thematisieren die SuS die Ehrlichkeitskategorie der Offenheit anhand einer Ehrlichkeitsregel, die formuliert werden kann als Alles direkt sagen. Bei zwei Schülerinnen (Lilian und Ilona) zeigt sich die Anwendung dieser Ehrlichkeitsregel besonders deutlich: Die Schülerinnen geben an, in gewissen Situationen hemmungslos alles zu sagen, was sie gerade denken.

Schließlich ist die Ehrlichkeitskategorie Ehrlichkeit ist Ehrlichkeit zu erwähnen, welche aus dem Datenmaterial gewonnen wurde. Mit dieser Ergänzung der drei Ehrlichkeitskategorien ,Wahrhaftigkeit", ,Aufrichtigkeit" und ,Offenheit" ist das selbstsprechende Verständnis von Ehrlichkeit gemeint. Lilian erwähnt diese Kategorie wörtlich und andere SuS beziehen sich darauf, wenn sie anhand von Unterrichts- und Schulbeispielen von ihren Ehrlichkeitspraxen berichten, ohne Ehrlichkeitskategorien zu benennen. Zudem untermauern die meisten SuS diese zusätzliche Ehrlichkeitskategorie damit, dass sich unterschiedliche Schwierigkeiten einstellten, über Ehrlichkeitspraxen zu sprechen (vgl. Kap. 11.2).

Die empirische Analyse der vier Ehrlichkeitskategorien (Wahrhaftigkeit, Aufrichtigkeit, Offenheit, selbstsprechendes Verständnis) verwies auf deren enge Definition von Ehrlichkeit, womit nur begrenzt gedeutete Ehrlichkeitspraxen erfasst werden können. Gleichzeitig lieferte die Analyse erste wichtige Hinweise zu den Eigenschaften von gedeuteten Ehrlichkeitspraxen, die sich in der Vielfältigkeit, den Variationen, der Kontext- und Situationsgebundenheit, der Personenabhängigkeit und der schulseitig teilweise nicht explizit thematisierten Tugend Ehrlichkeit abzeichnen. Diese Eigenschaften, an denen sich Verhaltensweisen orientieren, werden mittels expliziten und impliziten Ehrlichkeitsregeln eingehend und angemessen berücksichtigt. Dieses Vorgehen beleuchtet zudem die gedeuteten Ehrlichkeitspraxen. Ehrlichkeitsregeln zeichnen sich darüber hinaus dadurch aus, dass sie Verhaltensweisen identifizieren, die von sozialen Erwartungen geprägt sind. 


\subsection{Grenzen der Thematisierung von Ehrlichkeit}

,Ehrlichkeit‘ als Wort ist nicht im alltäglichen Sprachgebrauch von SuS zu finden, wenngleich alle SuS eine Definition von Ehrlichkeit artikulierten, die sich in den vorausgegangenen und nachfolgenden Aussagen des Interviewgesprächs nicht stringent durchzieht. Die alltagssprachliche Entfremdung des Wortes ,Ehrlichkeit' zeigt sich in zwei Beobachtungen. Zum einen verwendeten die SuS das Wort nicht explizit, beispielsweise wenn sie über ihre Ehrlichkeitspraxen während Schultests oder im Umgang mit Elternunterschriften berichteten. Zum anderen griffen einige SuS dieses Wort ebenfalls nicht auf, als die Interviewerin gegen Ende des Interviews die Frage nach dem Ehrlichkeitsverständnis stellte. Vielmehr umschrieben sie das Wort mit den konkretisierten Tätigkeiten. Auffallend sind die Abgrenzung ihrer gegenwärtigen Verhaltensweise zu jüngeren Altersstufen und das bewusste Hinweisen auf die Lehrperson- und Kontextabhängigkeit. In den Aussagen der SuS zeichnet sich eine Graduierung von ehrlichen Verhaltensweisen ab, die sich am Beispiel von Lilian besonders deutlich zeigt, wenn diese erklärt, dass sie zu ehrlich sei. Weiter ist die Konjunktion ,Wenn - dann' in den Aussagen der SuS regelmäßig zu erkennen. Damit ist ein Regeldenken dokumentiert, das in der neueren Forschung bisweilen unter den Stichworten ,schulische Logiken" oder als Spannungsfeld zwischen Schulordnung und Gleichaltrigenkultur artikuliert wird und das sich vornehmlich auf das Vorschulalter und die Primarschule bezieht (vgl. bspw. Jünger 2010; Boer und Deckert-Peaceman 2009). Weitere Schüleruntersuchungen mit Fokus auf jugendliche SuS sind an dieser Stelle anzuvisieren, welche das ,Regeldenken “ auf das Jugendalter zugänglich machen können.

Der knappe Einblick dahinein, wie die SuS Ehrlichkeit thematisieren, zeigt auf, dass ,Ehrlichkeit' kein eingespieltes Wort in der Alltagssprache der $\mathrm{SuS}$ ist, woraus sich offene Fragen ergeben:

- Wie kann das, was Erziehungsbeauftragte mit Ehrlichkeit meinen, in die Alltagssprache der SuS übersetzt werden?

- Wie können gezielt didaktische Mittel bei den SuS eingesetzt werden oder verbale Ermahnungen der Lehrpersonen nachhaltig wirken, ohne auf unwirksame Regelkataloge reduziert zu werden, wie das üblicherweise vorgeschlagen wird (vgl. bspw. Weidner 2004; Seydel 2003)?

- Wie soll in theoretischen und öffentlichen Erziehungsdebatten das Thema Ehrlichkeit behandelt werden, wenn man angemessen die Alltagssprache der SuS und deren Sichtweisen berücksichtigen will? 


\subsection{Verhaltensorientierte Ehrlichkeitsregeln im Schulalltag}

Die SuS berichten von einer breiten Palette an expliziten und impliziten Ehrlichkeitsregeln, an der sie ihre Verhaltensweisen in der Schule orientieren. Die Vielfalt der Regeln lässt sich anhand der sozialen Erwünschtheit in zwei Gruppen einteilen: konventionelle und unkonventionelle Ehrlichkeitsregeln. Die Sammelbezeichnung der zwei Gruppen verweist auf die Eigenart des Zuordnungskriteriums. In den konventionellen Ehrlichkeitsregeln sind diejenigen erfasst, welche der sozialen Erwartung entsprechen, die also implizit im schulischen Kontext erwartet und nicht sanktioniert werden. Den unkonventionellen Ehrlichkeitsregeln werden Regeln zugeordnet, welche die SuS alternativ zu den konventionellen Regeln entwickeln und welche zulässige Unehrlicheitsregeln erfassen. Mittels dieser systematischen Zuordnung der Ehrlichkeitsregeln ist ein sich annähernder Zugang zu den Ehrlichkeitspraxen eröffnet, wobei nicht beabsichtigt wird, Ehrlichkeitspraxen vollständig zu beleuchten. Denn auf Überschneidungen der zwei Gruppen und fließende Übergänge zwischen diesen verweisen insbesondere Berichte der SuS, in denen die Lehrperson situativ entscheidet, was unter Konvention fällt, indem etwa Hausaufgaben nicht immer kontrolliert werden.

$\mathrm{Zu}$ den konventionellen Ehrlichkeitsregeln zählen Nicht schummeln, Deklarieren von unerfüllten Hausaufgaben oder Elternunterschriften nicht selber setzen. Ein fließender Übergang zu unkonventionellen Ehrlichkeitsregeln ist festzustellen, welche sich primär auf die bereits erwähnte Entscheidungsachse der Lehrperson zurückführen lassen: ob diese ein Kontrollverhalten oder eine Bereitschaft aufweist, Sanktionen auszusprechen. Besonders im Rahmen von Schultests zeichnet sich dies klar ab. So berichtet Sandra, wie ihre Angst je nach Kontrollschärfe der Lehrperson variiert und Auslöser ist, ob sie sich getraut zu schummeln (vgl. zur Neubalancierung von Abhängigkeit und Autonomie bspw. Böhme 2009, S. 147). Gleichzeitig fordern SuS, dass die Lehrpersonen während Schultests kontrollieren. Eine Nichteinhaltung dieser Erwartung wertet beispielsweise Sandra als Legitimationsgrund, unlautere Hilfsmittel beizuziehen. In der Literatur zur Jugendforschung ist die Abgrenzung von der Kontrollfunktion der Erwachsenen als Entwicklungsschritt beschrieben, wobei eine spätere Wiedereinbindung als Integrationsschritt in die Gesellschaft geschildert wird (vgl. bspw. Terhart 1997b, S. 28ff.; Ferchhoff 2011; Scherr 2009, S. 65ff.).

Konventionelle Ehrlichkeitsregeln differenzieren sich zudem nach den fächerspezifischen Anforderungen und der Stellung des jeweiligen Faches im Fächerkanon. SuS lenken zum einen den Blick auf die vorhandenen Möglichkeiten, welche durch die Anerkennung und die hierarchische Stellung der Lehrperson und ihres Faches - Klassenlehrperson oder Fachlehrperson gegeben ist. Zum anderen bestimmen unterschiedlich geforderte Fachkompe- 
tenzen das Einhalten von konventionellen Ehrlichkeitsregeln. So berichten mehrere SuS, dass es in Französischtests leicht sei, ein aufgeschriebenes Wort unauffällig zu verwenden. Hingegen sei es schwieriger, Lösungsberichte bei mathematischen Aufgabenstellungen mit unerlaubten Hilfsmitteln zu erstellen. Zudem ist den Aussagen der SuS zu entnehmen, dass die Gestaltung des Schultests und das Prüfungsthema einen entscheidenden Einfluss auf Ehrlichkeitspraxen haben.

Weiter zeigen SuS auf, dass der Umgang mit unerledigten Hausaufgaben ebenfalls vom Kontrollverhalten der Lehrperson abhängig ist, was weiter oben mit dem Unterrichtsbereich der Schultests übereinstimmt. Hingegen ist der Umgang mit Elternunterschriften vom familiären Kontext, von den Beziehungen mit den Eltern und von deren Kontrollverhalten abhängig. Denn ungenügende Noten können $\mathrm{zu}$ Konflikten und Freizeitsanktionen führen, was am Beispiel von Stefan oder Clemens deutlich zu sehen ist.

Interessanterweise berichten die meisten SuS ausführlich und aufeinanderfolgend über die konventionellen Ehrlichkeitsregeln und deren Grenzbereiche. Dennoch ist in ihren Aussagen zu beobachten, dass konventionelle Ehrlichkeitsregeln punktuell überschritten werden. Diese Feststellung der grundsätzlichen Einhaltung von konventionellen Ehrlichkeitsregeln wie etwa Nicht schummeln steht den Befunden von Stamm zur Häufigkeit des Schummelns entgegen (vgl. bspw. Stamm 1995).

Selten legen die SuS die konventionellen Ehrlichkeitsregeln als ein in sich geschlossenes Regelwerk dar, üblicherweise führen sie Ausnahmen in Form von individuellen Reflexionen und Schul- sowie Unterrichtsbeispielen heran. In der Bezugsetzung der Gegenfolie zu konventionellen Ehrlichkeitsregeln spiegeln sich alternative Ehrlichkeitsregeln wider, welche als ,unkonventionelle Regeln' in dieser Arbeit zusammengefasst wurden. Das Spannungsfeld zwischen konventionellen und unkonventionellen Ehrlichkeitsregeln zeigt die Aussage von Valentina klar auf. Sie schildert Schummeln als Betrug - und gleichzeitig als etwas Schönes. In der Gleichzeitigkeit und im Wechselspiel zwischen konventionellen und unkonventionellen Ehrlichkeitsregeln spiegelt sich zudem ein Wechselspiel zwischen Vernunft und Gefühlsstimmungen, was für die Adoleszenz besonders bezeichnend ist (vgl. bspw. Terhart 1997b, S. 26ff.; Fend 2005, S. 205ff.). Am Beispiel von Valentina zeigt sich sehr deutlich, wie SuS unkonventionelle Ehrlichkeitsregeln normalisieren und legitimieren. Dadurch wird eine Eigenwelt der SuS eröffnet (vgl. Ziehe 2005).

Anders als die konventionellen zeichnen sich die unkonventionellen Ehrlichkeitsregeln durch eine Vielfältigkeit aus, die stark von den jeweiligen Situationen, Kontexten, Personen und gesetzten Akzentuierungen der SuS abhängig ist. In der Heterogenität der unkonventionellen Ehrlichkeitsregeln kristallisieren sich dennoch gewisse Gemeinsamkeiten heraus, die insbesondere an zwei Tendenzen von Ehrlichkeitsregeln festzumachen sind: Ehrlich- 
keitsregeln reagieren sowohl stark auf die Kontrolle der Lehrperson als auch auf kollegiale und kollektive Regeln. Diese zwei Tendenzen bekräftigen zwei Anhaltspunkte für Lehrpersonen: Ehrliches Verhalten der SuS kann von Lehrpersonen unterstützt werden, indem Aufgabenaufträge und konventionelle Schulregeln wenn möglich kontrolliert und eingehalten werden. Zudem zeichnete sich bezüglich des Nichtschummelns die spannende inhaltliche und formale Gestaltung der Schultests als ein wichtiges Kriterium bei den SuS ab. Als ein weiterer Anhaltspunkt zur Förderung von ehrlichen Verhaltensweisen erweist sich der Einbezug der aktiven Rolle der SuS in Erziehungsabsichten. Schließlich geht aus den Befunden hervor, dass potenzielle unehrliche Vorfälle nicht nur im Klassenverband, sondern ebenso individuell - und in bestimmten Situationen nur individuell - zu klären sind. Denn die Bedeutung der Kollegialität und der Kollektivität im Klassenverband stellte sich als sehr bedeutsam für die meisten SuS heraus, sodass SuS kollegiale Ehrlichkeitsregeln gegenüber den konventionellen Ehrlichkeitsregeln bevorzugen können.

Um zentrale Muster der unkonventionellen Ehrlichkeitsregeln darzustellen, drängt sich eine weitere inhaltliche Unterteilung in insgesamt vier Gruppen auf:

a) individuelle Ehrlichkeitsregeln

b) kollegiale Ehrlichkeitsregeln

c) unkonventionelle Ehrlichkeitsregeln mit einem Bezug zum Unterhaltungsaspekt ,Spaß‘ und zum Ernst

d) unkonventionelle Ehrlichkeitsregeln mit außerschulischem Bezug

Die letzten zwei Gruppen sprengen die vorgenommene Einteilung von individuellen und kollegialen Regeln, indem in jenen Gruppen der individuelle und der kollegiale Aspekt oft gleichgestellt sind. Hier dokumentiert sich die Schwierigkeit, soziale Realitäten begrifflich zu fassen.

a) Weitgehende Übereinstimmung in den Aussagen der SuS finden sich in den individuellen Ehrlichkeitsregeln des Gebrauchs von unlauteren Hilfsmitteln während Schultests. Einige SuS nennen die Bedeutung der guten Noten im Schulzeugnis für die Lehrstellensuche als Legitimationsgrund, um vereinzelt während Schultests zu schummeln.

Die Bedeutung von individuellen und unkonventionellen Ehrlichkeitsregeln ist bei folgenden Regeln hingegen heterogener: Informationen der Lehrperson vorenthalten, Elternunterschriften selber setzen oder Aufrichtigkeit nur auf Anfrage hin geben. Eindeutige Unterschiede präsentieren sich in der Anwendung der Regel Meinung direkt sagen mit oder ohne Respekt gegenüber SuS und Lehrpersonen.

b) Neben den individuellen Ehrlichkeitsregeln treten in einzelnen Unterrichtsbereichen kollegiale Ehrlichkeitsregeln auf. So orientiert sich Fabian an kollegialen Ehrlichkeitsregeln der gegenseitigen unlauteren Hilfestellung 
unter Kollegen während Schultests und setzt zugleich individuell unlautere Hilfsmittel ein.

$\mathrm{Zu}$ den kollegialen und unkonventionellen Ehrlichkeitsregeln lässt sich sowohl Unlautere Hilfsbereitschaft während Schultests zählen als auch das Nichtverpetzen von Mitschülerinnen und -schülern vor allem gegenüber Lehrpersonen. Diese Ansicht wird von den meisten SuS geteilt, wobei einzelne Aussagen auf Konfliktpotenziale mit kollegialen Regeln hinweisen. Die Regel Nicht verpetzen wird in bestimmten Alltagssituationen von der kollegialen und meist unausgesprochenen Verpflichtung gelöst und das üblicherweise verpönte Verpetzen legitimiert. Dies trifft in Alltagssituationen ein, in denen der Gerechtigkeitssinn tangiert ist, schwere Manipulationen voraussehbar sind, die Ernsthaftigkeit einer Situation sich präsentiert, unsympathische SuS betroffen sind oder jemand sich mit einer Aktivität bei der Lehrperson einschmeicheln will.

Insgesamt sind Schwierigkeiten der SuS zu beobachten, wenn sie sich den kollegialen Ehrlichkeitsregeln entgegenstellen. Das deutet aus einer individuellen Perspektive auf die Persönlichkeitsentwicklung und damit auf die Entfaltung des Selbstbewusstseins und der Identität der SuS hin (vgl. bspw. Scherr 2009, S. 65ff.; Terhart 1997b, S. 26ff.). Aus einer kollegialen Perspektive ist gleichzeitig die Bedeutung der Gruppendynamik und Gruppenzugehörigkeit angeschnitten. Bereits die klassische sozialpsychologischse Theoriebildung zur Gruppenzugehörigkeit erklärt die engen Zusammenhänge zwischen den einzelnen Peers gegenüber der Gruppe mit der geringen Durchlässigkeit von In- und Out-Groups (vgl. Haan 2007; Tajfel 1971).

c) Für die Legitimation von unkonventionellen Ehrlichkeitsregeln, welche sowohl kollegial als auch individuell sein können, ist der Unterhaltungscharakter bestimmend, was einzelne SuS mit ,Spaß gegenüber dem ,Ernst ${ }^{\star}$ ausdrücken. Ehrlichkeit ist in diesen Beispielen mit Offenheit und Transparenz gleichzusetzen, die indes in den geschilderten Situationen fehlten. Die Beispiele zeichnen sich meistens durch die Tätigkeit des ,Fertigmachens“ aus. So berichtet Nora, wie die ganze Klasse Spaß hat, stets den gleichen Schüler auszulachen und ,fertigzumachen', der seinerseits mitlacht. Dies ist ein besonderer Fall von kollegialer Ehrlichkeitsregel, weil die SuS das Mitlachen des Schülers als Zustimmung zur kollegialen Regel interpretieren und gleichzeitig die Offenheit seiner eigentlichen Gefühlslage verdeckt wird. In diesem Beispiel bestehen Überschneidungen zum Thema Mobbing, das hinsichtlich sozialer Kompetenzen breit erforscht ist (vgl. Schubarth 2010; Jannan 2010). Allerdings ist bislang das Thema der Ehrlichkeit in diesem Zusammenhang, der den wichtigen Aspekt der verdeckten oder vortäuschenden Verhaltensweisen betont, kaum aufgegriffen worden.

d) In einer vierten Gruppe sind diejenigen unkonventionellen Ehrlichkeitsregeln erfasst, welche über den Schulkontext hinausweisen. Diese Ehrlichkeits- 
regeln zeigen Bezüge zur Gesellschaft, zu Familie und Peers. So belegt das Beispiel von Regula, dass Schummeln durch die Anwendungsmöglichkeit von allen SuS legal wird. Das Verallgemeinerungsargument, das ebenso unkonventionelle Regeln legitimieren kann, unabhängig von deren Inhalten, verweist auf eine problematische Anwendung, welche sich auf andere Lebensbereiche wie Arbeit oder Familie übertragen lässt. Die so bezeichnete normative Regel ist auf die kantianische Prinzipienethik zurückzuführen (vgl. Kant [1797] 1966), die bis heute in den pädagogischen Ethikdebatten bedeutsam ist (vgl. Kesselring 2009), aber auch in Frage gestellt wird (vgl. Prange 2010; Horster und Oelkers 2005). Auf der Prinzipienethik basieren vornehmlich Debatten zu demokratischen Grundwerten (vgl. Zehetmair 1996; Fees 2000; Scherb 2004; Mokrosch und Regenbogen 2009; Schubarth et al. 2010).

Als zweiter außerschulischer Bezug steht die Familie, welche hinsichtlich soziokultureller Hintergründe die Ehrlichkeitsregeln der SuS mitprägt. So berichtet Marco von seinen Verhaltensweisen zu Hause, in denen er konventionelle Regeln bricht. In Marcos Aussagen ist das ,Gewissen“ enthalten. Diese Gefühlslage empfindet er als negativ. Nur Ilona verwendet dasselbe Wort im Schulkontext, wenn sie sich von unkonventionellen Ehrlichkeitsregeln in Schultests abgrenzt. Aus der Sprachverwendung ist zu vermuten, dass die soziokulturelle Herkunft durchdringt. Diese ist ebenfalls in den Bedeutungen zu erkennen, welche die SuS unterschiedlichen Situationen, Ehrlichkeitspraxen und Verhaltensweisen im Schulalltag zusprechen. So führt das fehlende Verständnis der Eltern für ungenügende Noten eher zu unkonventionellen Ehrlichkeitsregeln, was Berichte von einzelnen SuS belegen. Allerdings beobachtet Lara, dass alle SuS ungern ungenügende Noten zu Hause vorzeigen - und dennoch machen es viele.

Drittens zeigt sich insbesondere am Beispiel von Lilian, dass schulische Ehrlichkeitsregeln nicht mit denjenigen aus der Freizeit in Peer Groups übereinstimmen müssen. Neben dieser Diskrepanz zwischen kollegialen Ehrlichkeitsregeln in der Schule und der Freizeit in Peer Groups erwähnen andere SuS Zusammenhänge zwischen kollegialen Ehrlichkeitsregeln in der Schule und in den Peer Groups, womit belegt ist, dass eine Ähnlichkeit solcher Regeln über den schulischen Rahmen hinaus besteht. Dass kollegiale Regeln prinzipiell unabhängig vom Kontext Gültigkeit behalten, zeigen die bereits erwähnten theoretischen sozialpsychologischen Ansätze der Gruppenzugehörigkeit.

Die unkonventionellen Ehrlichkeitsregeln zeichnen sich noch stärker als die konventionellen Ehrlichkeitsregeln dadurch aus, dass sie nicht verschriftlicht und selten ausgesprochen werden. Einzelne Probleme der SuS, sich über Ehrlichkeitspraxen zu äußern, beruhen auf diesem Faktum, womit m. E. ein Tabuthema angeschnitten ist.

Die SuS kommen aus der gleichen Schulform und aus der gleichen Leistungsstufe, allerdings aus sozial unterschiedlich belasteten Schulgemeinden, 
sowohl aus Landgemeinden als auch aus der Stadt. Vor diesem Hintergrund zeichnen sich vorerst weitgehend homogene konventionelle Ehrlichkeitsregeln ab, die aufgrund der Vielfalt von unkonventionellen Ehrlichkeitsregeln innerhalb der einzelnen Klassen und über die Klassen hinaus bislang keine Anhaltspunkte zu einer Definition von Ehrlichkeit liefern. Aus diesem Grund ist vom Wunsch einer einheitlichen Definition Abstand zu nehmen.

Hingegen verweist die äußere Angepasstheit der SuS, die sich in der Orientierung an konventionellen Ehrlichkeitsregeln ausdrückt, auf eine Mittelschichtorientierung, wie sie Hitzler und Niederbacher bezogen auf Jugendaktivitäten feststellen (vgl. Hitzler und Niederbacher 2010). Allerdings ist damit nur tendenziell das Phänomen der Angepasstheit der SuS erklärt. Denn werden die Aktivitäten der SuS genauer betrachtet (was Ziel dieser Arbeit ist), welche sich an einer Vielzahl von unkonventionellen Ehrlichkeitsregeln orientieren, zeichnet sich ein anderes Bild ab: Unkonventionelle Ehrlichkeitsregeln werden im Verborgenen oder verdeckt angewandt, ohne dass Eltern oder Lehrpersonen etwas davon bemerken. Nach außen scheint ein Verhalten nach konventionellen Ehrlichkeitsregeln vorzuliegen, im Verborgenen spielt sich etwas anderes ab. Das Beispiel der Kollektivstrafe, die Otto auf sich genommen hat, obwohl er während der Kochstunde nicht die Eier auf die vorbeifahrenden Autos geworfen hat, verweist genau auf diese verborgenen Tätigkeiten. ${ }^{26}$ Die Lehrperson ist der Meinung, dass Otto den Vorfall zu verantworten hat, obwohl dem nicht so ist. Anhand der Vorder- und Hinterbühne erklärt Goffman das Phänomen der vorgetäuschten Verhaltensweisen (vgl. Goffman [1959] 1983, S. 99ff.).

Hier knüpft die ambivalente Eigenschaft der Sekundärtugend an, indem eine Ehrlichkeitsregel in einem gewissen Kontext erwünscht und dieselbe Ehrlichkeitsregel in einem anderen Kontext verwerflich ist. So ist etwa das Verheimlichen von Informationen über Hausaufgaben gegenüber der Lehrperson hinsichtlich der konventionellen Ehrlichkeitsregeln untersagt, hingegen sind jene bezüglich privater Angelegenheiten gegenüber der Lehrperson angebracht. Die wechselnde Relevanz von Ehrlichkeit und die damit verknüpfte schulalltägliche Herausforderung an die SuS ist ein zentraler Befund, der in der Tugendforschung sowie in der Schulforschung zu wenig berücksichtigt wird.

Der Grund, weshalb Lehrpersonen konventionelle Ehrlichkeitsregeln einzufordern haben, liegt im Bildungs- und Erziehungsauftrag: Unterricht muss funktionieren, und deshalb ist das Verhalten der SuS zu kontrollieren. Damit ist ein ungleiches Machtverhältnis bildungsstrukturell verankert, aus dem zu schliessen ist, dass bildungsstrukturelle Voraussetzungen und Bildungs- sowie Erziehungsanforderungen, welchen die Schule als Institution nachzukommen hat (Fend 2009), das Verhalten von SuS beeinflussen. Diese

26 Ein paralleles Beispiel von Thomas konnte aus der ersten Erhebungsphase gewonnen werden (vgl. Kap. 1). 
bewältigen unter den vorgegebenen Rahmenbedingungen und aufgrund der individuellen und kollegialen Bedürfnisse ihren schulischen Alltag, was in den Aussagen der SuS dokumentiert ist. Zudem sind Ursachen für unkonventionelle Ehrlichkeitsregeln im Entwicklungsprozess der SuS zu finden, welche vorhandene Freiräume zu erkunden und Verhaltensgrenzen $\mathrm{zu}$ testen haben. Unter dem Ansatz der produktiven Verarbeitung von sozialen Realitäten (vgl. Mansel und Hurrelmann 2003) wird erklärt, wie Heranwachsende alltägliche Herausforderungen aktiv angehen und Lösungen zu deren Bewältigung finden. Ausgehend von diesem Ansatz sind Normalisierungsprozesse oder Legitimierungsprozesse von unkonventionellen Regeln zu erklären.

Mit dem Ansatz der produktiven Verarbeitung lassen sich ebenso intuitive Verhaltensweisen der SuS erläutern. Am Beispiel von Marco ist dies ersichtlich. Als er trotz der kollegialen Regel des Vorenthaltens von Informationen unter großen Spannungen seinen Namen dem fordernden Lehrer schließlich sagt, orientiert er sich an einem Ende des Konflikts mit dem Lehrer, das geringe negative Folgen nach sich zieht: Mit der Angabe des Namens verspricht er sich, mögliche Sanktionen zu meiden, was tatsächlich eintritt.

\subsection{Verhaltensorientierte Ehrlichkeitsregeln in realen schulspezifischen Dilemmasituationen}

Das Kapitel zu realen Dilemmas geht von konventionellen, individuellen und kollegialen Ehrlichkeitsregeln und von unkonventionellen Ehrlichkeitsregeln aus. Dabei entsprechen die individuellen und kollegialen Ehrlichkeitsregeln den unkonventionellen Ehrlichkeitsregeln. Anders als die bisherigen fallübergreifenden Ausführungen fokussierte Kapitel 9 auf Ehrlichkeitsregeln im Zusammenhang von acht breit ausgewählten realen und fallimmanenten Dilemmasituationen. Damit sind folgende Themen aus dem Schulalltag tangiert: Hausaufgabenkontrolle, Schultests, Umgang mit Elternunterschriften und ungenügenden Noten, Stellenwert des Klassenverbandes sowie der darin bestehenden kollegialen Beziehungen und Verhaltensregeln gegenüber der Schulordnung. In einer akuten Dilemmasituation ist ein Entscheidungsprozess gefordert, aus dem notwendigerweise Verhaltensweisen resultieren. Folgende verhaltensleitende Ehrlichkeitsregeln, die sich in konventionelle (k), individuelle (i) und kollegiale (kl) Ehrlichkeitsregeln einordnen lassen, wurden aus den acht Dilemmasituationen gewonnen:

- Die eigene Meinung direkt sagen, ohne diese an die Meinung der anderen Peers anzupassen. (i) 
- Die kollegiale Offenheit grenzt sich auf die eingeweihten und betroffenen Personen ein. (i/kl)

- Partielle Täuschung ist erlaubt, um nicht die eigenen Misserfolge in Schultests vor den Eltern zu verantworten. (i)

- Für die erbrachten Schulleistungen einstehen und die Mutter überzeugen, dass ungenügende Noten nicht notwendigerweise mit ungenügendem schulischem Engagement zu tun haben. (i)

- Gelegentliches Zuflüstern von angefragten Lösungen während Schultests ist legitim. (kl)

- Sachverhalte, die durch die Lehrperson irrtümlicherweise ausgehen, müssen nicht richtiggestellt werden, wenn ein kollegialer Vorteil daraus zu erkennen ist. (kl)

- Eigene erfüllte Hausaufgaben sollen deklariert werden, auch wenn dadurch Mitschülerinnen und -schüler benachteiligt werden, die diese nicht erledigt haben. (i/k)

- Täuschung und (Not-)Lügen sind legitim, um kollektive und unkonventionelle Verhaltensweisen zu verdecken. (kl)

Die Auflistung der verhaltensorientierten Ehrlichkeitsregeln verweist auf die Vielfältigkeit, Differenziertheit, Situationsabhängigkeit, Kontextabhängigkeit und Widersprüchlichkeit von subjektiven Ehrlichkeitsregeln. Deshalb geht es nicht darum, eine vollständige Aufzählung der wichtigsten Ehrlichkeitsregeln zu präsentieren. Zudem soll die Zuordnung zu den Gruppen ,konventionelle‘, ,individuelle" und ,kollegiale Ehrlichkeitsregeln' auf die Tendenz hinweisen, dass konventionelle Ehrlichkeitsregeln in Dilemmasituationen stark unterrepräsentiert sind. Weiterhin handelt es sich hier nicht um eine scharfe Systematisierung der Regeln, zumal dies in gewissen Fällen schon deshalb nicht zu bewerkstelligen ist, weil einzelne Ehrlichkeitsregeln zu zwei der drei Überkategorien passen.

Diese ersten Befunde von verhaltensorientierten Ehrlichkeitsregeln sind durch die Komplexität der Dilemmasituation bedingt, welche neben Situationskontext, -ursachen und intervenierenden Maßnahmen zusätzlich in bestimmten Fällen auf unterschiedlichen Ebenen auftritt. Die Entscheidungsprozesse für einzelne Verhaltensregeln haben ihre Ursache in:

- Loyalität zu Schulkolleginnen und -kollegen

- Gerechtigkeitssinn

- Integrität

- Bedürfnis nach Anerkennung der ausgeführten Arbeit

- Empathie gegenüber Lehrpersonen und Eltern

- Schamgefühl

- Abhängigkeitsverhältnissen oder Unsicherheitsgefühl

Damit sind einzelne Tugenden wie Gerechtigkeit, Einfühlungsvermögen, Authentizität oder Fleiß in den Ursachen für Entscheidungsprozesse erfasst, 
die im Zusammenhang mit Ehrlichkeitspraxen stehen. In der Literatur weist Haydon darauf hin, dass einzelne Tugenden untereinander verbunden sind (vgl. Haydon 1999). Die Ursache des Abhängigkeitsverhältnisses verweist auf das Machtverhältnis von Lehrpersonen gegenüber SuS. Am Fallbeispiel von Fabian zeigt sich dieser Einfluss klar. Er überlegt sich die Möglichkeit der einmaligen Orientierung an einer unkonventionellen Regel, um unerledigte Hausaufgaben zu verbergen und damit einen Eintrag ins Kontaktheft $\mathrm{zu}$ vermeiden. Doch plötzlich deklariert er die unerledigten Hausaufgaben dem Lehrer mit der Begründung, dass er damit vielleicht keine Sanktion erhält. Damit leitet die Angst vor Sanktionen Fabians Verhaltensweise. Ein implizites Abhängigkeitsverhältnis zeichnet sich auch im Fallbeispiel von Marco ab, der durch partielle Täuschung der Eltern nicht zu seinen ungenügenden Noten steht. Demgegenüber zeigt Stefan ein größeres Selbstbewusstsein, indem er die ungenügenden Noten der Mutter offen zeigt und diese gleichzeitig überzeugt, dass die ungenügenden Noten nicht notwendigerweise mit fehlendem Lerneinsatz zu tun haben, sondern ebenso mit Prüfungssituationen.

Die Diskrepanzen im Umgang der SuS mit Abhängigkeitsverhältnissen, die besonders dann zum Ausdruck kommen, wenn Leistungserwartungen nicht erfüllt werden, verweisen auf die Entwicklungsphase der SuS. In der Literatur ist diese durch Stufenmodelle dargestellt (vgl. bspw. Kohlberg et al. 1995), womit grobe Entwicklungstendenzen erfasst werden. Doch vermögen solche Modelle nicht, situationsspezifische und komplexe Verhaltensweisen nachzuvollziehen, wie sie die vorliegenden Ergebnisse beleuchten. Gerade die situative Abhängigkeit von Verhaltensweise veranlasst, dass einzelne SuS gleichzeitig in mehreren Entwicklungsphasen stehen können. So zählt im oben erwähnten Beispiel Fabians Verhaltensweise, die sich nach Minimierung der Sanktionen orientiert, zur ersten Entwicklungsstufe Kohlbergs, die sich nach Strafe und Autorität richtet. Gleichzeitig orientiert Fabian seine Ehrlichkeitsregeln in anderen Situationen an gruppenspezifischen Übereinstimmungen, die der dritten Entwicklungsstufe entsprechen.

Besonders die Gleichzeitigkeit von unterschiedlichen Ehrlichkeitsregeln in einer Dilemmasituation verweist auf die Herausforderungen, denen die SuS während eines situativen Entscheidungsprozesses ausgesetzt sind. Die daraus resultierenden Verhaltensweisen folgen eigenen Logiken der SuS, was sich in den vornehmlich individuellen Ehrlichkeitsregeln abzeichnet. In der Gleichzeitigkeit der Ehrlichkeitsregeln erklärt sich zudem die Tatsache, weshalb sich eine Dilemmasituation darstellt, in der nicht von Beginn an klar ist, wie sich die SuS entscheiden werden.

Hauptsächlich die kollegialen Ehrlichkeitsregeln sind in den Dilemmasituationen stärker differenziert, sodass im Fallbeispiel von Christoph zwischen gänzlicher Offenheit gegenüber Dritten und bedingter Offenheit zwischen einzelnen SuS unterschieden wird. Innerhalb der kollegialen Ehrlichkeitsregeln zeigt sich zudem die Schwierigkeit, zu erkennen, ob alle betroffenen 
Kollegen freiwillig und aktiv den beschlossenen Regeln folgen oder ob ein passives Mitgehen festzustellen ist. Diese Differenzierung der kollegialen Ehrlichkeitsregeln ist noch wenig ausgearbeitet und bedarf weiterer Analysen.

Parallel zur Ausdifferenzierung der kollegialen Ehrlichkeitsregeln sind in den Dilemmasituationen eine Ausdifferenzierung der individuellen Ehrlichkeitsregeln und eine Weiterentwicklung dieser Regeln zu erkennen. So zeigt sich im Fallbeispiel von Lara ausgehend davon, dass sie sich schämt, die konventionelle Ehrlichkeitsregel Nicht lügen, welche zur individuellen Ehrlichkeitsregel mutiert und deshalb intrinsisch durch das negative Gefühl motiviert ist. Trotz der entwickelten individuellen Ehrlichkeitsregeln ist in jener Situation die kollegiale Regel ausschlaggebend, die anderen SuS durch legitimiertes Täuschen zu decken.

In den komplexen Entscheidungsprozessen von Dilemmasituationen zeichnen sich besonders in zwei Fallbeispielen von Marco Taktiken ab. Darin sind Taktiken täuschende Verhaltensweisen, die in einer einzelnen konkreten Situation eingesetzt werden (Heinz 1980). In Marcos Fall handelt es sich um punktuell, z. T. wiederholt und absichtlich eingesetzte täuschende Verhaltensweisen, mit denen er ein persönliches Ziel erreicht: Ungenügende Noten nicht zeigen und dem Freiheitsbedürfnis entgegen Schulregel nachgehen. Darin drückt sich eine Art aus, mit alltäglichen Anforderungen produktiv umzugehen, die in der Literatur dokumentiert ist (vgl. Heinze 1980).

Anders geht Stefan mit den hohen Leistungserwartungen um, welche seine Mutter an ihn stellt. Er erhöht das Lernengagement und argumentiert überzeugend gegenüber der skeptischen Mutter. Diese und andere situative Modi, welche sich an individuellen Ehrlichkeitsregeln orientieren, verweisen über die Thematik der Tugend Ehrlichkeit hinaus auf Verarbeitungsweisen der SuS gegenüber schulspezifischen Herausforderungen. Diese unterschiedlichen Verhaltensweisen sind von folgenden Faktoren geprägt, die aus den Dilemmasituationen gewonnen wurden:

- $\quad$ sich bietende Gelegenheiten

- Gefühlszustände

- kollegiale oder freundschaftliche Beziehungen

- Präsenz der Lehrperson

- $\quad$ aktive Kontrolle durch Lehrperson

- Überprüfung von Tatbeständen

- Vergabe und Überprüfung von Arbeitsaufträgen durch die Lehrperson

- Selbstdarstellung, Selbstwertgefühl oder Eigeninitiative

Ausgehend von diesen Faktoren zeichnet sich ab, dass Erklärungsansätze zur produktiven Verarbeitung des schulischen Alltags Probleme nach sich ziehen, die im engen Zusammenhang mit den Verarbeitungsweisen und der realen zu verarbeitenden Situation stehen. Anders ausgedrückt: Die Verarbei- 
tungsweise passt sich der alltäglichen Situation an und ist deshalb vielfältig, was wiederum schwer in Erklärungsansätze zu fassen ist. Vorliegende Befunde geben vorerst Hinweise darauf, worin die Herausforderungen des schulischen Alltags im Hinblick auf dedeuteten Ehrlichkeitspraxen bestehen.

Neben den benannten Ursachen weist das Zusammenwirken von unterschiedlichen subjektiv gedeuteten Ehrlichkeitspraxen auf komplexe, eigenwillige und widersprüchliche Strukturen von Ehrlichkeitspraxen hin. Umso grösser ist der Erklärungsbedarf, Ehrlichkeitspraxen im Speziellen und Schülerpraxen im Allgemeinen zu erfassen, um die Chancen und Grenzen von Erziehungsansätzen zu benennen.

Abschließend zeichnet sich der zentrale Zusammenhang zwischen unkonventionellen Ehrlichkeitsregeln und der Beziehungsebene insbesondere in der Analyse von Dilemmasituationen ab. Einzelne Hinweise darauf sind bereits in den fallübergreifenden Ehrlichkeitsregeln zu finden. Allerdings ist in den Entscheidungsprozessen der SuS klar zu erkennen, dass die unkonventionellen Ehrlichkeitsregeln immer in Bezug zu Personen (SuS, Peers, Lehrpersonen, Eltern) stehen. In diesem sozialen Gefüge bietet es sich für Erziehungsbeauftragte an, mittels Aushandlungsprozessen Regeln im Unterricht aufzustellen. Gerecht (2010) nennt die gute Beziehung zwischen Lernenden und Lehrperson als wichtigsten Faktor, um ,nichtkognitive Erziehungswirksamkeit‘ zu erzielen. Diesen Befund unterstreichen die vorliegenden Ergebnisse, jedoch nicht mit derselben Ausschließlichkeit: Trotz guter Beziehung zur Lehrperson können aus Gründen der Kollegialität Verhaltensweisen eintreten, die den Erziehungsbemühungen der Lehrperson widersprechen können. So zeichnet sich insbesondere in den Dilemmasituationen eine Begrenzung von schulischen Erziehungsansätzen ab. Denn bislang fehlen theoretische Erziehungsansätze, die u. a. den komplexen Ehrlichkeitspraxen und Entscheidungsprozessen der SuS gerecht werden.

\section{Zentrale Ergebnisse der Arbeit}

Die vorliegende Arbeit zeigte auf, dass der Tugend Ehrlichkeit als erwünschte Verhaltensweise in der Schulpraxis eine grosse Bedeutung zukommt, während der Tugendbegriff in den theoretischen Ansätzen der Schulpädagogik seit den 1960er Jahren weitgehend verschwunden ist. Zudem fehlen in der Schülerforschung empirische Daten, um aufzuzeigen, wie SuS, die als produktiv verarbeitende Subjekte verstanden werden, Tugenden erfahren und deuten. Punktuelle Befunde aus kleinen standardisierten Untersuchungen beziehen sich allein auf die Einstellung der SuS zum Schummeln (vgl. Stamm 1995) und Lügen: Beide Verhaltensweisen sind im Ergebnis gegen- 
über Lehrpersonen legitimer als gegenüber Eltern (vgl. Latzko 2006). Hingegen sind Einstellungen zu Sekundärtugenden in der standardisierten Jugendforschung eingehend untersucht und zeigen zwar einige interessante Befunde, nach denen Jugendliche Sekundärtugenden hoch einstufen. Allerdings setzen standardisierte Daten theoretische Vorannahmen fest und begrenzen sich auf die Untersuchung von Einstellungen. Damit wird weniger der Alltag der Heranwachsenden und ihre alltäglichen Verhaltensweisen untersucht, sondern vielmehr die sozialen Erwartungen, die Angepasstheit an gesellschaftlich gesetzte Tugenden sowie die Geltung der theoretischen Vorannahmen gemessen.

Vor diesem Hintergrund entstand das Interesse für das Forschungsvorhaben: In der Schulpädagogik fehlen qualitative Daten zu Tugenden aus dem Schulalltag und aus der Perspektive der SuS. Die vorliegende Arbeit hat die schulrelevante Einzeltugend Ehrlichkeit fokussiert und mit einer rekonstruktiven Forschungsmethode untersucht, wie SuS selbst Ehrlichkeitspraxen deuten und mit diesen umgehen. Zum einen leistet die Arbeit damit einen wichtigen Beitrag zur Schließung der empirischen Forschungslücke. Zum anderen untersucht das Forschungsprojekt die Tugend Ehrlichkeit als Erfahrungswert aus der Sichtweise der SuS und ermöglicht einen Einblick in gedeuteten Ehrlichkeitspraxen. Zudem sind Ehrlichkeitspraxen aufgrund der ambivalenten Eigenschaft von Ehrlichkeit schwer zu beobachten; zugleich sind Lehrpersonen aufgrund des Erziehungsauftrags der Volkschule angehalten, diese Praxen zu kontrollieren und zu sanktionieren.

Die Grundlagen der vorliegenden Arbeit habe ich anhand der interdisziplinären Klärung des Ehrlichkeitsbegriffs und der Kontextualisierung des Forschungsgegenstands (Deutungen der Jugendlichen von ihren Ehrlichkeitspraxen im Schulalltag) erarbeitet. Die Reflexionen und Erkenntnisse zu diesen Grundlagen fasse ich an dieser Stelle zusammen, was das theoretische Vorwissen für die empirische Untersuchung bildet.

Aus einer kritischen Auseinandersetzung mit dem Ehrlichkeitsbegriff in essayistischen, psychologischen, erziehungsphilosophischen, historischpädagogischen und in angelsächsischen Abhandlungen konnte ich folgende Schlüsse ziehen (Kap. 2):

- Der Ehrlichkeitsbegriff ist in essayistischen Publikationen zu finden, die weit verbreitet sind, aber keine oder ungenügende empirische und theoretische Bezüge aufweisen.

- Psychologische Untersuchungen beschäftigen sich mittels Experimenten und quantitativer Befragungen mit einem dualen Ehrlichkeitsbegriff. Unterschiedliche Untersuchungen gehen von einer Kontextabhängigkeit von Ehrlichkeit aus. Der Forschungsbereich der Lügenforschung ist dominierend, welcher zahlreiche Befunde, allerdings zu jeweils einem verbalen Aspekt der Unehrlichkeit liefert. Das Ziel der Lügenforschung besteht in der Identifizierung von Lügen, was trotz einer Kombination verschiede- 
ner methodischer Zugänge ein schwieriges Unterfangen mit nicht eindeutigen Fällen bleibt. Zusammenfassend sind in psychologischen Untersuchungen und insbesondere in der Lügenforschung ehrliche Verhaltensweisen unter einer negativen Folie, einseitig, dual, aber kontextabhängig beleuchtet.

- In der Theorie der Erziehung, die bis Mitte des 20. Jahrhunderts vorwiegend von erziehungsphilosophischen Ansätzen geprägt ist, galt Ehrlichkeit nicht immer als zentrale Tugend. So konnte ich am Beispiel von Platon und Aristoteles (zwei bis heute vielzitierte Klassiker des Tugendthemas) aufzeigen, dass Ehrlichkeit als ,Wahrhaftigkeit" nur bei Aristoteles als Charaktertugend vorkommt; Platon erwähnt sie gar nicht. Die Tugend Ehrlichkeit im Sinne von Verhaltensweisen erlebte im 18. Jahrhundert im Zusammenhang mit bürgerlichen Tugenden als gesellschaftlich anerkannte Tugend ihren Höhepunkt. Ehrlichkeit haben andere Autoren in den 1970er Jahren den Sekundärtugenden zugeordnet, weil Ehrlichkeit ohne Bezug zu einer anderen Tugend keine ethische Bedeutung habe: Mit Bezug auf die aristotelischen Charaktertugenden kann in gewissen Situationen Ehrlichkeit wegen einer Untertreibung oder Übertreibung negativ konnotiert werden. Mit diesem kurzen historischen Abriss ist eine kulturelle, wirtschaftliche und gesellschaftliche Abhängigkeit der Bedeutung von Tugenden angedeutet. Interessanterweise sind in der Geschichte der Theorie der Erziehung durchgehend Tugendkataloge und Tugendlisten bekannt (vgl. Gadebusch Bondio und Bettels 2012). Ich konnte zeigen, dass kaum Abhandlungen zur Einzeltugend Ehrlichkeit vorzufinden sind, welche Ehrlichkeit in einen Zusammenhang mit einer anderen Einzeltugend bringen. Zudem erklären die Autoren von Tugendlisten nicht die Bezüge zwischen Tugend und Einzeltugenden oder zwischen den Einzeltugenden. Weiter bietet der summative Aspekt der Tugend, der in Tugendlisten sichtbar wird, ungenügende Möglichkeiten, die komplexen, dynamischen und vielfältigen Strukturen von Ehrlichkeitspraxen zu erfassen. Zudem berücksichtigt die Erziehungsphilosophie grundsätzlich den Erziehungsalltag nicht und bietet keine umsetzbaren pädagogischen Ansätze.

- Obwohl Tugenden im Allgemeinen in der Erziehungsphilosophie seit der griechischen Antike ein Dauerthema sind, gibt es zur Einzeltugend Ehrlichkeit nur spärliche theoretische Ansätze.

- Wenngleich Tugenden aktuell in der angelsächsischen Erziehungsphilosophie und politischen Bildung ein zentrales Thema sind, befassen sich wenige Autoren mit Einzeltugenden. Zudem mag erstaunen, dass trotz der Etablierung des Tugendbegriffs in bildungspolitischen Debatten sowie der expliziten Nennung einzelner Tugenden im Erziehungsauftrag der öffentlichen Schulen nur wenige Autoren ihre Reflexionen zu Tugenden in einen schulrelevanten Kontext setzen. Dennoch gibt es verein- 
zelte angelsächsische Autoren, welche Einzeltugenden theoretisch analysieren. So schlägt White (1996) einen dreifachen Ehrlichkeitsbegriff vor. Graham (1999) verweist auf Zusammenhänge zwischen Tugenden, wobei er diese Erkenntnis nicht weiter ausarbeitet. Empirische Untersuchungen zu Tugenden im angelsächsischen Bildungsverlauf sind in der Schulpädagogik trotz der regen Tugenddebatten rar. Einzelne Studien zielen darauf, einen Überblick über die Einstellungen zu Tugenden zu leisten.

- Die Skizzierung des Ehrlichkeitsbegriffs in unterschiedlichen Fachbereichen bestätigt, dass dieser teilweise verkürzt verwendet wird. Zudem bestehen ungenügende theoretische Ansätze zum Ehrlichkeitsbegriff und empirische Daten zu Ehrlichkeitspraxen sowie deren Deutungen fehlen. Diese Daten sind jedoch sehr zentral, um den Schulalltag der SuS zu erfassen und zu verstehen.

Ausgehend von den limitierten theoretischen Möglichkeiten, den Ehrlichkeitsbegriff zu definieren, geht diese Arbeit von Whites dreifachem Ehrlichkeitsbegriff (White 1996) aus, den ich in Bezug zum Tugendbegriff als moralische Verhaltensweise (Reichenbach 2004 und 2008) gesetzt und durch erste empirische Befunde erweitert habe. Der so definierte Ehrlichkeitsbegriff hat den losen theoretischen Rahmen der vorliegenden Arbeit gebildet und ermöglicht, die ambivalente Eigenschaft von Ehrlichkeit zu berücksichtigen: Je nach Situation und Kontext werden ehrliche Verhaltensweisen als angebracht oder als unpassend beurteilt.

$\mathrm{Zu}$ den Grundlagen der vorliegenden Studie zählt neben der Bestimmung des Erziehungsbegriffs die Kontextualisierung des Forschungsgegenstands: Deutungen der Jugendlichen von ihren Ehrlichkeitspraxen im Schulalltag (Kap. 3). Der Forschungsgegenstand situiert sich erstens an der Schnittstelle zwischen Schülerforschung und Jugendforschung. Zweitens ist die Schule als Institution zu berücksichtigen, und drittens ist der öffentliche Erziehungsauftrag der Schule einzubeziehen.

$\mathrm{SuS}$ werden in der gegenwärtigen Schülerforschung als aktive Heranwachsende bezeichnet. In der Jugendforschung sind sie als produktiv verarbeitende Subjekte präzisiert, indem die Jugendlichen nicht nur Akteure, sondern fähig sind, soziale Realitäten anzugehen und unterschiedlich zu bewältigen. Insbesondere in der Jugendforschung sind Einstellungen zu Werten und Sekundärtugenden zu finden. Damit werden das Meinungsbild und die Überzeugungen erfasst, die zur Enttabuisierung eines Themas relevant sind (vgl. Nunner-Winkler et al. 2006, S. 215ff.). Jedoch fehlen neben Handlungsimplikationen Aussagen dazu, wie Jugendliche Verhaltensweisen deuten, die sie an Tugenden orientieren. Zudem gibt es bislang keine Befunde, insbesondere in der umstrittenen schulischen Erziehung, wie SuS Ehrlichkeits- oder Unehrlichkeitspraxen deuten. Diese Befunde wären jedoch entscheidend für Lehrpersonen, um die SuS besser zu verstehen. Die Jugendforschung liefert 
darüber hinaus wichtige Anhaltspunkte, welche den entwicklungspsychologischen und gesellschaftlich sich wandelnden Kontext sowie das individuelle Spannungsfeld zwischen Selbst- und Fremdbestimmung aufzeigen, mit denen sich Jugendliche auseinanderzusetzen haben.

Die Schule ist eine öffentliche und gesellschaftliche Institution, in der Lehrpersonen und Schulleiterinnen sowie Schulleiter gesellschaftlichen Funktionen der Schule nachzukommen haben, was den Schulalltag prägt. Zusammenfassend halte ich vier zentrale Schulfunktionen fest:

- Die kulturelle Reproduktion gibt an, was innerhalb einer Gesellschaft als moralisch zu verantworten ist und als ,normal' gilt, beispielsweise, dass eine Landessprache zu erwerben ist.

- Die Qualifikationsfunktion bezweckt eine Integration der SuS in Wirtschaft und Politik.

- Die Allokationsfunktion bezieht sich auf die Zuordnung von Schulabschluss und Arbeitsplatz.

- Die Integrations- und Legitimationsfunktion schließlich knüpft an die Reproduktionsfunktion an und geht expliziter auf die Einführung in die Gesellschaft ein, womit Regeln, Werte, Normen und Weltansichten reproduziert und legitimiert werden.

Schule ist also als Ort der Wissensvermittlung, der Sozialisation und Erziehung zu verstehen, wobei vom ,heimlichen Lehrplan' die Rede ist, wenn der Lehrplan nicht gesellschaftlich geprägte Funktionen der Schule explizit berücksichtigt. Daraus entsteht eine Spannung zwischen dem Ziel, gute Leistungen zu erreichen und gleichzeitig ehrliche Verhaltensweisen zu manifestieren, um ein Beispiel aus den vorgelegten Ergebnissen zu nennen. Unbeantwortet bleibt die Frage, inwiefern ungleiche Behandlung und Erziehung der SuS aufgrund der sozialen Herkunft - wie sie in der bildungsorientierten Ungleichheitsforschung belegt sind (vgl. bspw. Jünger 2010) - für deren Bewältigung von schulspezifischen Situationen prägend sind.

Wie für alle öffentlichen Schulen in der Schweiz besteht für diejenigen im Kanton Zürich ein öffentlicher Erziehungsauftrag, der allgemein formuliert ist und eine Förderung von ehrlichen Verhaltensweisen impliziert. Zudem ist Ehrlichkeit im Schulgesetz, in Schulordnungen, Zeugnissen und anderen schulischen Dokumenten aufgeführt und in der Schulpraxis unter gewissen Umständen auch von den Mitschülerinnen und -schülern erwünscht.

Mit dem Erziehungsauftrag berührte ich ein umstrittenes Thema der Erziehung: Soll Schule erziehen oder ist dies Sache des Elternhauses? Ein Blick auf theoretische Ansätze zur Erziehung, wie ich diesen in der vorliegenden Arbeit punktuell vollzog, verweist auf die Grenzen von theoretischen Erziehungsansätzen. Denn für theoretische Erziehungsansätze sind nicht eindimensionale, sondern komplexe, paradoxe, unbeabsichtigte und nichtkalku- 
lierbare Faktoren zu berücksichtigen, die schwer zu erfassen und zu systematisieren sind. $\mathrm{Zu}$ den aufgeführten Faktoren zählen auch die Deutungen von Ehrlichkeitspraxen, wozu die vorliegende Arbeit einen Beitrag leistet.

Aufgrund der Kontextualisierung des Forschungsgegenstands konnte ich das Ziel der vorliegenden Arbeit ausarbeiten: Deutungen der Jugendlichen von Ehrlichkeitspraxen im Schulkontext. Dabei geht es nicht um moralisches Handeln, sondern um Selbsteinsicht und Deutungen von schulspezifischen Ehrlichkeitspraxen. Zudem zielte das Forschungsvorhaben darauf, keine Einstellungen der SuS zu gewinnen, sondern Deutungen von Praxen und Verhaltensweisen.

Anhand der gewonnenen Erkenntnisse aus den aufgeführten Grundlagen der Arbeit formulierte ich die leitende Forschungsfrage (Kap. 4): Wie deuten 14- bis 15-Jährige unterschiedliche Ehrlichkeitspraxen im Schulalltag? Mit dieser Forschungsfrage war das Ziel verbunden, an einer schulrelevanten Einzeltugend ein moralisches Thema der Tugenderziehung, sozialwissenschaftlich, empirisch und differenziert zu untersuchen. Ein weiteres Ziel war es, Aussagen aus der Perspektive der SuS zu formulieren, und nicht aus der üblichen und bekannten Sichtweise der Erwachsenen. Die Erkundung der Sichtweise der SuS ermöglichte es, einen vertieften Einblick in deren Ehrlichkeitsorientierungen.

Angesichts der fehlenden theoretischen Voraussetzungen und aufgrund der Komplexität, Dynamik und Vielfältigkeit von Ehrlichkeitspraxen und deren Deutungen aus der Sicht von SuS wählte ich einen explorativen Forschungszugang. Aus den subjektiven Deutungen der SuS rekonstruierte ich Ehrlichkeitsregeln, an denen sie ihr Verhalten orientieren.

Basierend auf methodologischen Grundüberlegungen zu kontrolliertem Fremdverstehen und zu Erhebungs- und Auswertungsmethoden zeigte ich auf, dass das problemzentrierte Interview und die dokumentarische Methode adäquate Untersuchungsmethoden für die Erkundung des Forschungsgegenstands sind (vgl. Kap. 5). Zudem dokumentierte ich das Forschungsvorgehen und stellte es transparent dar.

Die vorliegenden Ergebnisse sind Teil eines dreiteiligen Forschungsprozesses zum Thema. Zudem sind die Kriterien zur Auswahl der SuS beschrieben und einige Informationen zu deren Person gegeben. Das Sampling umfasst 16 Interviews mit acht Schülerinnen und acht Schülern (14- bis 15Jährige) aus der dritten Oberstufenklasse Typ A. Ich wählte durchgehend ein Leistungstypus, um mögliche Differenzen in leistungsabhängigen Erziehungsabsichten und die damit zusammenhängenden unterschiedlichen Sanktionsweisen zu minimieren. Um die Klassendynamik adäquat zu erfassen und um eine möglichst grosse Bandbreite an Aussagen sicherzustellen, gewichtete ich das Stadt-Land-Verhältnis gleich ebenso wie das Verhältnis von SuS mit einem sozial benachteiligten und sozial privilegierten Schulhintergrund. Mit diesen Auswahlkriterien berücksichtigte ich zudem die verbreitete 
populäre Meinung, dass sozial wenig belastete und ländliche Schulen geringe Erziehungsherausforderung aufweisen. Schließlich dokumentierte und reflektierte ich das Erhebungs- und Auswertungsverfahren.

Teil II der Arbeit präsentierte die Ergebnisse der Interviewauswertung ausführlich (Kap. 6, 7, 8,9) und fasste sie in Kapitel 10 zusammen.

In Teil III dikutierte ich vorerst die empirischen Befunde und stellte sie in Bezug zum Forschungsstand der Schulpädagogik sowie der Jugendforschung, woraus ich Forschungsdesiderata formulierte (vgl. Kap. 11).

Nach dieser knappen Präsentation der Ergebnisse dieser Arbeit gehe ich direkt zum eingangs angekündigten dritten Schritt über, der die Bedeutung der Ergebnisse für die Schüler- und Jugendforschung aufzeigt und eine Anregung für weitere Forschungsprojekte gibt.

\section{Ausblick: Schüler- und Jugendforschung}

Die Ergebnisse untersuchte ich darauf hin, was sie für die Schüler- und Jugendforschung leisten können. Auf Anregungen für weitere Forschung ist bereits im Diskussionsteil (vgl. Kap. 11) hingewiesen worden, was ich an dieser Stelle zusammenfasse.

Die Ergebnisse verweisen auf vielfältige, kontext- und situationsabhängige, differenzierte Ehrlichkeitsregeln, womit die Komplexität von gedeuteten Ehrlichkeits- und Unehrlichkeitspraxen auf eine differenzierte Weise aufgezeigt wird. Dieses Ergebnis geht über Befunde wie ,mangelnde Bereitschaft zur Normbefolgung" (Nunner-Winkler et al. 2006, S. 215) hinaus. Anders zeigen die Befunde der vorliegenden Arbeit, dass ehrliches Verhalten grundsätzlich von den SuS erwünscht ist, wobei situations- und kontextabhängige Ausnahmen möglich sind und zu unkonventionellen Ehrlichkeitsregeln führen. Damit untermauern die Ergebnisse die Annahme, dass die Verhaltensweisen der untersuchten Jugendlichen weniger eine Verweigerung von Normbefolgung als vielmehr eine produktive Verarbeitung des schulischen Alltages darstellen. Denn die SuS haben sich zwischen den Anforderungen des Schulsystems, der Eltern, des Klassenverbandes und eigenen Präferenzen zurechtzufinden, was sich aus den Befunden als eine nicht zu unterschätzende Aufgabe erweist. Die Feststellung der produktiv verarbeitenden Haltung von $\mathrm{SuS}$ ist in einer weiteren Studie mit einer größeren Anzahl an Jugendlichen zu belegen, wodurch die Ausnahmesituationen und somit die unkonventionellen Ehrlichkeitsregeln umfangreicher analysiert werden könnten. Zudem wäre damit die Gleichzeitigkeit von unterschiedlichen Ehrlichkeitsregeln in einer realen Dilemmasituation gezielter herauszuarbeiten. 
Bereits die impliziten Ursachen für subjektive Entscheidungen von Ehrlichkeitsregeln in Dilemmasituationen geben Indizien für schulalltägliche Herausforderungen, die beispielsweise in der Loyalität zur Lehrperson oder zu den Mitschülerinnen und -schülern bestehen. Diese Indizien könnten in einer weiteren Untersuchung eingehender erforscht werden und so Aspekte von schulalltäglichen und subjektiv wahrgenommenen Herausforderungen differenzierter beschrieben und erklärt werden. Dadurch würden die bisherigen Studien zu Einstellungen gegenüber Tugenden mit einem weiteren Beitrag aus der rekonstruktiven Forschung bereichert.

Insbesondere Unehrlichkeitspraxen zeichnen sich dadurch aus, dass sie von Beobachtern nicht aufgedeckt werden sollen. Aus den Ergebnissen geht hervor, dass sich die SuS in der Schule zwischen einer Vorderbühne und einer Hinterbühne (Goffman [1959] 1983) hin und her bewegen, beispielsweise wenn Informationen vorenthalten werden (Hinterbühne), weil die Lehrperson sie nicht abfragt (Vorderbühne), oder wenn ein Schüler oder eine Schülerin die Lehrperson auf unkontrollierte Hausaufgaben hinweist (Vorderbühne) und die anderen SuS dies verschwiegen hatten, um diejenigen zu decken, welche die Hausaufgaben nicht gelöst hatten (Hinterbühne). Die letztgenannte Gruppe fungiert in jener Situation als verärgertes Publikum. Darin zeichnet sich einerseits eine Bewältigung des schulischen Alltags ab. Zum anderen zeigen solche Verhaltensweisen auf ein Herausfinden von Grenzen hin, was zur Charakteristik der Jugendzeit gehört (vgl. Terhart 1997b) und mit den gefundenen Daten untermauert wird. Zudem wird hier der Begriff ,Vorderbühne und Hinterbühne' dynamisch verstanden (Bennewitz und Meier 2010, S. 103f.), weil dieselben SuS einmal auf der Vorderbühne agieren und ein anderes Mal auf der Hinterbühne (vgl. bspw. Kap. 9.1.1, Lilian).

Die starke Betonung der Loyalität und des Gerechtigkeitsgefühls unterstützt üblicherweise unkonventionelle Ehrlichkeitsregeln und die damit zusammenhängenden kollegialen oder kollektiven Ehrlichkeitsregeln. Zugleich können Brüche zu dieser Beobachtung festgestellt werden, wenn beispielsweise die Loyalität gegenüber der Lehrperson stärker ist als die zu den Mitschülerinnen und -schülern. Hieran können weitere Untersuchungen anknüpfen, die solchen Brüchen mittels Gruppendiskussionen oder teilnehmender Beobachtung nachgehen. Damit wäre auch ein Beitrag zur Forschung zu Peer Groups geleistet, welche sich mit Loyalität und Gerechtigkeitssinn innerhalb einer Gruppe und gegenüber anderen Gruppen befasst und grundsätzlich einen stärkeren Gruppenzusammenhang innerhalb einer Gruppe bestätigt (vgl. Tajfel 1971).

Die Ergebnisse weisen weiter darauf hin, dass der Kontext Schule nur bestimmte Ehrlichkeitsregeln fördert und andere nicht. So zeichnet sich die Tendenz ab, dass schulspezifische Ehrlichkeitsregeln gefördert werden, die kontrolliert werden, wie etwa das Eingestehen von unerledigten Hausaufga- 
ben. Jedoch hängt damit die Förderung von kollegialen Ehrlichkeitsregeln zusammen, indem Mitschülerinnen und -schüler gedeckt werden. Schließlich ist es schwieriger, konventionelle Ehrlichkeitsregeln zu fördern, die im direkten Zusammenhang mit Schulleistungen oder Einträgen ins Kontaktheft stehen, als etwa den Umgang mit Unterschrift der Eltern oder Schummeln zu regeln. Zudem zeigen die Ergebnisse Überschneidungen zwischen Ehrlichkeitsregeln innerhalb der Schule, unter Peers und im Elternhaus. Um mögliche Eigenschaften von schulischen und freizeitorientierten Peer Groups zu differenzieren, wäre eine kontrastierende Untersuchung zu beiden Lebensräumen nötig. Mit einer solchen Untersuchung ließe sich die Konsistenz von schulischen und freizeitorientierten Verhaltensweisen bezüglich Ehrlichkeit oder anderen Tugenden gezielt überprüfen. Und schließlich ist es interessant, Gemeinsamkeiten und Unterschiede zwischen der Bedeutung von Ehrlichkeitsregeln in der Schule, im Elternhaus oder unter Peers zu erkunden, um die Eigenheiten der unterschiedlichen Kontexte und die damit verbundenen Eigenheiten des Verhaltens der Jugendlichen besser zu verstehen (vgl. Latzko 2006; Ziehe 2005; Brennewitz 2010).

Neuere Befunde der Jugendforschung verweisen auf eine Aufwertung der Sekundärtugenden unter den Jugendlichen. Die vorliegenden Ergebnisse bestätigen das optimistische Bild der gegenwärtigen Jugendlichen - und erweitern es. Denn konventionelle Ehrlichkeitsregeln sind von den SuS nicht in jeder Situation erwünscht. Unter bestimmten Umständen orientieren sich $\mathrm{SuS}$ an unkonventionellen Ehrlichkeitsregeln. Mit der Unterscheidung von konventionellen und unkonventionellen Ehrlichkeitsregeln wird ein Zweifaches betont: zum einen die Kapazität der SuS zur Differenzierung, zum anderen die ambivalente Eigenschaft der Sekundärtugend Ehrlichkeit. Ehrliches Verhalten ist nämlich nicht in sich gut oder schlecht, sondern situations- und kontextabhängig, woraus nicht Normen, aber die Angemessenheit der einsetzenden Ehrlichkeitsregel abzuleiten ist.

Die Ergebnisse verweisen auf das Spannungsfeld zwischen der Mündigkeitsförderung von SuS, welche auf die Persönlichkeitsentwicklung und Verarbeitungsfähigkeit schulischer Herausforderungen abzielt, und dem Erziehungsauftrag der Schule, der seine Funktion in der Integration der SuS in die Gesellschaft und deren Normsystem sieht. Dieses Spannungsfeld widerspiegelt sich besonders deutlich in den Ehrlichkeitsregeln innerhalb von Dilemmasituationen, welche die SuS unterschiedlich bewältigen.

In den Ergebnissen verweisen die SuS explizit auf leistungsstufenabhängige Verhaltensweisen bezüglich Ehrlichkeit. Allerdings lassen die gewonnenen Ehrlichkeitsregeln aufgrund ihrer Varietät nicht hinreichend auf typische Regeln von SuS der Sekundarstufe A schliessen. Für eine zukünftige Forschung wäre eine vergleichende Studie zwischen SuS aus unterschiedlichen Leistungsstufen anzuregen, die mögliche leistungsabhängige Eigenschaften 
von Ehrlichkeitsregeln generiert. Schulformen wie Kantonsschulen, Sekundar $\mathrm{B}$ und $\mathrm{C}^{27}$ wären zu berücksichtigen.

In der Forschung zur Bildungsungleichheit ist die Berücksichtigung der Herkunft von SuS zentral, was in der vorliegenden Untersuchung insofern ausgeführt wurde, als SuS aus möglichst unterschiedlich sozial belasteten Schulgemeinden gewählt wurden. Da ehrliche Verhaltensweisen mit dem kulturellen Hintergrund zusammenhängen können, ist anzunehmen, dass andere Ehrlichkeitsregeln in herkunftsvielfältigen Schulgemeinden vorzufinden sind. Zudem stellt sich die Frage, wie die Lehrpersonen mit der erwähnten Vielfalt oder mit möglichen geringeren Schulleistungen umgehen: ob mehr Sanktionen oder eher ein Ignorieren von unehrlichen Verhaltensweisen folgen. Das ist ein spannendes Thema für die empirische Schulforschung, weil eigentlich Integration und Förderung von ,Tugenden' im Erziehungsauftrag enthalten sind, aber gleichzeitig die kulturelle Herkunft zu berücksichtigen ist, was noch zu wenig untersucht wird. Anschließend an die Forschungsfrage der Leistungsabhängigkeit von Ehrlichkeitsregeln wäre demzufolge der Forschungsfrage nachzugehen, inwiefern herkunftsspezifische Aspekte der SuS Einfluss auf ihr ehrliches Verhalten haben. In einer qualitativen Studie könnten vorerst aufgrund eines kontrastierenden Samplings mögliche herkunftsspezifische Aspekte gezielt identifiziert und beschrieben werden. In einer quantitativen Studie bietet sich an, die Verteilung des Zusammenhangs zwischen Herkunft und Ehrlichkeitspraxen zu untersuchen. Damit wäre ein kritischer Beitrag geleistet, von dem ausgehend die Forderung nach Minimalmoral und nach gemeinsamen und überkulturellen Werten (vgl. bspw. Nunner-Winkler 2005, 2009) geprüft werden kann.

\section{Ausblick: Erziehungsanforderungen und theoretisch fundierte sowie praxisorientierte Tugenddebatten}

Die Ergebnisse tragen wesentlich zur Versachlichung der oft emotional geführten populären Tugenddebatten bei, indem sie Erfahrungswissen der SuS anhand von vielfältigen Ehrlichkeitsregeln präsentieren und reflektieren. Die SuS offenbaren ein anwendungsorientiertes Problembewusstsein für die Einzeltugend Ehrlichkeit. Sie zeigen eine grosse Bedeutung, aber differenzierte Deutung der Tugend Ehrlichkeit in der Schule auf. Aufgrund dieser Befunde ist zu prüfen, ob ein anwendungsorientiertes Bewusstsein und differenzierte

27 Beziehungsweise die entsprechenden neuen Leistungsstufen der Sekundarstufe I „E“ und „G“ (vgl. dazu Fussnote 16). 
Deutung bei anderen Tugenden im Schulkontext ebenso zu finden sind oder $\mathrm{ob}$ andere Faktoren relevanter sind. Der Befund, dass unterschiedliche Tugenden mit der Tugend Ehrlichkeit zusammenwirken und untereinander eine Wechselwirkung aufweisen - beispielsweise Zivilcourage und Aufrichtigkeit, gibt Anlass, die Zusammenhänge unterschiedlicher Tugenden weiter zu untersuchen, um mögliche Verhaltensmuster ausfindig zu machen.

Die Befunde bestätigen eine Komplexität von Ehrlichkeitspraxen und der damit zusammenhängenden Ehrlichkeitsregeln, woran nur Definitionen des Ehrlichkeitsbegriffs und die damit zusammenhängenden theoretischen Ansätze anknüpfen können, die diesen Anforderungen gerecht werden. Es bräuchte quasi eine eigene Theorie zu Verhaltensweisen von Jugendlichen.

Zudem steht bis heute eine systematische empirische Überprüfung der Grundannahme der Kontext- und Situationsabhängigkeit von Tugenden nicht nur in Bildungsinstitutionen, sondern auch in beruflichen oder politischen Institutionen aus. Einzelne Untersuchungen verweisen auf die Erwünschtheit von Tugenden (vgl. Brumlik 2011; Vorlauf 2010; Schönborn 2006; Rabich 2007), wobei ein Zugang zu deren Praxen und zu Verhaltensprozessen ungenügend beleuchtet ist.

Es wurde deutlich, dass Tugenden nicht im Allgemeinen, sondern als Einzeltugenden empirisch zu untersuchen sind. Denn insbesondere die komplexen Abläufe von Verhaltensweisen und die zugrundeliegenden Regeln erfordern es, mittels einer komplexen Anlage von Studien untersucht zu werden, wofür in einem ersten Schritt qualitative Forschungsmethoden geeignet sind und woran integrative Forschungsdesigns (vgl. Kelle 2008) anknüpfen können. Die empirische Befundlage der Tugendforschung ist auffallend dünn, obwohl Forschungsdesiderata beispielsweise im Hinblick auf die Berufsausbildung bestehen (vgl. Neuenschwander et al. 2010).

Aus den Ergebnissen geht klar hervor, dass die SuS ihre Ehrlichkeitsregeln nicht an Werte- und Tugendkatalogen orientieren. Vielmehr leiten sie sie aus ihrem Erfahrungswissen ab, das auf vielfältigen Faktoren basiert, welche sie aus ihrer gedeuteten Schulpraxis gewonnen haben. Falls Programme zur Förderung von Ehrlichkeitspraxen in der Schule erwünscht sind, sollten diese in den gängigen Schul- und Unterrichtsalltag implementiert sein. Denn der Befund, dass ehrliche Verhaltensweisen der SuS praxisorientiert sind, lässt auf die geringe Wirksamkeit von kognitiv angesetzten Ethikprogrammen schliessen (vgl. bspw. Kenngott 2010). Parallel zur gegenwärtigen Werteforschung (vgl. Schubarth et al. 2010), die sich weiterentwickelt hat und an Wertebildung und nicht mehr an Wertevermittlung ansetzt, gilt es auch in der Tugendforschung, von einer kognitiven Übertragung von Tugenden zu einer an das Schul- und Unterrichtsgeschehen anknüpfenden und schülerorientierten Tugendförderung überzugehen.

In der internationalen Forschung liegen neben Evaluationen von Programmen zur Förderung von Tugenden (vgl. Larson-Knight 2004) bisher nur 
wenige ertragreiche Befunde vor (vgl. Arthur et al. 2009). Zudem erscheint es fraglich, ob Tugenden, die vom kulturellen Kontext abhängig sind, über unterschiedliche Kulturen hinweg vergleichbar sind. Allerdings könnte eine solche Kontrastierung kulturspezifische Eigenheiten von Einzeltugenden besonders deutlich hervorheben.

Zukünftige Tugendforschung tut gut daran, die pädagogischen Prozesse, schulische Routinen und Praktiken und die Sichtweise der SuS mehr zu gewichten, um die Bedeutung von Tugenden in der Schule ursächlich zu verstehen und zu erklären.

Das Spektrum der empirischen Analysemöglichkeiten gilt es anzureichern, indem unterschiedliche Tugenden aus der Sichtweise der SuS, Lehrpersonen, Schulleiter, Eltern und Peers weiterverfolgt werden. Dann lässt sich die Bedeutung nicht nur von Ehrlichkeit, sondern ebenso von anderen Tugenden bei den Jugendlichen genauer erfassen, besser verstehen und letztlich auch erklären. 


\section{Bibliografie}

\section{Literaturverzeichnis}

Abendschön, Simone (2007): Demokratische Werte und Normen. In: Deth, Jan W. van (Hg.): Kinder und Politik. Politische Einstellungen von jungen Kindern im ersten Grundschuljahr. Wiesbaden: VS Verlag für Sozialwissenschaften, S. 161203.

Ahlborn, Hans-Ulrich (1996): Werteerziehung durch Vorbildlernen. Tugenden in moderner Sicht. Frankfurt a. M.: VAS.

Ahrbeck, Bernd (2004): Kinder brauchen Erziehung. Die vergessene pädagogische Verantwortung. Stuttgart: Kohlhammer.

Albert, Mathias (2010): Jugend 2010. Eine pragmatische Generation behauptet sich. 16. Shell Jugendstudie. Frankfurt a. M.: Fischer.

Ammann, Thomas (2004): Zur Berufszufriedenheit von Lehrerinnen. Erfahrungsbilanzen in der mittleren Berufsphase. Bad Heilbrunn/Obb.: Klinkhardt.

Aristoteles (2006): Nikomachische Ethik. Reinbek bei Hamburg: Rowohlt.

Arthur, James (2003): Education with Character. The Moral Economy of Schooling. London/New York: RoutledgeFalmer.

Arthur, James (2005): The Re-Emergence of Character Education in British Education Policy. In: British Journal of Educational Studies 53, S. 239-254.

Arthur, James; Harding, Robert; Godfrey Ray (Hg.) (2009): Citizens of Character. Main report. Canterbury. Online verfügbar unter http://www.learningforlife.org. uk/research-projects/learn-ing-for-life-research-reports/, zuletzt geprüft am 16.01.2012.

Asbrand, Barbara (2009): Qualitative Schulforschung. In: Sigrid Blömeke, Thorsten Bohl, Ludwig Haag, Gregor Lang-Wojtasik und Werner Sacher (Hg.): Handbuch Schule: Theorie - Organisation - Entwicklung. Bad Heilbrunn: W. Bertelsmann, S. 134-141.

Atkinson, Paul; Silverman, David (1997): Kundera's Immortality: The Interview Society and the Intervention of the Self. In: Qualitative Inquiry 3 (3), S. 304-322.

Baacke, Dieter (2004): Jugend und Jugendkulturen. Darstellung und Deutung. Weinheim: Juventa.

Bennewitz, Hedda (2010): Entwicklungslinien und Situation des qualitativen Forschungsansatzes in der Erziehungswissenschaft. In: Barbara Friebertshäuser, Antje Langer und Annedore Prengel (Hg.): Handbuch Qualitative Forschungsmethoden in der Erziehungswissenschaft. Weinheim: Juventa, S. 43-59.

Bennewitz, Hedda; Meier, Michael (2010): Zum Verhältnis von Jugend und Schule. Ethnographische Studien zu Peerkultur und Unterricht. In: Anna Brake und Helmut Bremer (Hg.): Alltagswelt Schule. Die soziale Herstellung schulischer Wirklichkeiten. Weinheim: Juventa, S. 97-110. 
Benthien, Claudia (2006): Die Kunst der Aufrichtigkeit im 17. Jahrhundert. Tübingen: Max Niemeyer.

Bergmann, Wolfgang (2005): Gute Autorität. Grundsätze einer zeitgemäßen Erziehung. Weinheim: Beltz.

Bernstein, Basil (1980): Studien zur sprachlichen Sozialisation. Düsseldorf: Schwann.

Berthold, Siegwart (2007): Lug und Trug. Zwei Unterrichtsvorschläge zum Thema Lüge. In: Schulmagazin 5 bis 1075 (2), S. 25-28.

Bessoth, Richard (2000): Unterrichtsqualität - oder: Was macht guten Unterricht aus? In: Pädagogische Führung 11 (1), S. 65-71.

Bieri Buschor, Christine; Forrer, Esther (2005): Überfachliche Kompetenzen junger Erwachsener am Übergang zwischen Schule und Beruf. Jugend- und Rekrutenbefragung als Beitrag zum Bildungsmonitoring. Zürich: Diss. Universität Zürich.

Bildungsdirektion des Kantons Zürichs (Hg.) (2010): Lehrplan für die Volksschule des Kantons Zürichs. Beschlüsse vom Bildungsrat zwischen 21.11.2002 und 18.12.2008: Online verfügbar unter http://www.sekzh.ch/upload/schule/lehrplan/, zuletzt geprüft am 16.01.2012.

Bildungsstatistik des Kantons Zürichs (Bista) (2011): Online verfügbar unter http://www.bista.zh.ch/vs/VS_Stufen.aspx, zuletzt aktualisiert am 31.05.2011, zuletzt geprüft am 16.01.2012.

Blumer, Herbert (1954): What's Wrong with Social Theory? In: ASR 19 (1), S. 3-10.

Boer, Heike; Deckert-Peaceman, Heike (2009): Kinder in der Schule. Zwischen Gleichaltrigenkultur und schulischer Ordnung. Wiesbaden: VS Verlag für Sozialwissenschaften.

Böhme, Jeanette (2008): Qualitative Schulforschung auf Konsolidierungskurs: interdisziplinäre Spannungen und Herausforderungen. In: Werner Helsper (Hg.): Handbuch der Schulforschung. Wiesbaden: VS Verlag für Sozialwissenschaften, S. $125-155$.

Böhme, Jeanette (2009): Schul- und Unterrichtsforschung. In: Werner Helsper (Hg.): Schule und Bildung im Wandel. Anthologie historischer und aktueller Perspektiven. Wiesbaden: VS Verlag für Sozialwissenschaften, S. 733-743.

Bohnsack, Ralf (2003): Dokumentarische Methode und sozialwissenschaftliche Hermeneutik. In: Zeitschrift für Erziehungswissenschaft 6 (4), S. 551-570.

Bohnsack, Ralf (2006): Das Gruppendiskussionsverfahren in der Forschungspraxis. Opladen: Barbara Budrich.

Bohnsack, Ralf (2007): Rekonstruktive Sozialforschung. Einführung in qualitative Methoden. Opladen: Barbara Budrich.

Bohnsack, Ralf (2008): Rekonstruktive Sozialforschung. Einführung in qualitative Methoden. Opladen: Barbara Budrich.

Bohnsack, Ralf; Loos, Peter; Schäffer, Burkhard; Städtler, Klaus; Wild, Bodo (1995): Die Suche nach Gemeinsamkeit und die Gewalt der Gruppe. Hooligans, Musikgruppen und andere Jugendcliquen. Opladen: Leske \& Budrich.

Bohnsack, Ralf; Nentwig-Gesemann, Iris; Nohl, Arnd-Michael (Hg.) (2007): Die dokumentarische Methode und ihre Forschungspraxis. Grundlagen qualitativer Sozialforschung. Wiesbaden: VS Verlag für Sozialwissenschaften.

Bohnsack, Ralf; Przyborski, Aglaja; Schäffer, Burkhart (Hg.) (2010): Das Gruppendiskussionsverfahren in der Forschungspraxis. Opladen: Barbara Budrich.

Bollnow, Otto Friedrich (1958): Wesen und Wandel der Tugenden. Frankfurt a. M.: Ullstein. 
Bonafaccia, Simone (2003): Die Tugend der Toleranz. Theoretische Aspekte einer auf der Achtung vor der Menschenwürde begründeten Tugend. Diss. Univ. Tübingen, 2003. Hamburg: Kovač.

Bönsch, Manfred (2002): Erziehung fängt in der Familie an - aber die Schule kann sie nicht allein fortsetzen! In: Die Deutsche Schule 94, S. 67-78.

Bormann, Inka; Haan, Gerhard (2008): Kompetenzen der Bildung für nachhaltige Entwicklung. Operationalisierung, Messung, Rahmenbedingungen, Befunde. Wiesbaden: VS Verlag für Sozialwissenschaften.

Bourdieu, Pierre (1982): Die feinen Unterschiede. Kritik der gesellschaftlichen Urteilskraft. Frankfurt a. M.: Suhrkamp.

Brake, Anna; Bremer, Helmut (Hg.) (2010): Alltagswelt Schule. Die soziale Herstellung schulischer Wirklichkeiten. Weinheim: Juventa.

Braun, Anna Katharina; Meier, Michaela (2004): Wie Gehirne laufen lernen oder: „Früh übt sich, wer ein Meister werden will!““. In: Zeitschrift für Pädagogik 50 (4), S. 507-520.

Breidenstein, Georg; Jergus, Kerstin (2005): Schule als „Job“? Beobachtungen aus der achten Klasse. In: Georg Breidenstein und Annedore Prengel (Hg.): Schulforschung und Kindheitsforschung - ein Gegensatz? Wiesbaden: VS Verlag für Sozialwissenschaften, S. 177-198.

Brennewitz, Hedda (2010): Szenen, Gruppen, Peers. Seelze: Friedrich.

Breuer, Franz; Reichertz, Jo (2001): Wissenschafts-Kriterien: Eine Moderation [40 Absätze]. In: Forum Qualitative Sozialforschung / Forum: Qualitative Social Research 2 (3), S. Art. 24: Online verfügbar unter http://www.qualitativeresearch.net/index.php/fqs/article/view/919/2007, zuletzt geprüft am 16.01.2012.

Brumlik, Micha (2002): Bildung und Glück. Versuch einer Theorie der Tugenden. Berlin: Philo.

Brumlik, Micha (2011): Tugend. In: Hans-Uwe Otto und Hans Thiersch (Hg.): Handbuch Soziale Arbeit. 4., völlig neu bearb. München: Reinhardt.

Brumlik, Micha (Hg.) (2007): Vom Missbrauch der Disziplin. Antworten der Wissenschaft auf Bernhard Bueb. Weinheim: Beltz.

Bucher, Anton A. (2001): Was Kinder glücklich macht. Historische, psychologische und empirische Annäherungen an Kindheitsglück. Weinheim: Juventa.

Bueb, Bernhard (2006): Lob der Disziplin. Eine Streitschrift. Berlin: List.

Bütow, Birgit (2006): Mädchen in Cliquen. Sozialräumliche Konstruktionsprozesse von Geschlecht in der weiblichen Adoleszenz. Weinheim: Juventa.

Carr, David (2007): Character in Teaching. In: British Journal of Educational Studies 55 (4), S. 369-289.

Chiapparini, Emanuela (2012, im Druck): Verantwortungsvolles Lügen? Stellenwert der "Tugend" Ehrlichkeit bei AbschlussschülerInnen der Zürcher Volksschule In: Maria Carla Gadebusch Bondio und Andrea Bettels (Hg.): Im Korsett der Tugenden. Moral und Geschlecht im kulturhistorischen Kontext. Bielefeld: Transcript.

Chiapparini, Emanuela (2011): Transkription der 16 Einzelinterviews mit Schülerinnen und Schülern an der Zürcher Volksschule. Datenmaterial. Zürich: Eigendruck.

Chiapparini, Emanuela (2001): Werterziehung der gegenwärtigen Jugend? Eine Untersuchung zur theoretischen Begründung, ausgehend von der historischen und gegenwärtigen Autonomieforderung mit Schwerpunkt der Werterziehung gemäss 
dem Ansatz von R. S. Peters. Lizenziatsarbeit. Zürich: Pädagogisches Institut der Universität Zürich.

COCON (2006). Schweizer Befragung von Kindern und Jugendlichen. Erste Ergebnisse (2006): Online verfügbar unter http://www.cocon.unizh.ch/de/agenda.html, zuletzt geprüft am 16.01.2012.

COCON (2007): Erste Ergebnisse der 2. Welle des Kinder- und Jugendsurveys COCON: Jugendliche in der Schweiz - was und wer ist ihnen wichtig? Jacobs Center. Zürich: Online verfügbar unter http://www.cocon.uzh.ch/de/publikationen news.html, zuletzt geprüft am 16.01.2012.

Dalbert, Claudia; Stöber, Joachim (2008): Forschung zur Schülerpersönlichkeit. In: Werner Helsper (Hg.): Handbuch der Schulforschung. Wiesbaden: VS Verlag für Sozialwissenschaften, S. 905-925.

Davidson, Jenny (2004): Hypocrisy and the politics of politeness. Manners and morals from Locke to Austen. Cambridge: Cambridge University Press.

Davies, Ian; Gorard, Stephen; McGuinn, Nick (2005): Citizenship Education and Character Education. Similarities and Contrasts. In: British Journal of Educational Studies 53 (3), S. 341-358.

Dietenberger, Marcus (2002): Moral, Bildung, Motivation. Eine Theorie moralischer Handlungskompetenz und ihre schulpädagogischen Bezüge. Weinheim: Beltz.

Dieth, Eugen; Schmid-Cadalbert, Christian (1986): Schwyzertütschi Dialäktschrift. Dieth-Schreibung. Aarau: Sauerländer.

Dittmar, Norbert (2009): Transkription. Ein Leitfaden mit Aufgaben für Studenten, Forscher und Laien. Wiesbaden: VS Verlag für Sozialwissenschaften.

Döbrich, Peter (2009): Stundenplan. In: Sigrid Blömeke, Thorsten Bohl, Ludwig Haag, Gregor Lang-Wojtasik und Werner Sacher (Hg.): Handbuch Schule: Theorie - Organisation - Entwicklung. Bad Heilbrunn: Klinkhardt, S. 368-372.

Dopfer, Kurt (2002): Rückkehr des verlorenen Menschen. In: Ernst Fehr (Hg.): Psychologische Grundlagen der Ökonomie. Über Vernunft und Eigennutz hinaus. Zürich: Neue Zürcher Zeitung, S. 99-111.

Dresing, Thorsten; Pehl, Thorsten (2011): Praxisbuch Transkription. Regelsysteme, Software und praktische Anleitungen für qualitative ForscherInnen. Marburg: Online verfügbar unter www.audiotranskription.de/praxisbuch (Datum des Downloads: 28.06.2011), zuletzt geprüft am 16.01.2012.

Drieschner, Elmar (2007): Erziehungsziel „Selbstständigkeit“. Grundlagen, Theorien und Probleme eines Leitbildes der Pädagogik. Wiesbaden: VS Verlag für Sozialwissenschaften.

Drilling, Matthias (2008): Gewalt an Schulen. Forschungsergebnisse und Handlungskonzepte. Zürich: Verlag Pestalozzianum.

Dubs, Rolf (Hg.) (2009): Lehrerverhalten. Einen Beitrag zur Interaktion von Lehrenden und Lernenden im Unterricht. Zürich: SKV.

Dudek, Peter (2010): Geschichte der Jugend. In: Heinz-Hermann Krüger (Hg.): Handbuch Kindheits- und Jugendforschung. Wiesbaden: VS Verlag für Sozialwissenschaften, S. 333-349.

Ecarius, Jutta (2011): Jugend und Sozialisation. Wiesbaden: VS Verlag für Sozialwissenschaften.

Eisner, Manuel; Ribeaud, Denis (2007): Zur Evaluation von Gewaltpräventionsmaßnahmen. Drei Analysen zur Wirksamkeit von Interventionen. Forschungsbericht aus der Reihe z-proso. Zürich: Online verfügbar unter http://www.ife.uzh.ch 
/fckeditor_downloads/file/Zproso/04_Publikationen/02_Forschungsberichte/02_ Vollstaendige_Berichte/FB_Nr6_MEisnerDRibeaud.pdf, zueltzt geprüft am 16.01.2012.

Ekman, Paul (2009): Telling lies. Clues to deceit in the marketplace, politics, and marriage. Reissued with a new chapter. New York: W.W. Norton.

Esslinger-Hinz, Ilona (Hg.) (2008): Spannungsfelder der Erziehung und Bildung. Ein Studienbuch zu grundlegenden Themenfeldern der Pädagogik. Baltmannsweiler: Schneider.

Eykmann, Walter; Seichter, Sabine (Hg.) (2007): Pädagogische Tugenden. Winfried Böhm zum 22. März 2007. Würzburg: Königshausen \& Neumann.

f4 (2004): Kostenloses Softwareprogramm für Transkriptionen: Online verfügbar unter http://www.audiotranskription.de/f4.htm, zuletzt geprüft am 16.01.2012.

Fees, Konrad (2000): Werte und Bildung. Wertorientierung im Pluralismus als Problem für Erziehung und Unterricht. Opladen: Leske \& Budrich.

Fellsches, Josef (1996): Lebenkönnen. Von Tugendtheorie zur Lebenskunst. Essen: Die Blaue Eule.

Fend, Helmut (2005): Entwicklungspsychologie des Jugendalters. Wiesbaden: VS Verlag für Sozialwissenschaften.

Fend, Helmut (2009): Neue Theorie der Schule. Wiesbaden: VS Verlag für Sozialwissenschaften.

Ferchhoff, Wilfried (2011): Jugend und Jugendkulturen im 21. Jahrhundert. Lebensformen und Lebensstile. Wiesbaden: VS Verlag für Sozialwissenschaften.

Flick, Uwe (2008): Konstruktivismus. In: Uwe Flick, Ernst v. Kardorff und Ines Steinke (Hg.): Qualitative Forschung. Ein Handbuch. Hamburg: Rowohlt, S. 150164.

Flick, Uwe (2009): Qualitative Sozialforschung. Eine Einführung. Reinbek bei Hamburg: Rowohlt.

Gadebusch Bondio, Maria Carla; Bettels, Andrea (Hg.) (2012, im Druck): Im Korsett der Tugenden. Moral und Geschlecht im kulturhistorischen Kontext. Bielefeld: Transcript.

Gallmann, Heinz (2010): Zürichdeutsches Wörterbuch. Zürich: Neue Zürcher Zeitung.

Gamm, Hans-Jochen (1988): Pädagogische Ethik. Versuche zur Analyse der erzieherischen Verhältnisse. Weinheim: Deutscher Studien-Verlag.

Garfinkel, Harold (1967): Studies in Ethnomethodology. Reprint. Englewood Cliffs: Prentice-Hall.

Garfinkel, Harold ([1961] 1981): Das Alltagswissen über soziale und innerhalb sozialer Strukturen. In: Arbeitsgruppe Bielerfelder Soziologie (Hg.): Alltagswissen, Interaktion und gesellschaftliche Wirklichkeit. Opladen: Westdeutscher Verlag.

Geitner, Ursula (1992): Die Sprache der Verstellung. Studien zum rhetorischen und anthropologischen Wissen im 17. und 18. Jahrhundert. Tübingen: Niemeyer.

Gerecht, Marius (2010): Schul- und Unterrichtsqualität und ihre erzieherischen Wirkungen. Eine Sekundäranalyse auf der Grundlage der pädagogischen Entwicklungsbilanzen. Münster: Waxmann.

Gerster, Petra; Nürnberger, Christian (2001): Der Erziehungsnotstand. Wie wir die Zukunft unserer Kinder retten. Berlin: Rowohlt.

Gesetz über die Information und den Datenschutz (IDG) (vom 12. Februar 2007) (2011): Online verfügbar unter http://www.zh.ch/internet/de/rechtliche_grundla- 
gen /gesetze/erlass.html?Open \&Ordnr=170.4, zuletzt aktualisiert am 01.01.2011, zuletzt geprüft am 16.01.2012.

Giesecke, Hermann (2005): Wie lernt man Werte? Grundlagen der Sozialerziehung. Weinheim: Juventa.

Giesinger, Johannes (2006): Erziehung der Gehirne? Willensfreiheit, Hirnforschung und Pädagogik. In: Zeitschrift für Erziehungswissenschaft 9 (1), S. 97-109. Online verfügbar unter http://www.pedocs.de/volltexte/2011/1502/pdf/Giesinger_Erziehung_der_Gehirne_D_A.pdf, zuletzt geprüft am 16.01.2012.

Glaser, Barney G. (1978): Theoretical sensitivity. Advances in the methodology of grounded theory. Mill Valley, CA: Sociology Press.

Goffman, Erving ([1959] 1983): Wir alle spielen Theater. Die Selbstdarstellung im Alltag. München: Piper.

Graefe, Nils (2004): Wie Kinder verantwortungsvolles Lügen lernen. In: Mainpost 60 (176), 2. August 2004, S. D1.

Groeben, Annemarie von der (2000): Höflichkeit - eine vergessene Tugend? In: Pädagogik 52 (5).

Grunder, Hans-Ulrich (1999): Mogeln, Spicken, Schummeln. In: Schulaktiv 1, S. 3031.

Haan, Gerhard de (Hg.) (2007): Qualitätsrahmen Demokratiepädagogik: demokratische Handlungskompetenz fördern, demokratische Schulqualität entwickeln. Weinheim: Beltz.

Hartshorne, Hugh (1926): First steps toward a scale for measuring attitudes. In: The Journal of Educational Psychology 17 (3), S. 145-162.

Hartshorne, Hugh; May, Mark A.; Maller, Julius B. (Hg.) (1929): Studies in the Nature of Character. New York, NY: MacMillan.

Haydon, Graham (1999): Values, virtues and violence: Education and the Public Understanding of Morality. Oxford: Blackwell.

Haydon, Graham (2001): Kommunitarismus, Liberalismus und moralische Erziehung. In: Zeitschrift für Pädagogik 47 (1), S. 1-12.

Heinze, Thomas (1980): Schülertaktiken. München: Urban \& Schwarzenberg.

Heitger, Marian (2009): Tugend ist nicht lehrbar! In: P̈̈D-Forum 37/28 (2), S. 52-55.

Helfferich, Cornelia (2009): Die Qualität der qualitativen Daten. Wiesbaden: VS Verlag für Sozialwissenschaften.

Helsper, Werner (2008): Schülerbiographie und Schulkarriere. In: Werner Helsper (Hg.): Handbuch der Schulforschung: VS Verlag für Sozialwissenschaften, S. 927-944.

Helsper, Werner; Böhme, Jeanette (2010): Jugend und Schule. In: Heinz-Hermann Krüger (Hg.): Handbuch Kindheits- und Jugendforschung: VS Verlag für Sozialwissenschaften, S. 567-596.

Hillmann, Karl-Heinz (2003): Wertwandel. Ursachen, Tendenzen, Folgen. Würzburg: Carolus.

Hitzler, Roland; Niederbacher, Arne (2010): Leben in Szenen. Formen juveniler Vergemeinschaftung heute. Wiesbaden: VS Verlag für Sozialwissenschaften.

Höffe, Otfried (1997): Platon, Politeia. Berlin: Akademie-Verlag.

Höffe, Otfried (2008): Lexikon der Ethik. München: C. H. Beck.

Hopf, Christel (1978): Die Pseudo-Exploration - Überlegungen zur Technik qualitativer Interviews in der Sozialforschung. In: Zeitschrift für Soziologie 7 (1), S. 97115 . 
Horster, Detlef; Oelkers, Jürgen (Hg.) (2005): Pädagogik und Ethik. Wiesbaden: VS Verlag für Sozialwissenschaften.

Horton, John; Mendus, Susan (1994): After MacIntyre. Critical perspectives on the work of Alasdair MacIntyre. Cambridge: Polity Press.

Hoyer, Timo (2005): Tugend und Erziehung. Die Grundlegung der Moralpädagogik in der Antike. Zugl.: Kassel, Univ., Habil.-Schr., 2004/2005. Bad Heilbrunn: Klinkhardt.

Hurrelmann, Klaus (2010): Lebensphase Jugend. Eine Einführung in die sozialwissenschaftliche Jugendforschung. Weinheim, München: Juventa.

Hurrelmann, Klaus; Albert, Mathias (2006): Jugend 2006. Eine pragmatische Generation unter Druck; 15. Shell Jugendstudie. Hg. v. Deutsche Shell. Frankfurt a. M.: Fischer.

Hurrelmann, Klaus; Bründel, Heidrun (Hg.) (2003): Einführung in die Kindheitsforschung. Weinheim: Beltz.

Jannan, Mustafa (2010): Das Anti-Mobbing-Buch. Gewalt an der Schule - vorbeugen, erkennen, handeln. Weinheim: Beltz.

Jugendbarometer (2010): Die Spider-Generation setzt auf Crowds statt Clouds. Credit Suisse Jugendbarometer Schlussbericht Schweiz. Unter Mitarbeit von Golder, Lukas; Longchamp, Claude; Imfeld, Martina; Kocher, Jonas; Tschöpe, Stephan; Ratelband-Pally, Silvia; Lanz, Simone. Hg. v. Credit Suisse, gfs.bern. Credit Suisse, gfs.bern. Bern (Bulletin der Credit Suisse): Online verfügbar unter https://emagazine.credit-suisse.com/app/topic/index.cfm?fuseaction=OpenTopic\&coid=284834\&lang=DE, zuletzt geprüft am 16.01.2012.

Jünger, Rahel (2010): Schule aus der Sicht von Kindern. In: Anna Brake und Helmut Bremer (Hg.): Alltagswelt Schule. Die soziale Herstellung schulischer Wirklichkeiten. Weinheim: Juventa, S. 159-183.

Junghänel, Günter; Schuttpelz, Peter; Tackmann, Sigrid (1987): Sozialistische Moralerziehung aus philosophisch-ethischer Sicht. Berlin: Volk \& Wissen.

Kant, Immanuel ([1797] 1966): Metaphysik der Sitten. Hamburg: Meiner.

Kelle, Udo (2008): Die Integration qualitativer und quantitativer Methoden in der empirischen Sozialforschung. Theoretische Grundlagen und methodologische Konzepte. Wiesbaden: VS Verlag für Sozialwissenschaften.

Kenngott, Eva-Maria (2010): Wertebildung in der Schule: Handlungsansätze und Beispiele. In: Wilfried Schubarth, Karsten Speck und Heinz Lynen von Berg (Hg.): Wertebildung in Jugendarbeit, Schule und Kommune. Bilanz und Perspektiven. Wiesbaden: VS Verlag für Sozialwissenschaften, S. 199-209.

Kesselring, Thomas (2009): Handbuch Ethik für Pädagogen. Grundlagen und Praxis. Darmstadt: WBG.

Kleemann, Frank; Krähnke, Uwe; Matuschek, Ingo (2009): Interpretative Sozialforschung. Eine praxisorientierte Einführung. Wiesbaden: VS Verlag für Sozialwissenschaften.

Kohlberg, Lawrence; Althof, Wolfgang; Noam, Gil G.; Oser, Fritz (1995): Die Psychologie der Moralentwicklung. Frankfurt a. M.: Suhrkamp.

Krüger, Heinz-Hermann; Deppe, Ulrike (2010): Mikroprozesse sozialer Ungleichheit an der Schnittstelle von schulischen Biographien und Peerorientierung. In: Heinz-Hermann Krüger, Ursula Rabe-Kleberg, Rolf-Torste Kramer und Jürgen Budde (Hg.): Bildungsungleichheit revisited. Bildung und soziale Ungleichheit 
vom Kindergarten bis zur Hochschule. Wiesbaden: VS Verlag für Sozialwissenschaften, S. 185-201.

Krumm, Volker (2003): Wie Lehrer Schüler disziplinieren. Ein Beitrag zur „Schwarzen Pädagogik“. In: Pädagogik 55 (12), S. 30-34.

Krummenacher, Martin (2006): Lügen ist ein kreatives Umgehen von (oder mit) Fakten. Lügentypische Fehlleistungen bei der Sprachproduktion. Bern: Diss. Universität Bern.

Kruse, Jan (2009): Reader „Einführung in die Qualitative Interviewforschung“. Freiburg: Online verfügbar unter http://www.soziologie.uni-freiburg.de/kruse, zuletzt geprüft am 16.01.2012.

Kunzman, Robert (2006): The Civic (and pedagogical) Virtue of Recognizing Reasonable Disagreement. In: Theory and Research in Social Education 34 (6), S. 162-182.

Kurt, Roland (2011): Hans-Georg Soeffner: Kultur als Halt und Handlung. In: Stephan Moebius und Quadflieg Dirk (Hg.): Kultur. Theorien der Gegenwart. Wiesbaden: VS Verlag für Sozialwissenschaften, S. 228-240.

Larson-Knight, Bonnie (2004): Erziehungsziel: Respekt. Aufbau einer respektvollen Schulkultur - eine Hilfe gegen Gewalt. In: Pädagogik 56 (9), S. 36-39.

Latzko, Brigitte (2006): Werteerziehung in der Schule. Regeln und Autorität im Schulalltag. Opladen: Barbara Budrich.

Lickona, Thomas (1991): Educating for Character. How our School can teach Respect and Responsibility. New York: Bantam Books.

MacIntyre, Alasdair (1981): After Virtue. A Study in Moral Theory. London: Duckworth.

Maier, Karl Ernst (1986): Grundriss moralischer Erziehung. Bad Heilbrunn: Klinkhardt.

Mannheim, Karl (1964): Beiträge zur Theorie der Weltanschauungsinterpretation. In: Karl Mannheim und Kurt Heinrich Wolff (Hg.): Wissenssoziologie. Berlin etc.: Luchterhand, S. 91-154.

Mannheim, Karl; Kettler, David (1980): Strukturen des Denkens. Frankfurt a. M.: Suhrkamp.

Mansel, Jürgen; Hurrelmann, Klaus (2003): Jugendforschung und Sozialisationstheorie. In: Jürgen Mansel, Hartmut M. Griese und Albert Scherr (Hg.): Theoriedefizite der Jugendforschung. Standortbestimmung und Perspektiven. Weinheim: Juventa, S. 75-90.

Martin, John (1997): Inventing Sincerity, Refashioning Prudence: The Discovery of the Individual in Renaissance Europe. In: The American Historical Review 102 (5), S. 1309-1342): Online verfügbar unter http://www.jstor.org/stable/2171065, zuletzt geprüft am 16.01.2012.

MAXQDA 10 (2010). The Art of Textanalysis (Softwarprogramm): Online verfügbar unter http://www.maxqda.de/index.php/maxqda, zuletzt geprüft am 16.01.2012.

Mayring, Philipp (2008): Qualitative Inhaltsanalyse. Grundlagen und Techniken. Weinheim: Beltz.

McLaughlin, Terence H.; Halstead, Mark J. (1999): Education in Character and Virtue. In: Mark J. Halstead und Terence H. McLaughlin (Hg.): Education in Morality. London: Routledge, S. 132-163.

Mecheril, Paul (2003): Prekäre Verhältnisse. Über natio-ethno-kulturelle (Mehrfach-) Zugehörigkeit. Münster: Waxmann. 
Mey, Günter; Mruck, Katja (2007): Qualitative Interviews. In: Gabriele Naderer und Eva Balzer (Hg.): Qualitative Marktforschung in Theorie und Praxis. Grundlagen, Methoden und Anwendungen. Wiesbaden: Betriebswirtschaftlicher Verlag Dr. Th. Gabler, S. 249-278.

Miebach, Bernhard (2010): Soziologische Handlungstheorie. Eine Einführung. Wiesbaden: VS Verlag für Sozialwissenschaften.

Mienert, Malte (2008): Total diffus. Erwachsenwerden in der jugendlichen Gesellschaft. Wiesbaden: VS Verlag für Sozialwissenschaften.

Moebius, Stephan (2011): Kultur. Theorien der Gegenwart. Wiesbaden: VS Verlag für Sozialwissenschaften: Online verfügbar unter http://sfx.metabib.ch/sfx locater?sid=ALEPH:EBI01\&genre=book\&isbn=978-3-531-920566\&id=doi:10.1007/ 978-3-531-92056-6, zuletzt geprüft am 16.01.2012.

Mokrosch, Reinhold; Regenbogen, Arnim (Hg.) (2009): Werte-Erziehung und Schule. Ein Handbuch für Unterrichtende. Göttingen, Niedersachs: Vandenhoeck \& Ruprecht.

Moser, Urs; Buff, Alex; Angelone, Domenico; Hollenweger, Judith (2011): Nach sechs Jahren Primarschule. Deutsch, Mathematik und motivational-emotionales Befinden am Ende der 6. Klasse. Zürich. Online verfügbar unter http://www.ibe. uzh.ch/aktuell/Lernstandserhebung_6Klas-seZH_Bericht.pdf, zuletzt geprüft am 16.01.2012.

Münch, Paul (1984): Ordnung, Fleiß und Sparsamkeit. Texte und Dokumente zur Entstehung der „bürgerlichen Tugenden“. Originalausg. München: Deutscher Taschenbuch Verlag.

Muri, Gabriela (1999): Aufbruch ins Wunderland? Ethnographische Recherchen in Zürcher Technoszenen 1988-1998. Zürich: Volkskundliches Seminar der Universität Zürich.

National Curriculum (NC) (1999). Online verfügbar unter http://curriculum.qca.org. uk, zuletzt geprüft am 13.12.2010.

National Curriculum (NC) (2008): Online verfügbar unter http://curriculum.qcda.gov. uk/key-stages-1-and-2/aims-values-and-purposes/values/index.aspx, zuletzt geprüft am 16.01.2012.

Neuenschwander, Markus P.; Frey, Mirjam; Gerber-Schenk, Michelle; Rottermann, Benno (2010): Übergang von der Schule in den Beruf im Kanton Zürich: Herausforderungen und Erfolgsfaktoren. Schlussbericht zur Zürcher Ergänzungsstudie Berufsbildung. Hg. v. PH FHNW: Online verfügbar unter http://www.ph.fhnw.ch /ife/projekte/ifeDocus/Publication_UEbergang_von_der_Schule_in_ID1880/\%C 3\%9Cbergang\%20von $\% 20$ der $\% 20$ Schule $\% 20$ in $\% 20$ den $\% 20$ Beruf $\% 20 \mathrm{im} \% 20$ Kanton\%20Z\%C3\%BCrich.pdf, zuletzt aktualisiert am 01.09.2010, zuletzt geprüft am 16.01.2012.

Nohl, Arnd-Michael (2008): Interview und dokumentarische Methode. Anleitungen für die Forschungspraxis. Wiesbaden: VS Verlag für Sozialwissenschaften.

Nunner-Winkler, Gertrud (2005): Anerkennung moralischer Normen. In: Wilhelm Heitmeyer und Peter Imbusch (Hg.): Integrationspotenziale einer modernen Gesellschaft. Wiesbaden: VS Verlag für Sozialwissenschaften, S. 157-178.

Nunner-Winkler, Gertrud (2009): Identität und Wertbindung. In: Vierteljahrsschrift für wissenschaftliche Pädagogik 85 (1), S. 9-25. 
Nunner-Winkler, Gertrud; Meyer-Nikele, Marion; Wohlrab, Doris (2006): Integration durch Moral. Moralische Motivation und Ziviltugenden Jugendlicher. Wiesbaden: VS Verlag für Sozialwissenschaften.

Nussbaum, Martha C.; Pauer-Studer, Herlinde (1999): Gerechtigkeit oder Das gute Leben. Frankfurt a. M.: Suhrkamp.

Oelkers, Jürgen (2001): Einführung in die Theorie der Erziehung. Weinheim: Beltz.

Oelkers, Jürgen (2004): Überzogene Erwartungen, fragile Praxis. In: Marianne Horstkemper, Annette Scheunpflug, Klaus-Jürgen Tillmann und Sabine Walper (Hg.): Aufwachsen. Die Entwicklung von Kindern und Jugendlichen. Seelze: Friedrich in Velber (10), S. 120-123.

Oelkers, Jürgen (2009a): Fächerkanon und Fachunterricht. In: Sigrid Blömeke, Thorsten Bohl, Ludwig Haag, Gregor Lang-Wojtasik und Werner Sacher (Hg.): Handbuch Schule: Theorie - Organisation - Entwicklung. Bad Heilbrunn, S. 305-313.

Oelkers, Jürgen (2009b): Rhetorik und Praxis: Grenzen in der Erziehung. Kolloquium "Grenzen der Didaktik". Universität Bern, 22.05.2009: Online verfügbar unter http://paed-services.uzh.ch/ user_down loads/1012/BernGrenzen.pdf, zuletzt geprüft am 16.01.2012.

Opaschowski, Horst W. (2006): Das Moses Prinzip. Die 10 Gebote des 21. Jahrhunderts. Gütersloh: Gütersloher Verlagshaus.

Oswald, Hans (2010): Was heißt qualitativ forschen? In: Barbara Friebertshäuser, Antje Langer und Annedore Prengel (Hg.): Handbuch Qualitative Forschungsmethoden in der Erziehungswissenschaft. Weinheim: Juventa, S. 183-201.

Palentien, Christian; Hurrelmann, Klaus (1998): Veränderte Jugend - veränderte Formen der Beteiligung Jugendlicher? In: Christian Palentien; Klaus Hurrelmann (Hg.): Jugend und Politik. Ein Handbuch für Forschung, Lehre und Praxis. Berlin 1998. In: Christian Palentien und Rolf Oerter (Hg.): Jugend und Politik. Ein Handbuch für Forschung, Lehre und Praxis. Neuwied: Luchterhand, S. 11-29.

Peterson, Christopher; Seligman, Martin E.P (2004): Character Strengths and Virtues. A Handbook and Classification. Washington, DC: American Psychological Association.

Pfaff, Nicolle (2006): Jugendkultur und Politisierung. Wiesbaden: VS Verlag für Sozialwissenschaften.

Pfaff, Nicolle (2008): Zum Verhältnis von Schule und Jugendkultur: Entfaltungskontext und Gegenwelt. In: Cathleen Grunert und Heinz-Hermann Krüger (Hg.): Jugend und Bildung. Modernisierungsprozesse und Strukturwandel von Erziehung und Bildung am Beginn des 21. Jahrhunderts. Opladen: Barbara Budrich, S. 165182.

Pieper, Josef (2004): Über die Tugenden. Klugheit, Gerechtigkeit, Tapferkeit, Mass. München: Kösel.

Plato (2001): Politeia. bearb. von Dietrich Kurz ; griech. Text von Emile Chambry ; deutsche Übersetzung von Friedrich Schleiermacher. Darmstadt: Wissenschaftliche Buchgesellschaft.

Polanyi, Michael (1985): Implizites Wissen. Frankfurt a. M.: Suhrkamp.

Prange, Klaus (2010): Die Ethik der Pädagogik. Zur Normativität erzieherischen Handelns. Paderborn, Zürich: Schöningh.

Prengel, Annedore; Freibertshäuser, Barbara; Langer, Antje (2010): Perspektiven qualitativer Forschung in der Erziehungswissenschaft - eine Einführung. In: Barbara Friebertshäuser, Antje Langer und Annedore Prengel (Hg.): Handbuch Qua- 
litative Forschungsmethoden in der Erziehungswissenschaft. Weinheim: Juventa, S. 17-39.

Przyborski, Aglaja; Wohlrab-Sahr, Monika (2009): Qualitative Sozialforschung. Ein Arbeitsbuch. München: Oldenbourg.

Rabich, Adalbert (2007): Hat der Politiker die Tugend Ehrlichkeit? Die Folgen unvollständiger Information, beispielhaft in den Fachbereichen Energie-Vorrat und -Verbrauch, Geldvermögen und -menge, Arbeitsvermögen und -einsatz. Norderstedt: GRIN.

Radtke, Frank-Olaf (1979): Unterrichtsbeobachtung und Subjektivität. Vorarbeiten für ein Verfahren kommunikativer Beobachtung. In: Bärbel Schön und Klaus Hurrelmann (Hg.): Schulalltag und Empirie. Neuere Ansätze in d. schul. u. berufl. Sozialisationsforschung. Weinheim, Basel: Beltz, S. 30-51.

Reckwitz, Andreas (2003): Grundelemente einer Theorie sozialer Praktiken. In: Zeitschrift für Soziologie 32 (4), S. 282-301.

Regenbogen, Gundela (2009): Wahrhaftigkeit: Ehrlichkeit, Echtheit und Aufrichtigkeit. In: Reinhold Mokrosch und Arnim Regenbogen (Hg.): Werte-Erziehung und Schule. Ein Handbuch für Unterrichtende. Göttingen, Niedersachs: Vandenhoeck \& Ruprecht, S. 61-69.

Reichenbach, Roland (2004): Die Bildung der Lehrperson: Bemerkungen aus der pädagogischen Provinz der Gegenwart. Vortrag an der Eröffnungsfeier der Pädagogischen Hochschule Zentralschweiz/Schwyz, 15. Oktober 2004: Online verfügbar unter http://www.phz.ch/seiten/doku mente/referate/phz_reichenbach1510 2004.pdf., zuletzt geprüft am 8.08.2009.

Reichenbach, Roland (2007): Tugend, Kompetenz, Wahrheit und Humbug. In: Engagement (Zeitschrift für Erziehung und Schule) (3), S. 195-202.

Reichenbach, Roland (2008): "Von dem erlaubten moralischen Schein" - zur Bedeutung von Zivilität und Anstand. In: Ingrid Plath, Heiko Breit und Ines Graudenz (Hg.): Kultur - Handlung - Demokratie: Dreiklang des Humanen. Wiesbaden: VS Verlag für Sozialwissenschaften, S. 98-107.

Reinders, Heinz (2005a): Qualitative Interviews mit Jugendlichen führen. Ein Leitfaden. München: Oldenbourg.

Reinders, Heinz (2005b): Jugend, Werte, Zukunft. Wertvorstellungen, Zukunftsperspektiven und soziales Engagement im Jugendalter. Stuttgart: Landesstiftung Baden-Württemberg.

Rekus, Jürgen (2007): Kompetenz - ein neuer Bildungsbegriff? In: Engagement (Zeitschrift für Erziehung und Schule), S. 155-160.

Ribeaud, Denis; Eisner, Manuel (2009): Entwicklung von Gewalterfahrungen Jugendlicher im Kanton Zürich. Oberentfelden: Sauerländer Verlage AG.

Riegel, Christine; Geisen, Thomas (Hg.) (2010): Jugend, Zugehörigkeit und Migration. Subjektpositionierung im Kontext von Jugendkultur, Ethnizitäts- und Geschlechterkonstruktionen. Wiesbaden: VS Verlag für Sozialwissenschaften.

Rohlfs, Carsten; Harring, Marius; Palentien, Christian (Hg.) (2008): KompetenzBildung. Soziale, emotionale und kommunikative Kompetenzen von Kindern und Jugendlichen. Wiesbaden: VS Verlag für Sozialwissenschaften.

Rost, Detlef H.; Sparfeldt, Jörn R. (2003): Allgemeines und spezifisches Mogeln in der Schule. In: Zeitschrift für Entwicklungspsychologie und Pädagogische Psychologie 35 (2), S. 65-74. 
Roth, Heinrich (1963): „Die realistische Wendung in der pädagogischen Forschung“ Antrittsvorlesung 1962. In: Die Deutsche Schule 55, S. 109-119.

Roth, Heinrich (1971): Entwicklung und Erziehung. Grundlagen einer Entwicklungspädagogik. Hannover: Schroedel.

Roth, Lutz (1983): Die Erfindung des Jugendlichen. München: Juventa.

Rutter, Michael; Hentig, Hartmut von (1980): Fünfzehntausend Stunden. Schulen und ihre Wirkung auf die Kinder. Weinheim: Beltz.

Scherb, Armin (2004): Werteerziehung und pluralistische Demokratie. Politikdidaktische Annäherungen an ein pädagogisches Konzepte für die öffentliche Schule. Zugl. Teildr. von: Eichstätt, Kath. Univ., Habil.-Schr. u.d.T.: Scherb, Armin: Wertorientierte politische Bildung und pluralistische Gesellschaft. Frankfurt a. M.: P. Lang.

Scherr, Albert (2009): Jugendsoziologie. Einführung in Grundlagen und Theorien. Wiesbaden: VS Verlag für Sozialwissenschaften.

Schmidt, Christiane (2010): Auswertungstechniken für Leitfadeninterviews. In: Barbara Friebertshäuser, Antje Langer und Annedore Prengel (Hg.): Handbuch Qualitative Forschungsmethoden in der Erziehungswissenschaft. Weinheim: Juventa, S. 473-486.

Schmieder, Jürgen (2010): Du sollst nicht lügen! Von einem, der auszog, ehrlich zu sein. München: Bertelsmann.

Schobinger, Viktor (2010): Züritüütsch. Züri: Schobinger.

Schönborn, Gregor (2006): Unternehmen „C“" - Leadership Values - ManagerTugenden als messbarer Erfolgsfaktor. In: Bruno Klauk: Mit Werten wirtschaften - mit Trends trumpfen. Band zur 12. Tagung der Gesellschaft für Angewandte Wirtschaftspsychologie e.V. an der Business and Information Technology School (BiTS) am 03. und 04.02.2006 in Iserlohn. Hg. v. Gesellschaft für Angewandte Wirtschaftspsychologie. Lengerich: Pabst Science Publ, S. 13-43.

Schubarth, Wilfried (2010): Gewalt und Mobbing an Schulen. Möglichkeiten der Prävention und Intervention. Stuttgart: Kohlhammer.

Schubarth, Wilfried; Speck, Karsten (2008): Einstellungen, Wohlbefinden, abweichendes Verhalten von Schülerinnen und Schülern. In: Werner Helsper (Hg.): Handbuch der Schulforschung. Wiesbaden: VS Verlag für Sozialwissenschaften, S. 965-978.

Schubarth, Wilfried; Speck, Karsten; Berg, Heinz Lynen von (Hg.) (2010): Wertebildung in Jugendarbeit, Schule und Kommune. Bilanz und Perspektiven. Wiesbaden: VS Verlag für Sozialwissenschaften.

Schultheis, Franz (2008): Kindheit und Jugend in der Schweiz. Ergebnisse des Nationalen Forschungsprogramms „Kindheit, Jugend und Generationenbeziehungen im gesellschaftlichen Wandel". Weinheim: Beltz.

Schütz, Alfred ([1932] 1960): Der sinnhafte Aufbau der sozialen Welt. Eine Einleitung in die verstehende Soziologie. Wien: Springer.

Schütz, Alfred (1971-1972): Wissenschaftliche Interpretation und Alltagsverständnis menschlichen Handelns. In: Alfred Schütz (Hg.): Gesammelte Werke. Das Problem der sozialen Wirklichkeit. Den Haag: Nijhoff(I), S. 3-54.

Schütze, Fritz (1978): Die Technik des narrativen Interviews in Interaktionsfeldstudien - dargestellt an einem Projekt zur Erforschung von kommunalen Machtstrukturen. Arbeitsberichte und Forschungsmaterialien. Bielefeld: Universität Bielefeld. Fakultät für Soziologie. 
Schütze, Fritz (1987): Das narrative Interview in Interaktionsfeldstudien I. Kurseinheit 1: dreifacher Kurs. Hagen: FernUniversität. Gesamthochschule.

Schütze, Fritz; Meinefeld, Werner; Springer, Werner; Weymann, Ansgar (1973): Grundlagetheoretische Voraussetzungen methodisch kontrolliertes Fremdverstehens. In: Arbeitsgruppe Bielefelder Soziologen (Hg.): Alltagswissen, Interaktion und gesellschaftliche Wirklichkeit. Reinbek bei Hamburg: Rowohlt, S. 433-495.

Schweitzer, Friedrich; Thiersch, Hans (Hg.) (1983): Jugendzeit - Schulzeit. Von den Schwierigkeiten, die Jugendliche und Schule miteinander haben. Weinheim: Beltz.

Schweizerische Akademie der Geistes- und Sozialwissenschaften (Hg.) (2010): A Statement on the Meaning, Quality Assessment, and Teaching of Qualitative Research Methods. Unter Mitarbeit von Manfred Max Bergmann, Thomas S. Eberle, Uwe Flick und et al. Bern: Schweizerische Akademie der Geisteswissenschaften.

Seydel, Otto (2003): Sekundärtugenden in der Schule. In: Pädagogik 55 (12), S. $25-$ 29.

Silbernagel, Peter (1997): „Alle sind autonom und keiner ist zufrieden.“ Ehrlichkeit, Fairneß und Glaubwürdigkeit - drei Unbekannte in der Autonomie-Diskussion. In: Bildung aktuell 48 (1), S. 16-18.

Silverman, David (2011): Doing Qualitative Research. Issues of Theory, Method and Practice. London: Sage.

Sommerfeld-Lethen, Caroline (2005): Wie moralisch werden? Kants moralistische Ethik. Freiburg: Alber.

Spaemann, Robert (1988/1989): Disziplin und das Problem der sekundären Tugenden. In: Scheidewege 18, S. 80-91.

Stamm, Margrit (1995): „Ich muss einfach spicken, weil ich gute Noten brauche...“ Ergebnisse einer Umfrage bei aargauischen Oberstufenschülerinnen und schülern zum Thema „Spicken“. In: Schweizer Lehrerinnen- und Lehrer-Zeitung (2), S. 8-11.

Strauß, Anselm L. ([1987] 1991): Grundlagen qualitativer Sozialforschung. Datenanalyse und Theoriebildung in der empirischen soziologischen Forschung. München: Fink.

Strobel-Eisele, Gabriele; Prange, Klaus (2008): Schule und pädagogisches Handeln. In: Barbara Rendtorff und Svenia Burckhart (Hg.): Schule, Jugend und Gesellschaft. Ein Studienbuch zur Pädagogik der Sekundarschule. Stuttgart: Kohlhammer, S. 218-228.

Tajfel, Henri (1971): Social Categorization and Intergroup Behaviour. In: European Journal of Social Psychology 1 (2), S. 149-178.

Tenorth, Heinz-Elmar (2007): Beltz Lexikon Pädagogik. Weinheim: Beltz.

Terhart, Ewald (1997a): Entwicklung und Situation des qualitativen Forschungsansatzes in der Erziehungswissenschaft. In: Barbara Friebertshäuser und Annedore Prengel (Hg.): Handbuch qualitative Forschungsmethoden in der Erziehungswissenschaft. Weinheim: Juventa, S. 27-42.

Terhart, Ewald (1997b): Lehr-Lern-Methoden. Eine Einführung in Probleme der methodischen Organisation von Lehren und Lernen. Weinheim: Juventa.

Thiersch, Hans (1978): Alltagshandeln und Sozialpädagogik. In: neue praxis 8 (1), S. 6-25. 
Thole, Werner (2008): Bildung und Kindheit. Pädagogik der Frühen Kindheit in Wissenschaft und Lehre. Leverkusen: Barbara Budrich.

Thole, Werner (2009): Die Wiederentdeckung der Erziehung und ihre Infragestellung durch die Neurobiologie. In: Werner Helsper (Hg.): Schule und Bildung im Wandel. Anthologie historischer und aktueller Perspektiven. Wiesbaden: VS Verlag für Sozialwissenschaften, S. 303-316.

Tillmann, Klaus-Jürgen (2010): Sozialisationstheorien. Eine Einführung in den Zusammenhang von Gesellschaft, Institution und Subjektwerdung. Reinbek: Rowohlt.

Trautwein, Ulrich; Kropf, Michaela (2004): Das Hausaufgabenverhalten und die Hausaufgabenmotivation von Schülern. In: Psychologie in Erziehung und Unterricht 51 (4), S. 285-295.

Trilling, Lionel (1972): Sincerity and Authenticity. The Charles Eliot Norton Lectures, 1969 - 1970. Cambridge: Harvard University Press.

Trotha, Trutz von (1982): Zur Entstehung von Jugend. In: Kölner Zeitschrift für Soziologie und Sozialpsychologie 34 (2), S. 254-277.

Trusler, John (1776): The Difference, between Words, esteemed Sysnonymous, in the English Language, and the proper Coice of them determined: together with so much of Abbé Girard's treatise. Dubblin: R. Moncrieffe.

Tugendhat, Ernst (1984): Probleme der Ethik. Stuttgart: Reclam.

Uhl, Siegfried (1996): Die Mittel der Moralerziehung und ihre Wirksamkeit. Bad Heilbrunn: Klinkhardt.

Uhl, Siegfried (1998): Werte-Erziehung als Auftrag aller Fächer in der Schule: Aufgabe, Methoden, Erfolgsaussichten. In: Jörg-Dieter Gauger und Ruth DölleOelmüller (Hg.): Sinnvermittlung, Orientierung, Werte-Erziehung. Bilanz und Perspektiven des Religions-, Philosophie- und Rechtskundeunterrichts an den Schulen der Bundesrepublik Deutschland. Sinnvermittlung, Orientierung, WerteErziehung. Bilanz und Perspektiven des Religions-, Philosophie- und Rechtskundeunterrichts an den Schulen der Bundesrepublik Deutschland. Sankt Augustin: Academia, S. 148-169.

Ulich, Klaus (2008): Schulische Akteure. In: Barbara Rendtorff und Svenia Burckhart (Hg.): Schule, Jugend und Gesellschaft. Ein Studienbuch zur Pädagogik der Sekundarschule. Stuttgart: Kohlhammer, S. 203-217.

Utz, Klaus (1997): Was Sie über Lügen, Stehlen, Weglaufen, Zündeln wissen sollten. Kindliche Botschaften. In: Kindergarten heute 27 (4), S. 30-33.

Volksschulamt (2004): Die Oberstufe der Volksschule im Kanton Zürich. Hg. v. Bildungsdirektion des Kantons Zürichs: Online verfügbar unter http://www.mba.zh.ch/downloads/2_0_berufsbildung/

Sekundarschule_Unterschiede.pdf, zuletzt geprüft am 16.01.2012.

Vorlauf, Johannes (2010): Von der Tugend der Achtsamkeit. Versuch einer Annäherung an ein Ethos der Sozialen Arbeit. In: Soziale Arbeit 59 (9), S. 347-353.

Wolf. Ursula (1999): Tugend und Glück. Was Planton und Aristoteles lehren. In: Ruthard Stäblein (Hg.): Glück und Gerechtigkeit. Moral am Ende des 2. Jahrhunderts. Frankfurt a. M.: Insel-Verlag, S. 27-40.

Wagner-Willi, Monika (2010): Rituelle Praxis im Spannungsfeld zwischen schulischer Institution und Peer Group. Gruppendiskussionen mit Schülern. In: Ralf Bohnsack, Aglaja Przyborski und Burkhart Schäffer (Hg.): Das Gruppendiskussionsverfahren in der Forschungspraxis. Opladen: Barbara Budrich, S. 45-56. 
Wehr, Helmut (2008): Spannungsfeld 4. Erziehung und Bildung zwischen Fremd- und Selbstbestimmung. In: Ilona Esslinger-Hinz (Hg.): Spannungsfelder der Erziehung und Bildung. Ein Studienbuch zu grundlegenden Themenfeldern der Pädagogik. Baltmannsweiler: Schneider, S. 56-71.

Weidner, Margit (2004): Erziehungsziel: Höflichkeit. In: Pädagogik 56 (9), S. 6-38.

Wetzstein, Thomas; Erbeldinger, Patricia Isabella; Hilgers, Judith; Eckert, Roland (2005): Jugendliche Cliquen. Zur Bedeutung der Cliquen und ihrer Herkunftsund Freizeitwelten. Wiesbaden: VS Verlag für Sozialwissenschaften.

White, Patricia (1996): Civic Virtues and Public Schooling. Educating Citizens for a democratic Society. New York: Teachers College Press Columbia University.

Wickert, Ulrich (1994): Der Ehrliche ist der Dumme. Über den Verlust der Werte / ein Essay. Hamburg: Hoffmann und Campe.

Wickert, Ulrich (2007): Gauner muss man Gauner nennen. Von der Sehnsucht nach verlässlichen Werten. München: Piper.

Winkler, Michael (2010): Erziehung. In: Heinz-Hermann Krüger (Hg.): Einführung in Grundbegriffe und Grundfragen der Erziehungswissenschaft. Opladen: Barbara Budrich, S. 57-78.

Wippermann, Carsten (2007): Wie ticken Jugendliche? Sinus-Milieustudie U27. Düsseldorf: Haus Altenberg.

Witzel, Andreas (1982): Verfahren der qualitativen Sozialforschung. Überblick und Alternativen. Frankfurt a. M.: Campus.

Witzel, Andreas (1985): Das problemzentrierte Interview. In: Gerd Jüttemann (Hg.): Qualitative Forschung in der Psychologie. Grundfragen, Verfahrensweisen, Anwendungsfelder. Weinheim: Beltz, S. 227-255.

Witzel, Andreas (2000): Das problemzentrierte Interview [25 Absätze]. Forum Qualitative Sozialforschung / Forum: Qualitative Social Research, 1(1), Art. 22, http://nbn-resol-ving.de/urn:nbn:de:0114-fqs0001228, zuletzt geprüft am 16.01.2012.

Wurz, Ernst (2007): Die Jugend braucht mehr Raum und Zeit. Ein Projektbericht und Ergebnisse der Befragung: Jugendwerte - aber welche? In: Engelbert Washietl und Eva Pfisterer (Hg.): Werte - aber welche? Die Werte-Debatte. Wien: LIT, S. 65-82.

Zehetmair, Hans (1996): Auf der Grundlage gemeinsamer Werte Erziehung in demokratischer Verantwortung. In: Schulreport (1), S. 13-14.

Ziehe, Thomas (2005): Die Eigenwelt der Jugendlichen und die Anerkennungskrise der Schule. In: Detlef Horster und Jürgen Oelkers (Hg.): Pädagogik und Ethik. Wiesbaden: VS Verlag für Sozialwissenschaften, S. 277-291.

Zinnecker, Jürgen (1975): Der heimliche Lehrplan. Untersuchungen zum Schulunterricht. Weinheim: Beltz.

Zinnecker, Jürgen (1978): Die Schule als Hinterbühne oder Nachrichten aus dem Unterleben der Schüler. In: Gerd-Bodo Reinert und Jürgen Zinnecker (Hg.): Schüler im Schulbetrieb. Berichte und Bilder vom Lernalltag, von Lernpausen und vom Lernen in den Pausen. Reinbek bei Hamburg: Rowohlt, S. 29-121.

Zürcher Kantonsrat (Hg.) (2005): Volksschulgesetz: Online verfügbar unter http:// www.bista.zh.ch/pub/downloads/Volksschulgesetz_7_2_05.pdf, zuletzt geprüft am 16.01.2012. 


\section{Verzeichnis des Datenmaterials ${ }^{28}$}

Datenmaterial aus der ersten Erhebungsphase (März bis Juni 2008):

Thematischer Ablauf und Transkriptionen von acht Einzelinterviews mit Schülerinnen und Schüler (SuS) und drei Einzelinterviews mit Klassenlehrpersonen aus der 3. Oberstufenklasse (Schultyp A, B, C) einer sozial belasteten städtischen Schulgemeinde.

\section{Datenmaterial aus der zweiten Erhebungsphase (Juni/Juli 2009):}

Thematischer Ablauf und Transkriptionen von sieben Einzelinterviews mit SuS, drei Gruppendiskussionen mit insgesamt 16 SuS und zwei Einzelinterviews mit Klassenlehrpersonen aus der 3. Oberstufenklasse (Schultyp A) einer sozial belasteten Schulgemeinde in der Agglomeration der Stadt Zürich und einer sozial kaum belasteten städtischen Schulgemeinde.

Datenmaterial aus der dritten Erhebungsphase (erste Hälfte des Schuljahres 2009/2010):

Die empirischen Ergebnisse aus der vorliegenden Untersuchung basieren auf der Auswertung der Einzelinterviews mit den SuS, wozu das Datenmaterial aus Transkriptionen und Interpretationsdossier besteht:

Transkriptionen der 16 Schülerinnen und Schülern:

a) Die 16 Transkripte sind als vollständiges Datenmaterial wiedergegeben (Chiapparini 2011), worüber das Inhaltsverzeichnis einen Überblick gibt:

\begin{tabular}{|l|l|l|l|}
\hline Klasse D & Klasse E & Klasse F & Klasse G \\
\hline Lilian .......... 2 & Regula .......71 & Ilona ....... 135 & Sandra .....201 \\
Valentina ... 18 & Lara ..........86 & Claire ...... 152 & Nora .........220 \\
Adrian ....... 33 & Fabian ......105 & Christoph. 169 & Stefan .......233 \\
Clemens .... 48 & Marco .......122 & Emil ........190 & David ........244 \\
\hline
\end{tabular}

b) 16 Interpretationsdossiers zu den Einzelinterviews der SuS:

Deckblatt, Interviewtagebuch, Transkription, Notizen während der Transkription, thematischer Verlauf, formulierende und reflexive Interpretation.

28 Das Datenmaterial aus den drei Erhebungsphasen ist nach Vereinbarung einsehbar und erhältlich. 
c) Audiomaterial zu 16 Einzelinterviews (EI) mit SuS, vier Gruppendiskussionen (GD), Einzelinterviews mit vier Klassenlehrpersonen (KL) und vier Fachlehrpersonen (FL)

\section{Anhang ${ }^{29}$}

\section{A Datenblatt}

Das Datenblatt füllten die SuS selbstständig und in Anwesenheit der Forscherin aus. Folgende Themen sind darin enthalten: Vorname, Alter, Geschlecht, Sprachkompetenzen, Beruf der Eltern, Anzahl Geschwister, Vereinszugehörigkeit, finanzielle Lage, Freizeitaktivitäten, vorgesehene Berufs- und Schulausbildung, Schulleistungen, Teilnahmebereitschaft für Gruppendiskussion oder Einzelinterview während oder außerhalb der Schulzeit.

\section{B Interviewdossier und Leitfaden}

Das Interviewdossier erfasst in Form einer Checkliste die einzelne Vorbereitungs-, Erhebungs- und Nacharbeitungsschritte der Einzelinterviews, wie Interviewvereinbarung und Postskriptum. Im Dossier ist ebenso der Leitfaden enthalten, der folgende Themenkreise berücksichtigt: Verhaltensweisen in Schulstunden, Schultests schreiben, Umgang mit Unterschriften der Eltern, Klassenzusammenhalt, Gerüchte in der Klasse, direkte Frage nach dem Ehrlichkeitsverständnis.

\section{Transkriptionsregeln}

Die Interviews sind in Anlehnung an die vorgeschlagenen Transkriptionsrichtlinien von Ralf Bohnsack und Aglaja Przyborski vertextlicht (vgl. dazu Bohnsack 2008, S. 235f.). Für die vorliegende Arbeit fanden folgende Transkriptionszeichen Anwendung:

\begin{tabular}{|l|l|}
\hline Zeichen & Erklärung \\
\hline viellei- & Abbruch eines Wortes oder Satzes \\
\hline$(?)$ & Unsicherheiten bei der Transkription, schwer verständliche Äußerungen \\
\hline ja & laut (in Relation zur üblichen Lautstärke des Sprechers/der Sprecherin) \\
\hline$(2)$ & Anzahl der Sekunden, die eine Pause dauert \\
\hline I & Beginn einer Überlappung \\
\hline
\end{tabular}

29 Das Datenblatt und das Interviewdossier ist nach Vereinbarung einsehbar und erhältlich. 


\begin{tabular}{|l|l|}
\hline$\ldots . .$. & wörtliche Rede innerhalb der Aussage \\
\hline$@ j a @$ & lachend gesprochen \\
\hline$@ . @$ & kurzes Auflachen \\
\hline$@ 3 @$ & Lachen, Dauer in Sekunden \\
\hline Großschreibung & $\begin{array}{l}\text { Alle Hauptwörter werden groß geschrieben, ebenso Neuansetzungen } \\
\text { eines Sprechers oder einer Sprecherin }\end{array}$ \\
\hline Satzzeichen & Satzzeichen werden zur besseren Lesbarkeit des Textes benutzt. \\
\hline
\end{tabular}

